\title{
Álgebras de incidência hereditárias por partes
}

Marcelo Moreira da Silva

\author{
Tese apresentada \\ ao \\ Instituto de Matemática e Estatística \\ da \\ Universidade de São Paulo \\ para \\ obtenção do título \\ de \\ Doutor em Ciências
}

\author{
Programa: Matemática \\ Orientador: Prof. Dr. Eduardo do Nascimento Marcos
}

Durante o desenvolvimento deste trabalho o autor recebeu auxílio financeiro da CAPES

São Paulo, dezembro de 2016 


\section{Resumo}

MOREIRA, M. Álgebras de incidência hereditárias por partes. 2016. 273 p. Tese (Doutorado) Instituto de Matemática e Estatística, Universidade de São Paulo, São Paulo, 2016.

Apresentamos um estudo das álgebras de incidência que são hereditárias por partes, as quais denominamos Phias, piecewise hereditary incidence algebras. Através da aljava com relações, descrevemos as Phias de tipo Dynkin e introduzimos uma nova família de Phias de tipo Dynkin extendido chamada família ANS, em referência a Assem, Nehring e Skowroński. Nessa descrição, o importante método foi o dos cortes em extensões triviais, os quais inspiraram a elaboração de um programa que concebe exatamente os cortes na extensão trivial dada que resultam em álgebras de incidência. Abordamos as Phias $K \Delta$ de tipo feixes, estudando o $K \Delta$-módulo sincero canônico $M$ e a álgebra de extensão por um ponto $K \Delta[M]$. Demonstramos que se $K Q / I$ é uma álgebra sincera, quaseinclinada canônica de tipo aljava e tipo de representação infinito, então os $K Q / I$-módulos sinceros são excepcionais. Essa conclusão permite construir uma gama de Phias $K \Delta[M]$ de tipo selvagem. Exploramos as Phias simplesmente conexas, provando uma resposta positiva para o problema de Skowroński para $K \Delta$ uma Phia de tipo $\mathcal{H}$, com grafo de objetos inclinantes $\mathcal{K}_{D^{b}(\mathcal{H})}$ conexo: o grupo $H^{1}(K \Delta)$ é trivial se, e somente se, a álgebra $K \Delta$ é simplesmente conexa. Na área homológica, determinamos um limitante superior da dimensão global forte das Phias; mais ainda, ampliamos esse resultado para as álgebras sinceras provando que dada uma álgebra sincera e hereditária por partes, sua dimensão global forte é menor ou igual a três.

Palavras-chave: álgebra de incidência, álgebra hereditária por partes, simplicidade conexa, dimensão global forte. 


\section{Abstract}

MOREIRA, M. Piecewise hereditary incidence algebras. 2016. 273 p. Tese (Doutorado) - Instituto de Matemática e Estatística, Universidade de São Paulo, São Paulo, 2016.

We present a study of incidence algebras that are piecewise hereditary, which we denominate Phias. By means of the quiver with relations, we describe Phias of Dynkin type and introduce a new family of Phias of extended Dynkin type, which we call ANS family, in reference to Assem, Nehring, and Skowroński. In this description, the important method was the one of cuts on trivial extensions, inspiring the writing of a program that shows exactly the cuts on the given trivial extension that result on incidence algebras. We approach sheaves type Phias $K \Delta$, studying the canonical sincere $K \Delta$-module $M$ and the one-point extension algebra $K \Delta[M]$. We show that if $K Q / I$ is a sincere, quasi-tilted canonical algebra of quiver type and infinite representation type, then sincere $K Q / I$ modules are exceptional. This conclusion allows the construction of a wide range of Phias $K \Delta[M]$ wild type. We explore the simply conectedeness of Phias, proving a positive answer of the so called Skowroński problem for $K \Delta$ a Phia $\mathcal{H}$ type, with connected quiver of tilting objects $\mathcal{K}_{D^{b}(\mathcal{H})}$ : the group $H^{1}(K \Delta)$ is trivial if, and only if, $K \Delta$ is a simply connected algebra. On homology, we determine an upper bound for the strong global dimension of Phias; furthermore, we extend this result for sincere algebras proving that the strong global dimension of a sincere piecewise hereditary algebra is less or equal to three.

Keywords: incidence algebra, piecewise hereditary algebra, simply connected, strong global dimension. 


\section{Sumário}

Introdução

1 Conceitos iniciais $\quad 5$

2 Conexidade simples $\quad 11$

3 Dimensão global $\quad 23$

4 Dimensão global forte 29

5 Álgebra de incidência hereditária por partes de tipo aljava 33

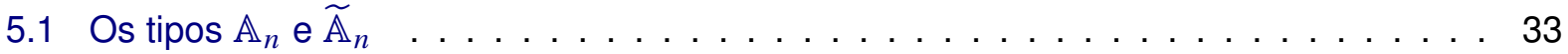

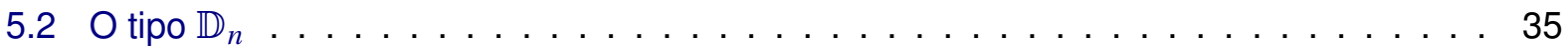

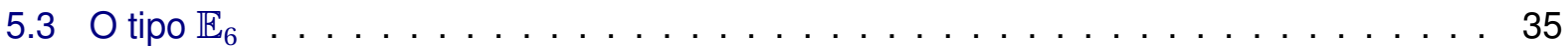

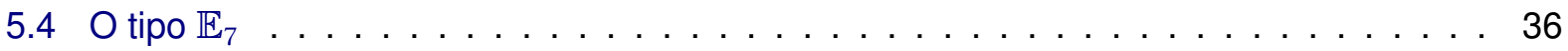

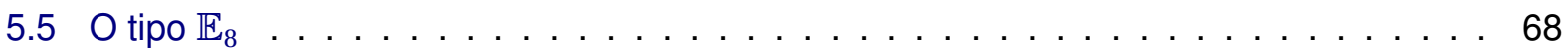

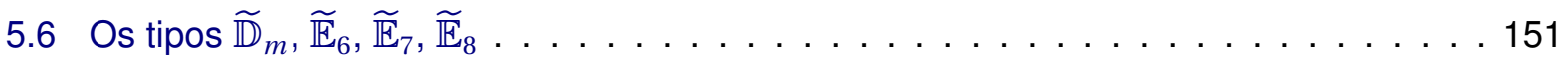

5.6.1 Álgebras minimais de tipo de representação infinito . . . . . . . . . . . . . 152

5.6.2 Família de Phias Assem-Nehring-Skowroński . . . . . . . . . . . . . . . . . . 158

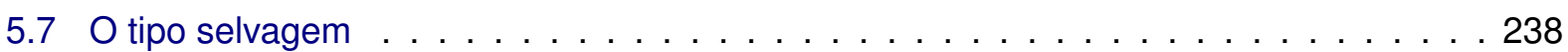

6 Álgebra de incidência hereditária por partes de tipo feixes 241

6.1 Extensão por um ponto . . . . . . . . . . . . . . . . . . . . . . . . 244

$\begin{array}{ll}\text { A Programa } & 251\end{array}$ 


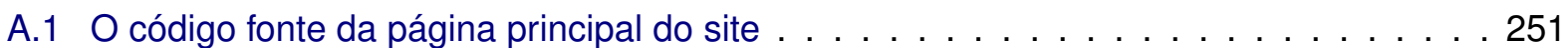

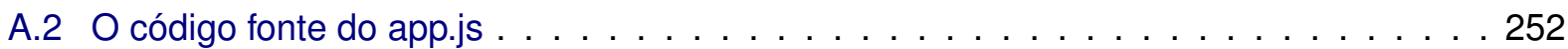

Referências Bibliográficas

Índice Remissivo 


\section{Introdução}

As álgebras de incidência foram introduzidas em meados da década de 1960 como uma maneira natural de estudar alguns problemas de combinatória. Nesse trabalho, concentramos o estudo nas álgebras associadas a um poset finito sobre um corpo algebricamente fechado. O termo poset vem do inglês, partial ordered set, e corresponde a um conjunto munido de uma ordem parcial (relação reflexiva, antissimétrica, e transitiva) entre seus elementos.

A descrição das álgebras é um dos problemas mais importantes na representação das álgebras de dimensão finita. Na década de 80, Assem e Happel começaram a caracterizar as álgebras hereditárias por partes com o trabalho "Generalized tilted algebras of type $\mathbb{A}_{n}$ ". Muitos matemáticos contribuíram na descrição das álgebras hereditárias por partes como Ringel [HR82], Keller [Kel91], Fernández [Fer99], Zacharia [HZ08], entre outros.

O propósito desta tese é o estudo das álgebras de incidência que são hereditárias por partes, as quais denominamos Phias, piecewise hereditary incidence algebras. Além disso, dadas álgebras $A$ e $B$, definimos a seguinte notação $A \cong \cong^{\prime} B$ para dizer que $A$ é derivadamente equivalente a $B$, ou seja, $\mathcal{D}^{b}(A) \cong \mathcal{D}^{b}(B)$ vistas como categorias trianguladas. Descrevemos as Phias por meio de sua aljava de relações, e nos capítulos dois, três e quatro mostramos resultados relacionados a esse assunto, destacando a simplicidade conexa e a dimensão global forte.

O tema é amplo e, portanto, nossa discussão será dividida em etapas. Para isso, utilizamos o principal teorema do artigo "A characterization of hereditary categories with tilting object" [Hap01], considerando assim, os seguintes casos:

1. $K \Delta \cong^{\prime} K Q$, em que $Q$ é uma aljava Dynkin

2. $K \Delta \cong^{\prime} K \widetilde{Q}$, em que $\widetilde{Q}$ é uma aljava Dynkin extendida

3. $K \Delta \cong^{\prime} K Q^{\prime}$, em que $Q^{\prime}$ não é uma aljava Dynkin estendida e nem Dynkin

4. $\mathcal{D}^{b}(K \Delta) \cong \mathcal{D}^{b}(\operatorname{Coh} \mathbb{X})$.

Os primeiros três itens são estudados no capítulo 5, e o último, no capítulo 6.

No capítulo 1, apresentamos alguns conceitos iniciais sobre álgebras de incidência e álgebras hereditárias por partes. 
No capítulo 2, exploramos as Phias simplesmente conexas afim de provar o seguinte problema de Skowroński:

Para quais álgebras $A$ temos $H^{1}(A)=0$ se, e somente, se $A$ é simplesmente conexa?

Demonstramos uma resposta positiva para as Phias $K \Delta \cong^{\prime} \mathcal{H}$ em que o grafo dos objetos inclinantes de $D^{b}(\mathcal{H})$ é conexo no seguinte teorema:

Teorema. Seja $K \Delta$ uma Phia tal que $K \Delta \cong^{\prime} \mathcal{H}$, com grafo de objetos inclinantes $\mathcal{K}_{D^{b}(\mathcal{H})}$ conexo. $O$ grupo $H^{1}(K \Delta)$ é trivial se, e somente se, a álgebra $K \Delta$ é simplesmente conexa.

Observamos que o teorema é válido para as Phias $K \Delta \cong^{\prime} K Q$.

O capítulo 3 é dedicado ao estudo da dimensão global das Phias. Em particular, conseguimos mostrar que as Phias de tipo de representação finito têm dimensão global menor ou igual a 2.

No século XXI, Skowroński, Happel e Zacharia introduziram uma nova propriedade homológica chamada de dimensão global forte. Um dos poucos trabalhos sobre a dimensão global forte de classes de álgebras foi do Kerner, Skowroński, Yamagata e Zacharia entitulado "Finitess of the strong global dimension of radical square zero algebras". Com esse intuito, no capítulo 4, determinamos o limitante superior da dimensão global forte das Phias. Mais ainda, ampliamos esse resultado para as álgebras sinceras com o teorema:

Teorema. Seja A uma álgebra sincera e hereditária por partes. Então, dimglf $A \leq 3$.

No capítulo 5 , tratamos o caso $K \Delta \cong^{\prime} K Q$, em que $Q$ é uma aljava Dynkin. Através da aljava com relações, descrevemos as Phias de tipo Dynkin e introduzimos uma nova família de Phias de tipo Dynkin extendido chamada família ANS, em referência a Assem, Nehring e Skowroński. Nessa descrição, o importante método foi o dos cortes admissíveis em extensões triviais, os quais inspiraram a elaboração de um programa que concebe exatamente os cortes admissíveis na extensão trivial dada que resultam em álgebras de incidência. Destacamos que estamos particularmente interessados em Phias cujas aljavas associadas admitem como grafos subjacentes diagramas que não são do tipo árvore, pois, caso contrário, a álgebra considerada será hereditária.

No capítulo 6, abordamos as Phias $K \Delta \cong$ Coh $\mathbb{X}$. Nesse contexto, estudamos o $K \Delta$-módulo sincero canônico $M$ e a álgebra de extensão por um ponto $K \Delta[M]$. Demonstramos que se $K Q / I$ é uma álgebra sincera, quase-inclinada canônica de tipo aljava e tipo de representação infinito, então os KQ/I-módulos sinceros são excepcionais. Essa conclusão permite obter uma gama de Phias $K \Delta[M]$ de tipo selvagem.

Gostaria de agradecer aos amigos Mayumi Makuta, Fernando Studzinski, Tanise Pierin, e a irmã Jacqueline Vallejo pelas correções e sugestões de todos os âmbitos da minha versão de língua 
portuguesa. Finalmente, agradeço ao meu orientador Prof. Dr. Eduardo do Nascimento Marcos pela minha formação de pesquisador e todo o trabalho da tese. 


\section{Capítulo 1}

\section{Conceitos iniciais}

Neste capítulo descreveremos algumas definições e resultados sobre as álgebras de incidência e as álgebras hereditárias por partes. A grande maioria dos conceitos da tese foram descritos na medida da necessidade.

Neste trabalho, consideraremos as álgebras $A$ de dimensão finita sobre um corpo $K$ algebricamente fechado e a categoria $\bmod A$ de módulos finitamente gerados à direita.

Há várias maneiras equivalentes de definir as álgebras de incidência. Antes disso, introduziremos o conceito de aljava.

\section{Definição 1.1 (aljava)}

Uma aljava $Q$ é uma quádrupla $\left(Q_{0}, Q_{1}, o(x), t(x)\right)$ tais que $Q_{0}$ é um conjunto cujos elementos são chamados de vértices, $Q_{1}$ é um outro conjunto em que os elementos são chamados de flechas, $o(x)$ é uma aplicação associando cada flecha a um vértice que se denominará origem da flecha e $t(x)$ é uma aplicação associando cada flecha a um vértice que se denominará término da flecha.

A aljava é finita quando os conjuntos $Q_{0}, Q_{1}$ são finitos.

Exemplo 1.2. Consideremos o exemplo: 
Assim, a aljava é $Q_{0}=\{1,2,3,4,5,6,7\}, \quad Q_{1}=$ $\left\{\alpha_{1}, \alpha_{2}, \alpha_{3}, \alpha_{4}, \alpha_{5}, \alpha_{6}\right\}$ e as aplicações são

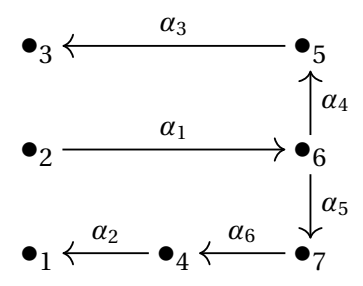

$$
\begin{aligned}
& o: Q_{1} \rightarrow Q_{0} \quad t: Q_{1} \rightarrow Q_{0} \\
& \alpha_{1} \mapsto o\left(\alpha_{1}\right)=2 \quad \alpha_{1} \mapsto t\left(\alpha_{1}\right)=6 \\
& \alpha_{2} \mapsto o\left(\alpha_{2}\right)=4 \quad \alpha_{2} \mapsto t\left(\alpha_{2}\right)=1 \\
& \alpha_{3} \mapsto o\left(\alpha_{3}\right)=5 \quad \alpha_{3} \mapsto t\left(\alpha_{3}\right)=3 \\
& \alpha_{4} \mapsto o\left(\alpha_{4}\right)=6 \quad \alpha_{4} \mapsto t\left(\alpha_{4}\right)=5 \\
& \alpha_{5} \mapsto o\left(\alpha_{5}\right)=6 \quad \alpha_{5} \mapsto t\left(\alpha_{5}\right)=7 \\
& \alpha_{6} \mapsto o\left(\alpha_{6}\right)=7 \quad \alpha_{6} \mapsto t\left(\alpha_{6}\right)=4
\end{aligned}
$$

Em seguida, definiremos caminho orientado em $Q$, que por simplicidade chamaremos apenas de caminho. Os vértices são os caminhos de comprimento zero, denotados por $e_{x}$, em que $x$ é um vértice de $Q$. As flechas são os caminhos de comprimento um. Para determinarmos os caminhos de comprimento maior ou igual a dois, precisamos definir o que é uma concatenação de duas flechas. Dadas $\lambda$ e $\beta$ flechas da aljava $Q$, a concatenação $\lambda \beta$ é um caminho de comprimento dois se $t(\lambda)=o(\beta)$. Caso contrário, não está definido um caminho. Em geral, dadas $\lambda_{1}, \cdots, \lambda_{n}$ flechas tais que $t\left(\lambda_{i}\right)=o\left(\lambda_{i+1}\right)$, para todo $i \in\{1, \cdots, n-1\}$, então $\lambda_{1} \cdots \lambda_{n}$ é um caminho de comprimento $n$ com início no vértice $o\left(\lambda_{1}\right)$ e final no vértice $t\left(\lambda_{n}\right)$. Em relação à concatenação entre vértices e flechas, se $e_{x}$ é o caminho de comprimento zero associado ao vértice $x$ e $\lambda$ é uma flecha, consideraremos a concatenação $e_{x} \lambda=\lambda$, se $o(\lambda)=x$, ou $\lambda e_{x}=\lambda$, se $t(\lambda)=x$. Assim, podemos concatenar um vértice com um caminho.

Já no exemplo anterior há um caminho de tamanho 3 começando no vértice 2 e terminando no vértice 3, representado pela sequência $\alpha_{1} \alpha_{4} \alpha_{3}$. Um caminho com o início igual ao seu término mas com tamanho maior ou igual a 1 , é chamado de ciclo. Uma aljava sem ciclos é chamada de acíclica.

Dados dois caminhos $\gamma$ e $\gamma^{\prime}$ em $Q$, dizemos que $\gamma$ e $\gamma^{\prime}$ são paralelos se eles têm a mesma origem e o mesmo término. Na próxima etapa relacionaremos uma aljava e uma álgebra sobre um corpo $K$.

\section{Definição 1.3 (álgebra de caminhos)}

Definimos a álgebra de caminhos $K Q$ como o espaço vetorial sobre um corpo $K$ que tem como base o conjunto de todos os caminhos formados na aljava $Q$, munido do produto de dois vetores $p=\beta_{1} \cdots \beta_{d}$ e $q=\lambda_{1} \cdots \lambda_{l}$ da base de $K Q$ definido por

$$
\beta_{1} \cdots \beta_{d} \lambda_{1} \cdots \lambda_{l}= \begin{cases}0 & \text { se } t\left(\beta_{d}\right) \neq o\left(\lambda_{1}\right) \\ p q & \text { se } t\left(\beta_{d}\right)=o\left(\lambda_{1}\right) .\end{cases}
$$

Exemplo 1.4. Um exemplo para entendermos melhor a álgebra de caminhos. Consideramos a aljava 
Q abaixo:

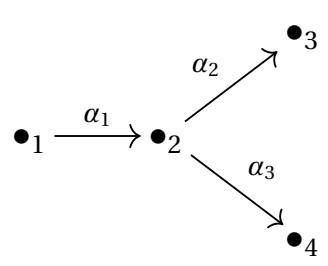

Sejam $5 \alpha_{1} \alpha_{2},-\alpha_{1}, 10 \alpha_{3}, 8 \alpha_{1} \alpha_{3}$ elementos da álgebra de caminhos $\mathbb{R} Q$. Assim, podemos fazer operações entre esses elementos como as operações a seguir:

$$
\begin{aligned}
& 5 \alpha_{1} \alpha_{2}-\alpha_{1} \\
& \left(-\alpha_{1}\right)\left(8 \alpha_{1} \alpha_{3}\right)=0 \\
& \left(-\alpha_{1}\right)\left(5 \alpha_{1} \alpha_{2}-\alpha_{1}\right)=0 \\
& \left(10 \alpha_{1}\right)\left(-\alpha_{3}\right)=-10 \alpha_{1} \alpha_{3} .
\end{aligned}
$$

Um elemento especial da álgebra de caminhos $K Q$ é o chamado relação. Dados $x, y$ vértices de uma aljava $Q$, uma relação de um vértice $x$ ao vértice $y$ é uma combinação linear $\rho=\sum_{i=1}^{m} k_{i} \gamma_{i}$, em que $k_{i} \in K$ e $\gamma_{i}$ é um caminho de no mínimo comprimento dois do vértice $x$ ao vértice $y$, para cada $1 \leq i \leq m$. O conjunto de relações em $Q$ geram um ideal $I$ na álgebra de caminhos $K Q$.

\section{Definição 1.5 (álgebra de incidência)}

Seja $(\Delta, \leq)$ um poset com $n$ elementos. A álgebra de incidência $K \Delta$ pode ser considerada como uma aljava $Q$ sem múltiplas flechas e com relações. $O$ conjunto $Q_{0}$ é formado pelos vértices associados aos pontos do poset $\Delta$ e $Q_{1}$ é formado pelas flechas $\alpha$ de um vértice $a$ a um vértice $b$, tais que $a \leq b$ e não existe a relação $a \leq c \leq b$, com $c \neq a$ e $c \neq b$.

Seja I o ideal gerado por todas as relações de comutatividade $\gamma-\gamma^{\prime}$, com $\gamma$ e $\gamma^{\prime}$ caminhos paralelos. A álgebra de incidência $K \Delta$ é $K Q / I$.

llustramos abaixo alguns exemplos de álgebras de incidência. A linha pontilhada denota uma relação. Neste primeiro, as linhas pontilhadas nas diagonais dos quadrados estão indicando as relações de comutatividade.

Exemplo 1.6.

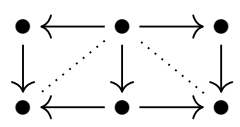

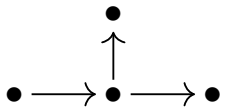

A próxima aljava possui duas relações, uma delas não é de comutatividade. Dessa forma, ela não está associada a nenhuma álgebra de incidência.

Exemplo 1.7.

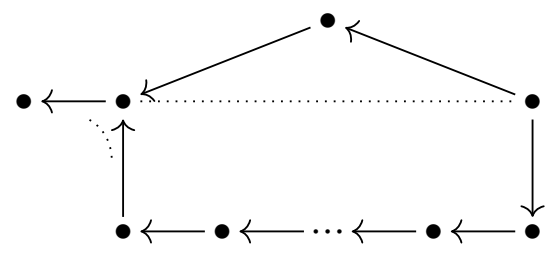


Outro exemplo de aljava que não está associada a uma álgebra de incidência:

Exemplo 1.8. $1 \longrightarrow 2 \longrightarrow \cdots \longrightarrow n-1 \longrightarrow n$ Supomos que esta aljava seja associada a uma $K \Delta$, pela existência dessa relação representada pela linha pontilhada, o elemento 1 não se relaciona com o elemento $n$. Isso contradiz a propriedade de transitividade do poset $\Delta$, consequentemente esse diagrama não pode representar uma álgebra de incidência.

Seja $\mathcal{A}$ uma $K$-categoria abeliana em que $K$ é um corpo, ou seja, $\mathcal{A}$ é uma categoria que satisfaz as seguintes condições:

- para cada par de objetos $X$ e $Y$ de $\mathcal{A}$, o conjunto dos morfismos $\operatorname{Hom}_{\mathcal{A}}(X, Y)$ tem uma estrutura de grupo abeliano;

- a composição dos morfismos é bilinear sobre $K$;

- existe o objeto zero;

- existe o produto e o coproduto finito dos objetos;

- cada morfismo $X \stackrel{f}{\rightarrow} Y$ admite um núcleo Nuc $f \stackrel{u}{\rightarrow} X$ e um conúcleo $Y \stackrel{p}{\rightarrow}$ CoNuc $f$;

- todo monomorfismo é um núcleo de um morfismo e todo epimorfismo é um conúcleo de um morfismo,

para mais detalhes recomendamos ver o livro do Freyd [Fre64].

No que segue, começamos uma sucinta revisão sobre a categoria derivada de $\mathcal{A}$. Primeiramente, lembramos que a categoria $\mathcal{K}(\mathcal{A})$ é o quociente da categoria dos complexos de $\mathcal{A}$ sobre o seu ideal em que é formado pelos morfismos homotópicos a zero.

\section{Definição 1.9}

Um morfismo $\phi: X \rightarrow Y$ entre complexos é homotópico a zero se existem morfismos

$$
\rho^{n}: X^{n} \rightarrow Y^{n-1}
$$

para todo $n \in \mathbb{Z}$ tal que

$$
\phi^{n}=d_{Y}^{n-1} \rho^{n}+\rho^{n+1} d_{X}^{n}
$$

em que, os morfismos $d^{i}$ são chamados de diferenciais e são associados aos complexos indicados.

Assim, a categoria derivada $\mathcal{D}(\mathcal{A})$ é obtida de $\mathcal{K}(\mathcal{A})$ via localização, com respeito ao conjunto de quase-isomorfismos, ou seja, dos morfismos $f: X \rightarrow Y$ da categoria dos complexos sobre $\mathcal{A}$, em que os morfismos induzidos pelas cohomologias $H^{i}(f): H^{i}(X) \rightarrow H^{i}(Y)$ são isomorfismos, para cada número inteiro $i$. Recordamos que a cohomologia $H^{i}$ de um complexo $X$ é $\operatorname{Nuc} d_{X}^{i} / \operatorname{Im} d_{X}^{i-1}$. 
No decorrer do texto, quando estivermos nos referindo a uma categoria derivada limitada da categoria $\bmod A$ de uma $K$-álgebra $A$, denotaremos por $\mathcal{D}^{b}(A)$. Além disso, assumimos que $\mathcal{A}$ é uma $K$-categoria abeliana, esqueleticamente pequena, conexa e que é Ext finita, ou seja, todos os espaços de morfismos e os espaços de extensões Ext $^{i}$ tenham dimensões finita sobre $K$.

Antes de definir uma categoria hereditária por partes, chamamos uma categoria abeliana $\mathcal{H}$ de hereditária se as extensões $\operatorname{Ext}_{\mathcal{H}}^{n}(X, Y)=0$ para $n \geq 2$ e quaisquer objetos $X$ e $Y$ de $\mathcal{H}$.

\section{Definição 1.10 (categoria hereditária por partes)}

A categoria abeliana $\mathcal{A}$ é hereditária por partes de tipo $\mathcal{H}$ se existir uma categoria abeliana hereditária $\mathcal{H}$ com um objeto inclinante tal que $\mathcal{D}^{b}(\mathcal{A})$ e $\mathcal{D}^{b}(\mathcal{H})$ são equivalentes como categorias trianguladas.

Quando dizemos que uma álgebra $A$ é hereditária por partes, queremos dizer que a categoria $\bmod A$ é hereditária por partes. Além disso, existe um objeto $T$ em $\mathcal{H}$ chamado de inclinante tal que $A=\left(\operatorname{End}_{\mathcal{H}} T\right)^{o p}$. A definição de objeto inclinante é a seguinte:

\section{Definição 1.11 (objeto inclinante)}

Seja $\mathcal{H}$ uma $K$-categoria abeliana, hereditária e Ext-finita. Dizemos que $T \in \mathcal{H}$ é um objeto inclinante de $\mathcal{H}$ se

i) $\operatorname{Ext}_{\mathcal{H}}^{1}(T, T)=0, e$

ii) para todo $X \in \mathcal{H}$ a condição $\operatorname{Ext}_{\mathcal{H}}^{1}(T, X)=0=\operatorname{Hom}_{\mathcal{H}}(T, X)$ implica que $X=0$.

Inspirado na definição de módulo inclinante.

\section{Definição 1.12 (módulo inclinante)}

Seja $A$ uma álgebra de dimensão finita sobre um corpo $K$. Dizemos que $T \in \bmod A$ é um módulo inclinante se satisfaz as seguintes condições:

i) $\operatorname{Ext}^{1}(T, T)=0$,

ii) a dimensão projetiva de $T$ é menor ou igual a $1, e$

iii) existe a sequência exata $0 \rightarrow A \rightarrow T^{1} \rightarrow T^{2} \rightarrow 0$ em que $T^{1}, T^{2} \in$ add $T$.

Enunciamos adiante um dos mais importantes teoremas sobre as álgebras hereditárias por partes.

Teorema 1.13 (Happel [Hap01]). Seja $\mathcal{H}$ uma $K$-categoria abeliana, hereditária, conexa e com objeto inclinante. Então $\mathcal{H}$ é derivadamente equivalente a $\bmod K Q$ ou derivadamente equivalente a Coh $\mathbb{X}$ para alguma reta projetiva com pesos $\mathbb{X}$.

Consequentemente, denominamos as álgebras $A \cong^{\prime} K Q$ de álgebras hereditárias por partes de tipo aljava ou de tipo $Q$. Como também, as álgebras $A \cong{ }^{\prime} \operatorname{Coh} \mathbb{X}$ chamamos de álgebras hereditárias por partes de tipo feixes. 
A fim de estudarmos as Phias de tipo aljava basta-nos caracterizar as álgebras de incidência inclinadas iteradas de tipo Q, uma vez que, segundo Happel, Rickard e Schofield ([HRS88]), o seguinte teorema pode ser obtido:

Teorema 1.14 (Happel-Rickard-Schofield). Sejam A uma $K$-álgebra de dimensão finita e $Q$ uma aljava finita sem ciclos orientados. Então A é hereditária por partes de tipo $Q$ se, e somente se, $A$ for uma álgebra inclinada iterada de tipo $Q$.

Recordamos que uma álgebra $A$ é dita inclinada iterada de tipo $Q$ se existirem uma sequência de álgebras $A=A_{0}, A_{1}, \ldots, A_{n}$, em que $A_{n}$ é a álgebra de caminhos da aljava $Q$, e uma sequência de módulos inclinantes $T_{A_{i}}^{i}$, para $0 \leq i<n$, tal que $A_{i+1}=\operatorname{End}\left(T_{A_{i}}^{i}\right)$, e se todo $A_{i}$-módulo indecomponível $M$ satisfizer $\operatorname{Hom}_{A_{i}}\left(T^{i}, M\right)=0$ ou $\operatorname{Ext}_{A_{i}}^{1}\left(T^{i}, M\right)=0$. 


\section{Capítulo 2}

\section{Conexidade simples}

A simplicidade conexa é uma característica de objeto que podemos encontrar em várias áreas na matemática, como na topologia, na geometria, etc. No caso da teoria de representação de álgebras de dimensão finita sobre um corpo algebricamente fechado, Gabriel e Bongartz, no trabalho "Covering spaces in representation theory", introduziram as álgebras simplesmente conexas para o caso de tipo de representação finito. Nós, em particular para as álgebras de incidência, iremos apresentar vários conceitos interligados com a simplicidade conexa que descreveremos nesse capítulo.

Gabriel e Bongartz definiram o conceito de álgebra simplesmente conexa usando a teoria de recobrimento universal. Inicialmente iremos definir a álgebra simplesmente conexa usando os grupos fundamentais das apresentações dessa álgebra.

Sejam $Q$ uma aljava conexa e sem ciclos orientados, e $I$ um ideal da álgebra de caminhos $K Q$ gerado por relações. Uma relação $\rho=\sum_{i=1}^{m} \lambda_{i} w_{i}$ é chamada de minimal se $m \geq 2$ e, para todo subconjunto $J$ próprio dos índices $\{1,2, \ldots, m\}$ temos que $\sum_{i \in J} \lambda_{i} w_{i}$ não é uma relação.

Dada uma flecha $\alpha$ de $x$ para $y$ em $Q$, denotamos por $\alpha^{-1}$ sua inversa formal, cuja origem é $y$ e cujo término é $x$.

Um passeio $\omega$ em $Q$ do vértice $x$ ao vértice $y$ é o produto $\alpha_{1}^{\epsilon_{1}} \alpha_{2}^{\epsilon_{2}} \ldots \alpha_{t}^{\epsilon_{t}}$ em que $\alpha_{i}$ são as flechas de $Q$ e $\epsilon_{i} \in\{-1,1\}$ para todo $i$. Convencionamos que o passeio trivial em $x \in Q_{0}$ é o caminho trivial em $x$ denotado por $e_{x}$. Um passeio que começa em $x$ e termina em $x$ é chamado de passeio cíclico ou passeio fechado.

Se $\omega_{1}$ é um passeio em $Q$ de $x$ para $y$ e $\omega_{2}$ é um passeio em $Q$ de $y$ para $z$, então $\omega_{1} \omega_{2}$ é um passeio em $Q$ de $x$ para $z$, denominado o produto dos passeios $\omega_{1}$ e $\omega_{2}$. Seja $\Omega(Q)$ o conjunto dos passeios de $Q$.

A relação de homotopia é a menor equivalência no conjunto de todos os passeios em $Q$ tais que:

(i) para cada flecha $x \stackrel{\alpha}{\rightarrow} y$, temos $\alpha \alpha^{-1} \sim e_{x}$ e $\alpha^{-1} \alpha \sim e_{y}$ 
(ii) para cada relação minimal $\sum \lambda_{i} w_{i}$, temos $w_{i} \sim w_{j}$ para todo $i, j$.

(iii) Se $\omega, \gamma, \omega_{1}, \omega_{2}$ são passeios em $Q$ tais que $\omega \sim \gamma$, então $\omega_{1} \omega \omega_{2} \sim \omega_{1} \gamma \omega_{2}$, sempre que tais produtos estiverem definidos.

Iremos usar essa relação de homotopia em $Q$ para definir grupo fundamental de uma álgebra $K Q / I$.

\section{Definição 2.1 (grupo fundamental)}

Sejam Q uma aljava conexa e sem ciclos orientados, e I ideal da álgebra de caminhos KQ gerado por relações.

O conjunto de todas as classes de equivalência de passeios cíclico num vértice base fixado $x_{0}$ é um grupo. Como não depende da escolha do vértice base, esse grupo chamamos de grupo fundamental de $(Q, I)$ e denotamos por $\pi_{1}(Q, I)$.

Pela definição acima, o grupo depende da apresentação da álgebra. Mas no caso das álgebras de incidência, é independente da apresentação. Isso foi demonstrado por Bardzell e Marcos no artigo " $H^{1}(\Lambda)$ and presentations of finite dimensional algebras".

Abaixo apresentaremos um exemplo de como calcular o grupo fundamental de uma álgebra de incidência.

Exemplo 2.2. Temos a relação minimal $\alpha_{2} \alpha_{4}-\alpha_{3} \alpha_{5}$ na aljava abaixo, então $\alpha_{2} \alpha_{4} \sim \alpha_{3} \alpha_{5}$. Assim, o passeio cíclico $\alpha_{2} \alpha_{4} \alpha_{5}^{-1} \alpha_{3}^{-1}$ é homotópico a $\alpha_{3} \alpha_{5} \alpha_{5}^{-1} \alpha_{3}^{-1} \sim e_{x_{0}}$.

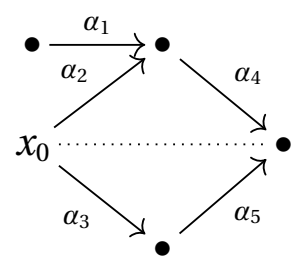

O passeio cíclico $\alpha_{2} \alpha_{1}^{-1} \alpha_{1} \alpha_{2}^{-1}$ também é homotópico a $e_{x_{0}}$, pela relação abaixo:

$$
\alpha_{2} \alpha_{1}^{-1} \alpha_{1} \alpha_{2}^{-1} \sim \alpha_{2} \alpha_{2}^{-1} \sim e_{x_{0}}
$$

Portanto, qualquer outro passeio cíclico vai ser homotópico à $e_{x_{0}}$. Implicando que o grupo fundamental dessa álgebra é trivial.

Dependendo da apresentação, o cálculo do grupo fundamental de uma álgebra pode ser bem difícil. Reynaud descreveu um algoritmo para essa tarefa no trabalho "Incidence algebras and algebraic fundamental group". Existe outras abordagens desse grupo, uma delas está no artigo do Farkas, Green e Marcos entitulado "Diagonalizable derivations of finite-dimensional algebras II".

Em "On some class of simply connected algebras", Assem e Skowroński descreveram as álgebras simplesmente conexas para as quais as suas aljavas não tem ciclos orientados. 


\section{Definição 2.3 (simplesmente conexa)}

Seja $A=K Q / I$ uma álgebra em que a aljava $Q$ não tem ciclos orientados. Chamamos a álgebra $A$ de simplesmente conexa, se para qualquer apresentação $\left(Q_{A}, I\right)$ de $A$, o grupo $\pi_{1}\left(Q_{A}, I\right)$ é o trivial.

Para as álgebras de incidência, nas quais o grupo fundamental não depende da apresentação, temos o exemplo acima de uma álgebra simplesmente conexa. Bustamante descreveu uma condição suficiente para que uma álgebra de incidência seja simplesmente conexa.

Teorema 2.4 (Bustamante [Bus02]). Seja $(\Delta, \leq)$ um poset finito. Se existe um elemento minimal ou maximal $x_{0}$ em $\Delta$, então

(i) álgebra de incidência $K \Delta$ é simplesmente conexa.

(ii) $H^{i}(K \Delta)=0$ para todo $i \geq 1$.

No resultado acima, observamos que a cohomologia de Hochschild foi abordada. Seja $A$ uma álgebra de dimensão finita sobre um corpo algebricamente fechado $K$, os grupos $H^{i}(A, A)=H^{i}(A)$ para $i \geq 0$ foram introduzidas por Hoschschild no trabalho "On the cohomology groups of an associative algebra" de 1946 e no qual a abordagem era sobre um anel comutativo. Ele obteve informações sobre a estrutura da álgebra $A$ através desses grupos serem triviais, por exemplo demonstrando que a álgebra é separável se, e somente se, os seus grupos de cohomologia de Hochschild são triviais. Inicialmente na apresentação desses grupos de cohomologia de $A$, precisamos saber a definição dos complexos associados à álgebra $A$. O complexo de Hochschild $C^{\bullet}=\left(C^{i}, d^{i}\right)_{i \in \mathbb{Z}}$ associado tem as seguintes informações:

- para $i<0, C^{i}=0$ e $d^{i}=0$;

- para $i=0$, definimos $C^{0}=A \mathrm{e}$

$$
d^{0}: A \rightarrow \operatorname{Hom}_{K}(A, A) \operatorname{com}\left(d^{0} x\right)(a)=a x-x a \text { para } x, a \in A
$$

- para $i>0, C^{i}=\operatorname{Hom}_{K}\left(A^{\otimes i}, A\right)$ em que denotamos $A^{\otimes i}$ por $i$-iésimo produto tensorial sobre $K$ de $A$ com ele mesmo e $d^{i}: C^{i} \rightarrow C^{i+1}$ com

$$
\begin{aligned}
\left(d^{i} f\right)\left(a_{1} \otimes \ldots \otimes a_{i+1}\right) & =a_{1} f\left(a_{2} \otimes \ldots \otimes a_{i+1}\right) \\
& +\sum_{j=1}^{i}(-1)^{j} f\left(a_{1} \otimes \ldots \otimes a_{j} a_{j+1} \otimes \ldots \otimes a_{i+1}\right) \\
& +(-1)^{i+1} f\left(a_{1} \otimes \ldots \otimes a_{i}\right) a_{i+1}
\end{aligned}
$$

para $f \in C^{i}$ e $a_{1}, \ldots, a_{i+1} \in A$. 
Assim, definimos $H^{i}(A)=H^{i}\left(C^{\bullet}\right)=\operatorname{Nuc} d^{i} / \operatorname{Im} d^{i-1}$ como o $i$-ésimo grupo de cohomologia de Hochschild de $A$ com coeficientes em $A$.

Vejamos algumas interpretações dos grupos $H^{0}(A)$ e $H^{1}(A) . \bigcirc H^{0}(A)=A^{A}=\{x \in A \mid a x=$ $x a \forall a \in A\}$ coincide com o centro de $A$. Seja $\operatorname{Der}(A)=\{\delta \in \operatorname{End}(A) \mid \delta(a b)=a \delta(b)+\delta(a) b\}$ o espaço vetorial sobre $K$ das derivações de $A$ sobre $A$. Seja $\operatorname{Der}^{\circ}(A)=\left\{\delta_{x} \in \operatorname{End}(A) \mid \delta_{x}(a)=\right.$ $a x-x a, x \in A\}$ o subespaço das $K$-derivações internas. Segue imediatamente da definição que $H^{1}(A)=\operatorname{Der}(A) / \operatorname{Der}^{\circ}(A)$.

Existe uma relação entre essa cohomologia e a simplicidade conexa de uma álgebra. Em 1993, no artigo "Simply connected algebras and Hochschild cohomologies", Skowroński propôs o seguinte problema:

Para quais álgebras $A$ temos $H^{1}(A)=0$ se, e somente se, $A$ é simplesmente conexa?

Ela foi respondida positivamente em vários tipos de álgebras. Vejamos um histórico desse assunto:

- Em 1986, Assem e Skowroński mostraram que as álgebras derivadamente equivalentes as álgebras canônicas mansas são simplesmente conexas e as álgebras hereditárias por partes de tipo $\widetilde{\mathbb{A}}_{n}$ não são simplesmente conexas, no artigo "On some classes of simply connected algebras".

- Em 1989, Happel mostrou no artigo "Hochschild cohomology of finite-dimensional algebras" que o problema é válido para as álgebras de representação dirigida.

- Em 1997, no artigo "Strongly simply connected Auslander algebras", Assem e Brown exibiram a validade para as álgebras auslander de álgebras de tipo de representação finito sobre um corpo algebricamente fechado de característica zero.

- Em 1998, no trabalho "Hochschild cohomology of piecewise hereditary algebras", Happel demonstrou que as álgebras de tipo de representação finito e hereditárias por partes de tipo aljava são simplesmente conexas se, e somente se, a aljava é de tipo árvore. Mais ainda, se as álgebras de tipo de representação finito e hereditárias por partes de tipo feixes não domésticos então essas álgebras são simplesmente conexas.

- Em 2001, surgiu outra afirmação positiva para as álgebras inclinadas mansas. Assem, Marcos e de la Peña demonstraram no artigo "The simple connectedness of a tame tilted algebra".

- Em 2002, no trabalho "Universal Galois Coverings of Self-Injective Algebras by Repetitive Algebras and Hochschild Cohomology", Redondo mostrou o resultado para as classes de álgebras autoinjetivas. 
- Em 2002, Assem, Coelho e Trepode asseguraram essa equivalência para as álgebras mansas quase-inclinadas em "Simply connected tame quasi-tilted algebras".

- Em 2004, Buchweitz e Liu provaram que o problema tem a resposta positiva para as álgebras de tipo de representação finito em "Hochschild Cohomology and Representation-finite Algebras".

- Em 2004, Assem e Lanzilotta mostraram a validade da equivalência para as álgebras mansas weakly shod no artigo "The simple connectedness of a tame weakly shod algebra".

- Em 2009, no artigo "The first Hochschild cohomology group of a schurian cluster-tilted algebra", Assem e Redondo demonstraram a validade do problema para as álgebras cluster inclinadas schurian.

- Em 2010, Le Meur assegurou o resultado para as álgebras derivadamente equivalente as álgebras hereditárias no trabalho "Topological invariants of piecewise hereditary algebras" e também no mesmo ano, para as álgebras weakly shod no artigo "Galois coverings of weakly shod algebras".

- Em 2013, no trabalho "Coverings of laura algebras: the standard case", Assem, Bustamante e Le Meur verificaram a validade do problema para as álgebras laura no caso standard.

Uma das principais perguntas no nosso trabalho é se a equivalência é válida para as Phias:

Seja $K \Delta$ uma Phia. $H^{1}(K \Delta)=0$ se, e somente se, $K \Delta$ é simplesmente conexa.

A implicação $K \Delta$ é simplesmente conexa então $H^{1}(K \Delta)=0$ já foi provada e para as álgebras de incidência em geral. No artigo "On The first Hochschild cohomology group of an algebra", De la Peña e Saorín mostraram o resultado abaixo:

Teorema 2.5 (De la Peña, Saorín [DS01]). Sejam $K \Delta$ uma álgebra de incidência e $\left(Q, I_{v}\right)$ uma apresentação de $K \Delta$. Então

$$
\operatorname{Hom}\left(\pi_{1}\left(Q, I_{v}\right), K^{+}\right) \cong H^{1}(K \Delta)
$$

Como a álgebra de incidência tem o seu grupo fundamental invariante por apresentação e com acréscimo de que a álgebra de incidência é simplesmente conexa, de posse desse teorema, obtemos $H^{1}(K \Delta)$ trivial.

Por outro lado, na implicação "se $H^{1}(K \Delta)=0$ então $K \Delta$ é simplesmente conexa" é importante o acréscimo da hipótese hereditária por partes na álgebra de incidência, isto é, $K \Delta$ ser Phia. Podemos ver no exemplo do plano projetivo, cuja a triangulação tem o complexo simplicial com o grupo fundamental isomorfo ao $\mathbb{Z}_{2}$ para um corpo de característica diferente de 2 . Sabendo que o grupo 
fundamental do complexo simplicial é isomorfo ao grupo fundamental do poset $\Delta$ associado a esse complexo, aplicando o teorema anterior:

$$
\operatorname{Hom}\left(\mathbb{Z}_{2}, K^{+}\right) \cong H^{1}(K \Delta) .
$$

Portanto, $K \Delta$ não é simplesmente conexa mas $H^{1}(K \Delta)=0$.

Seja $K \Delta$ uma Phia, começaremos o caminho para a demonstração em que "se $H^{1}(K \Delta)=0$ então $K \Delta$ é simplesmente conexa". O guia foi o artigo "Topological invariants of piecewise hereditary algebras" [Le 11] do Le Meur. Ele utilizou a seguinte equivalência de álgebras simplesmente conexas advinda do artigo "Algebras of polynomial growth" do Skowroński: seja $A$ uma álgebra triangular e conexa. Então $A$ é simplesmente conexa se, e somente se, não admite um recobrimento de Galois próprio. Em seguida iremos inserir os conceitos básicos sobre o recobrimento de Galois.

Vamos definir o funtor de recobrimento entre $K$-categorias. Uma $K$-categoria $\mathcal{C}$ é uma categoria que para quaisquer objetos $x$ e $y$ de $\mathcal{C}$, o conjunto $\operatorname{Hom}_{\mathcal{C}}(x, y)$ é um $K$-espaço vetorial e a composição de morfismos em $\mathcal{C}$ é $K$-bilinear. Uma categoria se chama pequena se os objetos formam um conjunto. É conveniente considerarmos uma álgebra $A=K Q / I$ como uma $K$-categoria em que os objetos são os vértices $Q_{0}$ e os morfismos são $\operatorname{Hom}_{A}\left(e_{i} A, e_{j} A\right)$ tais que $i, j \in Q_{0}$. Esse é um exemplo de $K$-categoria pequena.

\section{Definição 2.6 (categoria localmente limitada)}

Seja $\mathcal{C}$ uma $K$-categoria. Dizemos que $\mathcal{C}$ é localmente limitada se as seguintes propriedades são satisfeitas:

i) objetos distintos não são isomorfos;

ii) para cada objeto $x$ de $\mathcal{C}$, a $K$-álgebra $\operatorname{End}_{A}(x)$ é local;

iii) $\bigoplus_{y \in \mathcal{C}_{0}} \operatorname{Hom}_{\mathcal{C}}(x, y)$ tem dimensão finita para qualquer $x \in \mathcal{C}_{0}$;

iv) $\underset{y \in \mathcal{C}_{0}}{\bigoplus} \operatorname{Hom}_{\mathcal{C}}(y, x)$ tem dimensão finita para qualquer $x \in \mathcal{C}_{0}$.

Bongartz e Gabriel introduziram o recobrimento de Galois no artigo "Covering spaces in representation theory"[GB82] de 1982. Estudaram aplicações nas aljavas de Auslander-Reiten e relações com o grupo fundamental.

\section{Definição 2.7 (funtor de recobrimento)}

Um funtor de recobrimento é um funtor $K$-linear $F: \mathcal{C} \rightarrow \mathcal{C}^{\prime}$ entre $K$-categorias verificando as propriedades abaixo:

i) $F^{-1}(y) \neq \varnothing$ para todo objeto $y$ de $\mathcal{C}^{\prime}$;

ii) para quaisquer objetos $y_{0}, y_{1}$ de $\mathcal{C}^{\prime}$ e para todos objetos $x_{0}, x_{1}$ de $\mathcal{C}$ tais que $F\left(x_{0}\right)=y_{0} e$ $F\left(x_{1}\right)=y_{1}$, as aplicações induzidas por $F$ são as bijeções:

$\bigoplus_{x \in F^{-1}\left(y_{0}\right)} \operatorname{Hom}_{\mathcal{C}}\left(x, x_{1}\right) \rightarrow \operatorname{Hom}_{\mathcal{C}^{\prime}}\left(y_{0}, y_{1}\right) \quad \bigoplus_{x \in F^{-1}\left(y_{1}\right)} \operatorname{Hom}_{\mathcal{C}}\left(x_{0}, x\right) \rightarrow \operatorname{Hom}_{\mathcal{C}^{\prime}}\left(y_{0}, y_{1}\right)$ 
Para definirmos o recobrimento de Galois precisamos de dois elementos: um funtor de recobrimento e um grupo. Como seria a relação do grupo com o funtor? Quais exigências precisa ter o grupo?

Usaremos a linguagem da representação de álgebra, onde os conceitos se aplicam totalmente em categorias. Começaremos com o grupo de automorfismos Aut $A$ de uma álgebra $A=K Q / I$. Denotamos por $\operatorname{Aut}(Q, I)$ o grupo de simetrias de $Q$ fixando $I$, ou seja, $g: K Q \rightarrow K Q$ tal que $g(I)=I$. Mais ainda, o grupo $\operatorname{Aut}(Q, I)$ é um subgrupo de Aut $A$.

Outra noção da teoria de grupo é a órbita de um elemento. Dado $i \in Q_{0}$, consideramos $G i$ a órbita de $i$, ou seja, $G i$ é uma classe de equivalência, com respeito a relação $i^{\prime} \sim i$ se existe $g \in G$ tal que $i^{\prime}=g i$.

Seja $G$ um subgrupo de $\operatorname{Aut}(Q, I)$ então $G$ age em $\bmod A$ da seguinte forma: dados $X \epsilon$ $\bmod A$ e $g \in G$, então $X^{g} \in \bmod A$ tais que $i \stackrel{\alpha}{\rightarrow} j$, temos que $X^{g}(i)=X(g i) \stackrel{X(g \alpha)}{\longrightarrow} X(g j)$. Similarmente, para $f \in \operatorname{Hom}_{A}(X, Y)$, definimos $f^{g} \in \operatorname{Hom}_{A}\left(X^{g}, Y^{g}\right)$.

Dizemos que $G$ age livremente em $A$ se $g i=i$ para algum $i \in Q_{0}$ implica que $g=1$.

\section{Definição 2.8 (recobrimento de Galois)}

Seja $F: A \rightarrow A^{\prime}$ um funtor de recobrimento $K$-linear em que $A=K Q / I$. Chamamos $F$ de recobrimento galosiano de grupo $G$ se $F g=F$ e $G$ age livremente em $A$ tal que existe um diagrama comutativo de $K$-categorias:

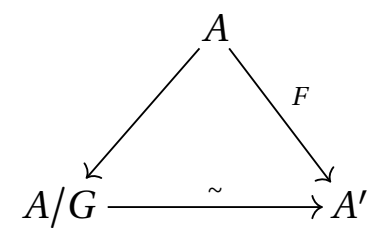

A $K$-categoria $A / G$ é formada pelas $G$-órbitas dos vértices $Q_{0}$ e para qualquer $a=G i$ e $b=G j$ tem-se que

$$
\operatorname{Hom}_{A / G}(a, b)=\bigoplus_{g \in G} \operatorname{Hom}_{A}(i, g j)
$$

Exemplo 2.9. Vejamos um exemplo de recobrimento de Galois com o grupo $\mathbb{Z}_{2}$. Sejam $Q$ e $Q^{\prime}$ as seguintes aljavas:
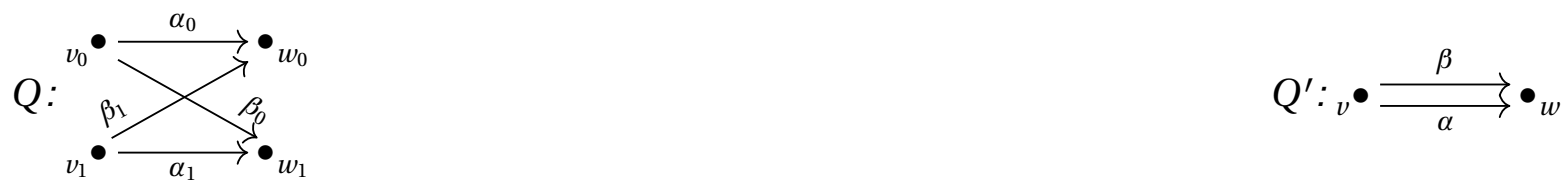

Consideramos o recobrimento de Galois de grupo $\mathbb{Z}_{2}=\{\overline{0}, \overline{1}\}$ abaixo: 


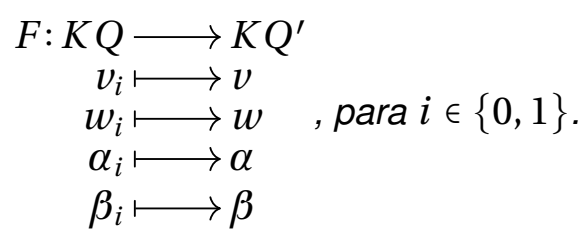

Agora, faltam dois conceitos relacionados com o recobrimento de Galois: recobrimento de Galois conexo e funtor push-down.

Uma $K$-categoria $\mathcal{C}$ é chamada de $K$-categoria conexa se, e somente se, não existe uma partição $E \sqcup F$ não trivial nos objetos tais que $\operatorname{Hom}_{\mathcal{C}}(x, y)=0$ e $\operatorname{Hom}_{\mathcal{C}}(y, x)=0$ para $x \in E$ e $y \in F$. Portanto, um recobrimento de Galois $F: A \rightarrow A^{\prime}$ é conexo quando $A$ e $A^{\prime}$ são conexas e localmente limitadas.

Dado uma $K$-categoria $A$, consideramos um $A$-módulo à direita $M$ por um funtor $K$-linear $M: A^{o p} \rightarrow$ MOD $K$, em que MOD $K$ é a categoria dos $K$-espaços vetoriais. Denominaremos a categoria dos $A$-módulos por MOD $A$. Seja $F: A \rightarrow A^{\prime}$ um recobrimento de Galois de $K$-categorias definida por ação de um grupo $G$. O recobrimento define o funtor exato $F_{\lambda}$ push-down. Nos objetos de MOD $A$ têm a seguinte associação:

$$
\begin{aligned}
F_{\lambda}: \operatorname{MOD} A & \longrightarrow \operatorname{MOD} A^{\prime} \\
(M: A \rightarrow \bmod K) & \longmapsto F_{\lambda}(M)(a)=\bigoplus_{x \in F^{-1}(a)} M(x)
\end{aligned}
$$

Falta determinar como é $F_{\lambda}(M)$ nos morfismos em MOD $A^{\prime}$. Seja $\alpha: a \rightarrow b$ em MOD $A^{\prime}$. Por $F$ ser $G$-invariante, para cada par $(x, y)$ de $F^{-1}(a) \times F^{-1}(b)$, existe um único $\alpha_{y, x} \in \operatorname{Hom}_{A}(x, y)$ tal que $\sum_{y \in F^{-1}(b)} F\left(\alpha_{y, x}\right)$ é igual a $\alpha$, para todo $x \in F^{-1}(a)$. Observando que $M\left(\alpha_{y, x}\right)$ é uma aplicação linear de $M(x)$ a $M(y)$, estabelece

$$
F_{\lambda}(M)(\alpha)=\left(M\left(\alpha_{y, x}\right)\right)_{(y, x) \in F^{-1}(b) \times F^{-1}(a)}: \bigoplus_{x \in F^{-1}(a)} M(x) \rightarrow \bigoplus_{y \in F^{-1}(b)} M(y)
$$

Nos morfismos de MOD $A, F_{\lambda}$ tem a definição abaixo:

$$
\begin{aligned}
F_{\lambda}: \operatorname{MOD} A & \longrightarrow \operatorname{MOD} A^{\prime} \\
(f: M \rightarrow N) & \longmapsto\left(F_{\lambda}(f)(a)=\operatorname{diag}\left\{f(x) \mid x \in F^{-1}(a)\right\}: \bigoplus_{x \in F^{-1}(a)} M(x) \rightarrow \bigoplus_{x \in F^{-1}(a)} N(x)\right)
\end{aligned}
$$

para cada $a \in A^{\prime}$, obtemos um morfismo $F_{\lambda}(f): F_{\lambda} M \rightarrow F_{\lambda} N$ em MOD $A^{\prime}$.

Dos resultados de Le Meur, precisaremos da construção de um recobrimento de Galois e sua relação com as equivalências derivadas. Nesse processo, utiliza o fato de que o grafo dos objetos inclinantes (definida a seguir) é conexa.

Definição 2.10 (grafo inclinante)

O grafo dos objetos inclinantes $\mathcal{K}_{\mathcal{H}}$ de uma categoria $\mathcal{H}$ tem os seus vértices associados com 
as isoclasses dos objetos inclinantes. Sejam $T, T^{\prime}$ objetos inclinantes não isomorfos, existe uma flecha $T \rightarrow T^{\prime}$ se $T=U \oplus X, T^{\prime}=U \oplus Y$, em que $X, Y$ são indecomponíveis não isomorfos, e existe a seguinte sequência exata curta:

$$
0 \longrightarrow X \longrightarrow \tilde{U} \longrightarrow Y \longrightarrow 0
$$

$\operatorname{com} \tilde{U} \in \operatorname{add} U$

No enunciado a seguir, observamos a existência da hipótese de que o grafo $\mathcal{K}_{D^{b}(\mathcal{H})}$ é conexo. Quase todas as categorias hereditárias $\mathcal{H}$ têm o grafo inclinante $\mathcal{K}_{D^{b}(\mathcal{H})}$ conexo ([HU05], [BMR $\left.{ }^{+} 06\right]$, $[B K L 10])$. Apenas o caso da categoria $\operatorname{Coh}(\mathbb{X})$ de tipo selvagem que é um problema em aberto [BKL10].

Teorema 2.11. Seja $K \Delta$ uma Phia tal que $K \Delta \cong^{\prime} \mathcal{H}$. Se $\mathcal{K}_{D^{b}(\mathcal{H})}$ é conexo e $H^{1}(K \Delta)=0$, então a álgebra $K \Delta$ é simplesmente conexa.

Demonstração A estratégia da prova é a construção de um recobrimento de Galois de $B=$ End $_{D^{b}(K \Delta)} T$ a partir de um recobrimento de Galois de $D^{b}(K \Delta)$, em que $T$ é objeto inclinante. Essa construção vai partir de um objeto inclinante de $D^{b}(K \Delta)$. Mostraremos que esse objeto inclinante de $D^{b}(K \Delta)$ tem umas propriedades especiais que chamaremos de galoisianas. Mais ainda, a conexidade do grafo de inclinantes de $D^{b}(\mathcal{H})$ vai ser importante para demonstrar que todos os objetos inclinantes de $D^{b}(K \Delta)$ têm essas propriedades especiais.

O primeiro passo é considerarmos um recobrimento de Galois qualquer $F: \mathcal{C} \rightarrow K \Delta$ com grupo $G$, e $\mathcal{C}$ localmente limitado e de dimensão global finita. A $G$-ação em $\bmod \mathcal{C}$ naturalmente define uma $G$-ação em $D^{b}(\bmod \mathcal{C})$, em que usaremos a mesma notação. O funtor push-down $F_{\lambda}$ induz um funtor exato $F_{\lambda}: D^{b}(\bmod \mathcal{C}) \rightarrow D^{b}(K \Delta)$ pela próxima proposição.

Proposição 2.12 (Le Meur [Le 11]). Existe um funtor exato $F_{\lambda}: D^{b}(\bmod \mathcal{C}) \rightarrow D^{b}(K \Delta)$ tal que o seguinte diagrama comuta:

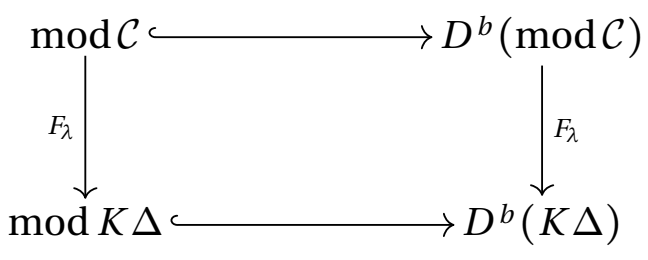

O funtor $F_{\lambda}: D^{b}(\bmod \mathcal{C}) \rightarrow D^{b}(K \Delta)$ é $G$-invariante e as seguintes aplicações são bijeções lineares para todo $X, Y$ objetos de $D^{b}(\bmod \mathcal{C})$ :

$$
\begin{aligned}
& \bigoplus_{g \in G} \operatorname{Hom}_{D^{b}(\bmod \mathcal{C})}\left(X^{g}, Y\right) \rightarrow \operatorname{Hom}_{D^{b}(K \Delta)}\left(F_{\lambda} X, F_{\lambda} Y\right) \\
& \bigoplus_{g \in G} \operatorname{Hom}_{D^{b}(\bmod \mathcal{C})}\left(X, Y^{g}\right) \rightarrow \operatorname{Hom}_{D^{b}(K \Delta)}\left(F_{\lambda} X, F_{\lambda} Y\right)
\end{aligned}
$$


Denominamos de propriedades galosianas sobre um objeto inclinante $T \in D^{b}(K \Delta)$ as seguintes condições:

$\left(H_{1}\right)$ para todo somando direto indecomponível $X$ de $T$, existe $\widetilde{X} \in D^{b}(\bmod \mathcal{C})$ tal que $F_{\lambda} \widetilde{X} \cong X$ em $D^{b}(K \Delta)$;

$\left(H_{2}\right) \quad \widetilde{X} \nsucceq g \widetilde{X}$ para todo somando direto indecomponível $X$ de $T$ e $g \in G \backslash\{1\}$;

$\left(H_{3}\right)$ Se $\psi: K \Delta \rightarrow K \Delta$ é um automorfismo tal que $\psi(x)=x$ para todo objeto $x$, então $\psi_{\lambda} X \cong$ $X$ em $D^{b}(K \Delta)$ para todo somando direto indecomponível $X$ de $T$.

Supondo que $K \Delta \cong^{\prime} \mathcal{H}$ e $\mathcal{K}_{D^{b}(\mathcal{H})}$ é conexo, o próximo resultado prova que qualquer objeto inclinante de $D^{b}(K \Delta)$ tem a propriedade galosiana $H_{1}$.

Proposição 2.13 (Le Meur [Le 11]). Seja $A \cong^{\prime} \mathcal{H}$ tal que $\mathcal{K}_{D^{b}(\mathcal{H})}$ é conexo. Fixado um recobrimento de Galois $F: \mathcal{C} \rightarrow$ A com grupo $G$ e $\mathcal{C}$ localmente limitado. Seja $T \in D^{b}(A)$ um objeto inclinante. Então:

a) para todo somando direto indecomponível $X$ de $T$, existe $\widetilde{X} \in D^{b}(\bmod \mathcal{C})$ tal que $F_{\lambda} \widetilde{X} \cong X$ em $D^{b}(K \Delta)$; Mais ainda, a classe

$$
\left\{\widetilde{X} \in D^{b}(\bmod \mathcal{C}) \mid F_{\lambda} \widetilde{X} \text { é um somando direto indecomponível de } T\right\}
$$

satisfaz as seguintes condições:

b) gera a categoria triangulada $D^{b}(\bmod \mathcal{C})$;

c) estável sobre a ação de $G$;

d) $\operatorname{Hom}_{D^{b}(\bmod \mathcal{C})}\left(\widetilde{X},{ }^{g} \widetilde{Y}[i]\right)=0$ para todo $\widetilde{X}, \widetilde{Y}$ da classe, em que $i \neq 0$ e $g \in G$.

Observamos que a proposição anterior prova que a classe

$$
\left\{\widetilde{X} \in D^{b}(\bmod \mathcal{C}) \mid F_{\lambda} \widetilde{X} \text { é um somando direto indecomponível de } T\right\}
$$

satisfaz várias condições importantes.

Agora, Le Meur prova que a propriedade galosiana $\mathrm{H}_{2}$ é satisfeita para qualquer objeto inclinante $T$ de $D^{b}(K \Delta)$.

Proposição 2.14 (Le Meur [Le 11]). Seja $A \cong \cong^{\prime} \mathcal{H}$ tal que $\mathcal{K}_{D^{b}(\mathcal{H})}$ é conexo. Fixado um recobrimento de Galois $F: \mathcal{C} \rightarrow$ A com grupo $G$ e $\mathcal{C}$ localmente limitado. Seja $X$ um somando direto indecomponível de um objeto inclinante $T \in D^{b}(A)$. Assumimos que $T$ tem a propriedade galosiana $H_{1}$. Então

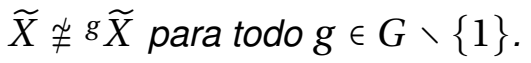

Por último, o resultado a seguir mostra que qualquer objeto inclinante $T$ de $D^{b}(K \Delta)$ tem a propriedade galosiana $\mathrm{H}_{3}$. 
Proposição 2.15 (Le Meur [Le 11]). Seja $A \cong^{\prime} \mathcal{H}$ tal que $\mathcal{K}_{D^{b}(\mathcal{H})}$ é conexo. Fixado um recobrimento de Galois $F: \mathcal{C} \rightarrow$ A com grupo $G$ e $\mathcal{C}$ localmente limitado. Seja $T \in D^{b}(A)$ um objeto inclinante.

Se $\psi: K \Delta \rightarrow K \Delta$ um automorfismo tal que $\psi(x)=x$ para todo objeto $x$, então $\psi_{\lambda} X \cong X$ em $D^{b}(K \Delta)$ para todo somando direto indecomponível $X$ de $T$.

Em seguida, Le Meur constrói um recobrimento de Galois para $B=\operatorname{End}_{D^{b}(K \Delta)} T$ com o mesmo grupo $G$ do recobrimento de Galois de $K \Delta$.

Lema 2.16 (Le Meur [Le 11]). Seja $A \cong \cong^{\prime} \mathcal{H}$ tal que $\mathcal{K}_{D^{b}(\mathcal{H})}$ é conexo. Fixado um recobrimento de Galois $F: \mathcal{C} \rightarrow A$ com grupo $G$ e $\mathcal{C}$ localmente limitado. Sejam $T \in D^{b}(A)$ um objeto inclinante e $B=\operatorname{End}_{D^{b}(A)} T$ em que $T=T_{1} \oplus \ldots \oplus T_{n}$ uma decomposição indecomponível.

Considere o isomorfismo $\lambda_{i}: F_{\lambda} \widetilde{T}_{i} \rightarrow T_{i}$ em que $\widetilde{T}_{i} \in D^{b}(\bmod \mathcal{C})$ é indecomponível para todo $i$. Assim, define um complexo limitado de $\mathcal{C}$-módulos $\widetilde{T}:=\bigoplus_{i, g}^{g} \widetilde{T}_{i}$ em que $g \in G$ e $i \in\{1, \ldots, n\}$.

Seja $\mathcal{C}^{\prime}$ a subcategoria plena de $D^{b}(\bmod \mathcal{C})$ em que os objetos são ${ }^{g} \widetilde{T}_{i}$ (para $g \in G$ e $i \in$ $\{1, \ldots, n\})$.

Então o funtor triangulado $F_{\lambda}: D^{b}(\bmod \mathcal{C}) \rightarrow D^{b}(A)$ induz um recobrimento conexo de Galois com grupo $G$ :

$$
\begin{aligned}
& F_{\widetilde{T}, \lambda}: \mathcal{C}^{\prime} \longrightarrow B \\
&{ }^{g} \widetilde{T}_{i} \longmapsto T_{i} \\
&{ }^{g} \widetilde{T}_{i} \stackrel{{ }^{u}}{\rightarrow}{ }^{h} \widetilde{T}_{j} \longmapsto T_{i} \stackrel{\lambda_{j} F_{\lambda}(u) \lambda_{i}^{-1}}{\longrightarrow} T_{j},
\end{aligned}
$$

Finalmente, temos o resultado relacionando os recobrimentos universais de $K \Delta$ com a álgebra $B=\operatorname{End}_{D^{b}(K \Delta)} T$.

Proposição 2.17 (Le Meur [Le 11]). Seja $A \cong \mathcal{H}$ tal que $\mathcal{K}_{D^{b}(\mathcal{H})}$ é conexo. Assumimos que $A$ admite um recobrimento universal $\widetilde{F}: \widetilde{\mathcal{C}} \rightarrow A$ e consideramos $T$ um objeto inclinante de $D^{b}(A)$. Então $\operatorname{End}_{D^{b}(A)} T$ admite um recobrimento universal com grupo isomorfo ao grupo de $\widetilde{F}$.

Agora podemos concluir o nosso teorema dividindo em dois casos dependendo do tipo da categoria abeliana hereditária $\mathcal{H}$.

Supomos que $\mathcal{H}=\bmod K Q$ em que $Q$ é uma aljava finita, conexa e sem ciclos orientados. Por $K \Delta \cong \mathcal{H}$, existe um objeto inclinante $T$ de $D^{b}(K \Delta)$ tal que $\mathcal{H}=\operatorname{End}_{D^{b}(K \Delta)} T$. Pela proposição 2.17, a álgebra $K Q$ admite um recobrimento universal de Galois com grupo isomorfo ao grupo fundamental de $K \Delta$. Como $H^{1}(K \Delta)=H^{1}(K Q)=0$, então $K Q$ é tipo árvore e simplesmente conexa. Logo o grupo fundamental de $K \Delta$ é o trivial como queríamos demonstrar.

O outro caso é quando $\mathcal{H}$ não tem objetos projetivos não nulos. Portanto, existe uma álgebra squid $S$ tal que $\mathcal{H} \cong S$. Consequentemente, existe um objeto inclinante $T$ de $D^{b}(K \Delta)$ tal que 
$S=\operatorname{End}_{D^{b}(K \Delta)} T$. Pela proposição 2.17, a álgebra $S$ admite um recobrimento universal de Galois com grupo isomorfo ao grupo fundamental de $K \Delta$. Pelo artigo do Le Meur [Le 11], $H^{1}(K \Delta)=$ $H^{1}(S)=0$, então $S$ é simplesmente conexa. Concluímos que o grupo fundamental de $K \Delta$ é o trivial. 


\section{Capítulo 3}

\section{Dimensão global}

A dimensão global da categoria abeliana $\mathcal{A}$ é por definição o supremo das dimensões projetivas de todos objetos $M$, em que:

$$
\operatorname{dp}_{\mathcal{A}} M=\sup \left\{d \in \mathbb{N} / \operatorname{Ext}_{\mathcal{A}}^{d}\left(M, M^{\prime}\right) \neq 0 \text { para algum } M^{\prime}\right\}
$$

Alguns dos pioneiros na apresentação dessa "medida" foram Cartan e Eilenberg no livro "Homological Algebra", de 1956.

Quando referimos à dimensão global das álgebras $A$, estamos considerando a dimensão global da categoria $\bmod A$. Dessa forma, considerando as álgebras hereditárias por partes, foi descoberto que a dimensão global dessas álgebras são finitas. Uma das exigências é que a categoria seja de comprimento finito, ou seja, cada objeto tem uma série de composição. Esse resultado aparece no artigo de Happel, Reiten e Smalø([HRS96]) descrito abaixo:

Teorema 3.1 (Happel-Reiten-Smalø[HRS96]). Seja $\mathcal{A}$ uma categoria abeliana com comprimento finito, hereditária por partes e com $n$ objetos simples não isomorfos. Então essa categoria possui dimensão global menor ou igual à $n$.

Uma pergunta interessante é se haveria um limitante superior especial para as álgebras de incidência hereditárias por partes, as Phias (Piecewise Hereditary Incidence Algebra). Encontramos a resposta positiva no artigo "Bounds on the global dimension of certain piecewise hereditary categories" [Lad08a] do Ladkani. Melhor, ele obteve outro limitante superior para a dimensão global das álgebras hereditárias por partes. Antes de enunciarmos esse teorema, alguns conceitos são necessários. Seja $G(\mathcal{A})$ um grafo dirigido cujos vértices correspondem às classes de isomorfismo de indecomponíveis de $\mathcal{A}$, e tal que existe a flecha $M \longrightarrow M^{\prime}$ entre os vértices $M$ e $M^{\prime}$, se $\operatorname{Hom}_{\mathcal{A}}\left(M, M^{\prime}\right) \neq 0$. Nesse grafo, um passeio entre dois vértices é uma sequência $\epsilon=\left(\epsilon_{0}, \ldots, \epsilon_{r-1}\right)$, em $\{-1,1\}^{r}$ e $r$ é um inteiro maior ou igual a um. Um $\epsilon$-caminho de $M$ para 
$M^{\prime}$ é uma sequência de vértices $M_{0}=M, M_{1}, \ldots, M_{r}=M^{\prime}$ tal que

$$
\begin{gathered}
M_{i} \longrightarrow M_{i+1} \text { em } G(\mathcal{A}) \text { se } \epsilon_{i}=1, \mathrm{e} \\
M_{i+1} \longrightarrow M_{i} \text { em } G(\mathcal{A}) \text { se } \epsilon_{i}=-1
\end{gathered}
$$

Teorema 3.2 (Ladkani [Lad08a]). Seja $\mathcal{A}$ uma categoria hereditária por partes de comprimento finito. Assumimos que existem $r \geq 1, \epsilon \in\{-1,1\}^{r}$ e um indecomponível $M_{0}$ tais que, para qualquer indecomponível $M$, exista um $\epsilon$-caminho de $M_{0}$ a $M$. Então

$$
\operatorname{dimgl} \mathcal{A} \leq r+1 \text { e } \operatorname{dp}_{\mathcal{A}} M+\operatorname{di}_{\mathcal{A}} M \leq r+2 \text {, para todo indecomponível } M .
$$

Observamos que a hipótese hereditária por partes é necessária, por exemplo, o resultado não vale para a classe das álgebras locais que, em geral, têm dimensão global infinita.

Uma das consequências do teorema diz respeito às chamadas álgebras sinceras. Lembramos que uma álgebra é dita sincera se admitir um indecomponível sincero em sua categoria de módulos, isto é, um indecomponível tal que todos os módulos simples ocorrem como fatores de sua série de composição. Um exemplo de álgebra sincera é a álgebra de incidência.

Proposição 3.3. Seja $K \Delta=K Q_{K \Delta} / I$ uma álgebra de incidência. Então $K \Delta$ é uma álgebra sincera.

Demonstração Precisamos mostrar a existência de um módulo $M$ indecomponível sincero sobre $K \Delta$. O candidato $M$ é a seguinte representação de módulo:

a) para cada vértice $a$ de $Q_{K \Delta}$ associamos $K$;

b) para cada flecha $\alpha: a \rightarrow b$ de $Q_{K \Delta}$ associamos a identidade $1: K \rightarrow K$.

Primeiro, mostraremos que $M$ é indecomponível. Para isso, estudaremos o End $M$. Consideramos $f=\left(f_{a}\right)_{a \in Q_{0}}$ um morfismo não nulo de End $M$. Assim, existe $f_{a}: K \rightarrow K$ não nulo para algum $a \in Q_{0}$, implicando que $f_{a}$ é um isomorfismo. Dado uma flecha $\alpha: a \rightarrow b$, temos que $f_{a} 1=1 f_{b}$. Caso a flecha é na outra direção, obtemos o mesmo resultado. Dessa forma, não importando a direção da flecha concluímos que $f_{a}=f_{b}$. Logo, como o grafo é conexo, temos sempre um passeio ligando o vértice $a$ a qualquer vértice $c$ :

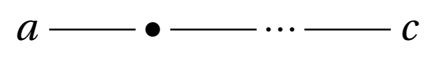

Por um processo finito, concluímos que $f_{a}=f_{c}$ para todo vértice $c$ de $Q_{K \Delta}$ e consequentemente End $M \cong K$. Então $M$ é um módulo indecomponível.

O próximo passo é mostrar que $M$ é um módulo sincero. Uma definição equivalente de um módulo sincero é satisfazer $\operatorname{Hom}_{K \Delta}(K \Delta e, M) \neq 0$ para todo idempotente $e$ da álgebra $K \Delta$. $\mathrm{O}$ 
nosso candidato $M$ claramente satisfaz essa condição:

$$
\operatorname{Hom}_{K \Delta}(K \Delta e, M) \cong M e \neq 0 \quad \text { para todo idempotente } e \text {. }
$$

Portanto, $M$ é um módulo sincero indecomponível sobre a álgebra de incidência $K \Delta$, denominamos esse módulo de sincero canônico. Assim, a álgebra de incidência é uma álgebra sincera.

Confirmamos que as Phias são sinceras, portanto o resultado abaixo é aplicável a elas.

Corolário 3.4 (Ladkani [Lad08a]). Seja A uma álgebra de dimensão finita, hereditária por partes e sincera. Então dimgl $A \leq 3$ e dp $M+\operatorname{di} M \leq 4$, para qualquer indecomponível $M \operatorname{de} \bmod A$.

As Phias de tipo de representação finito têm um limitante menor ainda, a dimensão global delas são menores ou iguais a dois. Antes de provar essa afirmação, precisaremos da definição de módulo dirigido.

\section{Definição 3.5 (módulo dirigido)}

Considere abaixo um ciclo na categoria de módulos:

$$
M_{0} \stackrel{f_{1}}{\rightarrow} M_{1} \stackrel{f_{2}}{\rightarrow} M_{2} \stackrel{f_{3}}{\rightarrow} \ldots \rightarrow M_{t-1} \stackrel{f_{t}}{\rightarrow} M_{t} \cong M_{0}
$$

em que $f_{i}$ são morfismos não nulos entre indecomponíveis e não são isomorfismos, com $1 \leq i \leq$ $t$.

Seja $M$ um módulo indecomponível, dizemos que $M$ é dirigido se não pertence a nenhum ciclo.

Happel mostrou no artigo "On the derived category of a finite-dimensional algebra" [Hap87] que uma álgebra $A$ de representação finita e hereditária por partes tem $\bmod A$ dirigida, quer dizer, todos os $A$-módulos são dirigidos. Para uma álgebra $A$ dirigida, tome um indecomponível $M$ de mod $A$, se existir um não-isomorfismo $M \stackrel{f}{\rightarrow} M$ não nulo então $M$ pertence a um ciclo. Isso contradiz o fato de que $A$ é dirigida. Assim, observamos que End $M$ é isomorfa a $K$ para qualquer $A$-módulo $M$ indecomponível.

Agora, usando o teorema de Ringel [Rin84] abaixo:

Teorema 3.6 (Ringel [Rin84]). Seja A uma álgebra tendo um módulo indecomponível sincero e dirigido. Então $A$ é uma álgebra inclinada.

Obtemos o próximo resultado:

Proposição 3.7. Seja A uma álgebra phia de representação finita. Então A é uma álgebra inclinada, consequentemente tem $\operatorname{dimgl} A \leq 2$. 
Demonstração Para usar o teorema do Ringel, precisamos mostrar que a álgebra phia $A$ tem um módulo indecomponível sincero e dirigido. Sabemos que $A$ é uma álgebra sincera, então possui um indecomponível sincero. Como $A$ é uma álgebra phia de representação finita, esse indecomponível sincero é dirigido, graças ao resultado do Happel.

Chegamos a conclusão que $A$ é uma álgebra inclinada. A dimensão global dessa álgebra é menor ou igual a dois, consequência do teorema 5.2 do artigo "Tilted algebras" [HR82].

Outra ligação que podemos fazer com a dimensão global é com as álgebras de incidência fortemente simplesmente conexas . Antes de mostrar os resultados visualizando essa ligação, introduziremos uma família de álgebras com dimensão global igual a três chamada de álgebras críticas.

\section{Definição 3.8 (álgebra crítica [BFT11])}

Dizemos que uma álgebra $B$ é crítica se satisfaz as seguintes propriedades:

(i) A aljava de $B$ tem uma única fonte $a$ e um único poço $b$.

(ii) Sejam $S_{a}$ o módulo simples associado a fonte $a$ e $S_{b}$ o módulo simples associado ao poço $b$, tem-se que a dp $S_{a}=3$ e a di $S_{b}=3$. Se $S$ é um simples associado a qualquer outro vértice então $\operatorname{dp} S \leq 2$ e di $S \leq 2$.

(iii) Considere a resolução projetiva minimal do simples $S_{a}$ abaixo:

$$
0 \longrightarrow P_{3} \longrightarrow P_{2} \longrightarrow P_{1} \longrightarrow P_{0} \longrightarrow S_{a} \longrightarrow 0
$$

Dado o $B$-módulo $P=\bigoplus_{k=0}^{3} P_{k}$, todos os projetivos indecomponíveis estão em add $P$, e cada projetivo indecomponível é um somando direto de exatamente um $P_{k}$, para $k \in\{0, \ldots, 3\}$.

(iv) Considere a resolução injetiva minimal do simples $S_{b}$ abaixo:

$$
0 \longrightarrow S_{b} \longrightarrow I_{0} \longrightarrow I_{1} \longrightarrow I_{2} \longrightarrow I_{3} \longrightarrow 0
$$

Considere ainda o $B$-módulo $I=\bigoplus_{k=0}^{3} I_{k}$. Então todos os injetivos indecomponíveis estão em add $I$, e cada injetivo indecomponível é um somando direto de exatamente um $I_{k}$, para $k \in$ $\{0, \ldots, 3\}$.

(v) B não contém qualquer subcategoria própria e plena que satisfaz i), ii), iii) e iv).

Observamos que o item v) diz que a álgebra $B$ é minimal no sentido de que não contém nenhuma subcategoria própria e plena que tem dimensão global igual a três, principalmente em razão ao item ii).

Veremos abaixo a descrição das aljavas com relações de todas as álgebras críticas do trabalho "A criterion for global dimension two for strongly simply connected schurian algebras" [BFT11].

Proposição 3.9 (Bordino-Fernández-Trepode [BFT11]). Seja B uma álgebra crítica. Então a álgebra tem uma das seguintes apresentações por aljava e relações. 

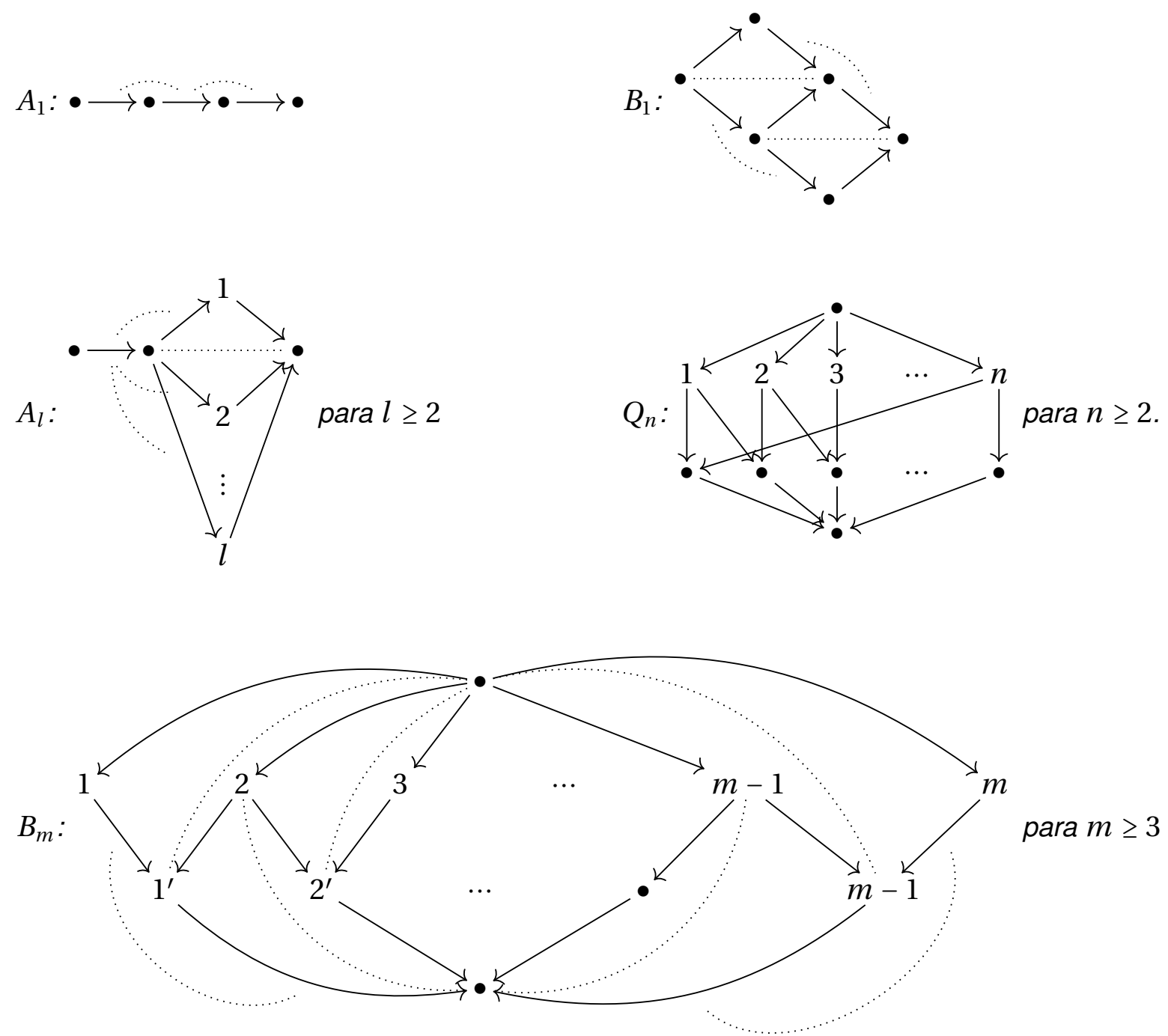

No caso do $Q_{n}$, as relações são dadas declarando que dois caminhos paralelos são iguais.

Através dessa proposição, observamos que a única álgebra de incidência crítica é a $Q_{n}$. Essa conclusão se deve as apresentações das álgebras $A_{1}, A_{l}, B_{1}$ e $B_{l}$ terem relações que não são de comutatividade.

Agora podemos enunciar um teorema do trabalho da Bordino, Fernández e Trepode [BFT11] e que iremos usar em nossa proposição.

Teorema 3.10 (Bordino-Fernández-Trepode [BFT11]). Seja A uma álgebra schurian fortemente simplesmente conexa com dimensão global maior ou igual a três. Então existe uma subcategoria $B$ plena de $A$ tal que $B$ é critica.

É interessante achar condições sobre as álgebras que nos permita concluir algo sobre a dimen- 
são global delas. Nesse intuito, provamos um resultado para as álgebras de incidência. Vejamos a seguir:

Proposição 3.11. Seja $K \Delta$ uma álgebra de incidência fortemente simplesmente conexa. Então a dimensão global de $K \Delta$ é menor ou igual a dois.

Demonstração Lembramos que uma álgebra de incidência é uma álgebra schurian. Se a dimensão global de $K \Delta$ for maior ou igual a três então ela vai conter uma subcategoria plena $B$ que é crítica. Por ser uma álgebra de incidência, $B$ é uma $Q_{n}$ e assim, a álgebra de incidência $K \Delta$ conteria uma coroa. Isso contradiz o seguinte resultado:

Teorema 3.12 (Assem-Castonguay-Marcos-Trepode [ACMT05]). Seja K $\Delta$ uma álgebra de incidência, $K \Delta$ é fortemente simplesmente conexa se, e somente, se $K \Delta$ não contém uma coroa.

Logo não existe uma álgebra de incidência fortemente simplesmente conexa com dimensão global maior igual a três. Com isso, obtemos que uma álgebra de incidência fortemente simplesmente conexa tem dimensão global menor ou igual a dois. 


\section{Capítulo 4}

\section{Dimensão global forte}

Nos capítulos anteriores, estudamos a dimensão global das Phias e as Phias simplesmente conexas. Nessa etapa, exploraremos a dimensão global forte das Phias. Onde, inicialmente, trabalharemos com as Phias que possuem um ponto extremante no poset associado. Ao final, mostraremos um limitante da dimensão global forte para a família das Phias. E mais, demonstraremos que é válido para todas as álgebras sinceras hereditárias por partes.

A definição da dimensão global forte de uma álgebra $A$ é inspirada na dimensão global, porém o ambiente é na categoria homotópica da álgebra. Antes de mostrar a definição, precisamos saber o que significa o comprimento de um complexo limitado de módulos em uma categoria particular. Seja $\mathcal{P}_{A}$ a subcategoria plena de $\bmod A$ consistindo dos módulos projetivos. Consideramos $C^{b}\left(\mathcal{P}_{A}\right)$ a categoria de complexos limitados sobre $\mathcal{P}_{A}$ e por $K^{b}\left(\mathcal{P}_{A}\right)$ a categoria homotópica correspondente. Se $P=\left(P^{i}, d^{i}\right) \in K^{b}\left(\mathcal{P}_{A}\right)$ é não nulo, existem $r \leq s$ tais que $P^{i}=0$ para todo $i<r$ e $P^{i}=0$ para todo $s<i$. Assim, o comprimento de $P$ é definido como $1(P)=s-r$.

\section{Definição 4.1 (dimensão global forte [HZ08])}

Seja A uma álgebra de dimensão finita. Então, definimos a dimensão global forte de A por

$$
\operatorname{dimglf} A=\sup \left\{1(P) \mid P \in K^{b}\left(\mathcal{P}_{A}\right) \text { indecomponível }\right\} \text {. }
$$

No artigo "A homological characterization of piecewise hereditary algebras" [HZ08], Happel e Zacharia explicam a leve mudança da definição que foi dado por Skowroński no trabalho "On algebras with finite strong dimension" em que define o comprimento do complexo $P$ em $C^{b}\left(\mathcal{P}_{A}\right)$. Esse trabaIho foi um dos primeiros que buscavam condições para finitude dessa nova dimensão. Outro trabalho especial nesse sentido, foi de Kerner, Skowroński, Yamagata e Zacharia entitulado "Finitess of the strong global dimension of radical square zero algebras".

Prosseguindo com o raciocínio, destacaremos o teorema que fornece uma importância do estudo da dimensão global forte para as Phias. 
Teorema 4.2 (Happel-Zacharia [HZ08]). Seja A uma álgebra de dimensão finita. A álgebra é hereditária por partes se, e somente, se $\operatorname{dimglf} A$ é finita.

Dessa forma fica claro que as Phias têm dimensão global forte finita. Com esse fato, o caminho para examinar qual seria a dimensão ou a existência de um limitante foi utilizar o teorema abaixo.

Teorema 4.3 (Alvares, Le Meur, Marcos [MLA14]). Seja $\mathcal{T}$ uma categoria triangulada que é equivalente triangularmente a uma categoria derivada limitada de uma categoria abeliana hereditária. Seja $T \in \mathcal{T}$ um objeto inclinante.

Então existe uma subcategoria plena e aditiva $\mathcal{H} \subset \mathcal{T}$ que é hereditária e abeliana, tais que 0 mergulho $\mathcal{H} \hookrightarrow \mathcal{T}$ estende a equivalência triangulada $D^{b}(\mathcal{H}) \cong \mathcal{T}$,e

$$
T \in \bigsqcup_{m=0}^{l} \mathcal{H}[m]
$$

para algum inteiro $l \geq 0$. Mais ainda, existe um $\operatorname{par}(\mathcal{H}, l)$ verificando que $\operatorname{dimglf}\left(\operatorname{End}_{\mathcal{T}} T\right)^{o p}=l+2$

Portanto, nas demonstrações dos nossos resultados quando escrevemos $A \cong{ }^{\prime} \mathcal{H}$ estamos considerando o $\operatorname{par}(\mathcal{H}, l)$ fornecido por esse teorema.

Inspirado no artigo "Jordan Hölder theorems for derived module categories of piecewise hereditary algebras" [HKL12], Alvares, Le Meur e Marcos propuseram uma definição alternativa no trabalho "The strong global dimension of piecewise hereditary algebras" a ser publicado [MLA14].

Lema 4.4 (Alvares, Le Meur, Marcos [MLA14]). Seja $T \in D^{b}(\mathcal{H})$ um objeto inclinante tal que $A=$ (End $T)^{o p}$. Dado um objeto $Y$ de $D^{b}(\mathcal{H})$, definimos

$$
\begin{aligned}
& \ell_{T}^{+}(Y)=\sup \left\{n \in \mathbb{Z} \mid \operatorname{Hom}_{D^{b}(\mathcal{H})}(Y, T[n]) \neq 0\right\} \\
& \ell_{T}^{-}(Y)=\inf \left\{n \in \mathbb{Z} \mid \operatorname{Hom}_{D^{b}(\mathcal{H})}(T[n], Y) \neq 0\right\} .
\end{aligned}
$$

Então temos que $\operatorname{dimglf} A=\sup \left\{\ell_{T}^{+}(Y)-\ell_{T}^{-}(Y) \mid Y \in D^{b}(\mathcal{H})\right\}$.

Seja $K \Delta \cong K Q_{K \Delta} / I$ uma álgebra de incidência. Consideramos um elemento $x \in\left(K Q_{K \Delta}\right)_{0}$ tal que $x$ é a origem de um caminho não nulo em $K Q_{K \Delta} / I$ para todo $y \in\left(K Q_{K \Delta}\right)_{0}$. Denotamos $x$ de elemento minimal. Dualmente, definimos o elemento maximal.

A princípio reparamos que as álgebras de incidência $K \Delta \cong \cong^{\prime} \mathcal{H}$ com elemento maximal ou minimal têm um comportamento particular na distribuição dos somandos indecomponíveis dos objetos inclinantes $T$ tal que $\left(\operatorname{End}_{D^{b}(\mathcal{H})} T\right)^{o p} \cong K \Delta$. Essa observação gerou o primeiro resultado para o nosso objetivo.

Proposição 4.5. Seja $K \Delta$ uma Phia com elemento minimal ou maximal $x$. Então dimglf $K \Delta \leq 3$. 
Demonstração Supomos que a Phia $K \Delta$ tem o elemento minimal $x$, a demonstração é análoga para a existência de elemento maximal.

Seja $K \Delta \cong \cong^{\prime} \mathcal{H}$. Consideramos $T=T_{0} \oplus \ldots \oplus T_{n}$ o objeto inclinante de $D^{b}(\mathcal{H})$ tal que (End $T)^{o p} \cong K \Delta$. Mais ainda, denotamos o $T_{0}$ representando o elemento minimal $x$.

Seja $D^{b}(\mathcal{H})=\bigsqcup_{m \in \mathbb{Z}} \mathcal{H}[m]$ com morfismos não nulos $\mathcal{H}[i] \rightarrow \mathcal{H}[j]$ somente se $j-i \in\{0,1\}$. A menos de translação, supomos que $T_{0} \in \mathcal{H}[0]$.

Por ser elemento minimal, temos que $\operatorname{Hom}\left(T_{0}, T_{j}\right)$ é não nulo para todo $j \in\{0, \ldots, n\}$. Consideramos $T_{j}=T_{j}^{\prime}[m] \in \mathcal{H}[m]$ tal que $T_{j}^{\prime} \in \mathcal{H}[0]$. A categoria $\mathcal{H}$ é hereditária, então $\operatorname{Hom}\left(T_{0}, T_{j}^{\prime}[m]\right)$ não nulo implica que $m \in\{0,1\}$.

Consequentemente, provamos que dimglf $K \Delta \leq 3$.

Essa proposição nos lançou à busca para provar um limitante para a família das Phias. Pensamos na ideia de forçar um elemento minimal em todo poset $\Delta$ associado da Phia $K \Delta$, gerando uma álgebra de incidência $K \Delta^{\prime}$ com o poset $\Delta^{\prime}=\Delta \cup\{x\}$, em que $\{x\}$ é o elemento minimal. Essa álgebra de incidência $K \Delta^{\prime}$ é a extensão por um ponto de $K \Delta$. Se $K \Delta^{\prime}$ fosse Phia, então usaríamos a proposição e provaríamos que dimglf $K \Delta \leq 3$ aplicando o teorema de Happel e Zacharia [ZH09]. A dificuldade é provar que $K \Delta^{\prime}$ é hereditária por partes. No capítulo 6, detalharemos mais sobre esse problema.

Teorema 4.6. Seja A uma álgebra sincera e hereditária por partes. Então dimglf $A \leq 3$.

Demonstração Consideramos $M$ o módulo sincero e $A=P_{1} \oplus \ldots \oplus P_{n}$ a álgebra com a sua decomposição em projetivos indecomponíveis.

Por hipótese, existe um funtor quase-inverso $F: D^{b}(A) \rightarrow D^{b}(\mathcal{H})$ que faz a equivalência triangulada, em que $\mathcal{H}$ é a categoria hereditária.

Usando a propriedade do módulo sincero, para cada $i \in\{1, \ldots, n\}$ temos que:

$$
0 \neq \operatorname{Hom}_{A}\left(P_{i}, M\right)=\operatorname{Hom}_{D^{b}(A)}\left(P_{i}, M\right) \cong \operatorname{Hom}_{D^{b}(\mathcal{H})}\left(F P_{i}, F M\right) .
$$

Seja $F A=T$ o objeto inclinante em $D^{b}(\mathcal{H})$ tal que $(\text { End } T)^{o p} \cong A$. Para cada $i \in\{1, \ldots, n\}$, consideramos $F P_{i}=T_{i}\left[m_{i}\right]$ o somando indecomponível de $T$ em que $T_{i} \in \mathcal{H}[0]$ tal que $D^{b}(\mathcal{H})=$ $\bigsqcup_{m \in \mathbb{Z}} \mathcal{H}[m]$. Também denotamos $F M=M^{\prime}[m]$ em que $M^{\prime} \in \mathcal{H}[0]$.

Agora, para cada $i \in\{1, \ldots, n\}$ obtemos o seguinte:

$$
0 \neq \operatorname{Hom}_{D^{b}(\mathcal{H})}\left(F P_{i}, F M\right)=\operatorname{Hom}_{D^{b}(\mathcal{H})}\left(T_{i}\left[m_{i}\right], M^{\prime}[m]\right) .
$$

Portanto $m_{i}=m$ ou $m_{i}=m-1$, para cada $i \in\{1, \ldots, n\}$, implicando que os somandos indecomponíveis de $T$ estão em $\mathcal{H}[m-1]$ ou $\mathcal{H}[m]$. Consequentemente $\operatorname{dimglf} A \leq 3$ como queríamos 
demonstrar.

Enunciamos abaixo um corolário natural.

Corolário 4.7. Seja $K \Delta$ uma Phia. Então dimglf $K \Delta \leq 3$.

Outro corolário é o que já foi provado por Ladkani em [Lad08a].

Corolário 4.8. Seja A uma álgebra sincera e hereditária por partes. Então $\operatorname{dimgl} A \leq 3$.

Demonstração Isso é uma consequência da desigualdade $\operatorname{dimgl} A \leq \operatorname{dimglf} A$.

Exemplo 4.9. Vamos mostrar um exemplo de uma Phia que tem a dimensão global igual a três, e por isso equipara a sua dimensão global forte:

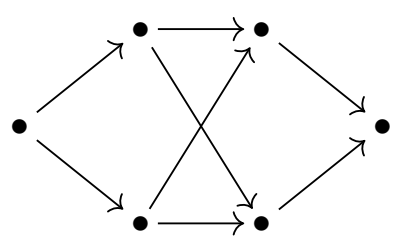

A aljava acima foi retirada do artigo do Ladkani [Lad08a]. No capítulo das Phias de tipo aljava, vamos explicar que essa álgebra de incidência é hereditária por partes e mostraremos o tipo dela.

Pelo resultado 3.11, a família das Phias que tem as dimensões global e global forte iguais a três não são fortemente simplesmente conexa. Será que essa família pode ser mais caracterizada?

Outra pergunta que podemos fazer nesse capítulo é sobre a "posição" da imagem do módulo sincero $M$ sobre a Phia $K \Delta$ em $D^{b}(\mathcal{H})$. Formulamos com mais detalhes:

Seja o funtor quase-inverso $F: D^{b}(K \Delta) \rightarrow D^{b}(\mathcal{H})$ que faz a equivalência triangulada, em que $\mathcal{H}$ é a categoria hereditária. Denotamos, a menos por translação, o objeto inclinante $T$ em $\mathcal{H}[0] \sqcup \mathcal{H}[1]$. Consideramos $F M=M^{\prime}[m]$ em que $M^{\prime} \in \mathcal{H}[0]$, dessa forma, qual seria o número natural $m$ ? 


\section{Capítulo 5}

\section{Álgebra de incidência hereditária por partes de tipo aljava}

Neste capítulo, algumas famílias de Phia serão totalmente descritas. Seja $K \Delta$ uma Phia de tipo aljava, então:
a) $K \Delta \cong^{\prime} K Q$, em que $Q$ é uma aljava Dynkin,
b) $K \Delta \cong^{\prime} K \widetilde{Q}$, em que $\widetilde{Q}$ é uma aljava Dynkin estendida.
c) $K \Delta \cong^{\prime} K Q^{\prime}$, em que $Q^{\prime}$ não é uma aljava Dynkin estendida e nem Dynkin.

\subsection{Os tipos $\mathbb{A}_{n}$ e $\widetilde{\mathbb{A}}_{n}$}

Dedicaremos esta seção ao estudo das álgebras de incidência que são adicionalmente hereditárias por partes e gentis ([ASS06]).

\section{Definição 5.1}

Seja $A$ uma álgebra com $Q_{A}$ acíclica. A álgebra $A \simeq K Q_{A} / I$ é chamada gentil se a aljava com relações $\left(Q_{A}, I\right)$ satisfizer as seguintes propriedades:

1. Cada vértice deve ser a origem e o término de no máximo duas flechas.

2. Para cada flecha $\alpha$ da aljava, devem existir no máximo uma flecha $\beta$ e uma flecha $\gamma$ tais que $\alpha \beta \notin I$ e $\gamma \alpha \notin I$.

3. Para cada flecha $\alpha$ da aljava, devem existir no máximo uma flecha $\delta$ e uma flecha $\zeta$ tais que $\alpha \delta \in I$ e $\zeta \alpha \in I$.

4. O ideal I deve ser gerado por caminhos de tamanho dois.

Denotaremos por $\bar{Q}$, o grafo que se obtém da aljava $Q$ desconsiderando a orientação das flechas e é normalmente chamado de grafo adjacente de $Q$. Assim, pela proposição a seguir, caracterizamos 
as álgebras de incidência gentis.

Proposição 5.2. Toda álgebra de incidência $K \Delta=K Q / I$ gentil é hereditária.

Demonstração Mostraremos que o ideal admissível I é zero. Supomos que não, então a álgebra de incidência tem pelo menos uma relação de comutatividade. Seja a seguinte subaljava de $Q$ :

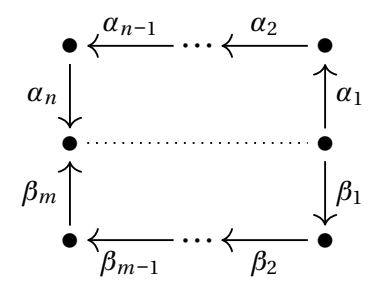

Portanto $\alpha_{1} \alpha_{2} \cdots \alpha_{n}-\beta_{1} \beta_{2} \cdots \beta_{m} \in I$, em que $m, n \geq 2$. Como $K \Delta$ é uma álgebra gentil, o ideal $I$ é gerado pelos caminhos de comprimento dois. Mas a relação de comutatividade não é um caminho, contradizendo a suposição de que $I$ não é zero.

Logo, a álgebra de incidência gentil é hereditária.

Podemos enunciar o seguinte resultado:

Corolário 5.3. Se uma Phia $K \Delta$ for gentil, então $K \Delta \cong K Q$, em que $\bar{Q}=\mathbb{A}_{n}$ ou $\bar{Q}=\widetilde{\mathbb{A}}_{n}$.

Demonstração Pela proposição anterior, concluímos que $K \Delta$ é hereditária, isto é, $K \Delta$ é isomorfa a $K Q$. Isso implica que $K Q$ é gentil, pois $K \Delta$ é uma álgebra gentil, por hipótese.

Supomos que existe a seguinte subaljava de $Q$

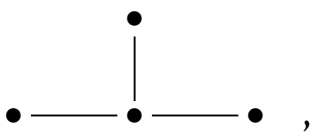

com as arestas orientadas respeitando a primeira condição da definição de álgebra gentil. Analisaremos um caso de orientação dessas arestas, os outros casos são similares. Seja

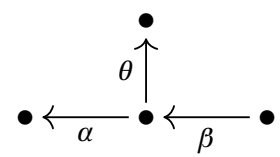

Pela quarta condição da definição de álgebra gentil, o ideal $I$ é gerado por caminhos de comprimento dois. Na subaljava acima, temos os caminhos $\beta \alpha$ e $\beta \theta$ ambos de comprimento dois. Assim, $\beta \alpha \in I$ ou $\beta \theta \in I$. Uma vez que nossa álgebra $K Q$ tem o ideal $I$ vazio, esse caso não acontece.

Logo, temos apenas as duas opções abaixo: 


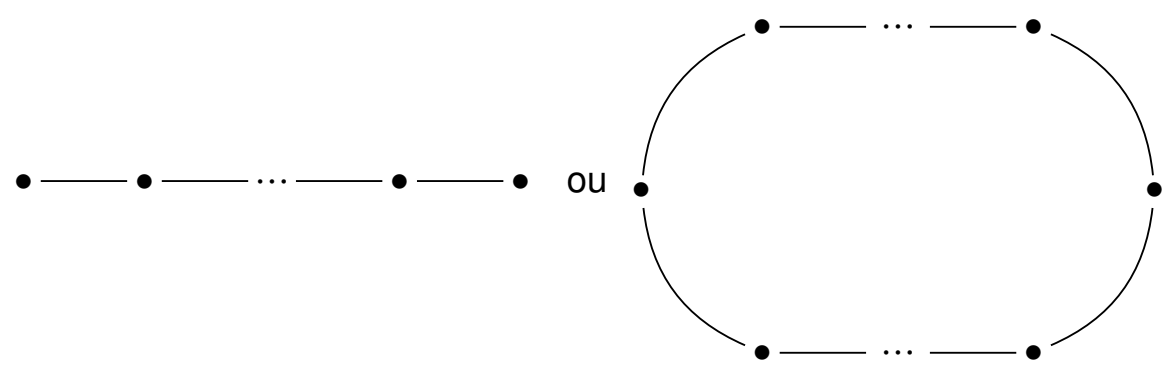

Concluímos que $\overline{Q_{A}}=\mathbb{A}_{n}$ ou $\overline{Q_{A}}=\widetilde{\mathbb{A}}_{n}$.

Esses dois primeiros resultados e o fato de que a propriedade da álgebra ser gentil é invariante por equivalência derivada [SZ03] obtemos esses corolários.

Corolário 5.4. Se $K \Delta$ é uma Phia do tipo $\mathbb{A}_{n}$, então $K \Delta \cong K Q$, em que $\bar{Q}=\mathbb{A}_{n}$.

Corolário 5.5. Se $K \Delta$ é uma Phia do tipo $\widetilde{\mathbb{A}}_{n}$, então $K \Delta \cong K Q$, em que $\bar{Q}=\widetilde{\mathbb{A}}_{n}$.

\subsection{O tipo $\mathbb{D}_{n}$}

A caracterização das álgebras hereditárias por partes de tipo $\mathbb{D}_{n}$ foi feita por Keller no artigo [Kel91]. Em sua tese ([Fer99]), Elsa Fernández obteve essa mesma caracterização através de uma ferramenta alternativa, o conceito de extensão trivial. Com base nesse material, é possível enunciar o seguinte resultado:

Teorema 5.6. Seja $K \Delta=K Q / I$ uma álgebra phia do tipo $\mathbb{D}_{n}$ tal que $\bar{Q}$ não é uma árvore. Então $K \Delta$ ou $K \Delta^{o p}$ é isomorfa a

1.

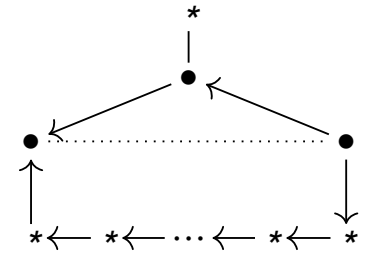

4.

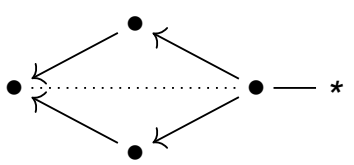

2.

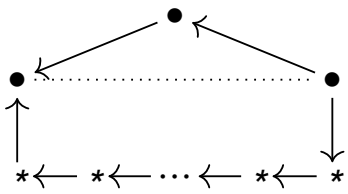

5.

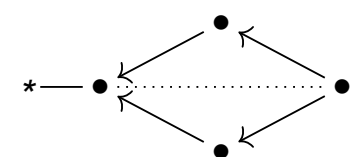

3.

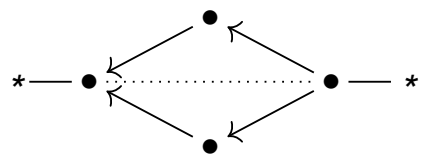

Observamos que aos vértices * dos grafos acima podemos anexar um diagrama da forma

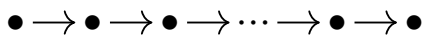

\subsection{0 tipo $\mathbb{E}_{6}$}

Os primeiros esforços para classificar as álgebras hereditárias por partes de tipo $\mathbb{E}_{6}$ foram feitos por Happel, em [Hap85]. Anos mais tarde, em sua tese, Elsa Fernández obteve algumas atualizações em relação a essa classificação, determinando uma descrição completa para essa classe de 
álgebras. Esse problema foi também tratado computacionalmente pelo projeto de Roggon [Rog95], que mostra a quantidade exata dessas álgebras a menos de isomorfismos. Particularmente sobre as álgebras hereditárias por partes de tipo $\mathbb{E}_{6}$ que também são álgebras de incidência, podemos enunciar o seguinte teorema:

Teorema 5.7. Seja $K \Delta=K Q / I$ uma álgebra phia do tipo $\mathbb{E}_{6}$ tal que $\bar{Q}$ não é uma árvore. Então $K \Delta$ ou $K \Delta^{o p}$ é isomorfa a

1.
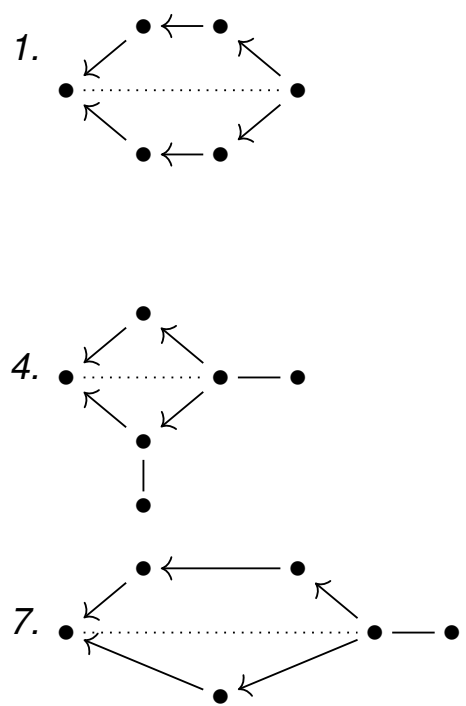

2.

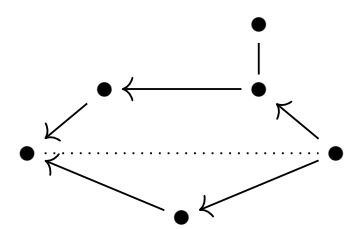

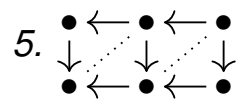

3.

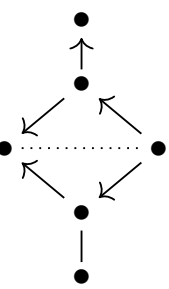

6.

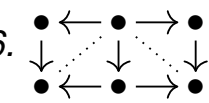

Nas aljavas acima têm arestas, as quais no desenho não estão orientadas. Uma observação sobre as arestas é que se pode considerar qualquer uma das duas possíveis orientações.

\subsection{O tipo $\mathbb{E}_{7}$}

A classe das álgebras inclinadas iteradas de tipo $\mathbb{E}_{7}$ possui uma relação importante com as extensões triviais de tipo de representação finito de classe de Cartan $\mathbb{E}_{7}$. Fernández [Fer99], em sua tese, descreve minuciosamente sobre essas extensões triviais. Recordamos o que é uma extensão trivial de uma álgebra. 


\section{Definição 5.8}

Seja A uma álgebra sobre $K$. Denominamos o dual de $A$ como $D(A)=\operatorname{Hom}_{K}(A, K)$, com a seguinte estrutura de $A$-A bimódulo

$$
(a f)(b)=f(b a), \quad(f a)(b)=f(a b), \quad \text { para quaisquer } a, b \in A \text { e } f \in D(A) .
$$

A extensão trivial da álgebra $A$ é a álgebra $T(A)=A \ltimes D(A)$, cuja estrutura de $K$-espaço vetorial é a de $A \times D(A)$ e a multiplicação é dada por:

$$
(a, f)(b, g)=(a b, a g+f b), \quad \text { para quaisquer } a, b \in A \text { e } f, g \in D(A) \text {. }
$$

A extensão trivial é uma álgebra autoinjetiva. Denominamos uma álgebra $B$ de autoinjetiva quando $B$ é um $B$-módulo injetivo. Pelo isomorfismo abaixo

$$
\begin{aligned}
& T(A) \longrightarrow D(T(A)) \\
& (a, f) \longmapsto((b, g) \mapsto f(b)+g(a)),
\end{aligned}
$$

obtemos que $T(A)$ é um $T(A)$-módulo injetivo. Portando, $T(A)$ é autoinjetiva. Com esse isomorfismo, mostramos também que a extensão trivial é uma álgebra simétrica.

Nesse trabalho, consideraremos as extensões triviais de tipo de representação finito e conexas. Vamos dividi-las em classes, chamadas de Cartan.

A classe de Cartan de uma álgebra $B$ autoinjetiva de tipo de representação finito foi introduzida por Riedtmann em 1980 pelo artigo "Algebren, Darstellungskocher, Ueberlagerungen und Zuruck". Consideramos $(\Gamma, \tau)$ a aljava estável de Auslander-Reiten da álgebra $B$. Isto é, em particular, a translação $\tau$ e sua inversa estão definidas em toda aljava $\Gamma$. Sejam $Q$ um diagrama Dynkin e $G$ um grupo de automorfismo de $\mathbb{Z} Q$. Riedtmann relacionava $(\Gamma, \tau)$ com $\mathbb{Z} Q / G$ por um isomorfismo, tal que a ação de $G$ em $\mathbb{Z} Q$ é admissível, ou seja, se cada órbita de $G$ encontra $\{x\} \cup x^{-}$em no máximo um vértice e encontra $\{x\} \cup x^{+}$em no máximo um vértice para cada $x$ de $(\mathbb{Z} Q)_{0}$. $O$ tipo Dynkin de $Q$ é unicamente determinado e chamamos de classe de Cartan de $B$.

Com a classificação de todas as extensões triviais de tipo de representação finito em conjunto com o próximo resultado, teremos uma associação com as álgebras inclinadas iteradas.

Teorema 5.9 (Assem-Happel-Roldán [AHR84]). Seja A uma álgebra. As seguintes condições são equivalentes:

a) $T(A)$ é de tipo de representação finito de classe de Cartan $Q$;

b) A é inclinada iterada de tipo Dynkin $Q$.

O foco do estudo é a aljava com relações da extensão trivial $T(A)$ de uma álgebra schurian $A \simeq K Q_{A} / I$. Para entender a descrição de sua aljava e de suas relações, precisamos do conceito 
de caminho maximal. Um caminho $p$ em $K Q_{A} / I$ se chamará maximal se $p \neq 0$ e $\alpha p=0=p \alpha$ para toda flecha $\alpha$ de $Q_{A}$. Mais ainda, nos teoremas de Fernández, usam-se as extensões triviais de álgebras schurian, isto é, uma álgebra que satisfaz $\operatorname{dim}_{K}\left(\operatorname{Hom}_{K}\left(P, P^{\prime}\right)\right) \leq 1$ para quaisquer $P, P^{\prime}$ projetivos indecomponíveis. Se não for schurian, sabe-se que a extensão trivial é de tipo de representação infinito [Fer99]. Como o nosso interesse é em tipo de representação finito, os enunciados serão específicos para as álgebras schurian.

O diagrama de $T(A)$ é descrito no seguinte teorema.

Teorema 5.10 ([Fer99]). Se $A=K Q_{A} / I$ é uma álgebra schurian, então a aljava de $T(A)$ é dada por:

a) $\left(Q_{T(A)}\right)_{0}=\left(Q_{A}\right)_{0}$,

b) $\left(Q_{T(A)}\right)_{1}=\left(Q_{A}\right)_{1} \cup\left\{\beta_{p_{1}}, \ldots, \boldsymbol{\beta}_{p_{t}}\right\}$, em que $\left\{p_{1}, \ldots, p_{t}\right\}$ é um conjunto maximal de caminhos maximais linearmente independentes. Mais ainda, para cada $i, \beta_{p_{i}}$ é uma flecha tal que $o\left(\beta_{p_{i}}\right)=$ $t\left(p_{i}\right)$ e $t\left(\beta_{p_{i}}\right)=o\left(p_{i}\right)$.

Seja $M=\left\{p_{1}, \ldots, p_{t}\right\}$ um conjunto maximal de caminhos maximais linearmente independentes. Falta descrever as relações da apresentação associada a $T(A)$ e para isso precisaremos de duas definições relacionadas aos caminhos maximais.

\section{Definição 5.11}

Diremos que um ciclo orientado $C$ de $Q_{T(A)}$ é elementar se verificar que $C=\beta_{p_{i}} \alpha_{1} \alpha_{2} \ldots \alpha_{n}$, em que $p_{i} \in M$ e $\alpha_{1} \ldots \alpha_{n}=k p_{i}$, para algum $k \in K$. Note que o comprimento de $C$ é pelo menos dois.

Agora, vamos definir o complemento de um caminho dentro do ciclo elementar.

\section{Definição 5.12}

Diremos que um caminho $q$ de $Q_{T(A)}$ admite um suplemento se estiver composto por flechas de um ciclo elementar $C$, o qual tem comprimento maior ou igual ao comprimento de $q$. Em outras palavras, um caminho admite um suplemento quando é um subcaminho de um ciclo elementar. Assim, chamaremos o suplemento de $q$ em $C$ o caminho trivial $e_{o(q)}$, se $o(q)=t(q)$, ou o caminho formado pelas flechas restantes de $C$, caso contrário.

Vejamos essas definições em um exemplo.

Exemplo 5.13. Seja A uma álgebra de incidência cujo o diagrama de Hasse é: 


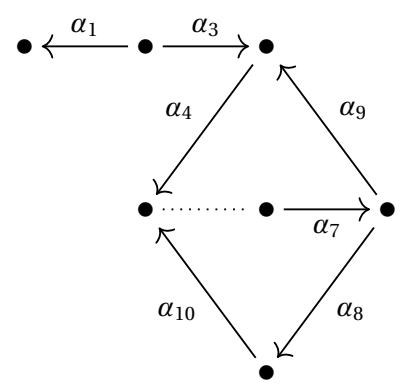

Relembramos que a linha pontilhada representa uma relação da apresentação da álgebra. Nesse exemplo, temos apenas a relação $\alpha_{7} \alpha_{8} \alpha_{10}-\alpha_{7} \alpha_{9} \alpha_{4}$.

Os caminhos $p_{1}=\alpha_{3} \alpha_{4}, p_{2}=\alpha_{7} \alpha_{8} \alpha_{10}=\alpha_{7} \alpha_{9} \alpha_{4}$ e $p_{3}=\alpha_{1}$ são os maximais e linearmente independentes da apresentação de $A$.

Assim, os ciclos elementares são $C_{1}=\alpha_{6} \alpha_{3} \alpha_{4}, C_{2}=\alpha_{5} \alpha_{7} \alpha_{8} \alpha_{10}, C_{3}=\alpha_{5} \alpha_{7} \alpha_{9} \alpha_{4}$, e $C_{4}=\alpha_{2} \alpha_{1}$ em que $\alpha_{6}=\beta_{p_{1}}, \alpha_{5}=\beta_{p_{2}}$ e $\alpha_{2}=\beta_{p_{3}}$. Dessa forma, a aljava da extensão trivial $T(A)$ associado a álgebra $A$ é:

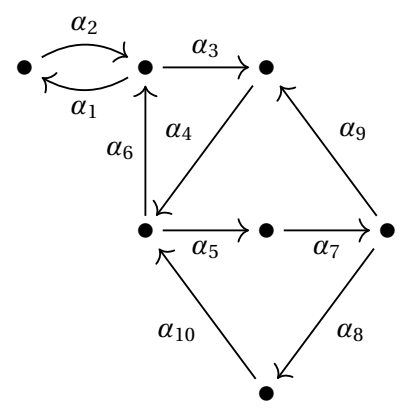

O caminho $q=\alpha_{2} \alpha_{3}$ não admite um suplemento pois não existe ciclo elementar $C$ de $Q_{T(A)}$ que tenha as flechas $\alpha_{2}$ e $\alpha_{3}$ na sua composição. Já o caminho $q^{\prime}=\alpha_{5} \alpha_{7}$ admite dois suplementos: $\alpha_{8} \alpha_{10}$ do ciclo $C_{2}$ e $\alpha_{9} \alpha_{4}$ do ciclo $C_{3}$.

Seja $T(A)$ uma extensão trivial de tipo de representação finito, dessa forma $A$ é uma álgebra schurian. Mais ainda, $A$ é uma álgebra de tipo de representação finito. Sabemos que uma álgebra schurian, triangular e de tipo de representação finito é isomorfa a uma álgebra schurian em que os caminhos paralelos são iguais. No seguinte teorema, descreveremos as relações da apresentação de $T(A)$ utilizando essa observação.

Teorema 5.14 (Fernández [Fer99]). Seja $A \simeq K Q_{A} / I$ uma álgebra schurian em que os caminhos paralelos são iguais. Seja $I_{T(A)}$ o ideal de $K Q_{T(A)}$ cujos geradores descrevemos em seguida:

a) os caminhos formados por $n+1$ flechas de um ciclo elementar de comprimento $n$,

b) os caminhos cujas flechas não pertencem a um mesmo ciclo elementar,

c) a diferença $\gamma-\gamma^{\prime}$ de caminhos $\gamma, \gamma^{\prime}$ com a mesma origem e o mesmo término e tendo o suplemento comum no ciclo elementar orientado de $Q_{T(A)}$. 
Para futuras referências no texto, chamaremos as relações de tipo 1, de tipo 2 e de tipo 3 referindo aos caminhos dos itens a), b) e c), respectivamente.

No próximo exemplo, mostraremos uma álgebra $B$ schurian que não é de incidência.

Exemplo 5.15. Vamos aplicar os teoremas na álgebra $B$ abaixo e exibir uma apresentação da extensão trivial $T(B)$.

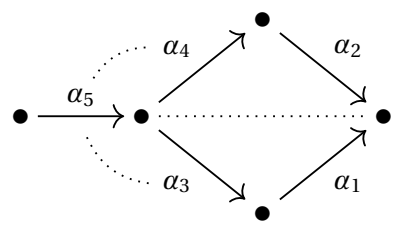

Os caminhos maximais são $p_{1}=\alpha_{5}$ e $p_{2}=\alpha_{4} \alpha_{2}=\alpha_{3} \alpha_{1}$. Portanto, a aljava associada a $T(B)$ é:

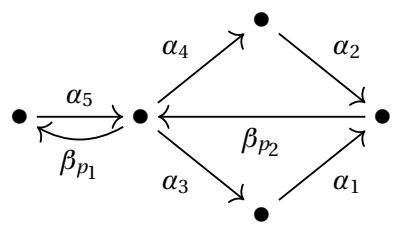

com as relações de tipo 1: $\alpha_{5} \beta_{p_{1}} \alpha_{5}, \beta_{p_{1}} \alpha_{5} \beta_{p_{1}}, \alpha_{4} \alpha_{2} \beta_{p_{2}} \alpha_{4}, \ldots, \beta_{p_{2}} \alpha_{3} \alpha_{1} \beta_{p_{2}}$. Agora, tem as seguintes relações do tipo 2: $\alpha_{5} \alpha_{4}, \alpha_{5} \alpha_{3}, \beta_{p_{2}} \beta_{p_{1}}, \alpha_{2} \beta_{p_{2}} \alpha_{3}$ e $\alpha_{1} \beta_{p_{2}} \alpha_{4}$. Finalmente, a relação do tipo 3 é $\alpha_{4} \alpha_{2}-\alpha_{3} \alpha_{1}$

Neste capítulo, usaremos um procedimento importante sobre uma extensão trivial $\Gamma=T(A)$ chamado corte. Para descrição desse método é necessário de duas definições.

\section{Definição 5.16 (corte [FP06])}

Seja $\Gamma \cong K Q_{\Gamma} / I$ uma extensão trivial. Um subconjunto do conjunto de flechas da aljava $Q_{\Gamma}$ é chamado de corte de $\Gamma$ se contém exatamente uma flecha de cada ciclo elementar dessa extensão trivial.

\section{Definição 5.17 ([FP06])}

Seja $\Gamma \cong K Q_{\Gamma} /$ I uma extensão trivial. $A$ álgebra quociente de $\Gamma$ por um corte de $\Gamma$ é a álgebra da forma $K Q_{\Gamma} /\left\langle I \cup \Sigma>\right.$, em que $\Sigma$ é o corte. Chamaremos $K Q_{\Gamma} /\langle I \cup \Sigma\rangle$ da álgebra resultante do corte $\Sigma$ de $K Q_{\Gamma}$.

Observando o Teorema 5.14 e a definição do corte, a álgebra $A^{\prime}$ resultante de qualquer corte não precisa ter as relações de tipo 1 . Por isso, no estudo dessas álgebras daremos a atenção nas relações do tipo 2 e do tipo 3 . Vejamos mais uma característica da álgebra $A^{\prime}$. 
Proposição 5.18 ([Fer99]). Seja A uma álgebra schurian triangular. Se $A^{\prime}$ for obtida de $T(A)$ por um corte, então $A^{\prime}$ será schurian.

Portanto, o processo de eliminar exatamente uma flecha de cada ciclo elementar $C_{1}, \ldots, C_{m}$ de $T(A)$ tem como resultado uma álgebra $A^{\prime}$ schurian. Assim, satisfaz a hipótese da próxima proposição.

Proposição 5.19 ([Fer99]). Seja $A^{\prime}$ uma álgebra resultante de um corte de $T(A)$. Se $A^{\prime}$ é uma álgebra schurian, então $T\left(A^{\prime}\right) \simeq T(A)$.

Vimos que o nosso elemento inicial de estudo é a extensão trivial $T(A)$ de tipo de representação finito. Já observamos anteriormente que nesse caso $A$ é schurian. Um detalhe da proposição anterior é a exigência de uma álgebra $A$ triangular, isto é, que $Q_{A}$ não tem ciclos orientados. $O$ próximo teorema nos ajuda a garantir a triangulariedade dessa álgebra.

Teorema 5.20 (Yamagata). Seja $T(A)$ uma extensão trivial de uma álgebra $A$. Se $T(A)$ for de tipo de representação finito, então $A$ será triangular.

Uma curiosidade natural é se considerarmos uma álgebra $B^{\prime}$ tal que $T\left(B^{\prime}\right) \simeq \Gamma$ é de tipo de representação finito, então, pelos resultados anteriores, $B^{\prime}$ é schurian e triangular. $O$ resultado abaixo, aprenderemos uma maneira de como obter essa álgebra $B^{\prime}$ através de $\Gamma$.

Proposição 5.21 ([Fer99]). Seja $\Gamma=T(A)$, com $A$ schurian. Se $A^{\prime}$ é uma álgebra schurian e $T\left(A^{\prime}\right) \simeq \Gamma$, então $A^{\prime}$ é obtida através de um corte de $\Gamma$.

Com essa série de proposições, fechamos o seguinte raciocínio: de uma família $\mathcal{E}$ de extensões triviais $T(A)$ de tipo de representação finito, o corte gera álgebras $A^{\prime}$ schurian tal que $T\left(A^{\prime}\right)$ é isomorfo a $T(A)$. Por outro lado, de uma família $\mathcal{S}$ de álgebras $B^{\prime}$ schurian tal que $T\left(B^{\prime}\right) \simeq T(A)$ então $B^{\prime}$ é resultante de um corte de $T(A)$. Como se o corte fosse uma aplicação sobrejetora

$$
\begin{array}{r}
\text { corte: } \mathcal{E} \longrightarrow \mathcal{S} \\
T(A) \longmapsto A^{\prime} .
\end{array}
$$

Obtemos um "terreno mais seguro" para trabalhar com as extensões triviais. Considere uma extensão trivial $\Gamma$ de tipo de representação finito de classe de Cartan $\mathbb{E}_{7}$. Todas as álgebras $A$ resultante de um corte têm as suas extensões triviais isomorfas a $\Gamma$. Portanto, a extensão trivial de $A$ é de tipo de representação finito de classe de Cartan $\mathbb{E}_{7}$, implicando que a álgebra $A$ é inclinada iterada de tipo $\mathbb{E}_{7}$ pelo Teorema 5.9.

O nosso trabalho é identificar todas as álgebras $A$ que são de incidência para cada extensão trivial $\Gamma$ de tipo de representação finito de classe de Cartan $\mathbb{E}_{7}$. Primeiramente, queremos separar as álgebras $A$ tais que suas aljavas existam apenas relações de comutatividade. Para esse fim, precisamos eliminar pelo menos uma flecha que pertença no mínimo a dois ciclos elementares da extensão trivial. 
Lema 5.22. Seja $\Gamma$ uma extensão trivial de uma álgebra schurian em que os caminhos paralelos são iguais. Tome uma álgebra $A \simeq K Q_{A} / I$ obtida por um corte em $\Gamma$. Se uma flecha do corte pertence a dois ciclos elementares de $Q_{\Gamma}$, no mínimo, então existe uma relação do tipo 3 em I.

Demonstração Sejam $B$ a álgebra schurian em que os caminhos com a mesma origem e o mesmo término são iguais, e a extensão trivial $\Gamma=T(B)$. Sem perda de generalidade, tomaremos uma extensão trivial $\Gamma$ associada a uma aljava com apenas dois ciclos elementares $C_{1}=$ $\theta_{1} \theta_{2} \ldots \theta_{l} \alpha_{l+1} \ldots \alpha_{n}$ e $C_{2}=\theta_{1} \theta_{2} \ldots \theta_{l} \alpha_{l+1}^{\prime} \ldots \alpha_{m}^{\prime}$. Assim, seja $Q_{\Gamma}$ o grafo abaixo:

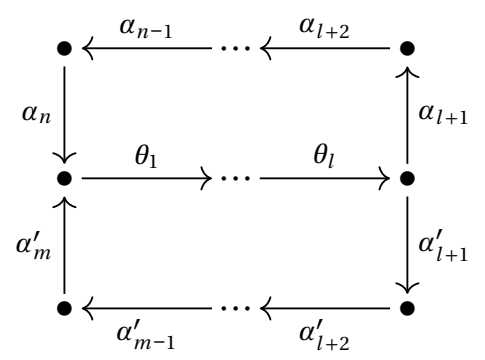

com as relações do tipo 1,2 e 3

$$
\begin{aligned}
& \theta_{1} \ldots \theta_{l} \alpha_{l+1} \ldots \alpha_{n} \theta_{1} ; \ldots ; \alpha_{n} \theta_{1} \ldots \theta_{l} \alpha_{l+1} \ldots \alpha_{n} \\
& \theta_{1} \ldots \theta_{l} \alpha_{l+1}^{\prime} \ldots \alpha_{m}^{\prime} \theta_{1} ; \ldots ; \alpha_{m}^{\prime} \theta_{1} \ldots \theta_{l} \alpha_{l+1}^{\prime} \ldots \alpha_{m}^{\prime} \\
& \alpha_{m}^{\prime} \theta_{1} \ldots \theta_{l} \alpha_{l+1} ; \alpha_{n} \theta_{1} \ldots \theta_{l} \alpha_{l+1}^{\prime} \\
& \alpha_{l+1} \ldots \alpha_{n}-\alpha_{l+1}^{\prime} \ldots \alpha_{m}^{\prime}
\end{aligned}
$$

Agora, eliminando alguma flecha $\theta_{i}$ para $i \in\{1, \ldots, l\}$ que pertence aos dois ciclos elementares $C_{1}$ e $C_{2}$, obtemos $I_{A}$ com apenas a relação do tipo 3. Portanto a apresentação de $A$ tem apenas a relação $\alpha_{l+1} \alpha_{l+2} \ldots \alpha_{n}-\alpha_{l+1}^{\prime} \alpha_{l+2}^{\prime} \ldots \alpha_{m}^{\prime}$, como desejado.

O próximo passo é obter as álgebras $A \simeq K Q_{A} / I$ cujo ideal é o ideal gerado por todas as relações de comutatividade e tal que $T(A)$ é isomorfa a $\Gamma$. Considere abaixo, o grafo associado a $\Gamma$ de tipo de representação finito de classe de Cartan $\mathbb{E}_{7}$, obtido do trabalho da Fernández [Fer99]:

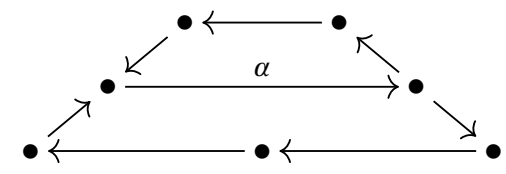

Para utilizarmos o lema anterior, queremos identificar os ciclos elementares de $Q_{\Gamma}$. Nesse caso, só há dois ciclos elementares e a única flecha em comum nesses ciclos é $\alpha$. Nos resta apenas uma álgebra $A$, fruto da eliminação da flecha $\alpha$, representada pelo diagrama:

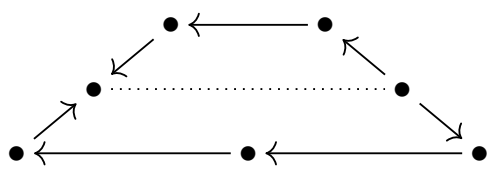


Observamos que a apresentação acima é de uma álgebra de incidência. Dessa forma, pelos argumentos anteriores, $A$ é hereditária por partes de tipo $\mathbb{E}_{7}$.

Iremos mostrar um grafo de uma extensão trivial $\Gamma$ com mais ciclos elementares. Seja o diagrama abaixo, associado a $\Gamma$ de tipo de representação finito de classe de Cartan $\mathbb{E}_{7}$, extraído do trabalho da Fernández [Fer99]:

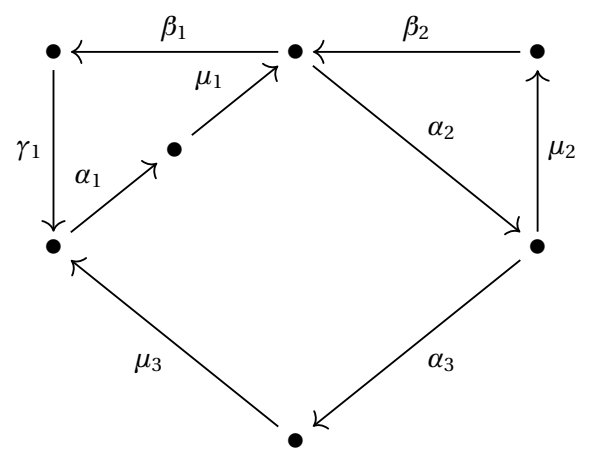

Analisando o grafo acima, existem três ciclos elementares: $C_{1}=\alpha_{1} \mu_{1} \beta_{1} \gamma_{1}, C_{2}=\alpha_{2} \mu_{2} \beta_{2}$ e $C_{3}=$ $\alpha_{3} \mu_{3} \alpha_{1} \mu_{1} \alpha_{2}$. Seguindo o processo explicado anteriormente, apresentaremos as álgebras $A$ que se obtêm eliminando exatamente uma flecha de cada ciclo elementar não nulo de $Q_{\Gamma}$, em conjunto com o lema. Usando uma combinatória simples, existem seis álgebras $A$ cujas presentações têm relações de comutatividade.

Eliminando $\alpha_{1}$ e $\mu_{2}$, obtemos a álgebra $A_{1}$. Observe que $\mu_{2}$ pertence apenas ao ciclo elementar $C_{2}$ e $\alpha_{1}$ pertence aos ciclos elementares $C_{1}$ e $C_{3}$, como o lema exige.

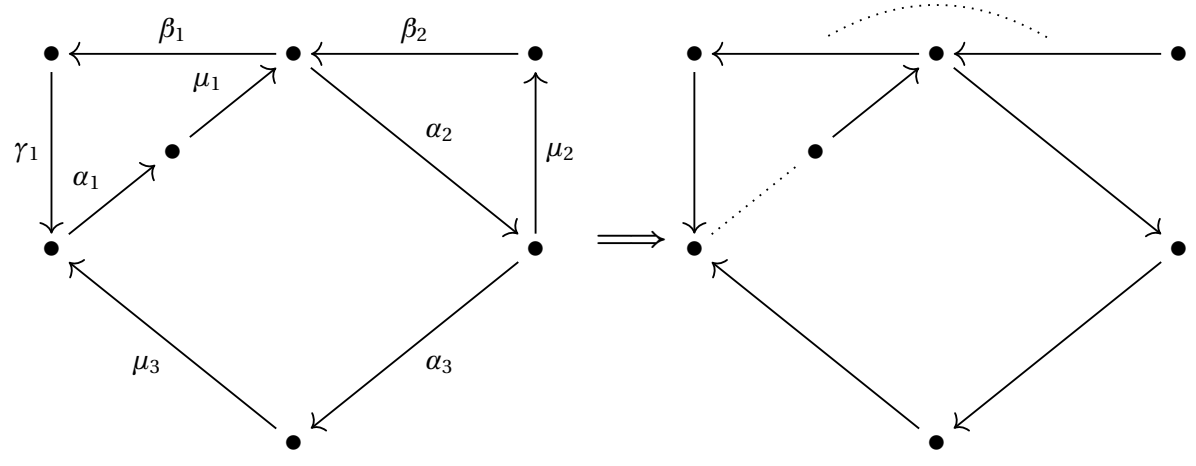

Essa presentação não é uma álgebra de incidência pois tem a relação $\beta_{2} \beta_{1}$. Eliminando $\mu_{1}$ e $\mu_{2}$, de forma análoga, obtemos uma álgebra $A_{2}$, que não é de incidência.

Na terceira combinação, retirando $\alpha_{1}$ e $\beta_{2}$, exibimos o grafo que representa a álgebra $A_{3}$. Veja que $\beta_{2}$ está no ciclo elementar $C_{2}$ e $\alpha_{1}$ está nos ciclos elementares $C_{1}$ e $C_{3}$. 


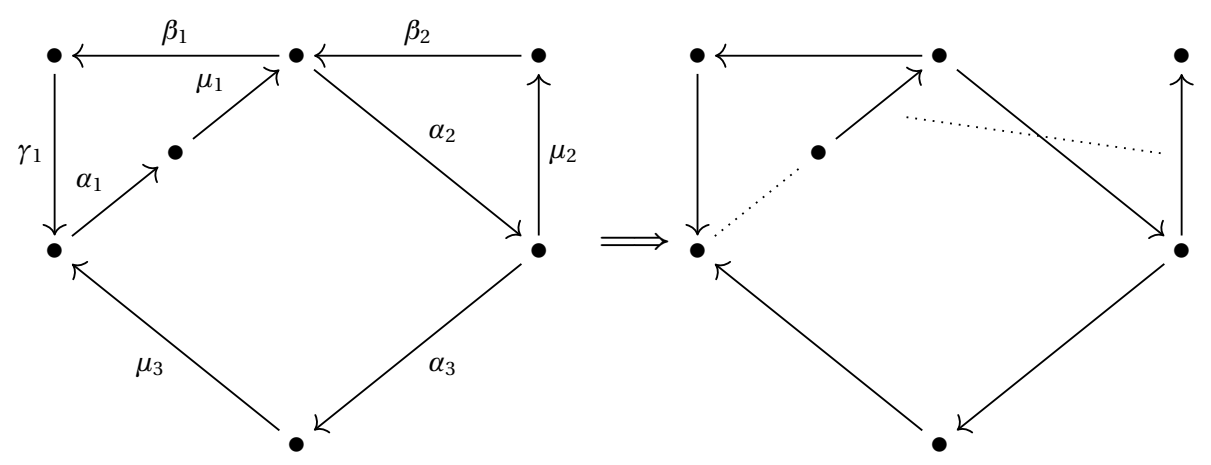

Retirando $\mu_{1}$ e $\beta_{2}$, obtemos a seguinte álgebra $A_{4}$ :
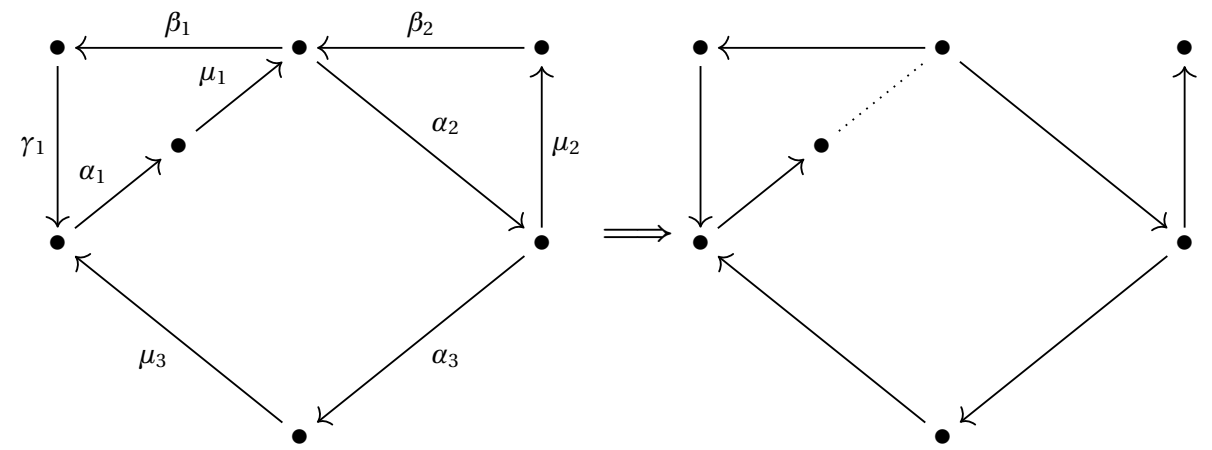

Note que $\beta_{2}$ pertence ao ciclo elementar $C_{2}$ e a flecha $\mu_{1}$ está nos ciclos elementares $C_{1}$ e $C_{3}$.

Na quinta combinação, a exclusão de $\alpha_{2}$ e $\beta_{1}$, resulta na álgebra $A_{5}$.

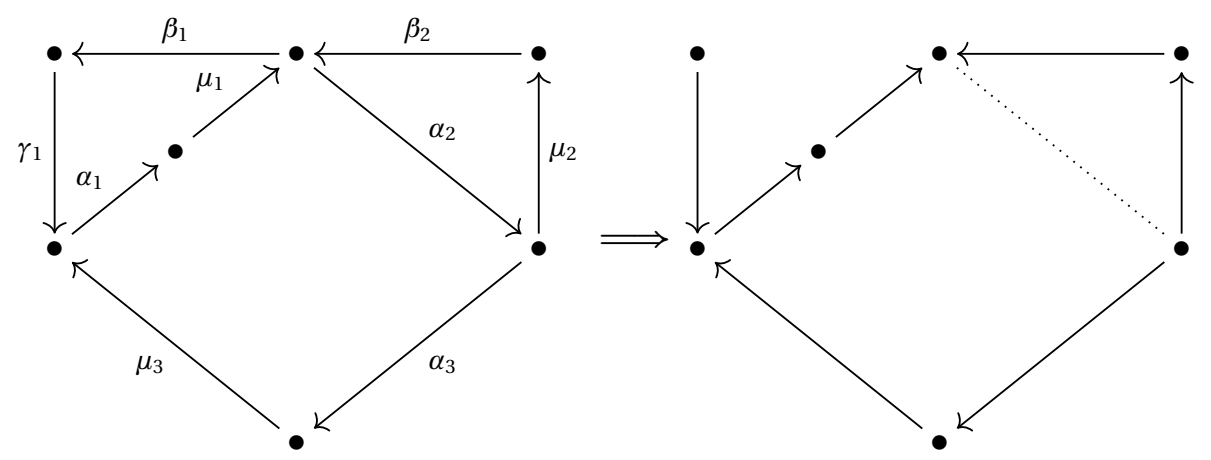

A flecha $\beta_{1}$ está no ciclo elementar $C_{1}$ e $\alpha_{2}$ pertence aos ciclos elementares $C_{2}$ e $C_{3}$.

Finalmente, eliminamos $\alpha_{2}$ e $\gamma_{1}$, originando a álgebra $A_{6}$ no que segue:

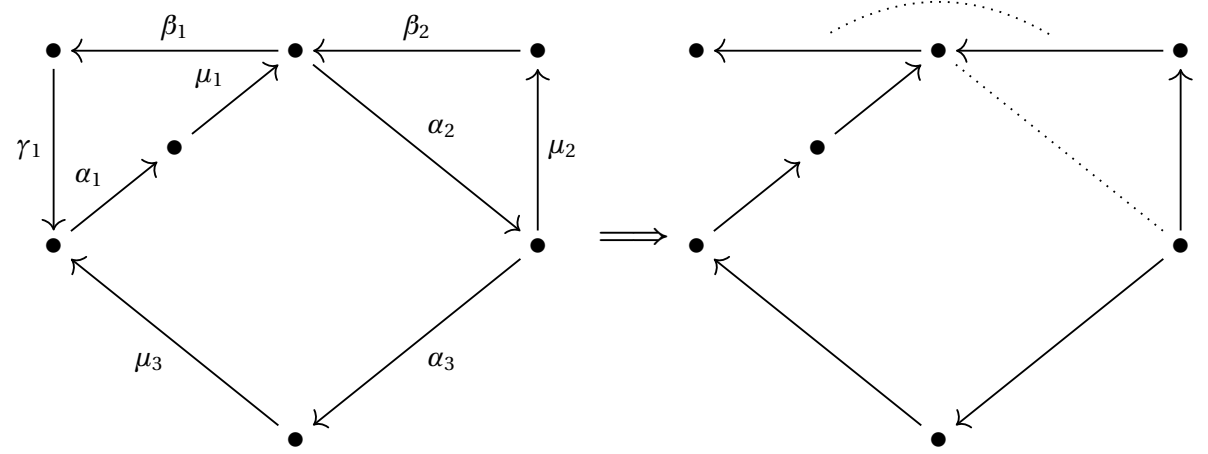


Observamos que $\gamma_{1}$ está no ciclo elementar $C_{1}$ e $\alpha_{2}$ pertence aos ciclos elementares $C_{2}$ e $C_{3}$.

Portanto, as álgebras $A_{4}$ e $A_{5}$ são de incidência e, consequentemente, vão entrar na lista do nosso teorema das álgebras hereditárias por partes de tipo $\mathbb{E}_{7}$.

Surge uma questão sobre a existência de um corte na extensão trivial que resulte numa álgebra de incidência. Observamos no exemplo e no próximo lema, que dificilmente existe um padrão geral. Deparamos com diversas formas de diagramas de extensões triviais e conseguimos achar alguns padrões particulares nos cortes para obter as álgebras de incidência. Reunimos esses padrões em lemas que chamaremos de lemas de corte. Antes de começar a mostra-los, introduziremos alguns conceitos.

Para cada vértice $h$ da aljava $Q_{\Gamma}$, seja $C_{h}$ o conjunto de todos os ciclos elementares $C$, não nulos em $\Gamma$, com a origem e o término deles em $h$.

Lema 5.23. Seja $Q_{\Gamma}$ um diagrama planar associado com quatro ciclos elementares. Se esse diagrama tiver um vértice a tais que $C_{a}=\left\{C_{1}, C_{2}, C_{3}, C_{4}\right\}$ e tem quatro arestas em $a$, então existem apenas dois cortes, ou seja, extraímos duas álgebras de incidência com relações.

Demonstração Consideramos uma extensão trivial $\Gamma$ associada a uma aljava com quatro ciclos elementares. Assim, seja $Q_{\Gamma}$ como abaixo:

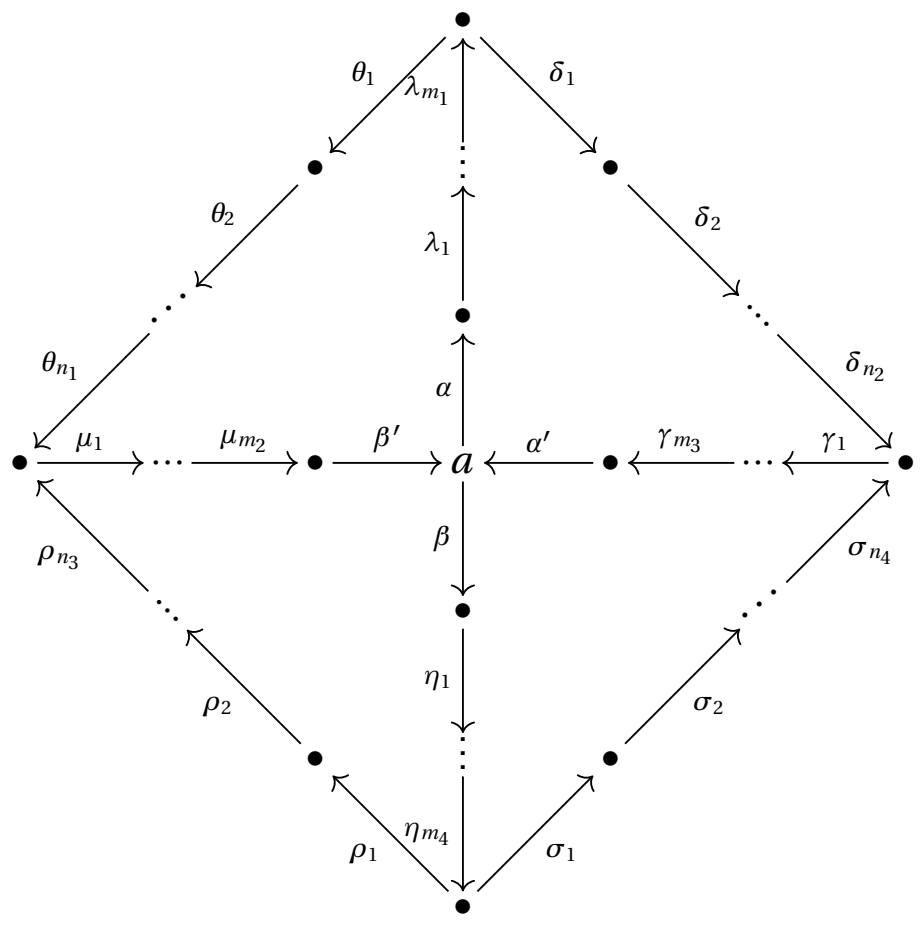

Descreveremos os geradores do ideal admissível $I_{\Gamma}$. Para facilitar a leitura, denotamos os caminhos $\lambda=\lambda_{1} \ldots \lambda_{m_{1}}, \theta=\theta_{1} \ldots \theta_{n_{1}}, \mu=\mu_{1} \ldots \mu_{m_{2}}, \eta=\eta_{1} \ldots \eta_{m_{4}}, \rho=\rho_{1} \ldots \rho_{n_{3}}, \delta=\delta_{1} \ldots \delta_{n_{2}}$, $\gamma=\gamma_{1} \ldots \gamma_{m_{3}}$ e $\sigma=\sigma_{1} \ldots \sigma_{n_{4}}$ : 
Temos as relações do tipo 1 relacionadas ao ciclo elementar $\alpha \lambda \theta \mu \beta^{\prime}$ :

$\alpha \lambda \theta \mu \beta^{\prime} \alpha=\lambda \theta \mu \beta^{\prime} \alpha \lambda_{1}=\ldots=\lambda_{m_{1}} \theta \mu \beta^{\prime} \alpha \lambda=\theta \mu \beta^{\prime} \alpha \lambda \theta_{1}=\ldots=\theta_{n_{1}} \mu \beta^{\prime} \alpha \lambda \theta=$ $\mu \beta^{\prime} \alpha \lambda \theta \mu_{1}=\ldots=\mu_{m_{2}} \beta^{\prime} \alpha \lambda \theta \mu=\beta^{\prime} \alpha \lambda \theta \mu \beta^{\prime}$.

Abaixo, são descritas as relações do tipo 1 relacionadas ao ciclo elementar $\alpha \lambda \delta \gamma \alpha^{\prime}$ :

$\alpha \lambda \delta \gamma \alpha^{\prime} \alpha=\lambda \delta \gamma \alpha^{\prime} \alpha \lambda_{1}=\ldots=\lambda_{m_{1}} \delta \gamma \alpha^{\prime} \alpha \lambda=\delta \gamma \alpha^{\prime} \alpha \lambda \delta_{1}=\ldots=\delta_{n_{2}} \gamma \alpha^{\prime} \alpha \lambda \delta=\gamma \alpha^{\prime} \alpha \lambda \delta \gamma_{1}=\ldots=$ $\gamma_{m_{3}} \alpha^{\prime} \alpha \lambda \delta \gamma=\alpha^{\prime} \alpha \lambda \delta \gamma \alpha^{\prime}$

Como também, estão logo em seguida, as relações do tipo 1 relacionadas ao ciclo elementar $\beta \eta \rho \mu \beta^{\prime}$ :

$\beta \eta \rho \mu \beta^{\prime} \beta=\eta \rho \mu \beta^{\prime} \beta \eta_{1}=\ldots=\eta_{m_{4}} \rho \mu \beta^{\prime} \beta \eta=\rho \mu \beta^{\prime} \beta \eta \rho_{1}=\ldots=\rho_{n_{3}} \mu \beta^{\prime} \beta \eta \rho=$ $\mu \beta^{\prime} \beta \eta \rho \mu_{1}=\ldots=\mu_{m_{2}} \beta^{\prime} \beta \eta \rho \mu=\beta^{\prime} \beta \eta \rho \mu \beta^{\prime}$.

Finalmente, as relações do tipo 1 relacionadas ao ciclo elementar $\beta \eta \sigma \gamma \alpha^{\prime}$ :

$$
\begin{aligned}
& \beta \eta \sigma \gamma \alpha^{\prime} \beta=\eta \sigma \gamma \alpha^{\prime} \beta \eta_{1}=\ldots=\eta_{m_{4}} \sigma \gamma \alpha^{\prime} \beta \eta=\sigma \gamma \alpha^{\prime} \beta \eta \sigma_{1}=\ldots=\sigma_{n_{4}} \gamma \alpha^{\prime} \beta \eta \sigma= \\
& \gamma \alpha^{\prime} \beta \eta \sigma \gamma_{1}=\ldots=\gamma_{m_{3}} \alpha^{\prime} \beta \eta \sigma \gamma=\alpha^{\prime} \beta \eta \sigma \gamma \alpha^{\prime} .
\end{aligned}
$$

Falta mostrar as relações do tipo 2 e 3 , que são as seguintes:

$$
\begin{aligned}
& \alpha \lambda \theta-\beta \eta \rho ; \theta \mu \beta^{\prime}-\delta \gamma \alpha^{\prime} ; \alpha \lambda \delta-\beta \eta \sigma ; \rho \mu \beta^{\prime}-\sigma \gamma \alpha^{\prime} ; \\
& \theta_{n_{1}} \mu \beta^{\prime} \beta ; \rho_{n_{3}} \mu \beta^{\prime} \alpha ; \delta_{n_{2}} \gamma \alpha^{\prime} \beta ; \sigma_{n_{4}} \gamma \alpha^{\prime} \alpha
\end{aligned}
$$

Fazendo o corte nas flechas $\alpha$ e $\beta$, obtemos a aljava ao lado com as relações de comutatividade $\theta \mu \beta^{\prime}-\delta \gamma \alpha^{\prime}$ e $\rho \mu \beta^{\prime}-\sigma \gamma \alpha^{\prime}$.

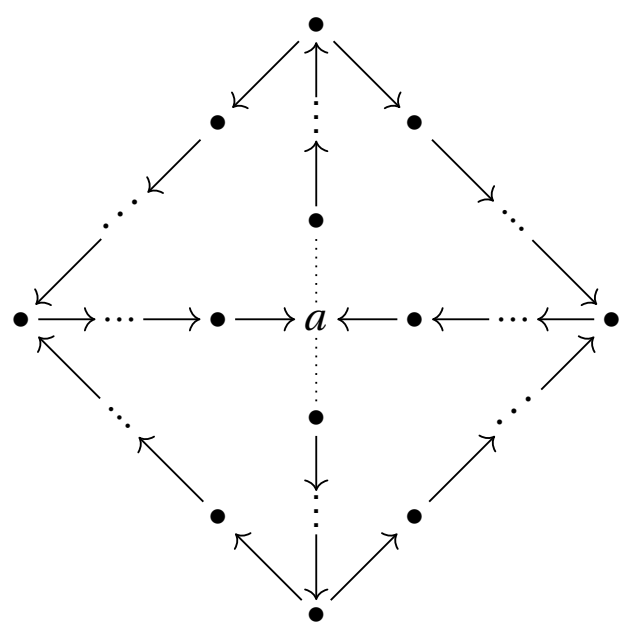

A segunda álgebra de incidência com rela- resultando na aljava ao lado com as relações de ções é obtida com o corte nas flechas $\alpha^{\prime}$ e $\beta^{\prime}$, comutatividade $\alpha \lambda \theta-\beta \eta \rho$ e $\alpha \lambda \delta-\beta \eta \sigma$. 


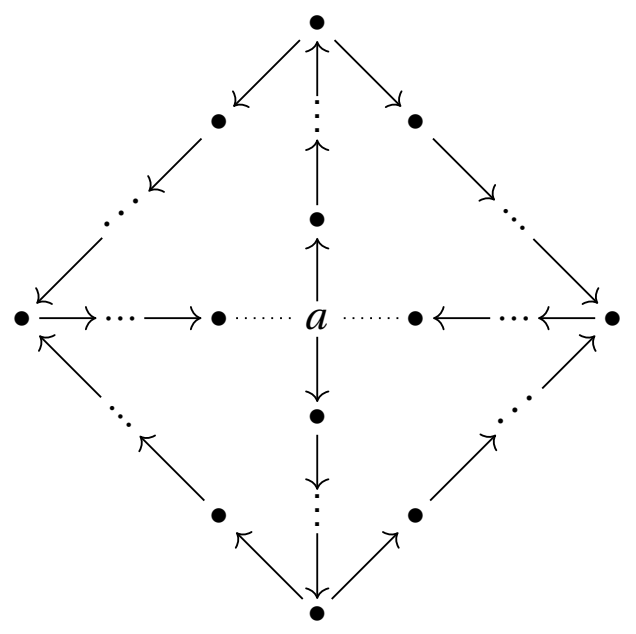

Agora, observamos nas relações da extensão trivial $K Q_{\Gamma} / I_{\Gamma}$ que qualquer outro corte diferente das duas mostradas anteriormente não conseguiremos álgebras de incidência com relações de comutatividade. Como no caso do corte das flechas $\delta_{1}, \beta^{\prime}, \sigma_{n_{4}}$, mostrada abaixo:

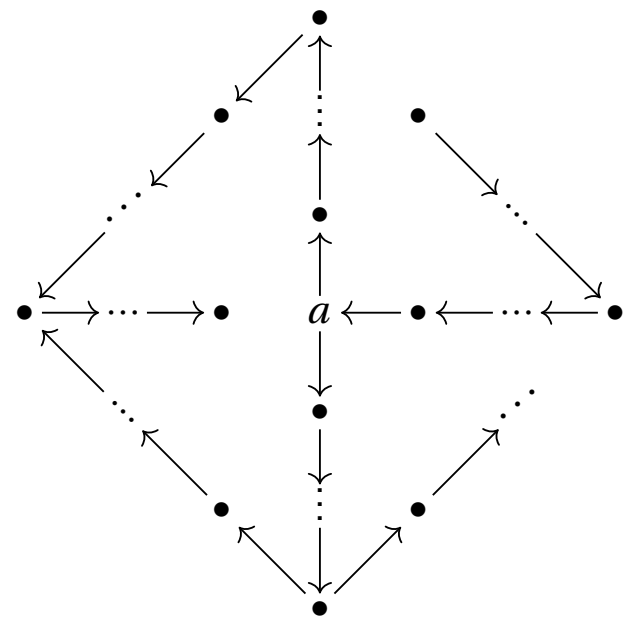

com as relações $\alpha \lambda \theta-\beta \eta \rho$ e $\delta_{n_{2}} \gamma \alpha^{\prime} \beta$.

Na seção 5.6 apresentamos mais dois lemas de corte 5.42 e 5.39 .

Esses lemas inspiraram a busca por um algoritmo geral do corte. Conseguimos dar um passo a mais, no caminho computacional, elaboramos um programa que concebe exatamente os cortes na extensão trivial dada que resultam em álgebras de incidência. Além disso, implementamos esse algoritmo no site http://www.ime.usp.br/ celovg/. No apêndice A, apresentamos todo o código fonte do programa. Adiante, vamos detalhar mais esse trabalho.

O primeiro passo é nomenclar as flechas da aljava da extensão trivial. Começamos $\operatorname{com} \alpha_{1}, \alpha_{2}$, $\alpha_{3}, \ldots$ nas flechas que compõem as relações do tipo 2 . A nomenclatura das flechas que não estão nesse conjunto são livres. O próximo passo é colocar as informações necessárias da extensão trivial no programa. Esses dados iniciais são:

- existem quantas relações de tipo 2; 
- números de ciclos elementares;

- quantas flechas estão envolvidas nas relações do tipo 2.

Logo em seguida, o programa exibe uma tabela preenchida com caixas de seleção. Nessa tabela, as colunas representam respectivamente as relações de tipo 2 e os ciclos elementares, e as linhas representam as flechas envolvidas nesses elementos. O usuário clica na célula da tabela indicada se a flecha está na(s) relação(ões) ou se pertence ao(s) ciclo(s), e deixa desmarcada caso contrário. Aperta o botão Processar e, em seguida, é mostrada a solução. A solução são os cortes que originam as álgebras de incidências.

A tese [Fer99] da Fernández mostrou todas as extensões triviais do tipo $\mathbb{E}_{7}$, no total de 72 . Assim, para cada extensão trivial da lista $\mathbb{E}_{7}$, fizemos o procedimento descrito nos dois exemplos anteriores, resultando no teorema principal da seção. Esse foi um trabalho totalmente manual e foi útil para termos ideias para o programa, e para alguns resultados anteriores. Com intuito de deixar a demonstração mais enxuta, utilizamos o programa para cada extensão trivial.

Teorema 5.24. Seja $K \Delta=K Q / I$ uma álgebra de incidência e iterada inclinada de tipo $\mathbb{E}_{7}$ tal que $\bar{Q}$ não é uma árvore. Então $K \Delta$ ou $K \Delta^{o p}$ é isomorfa a
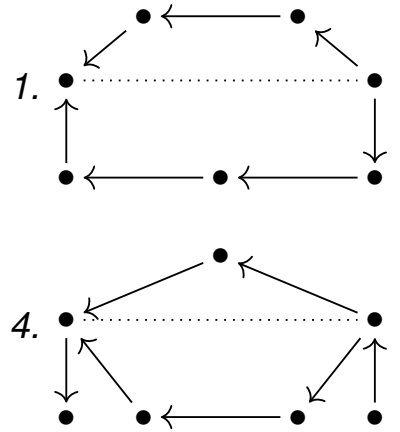

7.

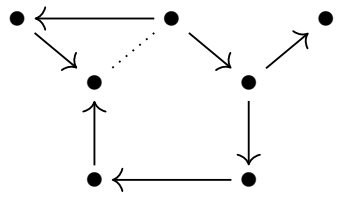

10.

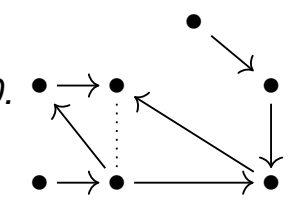

13.

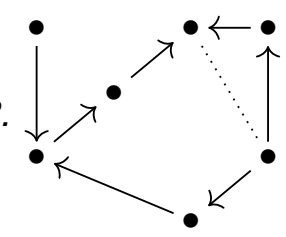

16.

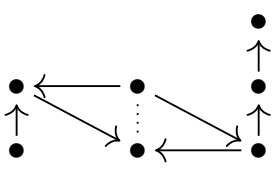

2.
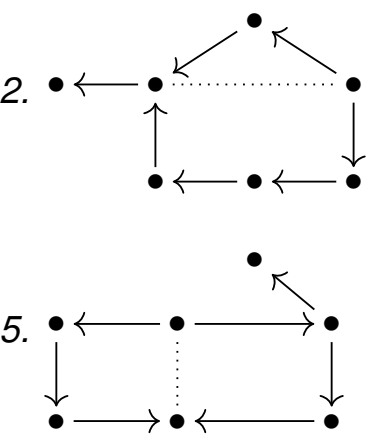

8.

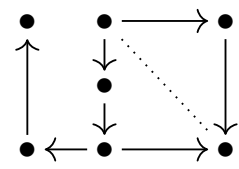

11.

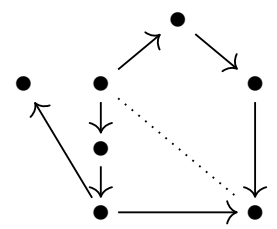

14.

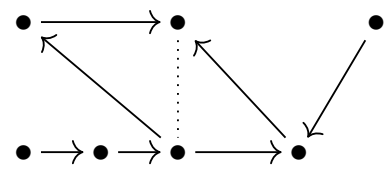

17.

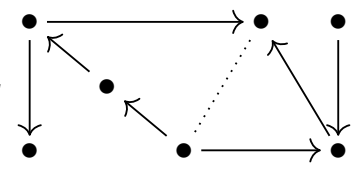

3.

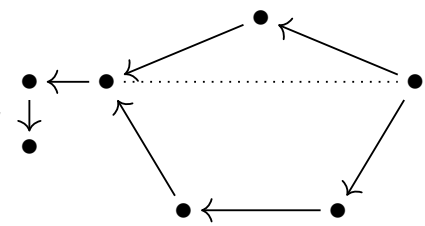

6.

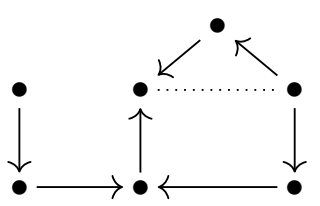

9.<smiles>CC1CCCCC1C</smiles>

12.

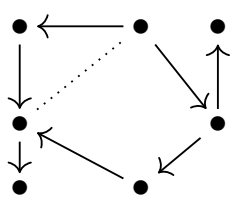

15.

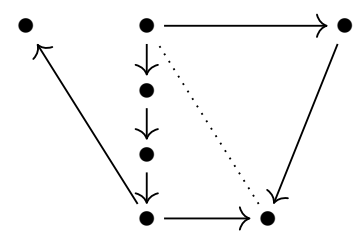

18.

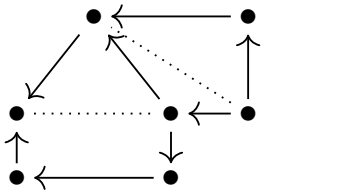



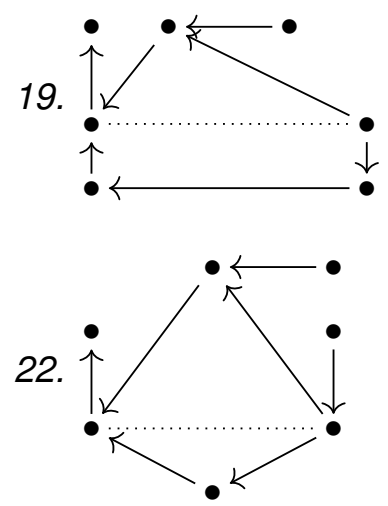

25.

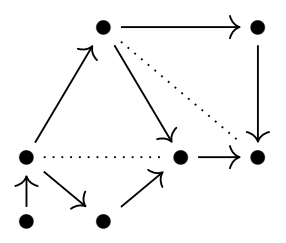

28.

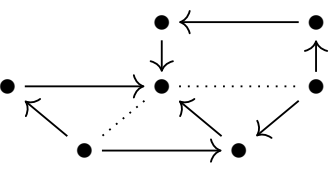

31.

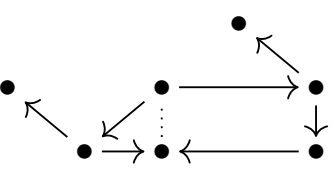

34.

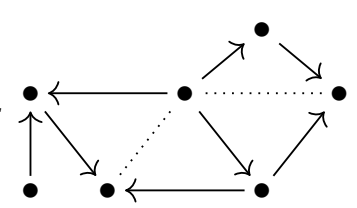

37.

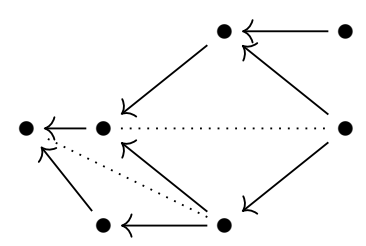

40.

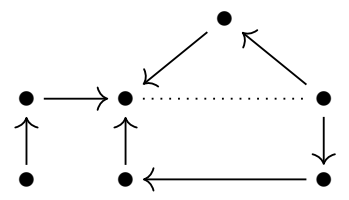

43.

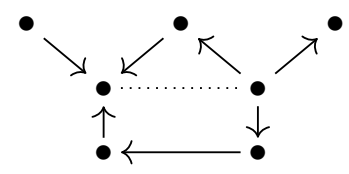

46.

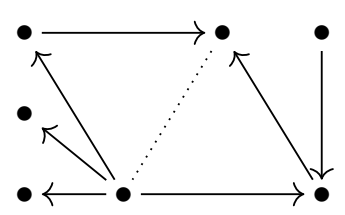

20.

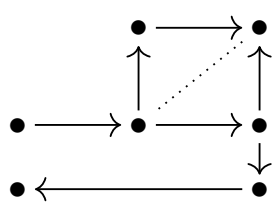

23.

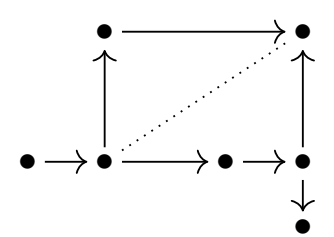

26.

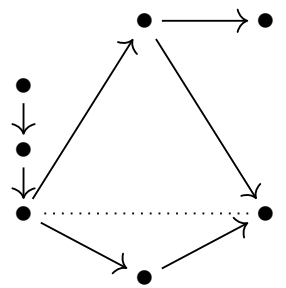

29.

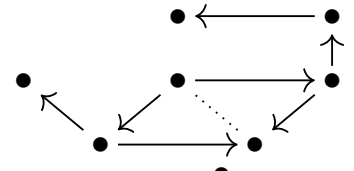

32.

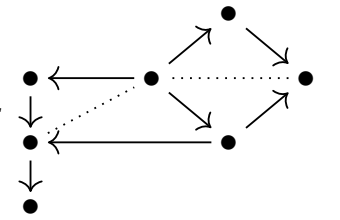

35.

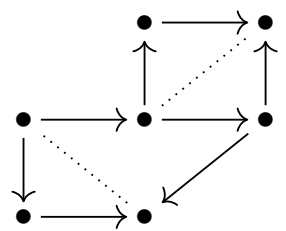

38.

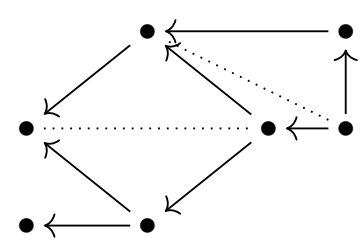

44.

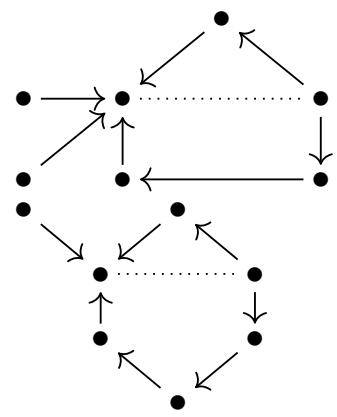

47.

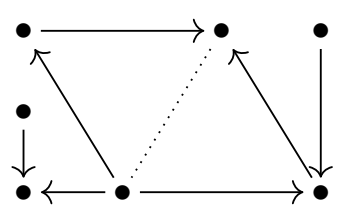

21.

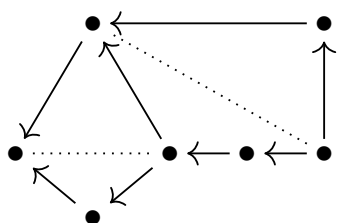

24.

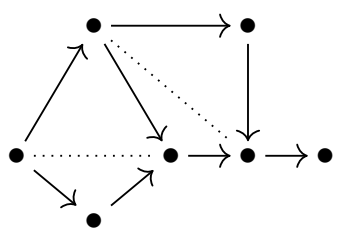

27.

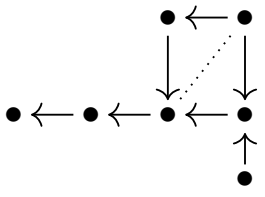

30.

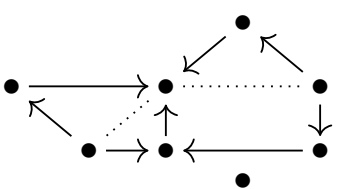

33.

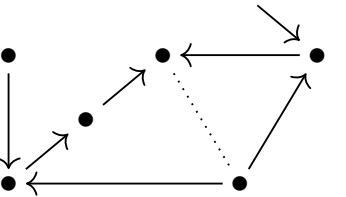

36.

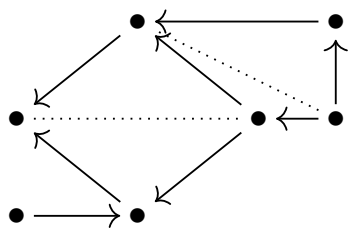

39.

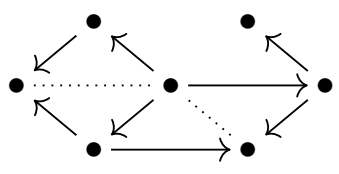

42.

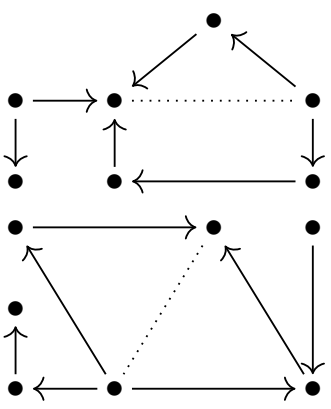

48.

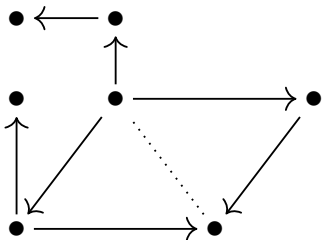


49.

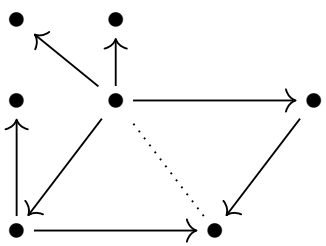

52.

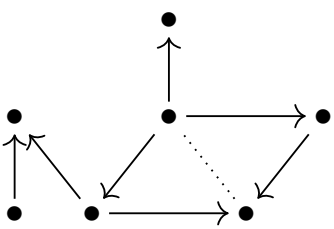

58.

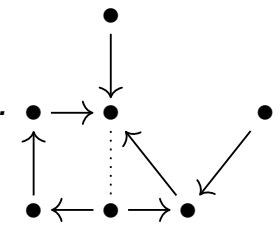

61.

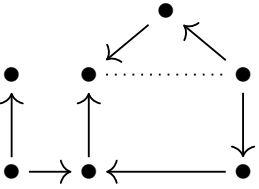

64.

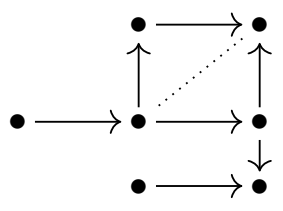

67.

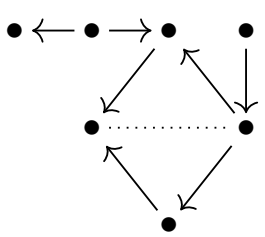

70.

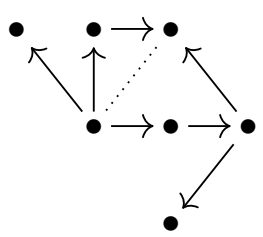

73.

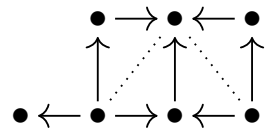

50.

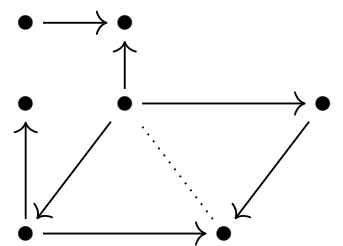

53.

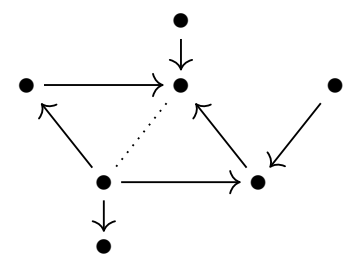

56.

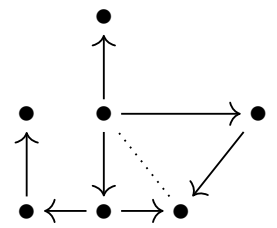

59.

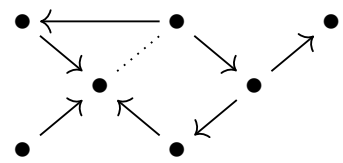

62.

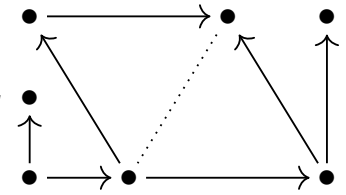

65.

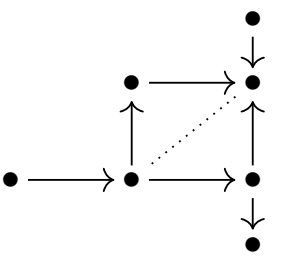

68.

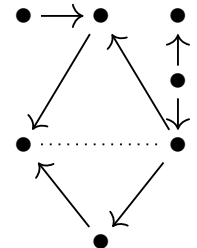

71.

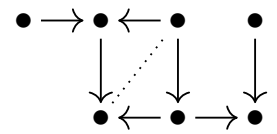

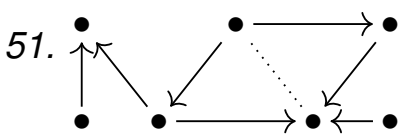

54.

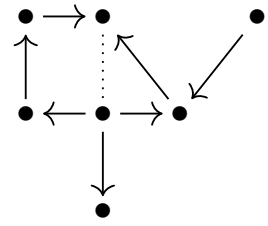

57.

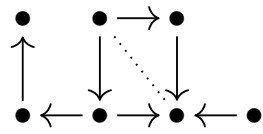

60.

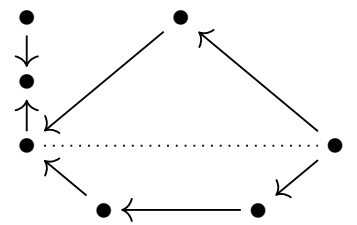

63.

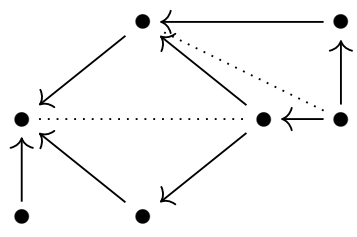

6.

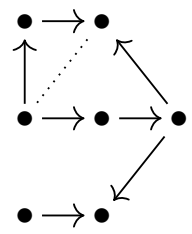

69.

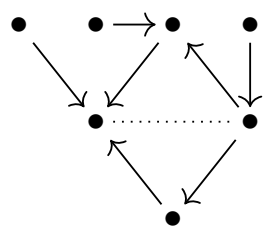

72.

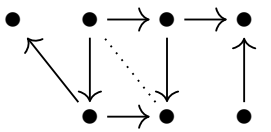

Demonstração Colocaremos as informações necessárias de cada extensão trivial no programa e exibiremos as soluções não hereditárias.

Começamos com a lista ordenada 3.2.7 [Fer99] que possui 27 extensões triviais. Dentre essas, 
observamos que apenas as extensões triviais (1), (2) e (3), através do corte, resultaram também em álgebra de incidência hereditárias.

(1)

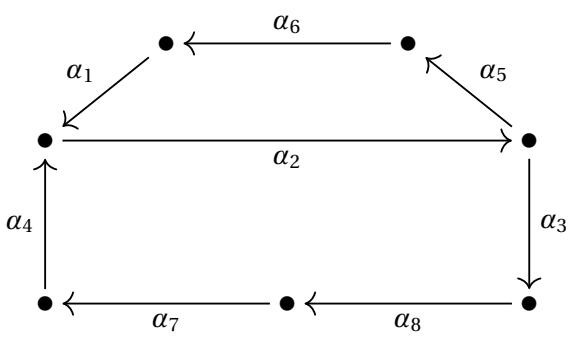

(2)

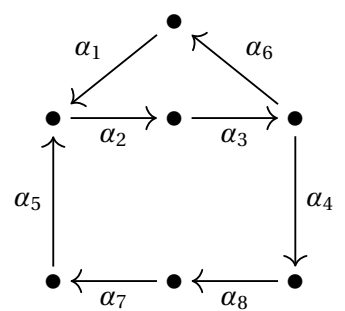

(3)

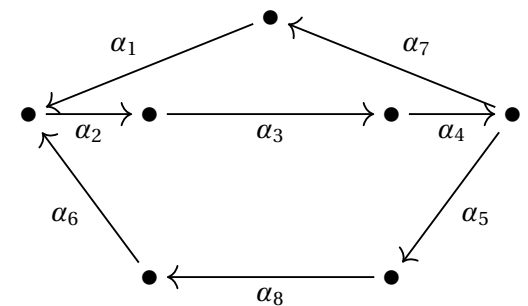

(4)

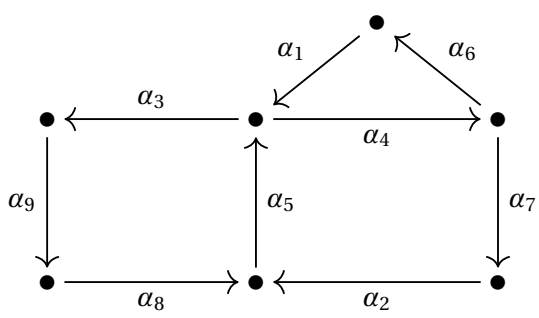

(5)

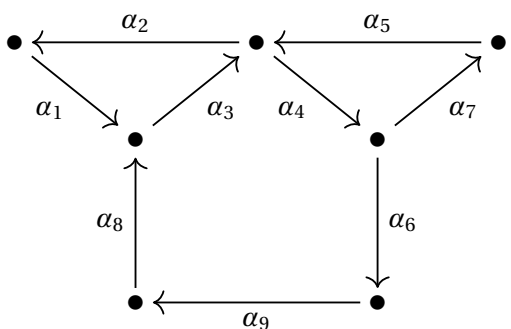

(6)

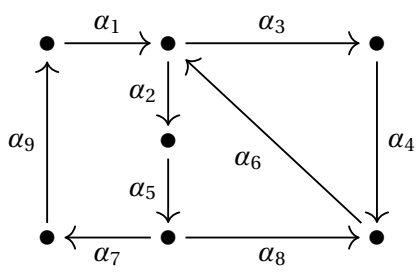

Essa álgebra é exatamente um dos exemplos trabaIhados no texto. As relações do tipo 2 são: $r 1=$ $\alpha_{1} \alpha_{2} \alpha_{3}$ e $r 2=\alpha_{4} \alpha_{2} \alpha_{5}$. Os ciclos elementares são: $C_{1}=\alpha_{1} \alpha_{2} \alpha_{5} \alpha_{6}$ e $C_{2}=\alpha_{4} \alpha_{2} \alpha_{3} \alpha_{8} \alpha_{7}$. Portanto temos apenas a solução 1 do teorema.

Essa extensão trivial tem as seguintes relações do tipo 2: $r 1=\alpha_{1} \alpha_{2} \alpha_{3} \alpha_{4}$ e $r 2=\alpha_{5} \alpha_{2} \alpha_{3} \alpha_{6}$.

Os ciclos elementares são: $C_{1}=\alpha_{1} \alpha_{2} \alpha_{3} \alpha_{6}$ e $C_{2}=$ $\alpha_{5} \alpha_{2} \alpha_{3} \alpha_{4} \alpha_{8} \alpha_{7}$. Resultando na álgebra 2 e a sua álgebra dual.

As relações do tipo 2 são: $r 1=\alpha_{1} \alpha_{2} \alpha_{3} \alpha_{4} \alpha_{5}$ e $r 2=\alpha_{6} \alpha_{2} \alpha_{3} \alpha_{4} \alpha_{7}$. Os ciclos elementares são: $C_{1}=$ $\alpha_{1} \alpha_{2} \alpha_{3} \alpha_{4} \alpha_{7}$ e $C_{2}=\alpha_{6} \alpha_{2} \alpha_{3} \alpha_{4} \alpha_{5} \alpha_{8}$. Logo, obtemos as álgebras 3,4 e a álgebra dual da 3 .

As relações do tipo 2 são: $r 1=\alpha_{1} \alpha_{3}, r 2=\alpha_{1} \alpha_{4} \alpha_{7}$, $r 3=\alpha_{8} \alpha_{5} \alpha_{4}, r 4=\alpha_{2} \alpha_{5} \alpha_{3}$ e $r 5=\alpha_{5} \alpha_{4} \alpha_{6}$. Os ciclos elementares são: $C_{1}=\alpha_{1} \alpha_{4} \alpha_{6}, C_{2}=\alpha_{3} \alpha_{9} \alpha_{8} \alpha_{5}$ e $C_{3}=\alpha_{7} \alpha_{2} \alpha_{5} \alpha_{4}$. Temos as soluções 5 e 6 do teorema.

As relações do tipo 2 são: $r 1=\alpha_{5} \alpha_{2}, r 2=\alpha_{5} \alpha_{4} \alpha_{6}$, $r 3=\alpha_{3} \alpha_{4} \alpha_{7}, r 4=\alpha_{8} \alpha_{3} \alpha_{2}$ e $r 5=\alpha_{1} \alpha_{3} \alpha_{4}$. Os ciclos elementares são: $C_{1}=\alpha_{2} \alpha_{1} \alpha_{3}, C_{2}=\alpha_{5} \alpha_{4} \alpha_{7}$ e $C_{3}=\alpha_{3} \alpha_{4} \alpha_{6} \alpha_{9} \alpha_{8}$. Portanto, aparecem a álgebra 7 e a sua álgebra dual.

As relações do tipo 2 são: $r 1=\alpha_{1} \alpha_{3}, r 2=\alpha_{4} \alpha_{6} \alpha_{2}$, $r 3=\alpha_{8} \alpha_{6} \alpha_{3}, r 4=\alpha_{6} \alpha_{2} \alpha_{5} \alpha_{7}$ e $r 5=\alpha_{1} \alpha_{2} \alpha_{5} \alpha_{8}$. Os ciclos elementares são: $C_{1}=\alpha_{1} \alpha_{2} \alpha_{5} \alpha_{7} \alpha_{9}, C_{2}=$ $\alpha_{3} \alpha_{4} \alpha_{6}$ e $C_{3}=\alpha_{6} \alpha_{2} \alpha_{5} \alpha_{8}$. Logo temos as álgebras 8 e 9 do teorema. 
(7)

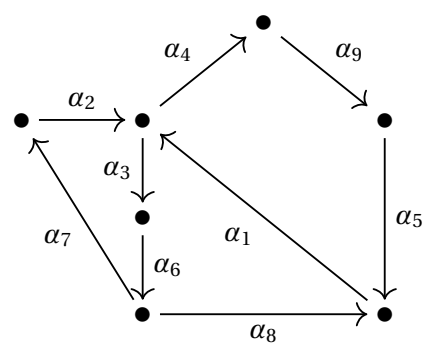

(8)

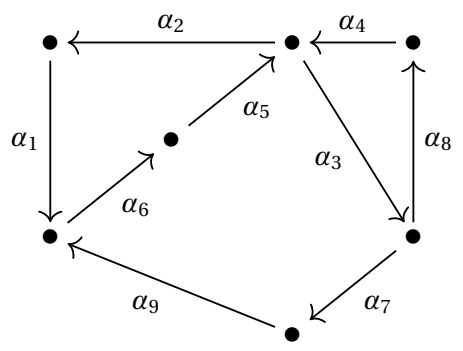

(9)

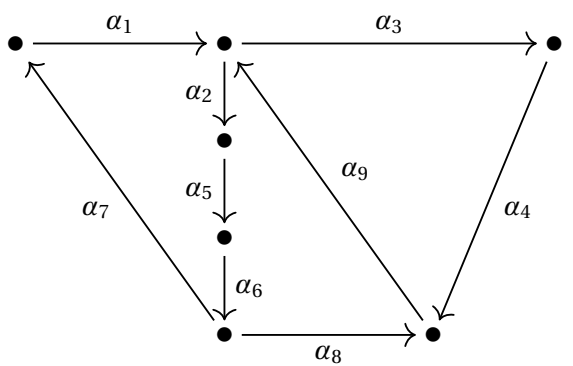

(10)

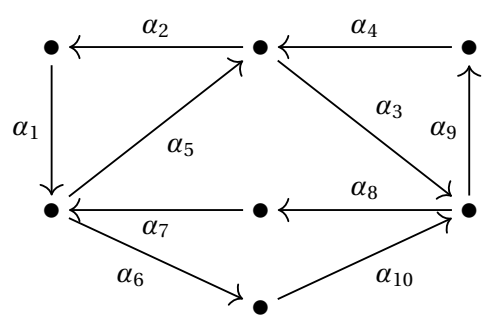

(11)

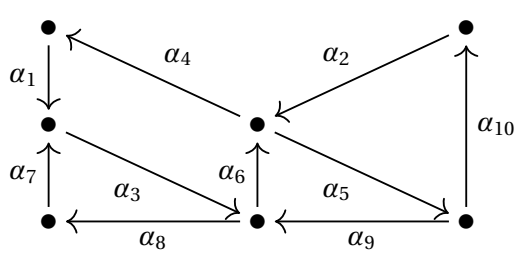

(12)

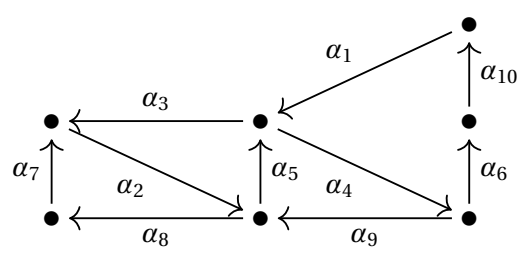

As relações do tipo 2 são: $r 1=\alpha_{2} \alpha_{4}, r 2=$ $\alpha_{2} \alpha_{3} \alpha_{6} \alpha_{8}, r 3=\alpha_{1} \alpha_{3} \alpha_{6} \alpha_{7}, r 4=\alpha_{5} \alpha_{1} \alpha_{3}$ e $r 5=$ $\alpha_{8} \alpha_{1} \alpha_{4}$. Os ciclos elementares são: $C_{1}=\alpha_{2} \alpha_{3} \alpha_{6} \alpha_{7}$, $C_{2}=\alpha_{4} \alpha_{9} \alpha_{5} \alpha_{1}$ e $C_{3}=\alpha_{1} \alpha_{3} \alpha_{6} \alpha_{8}$. Obtemos as álgebras 10 e 11 do teorema.

As relações do tipo 2 são: $r 1=\alpha_{4} \alpha_{2}, \quad r 2=$ $\alpha_{1} \alpha_{6} \alpha_{5} \alpha_{3}, r 3=\alpha_{9} \alpha_{6} \alpha_{5} \alpha_{2}, r 4=\alpha_{5} \alpha_{3} \alpha_{8}$ e $r 5=$ $\alpha_{4} \alpha_{3} \alpha_{7}$. Os ciclos elementares são: $C_{1}=\alpha_{3} \alpha_{8} \alpha_{4}$, $C_{2}=\alpha_{5} \alpha_{3} \alpha_{7} \alpha_{9} \alpha_{6}$ e $C_{3}=\alpha_{6} \alpha_{5} \alpha_{2} \alpha_{1}$. O programa mostra as soluções 12 e 13 do teorema.

As relações do tipo 2 são: $r 1=\alpha_{1} \alpha_{3}, \quad r 2=$ $\alpha_{1} \alpha_{2} \alpha_{5} \alpha_{6} \alpha_{8}, r 3=\alpha_{9} \alpha_{2} \alpha_{5} \alpha_{6} \alpha_{7}, r 4=\alpha_{8} \alpha_{9} \alpha_{3} \mathrm{e}$ $r 5=\alpha_{4} \alpha_{9} \alpha_{2}$. Os ciclos elementares são: $C_{1}=$ $\alpha_{3} \alpha_{4} \alpha_{9}, C_{2}=\alpha_{9} \alpha_{2} \alpha_{5} \alpha_{6} \alpha_{8}$ e $C_{3}=\alpha_{1} \alpha_{2} \alpha_{5} \alpha_{6} \alpha_{7}$. O programa mostra as soluções 14 e 15 do teorema.

As relações do tipo 2 são: $r 1=\alpha_{4} \alpha_{2}, \quad r 2=$ $\alpha_{4} \alpha_{3} \alpha_{8}, r 3=\alpha_{5} \alpha_{3} \alpha_{9}, r 4=\alpha_{1} \alpha_{5} \alpha_{3}, r 5=\alpha_{7} \alpha_{5} \alpha_{2}$, $r 6=\alpha_{1} \alpha_{6}, r 7=\alpha_{10} \alpha_{9}, r 8=\alpha_{10} \alpha_{8} \alpha_{7} \alpha_{5}$ e $r 9=$ $\alpha_{3} \alpha_{8} \alpha_{7} \alpha_{6}$. Os ciclos elementares são: $C_{1}=\alpha_{2} \alpha_{1} \alpha_{5}$, $C_{2}=\alpha_{5} \alpha_{3} \alpha_{8} \alpha_{7}, C_{3}=\alpha_{6} \alpha_{10} \alpha_{8} \alpha_{7}$ e $C_{4}=\alpha_{9} \alpha_{4} \alpha_{3}$. E não tem nenhum corte que resulte numa álgebra de incidência.

As relações do tipo 2 são: $r 1=\alpha_{1} \alpha_{3} \alpha_{8}, r 2=$ $\alpha_{7} \alpha_{3} \alpha_{6}, r 3=\alpha_{2} \alpha_{4}, r 4=\alpha_{9} \alpha_{6} \alpha_{4}, r 5=\alpha_{3} \alpha_{6} \alpha_{5}$, $r 6=\alpha_{9} \alpha_{8}, r 7=\alpha_{2} \alpha_{5} \alpha_{9}$ e $r 8=\alpha_{6} \alpha_{5} \alpha_{10}$. Os ciclos elementares são: $C_{1}=\alpha_{10} \alpha_{2} \alpha_{5}, C_{2}=\alpha_{5} \alpha_{9} \alpha_{6}$, $C_{3}=\alpha_{4} \alpha_{1} \alpha_{3} \alpha_{6}$ e $C_{4}=\alpha_{7} \alpha_{3} \alpha_{8}$. Logo, existe a álgebra dual da solução 21 .

As relações do tipo 2 são: $r 1=\alpha_{3} \alpha_{2} \alpha_{8}, r 2=$ $\alpha_{7} \alpha_{2} \alpha_{5}, r 3=\alpha_{9} \alpha_{8}, r 4=\alpha_{1} \alpha_{3}, r 5=\alpha_{2} \alpha_{5} \alpha_{4}$, $r 6=\alpha_{9} \alpha_{5} \alpha_{3}, r 7=\alpha_{5} \alpha_{4} \alpha_{6}$ e $r 8=\alpha_{1} \alpha_{4} \alpha_{9}$. Os ciclos elementares são: $C_{1}=\alpha_{1} \alpha_{4} \alpha_{10} \alpha_{6}, C_{2}=\alpha_{5} \alpha_{4} \alpha_{9}$, $C_{3}=\alpha_{3} \alpha_{2} \alpha_{5}$ e $C_{4}=\alpha_{7} \alpha_{2} \alpha_{8}$. Obtemos a álgebra 16. 
(13)

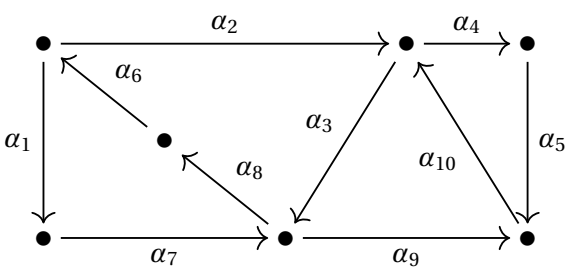

(14)

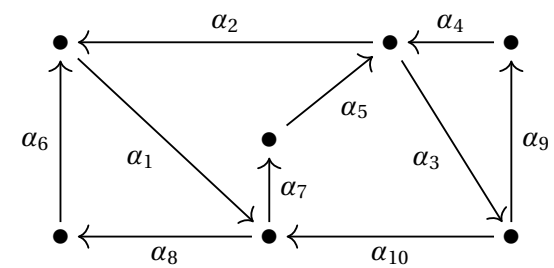

(15)

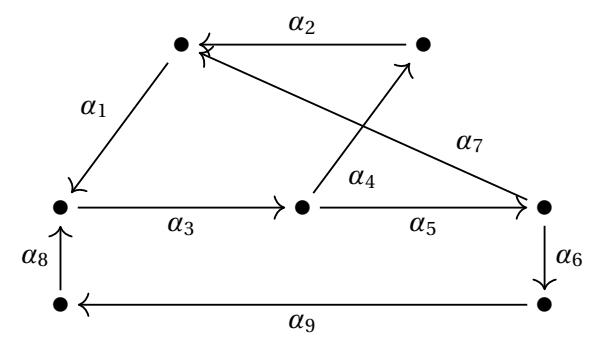

(16)

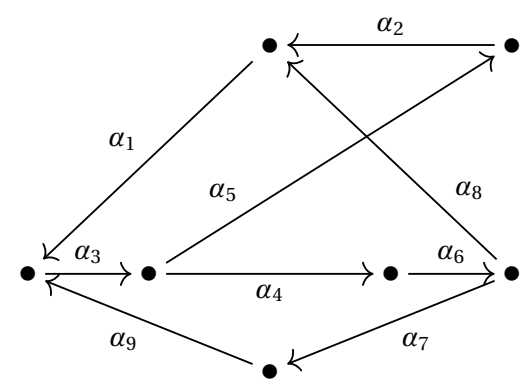

(17)

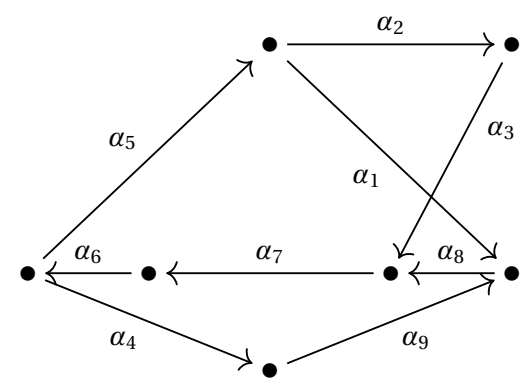

As relações do tipo 2 são: $r 1=\alpha_{2} \alpha_{4}, r 2=\alpha_{1} \alpha_{3} \alpha_{8}$, $r 3=\alpha_{7} \alpha_{3} \alpha_{6}, r 4=\alpha_{9} \alpha_{8}, r 5=\alpha_{3} \alpha_{6} \alpha_{5}, r 6=$ $\alpha_{9} \alpha_{6} \alpha_{4}, r 7=\alpha_{2} \alpha_{5} \alpha_{9}$ e $r 8=\alpha_{6} \alpha_{5} \alpha_{10}$. Os ciclos elementares são: $C_{1}=\alpha_{4} \alpha_{1} \alpha_{3} \alpha_{6}, C_{2}=\alpha_{2} \alpha_{5} \alpha_{10}$, $C_{3}=\alpha_{6} \alpha_{5} \alpha_{9}$ e $C_{4}=\alpha_{7} \alpha_{3} \alpha_{8}$. O programa mostra apenas a álgebra 17.

Essa extensão trivial tem as seguintes relações do tipo 2: $r 1=\alpha_{6} \alpha_{1} \alpha_{7}, r 2=\alpha_{2} \alpha_{1} \alpha_{8}, r 3=\alpha_{10} \alpha_{8}$, $r 4=\alpha_{1} \alpha_{7} \alpha_{5} \alpha_{3}, r 5=\alpha_{10} \alpha_{7} \alpha_{5} \alpha_{2}, r 6=\alpha_{4} \alpha_{2}, r 7=$ $\alpha_{4} \alpha_{3} \alpha_{10}$ e $r 8=\alpha_{5} \alpha_{3} \alpha_{9}$.

Os ciclos elementares são: $C_{1}=\alpha_{2} \alpha_{1} \alpha_{7} \alpha_{5}, C_{2}=$ $\alpha_{6} \alpha_{1} \alpha_{8}, C_{3}=\alpha_{10} \alpha_{7} \alpha_{5} \alpha_{3}$ e $C_{4}=\alpha_{4} \alpha_{3} \alpha_{9}$. Portanto não temos nenhuma álgebra de incidência originada pelo corte.

Essa extensão trivial tem as seguintes relações do tipo 2: $r 1=\alpha_{1} \alpha_{3} \alpha_{5} \alpha_{6}, r 2=\alpha_{8} \alpha_{3} \alpha_{5} \alpha_{7}, r 3=$ $\alpha_{8} \alpha_{3} \alpha_{4}, r 4=\alpha_{2} \alpha_{1} \alpha_{3} \alpha_{5}$ e $r 5=\alpha_{7} \alpha_{1} \alpha_{3} \alpha_{4}$.

Os ciclos elementares são: $C_{1}=\alpha_{1} \alpha_{3} \alpha_{4} \alpha_{2}, C_{2}=$ $\alpha_{1} \alpha_{3} \alpha_{5} \alpha_{7}$ e $C_{3}=\alpha_{3} \alpha_{5} \alpha_{6} \alpha_{9} \alpha_{8}$. Assim, resultam nas soluções 18,19 e 20.

As relações do tipo 2 são: $r 1=\alpha_{9} \alpha_{3} \alpha_{5}, r 2=$ $\alpha_{9} \alpha_{3} \alpha_{4} \alpha_{6} \alpha_{8}, r 3=\alpha_{1} \alpha_{3} \alpha_{4} \alpha_{6} \alpha_{7}, r 4=\alpha_{2} \alpha_{1} \alpha_{3} \alpha_{4}$ e $r 5=\alpha_{8} \alpha_{1} \alpha_{3} \alpha_{5}$. Os ciclos elementares são: $C_{1}=$ $\alpha_{3} \alpha_{5} \alpha_{2} \alpha_{1}, C_{2}=\alpha_{3} \alpha_{4} \alpha_{6} \alpha_{8} \alpha_{1}$ e $C_{3}=\alpha_{3} \alpha_{4} \alpha_{6} \alpha_{7} \alpha_{9}$. Portanto, temos as soluções 21, 22 e 23.

As relações do tipo 2 são: $r 1=\alpha_{1} \alpha_{8} \alpha_{7} \alpha_{6} \alpha_{4}, r 2=$ $\alpha_{3} \alpha_{7} \alpha_{6} \alpha_{4}, r 3=\alpha_{9} \alpha_{8} \alpha_{7} \alpha_{6} \alpha_{5}, r 4=\alpha_{8} \alpha_{7} \alpha_{6} \alpha_{5} \alpha_{2} \mathrm{e}$ $r 5=\alpha_{3} \alpha_{7} \alpha_{6} \alpha_{5} \alpha_{1}$. Os ciclos elementares são: $C_{1}=$ $\alpha_{2} \alpha_{3} \alpha_{7} \alpha_{6} \alpha_{5}, C_{2}=\alpha_{1} \alpha_{8} \alpha_{7} \alpha_{6} \alpha_{5}$ e $C_{3}=\alpha_{4} \alpha_{9} \alpha_{8} \alpha_{7} \alpha_{6}$. O programa exibe as soluções $24,25,26$ e 27 . 
(18)

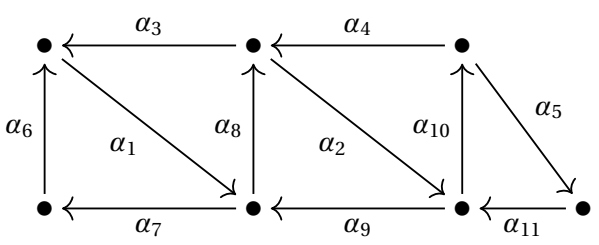

(19)

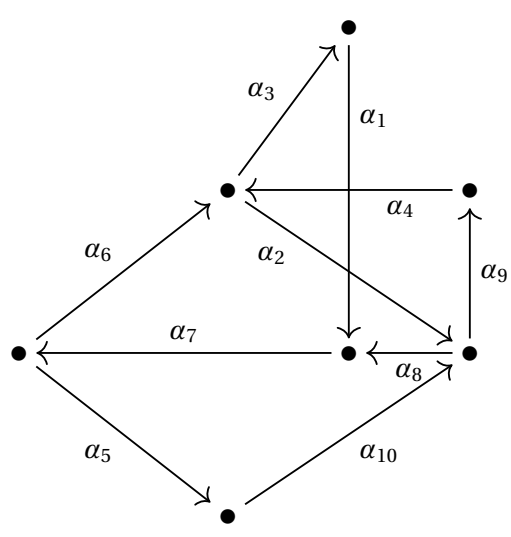

(20)

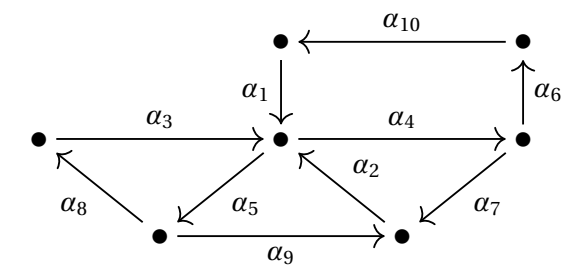

(21)

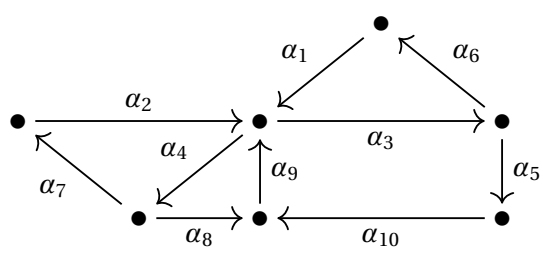

(22)

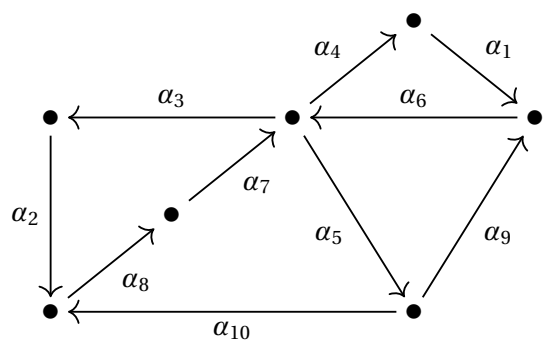

As relações do tipo 2 são: $r 1=\alpha_{6} \alpha_{1} \alpha_{8}, r 2=$ $\alpha_{3} \alpha_{1} \alpha_{7}, r 3=\alpha_{1} \alpha_{8} \alpha_{2}, r 4=\alpha_{9} \alpha_{8} \alpha_{3}, r 5=\alpha_{8} \alpha_{2} \alpha_{10}$, $r 6=\alpha_{4} \alpha_{2} \alpha_{9}, r 7=\alpha_{2} \alpha_{10} \alpha_{5}, r 8=\alpha_{11} \alpha_{10} \alpha_{4}, r 9=$ $\alpha_{11} \alpha_{9}, r 10=\alpha_{9} \alpha_{7}$ e $r 11=\alpha_{4} \alpha_{3}$. Os ciclos elementares são: $C_{1}=\alpha_{1} \alpha_{7} \alpha_{6}, C_{2}=\alpha_{1} \alpha_{8} \alpha_{3}, C_{3}=\alpha_{9} \alpha_{8} \alpha_{2}$, $C_{4}=\alpha_{2} \alpha_{10} \alpha_{4}$ e $C_{5}=\alpha_{11} \alpha_{10} \alpha_{5}$. Não temos nenhuma álgebra de incidência originada por corte.

As relações do tipo 2 são: $r 1=\alpha_{10} \alpha_{8} \alpha_{7} \alpha_{6}, r 2=$ $\alpha_{2} \alpha_{8} \alpha_{7} \alpha_{5}, r 3=\alpha_{10} \alpha_{9}, r 4=\alpha_{1} \alpha_{7} \alpha_{5}, r 5=\alpha_{4} \alpha_{3}$, $r 6=\alpha_{6} \alpha_{2} \alpha_{9}, r 7=\alpha_{4} \alpha_{2} \alpha_{8}, r 8=\alpha_{8} \alpha_{7} \alpha_{6} \alpha_{3} \mathrm{e}$ $r 9=\alpha_{1} \alpha_{7} \alpha_{6} \alpha_{2}$. Os ciclos elementares são: $C_{1}=$ $\alpha_{6} \alpha_{3} \alpha_{1} \alpha_{7}, C_{2}=\alpha_{4} \alpha_{2} \alpha_{9}, C_{3}=\alpha_{6} \alpha_{2} \alpha_{8} \alpha_{7}$ e $C_{4}=$ $\alpha_{8} \alpha_{7} \alpha_{5} \alpha_{10}$. Dessa extensão trivial não resulta em nenhuma álgebra de incidência.

As relações do tipo 2 são: $r 1=\alpha_{3} \alpha_{5} \alpha_{9}, r 2=$ $\alpha_{2} \alpha_{5} \alpha_{8}, r 3=\alpha_{3} \alpha_{4}, r 4=\alpha_{1} \alpha_{5}, r 5=\alpha_{9} \alpha_{2} \alpha_{4}$, $r 6=\alpha_{7} \alpha_{2} \alpha_{5}, r 7=\alpha_{2} \alpha_{4} \alpha_{6}$ e $r 8=\alpha_{1} \alpha_{4} \alpha_{7}$. Os ciclos elementares são: $C_{1}=\alpha_{1} \alpha_{4} \alpha_{6} \alpha_{10}, C_{2}=\alpha_{5} \alpha_{8} \alpha_{3}$, $C_{3}=\alpha_{9} \alpha_{2} \alpha_{5}$ e $C_{4}=\alpha_{7} \alpha_{2} \alpha_{4}$. Conseguimos as soluções 28 e 29.

As relações do tipo 2 são: $r 1=\alpha_{9} \alpha_{3} \alpha_{6}, r 2=$ $\alpha_{1} \alpha_{3} \alpha_{5}, r 3=\alpha_{1} \alpha_{4}, r 4=\alpha_{10} \alpha_{9} \alpha_{4}, r 5=\alpha_{8} \alpha_{9} \alpha_{3}$, $r 6=\alpha_{9} \alpha_{4} \alpha_{7}$ e $r 7=\alpha_{2} \alpha_{4} \alpha_{8}$. Os ciclos elementares são: $C_{1}=\alpha_{3} \alpha_{6} \alpha_{1}, C_{2}=\alpha_{3} \alpha_{5} \alpha_{10} \alpha_{9}, C_{3}=\alpha_{9} \alpha_{4} \alpha_{8}$ e $C_{4}=\alpha_{2} \alpha_{4} \alpha_{7}$. Portanto, aparecem as álgebras $30 \mathrm{e}$ 31 .

As relações do tipo 2 são: $r 1=\alpha_{1} \alpha_{6} \alpha_{5}, r 2=$ $\alpha_{9} \alpha_{6} \alpha_{4}, r 3=\alpha_{7} \alpha_{4}, r 4=\alpha_{6} \alpha_{3}, r 5=\alpha_{6} \alpha_{5} \alpha_{10}$, $r 6=\alpha_{7} \alpha_{5} \alpha_{9}, r 7=\alpha_{10} \alpha_{8} \alpha_{7} \alpha_{3}$ e $r 8=\alpha_{2} \alpha_{8} \alpha_{7} \alpha_{5}$. Os ciclos elementares são: $C_{1}=\alpha_{1} \alpha_{6} \alpha_{4}, C_{2}=$ $\alpha_{8} \alpha_{7} \alpha_{5} \alpha_{10}, C_{3}=\alpha_{8} \alpha_{7} \alpha_{3} \alpha_{2}$ e $C_{4}=\alpha_{5} \alpha_{9} \alpha_{6}$. O programa exibe as soluções 32 e 33 . 
(23)

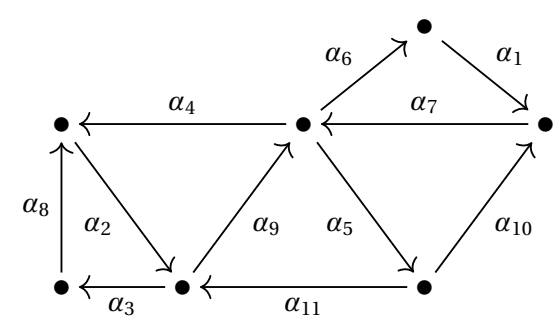

(24)

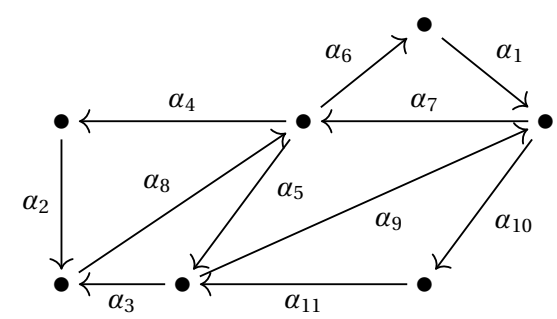

(25)

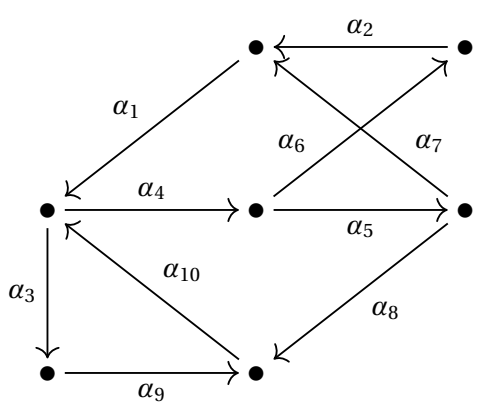

(26)

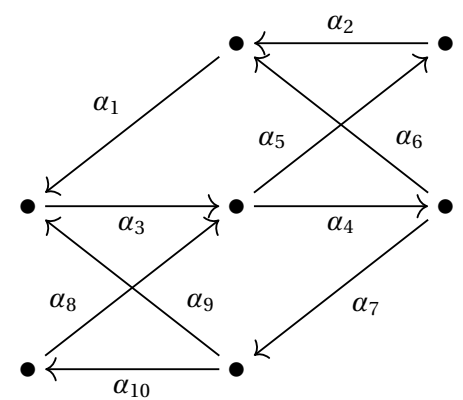

(27)

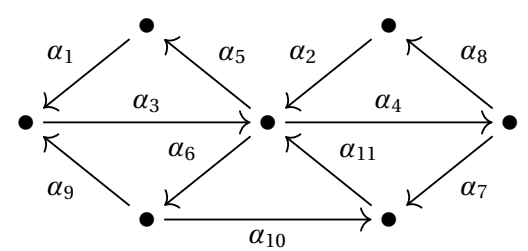

As relações do tipo 2 são: $r 1=\alpha_{1} \alpha_{7} \alpha_{5}, r 2=$ $\alpha_{10} \alpha_{7} \alpha_{6}, r 3=\alpha_{9} \alpha_{6}, r 4=\alpha_{7} \alpha_{4}, r 5=\alpha_{7} \alpha_{5} \alpha_{11}, r 6=$ $\alpha_{9} \alpha_{5} \alpha_{10}, r 7=\alpha_{11} \alpha_{3}, r 8=\alpha_{11} \alpha_{9} \alpha_{4}, r 9=\alpha_{2} \alpha_{9} \alpha_{5}$, $r 10=\alpha_{8} \alpha_{2} \alpha_{9}$ e $r 11=\alpha_{4} \alpha_{2} \alpha_{3}$. Os ciclos elementares são: $C_{1}=\alpha_{6} \alpha_{1} \alpha_{7}, C_{2}=\alpha_{5} \alpha_{10} \alpha_{7}, C_{3}=\alpha_{5} \alpha_{11} \alpha_{9}$, $C_{4}=\alpha_{9} \alpha_{4} \alpha_{2}$ e $C_{5}=\alpha_{2} \alpha_{3} \alpha_{8}$. Portanto, conseguimos a álgebra 34 .

As relações do tipo 2 são: $r 1=\alpha_{1} \alpha_{7} \alpha_{5}, r 2=$ $\alpha_{9} \alpha_{7} \alpha_{6}, r 3=\alpha_{1} \alpha_{10}, r 4=\alpha_{8} \alpha_{6}, r 5=\alpha_{7} \alpha_{4}$, $r 6=\alpha_{11} \alpha_{3}, r 7=\alpha_{5} \alpha_{9} \alpha_{10}, r 8=\alpha_{11} \alpha_{9} \alpha_{7}, r 9=$ $\alpha_{8} \alpha_{5} \alpha_{9}, r 10=\alpha_{7} \alpha_{5} \alpha_{3}, r 11=\alpha_{2} \alpha_{8} \alpha_{5}$ e $r 12=$ $\alpha_{3} \alpha_{8} \alpha_{4}$. Os ciclos elementares são: $C_{1}=\alpha_{1} \alpha_{7} \alpha_{6}$, $C_{2}=\alpha_{10} \alpha_{11} \alpha_{9}, C_{3}=\alpha_{9} \alpha_{7} \alpha_{5}, C_{4}=\alpha_{5} \alpha_{3} \alpha_{8} \mathrm{e}$ $C_{5}=\alpha_{8} \alpha_{4} \alpha_{2}$. Não temos soluções.

Essa extensão trivial tem as seguintes relações do tipo 2: $r 1=\alpha_{9} \alpha_{10} \alpha_{4}, r 2=\alpha_{8} \alpha_{10} \alpha_{3}, r 3=\alpha_{1} \alpha_{4} \alpha_{5} \alpha_{8}$, $r 4=\alpha_{10} \alpha_{4} \alpha_{6}, r 5=\alpha_{10} \alpha_{4} \alpha_{5} \alpha_{7}, r 6=\alpha_{1} \alpha_{3}, r 7=$ $\alpha_{2} \alpha_{1} \alpha_{4} \alpha_{5}$ e $r 8=\alpha_{7} \alpha_{1} \alpha_{4} \alpha_{6}$.

Os ciclos elementares são: $C_{1}=\alpha_{3} \alpha_{9} \alpha_{10}, C_{2}=$ $\alpha_{8} \alpha_{10} \alpha_{4} \alpha_{5}, C_{3}=\alpha_{6} \alpha_{2} \alpha_{1} \alpha_{4}$ e $C_{4}=\alpha_{7} \alpha_{1} \alpha_{4} \alpha_{5}$. Logo os cortes resultam nas álgebras 35 e 36 .

As relações do tipo 2 são: $r 1=\alpha_{1} \alpha_{3} \alpha_{4} \alpha_{7}, r 2=$ $\alpha_{9} \alpha_{3} \alpha_{5}, r 3=\alpha_{9} \alpha_{3} \alpha_{4} \alpha_{6}, r 4=\alpha_{8} \alpha_{5}, r 5=\alpha_{8} \alpha_{4} \alpha_{6}$, $r 6=\alpha_{2} \alpha_{1} \alpha_{3} \alpha_{4}, r 7=\alpha_{6} \alpha_{1} \alpha_{3} \alpha_{5}, r 8=\alpha_{3} \alpha_{4} \alpha_{7} \alpha_{10}$ e $r 9=\alpha_{8} \alpha_{4} \alpha_{7} \alpha_{9}$. Os ciclos elementares são: $C_{1}=$ $\alpha_{5} \alpha_{2} \alpha_{1} \alpha_{3}, C_{2}=\alpha_{6} \alpha_{1} \alpha_{3} \alpha_{4}, C_{3}=\alpha_{3} \alpha_{4} \alpha_{7} \alpha_{9}$ e $C_{4}=$ $\alpha_{4} \alpha_{7} \alpha_{10} \alpha_{8}$. Conseguimos as soluções 37 e 38 .

As relações do tipo 2 são: $r 1=\alpha_{1} \alpha_{3} \alpha_{6}, r 2=$ $\alpha_{9} \alpha_{3} \alpha_{5}, \quad r 3=\alpha_{2} \alpha_{5}, \quad r 4=\alpha_{11} \alpha_{5}, \quad r 5=\alpha_{3} \alpha_{4}$, $r 6=\alpha_{3} \alpha_{6} \alpha_{10}, r 7=\alpha_{11} \alpha_{6} \alpha_{9}, \quad r 8=\alpha_{10} \alpha_{11} \alpha_{4}$, $r 9=\alpha_{7} \alpha_{11} \alpha_{6}, r 10=\alpha_{11} \alpha_{4} \alpha_{8}, r 11=\alpha_{2} \alpha_{4} \alpha_{7} \mathrm{e}$ $r 12=\alpha_{2} \alpha_{6}$. Os ciclos elementares são: $C_{1}=$ $\alpha_{1} \alpha_{3} \alpha_{5}, C_{2}=\alpha_{2} \alpha_{4} \alpha_{8}, C_{3}=\alpha_{3} \alpha_{6} \alpha_{9}, C_{4}=\alpha_{6} \alpha_{10} \alpha_{11}$ e $C_{5}=\alpha_{4} \alpha_{7} \alpha_{11}$. Portanto, obtemos a álgebra 39 e a sua álgebra dual .

O último passo é fazer o mesmo processo para a lista enumerada do teorema 3.3.16 do trabalho 
de Fernández [Fer99]. O corte em cada uma dessas extensões triviais resultaram em álgebras de incidência hereditárias apenas nas álgebras (1), (2), (3), (5), (6), (8), (10), (11), (12), (13), (14), (16), (27), (28) e (29).

Algumas extensões triviais têm um vértice $\square$. No vértice $\square$ é anexado uma extensão trivial da classe de Cartan de tipo $A_{n}$, o número $n$ é igual a quantidade de vértices • que faltam para completar sete $\left(\mathbb{E}_{7}\right)$. O primeiro passo é fazer o vértice $\square$ como um normal $\bullet$, ver as relações do tipo 2 e os ciclos elementares. O próximo passo é listar as possíveis extensões triviais que podem ser anexadas no vértice $\square$, completar as informações sobre as relações do tipo 2 e os ciclos elementares da extensão trivial com sete vértices. Conforme a lista abaixo, anexaremos apenas as extensões triviais de tipo $A_{3}$. Com o intuito de resumir a demonstração, faremos algumas observações:

- No tipo $A_{3}$, duas opções aparecem para anexar no vértice $\square$,

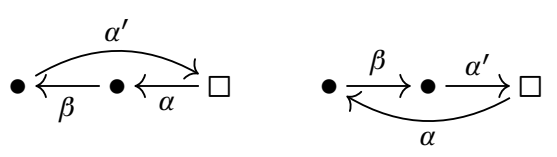

A flecha $\beta$ não faz parte das relações de tipo 2 , as flechas $\alpha^{\prime}$ têm o mesmo término, as flechas $\alpha$ têm a mesma origem, então colocaremos uma aresta (sem orientação) no lugar da flecha $\beta$ e calcularemos a presença dos cortes que origina as álgebras de incidência na extensão trivial com esse anexo:

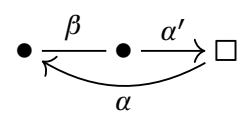

Assim, estamos analisando os dois casos equivalentes e a aresta deve ser orientada conformes essas opções.

- Existem duas opções equivalentes de extensões triviais da classe de Cartan de tipo $A_{3}$ :
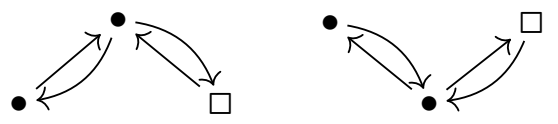

- E a última opção de extensão trivial da classe de Cartan de tipo $A_{3}$ é:

(1)

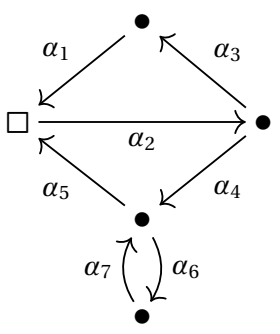

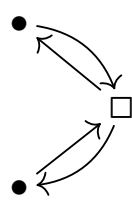

As relações do tipo 2 são: $r 1=\alpha_{5} \alpha_{2} \alpha_{3}, r 2=$ $\alpha_{1} \alpha_{2} \alpha_{4}, r 3=\alpha_{4} \alpha_{6}$ e $r 4=\alpha_{7} \alpha_{5}$.

Os ciclos elementares são: $C_{1}=\alpha_{2} \alpha_{3} \alpha_{1}, C_{2}=$ $\alpha_{2} \alpha_{4} \alpha_{5}$ e $C_{3}=\alpha_{6} \alpha_{7}$ 

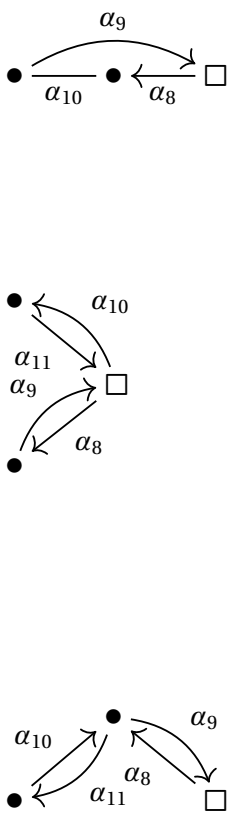

(2)
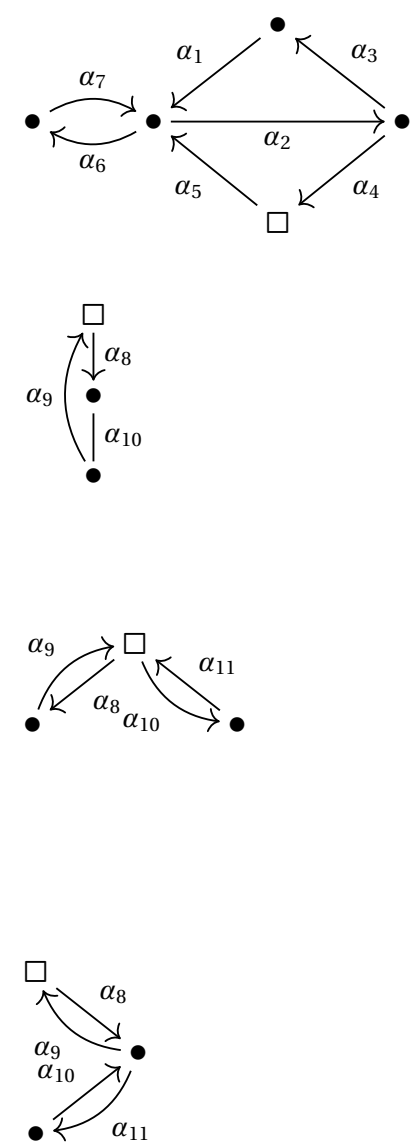

Completando, temos mais essas relações do tipo 2: $r 5=\alpha_{1} \alpha_{8}, r 6=\alpha_{5} \alpha_{8}$ e $r 7=\alpha_{9} \alpha_{2}$.

E o ciclo elementar é $C_{4}=\alpha_{8} \alpha_{10} \alpha_{9}$. O programa exibe uma solução hereditária.

Nesse caso, temos mais essas relações do tipo 2: $r 5=\alpha_{1} \alpha_{8}, r 6=\alpha_{5} \alpha_{8}, r 7=\alpha_{9} \alpha_{2}, r 8=\alpha_{1} \alpha_{10}, r 9=$ $\alpha_{5} \alpha_{10}, r 10=\alpha_{11} \alpha_{2}, r 11=\alpha_{9} \alpha_{10}$ e $r 12=\alpha_{11} \alpha_{8}$. E os ciclos elementares são $C_{4}=\alpha_{8} \alpha_{9}$ e $C_{5}=$ $\alpha_{11} \alpha_{10}$. Nesse caso, obtemos uma solução hereditária.

Nesse anexo, temos mais essas relações do tipo 2: $r 5=\alpha_{1} \alpha_{8}, r 6=\alpha_{5} \alpha_{8}, r 7=\alpha_{9} \alpha_{2}, r 8=\alpha_{10} \alpha_{9} \mathrm{e}$ $r 9=\alpha_{8} \alpha_{11}$.

$\mathrm{E}$ os ciclos elementares são $C_{4}=\alpha_{8} \alpha_{9}$ e $C_{5}=$ $\alpha_{11} \alpha_{10}$. Logo, temos um corte que origina uma álgebra de incidência hereditária.

Seguindo o raciocínio anterior, sejam as relações do tipo 2: $r 1=\alpha_{1} \alpha_{2} \alpha_{4}, r 2=\alpha_{5} \alpha_{2} \alpha_{3}, r 3=\alpha_{1} \alpha_{6}, r 4=$ $\alpha_{5} \alpha_{6}$ e $r 5=\alpha_{7} \alpha_{2}$.

Os ciclos elementares são: $C_{1}=\alpha_{1} \alpha_{2} \alpha_{3}, C_{2}=$ $\alpha_{2} \alpha_{4} \alpha_{5}$ e $C_{3}=\alpha_{6} \alpha_{7}$.

Completando, temos mais essas relações do tipo 2 : $r 6=\alpha_{4} \alpha_{8}$ e $r 7=\alpha_{9} \alpha_{5}$.

E o ciclo elementar é $C_{4}=\alpha_{8} \alpha_{10} \alpha_{9}$. O programa exibe apenas uma solução hereditária.

Nesse caso, temos mais essas relações do tipo 2: $r 6=\alpha_{4} \alpha_{8}, r 7=\alpha_{9} \alpha_{5}, r 8=\alpha_{4} \alpha_{10}, r 9=\alpha_{11} \alpha_{5}$, $r 10=\alpha_{9} \alpha_{10}$ e $r 11=\alpha_{11} \alpha_{8}$.

$\mathrm{E}$ os ciclos elementares são $C_{4}=\alpha_{8} \alpha_{9}$ e $C_{5}=$ $\alpha_{11} \alpha_{10}$. Nesse caso, obtemos uma solução hereditária.

Nesse anexo, temos mais essas relações do tipo 2: $r 6=\alpha_{4} \alpha_{8}, r 7=\alpha_{9} \alpha_{5}, r 8=\alpha_{10} \alpha_{9}$ e $r 9=\alpha_{8} \alpha_{11}$. $\mathrm{E}$ os ciclos elementares são $C_{4}=\alpha_{8} \alpha_{9}$ e $C_{5}=$ $\alpha_{10} \alpha_{11}$. Logo, temos um corte que origine uma álgebra de incidência hereditária. 
(3)
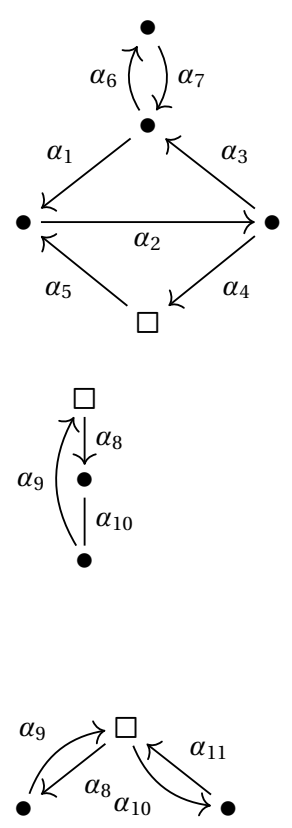

(4)

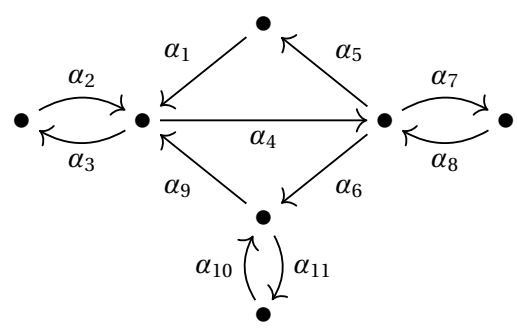

(5)
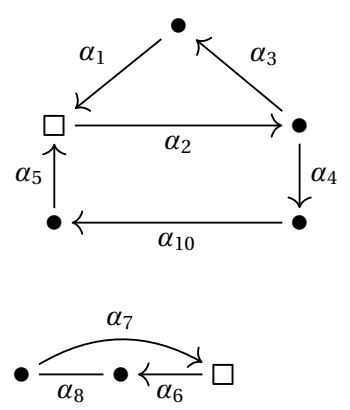

Analogamente aos dois anteriores, temos as relações do tipo 2: $r 1=\alpha_{1} \alpha_{2} \alpha_{4}, r 2=\alpha_{5} \alpha_{2} \alpha_{3}, r 3=$ $\alpha_{3} \alpha_{6}$ e $r 4=\alpha_{7} \alpha_{1}$. Os ciclos elementares são: $C_{1}=\alpha_{2} \alpha_{3} \alpha_{1}, C_{2}=\alpha_{2} \alpha_{4} \alpha_{5}$ e $C_{3}=\alpha_{6} \alpha_{7}$.

Completando, temos mais essas relações do tipo 2 : $r 5=\alpha_{4} \alpha_{8}$ e $r 6=\alpha_{9} \alpha_{5}$.

E o ciclo elementar é $C_{4}=\alpha_{8} \alpha_{10} \alpha_{9}$. O programa exibe duas soluções hereditárias.

Nesse caso, temos mais essas relações do tipo 2: $r 5=\alpha_{4} \alpha_{8}, r 6=\alpha_{9} \alpha_{5}, r 7=\alpha_{4} \alpha_{10}, r 8=\alpha_{11} \alpha_{5}$, $r 9=\alpha_{9} \alpha_{10}$ e $r 10=\alpha_{11} \alpha_{8}$.

$\mathrm{E}$ os ciclos elementares são $C_{4}=\alpha_{8} \alpha_{9}$ e $C_{5}=$ $\alpha_{11} \alpha_{10}$. Nesse caso, obtemos duas soluções hereditárias.

Nesse anexo, temos mais essas relações do tipo 2: $r 5=\alpha_{4} \alpha_{8}, r 6=\alpha_{9} \alpha_{5}, r 7=\alpha_{10} \alpha_{9}$ e $r 8=\alpha_{8} \alpha_{11}$. $\mathrm{E}$ os ciclos elementares são $C_{4}=\alpha_{8} \alpha_{9}$ e $C_{5}=$ $\alpha_{10} \alpha_{11}$. Logo, temos dois cortes que originam duas álgebras de incidência hereditária.

As relações do tipo 2 são: $r 1=\alpha_{1} \alpha_{4} \alpha_{6}, r 2=$ $\alpha_{9} \alpha_{4} \alpha_{5}, r 3=\alpha_{2} \alpha_{4}, r 4=\alpha_{1} \alpha_{3}, r 5=\alpha_{9} \alpha_{3}$, $r 6=\alpha_{4} \alpha_{7}, r 7=\alpha_{8} \alpha_{6}, r 8=\alpha_{8} \alpha_{5}, r 9=\alpha_{10} \alpha_{9} \mathrm{e}$ $r 10=\alpha_{6} \alpha_{11}$.

Os ciclos elementares são: $C_{1}=\alpha_{1} \alpha_{4} \alpha_{5}, C_{2}=$ $\alpha_{4} \alpha_{6} \alpha_{9}, C_{3}=\alpha_{2} \alpha_{3}, C_{4}=\alpha_{7} \alpha_{8}$ e $C_{5}=\alpha_{10} \alpha_{11}$. Não apresenta nenhuma álgebra de incidência.

As relações do tipo 2 são: $r 1=\alpha_{1} \alpha_{2} \alpha_{4}$ e $r 2=$ $\alpha_{5} \alpha_{2} \alpha_{3}$.

Os ciclos elementares são: $C_{1}=\alpha_{2} \alpha_{3} \alpha_{1}$ e $C_{2}=$ $\alpha_{2} \alpha_{4} \alpha_{10} \alpha_{5}$.

Completando, temos mais essas relações do tipo 2 : $r 3=\alpha_{1} \alpha_{6}, r 4=\alpha_{5} \alpha_{6}$ e $r 5=\alpha_{7} \alpha_{2}$.

E o ciclo elementar é $C_{3}=\alpha_{6} \alpha_{8} \alpha_{7}$. O programa exibe uma solução hereditária e a solução 40 . 

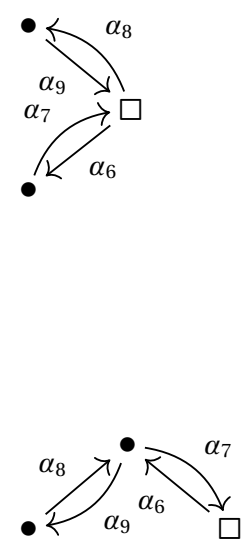

(6)
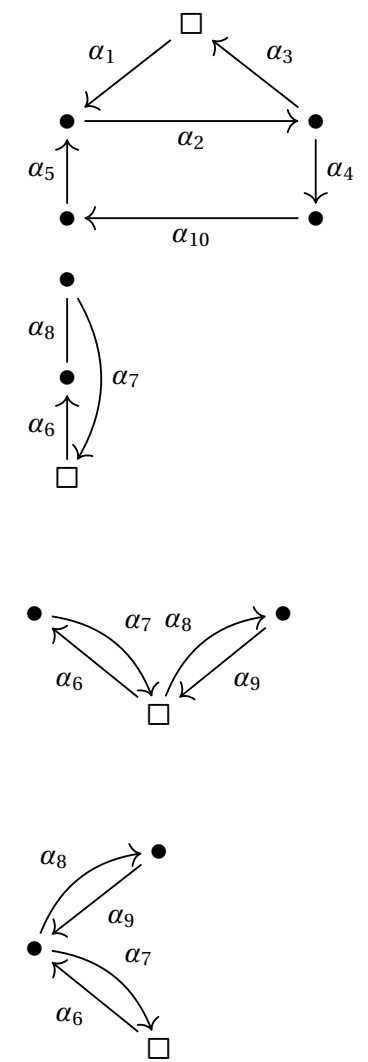

(7)

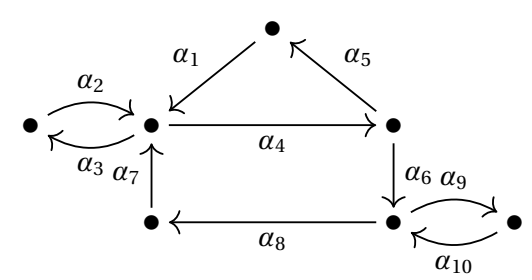

Nesse caso, temos mais essas relações do tipo 2: $r 3=\alpha_{1} \alpha_{6}, r 4=\alpha_{5} \alpha_{6}, r 5=\alpha_{7} \alpha_{2}, r 6=\alpha_{1} \alpha_{8}, r 7=$ $\alpha_{5} \alpha_{8}, r 8=\alpha_{9} \alpha_{2}, r 9=\alpha_{7} \alpha_{8}$ e $r 10=\alpha_{9} \alpha_{6}$.

E os ciclos elementares são $C_{3}=\alpha_{6} \alpha_{7}$ e $C_{4}=\alpha_{9} \alpha_{8}$. Nesse caso, obtemos uma solução hereditária e a solução 41.

Nesse anexo, temos mais essas relações do tipo 2: $r 3=\alpha_{1} \alpha_{6}, r 4=\alpha_{5} \alpha_{6}, r 5=\alpha_{7} \alpha_{2}, r 6=\alpha_{8} \alpha_{7} \mathrm{e}$ $r 7=\alpha_{6} \alpha_{9}$.

E os ciclos elementares são $C_{3}=\alpha_{6} \alpha_{7}$ e $C_{4}=\alpha_{9} \alpha_{8}$. Logo, temos dois cortes que originam uma álgebra de incidência hereditária e a álgebra 42 .

As relações do tipo 2 são: $r 1=\alpha_{1} \alpha_{2} \alpha_{4}$ e $r 2=$ $\alpha_{5} \alpha_{2} \alpha_{3}$.

Os ciclos elementares são: $C_{1}=\alpha_{2} \alpha_{3} \alpha_{1}$ e $C_{2}=$ $\alpha_{2} \alpha_{4} \alpha_{10} \alpha_{5}$

Completando, temos mais essas relações do tipo 2: $r 3=\alpha_{3} \alpha_{6}$ e $r 4=\alpha_{7} \alpha_{1}$.

E o ciclo elementar é $C_{3}=\alpha_{6} \alpha_{8} \alpha_{7}$. O programa exibe duas soluções hereditárias.

Nesse caso, temos mais essas relações do tipo 2: $r 3=\alpha_{3} \alpha_{6}, r 4=\alpha_{7} \alpha_{1}, r 5=\alpha_{3} \alpha_{8}, r 6=\alpha_{9} \alpha_{1}, r 7=$ $\alpha_{9} \alpha_{6}$ e $r 8=\alpha_{7} \alpha_{8}$.

E os ciclos elementares são $C_{3}=\alpha_{8} \alpha_{9}$ e $C_{4}=\alpha_{7} \alpha_{6}$. Logo obtemos duas soluções hereditárias.

Nesse anexo, temos mais essas relações do tipo 2: $r 3=\alpha_{3} \alpha_{6}, r 4=\alpha_{7} \alpha_{1}, r 5=\alpha_{9} \alpha_{7}$ e $r 6=\alpha_{6} \alpha_{8}$.

E os ciclos elementares são $C_{3}=\alpha_{8} \alpha_{9}$ e $C_{4}=\alpha_{7} \alpha_{6}$. Portanto, temos mais duas álgebras hereditárias.

As relações do tipo 2 são: $r 1=\alpha_{1} \alpha_{4} \alpha_{6}, r 2=$ $\alpha_{7} \alpha_{4} \alpha_{5}, r 3=\alpha_{2} \alpha_{4}, r 4=\alpha_{1} \alpha_{3}, r 5=\alpha_{7} \alpha_{3}$, $r 6=\alpha_{6} \alpha_{9}$ e $r 7=\alpha_{10} \alpha_{8}$. Os ciclos elementares são: $C_{1}=\alpha_{1} \alpha_{4} \alpha_{5}, C_{2}=\alpha_{4} \alpha_{6} \alpha_{8} \alpha_{7}, C_{3}=\alpha_{2} \alpha_{3} \mathrm{e}$ $C_{4}=\alpha_{9} \alpha_{10}$. Logo, o programa mostra nenhuma solução. 
(8)

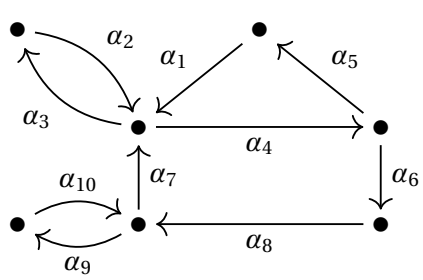

(9)

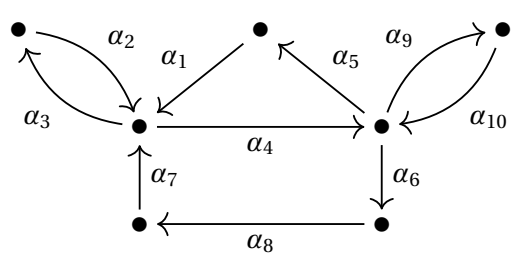

(10)

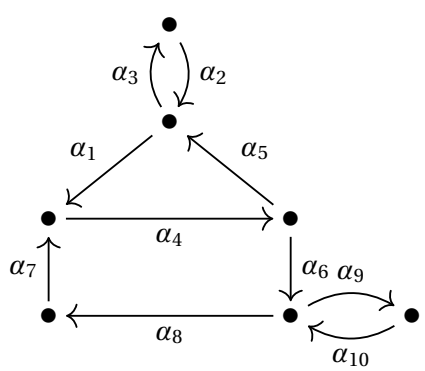

(11)

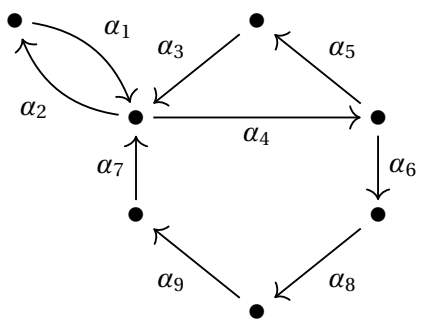

(12)

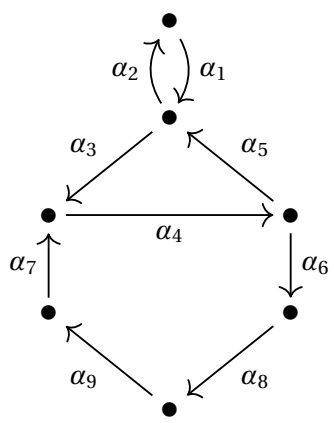

(13)

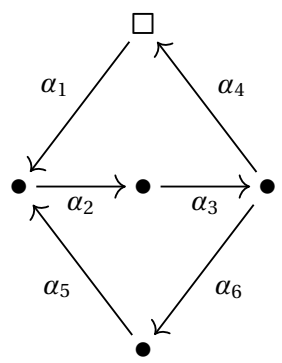

As relações do tipo 2 são: $r 1=\alpha_{1} \alpha_{4} \alpha_{6}, r 2=$ $\alpha_{7} \alpha_{4} \alpha_{5}, r 3=\alpha_{2} \alpha_{4}, r 4=\alpha_{1} \alpha_{3}, r 5=\alpha_{7} \alpha_{3}$, $r 6=\alpha_{8} \alpha_{9}$ e $r 7=\alpha_{10} \alpha_{7}$. Os ciclos elementares são: $C_{1}=\alpha_{1} \alpha_{4} \alpha_{5}, C_{2}=\alpha_{4} \alpha_{6} \alpha_{8} \alpha_{7}, C_{3}=\alpha_{2} \alpha_{3} \mathrm{e}$ $C_{4}=\alpha_{9} \alpha_{10}$. Não obtemos nenhuma álgebra de incidência não hereditária.

As relações do tipo 2 são: $r 1=\alpha_{1} \alpha_{4} \alpha_{6}, r 2=$ $\alpha_{7} \alpha_{4} \alpha_{5}, r 3=\alpha_{2} \alpha_{4}, r 4=\alpha_{1} \alpha_{3}, r 5=\alpha_{7} \alpha_{3}, r 6=$ $\alpha_{4} \alpha_{9}, r 7=\alpha_{10} \alpha_{5}$ e $r 8=\alpha_{6} \alpha_{7}$. Os ciclos elementares são: $C_{1}=\alpha_{1} \alpha_{4} \alpha_{5}, C_{2}=\alpha_{4} \alpha_{6} \alpha_{8} \alpha_{7}, C_{3}=\alpha_{2} \alpha_{3}$ e $C_{4}=\alpha_{9} \alpha_{10}$. Logo, o programa mostra a álgebra 43 .

As relações do tipo 2 são: $r 1=\alpha_{1} \alpha_{4} \alpha_{6}, r 2=$ $\alpha_{7} \alpha_{4} \alpha_{5}, r 3=\alpha_{5} \alpha_{3}, r 4=\alpha_{2} \alpha_{1}, r 5=\alpha_{10} \alpha_{8} \mathrm{e}$ $r 6=\alpha_{6} \alpha_{9}$. Os ciclos elementares são: $C_{1}=\alpha_{1} \alpha_{4} \alpha_{5}$, $C_{2}=\alpha_{4} \alpha_{6} \alpha_{8} \alpha_{7}, C_{3}=\alpha_{2} \alpha_{3}$ e $C_{4}=\alpha_{9} \alpha_{10}$. Portanto não tem nenhuma álgebra de incidência não hereditária.

As relações do tipo 2 são: $r 1=\alpha_{3} \alpha_{4} \alpha_{6}, r 2=$ $\alpha_{7} \alpha_{4} \alpha_{5}, r 3=\alpha_{1} \alpha_{4}, r 4=\alpha_{7} \alpha_{2}$ e $r 5=\alpha_{3} \alpha_{2}$. Os ciclos elementares são: $C_{1}=\alpha_{3} \alpha_{4} \alpha_{5}, C_{2}=$ $\alpha_{4} \alpha_{6} \alpha_{8} \alpha_{9} \alpha_{7}$ e $C_{3}=\alpha_{1} \alpha_{2}$. Obtemos a solução 44 .

As relações do tipo 2 são: $r 1=\alpha_{3} \alpha_{4} \alpha_{6}, r 2=$ $\alpha_{7} \alpha_{4} \alpha_{5}, r 3=\alpha_{5} \alpha_{2}$ e $r 4=\alpha_{1} \alpha_{3}$. Os ciclos elementares são: $C_{1}=\alpha_{3} \alpha_{4} \alpha_{5}, C_{2}=\alpha_{4} \alpha_{6} \alpha_{8} \alpha_{9} \alpha_{7}$ e $C_{3}=\alpha_{1} \alpha_{2}$. Portanto, não temos nenhuma solução.

Essa extensão trivial tem as seguintes relações do tipo 2: $r 1=\alpha_{5} \alpha_{2} \alpha_{3} \alpha_{4}$ e $r 2=\alpha_{1} \alpha_{2} \alpha_{3} \alpha_{6}$.

Os ciclos elementares são: $C_{1}=\alpha_{1} \alpha_{2} \alpha_{3} \alpha_{4}$ e $C_{2}=$ $\alpha_{2} \alpha_{3} \alpha_{6} \alpha_{5}$. 

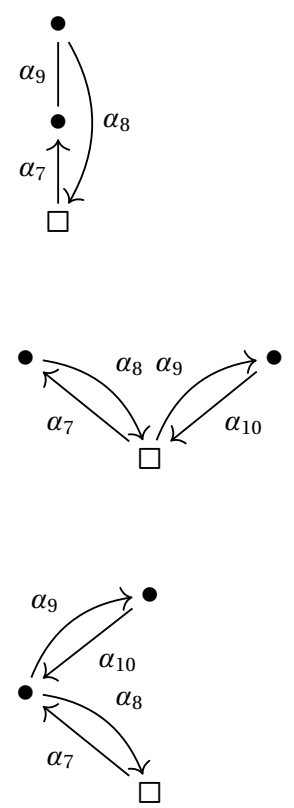

(14)

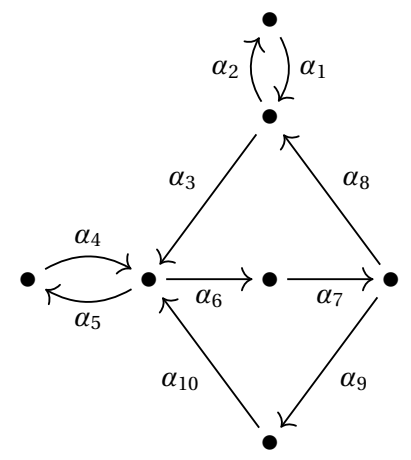

(15)

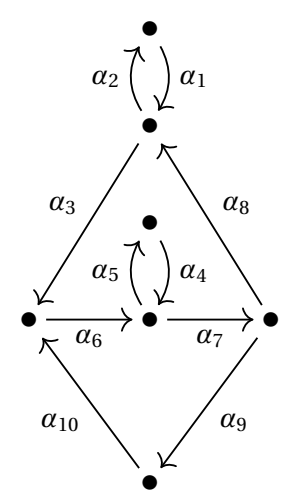

(16)

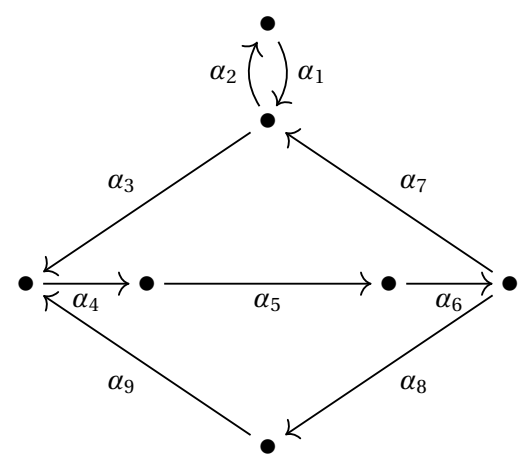

Completando, temos mais essas relações do tipo 2: $r 3=\alpha_{4} \alpha_{7}$ e $r 4=\alpha_{8} \alpha_{1}$.

E o ciclo elementar é $C_{3}=\alpha_{7} \alpha_{9} \alpha_{8}$. O programa exibe duas soluções hereditárias.

Nesse caso, temos mais essas relações do tipo 2: $r 3=\alpha_{4} \alpha_{7}, r 4=\alpha_{8} \alpha_{1}, r 5=\alpha_{4} \alpha_{9}, r 6=\alpha_{10} \alpha_{1}$, $r 7=\alpha_{10} \alpha_{7}$ e $r 8=\alpha_{8} \alpha_{9}$.

E os ciclos elementares são $C_{3}=\alpha_{9} \alpha_{10}$ e $C_{4}=\alpha_{8} \alpha_{7}$. Logo obtemos duas soluções hereditárias.

Nesse anexo, temos mais essas relações do tipo 2: $r 3=\alpha_{4} \alpha_{7}, r 4=\alpha_{8} \alpha_{1}, r 5=\alpha_{10} \alpha_{8}$ e $r 6=\alpha_{7} \alpha_{9}$.

E os ciclos elementares são $C_{3}=\alpha_{9} \alpha_{10}$ e $C_{4}=\alpha_{8} \alpha_{7}$. Portanto, temos mais duas álgebras hereditárias.

As relações do tipo 2 são: $r 1=\alpha_{3} \alpha_{6} \alpha_{7} \alpha_{9}, r 2=$ $\alpha_{10} \alpha_{6} \alpha_{7} \alpha_{8}, r 3=\alpha_{4} \alpha_{6}, r 4=\alpha_{10} \alpha_{5}, r 5=\alpha_{3} \alpha_{5}, r 6=$ $\alpha_{8} \alpha_{2}$ e $r 7=\alpha_{1} \alpha_{3}$. Os ciclos elementares são: $C_{1}=$ $\alpha_{3} \alpha_{6} \alpha_{7} \alpha_{8}, C_{2}=\alpha_{6} \alpha_{7} \alpha_{9} \alpha_{10}, C_{3}=\alpha_{1} \alpha_{2}$ e $C_{4}=\alpha_{5} \alpha_{4}$. Logo, não temos soluções não hereditárias.

As relações do tipo 2 são: $r 1=\alpha_{3} \alpha_{6} \alpha_{7} \alpha_{9}, r 2=$ $\alpha_{10} \alpha_{6} \alpha_{7} \alpha_{8}, r 3=\alpha_{6} \alpha_{5}, r 4=\alpha_{4} \alpha_{7}, r 5=\alpha_{8} \alpha_{2}$ e $r 6=$ $\alpha_{1} \alpha_{3}$. Os ciclos elementares são: $C_{1}=\alpha_{3} \alpha_{6} \alpha_{7} \alpha_{8}$, $C_{2}=\alpha_{6} \alpha_{7} \alpha_{9} \alpha_{10}, C_{3}=\alpha_{1} \alpha_{2}$ e $C_{4}=\alpha_{5} \alpha_{4}$. Não apresenta álgebras de incidência originadas pelos cortes.

As relações do tipo 2 são: $r 1=\alpha_{3} \alpha_{4} \alpha_{5} \alpha_{6} \alpha_{8}, r 2=$ $\alpha_{9} \alpha_{4} \alpha_{5} \alpha_{6} \alpha_{7}, r 3=\alpha_{7} \alpha_{2}$ e $r 4=\alpha_{1} \alpha_{3}$. Os ciclos elementares são: $C_{1}=\alpha_{3} \alpha_{4} \alpha_{5} \alpha_{6} \alpha_{7}, C_{2}=\alpha_{4} \alpha_{5} \alpha_{6} \alpha_{8} \alpha_{9}$ e $C_{3}=\alpha_{1} \alpha_{2}$. Portanto não temos soluções não hereditárias. 
(17)
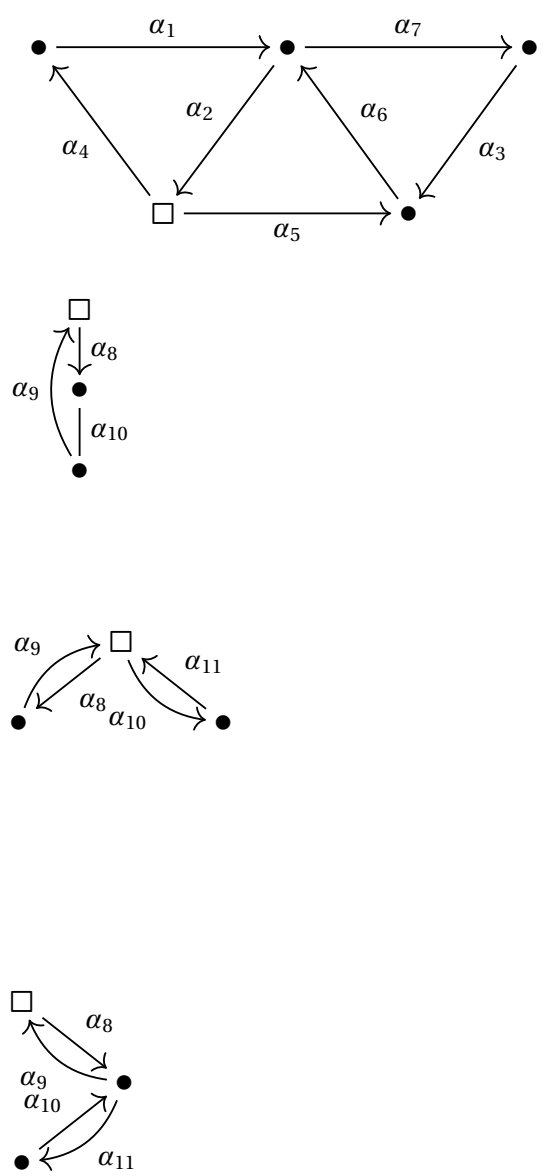

(18)
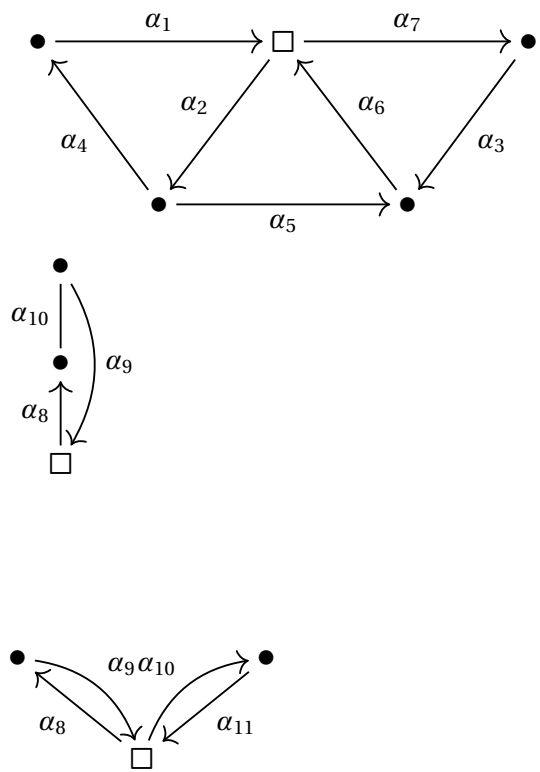

As relações do tipo 2 são: $r 1=\alpha_{1} \alpha_{2} \alpha_{5}, r 2=$ $\alpha_{6} \alpha_{2} \alpha_{4}, r 3=\alpha_{1} \alpha_{7}, r 4=\alpha_{5} \alpha_{6} \alpha_{7}$ e $r 5=\alpha_{3} \alpha_{6} \alpha_{2}$.

Os ciclos elementares são: $C_{1}=\alpha_{1} \alpha_{2} \alpha_{4}, C_{2}=$ $\alpha_{2} \alpha_{5} \alpha_{6}$ e $C_{3}=\alpha_{3} \alpha_{6} \alpha_{7}$.

Completando, temos mais essas relações do tipo 2: $r 6=\alpha_{2} \alpha_{8}, r 7=\alpha_{9} \alpha_{5}$ e $r 8=\alpha_{9} \alpha_{4}$.

E o ciclo elementar é $C_{4}=\alpha_{8} \alpha_{10} \alpha_{9}$. O programa exibe a solução 45 .

Nesse caso, temos mais essas relações do tipo 2: $r 6=\alpha_{2} \alpha_{8}, r 7=\alpha_{9} \alpha_{5}, r 8=\alpha_{9} \alpha_{4}, r 9=\alpha_{2} \alpha_{10}$, $r 10=\alpha_{11} \alpha_{5}, r 11=\alpha_{11} \alpha_{4}, r 12=\alpha_{9} \alpha_{10}$ e $r 13=$ $\alpha_{11} \alpha_{8}$.

$\mathrm{E}$ os ciclos elementares são $C_{4}=\alpha_{8} \alpha_{9}$ e $C_{5}=$ $\alpha_{11} \alpha_{10}$. Nesse caso, obtemos a solução 46 .

Nesse anexo, temos mais essas relações do tipo 2: $r 6=\alpha_{2} \alpha_{8}, r 7=\alpha_{9} \alpha_{5}, r 8=\alpha_{9} \alpha_{4}, r 9=\alpha_{10} \alpha_{9} \mathrm{e}$ $r 10=\alpha_{8} \alpha_{11}$.

$\mathrm{E}$ os ciclos elementares são $C_{4}=\alpha_{8} \alpha_{9}$ e $C_{5}=$ $\alpha_{10} \alpha_{11}$. Logo, temos um corte que origina a álgebra 47.

As relações do tipo 2 são: $r 1=\alpha_{1} \alpha_{2} \alpha_{5}, r 2=$ $\alpha_{6} \alpha_{2} \alpha_{4}, r 3=\alpha_{1} \alpha_{7}, r 4=\alpha_{5} \alpha_{6} \alpha_{7}$ e $r 5=\alpha_{3} \alpha_{6} \alpha_{2}$.

Os ciclos elementares são: $C_{1}=\alpha_{1} \alpha_{2} \alpha_{4}, C_{2}=$ $\alpha_{2} \alpha_{5} \alpha_{6}$ e $C_{3}=\alpha_{3} \alpha_{6} \alpha_{7}$.

Completando, temos mais essas relações do tipo 2: $r 6=\alpha_{1} \alpha_{8}, r 7=\alpha_{6} \alpha_{8}, r 8=\alpha_{9} \alpha_{2}$ e $r 9=\alpha_{9} \alpha_{7}$.

E o ciclo elementar é $C_{4}=\alpha_{8} \alpha_{10} \alpha_{9}$. O programa exibe a álgebra 48 e a sua álgebra dual.

Nesse caso, temos mais essas relações do tipo 2: $r 6=\alpha_{1} \alpha_{8}, r 7=\alpha_{6} \alpha_{8}, r 8=\alpha_{9} \alpha_{2}, r 9=\alpha_{9} \alpha_{7}, r 10=$ $\alpha_{1} \alpha_{10}, r 11=\alpha_{6} \alpha_{10}, r 12=\alpha_{11} \alpha_{2}, r 13=\alpha_{11} \alpha_{7}$, $r 14=\alpha_{11} \alpha_{8}$ e $r 15=\alpha_{9} \alpha_{10}$.

E os ciclos elementares são $C_{4}=\alpha_{10} \alpha_{11}$ e $C_{5}=$ $\alpha_{9} \alpha_{8}$. Logo obtemos a álgebra 49 e a sua álgebra dual. 


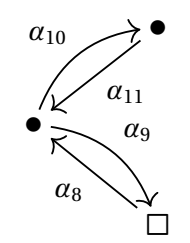

(19)

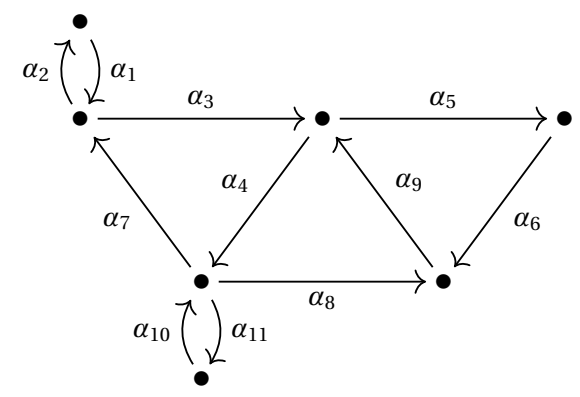

(20)

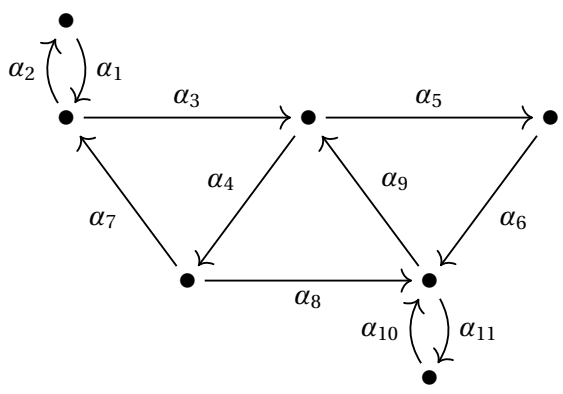

(21)

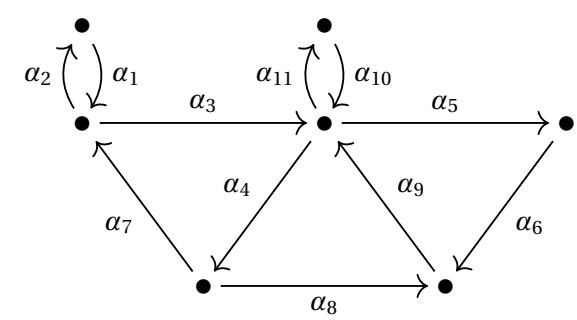

(22)

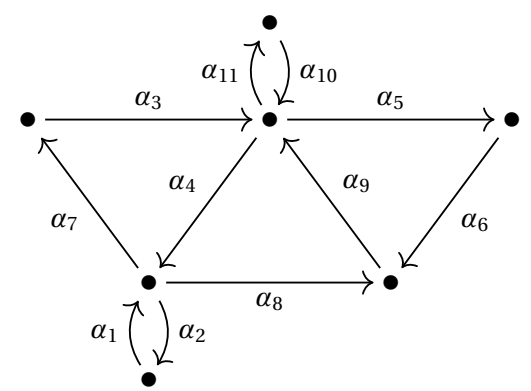

Nesse anexo, temos mais essas relações do tipo 2: $r 6=\alpha_{1} \alpha_{8}, r 7=\alpha_{6} \alpha_{8}, r 8=\alpha_{9} \alpha_{2}, r 9=\alpha_{9} \alpha_{7}, r 10=$ $\alpha_{11} \alpha_{9}$ e $r 11=\alpha_{8} \alpha_{10}$.

$\mathrm{E}$ os ciclos elementares são $C_{4}=\alpha_{10} \alpha_{11}$ e $C_{5}=$ $\alpha_{9} \alpha_{8}$. Portanto, temos a álgebra 50 e a sua álgebra dual.

As relações do tipo 2 são: $r 1=\alpha_{3} \alpha_{4} \alpha_{8}, r 2=$ $\alpha_{9} \alpha_{4} \alpha_{7}, r 3=\alpha_{8} \alpha_{9} \alpha_{5}, r 4=\alpha_{6} \alpha_{9} \alpha_{4}, r 5=\alpha_{4} \alpha_{11}$, $r 6=\alpha_{10} \alpha_{7}, r 7=\alpha_{10} \alpha_{8}, r 8=\alpha_{1} \alpha_{3}, r 9=\alpha_{7} \alpha_{2}$ e $r 10=\alpha_{3} \alpha_{5}$. Os ciclos elementares são: $C_{1}=$ $\alpha_{3} \alpha_{4} \alpha_{7}, C_{2}=\alpha_{4} \alpha_{8} \alpha_{9}, C_{3}=\alpha_{6} \alpha_{9} \alpha_{5}, C_{4}=\alpha_{10} \alpha_{11}$ e $C_{5}=\alpha_{2} \alpha_{1}$. Portanto, não temos nenhuma álgebra.

As relações do tipo 2 são: $r 1=\alpha_{3} \alpha_{4} \alpha_{8}, r 2=$ $\alpha_{9} \alpha_{4} \alpha_{7}, r 3=\alpha_{8} \alpha_{9} \alpha_{5}, r 4=\alpha_{6} \alpha_{9} \alpha_{4}, r 5=\alpha_{8} \alpha_{11}$, $r 6=\alpha_{6} \alpha_{11}, r 7=\alpha_{10} \alpha_{9}, r 8=\alpha_{1} \alpha_{3}, r 9=\alpha_{7} \alpha_{2}$ e $r 10=\alpha_{3} \alpha_{5}$. Os ciclos elementares são: $C_{1}=$ $\alpha_{3} \alpha_{4} \alpha_{7}, C_{2}=\alpha_{4} \alpha_{8} \alpha_{9}, C_{3}=\alpha_{6} \alpha_{9} \alpha_{5}, C_{4}=\alpha_{10} \alpha_{11}$ e $C_{5}=\alpha_{2} \alpha_{1}$. O programa mostra apenas a solução 51 .

As relações do tipo 2 são: $r 1=\alpha_{3} \alpha_{4} \alpha_{8}, r 2=$ $\alpha_{9} \alpha_{4} \alpha_{7}, r 3=\alpha_{8} \alpha_{9} \alpha_{5}, r 4=\alpha_{6} \alpha_{9} \alpha_{4}, r 5=\alpha_{3} \alpha_{11}$, $r 6=\alpha_{9} \alpha_{11}, r 7=\alpha_{10} \alpha_{5}, r 8=\alpha_{1} \alpha_{3}, r 9=\alpha_{7} \alpha_{2}$, $r 10=\alpha_{3} \alpha_{5}$ e $r 11=\alpha_{10} \alpha_{4}$. Os ciclos elementares são: $C_{1}=\alpha_{3} \alpha_{4} \alpha_{7}, C_{2}=\alpha_{4} \alpha_{8} \alpha_{9}, C_{3}=\alpha_{6} \alpha_{9} \alpha_{5}$, $C_{4}=\alpha_{10} \alpha_{11}$ e $C_{5}=\alpha_{2} \alpha_{1}$. Portanto, o corte resulta na solução 52 .

As relações do tipo 2 são: $r 1=\alpha_{3} \alpha_{4} \alpha_{8}, r 2=$ $\alpha_{9} \alpha_{4} \alpha_{7}, r 3=\alpha_{8} \alpha_{9} \alpha_{5}, r 4=\alpha_{6} \alpha_{9} \alpha_{4}, r 5=\alpha_{3} \alpha_{11}$, $r 6=\alpha_{9} \alpha_{11}, r 7=\alpha_{10} \alpha_{5}, r 8=\alpha_{1} \alpha_{7}, r 9=\alpha_{4} \alpha_{2}$, $r 10=\alpha_{3} \alpha_{5}, r 11=\alpha_{10} \alpha_{4}$ e $r 12=\alpha_{1} \alpha_{8}$. Os ciclos elementares são: $C_{1}=\alpha_{3} \alpha_{4} \alpha_{7}, C_{2}=\alpha_{4} \alpha_{8} \alpha_{9}$, $C_{3}=\alpha_{6} \alpha_{9} \alpha_{5}, C_{4}=\alpha_{10} \alpha_{11}$ e $C_{5}=\alpha_{2} \alpha_{1}$. Obtemos a álgebra de incidência 53 . 
(23)

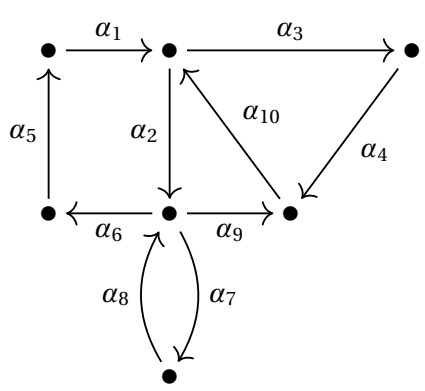

(24)

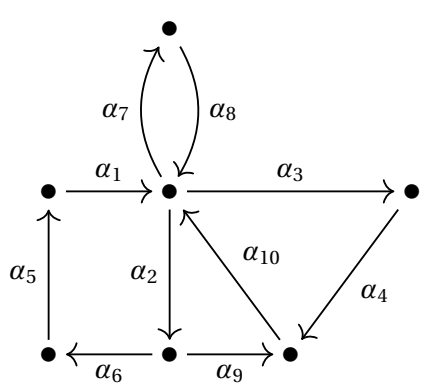

(25)

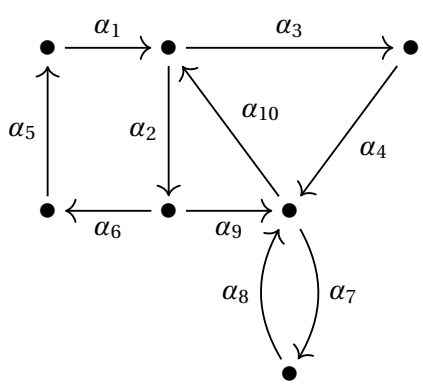

(26)

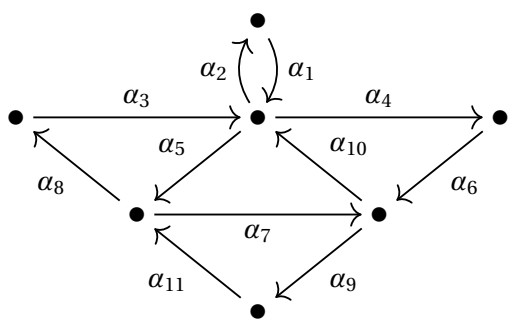

(27)

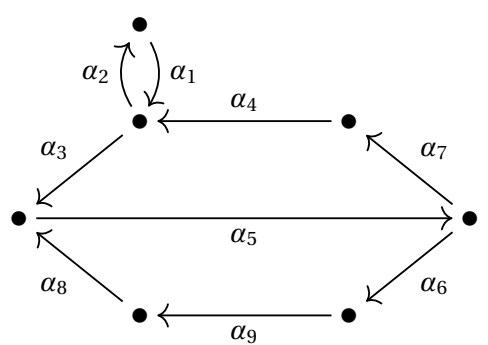

(28)

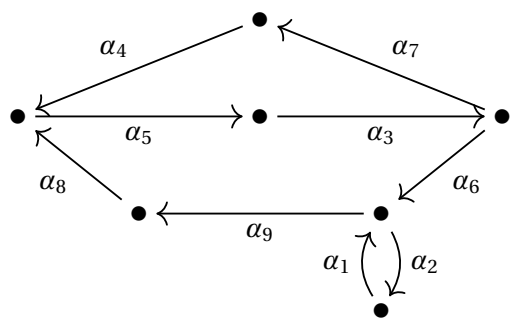

As relações do tipo 2 são: $r 1=\alpha_{1} \alpha_{2} \alpha_{9}, r 2=$ $\alpha_{10} \alpha_{2} \alpha_{6}, r 3=\alpha_{9} \alpha_{10} \alpha_{3}, r 4=\alpha_{4} \alpha_{10} \alpha_{2}, r 5=\alpha_{1} \alpha_{3}$, $r 6=\alpha_{8} \alpha_{9}, r 7=\alpha_{8} \alpha_{6}$ e $r 8=\alpha_{2} \alpha_{7}$. Os ciclos elementares são: $C_{1}=\alpha_{1} \alpha_{2} \alpha_{6} \alpha_{5}, C_{2}=\alpha_{3} \alpha_{4} \alpha_{10}$, $C_{3}=\alpha_{10} \alpha_{2} \alpha_{9}$ e $C_{4}=\alpha_{8} \alpha_{7}$. Logo, o programa exibe a álgebra 54 .

As relações do tipo 2 são: $r 1=\alpha_{1} \alpha_{2} \alpha_{9}, r 2=$ $\alpha_{10} \alpha_{2} \alpha_{6}, r 3=\alpha_{9} \alpha_{10} \alpha_{3}, r 4=\alpha_{4} \alpha_{10} \alpha_{2}, r 5=\alpha_{1} \alpha_{3}$, $r 6=\alpha_{8} \alpha_{2}, r 7=\alpha_{8} \alpha_{3}, r 8=\alpha_{10} \alpha_{7}$ e $r 9=\alpha_{1} \alpha_{7}$. Os ciclos elementares são: $C_{1}=\alpha_{1} \alpha_{2} \alpha_{6} \alpha_{5}, C_{2}=$ $\alpha_{3} \alpha_{4} \alpha_{10}, C_{3}=\alpha_{10} \alpha_{2} \alpha_{9}$ e $C_{4}=\alpha_{8} \alpha_{7}$. Nessa extensão trivial, originam duas soluções 55 e 56.

As relações do tipo 2 são: $r 1=\alpha_{1} \alpha_{2} \alpha_{9}, r 2=$ $\alpha_{10} \alpha_{2} \alpha_{6}, r 3=\alpha_{9} \alpha_{10} \alpha_{3}, r 4=\alpha_{4} \alpha_{10} \alpha_{2}, r 5=\alpha_{1} \alpha_{3}$, $r 6=\alpha_{8} \alpha_{10}, r 7=\alpha_{4} \alpha_{7}$ e $r 8=\alpha_{9} \alpha_{7}$. Os ciclos elementares são: $C_{1}=\alpha_{1} \alpha_{2} \alpha_{6} \alpha_{5}, C_{2}=\alpha_{3} \alpha_{4} \alpha_{10}$, $C_{3}=\alpha_{10} \alpha_{2} \alpha_{9}$ e $C_{4}=\alpha_{8} \alpha_{7}$. Aqui, obtemos apenas a álgebra 57.

As relações do tipo 2 são: $r 1=\alpha_{11} \alpha_{7} \alpha_{10}, r 2=$ $\alpha_{5} \alpha_{7} \alpha_{9}, r 3=\alpha_{3} \alpha_{5} \alpha_{7}, r 4=\alpha_{10} \alpha_{5} \alpha_{8}, r 5=\alpha_{7} \alpha_{10} \alpha_{4}$, $r 6=\alpha_{6} \alpha_{10} \alpha_{5}, r 7=\alpha_{3} \alpha_{2}, r 8=\alpha_{10} \alpha_{2}, r 9=\alpha_{1} \alpha_{4}$, $r 10=\alpha_{1} \alpha_{5}, r 11=\alpha_{3} \alpha_{4}, r 12=\alpha_{6} \alpha_{9}$ e $r 13=\alpha_{11} \alpha_{8}$. Os ciclos elementares são: $C_{1}=\alpha_{5} \alpha_{8} \alpha_{3}, C_{2}=$ $\alpha_{5} \alpha_{7} \alpha_{10}, C_{3}=\alpha_{10} \alpha_{4} \alpha_{6}, C_{4}=\alpha_{9} \alpha_{11} \alpha_{7}$ e $C_{5}=\alpha_{1} \alpha_{2}$. Não temos nenhuma solução.

As relações do tipo 2 são: $r 1=\alpha_{3} \alpha_{5} \alpha_{6}, r 2=$ $\alpha_{8} \alpha_{5} \alpha_{7}, r 3=\alpha_{4} \alpha_{2}$ e $r 4=\alpha_{1} \alpha_{3}$. Os ciclos elementares são: $C_{1}=\alpha_{7} \alpha_{4} \alpha_{3} \alpha_{5}, C_{2}=\alpha_{5} \alpha_{6} \alpha_{9} \alpha_{8} \mathrm{e}$ $C_{3}=\alpha_{1} \alpha_{2}$. O programa não mostra solução que origine uma álgebra de incidência não hereditária.

As relações do tipo 2 são: $r 1=\alpha_{4} \alpha_{5} \alpha_{3} \alpha_{6}, r 2=$ $\alpha_{8} \alpha_{5} \alpha_{3} \alpha_{7}, r 3=\alpha_{6} \alpha_{2}$ e $r 4=\alpha_{1} \alpha_{9}$. Os ciclos elementares são: $C_{1}=\alpha_{5} \alpha_{3} \alpha_{7} \alpha_{4}, C_{2}=\alpha_{9} \alpha_{8} \alpha_{5} \alpha_{3} \alpha_{6}$ e $C_{3}=\alpha_{1} \alpha_{2}$. Novamente, o programa não mostra solução não hereditária. 
(29)

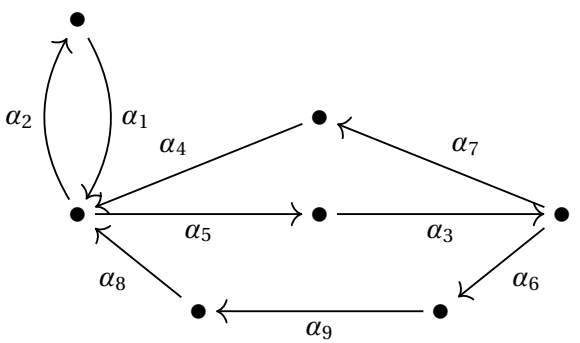

(30)

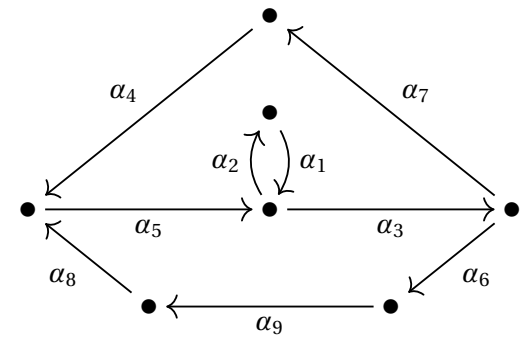

(31)

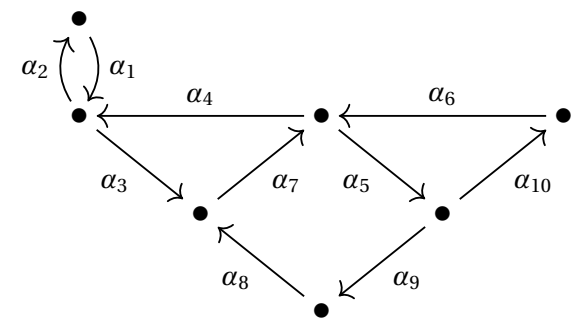

(32)

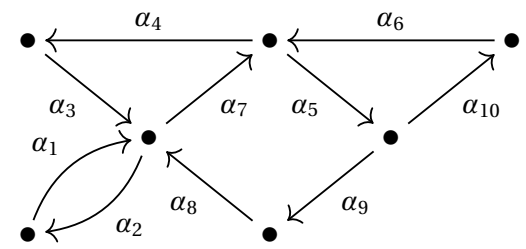

(33)

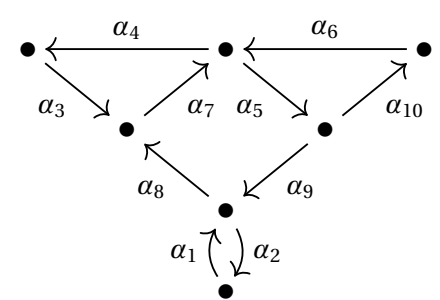

(34)

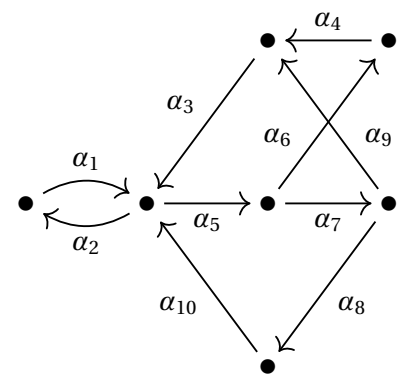

As relações do tipo 2 são: $r 1=\alpha_{4} \alpha_{5} \alpha_{3} \alpha_{6}, r 2=$ $\alpha_{8} \alpha_{5} \alpha_{3} \alpha_{7}, r 3=\alpha_{8} \alpha_{2}, r 4=\alpha_{4} \alpha_{2}$ e $r 5=\alpha_{1} \alpha_{5}$. Os ciclos elementares são: $C_{1}=\alpha_{5} \alpha_{3} \alpha_{7} \alpha_{4}, C_{2}=$ $\alpha_{9} \alpha_{8} \alpha_{5} \alpha_{3} \alpha_{6}$ e $C_{3}=\alpha_{1} \alpha_{2}$. Portanto, obtemos a álgebra 58.

As relações do tipo 2 são: $r 1=\alpha_{4} \alpha_{5} \alpha_{3} \alpha_{6}, r 2=$ $\alpha_{8} \alpha_{5} \alpha_{3} \alpha_{7}, r 3=\alpha_{5} \alpha_{2}$ e $r 4=\alpha_{1} \alpha_{3}$. Os ciclos elementares são: $C_{1}=\alpha_{5} \alpha_{3} \alpha_{7} \alpha_{4}, C_{2}=\alpha_{9} \alpha_{8} \alpha_{5} \alpha_{3} \alpha_{6}$ e $C_{3}=\alpha_{1} \alpha_{2}$. Logo, temos as soluções 59 e 60 .

As relações do tipo 2 são: $r 1=\alpha_{3} \alpha_{7} \alpha_{5}, r 2=$ $\alpha_{8} \alpha_{7} \alpha_{4}, r 3=\alpha_{7} \alpha_{5} \alpha_{10}, r 4=\alpha_{6} \alpha_{5} \alpha_{9}, r 5=\alpha_{6} \alpha_{4}$, $r 6=\alpha_{4} \alpha_{2}$ e $r 7=\alpha_{1} \alpha_{3}$. Os ciclos elementares são: $C_{1}=\alpha_{4} \alpha_{3} \alpha_{7}, C_{2}=\alpha_{10} \alpha_{6} \alpha_{5}, C_{3}=\alpha_{7} \alpha_{5} \alpha_{9} \alpha_{8}$ e $C_{4}=\alpha_{1} \alpha_{2}$. Portanto, o programa mostra a álgebra dual da solução 60 .

As relações do tipo 2 são: $r 1=\alpha_{3} \alpha_{7} \alpha_{5}, r 2=$ $\alpha_{8} \alpha_{7} \alpha_{4}, r 3=\alpha_{7} \alpha_{5} \alpha_{10}, r 4=\alpha_{6} \alpha_{5} \alpha_{9}, r 5=\alpha_{6} \alpha_{4}$, $r 6=\alpha_{3} \alpha_{2}, r 7=\alpha_{1} \alpha_{7}$ e $r 8=\alpha_{8} \alpha_{2}$. Os ciclos elementares são: $C_{1}=\alpha_{4} \alpha_{3} \alpha_{7}, C_{2}=\alpha_{10} \alpha_{6} \alpha_{5}$, $C_{3}=\alpha_{7} \alpha_{5} \alpha_{9} \alpha_{8}$ e $C_{4}=\alpha_{1} \alpha_{2}$. Assim, temos a álgebra 61 .

As relações do tipo 2 são: $r 1=\alpha_{3} \alpha_{7} \alpha_{5}, r 2=$ $\alpha_{8} \alpha_{7} \alpha_{4}, r 3=\alpha_{7} \alpha_{5} \alpha_{10}, r 4=\alpha_{6} \alpha_{5} \alpha_{9}, r 5=\alpha_{6} \alpha_{4}$, $r 6=\alpha_{9} \alpha_{2}$ e $r 7=\alpha_{1} \alpha_{8}$. Os ciclos elementares são: $C_{1}=\alpha_{4} \alpha_{3} \alpha_{7}, C_{2}=\alpha_{10} \alpha_{6} \alpha_{5}, C_{3}=\alpha_{7} \alpha_{5} \alpha_{9} \alpha_{8}$ e $C_{4}=\alpha_{1} \alpha_{2}$. Não temos solução para esse caso.

As relações do tipo 2 são: $r 1=\alpha_{3} \alpha_{5} \alpha_{7} \alpha_{8}, r 2=$ $\alpha_{10} \alpha_{5} \alpha_{7} \alpha_{9}, r 3=\alpha_{10} \alpha_{5} \alpha_{6}, r 4=\alpha_{9} \alpha_{3} \alpha_{5} \alpha_{6}, r 5=$ $\alpha_{4} \alpha_{3} \alpha_{5} \alpha_{7}, r 6=\alpha_{1} \alpha_{5}, r 7=\alpha_{3} \alpha_{2}$ e $r 8=\alpha_{10} \alpha_{2}$. Os ciclos elementares são: $C_{1}=\alpha_{3} \alpha_{5} \alpha_{6} \alpha_{4}, C_{2}=$ $\alpha_{3} \alpha_{5} \alpha_{7} \alpha_{9}, C_{3}=\alpha_{5} \alpha_{7} \alpha_{8} \alpha_{10}$ e $C_{4}=\alpha_{1} \alpha_{2}$. O programa mostra as soluções 62 e 63 . 
(35)

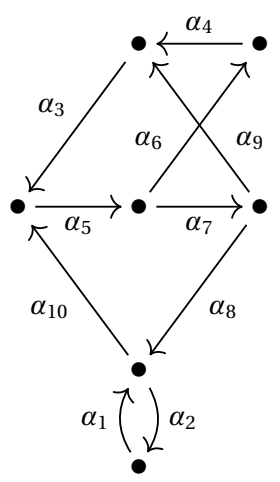

(36)

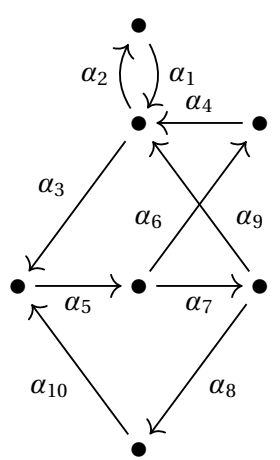

(37)

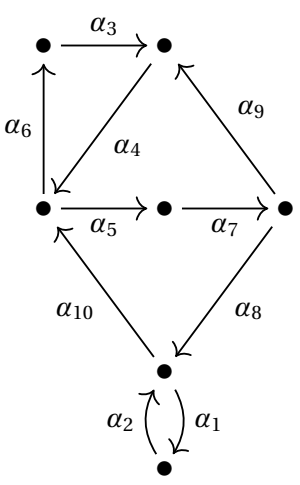

(38)

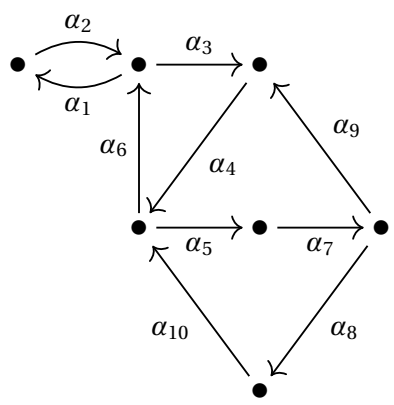

(39)

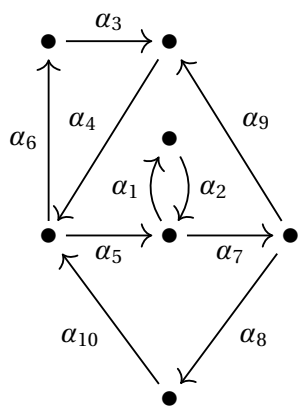

As relações do tipo 2 são: $r 1=\alpha_{3} \alpha_{5} \alpha_{7} \alpha_{8}, r 2=$ $\alpha_{10} \alpha_{5} \alpha_{7} \alpha_{9}, r 3=\alpha_{10} \alpha_{5} \alpha_{6}, r 4=\alpha_{9} \alpha_{3} \alpha_{5} \alpha_{6}, r 5=$ $\alpha_{4} \alpha_{3} \alpha_{5} \alpha_{7}, r 6=\alpha_{8} \alpha_{2}$ e $r 7=\alpha_{1} \alpha_{10}$. Os ciclos elementares são: $C_{1}=\alpha_{3} \alpha_{5} \alpha_{6} \alpha_{4}, C_{2}=\alpha_{3} \alpha_{5} \alpha_{7} \alpha_{9}$, $C_{3}=\alpha_{5} \alpha_{7} \alpha_{8} \alpha_{10}$ e $C_{4}=\alpha_{1} \alpha_{2}$. Logo, conseguimos apenas um corte resultando na álgebra 64 .

As relações do tipo 2 são: $r 1=\alpha_{3} \alpha_{5} \alpha_{7} \alpha_{8}, r 2=$ $\alpha_{10} \alpha_{5} \alpha_{7} \alpha_{9}, r 3=\alpha_{10} \alpha_{5} \alpha_{6}, r 4=\alpha_{9} \alpha_{3} \alpha_{5} \alpha_{6}, r 5=$ $\alpha_{4} \alpha_{3} \alpha_{5} \alpha_{7}, r 6=\alpha_{4} \alpha_{2}, r 7=\alpha_{1} \alpha_{3}$ e $r 8=\alpha_{9} \alpha_{2}$. Os ciclos elementares são: $C_{1}=\alpha_{3} \alpha_{5} \alpha_{6} \alpha_{4}, C_{2}=$ $\alpha_{3} \alpha_{5} \alpha_{7} \alpha_{9}, C_{3}=\alpha_{5} \alpha_{7} \alpha_{8} \alpha_{10}$ e $C_{4}=\alpha_{1} \alpha_{2}$. Portanto, obtemos a álgebra 65 .

As relações do tipo 2 são: $r 1=\alpha_{4} \alpha_{5} \alpha_{7} \alpha_{8}, r 2=$ $\alpha_{10} \alpha_{5} \alpha_{7} \alpha_{9}, r 3=\alpha_{9} \alpha_{4} \alpha_{6}, r 4=\alpha_{3} \alpha_{4} \alpha_{5}, r 5=\alpha_{10} \alpha_{6}$, $r 6=\alpha_{2} \alpha_{10}$ e $r 7=\alpha_{8} \alpha_{1}$. Os ciclos elementares são: $C_{1}=\alpha_{3} \alpha_{4} \alpha_{6}, C_{2}=\alpha_{9} \alpha_{4} \alpha_{5} \alpha_{7}, C_{3}=\alpha_{5} \alpha_{7} \alpha_{8} \alpha_{10} \mathrm{e}$ $C_{4}=\alpha_{1} \alpha_{2}$. O programa exibe a solução 66 .

As relações do tipo 2 são: $r 1=\alpha_{4} \alpha_{5} \alpha_{7} \alpha_{8}, r 2=$ $\alpha_{10} \alpha_{5} \alpha_{7} \alpha_{9}, r 3=\alpha_{9} \alpha_{4} \alpha_{6}, r 4=\alpha_{3} \alpha_{4} \alpha_{5}, r 5=\alpha_{10} \alpha_{6}$, $r 6=\alpha_{2} \alpha_{3}$ e $r 7=\alpha_{6} \alpha_{1}$. Os ciclos elementares são: $C_{1}=\alpha_{3} \alpha_{4} \alpha_{6}, C_{2}=\alpha_{9} \alpha_{4} \alpha_{5} \alpha_{7}, C_{3}=\alpha_{5} \alpha_{7} \alpha_{8} \alpha_{10} \mathrm{e}$ $C_{4}=\alpha_{1} \alpha_{2}$. Logo, obtemos a álgebra 67 .

As relações do tipo 2 são: $r 1=\alpha_{4} \alpha_{5} \alpha_{7} \alpha_{8}, r 2=$ $\alpha_{10} \alpha_{5} \alpha_{7} \alpha_{9}, r 3=\alpha_{9} \alpha_{4} \alpha_{6}, r 4=\alpha_{3} \alpha_{4} \alpha_{5}, r 5=\alpha_{10} \alpha_{6}$, $r 6=\alpha_{2} \alpha_{7}$ e $r 7=\alpha_{5} \alpha_{1}$. Os ciclos elementares são: $C_{1}=\alpha_{3} \alpha_{4} \alpha_{6}, C_{2}=\alpha_{9} \alpha_{4} \alpha_{5} \alpha_{7}, C_{3}=\alpha_{5} \alpha_{7} \alpha_{8} \alpha_{10} \mathrm{e}$ $C_{4}=\alpha_{1} \alpha_{2}$. Portanto, dessa extensão trivial, origina a álgebra 68. 
(40)

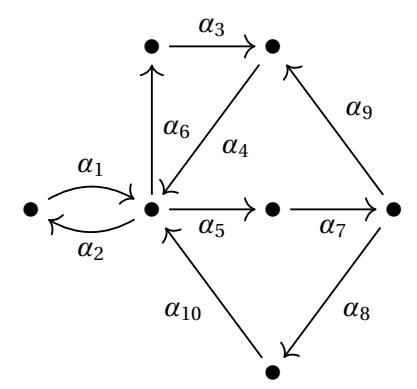

(41)

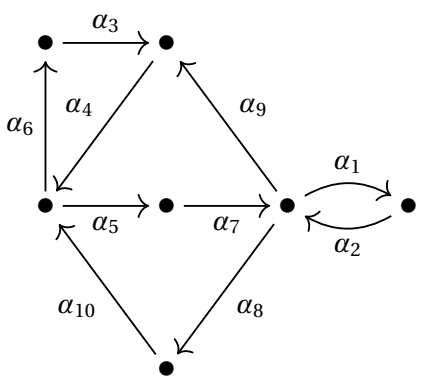

(42)

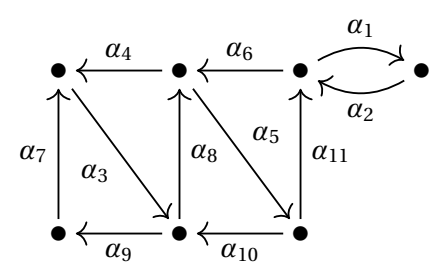

(43)

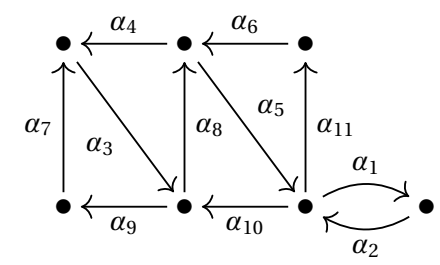

(44)

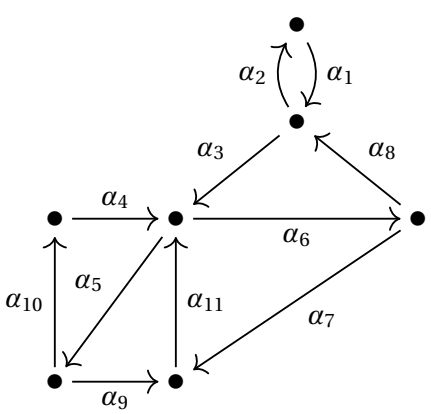

As relações do tipo 2 são: $r 1=\alpha_{4} \alpha_{5} \alpha_{7} \alpha_{8}, r 2=$ $\alpha_{10} \alpha_{5} \alpha_{7} \alpha_{9}, r 3=\alpha_{9} \alpha_{4} \alpha_{6}, r 4=\alpha_{3} \alpha_{4} \alpha_{5}, r 5=\alpha_{10} \alpha_{6}$, $r 6=\alpha_{1} \alpha_{5}, r 7=\alpha_{1} \alpha_{6}, r 8=\alpha_{4} \alpha_{2}$ e $r 9=\alpha_{10} \alpha_{2}$. Os ciclos elementares são: $C_{1}=\alpha_{3} \alpha_{4} \alpha_{6}, C_{2}=$ $\alpha_{9} \alpha_{4} \alpha_{5} \alpha_{7}, C_{3}=\alpha_{5} \alpha_{7} \alpha_{8} \alpha_{10}$ e $C_{4}=\alpha_{1} \alpha_{2}$. Obtemos as álgebras de incidência 69 e 70.

As relações do tipo 2 são: $r 1=\alpha_{4} \alpha_{5} \alpha_{7} \alpha_{8}, r 2=$ $\alpha_{10} \alpha_{5} \alpha_{7} \alpha_{9}, r 3=\alpha_{9} \alpha_{4} \alpha_{6}, r 4=\alpha_{3} \alpha_{4} \alpha_{5}, r 5=\alpha_{10} \alpha_{6}$, $r 6=\alpha_{7} \alpha_{1}, r 7=\alpha_{2} \alpha_{9}$ e $r 8=\alpha_{2} \alpha_{8}$. Os ciclos elementares são: $C_{1}=\alpha_{3} \alpha_{4} \alpha_{6}, C_{2}=\alpha_{9} \alpha_{4} \alpha_{5} \alpha_{7}$, $C_{3}=\alpha_{5} \alpha_{7} \alpha_{8} \alpha_{10}$ e $C_{4}=\alpha_{1} \alpha_{2}$. Não temos solução para esse caso.

As relações do tipo 2 são: $r 1=\alpha_{7} \alpha_{3} \alpha_{8}, r 2=$ $\alpha_{4} \alpha_{3} \alpha_{9}, r 3=\alpha_{3} \alpha_{8} \alpha_{5}, r 4=\alpha_{10} \alpha_{8} \alpha_{4}, r 5=\alpha_{8} \alpha_{5} \alpha_{11}$, $r 6=\alpha_{11} \alpha_{6} \alpha_{5}, r 7=\alpha_{6} \alpha_{4}, r 8=\alpha_{10} \alpha_{9}, r 9=\alpha_{11} \alpha_{1}$ e $r 10=\alpha_{2} \alpha_{6}$. Os ciclos elementares são: $C_{1}=$ $\alpha_{7} \alpha_{3} \alpha_{9}, C_{2}=\alpha_{3} \alpha_{8} \alpha_{5}, C_{3}=\alpha_{8} \alpha_{5} \alpha_{10}, C_{4}=\alpha_{6} \alpha_{5} \alpha_{11}$ e $C_{5}=\alpha_{1} \alpha_{2}$. O programa exibe apenas o corte que origina a álgebra 71 .

As relações do tipo 2 são: $r 1=\alpha_{7} \alpha_{3} \alpha_{8}, r 2=$ $\alpha_{4} \alpha_{3} \alpha_{9}, r 3=\alpha_{3} \alpha_{8} \alpha_{5}, r 4=\alpha_{10} \alpha_{8} \alpha_{4}, r 5=\alpha_{8} \alpha_{5} \alpha_{11}$, $r 6=\alpha_{11} \alpha_{6} \alpha_{5}, r 7=\alpha_{6} \alpha_{4}, r 8=\alpha_{10} \alpha_{9}, r 9=\alpha_{2} \alpha_{10}$, $r 10=\alpha_{2} \alpha_{11}$ e $r 11=\alpha_{5} \alpha_{1}$.

Os ciclos elementares são: $C_{1}=\alpha_{7} \alpha_{3} \alpha_{9}, C_{2}=$ $\alpha_{3} \alpha_{8} \alpha_{5}, C_{3}=\alpha_{8} \alpha_{5} \alpha_{10}, C_{4}=\alpha_{6} \alpha_{5} \alpha_{11}$ e $C_{5}=\alpha_{1} \alpha_{2}$. Portanto não temos solução para esse caso.

Essa extensão trivial tem as seguintes relações do tipo 2: $r 1=\alpha_{3} \alpha_{6} \alpha_{7}, r 2=\alpha_{11} \alpha_{6} \alpha_{8}, r 3=\alpha_{7} \alpha_{11} \alpha_{5}$, $r 4=\alpha_{9} \alpha_{11} \alpha_{6}, r 5=\alpha_{11} \alpha_{5} \alpha_{10}, r 6=\alpha_{4} \alpha_{5} \alpha_{9}, r 7=$ $\alpha_{4} \alpha_{6}, r 8=\alpha_{3} \alpha_{5}, r 9=\alpha_{1} \alpha_{3}$ e $r 10=\alpha_{8} \alpha_{2}$.

Os ciclos elementares são: $C_{1}=\alpha_{10} \alpha_{4} \alpha_{5}, C_{2}=$ $\alpha_{5} \alpha_{9} \alpha_{11}, C_{3}=\alpha_{11} \alpha_{6} \alpha_{7}, C_{4}=\alpha_{6} \alpha_{8} \alpha_{3}$ e $C_{5}=\alpha_{1} \alpha_{2}$. Essa extensão trivial origina a solução 72 . 
(45)

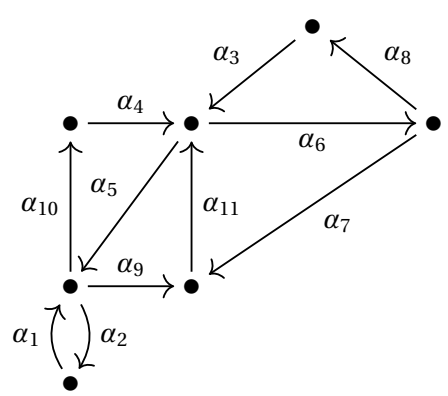

Essa extensão trivial tem as seguintes relações do tipo 2: $r 1=\alpha_{3} \alpha_{6} \alpha_{7}, r 2=\alpha_{11} \alpha_{6} \alpha_{8}, r 3=\alpha_{7} \alpha_{11} \alpha_{5}$, $r 4=\alpha_{9} \alpha_{11} \alpha_{6}, r 5=\alpha_{11} \alpha_{5} \alpha_{10}, r 6=\alpha_{4} \alpha_{5} \alpha_{9}, r 7=$ $\alpha_{4} \alpha_{6}, r 8=\alpha_{3} \alpha_{5}, r 9=\alpha_{1} \alpha_{10}, r 10=\alpha_{5} \alpha_{2}$ e $r 11=$ $\alpha_{1} \alpha_{9}$

Os ciclos elementares são: $C_{1}=\alpha_{10} \alpha_{4} \alpha_{5}, C_{2}=$ $\alpha_{5} \alpha_{9} \alpha_{11}, C_{3}=\alpha_{11} \alpha_{6} \alpha_{7}, C_{4}=\alpha_{6} \alpha_{8} \alpha_{3}$ e $C_{5}=\alpha_{1} \alpha_{2}$. Logo, dessa extensão trivial, obtemos a álgebra 73.

\subsection{0 tipo $\mathbb{E}_{8}$}

Na seção anterior, explicamos os resultados e os métodos aplicados na identificação de todas as álgebras de incidência que não são hereditárias e são derivadamente equivalentes a $K \mathbb{E}_{7}$. Repetiremos toda a sistemática para o caso $\mathbb{E}_{8}$.

As extensões triviais de tipo de representação finito de classe de Cartan $\mathbb{E}_{8}$ são álgebras inclinadas iteradas de tipo $\mathbb{E}_{8}$, e vice-versa. Pela tese de Elsa Fernández [Fer99], temos a listagem de todas as extensões triviais desse tipo, contabilizando 251. A partir disso, apresentaremos todas as álgebras de incidência não hereditárias originadas do corte de cada extensão trivial, como fizemos para $\mathbb{E}_{7}$. Fizemos o trabalho manual de encontrar as álgebras de incidência através dos cortes para cada uma das extensões triviais, só que para escrever a demonstração preferimos usar o programa deixando mais enxuta a prova.

Teorema 5.25. Seja $K \Delta=K Q / I$ uma álgebra de incidência e iterada inclinada de tipo $\mathbb{E}_{8}$ tal que $\bar{Q}$ não é uma árvore. Então $K \Delta$ ou $K \Delta^{o p}$ é isomorfa a

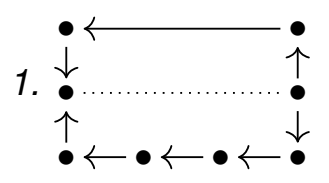

2.

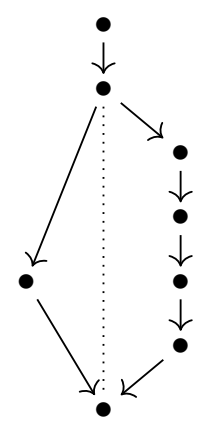

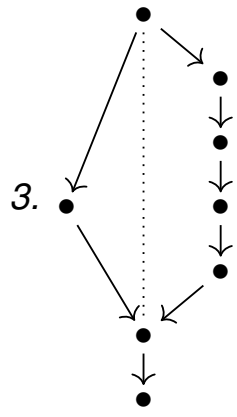



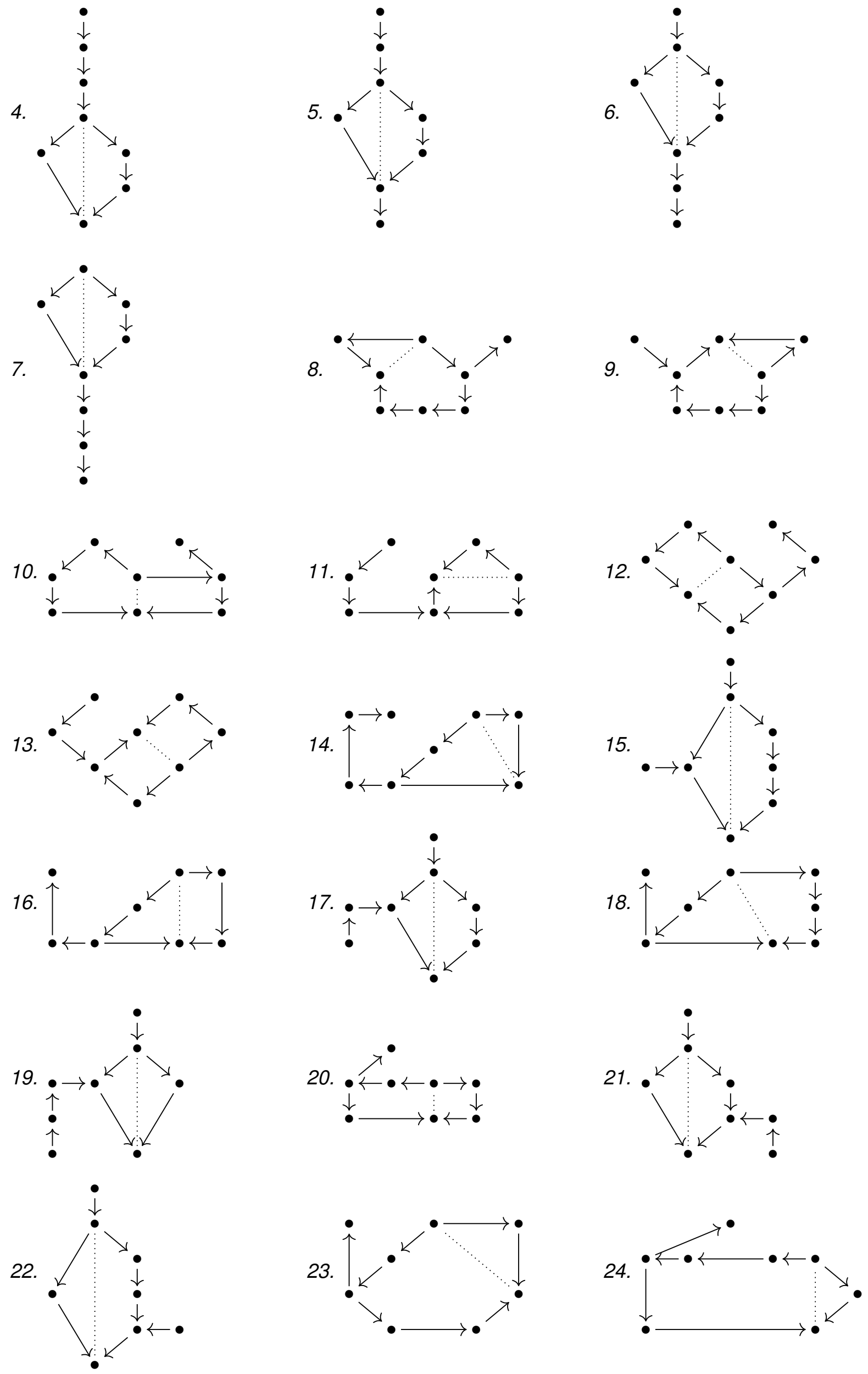

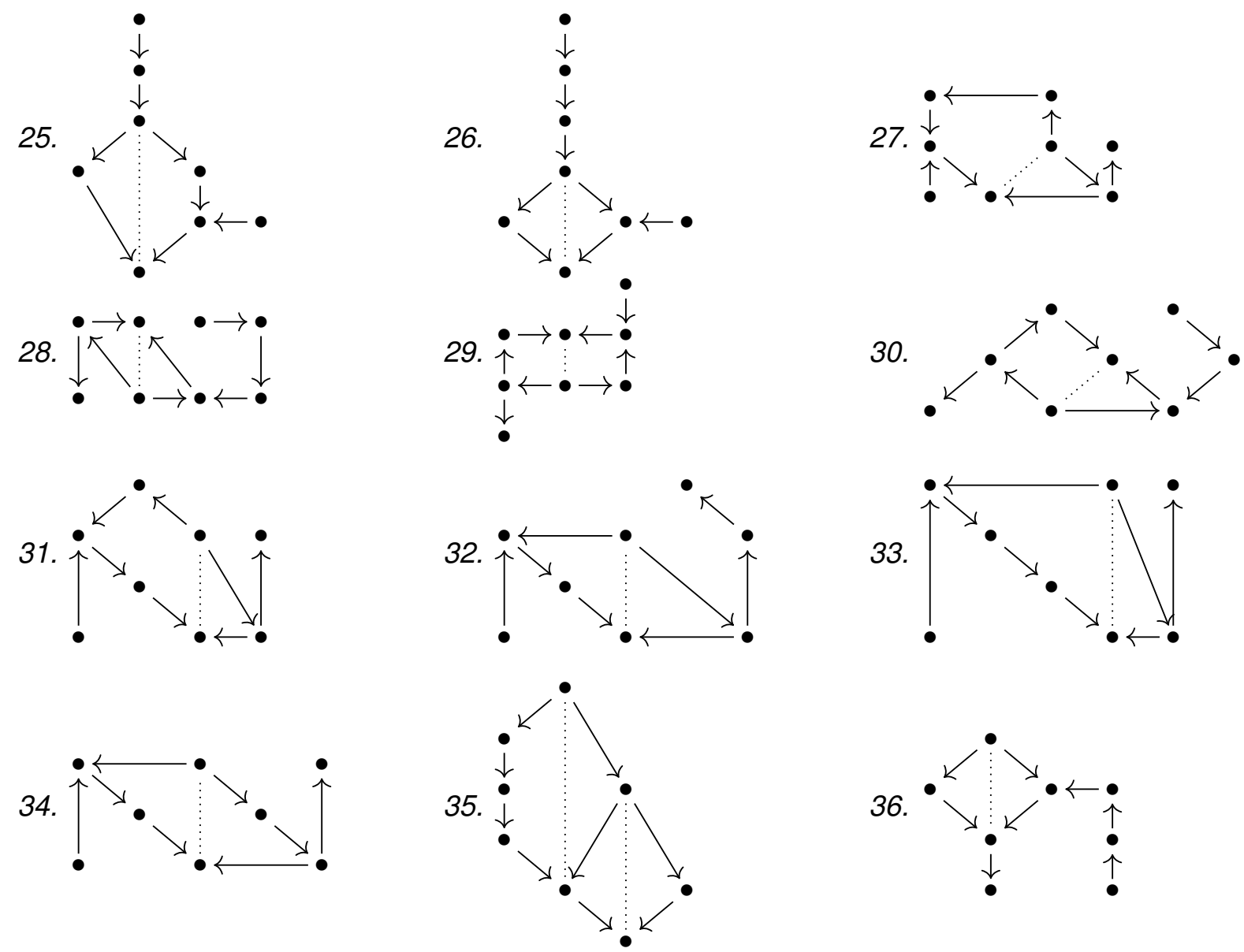

37.
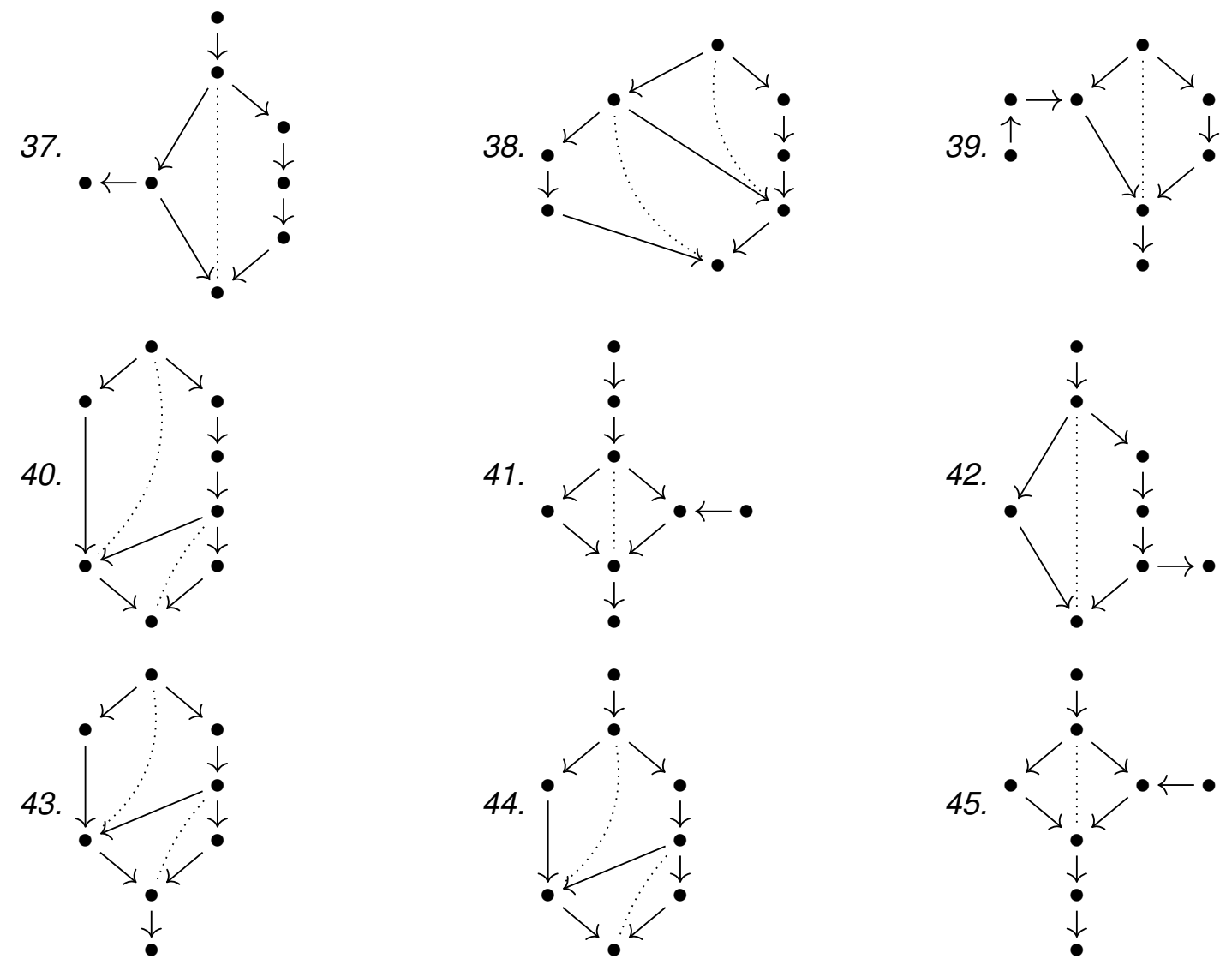
46.

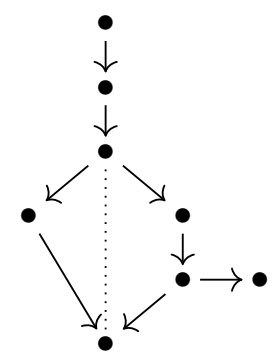

49.

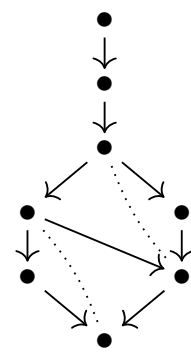

52.

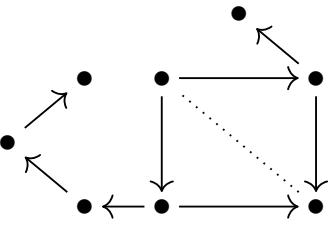

55.

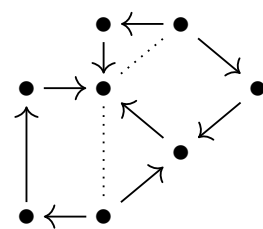

58.

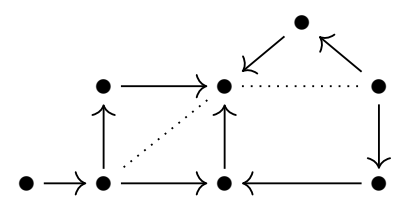

61. $\stackrel{\bullet}{\longleftarrow} \longrightarrow$
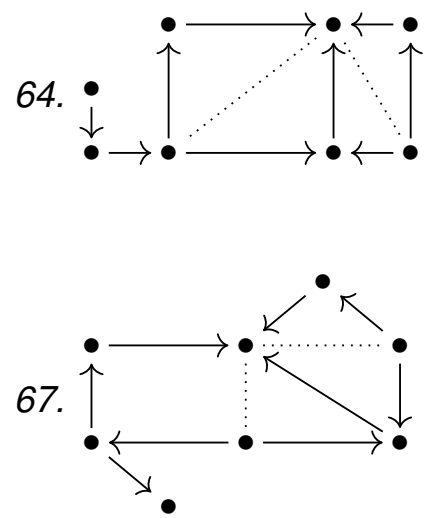

47.

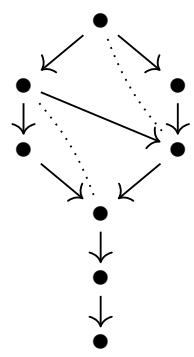

50.

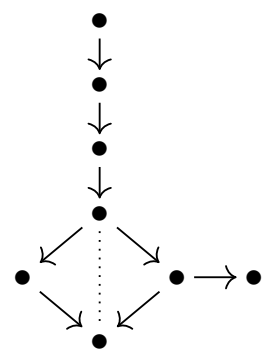

53.

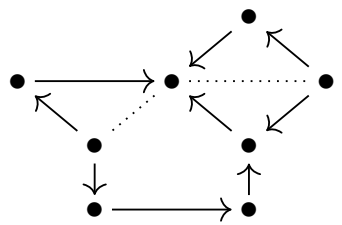

59.

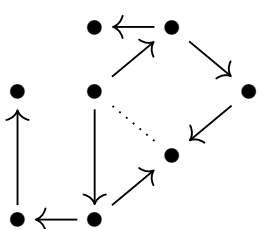

62.
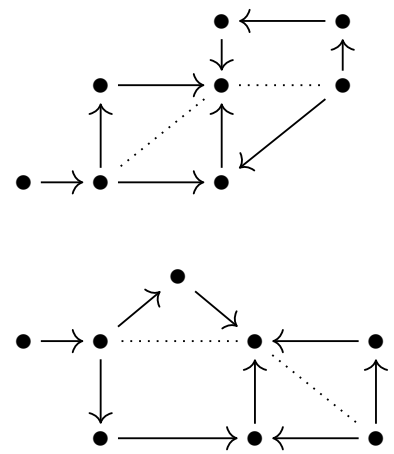

65.

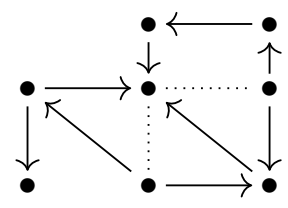

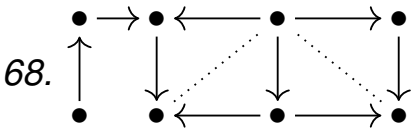

48.

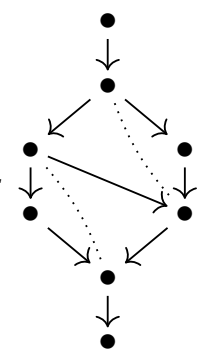

54.
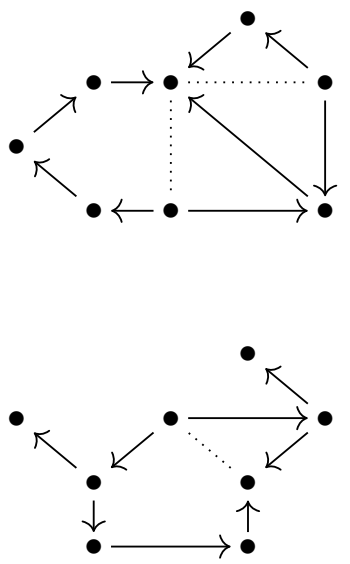

57.

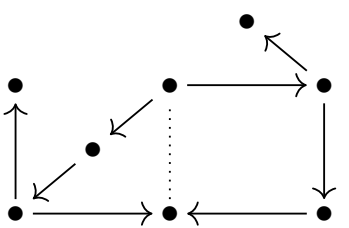

63.

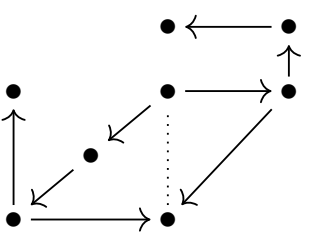

66.

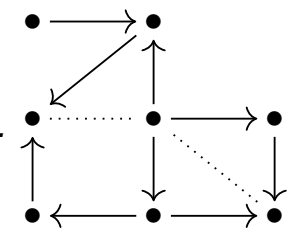

69.

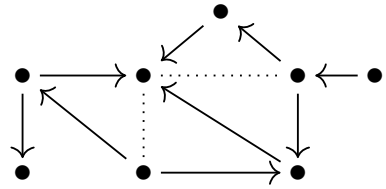




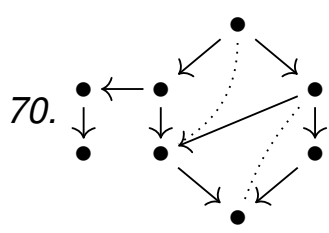

71.

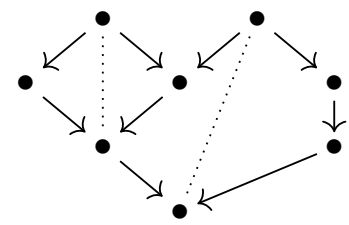

74.

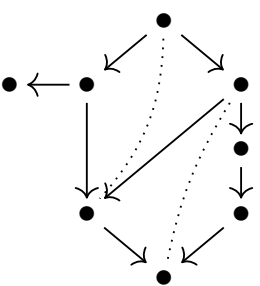

76.

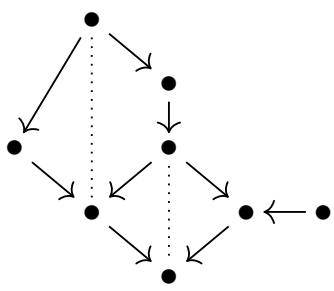

79.

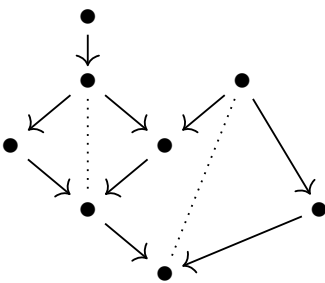

82.

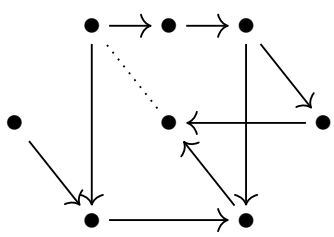

85.
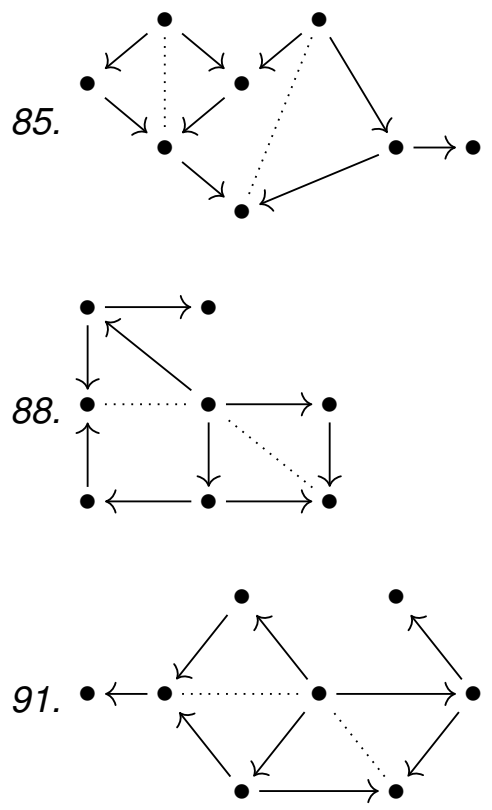

83.

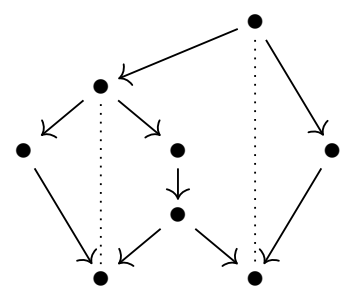

80.

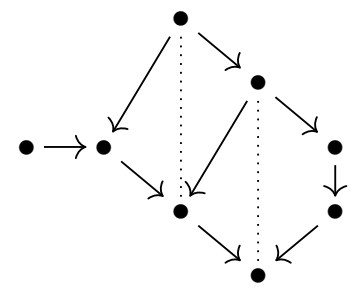

86.

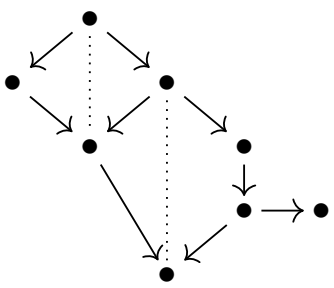

89.
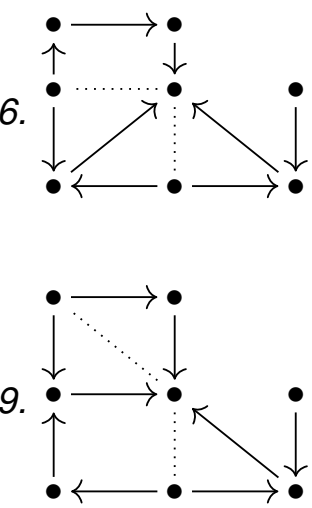

92. $\stackrel{\bullet}{\rightarrow} \uparrow \longleftarrow$
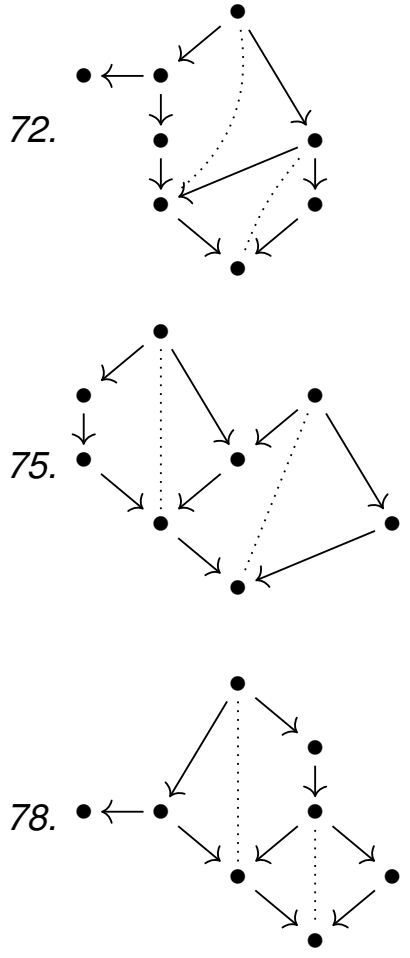

81.

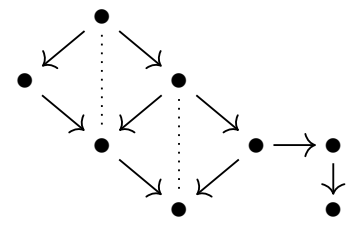

84.

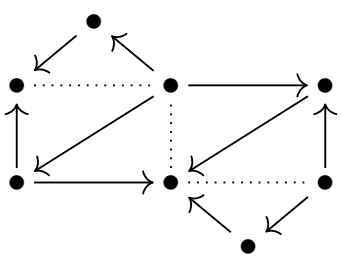

87.

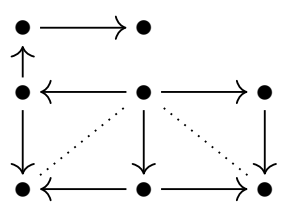

90.

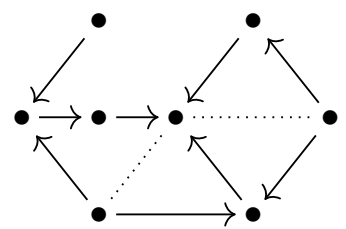

93.

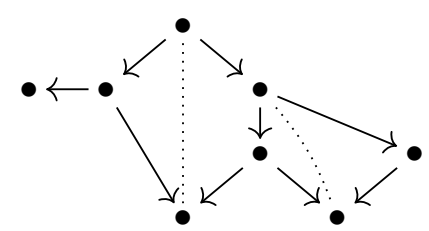




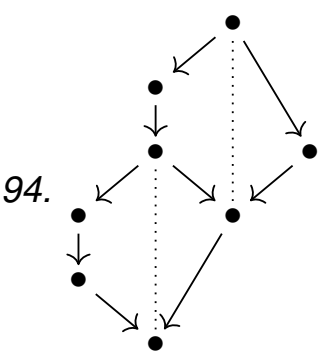

95.

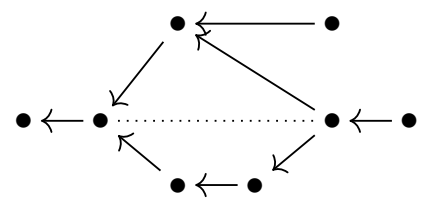

97.

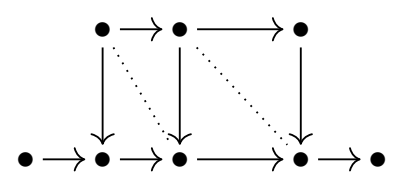

100.

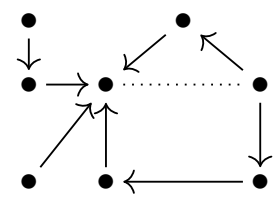

103.

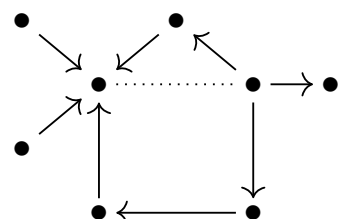

106.

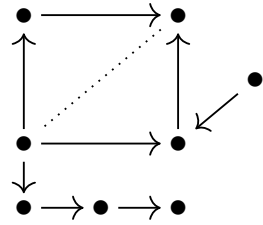

109

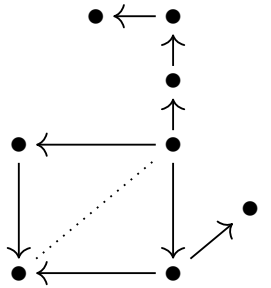

112.

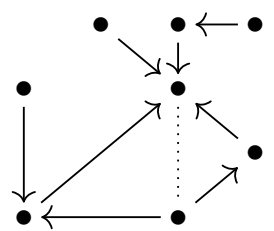

115.

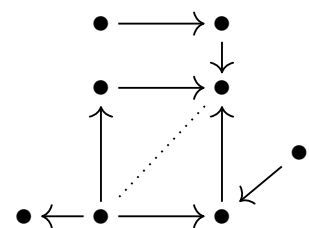

98.

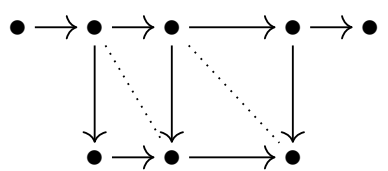

101.
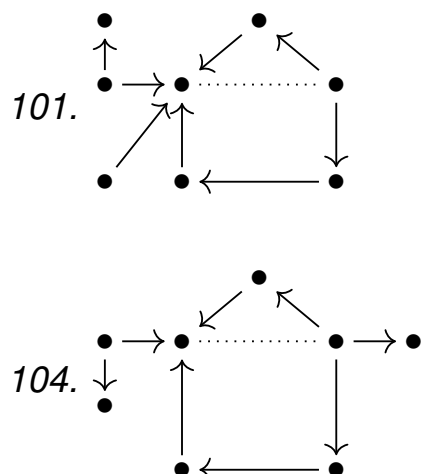

110.
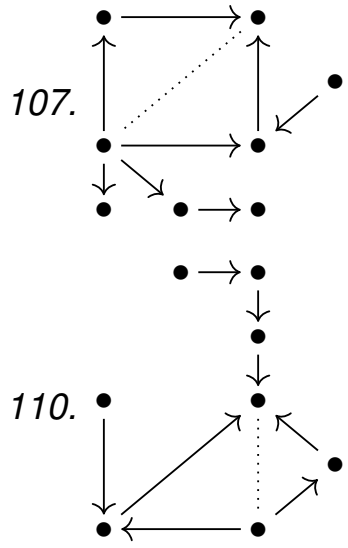

113.

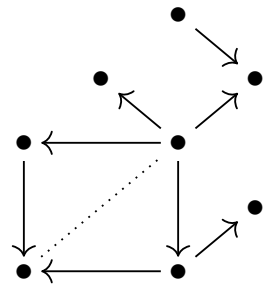

116

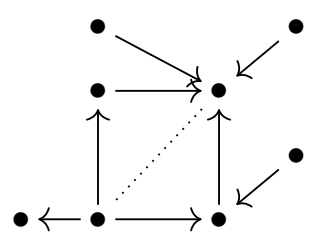

96.

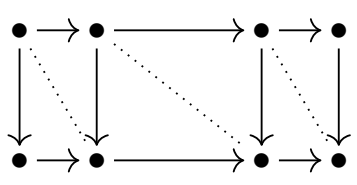

99.

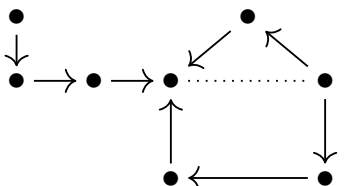

102.

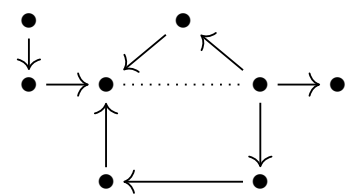

105.

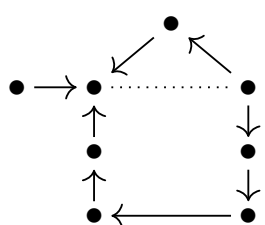

108.

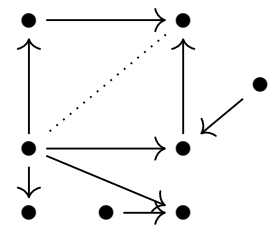

111

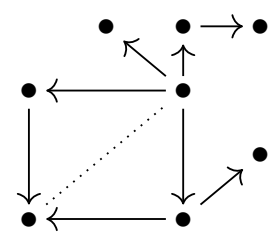

114

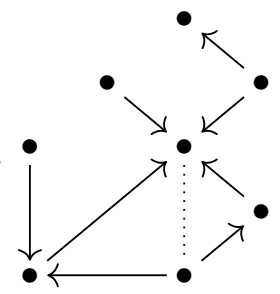

117.

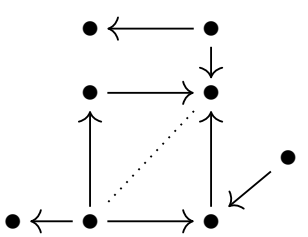


118.
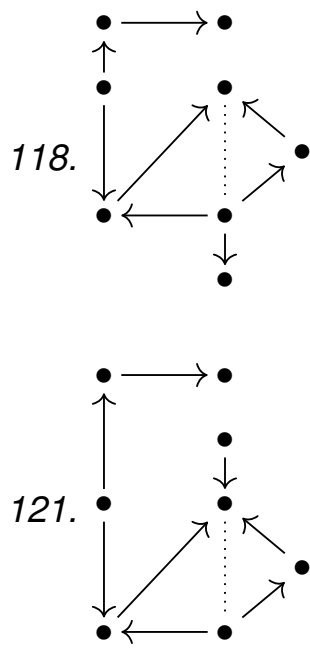

124

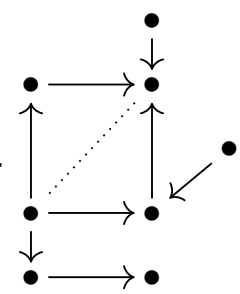

127.
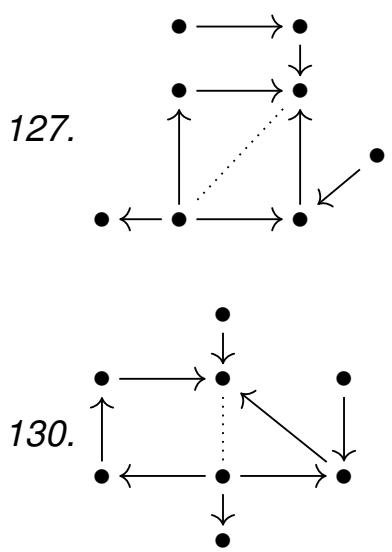

133.
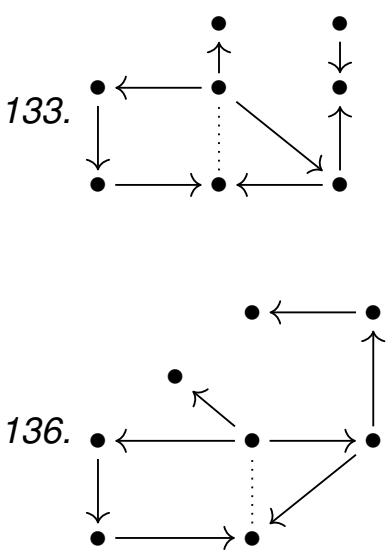

119.

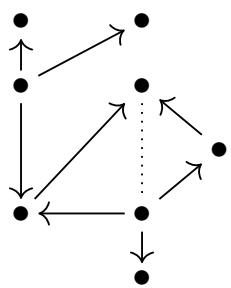

122.

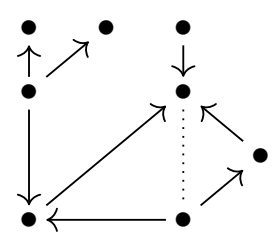

125.

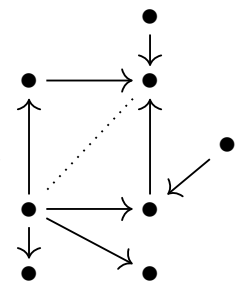

128.

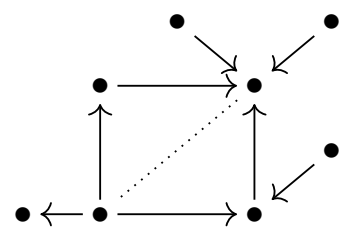

131.

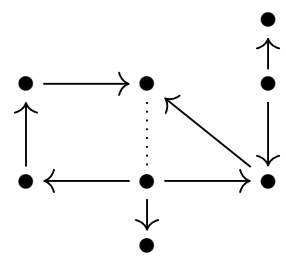

134.

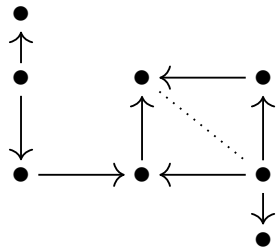

137

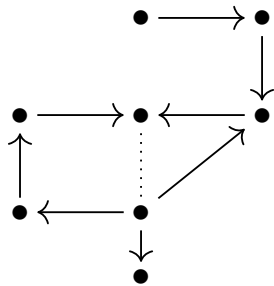

120.

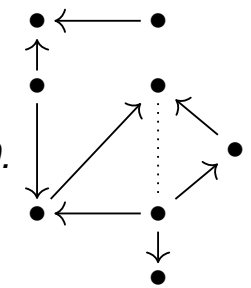

123.

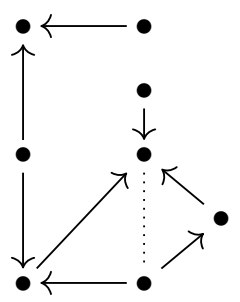

126

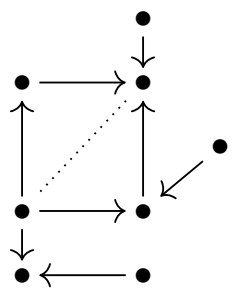

129.

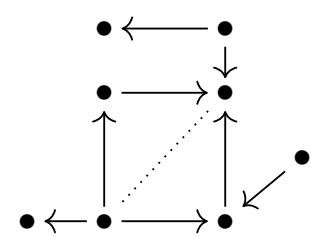

132.

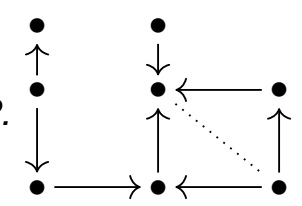

135.

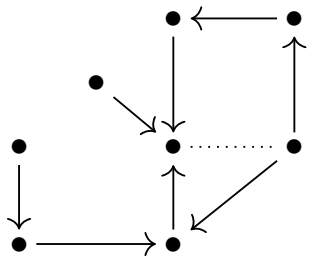

138.

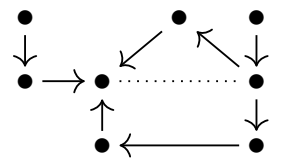


139.

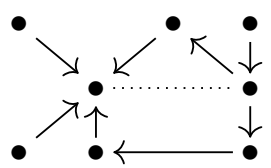

142.

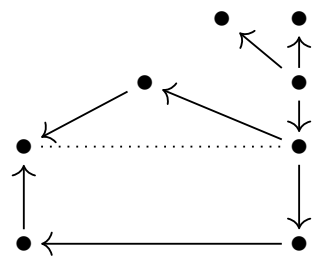

145.

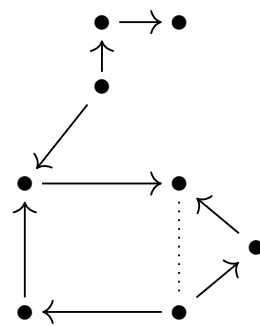

148.
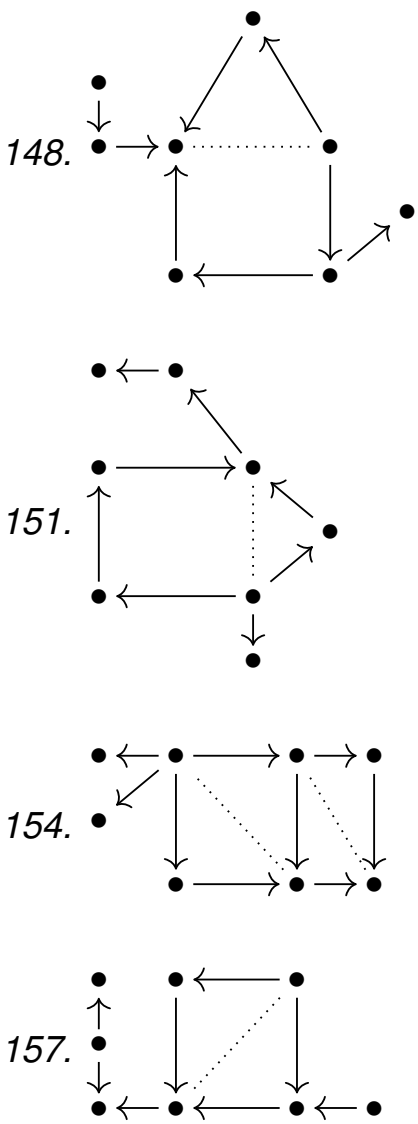

160.

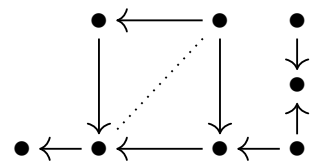

140.

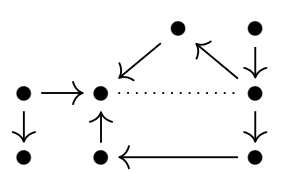

143.

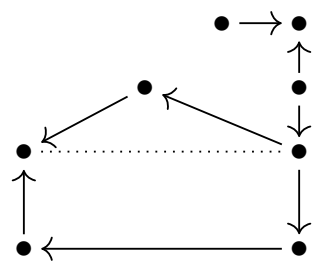

146.

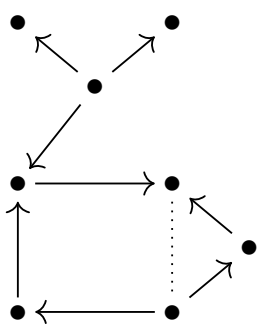

149.

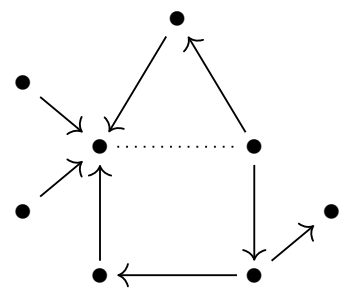

152.

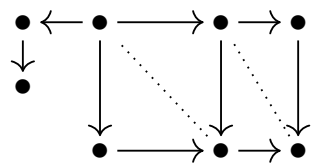

155.

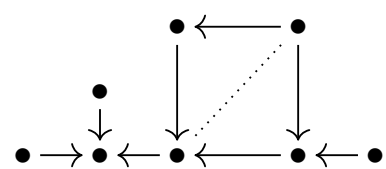

158.

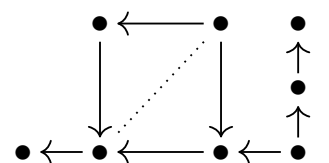

161.

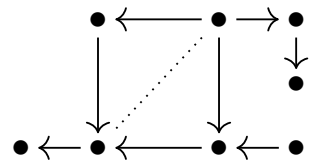

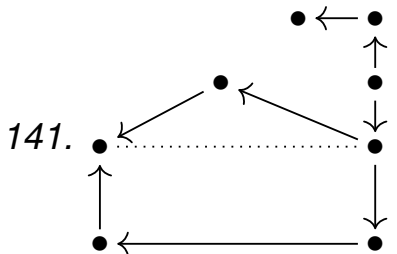
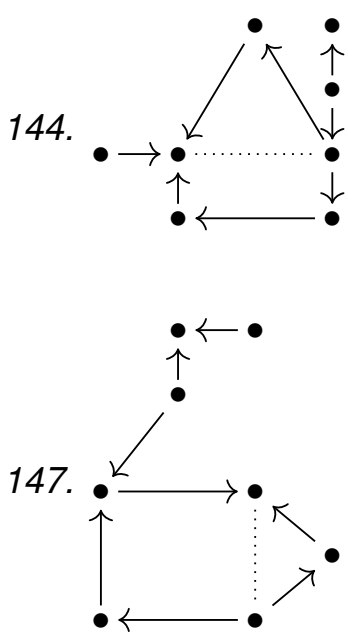

150.

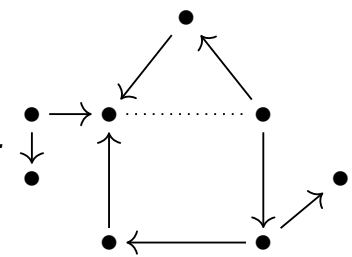

153.

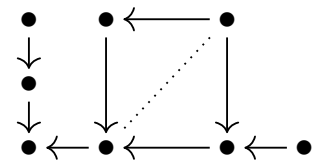

156.

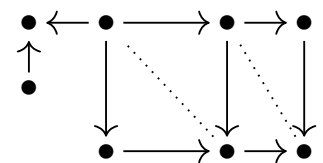

159.

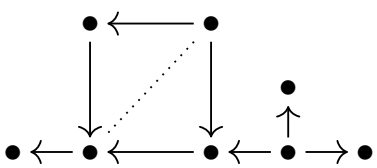

162.

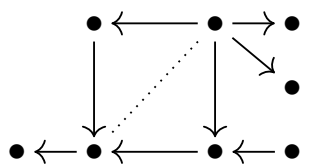


163.

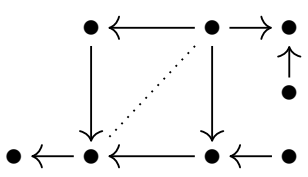

166.

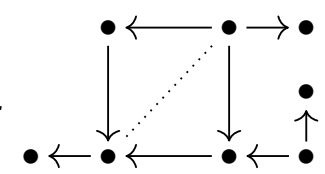

169.
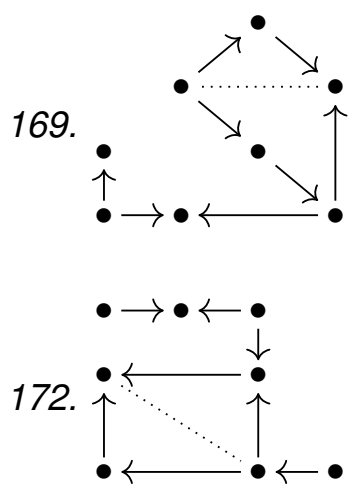

175

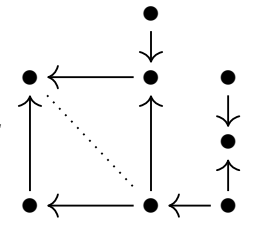

178.

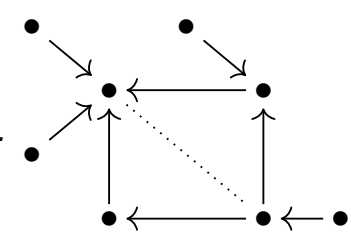

181.
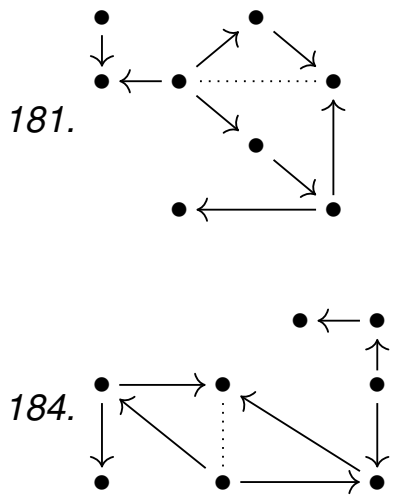

187.

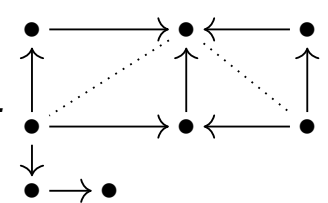

164.

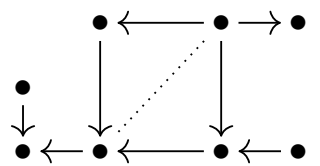

167.

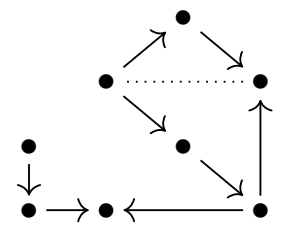

170

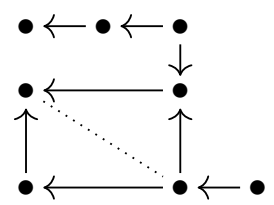

173.

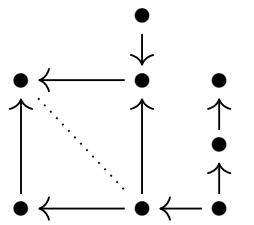

176.

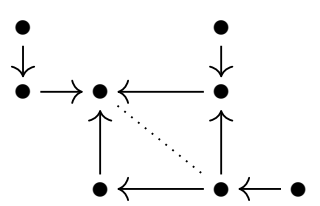

179.

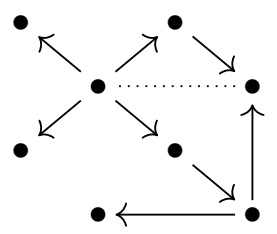

182.

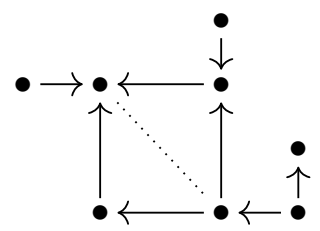

185.

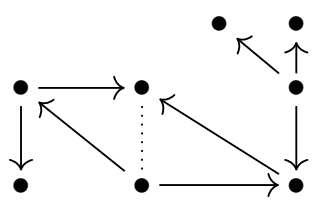

188.

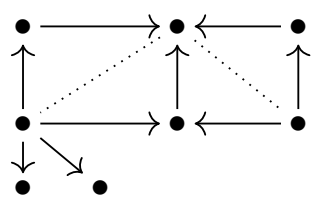

165.

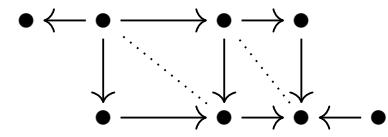

168.

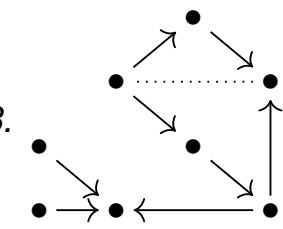

171.
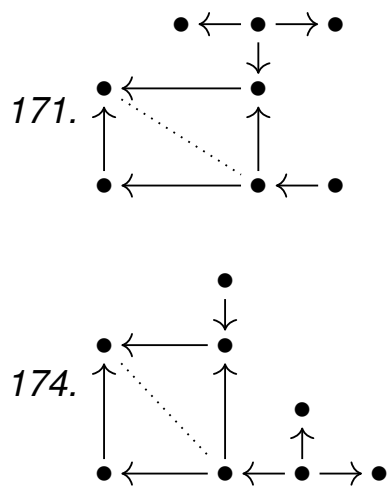

177.

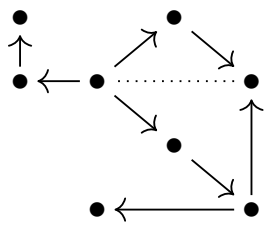

180.

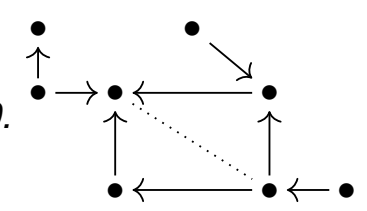

183.

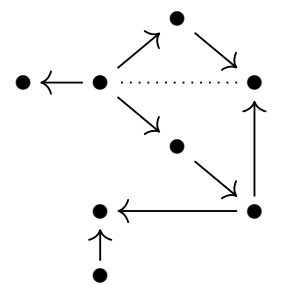

186.

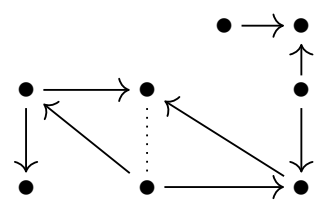

189.

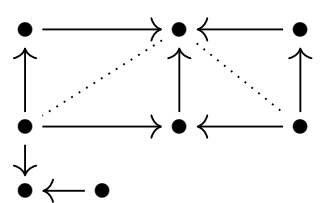




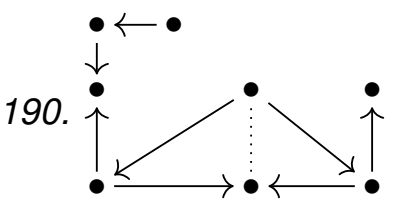

193.

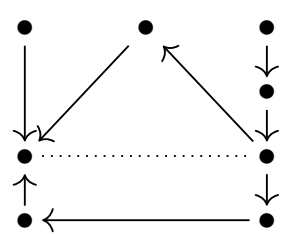

196.

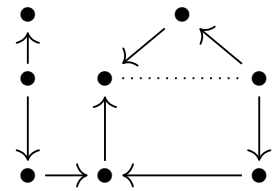

199.

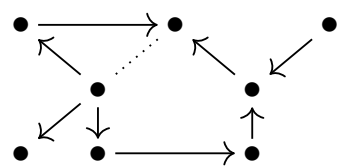

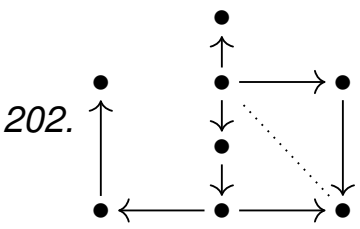

205.

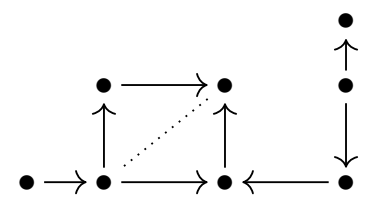

208.

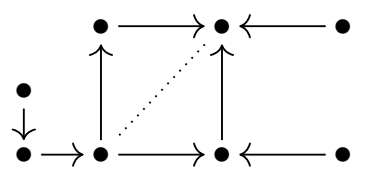

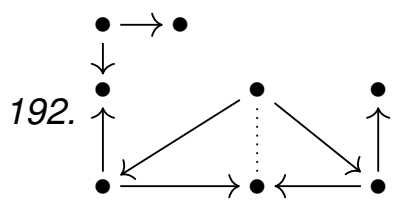

194.

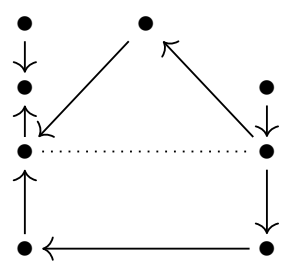

197.

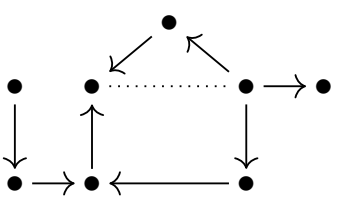

203.

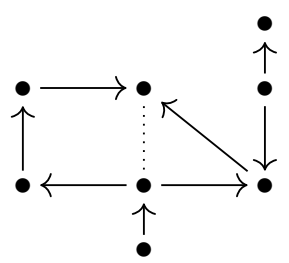

206.

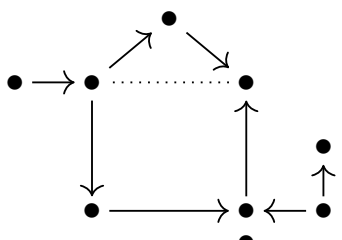

209.

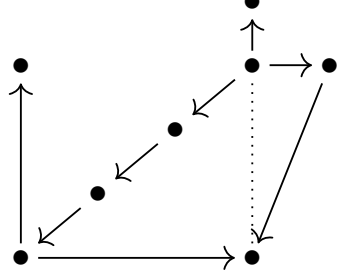

212.

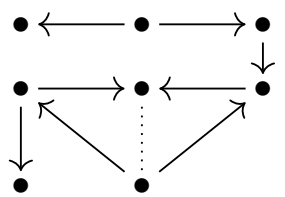

195.

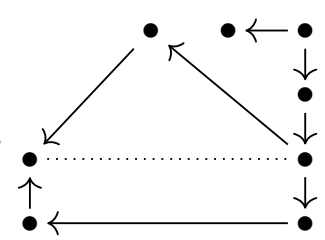

198.

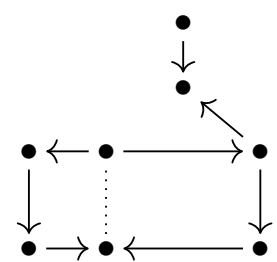

201.

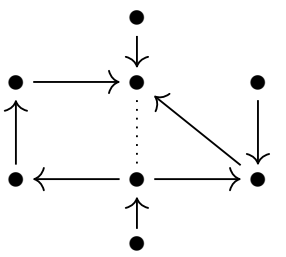

204.

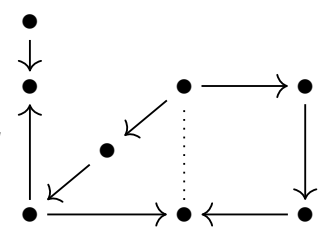

207.

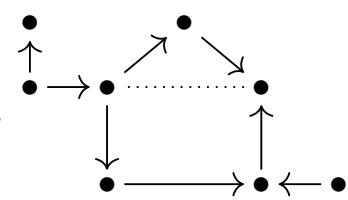

210.

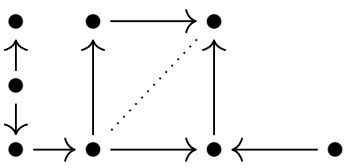

213. 


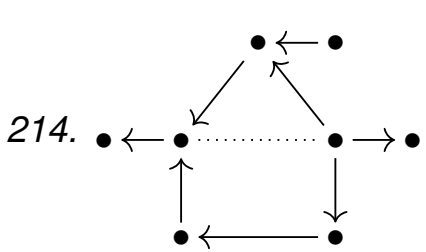

215.
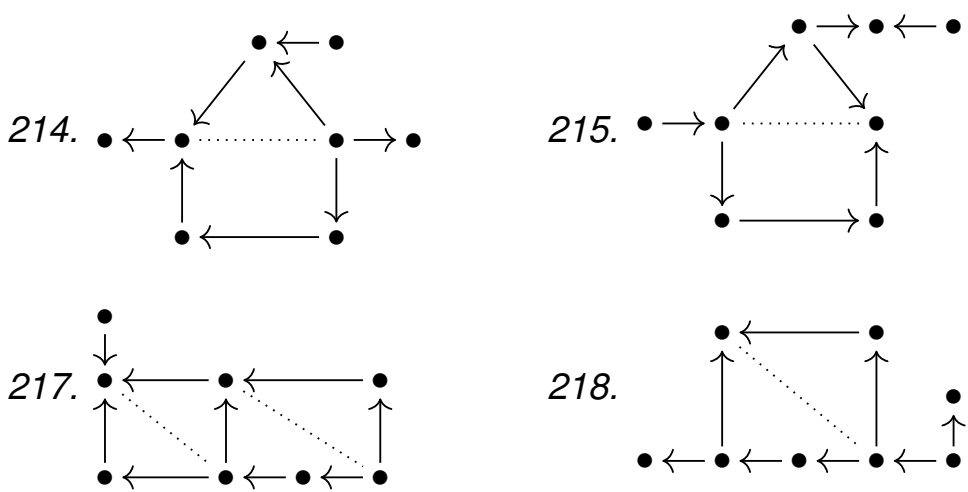

220.

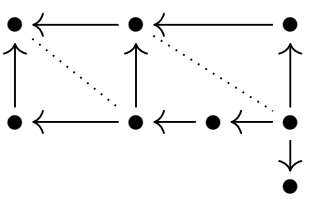

223.

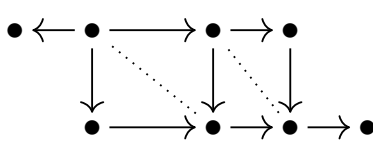

226.

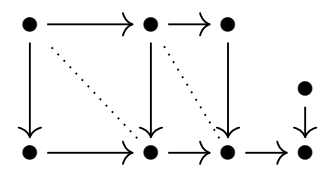

218.

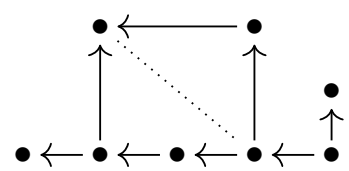

221.

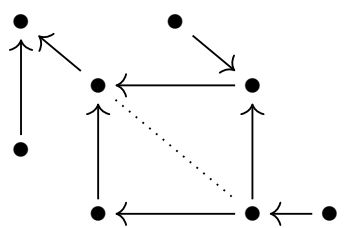

224

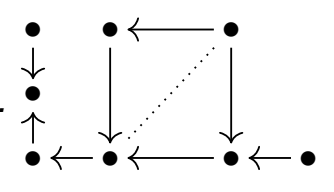

227.

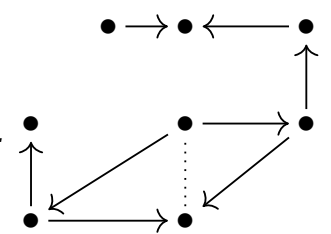

230.

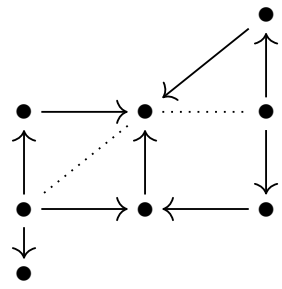

233.

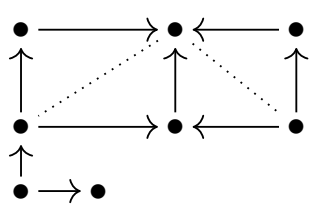

236.

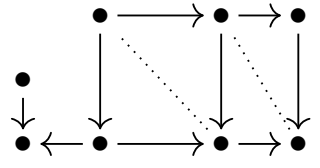

235

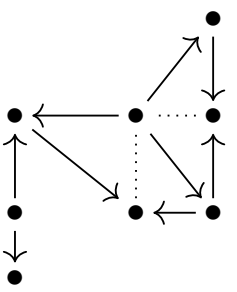

238.

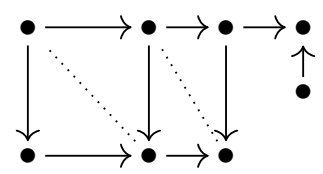

216.

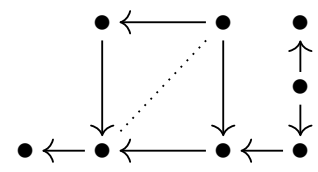

219.

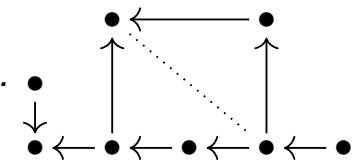

222.

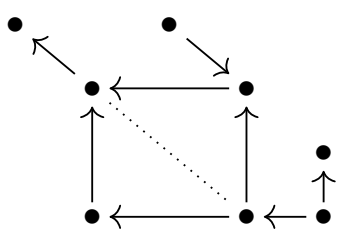

225.

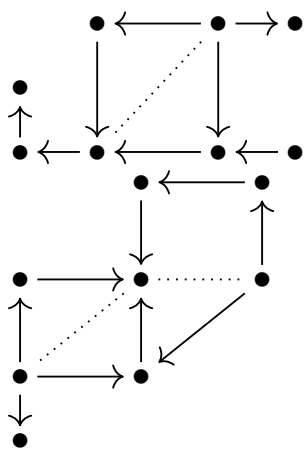

231.

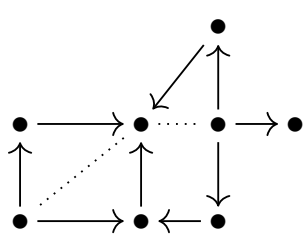

234.
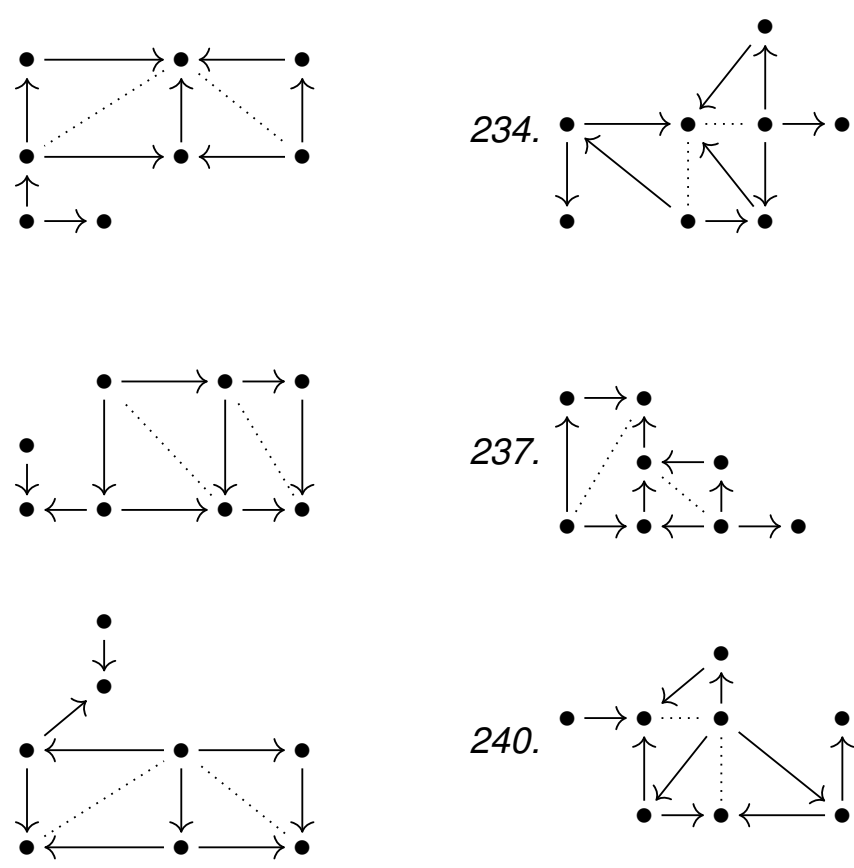

237.

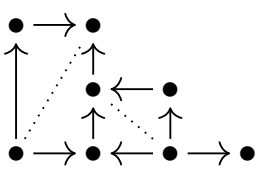

240.

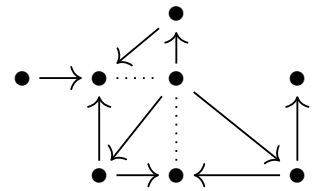


241.

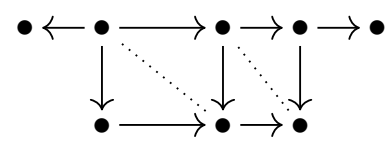

242.

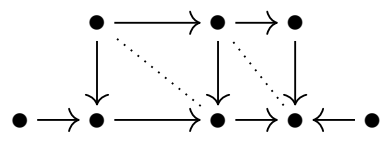

Demonstração A sistemática da demonstração é analisar cada extensão trivial, exibindo as suas relações de tipo 2 e os seus ciclos elementares. Com essas informações, aplicamos no nosso programa e retiramos os cortes originando as álgebras de incidência. Assim, mostramos na lista do nosso teorema apenas as álgebras de incidência não hereditárias.

Iniciaremos com a lista enumerada do corolário 3.2.9 da tese [Fer99], dentre essas extensões triviais apenas as álgebras (1), (2) e (3), podemos obter álgebras de incidência hereditárias através do corte.

(1)

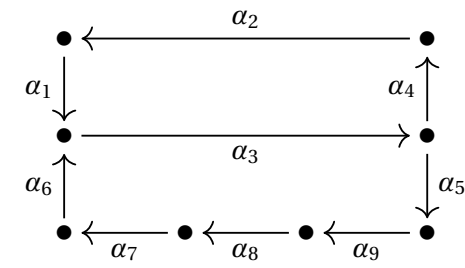

(2)

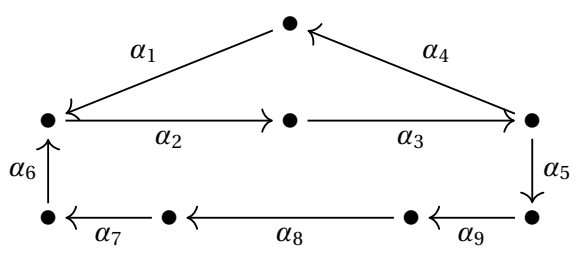

(3)

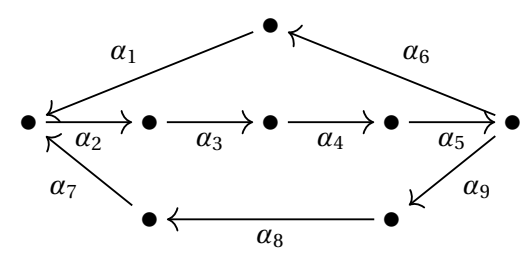

(4)

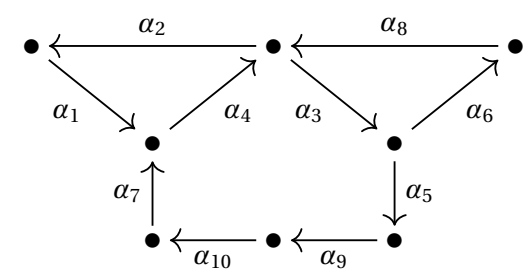

(5)

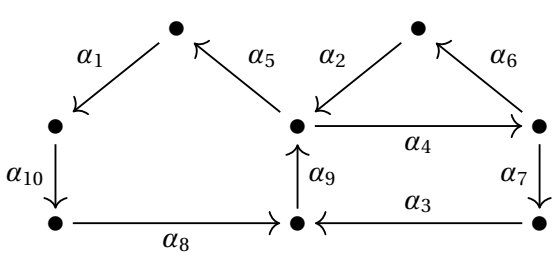

As relações do tipo 2 são: $r 1=\alpha_{1} \alpha_{3} \alpha_{5}$ e $r 2=$ $\alpha_{6} \alpha_{3} \alpha_{4}$.

Os ciclos elementares são: $C_{1}=\alpha_{1} \alpha_{3} \alpha_{4} \alpha_{2}$ e $C_{2}=$ $\alpha_{3} \alpha_{5} \alpha_{9} \alpha_{8} \alpha_{7} \alpha_{6}$. Portanto temos apenas a solução 1 do teorema.

As relações do tipo 2 são: $r 1=\alpha_{1} \alpha_{2} \alpha_{3} \alpha_{5}$ e $r 2=$ $\alpha_{6} \alpha_{2} \alpha_{3} \alpha_{4}$.

Os ciclos elementares são: $C_{1}=\alpha_{1} \alpha_{2} \alpha_{3} \alpha_{4}$ e $C_{2}=$ $\alpha_{2} \alpha_{3} \alpha_{5} \alpha_{9} \alpha_{8} \alpha_{7} \alpha_{6}$. Assim, o programa mostra as soluções 2 e 3 do teorema.

As relações do tipo 2 são: $r 1=\alpha_{1} \alpha_{2} \alpha_{3} \alpha_{4} \alpha_{5} \alpha_{9}$ e $r 2=\alpha_{7} \alpha_{2} \alpha_{3} \alpha_{4} \alpha_{5} \alpha_{6}$.

Os ciclos elementares são: $C_{1}=\alpha_{2} \alpha_{3} \alpha_{4} \alpha_{5} \alpha_{9} \alpha_{8} \alpha_{7}$ e $C_{2}=\alpha_{1} \alpha_{2} \alpha_{3} \alpha_{4} \alpha_{5} \alpha_{6}$. Logo, obtemos as álgebras 4 , 5,6 e 7 .

As relações do tipo 2 são: $r 1=\alpha_{7} \alpha_{4} \alpha_{2}, r 2=$ $\alpha_{1} \alpha_{4} \alpha_{3}, r 3=\alpha_{4} \alpha_{3} \alpha_{6}, r 4=\alpha_{8} \alpha_{3} \alpha_{5}$ e $r 5=\alpha_{8} \alpha_{2}$.

Os ciclos elementares são: $C_{1}=\alpha_{2} \alpha_{1} \alpha_{4}, C_{2}=$ $\alpha_{4} \alpha_{3} \alpha_{5} \alpha_{9} \alpha_{10} \alpha_{7}$ e $C_{3}=\alpha_{3} \alpha_{6} \alpha_{8}$. O programa exibe os cortes que originam as álgebras 8 e 9 .

As relações do tipo 2 são: $r 1=\alpha_{2} \alpha_{4} \alpha_{7}, r 2=$ $\alpha_{9} \alpha_{4} \alpha_{6}, r 3=\alpha_{3} \alpha_{9} \alpha_{5}, r 4=\alpha_{8} \alpha_{9} \alpha_{4}$ e $r 5=\alpha_{2} \alpha_{5}$.

Os ciclos elementares são: $C_{1}=\alpha_{2} \alpha_{4} \alpha_{6}, C_{2}=$ $\alpha_{1} \alpha_{10} \alpha_{8} \alpha_{9} \alpha_{5}$ e $C_{3}=\alpha_{4} \alpha_{7} \alpha_{3} \alpha_{9}$. Portanto obtemos as álgebras 10 e 11. 
(6)

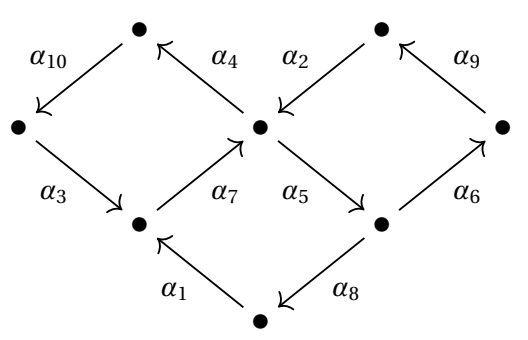

(7)

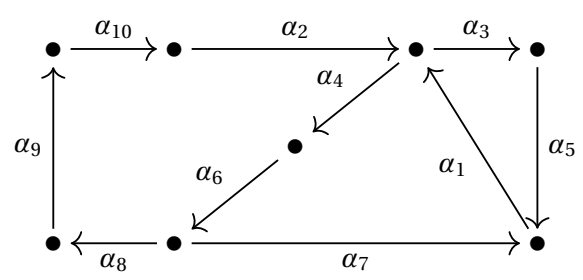

(8)

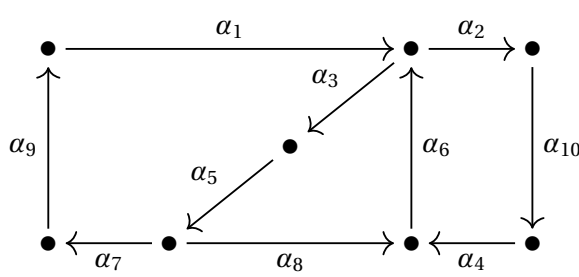

(9)

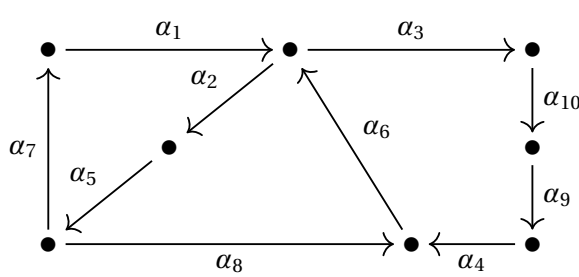

(10)

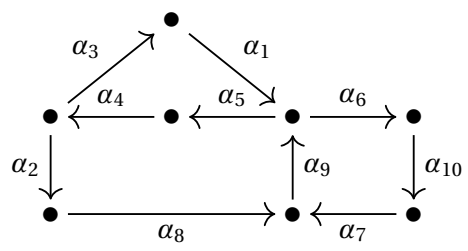

(11)

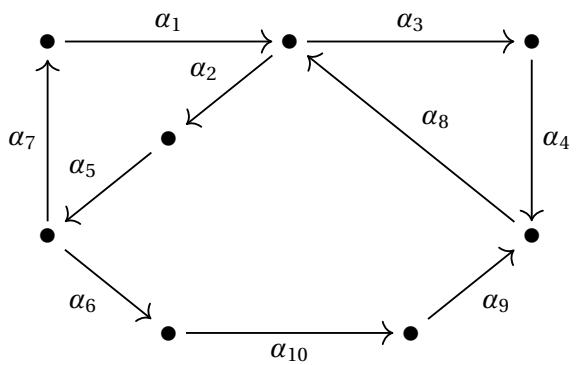

As relações do tipo 2 são: $r 1=\alpha_{3} \alpha_{7} \alpha_{5}, r 2=$ $\alpha_{1} \alpha_{7} \alpha_{4}, r 3=\alpha_{2} \alpha_{5} \alpha_{8}, r 4=\alpha_{7} \alpha_{5} \alpha_{6}$ e $r 5=\alpha_{2} \alpha_{4}$.

Os ciclos elementares são: $C_{1}=\alpha_{10} \alpha_{3} \alpha_{7} \alpha_{4}, C_{2}=$ $\alpha_{2} \alpha_{5} \alpha_{6} \alpha_{9}$ e $C_{3}=\alpha_{7} \alpha_{5} \alpha_{8} \alpha_{1}$. Assim, temos as soluções 12 e 13 .

As relações do tipo 2 são: $r 1=\alpha_{2} \alpha_{4} \alpha_{6} \alpha_{7}, r 2=$ $\alpha_{1} \alpha_{4} \alpha_{6} \alpha_{8}, r 3=\alpha_{7} \alpha_{1} \alpha_{3}, r 4=\alpha_{5} \alpha_{1} \alpha_{4}$ e $r 5=\alpha_{2} \alpha_{3}$. Os ciclos elementares são: $C_{1}=\alpha_{10} \alpha_{2} \alpha_{4} \alpha_{6} \alpha_{8} \alpha_{9}$, $C_{2}=\alpha_{4} \alpha_{6} \alpha_{7} \alpha_{1}$ e $C_{3}=\alpha_{3} \alpha_{5} \alpha_{1}$. Logo, o programa exibe os cortes para obter as álgebras 14 e 15.

As relações do tipo 2 são: $r 1=\alpha_{1} \alpha_{3} \alpha_{5} \alpha_{8}, r 2=$ $\alpha_{6} \alpha_{3} \alpha_{5} \alpha_{7}, r 3=\alpha_{8} \alpha_{6} \alpha_{2}, r 4=\alpha_{4} \alpha_{6} \alpha_{3}$ e $r 5=\alpha_{1} \alpha_{2}$. Os ciclos elementares são: $C_{1}=\alpha_{1} \alpha_{3} \alpha_{5} \alpha_{7} \alpha_{9}, C_{2}=$ $\alpha_{6} \alpha_{3} \alpha_{5} \alpha_{8}$ e $C_{3}=\alpha_{2} \alpha_{10} \alpha_{4} \alpha_{6}$. A partir disso, temos as soluções 16 e 17 .

As relações do tipo 2 são: $r 1=\alpha_{1} \alpha_{2} \alpha_{5} \alpha_{8}, r 2=$ $\alpha_{6} \alpha_{2} \alpha_{5} \alpha_{7}, r 3=\alpha_{8} \alpha_{6} \alpha_{3}, r 4=\alpha_{4} \alpha_{6} \alpha_{2}$ e $r 5=\alpha_{1} \alpha_{3}$. Os ciclos elementares são: $C_{1}=\alpha_{1} \alpha_{2} \alpha_{5} \alpha_{7}, C_{2}=$ $\alpha_{6} \alpha_{2} \alpha_{5} \alpha_{8}$ e $C_{3}=\alpha_{10} \alpha_{9} \alpha_{4} \alpha_{6} \alpha_{3}$. Logo, obtemos as álgebras 18 e 19 .

As relações do tipo 2 são: $r 1=\alpha_{1} \alpha_{5} \alpha_{4} \alpha_{2}, r 2=$ $\alpha_{9} \alpha_{5} \alpha_{4} \alpha_{3}, r 3=\alpha_{7} \alpha_{9} \alpha_{5}, r 4=\alpha_{8} \alpha_{9} \alpha_{6}$ e $r 5=\alpha_{1} \alpha_{6}$. Os ciclos elementares são: $C_{1}=\alpha_{1} \alpha_{5} \alpha_{4} \alpha_{3}, C_{2}=$ $\alpha_{2} \alpha_{8} \alpha_{9} \alpha_{5} \alpha_{4}$ e $C_{3}=\alpha_{6} \alpha_{10} \alpha_{7} \alpha_{9}$. Portanto, o programa mostra as álgebras 20 e 21 .

As relações do tipo 2 são: $r 1=\alpha_{1} \alpha_{2} \alpha_{5} \alpha_{6}, r 2=$ $\alpha_{8} \alpha_{2} \alpha_{5} \alpha_{7}, r 3=\alpha_{4} \alpha_{8} \alpha_{2}, r 4=\alpha_{9} \alpha_{8} \alpha_{3}$ e $r 5=\alpha_{1} \alpha_{3}$. Os ciclos elementares são: $C_{1}=\alpha_{1} \alpha_{2} \alpha_{5} \alpha_{7}, C_{2}=$ $\alpha_{8} \alpha_{2} \alpha_{5} \alpha_{6} \alpha_{10} \alpha_{9}$ e $C_{3}=\alpha_{3} \alpha_{4} \alpha_{8}$. O programa exibe as soluções 22 e 23 . 
(12)

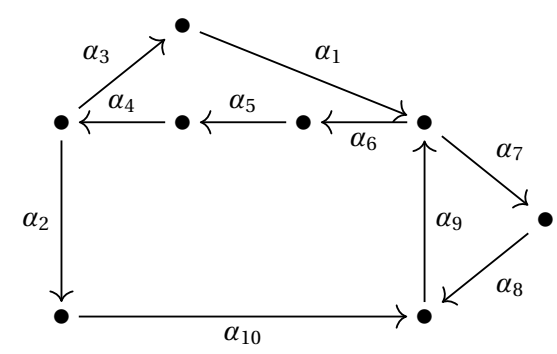

(13)

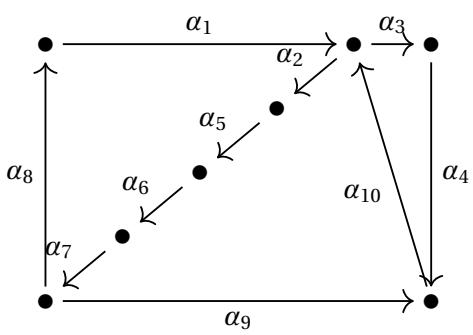

(14)

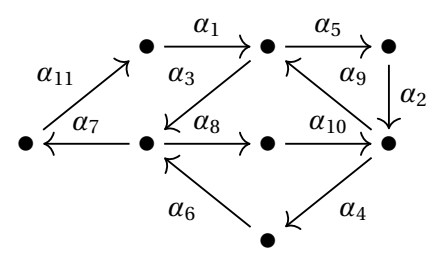

(15)

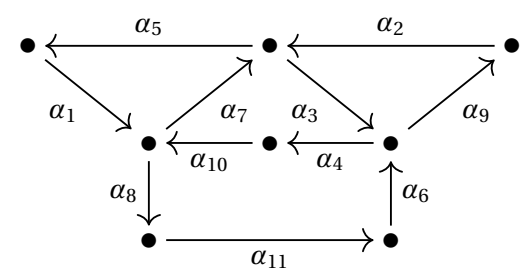

(16)

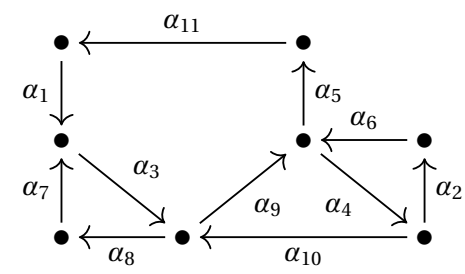

As relações do tipo 2 são: $r 1=\alpha_{1} \alpha_{6} \alpha_{5} \alpha_{4} \alpha_{2}, r 2=$ $\alpha_{9} \alpha_{6} \alpha_{5} \alpha_{4} \alpha_{3}, r 3=\alpha_{8} \alpha_{9} \alpha_{6}, r 4=\alpha_{10} \alpha_{9} \alpha_{7}$ e $r 5=$ $\alpha_{1} \alpha_{7}$.

Os ciclos elementares são: $C_{1}=\alpha_{3} \alpha_{1} \alpha_{6} \alpha_{5} \alpha_{4}, C_{2}=$ $\alpha_{2} \alpha_{10} \alpha_{9} \alpha_{6} \alpha_{5} \alpha_{4}$ e $C_{3}=\alpha_{7} \alpha_{8} \alpha_{9}$. Logo, temos as álgebras 24 e 25 .

As relações do tipo 2 são: $r 1=\alpha_{1} \alpha_{2} \alpha_{5} \alpha_{6} \alpha_{7} \alpha_{9}$, $r 2=\alpha_{10} \alpha_{2} \alpha_{5} \alpha_{6} \alpha_{7} \alpha_{8}, r 3=\alpha_{9} \alpha_{10} \alpha_{3}, r 4=\alpha_{4} \alpha_{10} \alpha_{2}$ e $r 5=\alpha_{1} \alpha_{3}$.

Os ciclos elementares são: $C_{1}=\alpha_{1} \alpha_{2} \alpha_{5} \alpha_{6} \alpha_{7} \alpha_{8}$, $C_{2}=\alpha_{10} \alpha_{2} \alpha_{5} \alpha_{6} \alpha_{7} \alpha_{9}$ e $C_{3}=\alpha_{3} \alpha_{4} \alpha_{10}$. Assim, conseguimos dois cortes resultando na álgebra 26 e na álgebra dual da 2 .

As relações do tipo 2 dessa extensão trivial são: $r 1=$ $\alpha_{1} \alpha_{3} \alpha_{8}, r 2=\alpha_{9} \alpha_{3} \alpha_{7}, r 3=\alpha_{2} \alpha_{9} \alpha_{3}, r 4=\alpha_{10} \alpha_{9} \alpha_{5}$, $r 5=\alpha_{3} \alpha_{8} \alpha_{10} \alpha_{4}, r 6=\alpha_{6} \alpha_{8} \alpha_{10} \alpha_{9}, r 7=\alpha_{6} \alpha_{7}, r 8=$ $\alpha_{1} \alpha_{5}$ e $r 9=\alpha_{2} \alpha_{4}$.

Os ciclos elementares são: $C_{1}=\alpha_{1} \alpha_{3} \alpha_{7} \alpha_{11}, C_{2}=$ $\alpha_{3} \alpha_{8} \alpha_{10} \alpha_{9}, C_{3}=\alpha_{8} \alpha_{10} \alpha_{4} \alpha_{6}$ e $C_{4}=\alpha_{5} \alpha_{2} \alpha_{9}$. Não tem corte que origine uma álgebra de incidência.

As relações do tipo 2 dessa extensão trivial são: $r 1=$ $\alpha_{1} \alpha_{7} \alpha_{3}, r 2=\alpha_{10} \alpha_{7} \alpha_{5}, r 3=\alpha_{7} \alpha_{3} \alpha_{9}, r 4=\alpha_{2} \alpha_{3} \alpha_{4}$, $r 5=\alpha_{3} \alpha_{4} \alpha_{10} \alpha_{8}, r 6=\alpha_{6} \alpha_{10} \alpha_{4} \alpha_{7}, r 7=\alpha_{2} \alpha_{5}, r 8=$ $\alpha_{1} \alpha_{8}$ e $r 9=\alpha_{6} \alpha_{9}$.

Os ciclos elementares são: $C_{1}=\alpha_{7} \alpha_{5} \alpha_{1}, C_{2}=$ $\alpha_{7} \alpha_{3} \alpha_{4} \alpha_{10}, C_{3}=\alpha_{4} \alpha_{10} \alpha_{8} \alpha_{11} \alpha_{6}$ e $C_{4}=\alpha_{2} \alpha_{3} \alpha_{9}$. O programa não mostra solução para esse caso.

As relações do tipo 2 são: $r 1=\alpha_{1} \alpha_{3} \alpha_{8}, r 2=$ $\alpha_{7} \alpha_{3} \alpha_{9}, r 3=\alpha_{3} \alpha_{9} \alpha_{4}, r 4=\alpha_{10} \alpha_{9} \alpha_{5}, r 5=\alpha_{9} \alpha_{4} \alpha_{2}$, $r 6=\alpha_{6} \alpha_{4} \alpha_{10}, r 7=\alpha_{10} \alpha_{8}$ e $r 8=\alpha_{6} \alpha_{5}$.

Os ciclos elementares são: $C_{1}=\alpha_{11} \alpha_{1} \alpha_{3} \alpha_{9} \alpha_{5}, C_{2}=$ $\alpha_{7} \alpha_{3} \alpha_{8}, C_{3}=\alpha_{10} \alpha_{9} \alpha_{4}$ e $C_{4}=\alpha_{6} \alpha_{4} \alpha_{2}$. Nesse caso, temos a solução 27 . 
(17)

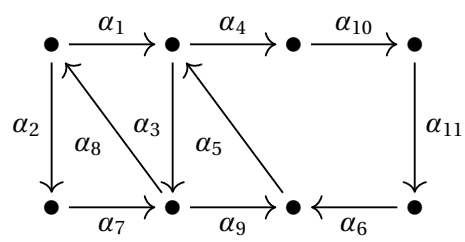

(18)

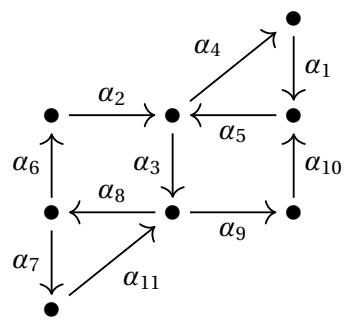

(19)

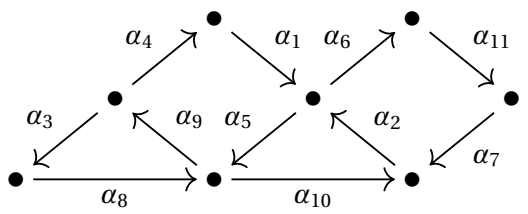

(20)

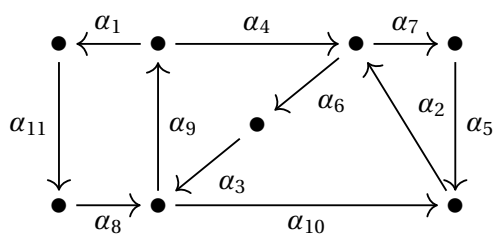

(21)

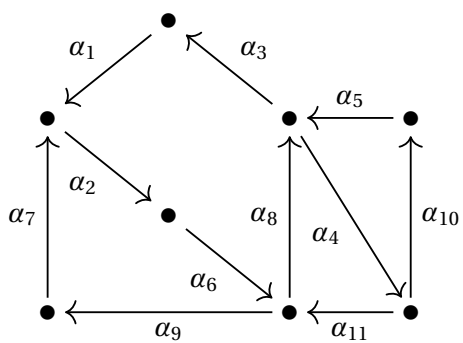

(22)

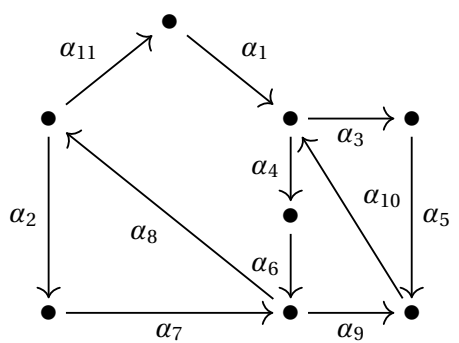

As relações do tipo 2 são: $r 1=\alpha_{7} \alpha_{8} \alpha_{1}, r 2=$ $\alpha_{3} \alpha_{8} \alpha_{2}, r 3=\alpha_{1} \alpha_{3} \alpha_{9}, r 4=\alpha_{10} \alpha_{3} \alpha_{8}, r 5=\alpha_{9} \alpha_{5} \alpha_{4}$, $r 6=\alpha_{6} \alpha_{5} \alpha_{3}, r 7=\alpha_{7} \alpha_{9}$ e $r 8=\alpha_{1} \alpha_{4}$.

Os ciclos elementares são: $C_{1}=\alpha_{2} \alpha_{7} \alpha_{8}, C_{2}=$ $\alpha_{8} \alpha_{1} \alpha_{3}, C_{3}=\alpha_{9} \alpha_{5} \alpha_{3}$ e $C_{4}=\alpha_{6} \alpha_{5} \alpha_{4} \alpha_{10} \alpha_{11}$. Portanto, obtemos a álgebra 28.

As relações do tipo 2 são: $r 1=\alpha_{1} \alpha_{5} \alpha_{3}, r 2=$ $\alpha_{10} \alpha_{5} \alpha_{4}, r 3=\alpha_{5} \alpha_{3} \alpha_{8}, r 4=\alpha_{2} \alpha_{3} \alpha_{9}, r 5=\alpha_{3} \alpha_{8} \alpha_{7}$, $r 6=\alpha_{11} \alpha_{8} \alpha_{6}, r 7=\alpha_{11} \alpha_{9}$ e $r 8=\alpha_{2} \alpha_{4}$.

Os ciclos elementares são: $C_{1}=\alpha_{1} \alpha_{5} \alpha_{4}, C_{2}=$ $\alpha_{2} \alpha_{3} \alpha_{8} \alpha_{6}, C_{3}=\alpha_{8} \alpha_{7} \alpha_{11}$ e $C_{4}=\alpha_{3} \alpha_{9} \alpha_{10} \alpha_{5}$. O programa mostra o corte que origina a solução 29 .

As relações do tipo 2 são: $r 1=\alpha_{8} \alpha_{9} \alpha_{4}, r 2=$ $\alpha_{5} \alpha_{9} \alpha_{3}, r 3=\alpha_{1} \alpha_{5} \alpha_{10}, r 4=\alpha_{2} \alpha_{5} \alpha_{9}, r 5=\alpha_{10} \alpha_{2} \alpha_{6}$, $r 6=\alpha_{7} \alpha_{2} \alpha_{5}, r 7=\alpha_{6} \alpha_{10}$ e $r 8=\alpha_{1} \alpha_{6}$.

Os ciclos elementares são: $C_{1}=\alpha_{3} \alpha_{8} \alpha_{9}, C_{2}=$ $\alpha_{4} \alpha_{1} \alpha_{5} \alpha_{9}, C_{3}=\alpha_{6} \alpha_{11} \alpha_{7} \alpha_{2}$ e $C_{4}=\alpha_{5} \alpha_{10} \alpha_{2}$. Logo, obtemos a álgebra 30 .

As relações do tipo 2 são: $r 1=\alpha_{8} \alpha_{9} \alpha_{4}, r 2=$ $\alpha_{3} \alpha_{9} \alpha_{1}, r 3=\alpha_{4} \alpha_{6} \alpha_{3} \alpha_{10}, r 4=\alpha_{2} \alpha_{6} \alpha_{3} \alpha_{9}, r 5=$ $\alpha_{10} \alpha_{2} \alpha_{7}, r 6=\alpha_{5} \alpha_{2} \alpha_{6}, r 7=\alpha_{4} \alpha_{7}$ e $r 8=\alpha_{8} \alpha_{10}$.

Os ciclos elementares são: $C_{1}=\alpha_{1} \alpha_{11} \alpha_{8} \alpha_{9}, C_{2}=$ $\alpha_{9} \alpha_{4} \alpha_{6} \alpha_{3}, C_{3}=\alpha_{6} \alpha_{3} \alpha_{10} \alpha_{2}$ e $C_{4}=\alpha_{2} \alpha_{7} \alpha_{5}$. Portanto, não temos solução para esse caso.

As relações do tipo 2 são: $r 1=\alpha_{1} \alpha_{2} \alpha_{6} \alpha_{9}, r 2=$ $\alpha_{7} \alpha_{2} \alpha_{6} \alpha_{8}, r 3=\alpha_{6} \alpha_{8} \alpha_{4}, r 4=\alpha_{11} \alpha_{8} \alpha_{3}, r 5=$ $\alpha_{8} \alpha_{4} \alpha_{10}, r 6=\alpha_{5} \alpha_{4} \alpha_{11}, r 7=\alpha_{11} \alpha_{9}$ e $r 8=\alpha_{5} \alpha_{3}$.

Os ciclos elementares são: $C_{1}=\alpha_{3} \alpha_{1} \alpha_{2} \alpha_{6} \alpha_{8}, C_{2}=$ $\alpha_{2} \alpha_{6} \alpha_{9} \alpha_{7}, C_{3}=\alpha_{8} \alpha_{4} \alpha_{11}$ e $C_{4}=\alpha_{10} \alpha_{5} \alpha_{4}$. Logo, o programa mostra a álgebra de incidência 31 .

As relações do tipo 2 são: $r 1=\alpha_{6} \alpha_{8} \alpha_{2}, r 2=$ $\alpha_{7} \alpha_{8} \alpha_{11}, r 3=\alpha_{1} \alpha_{4} \alpha_{6} \alpha_{9}, r 4=\alpha_{10} \alpha_{4} \alpha_{6} \alpha_{8}, r 5=$ $\alpha_{9} \alpha_{10} \alpha_{3}, r 6=\alpha_{5} \alpha_{10} \alpha_{4}, r 7=\alpha_{7} \alpha_{9}$ e $r 8=\alpha_{1} \alpha_{3}$.

Os ciclos elementares são: $C_{1}=\alpha_{1} \alpha_{4} \alpha_{6} \alpha_{8} \alpha_{11}, C_{2}=$ $\alpha_{8} \alpha_{2} \alpha_{7}, C_{3}=\alpha_{9} \alpha_{10} \alpha_{4} \alpha_{6}$ e $C_{4}=\alpha_{3} \alpha_{5} \alpha_{10}$. Com isso, o programa mostra nenhuma solução. 
(23)

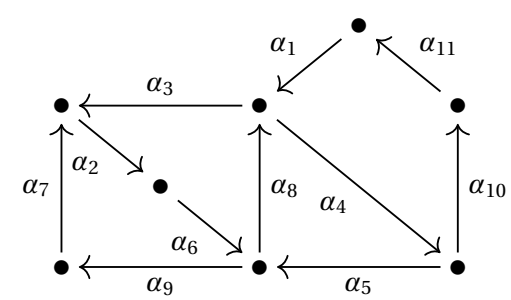

(24)

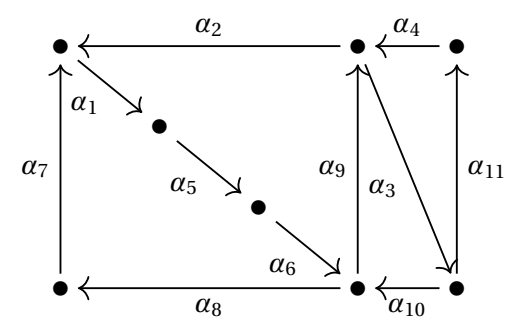

(25)

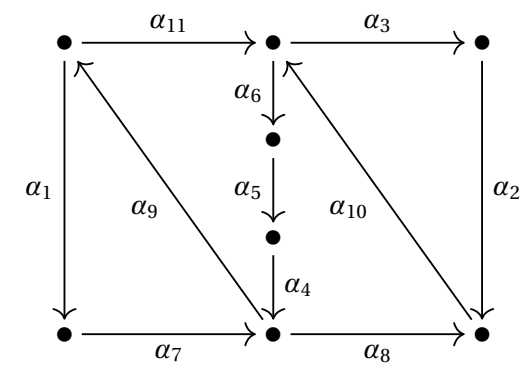

(26)

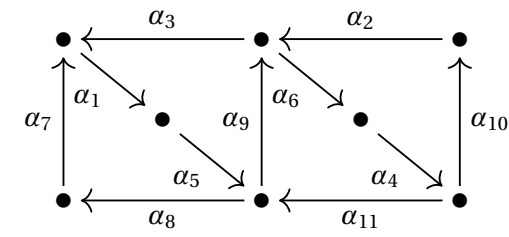

(27)

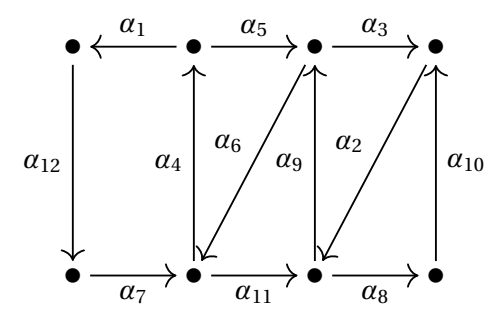

As relações do tipo 2 dessa extensão trivial são: $r 1=$ $\alpha_{1} \alpha_{4} \alpha_{5}, r 2=\alpha_{8} \alpha_{4} \alpha_{10}, r 3=\alpha_{5} \alpha_{8} \alpha_{3}, r 4=\alpha_{6} \alpha_{8} \alpha_{4}$, $r 5=\alpha_{3} \alpha_{2} \alpha_{6} \alpha_{9}, r 6=\alpha_{7} \alpha_{2} \alpha_{6} \alpha_{8}, r 7=\alpha_{1} \alpha_{3}$ e $r 8=$ $\alpha_{5} \alpha_{9}$.

Os ciclos elementares são: $C_{1}=\alpha_{1} \alpha_{4} \alpha_{10} \alpha_{11}, C_{2}=$ $\alpha_{4} \alpha_{5} \alpha_{8}, C_{3}=\alpha_{3} \alpha_{2} \alpha_{6} \alpha_{8}$ e $C_{4}=\alpha_{2} \alpha_{6} \alpha_{9} \alpha_{7}$. O programa exibe um corte que origina a solução 32 .

As relações do tipo 2 dessa extensão trivial são: $r 1=\alpha_{4} \alpha_{3} \alpha_{10}, r 2=\alpha_{9} \alpha_{3} \alpha_{11}, r 3=\alpha_{10} \alpha_{9} \alpha_{2}$, $r 4=\alpha_{6} \alpha_{9} \alpha_{3}, r 5=\alpha_{2} \alpha_{1} \alpha_{5} \alpha_{6} \alpha_{8}, r 6=\alpha_{7} \alpha_{1} \alpha_{5} \alpha_{6} \alpha_{9}$, $r 7=\alpha_{4} \alpha_{2}$ e $r 8=\alpha_{10} \alpha_{8}$.

Os ciclos elementares são: $C_{1}=\alpha_{2} \alpha_{1} \alpha_{5} \alpha_{6} \alpha_{9}, C_{2}=$ $\alpha_{1} \alpha_{5} \alpha_{6} \alpha_{8} \alpha_{7}, C_{3}=\alpha_{10} \alpha_{9} \alpha_{3}$ e $C_{4}=\alpha_{3} \alpha_{11} \alpha_{4}$. Portanto obtemos a solução 33 .

As relações do tipo 2 são: $r 1=\alpha_{7} \alpha_{9} \alpha_{11}, r 2=$ $\alpha_{4} \alpha_{9} \alpha_{1}, r 3=\alpha_{11} \alpha_{6} \alpha_{5} \alpha_{4} \alpha_{8}, r 4=\alpha_{10} \alpha_{6} \alpha_{5} \alpha_{4} \alpha_{9}$, $r 5=\alpha_{8} \alpha_{10} \alpha_{3}, r 6=\alpha_{2} \alpha_{10} \alpha_{6}, r 7=\alpha_{11} \alpha_{3}$ e $r 8=$ $\alpha_{7} \alpha_{8}$.

Os ciclos elementares são: $C_{1}=\alpha_{11} \alpha_{6} \alpha_{5} \alpha_{4} \alpha_{9}, C_{2}=$ $\alpha_{1} \alpha_{7} \alpha_{9}, C_{3}=\alpha_{8} \alpha_{10} \alpha_{6} \alpha_{5} \alpha_{4}$ e $C_{4}=\alpha_{3} \alpha_{2} \alpha_{10}$. Não obtemos nenhum corte.

As relações do tipo 2 são: $r 1=\alpha_{7} \alpha_{1} \alpha_{5} \alpha_{9}, r 2=$ $\alpha_{3} \alpha_{1} \alpha_{5} \alpha_{8}, r 3=\alpha_{5} \alpha_{9} \alpha_{6}, r 4=\alpha_{11} \alpha_{9} \alpha_{3}, r 5=$ $\alpha_{9} \alpha_{6} \alpha_{4} \alpha_{10}, r 6=\alpha_{2} \alpha_{6} \alpha_{4} \alpha_{11}, r 7=\alpha_{2} \alpha_{3}$ e $r 8=$ $\alpha_{11} \alpha_{8}$.

Os ciclos elementares são: $C_{1}=\alpha_{1} \alpha_{5} \alpha_{8} \alpha_{7}, C_{2}=$ $\alpha_{3} \alpha_{1} \alpha_{5} \alpha_{9}, C_{3}=\alpha_{9} \alpha_{6} \alpha_{4} \alpha_{11}$ e $C_{4}=\alpha_{2} \alpha_{6} \alpha_{4} \alpha_{10}$. O programa exibe o corte que origina a solução 34 .

As relações do tipo 2 são: $r 1=\alpha_{7} \alpha_{4} \alpha_{5}, r 2=$ $\alpha_{6} \alpha_{4} \alpha_{1}, r 3=\alpha_{5} \alpha_{6} \alpha_{11}, r 4=\alpha_{9} \alpha_{6} \alpha_{4}, r 5=\alpha_{11} \alpha_{9} \alpha_{3}$, $r 6=\alpha_{2} \alpha_{9} \alpha_{6}, r 7=\alpha_{3} \alpha_{2} \alpha_{8}, r 8=\alpha_{10} \alpha_{2} \alpha_{9}, r 9=$ $\alpha_{7} \alpha_{11}, r 10=\alpha_{11} \alpha_{8}$ e $r 11=\alpha_{5} \alpha_{3}$.

Os ciclos elementares dessa extensão trivial são: $C_{1}=\alpha_{1} \alpha_{12} \alpha_{7} \alpha_{4}, C_{2}=\alpha_{5} \alpha_{6} \alpha_{4}, C_{3}=\alpha_{6} \alpha_{11} \alpha_{9}$, $C_{4}=\alpha_{9} \alpha_{3} \alpha_{2}$ e $C_{5}=\alpha_{2} \alpha_{8} \alpha_{10}$. O programa não mostra solução. 
(28)

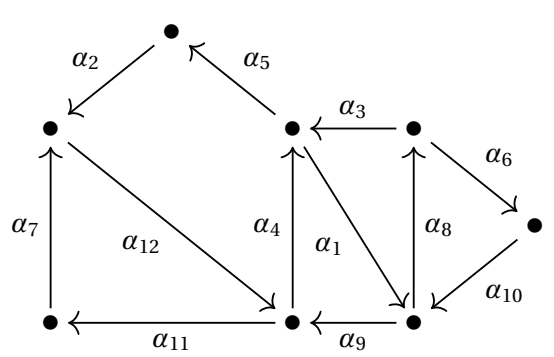

(29)

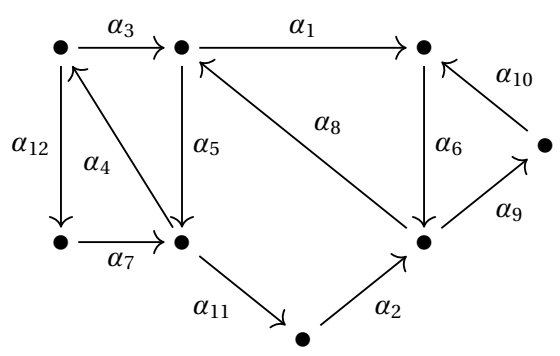

(30)

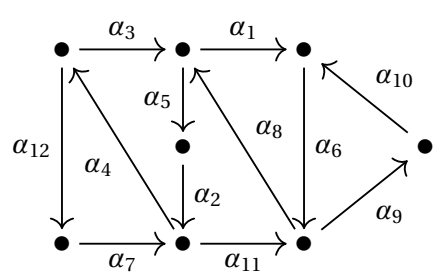

(31)

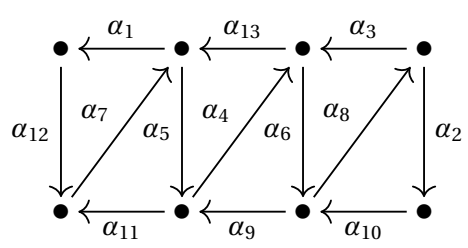

As relações do tipo 2 dessa extensão trivial são: $r 1=\alpha_{7} \alpha_{12} \alpha_{4}, r 2=\alpha_{2} \alpha_{12} \alpha_{11}, r 3=\alpha_{12} \alpha_{4} \alpha_{1}$, $r 4=\alpha_{9} \alpha_{4} \alpha_{5}, r 5=\alpha_{4} \alpha_{1} \alpha_{8}, r 6=\alpha_{3} \alpha_{1} \alpha_{9}, r 7=$ $\alpha_{1} \alpha_{8} \alpha_{6}, r 8=\alpha_{10} \alpha_{8} \alpha_{3}, r 9=\alpha_{9} \alpha_{11}, r 10=\alpha_{3} \alpha_{5} \mathrm{e}$ $r 11=\alpha_{10} \alpha_{9}$.

Os ciclos elementares são: $C_{1}=\alpha_{11} \alpha_{7} \alpha_{12}, C_{2}=$ $\alpha_{12} \alpha_{4} \alpha_{5} \alpha_{2}, C_{3}=\alpha_{4} \alpha_{1} \alpha_{9}, C_{4}=\alpha_{1} \alpha_{8} \alpha_{3}$ e $C_{5}=$ $\alpha_{8} \alpha_{6} \alpha_{10}$. Não obtemos solução.

As relações do tipo 2 dessa extensão trivial são: $r 1=$ $\alpha_{10} \alpha_{6} \alpha_{8}, r 2=\alpha_{1} \alpha_{6} \alpha_{9}, r 3=\alpha_{6} \alpha_{8} \alpha_{5}, r 4=\alpha_{2} \alpha_{8} \alpha_{1}$, $r 5=\alpha_{8} \alpha_{5} \alpha_{4}, r 6=\alpha_{3} \alpha_{5} \alpha_{11}, r 7=\alpha_{5} \alpha_{4} \alpha_{12}, r 8=$ $\alpha_{7} \alpha_{4} \alpha_{3}, r 9=\alpha_{3} \alpha_{1}, r 10=\alpha_{2} \alpha_{9}$ e $r 11=\alpha_{7} \alpha_{11}$.

Os ciclos elementares são: $C_{1}=\alpha_{12} \alpha_{7} \alpha_{4}, C_{2}=$ $\alpha_{4} \alpha_{3} \alpha_{5}, C_{3}=\alpha_{5} \alpha_{11} \alpha_{2} \alpha_{8}, C_{4}=\alpha_{8} \alpha_{1} \alpha_{6}$ e $C_{5}=$ $\alpha_{6} \alpha_{9} \alpha_{10}$. O programa não mostre nenhum corte.

As relações do tipo 2 dessa extensão trivial são: $r 1=\alpha_{7} \alpha_{4} \alpha_{3}, r 2=\alpha_{2} \alpha_{4} \alpha_{12}, r 3=\alpha_{3} \alpha_{5} \alpha_{2} \alpha_{11}$, $r 4=\alpha_{8} \alpha_{5} \alpha_{2} \alpha_{4}, r 5=\alpha_{11} \alpha_{8} \alpha_{1}, r 6=\alpha_{6} \alpha_{8} \alpha_{5}, r 7=$ $\alpha_{1} \alpha_{6} \alpha_{9}, r 8=\alpha_{10} \alpha_{6} \alpha_{8}, r 9=\alpha_{7} \alpha_{11}, r 10=\alpha_{3} \alpha_{1} \mathrm{e}$ $r 11=\alpha_{11} \alpha_{9}$.

Os ciclos elementares são: $C_{1}=\alpha_{12} \alpha_{7} \alpha_{4}, C_{2}=$ $\alpha_{4} \alpha_{3} \alpha_{5} \alpha_{2}, C_{3}=\alpha_{5} \alpha_{2} \alpha_{11} \alpha_{8}, C_{4}=\alpha_{8} \alpha_{1} \alpha_{6}$ e $C_{5}=$ $\alpha_{6} \alpha_{9} \alpha_{10}$. Nesse caso, não temos nenhum corte.

Nessa extensão trivial, temos as seguintes relações do tipo 2: $r 1=\alpha_{12} \alpha_{7} \alpha_{5}, r 2=\alpha_{11} \alpha_{7} \alpha_{1}, r 3=$ $\alpha_{7} \alpha_{5} \alpha_{4}, r 4=\alpha_{13} \alpha_{5} \alpha_{11}, r 5=\alpha_{5} \alpha_{4} \alpha_{6}, r 6=$ $\alpha_{9} \alpha_{4} \alpha_{13}, r 7=\alpha_{4} \alpha_{6} \alpha_{8}, r 8=\alpha_{3} \alpha_{6} \alpha_{9}, r 9=\alpha_{6} \alpha_{8} \alpha_{2}$, $r 10=\alpha_{10} \alpha_{8} \alpha_{3}, r 11=\alpha_{9} \alpha_{11}, r 12=\alpha_{10} \alpha_{9}, r 13=$ $\alpha_{3} \alpha_{13}$ e $r 14=\alpha_{13} \alpha_{1}$.

Os ciclos elementares são: $C_{1}=\alpha_{1} \alpha_{12} \alpha_{7}, C_{2}=$ $\alpha_{7} \alpha_{5} \alpha_{11}, C_{3}=\alpha_{13} \alpha_{5} \alpha_{4}, C_{4}=\alpha_{4} \alpha_{6} \alpha_{9}, C_{5}=\alpha_{3} \alpha_{6} \alpha_{8}$ e $C_{6}=\alpha_{8} \alpha_{2} \alpha_{10}$. Não obtemos álgebra de incidência originada de corte. 
(32)

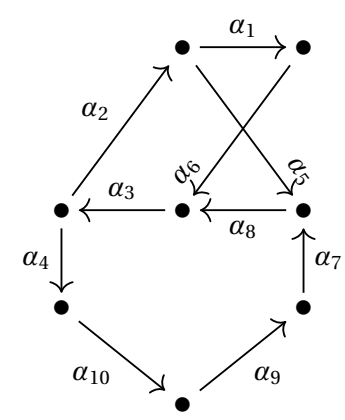

(33)

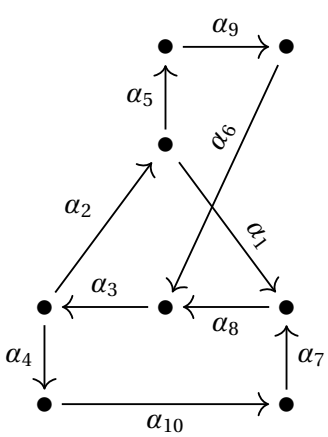

(34)

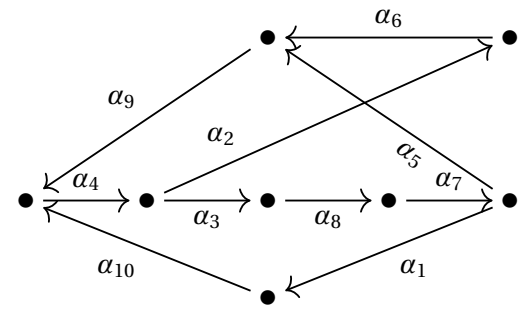

(35)

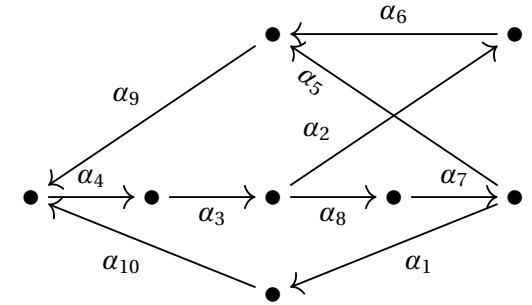

(36)

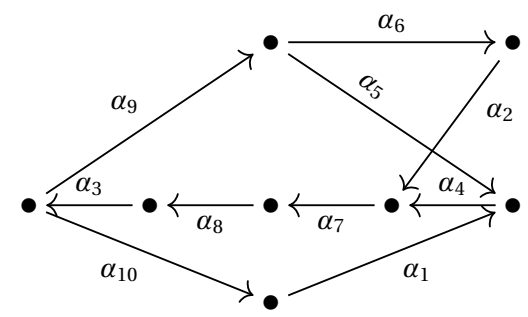

Nessa extensão trivial, temos as seguintes relações do tipo 2: $r 1=\alpha_{6} \alpha_{3} \alpha_{2} \alpha_{5}, r 2=\alpha_{8} \alpha_{3} \alpha_{2} \alpha_{1}, r 3=$ $\alpha_{5} \alpha_{8} \alpha_{3} \alpha_{4}, r 4=\alpha_{7} \alpha_{8} \alpha_{3} \alpha_{2}$ e $r 5=\alpha_{6} \alpha_{3} \alpha_{4}$.

Os ciclos elementares são: $C_{1}=\alpha_{1} \alpha_{6} \alpha_{3} \alpha_{2}, C_{2}=$ $\alpha_{2} \alpha_{5} \alpha_{8} \alpha_{3}$ e $C_{3}=\alpha_{3} \alpha_{4} \alpha_{10} \alpha_{9} \alpha_{7} \alpha_{8}$. O programa exibe as soluções 35,36 e 37 .

Nessa extensão trivial, temos as seguintes relações do tipo 2: $r 1=\alpha_{6} \alpha_{3} \alpha_{2} \alpha_{1}, r 2=\alpha_{8} \alpha_{3} \alpha_{2} \alpha_{5}, r 3=$ $\alpha_{7} \alpha_{8} \alpha_{3} \alpha_{2}, r 4=\alpha_{1} \alpha_{8} \alpha_{3} \alpha_{4}$ e $r 5=\alpha_{6} \alpha_{3} \alpha_{4}$.

Os ciclos elementares são: $C_{1}=\alpha_{9} \alpha_{6} \alpha_{3} \alpha_{2} \alpha_{5}, C_{2}=$ $\alpha_{2} \alpha_{1} \alpha_{8} \alpha_{3}$ e $C_{3}=\alpha_{3} \alpha_{4} \alpha_{10} \alpha_{7} \alpha_{8}$. Portanto, obtemos as álgebras 38, 39 e na álgebra dual da 39.

As relações do tipo 2 são: $r 1=\alpha_{6} \alpha_{9} \alpha_{4} \alpha_{3}, r 2=$ $\alpha_{5} \alpha_{9} \alpha_{4} \alpha_{2}, r 3=\alpha_{9} \alpha_{4} \alpha_{3} \alpha_{8} \alpha_{7} \alpha_{1}, r 4=\alpha_{10} \alpha_{4} \alpha_{2}$ e $r 5=\alpha_{10} \alpha_{4} \alpha_{3} \alpha_{8} \alpha_{7} \alpha_{5}$.

Os ciclos elementares são: $C_{1}=\alpha_{6} \alpha_{9} \alpha_{4} \alpha_{2}, C_{2}=$ $\alpha_{9} \alpha_{4} \alpha_{3} \alpha_{8} \alpha_{7} \alpha_{5}$ e $C_{3}=\alpha_{4} \alpha_{3} \alpha_{8} \alpha_{7} \alpha_{1} \alpha_{10}$. Logo, o programa exibe as soluções 40,41 e 42 .

As relações do tipo 2 são: $r 1=\alpha_{6} \alpha_{9} \alpha_{4} \alpha_{3} \alpha_{8}, r 2=$ $\alpha_{5} \alpha_{9} \alpha_{4} \alpha_{3} \alpha_{2}, r 3=\alpha_{9} \alpha_{4} \alpha_{3} \alpha_{8} \alpha_{7} \alpha_{1}, r 4=\alpha_{10} \alpha_{4} \alpha_{3} \alpha_{2}$ e $r 5=\alpha_{10} \alpha_{4} \alpha_{3} \alpha_{8} \alpha_{7} \alpha_{5}$.

Os ciclos elementares são: $C_{1}=\alpha_{6} \alpha_{9} \alpha_{4} \alpha_{3} \alpha_{2}, C_{2}=$ $\alpha_{9} \alpha_{4} \alpha_{3} \alpha_{8} \alpha_{7} \alpha_{5}$ e $C_{3}=\alpha_{4} \alpha_{3} \alpha_{8} \alpha_{7} \alpha_{1} \alpha_{10}$. Nesse caso, obtemos as soluções $43,44,45$ e 46 .

As relações do tipo 2 são: $r 1=\alpha_{2} \alpha_{7} \alpha_{8} \alpha_{3} \alpha_{9} \alpha_{5}$, $r 2=\alpha_{4} \alpha_{7} \alpha_{8} \alpha_{3} \alpha_{9} \alpha_{6}, r 3=\alpha_{5} \alpha_{4} \alpha_{7} \alpha_{8} \alpha_{3} \alpha_{10}, r 4=$ $\alpha_{1} \alpha_{4} \alpha_{7} \alpha_{8} \alpha_{3} \alpha_{9}$ e $r 5=\alpha_{2} \alpha_{7} \alpha_{8} \alpha_{3} \alpha_{10}$.

Os ciclos elementares são: $C_{1}=\alpha_{6} \alpha_{2} \alpha_{7} \alpha_{8} \alpha_{3} \alpha_{9}$, $C_{2}=\alpha_{9} \alpha_{5} \alpha_{4} \alpha_{7} \alpha_{8} \alpha_{3}$ e $C_{3}=\alpha_{4} \alpha_{7} \alpha_{8} \alpha_{3} \alpha_{10} \alpha_{1}$. O programa exibe os cortes que resultam nas álgebras 47 , 48, 49, 50 e na dual da álgebra 50 . 
(37)

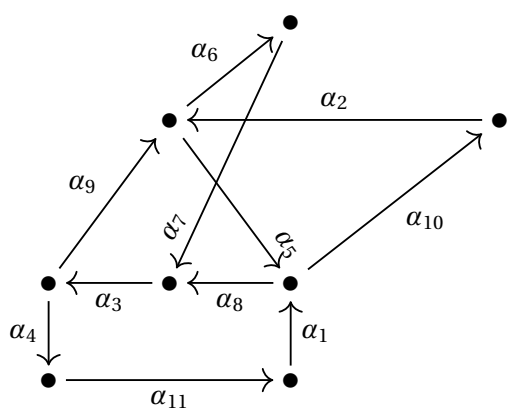

(38)

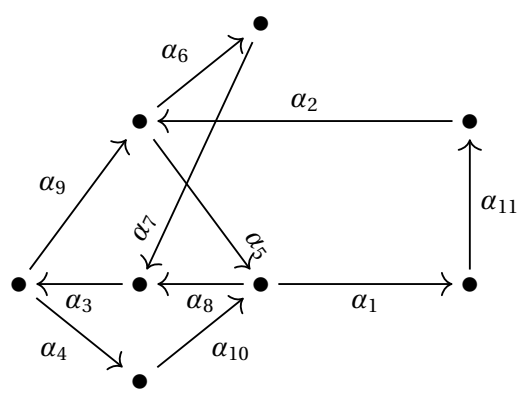

(39)

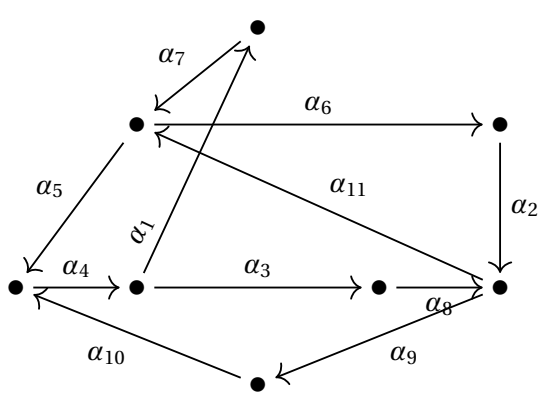

(40)

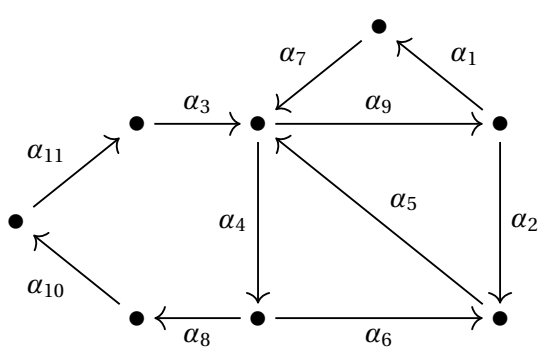

(41)

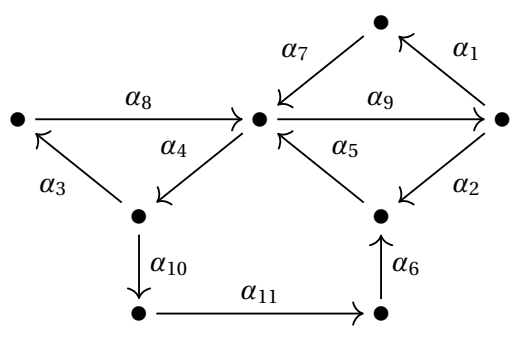

As relações do tipo 2 são: $r 1=\alpha_{7} \alpha_{3} \alpha_{9} \alpha_{5}, r 2=$ $\alpha_{8} \alpha_{3} \alpha_{9} \alpha_{6}, r 3=\alpha_{9} \alpha_{5} \alpha_{10}, r 4=\alpha_{2} \alpha_{5} \alpha_{8}, r 5=$ $\alpha_{1} \alpha_{8} \alpha_{3} \alpha_{9}, r 6=\alpha_{7} \alpha_{3} \alpha_{4}, r 7=\alpha_{5} \alpha_{8} \alpha_{3} \alpha_{4}, r 8=$ $\alpha_{1} \alpha_{10}$ e $r 9=\alpha_{2} \alpha_{6}$.

Os ciclos elementares são: $C_{1}=\alpha_{9} \alpha_{6} \alpha_{7} \alpha_{3}, C_{2}=$ $\alpha_{10} \alpha_{2} \alpha_{5}, C_{3}=\alpha_{5} \alpha_{8} \alpha_{3} \alpha_{9}$ e $C_{4}=\alpha_{4} \alpha_{11} \alpha_{1} \alpha_{8} \alpha_{3}$. Não obtemos nenhuma álgebra de incidência.

Essa extensão trivial tem as seguintes relações do tipo 2: $r 1=\alpha_{7} \alpha_{3} \alpha_{9} \alpha_{5}, r 2=\alpha_{8} \alpha_{3} \alpha_{9} \alpha_{6}, r 3=$ $\alpha_{9} \alpha_{5} \alpha_{1}, r 4=\alpha_{2} \alpha_{5} \alpha_{8}, r 5=\alpha_{10} \alpha_{8} \alpha_{3} \alpha_{9}, r 6=$ $\alpha_{7} \alpha_{3} \alpha_{4}, r 7=\alpha_{5} \alpha_{8} \alpha_{3} \alpha_{4}, r 8=\alpha_{10} \alpha_{1}$ e $r 9=\alpha_{2} \alpha_{6}$.

Os ciclos elementares são: $C_{1}=\alpha_{9} \alpha_{6} \alpha_{7} \alpha_{3}, C_{2}=$ $\alpha_{1} \alpha_{11} \alpha_{2} \alpha_{5}, C_{3}=\alpha_{5} \alpha_{8} \alpha_{3} \alpha_{9}$ e $C_{4}=\alpha_{10} \alpha_{8} \alpha_{3} \alpha_{4}$. Portanto, não tem nenhum corte.

Essa extensão trivial tem as seguintes relações do tipo 2: $r 1=\alpha_{7} \alpha_{5} \alpha_{4} \alpha_{3}, r 2=\alpha_{11} \alpha_{5} \alpha_{4} \alpha_{1}, r 3=$ $\alpha_{8} \alpha_{11} \alpha_{6}, r 4=\alpha_{2} \alpha_{11} \alpha_{5}, r 5=\alpha_{5} \alpha_{4} \alpha_{3} \alpha_{8} \alpha_{9}, r 6=$ $\alpha_{10} \alpha_{4} \alpha_{1}, r 7=\alpha_{10} \alpha_{4} \alpha_{3} \alpha_{8} \alpha_{11}, r 8=\alpha_{2} \alpha_{9}$ e $r 9=$ $\alpha_{7} \alpha_{6}$.

Os ciclos elementares são: $C_{1}=\alpha_{7} \alpha_{5} \alpha_{4} \alpha_{1}, C_{2}=$ $\alpha_{5} \alpha_{4} \alpha_{3} \alpha_{8} \alpha_{11}, C_{3}=\alpha_{11} \alpha_{6} \alpha_{2}$ e $C_{4}=\alpha_{9} \alpha_{10} \alpha_{4} \alpha_{3} \alpha_{8}$. Logo, não obtemos nenhuma álgebra de incidência.

Essa extensão trivial tem as seguintes relações do tipo 2: $r 1=\alpha_{3} \alpha_{4} \alpha_{6}, r 2=\alpha_{5} \alpha_{4} \alpha_{8}, r 3=\alpha_{6} \alpha_{5} \alpha_{9}$, $r 4=\alpha_{2} \alpha_{5} \alpha_{4}, r 5=\alpha_{7} \alpha_{9} \alpha_{2}, r 6=\alpha_{5} \alpha_{9} \alpha_{1}, r 7=\alpha_{3} \alpha_{9}$ e $r 8=\alpha_{7} \alpha_{4}$.

Os ciclos elementares são: $C_{1}=\alpha_{4} \alpha_{8} \alpha_{10} \alpha_{11} \alpha_{3}$, $C_{2}=\alpha_{9} \alpha_{1} \alpha_{7}, C_{3}=\alpha_{9} \alpha_{2} \alpha_{5}$ e $C_{4}=\alpha_{5} \alpha_{4} \alpha_{6}$. Assim, o programa nos mostra duas soluções: 51 e 52 .

Essa extensão trivial tem as seguintes relações do tipo 2: $r 1=\alpha_{6} \alpha_{5} \alpha_{9}, r 2=\alpha_{2} \alpha_{5} \alpha_{4}, r 3=\alpha_{5} \alpha_{9} \alpha_{1}$, $r 4=\alpha_{7} \alpha_{9} \alpha_{2}, r 5=\alpha_{5} \alpha_{4} \alpha_{3}, r 6=\alpha_{8} \alpha_{4} \alpha_{10}, r 7=$ $\alpha_{8} \alpha_{9}$ e $r 8=\alpha_{7} \alpha_{4}$.

Os ciclos elementares são: $C_{1}=\alpha_{4} \alpha_{10} \alpha_{11} \alpha_{6} \alpha_{5}$, $C_{2}=\alpha_{5} \alpha_{9} \alpha_{2}, C_{3}=\alpha_{9} \alpha_{1} \alpha_{7}$ e $C_{4}=\alpha_{4} \alpha_{3} \alpha_{8}$. Portanto, obtemos as álgebras de incidência 53 e 54 . 
(42)

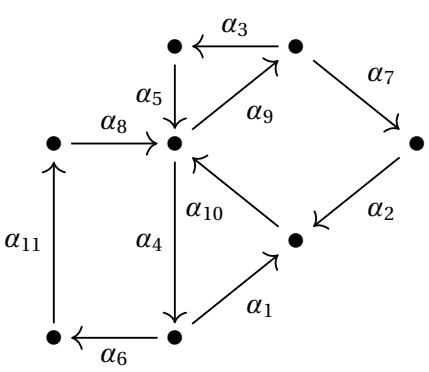

(43)

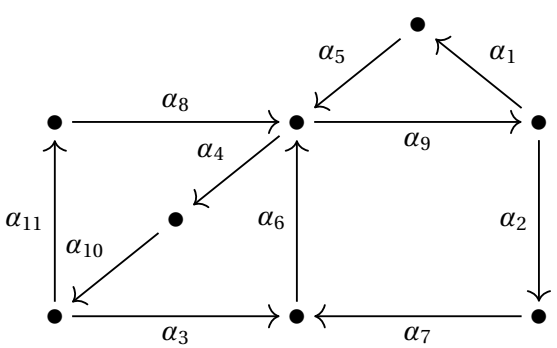

(44)

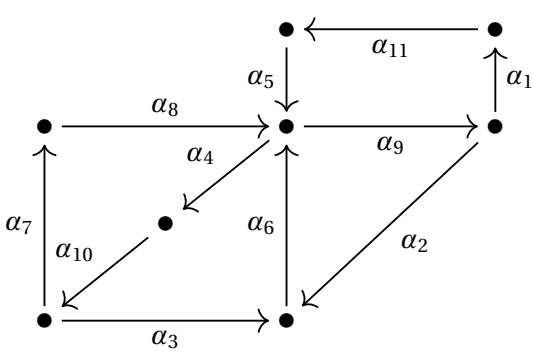

(45)

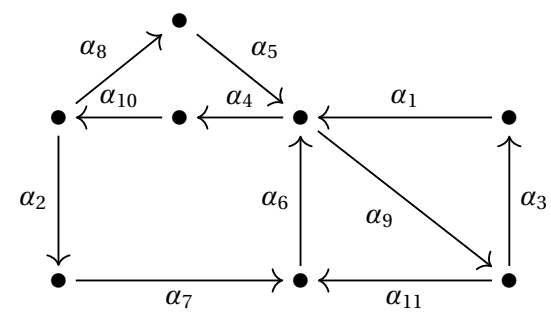

(46)

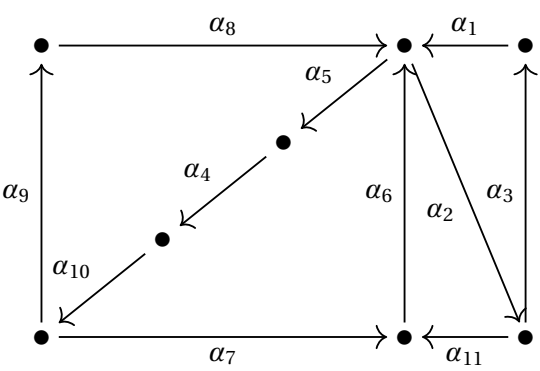

Essa extensão trivial tem as seguintes relações do tipo 2: $r 1=\alpha_{8} \alpha_{4} \alpha_{1}, r 2=\alpha_{10} \alpha_{4} \alpha_{6}, r 3=\alpha_{1} \alpha_{10} \alpha_{9}$, $r 4=\alpha_{2} \alpha_{10} \alpha_{4}, r 5=\alpha_{10} \alpha_{9} \alpha_{3}, r 6=\alpha_{5} \alpha_{9} \alpha_{7}, r 7=$ $\alpha_{5} \alpha_{4}$ e $r 8=\alpha_{8} \alpha_{9}$.

Os ciclos elementares são: $C_{1}=\alpha_{4} \alpha_{6} \alpha_{11} \alpha_{8}, C_{2}=$ $\alpha_{9} \alpha_{7} \alpha_{2} \alpha_{10}, C_{3}=\alpha_{10} \alpha_{4} \alpha_{1}$ e $C_{4}=\alpha_{5} \alpha_{9} \alpha_{3}$. Logo, temos as soluções 55 e 56 .

Essa extensão trivial tem as seguintes relações do tipo 2: $r 1=\alpha_{8} \alpha_{4} \alpha_{10} \alpha_{3}, r 2=\alpha_{6} \alpha_{4} \alpha_{10} \alpha_{11}, r 3=$ $\alpha_{3} \alpha_{6} \alpha_{9}, r 4=\alpha_{7} \alpha_{6} \alpha_{4}, r 5=\alpha_{6} \alpha_{9} \alpha_{1}, r 6=\alpha_{5} \alpha_{9} \alpha_{2}$, $r 7=\alpha_{8} \alpha_{9}$ e $r 8=\alpha_{5} \alpha_{4}$.

Os ciclos elementares são: $C_{1}=\alpha_{3} \alpha_{6} \alpha_{4} \alpha_{10}, C_{2}=$ $\alpha_{10} \alpha_{11} \alpha_{8} \alpha_{4}, C_{3}=\alpha_{6} \alpha_{9} \alpha_{2} \alpha_{7}$ e $C_{4}=\alpha_{5} \alpha_{9} \alpha_{1}$. Assim, o programa nos mostra as soluções 57 e 58 .

Essa extensão trivial tem as seguintes relações do tipo 2: $r 1=\alpha_{8} \alpha_{4} \alpha_{10} \alpha_{3}, r 2=\alpha_{6} \alpha_{4} \alpha_{10} \alpha_{7}, r 3=$ $\alpha_{3} \alpha_{6} \alpha_{9}, r 4=\alpha_{2} \alpha_{6} \alpha_{4}, r 5=\alpha_{6} \alpha_{9} \alpha_{1}, r 6=\alpha_{5} \alpha_{9} \alpha_{2}$, $r 7=\alpha_{8} \alpha_{9}$ e $r 8=\alpha_{5} \alpha_{4}$.

Os ciclos elementares são: $C_{1}=\alpha_{4} \alpha_{10} \alpha_{7} \alpha_{8}, C_{2}=$ $\alpha_{5} \alpha_{9} \alpha_{1} \alpha_{11}, C_{3}=\alpha_{4} \alpha_{10} \alpha_{3} \alpha_{6}$ e $C_{4}=\alpha_{6} \alpha_{9} \alpha_{2}$. Portanto, obtemos as álgebras de incidência 59 e 60.

Essa extensão trivial tem as seguintes relações do tipo 2: $r 1=\alpha_{7} \alpha_{6} \alpha_{9}, r 2=\alpha_{11} \alpha_{6} \alpha_{4}, r 3=\alpha_{6} \alpha_{4} \alpha_{10} \alpha_{8}$, $r 4=\alpha_{5} \alpha_{4} \alpha_{10} \alpha_{2}, r 5=\alpha_{1} \alpha_{9} \alpha_{11}, r 6=\alpha_{6} \alpha_{9} \alpha_{3}, r 7=$ $\alpha_{5} \alpha_{9}$ e $r 8=\alpha_{1} \alpha_{4}$.

Os ciclos elementares são: $C_{1}=\alpha_{4} \alpha_{10} \alpha_{2} \alpha_{7} \alpha_{6}, C_{2}=$ $\alpha_{4} \alpha_{10} \alpha_{8} \alpha_{5}, C_{3}=\alpha_{3} \alpha_{1} \alpha_{9}$ e $C_{4}=\alpha_{9} \alpha_{11} \alpha_{6}$. Com isso, temos as álgebras de incidência 61 e 62 .

Essa extensão trivial tem as seguintes relações do tipo 2: $r 1=\alpha_{6} \alpha_{5} \alpha_{4} \alpha_{10} \alpha_{9}, r 2=\alpha_{8} \alpha_{5} \alpha_{4} \alpha_{10} \alpha_{7}, r 3=$ $\alpha_{7} \alpha_{6} \alpha_{2}, r 4=\alpha_{11} \alpha_{6} \alpha_{5}, r 5=\alpha_{6} \alpha_{2} \alpha_{3}, r 6=\alpha_{1} \alpha_{2} \alpha_{11}$, $r 7=\alpha_{1} \alpha_{5}$ e $r 8=\alpha_{8} \alpha_{2}$.

Os ciclos elementares são: $C_{1}=\alpha_{8} \alpha_{5} \alpha_{4} \alpha_{10} \alpha_{9}, C_{2}=$ $\alpha_{5} \alpha_{4} \alpha_{10} \alpha_{7} \alpha_{6}, C_{3}=\alpha_{6} \alpha_{2} \alpha_{11}$ e $C_{4}=\alpha_{1} \alpha_{2} \alpha_{3}$. Logo obtemos as soluções 63 e 64 . 
(47)

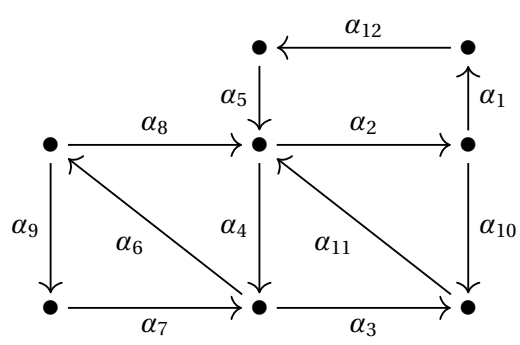

(48)

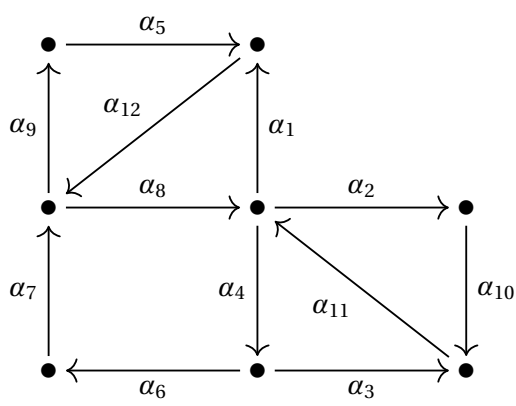

(49)

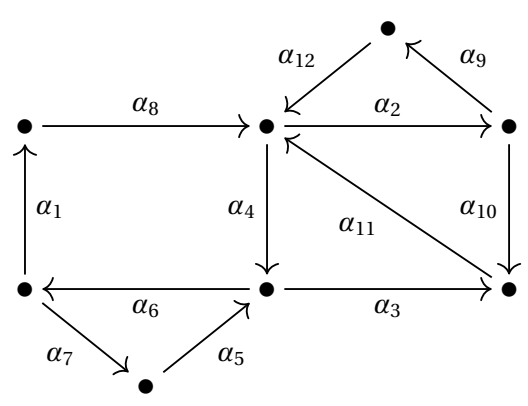

(50)

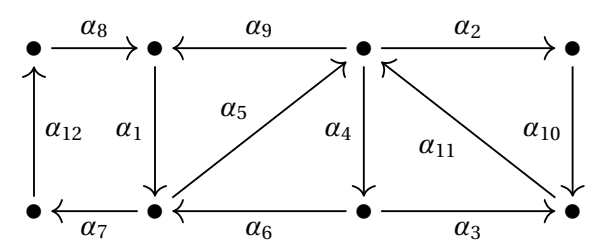

Essa extensão trivial tem as seguintes relações do tipo 2: $r 1=\alpha_{7} \alpha_{6} \alpha_{8}, r 2=\alpha_{4} \alpha_{6} \alpha_{9}, r 3=\alpha_{8} \alpha_{4} \alpha_{3}$, $r 4=\alpha_{11} \alpha_{4} \alpha_{6}, r 5=\alpha_{3} \alpha_{11} \alpha_{2}, r 6=\alpha_{10} \alpha_{11} \alpha_{4}, r 7=$ $\alpha_{11} \alpha_{2} \alpha_{1}, r 8=\alpha_{5} \alpha_{2} \alpha_{10}, r 9=\alpha_{5} \alpha_{4}, r 10=\alpha_{8} \alpha_{2} \mathrm{e}$ $r 11=\alpha_{7} \alpha_{3}$.

Os ciclos elementares são: $C_{1}=\alpha_{2} \alpha_{1} \alpha_{12} \alpha_{5}, C_{2}=$ $\alpha_{2} \alpha_{10} \alpha_{11}, C_{3}=\alpha_{11} \alpha_{4} \alpha_{3}, C_{4}=\alpha_{8} \alpha_{4} \alpha_{6}$ e $C_{5}=$ $\alpha_{6} \alpha_{9} \alpha_{7}$. Portanto, temos apenas a álgebra 65.

Essa extensão trivial tem as seguintes relações do tipo 2: $r 1=\alpha_{7} \alpha_{8} \alpha_{1}, r 2=\alpha_{12} \alpha_{8} \alpha_{4}, r 3=\alpha_{1} \alpha_{12} \alpha_{9}$, $r 4=\alpha_{5} \alpha_{12} \alpha_{8}, r 5=\alpha_{8} \alpha_{4} \alpha_{3}, r 6=\alpha_{11} \alpha_{4} \alpha_{6}, r 7=$ $\alpha_{3} \alpha_{11} \alpha_{2}, r 8=\alpha_{10} \alpha_{11} \alpha_{4}, r 9=\alpha_{7} \alpha_{9}, r 10=\alpha_{8} \alpha_{2} \mathrm{e}$ $r 11=\alpha_{11} \alpha_{1}$.

Os ciclos elementares são: $C_{1}=\alpha_{8} \alpha_{4} \alpha_{6} \alpha_{7}, C_{2}=$ $\alpha_{8} \alpha_{1} \alpha_{12}, C_{3}=\alpha_{12} \alpha_{9} \alpha_{5}, C_{4}=\alpha_{2} \alpha_{10} \alpha_{11}$ e $C_{5}=$ $\alpha_{11} \alpha_{4} \alpha_{3}$. O programa mostra a solução 66 .

Essa extensão trivial tem as seguintes relações do tipo 2: $r 1=\alpha_{5} \alpha_{6} \alpha_{1}, r 2=\alpha_{4} \alpha_{6} \alpha_{7}, r 3=\alpha_{8} \alpha_{4} \alpha_{3}$, $r 4=\alpha_{11} \alpha_{4} \alpha_{6}, r 5=\alpha_{3} \alpha_{11} \alpha_{2}, r 6=\alpha_{10} \alpha_{11} \alpha_{4}, r 7=$ $\alpha_{11} \alpha_{2} \alpha_{9}, r 8=\alpha_{12} \alpha_{2} \alpha_{10}, r 9=\alpha_{8} \alpha_{2}, r 10=\alpha_{12} \alpha_{4} \mathrm{e}$ $r 11=\alpha_{5} \alpha_{3}$.

Os ciclos elementares são: $C_{1}=\alpha_{4} \alpha_{6} \alpha_{1} \alpha_{8}, C_{2}=$ $\alpha_{2} \alpha_{9} \alpha_{12}, C_{3}=\alpha_{2} \alpha_{10} \alpha_{11}, C_{4}=\alpha_{11} \alpha_{4} \alpha_{3}$ e $C_{5}=$ $\alpha_{5} \alpha_{6} \alpha_{7}$. Portanto, obtemos a álgebra de incidência 67.

Essa extensão trivial tem as seguintes relações do tipo 2: $r 1=\alpha_{8} \alpha_{1} \alpha_{5}, r 2=\alpha_{9} \alpha_{1} \alpha_{7}, r 3=\alpha_{1} \alpha_{5} \alpha_{4}$, $r 4=\alpha_{6} \alpha_{5} \alpha_{9}, r 5=\alpha_{5} \alpha_{4} \alpha_{3}, r 6=\alpha_{11} \alpha_{4} \alpha_{6}, r 7=$ $\alpha_{3} \alpha_{11} \alpha_{2}, r 8=\alpha_{10} \alpha_{11} \alpha_{4}, r 9=\alpha_{6} \alpha_{7}, r 10=\alpha_{11} \alpha_{9} \mathrm{e}$ $r 11=\alpha_{5} \alpha_{2}$.

Os ciclos elementares dessa extensão trivial são: $C_{1}=\alpha_{12} \alpha_{8} \alpha_{1} \alpha_{7}, C_{2}=\alpha_{1} \alpha_{5} \alpha_{9}, C_{3}=\alpha_{5} \alpha_{4} \alpha_{6}$, $C_{4}=\alpha_{3} \alpha_{11} \alpha_{4}$ e $C_{5}=\alpha_{11} \alpha_{2} \alpha_{10}$. Logo, temos a solução 68 . 
(51)

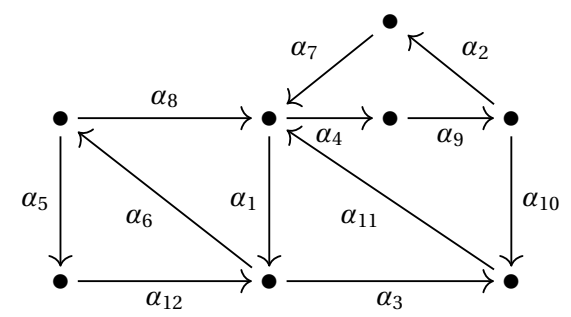

(52)

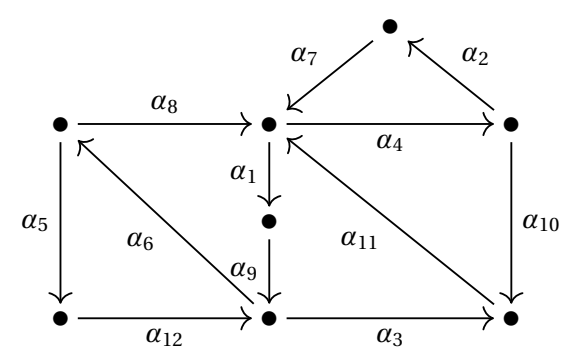

(53)

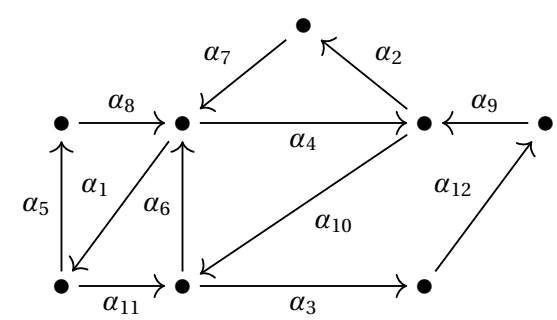

(54)

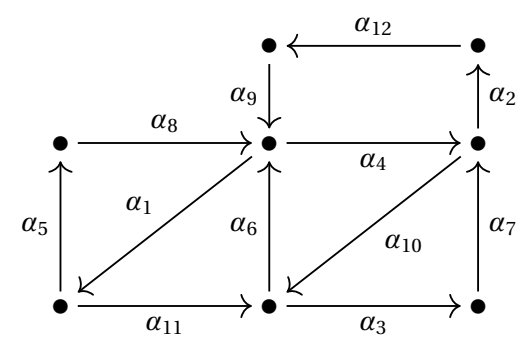

Essa extensão trivial tem as seguintes relações do tipo 2: $r 1=\alpha_{7} \alpha_{4} \alpha_{9} \alpha_{10}, r 2=\alpha_{11} \alpha_{4} \alpha_{9} \alpha_{2}, r 3=$ $\alpha_{10} \alpha_{11} \alpha_{1}, r 4=\alpha_{3} \alpha_{11} \alpha_{4}, r 5=\alpha_{11} \alpha_{1} \alpha_{6}, r 6=$ $\alpha_{8} \alpha_{1} \alpha_{3}, r 7=\alpha_{1} \alpha_{6} \alpha_{5}, r 8=\alpha_{12} \alpha_{6} \alpha_{8}, r 9=\alpha_{8} \alpha_{4}$, $r 10=\alpha_{7} \alpha_{1}$ e $r 11=\alpha_{12} \alpha_{3}$.

Os ciclos elementares são: $C_{1}=\alpha_{7} \alpha_{4} \alpha_{9} \alpha_{2}, C_{2}=$ $\alpha_{4} \alpha_{9} \alpha_{10} \alpha_{11}, C_{3}=\alpha_{11} \alpha_{1} \alpha_{3}, C_{4}=\alpha_{8} \alpha_{1} \alpha_{6}$ e $C_{5}=$ $\alpha_{6} \alpha_{5} \alpha_{12}$. O programa exibe a álgebra de incidência 69.

As relações do tipo 2 são: $r 1=\alpha_{8} \alpha_{1} \alpha_{9} \alpha_{3}, r 2=$ $\alpha_{11} \alpha_{1} \alpha_{9} \alpha_{6}, r 3=\alpha_{9} \alpha_{6} \alpha_{5}, r 4=\alpha_{12} \alpha_{6} \alpha_{8}, r 5=$ $\alpha_{3} \alpha_{11} \alpha_{4}, \quad r 6=\alpha_{10} \alpha_{11} \alpha_{1}, r 7=\alpha_{11} \alpha_{4} \alpha_{2}, \quad r 8=$ $\alpha_{7} \alpha_{4} \alpha_{10}, r 9=\alpha_{8} \alpha_{4}, r 10=\alpha_{7} \alpha_{1}$ e $r 11=\alpha_{12} \alpha_{3}$.

Os ciclos elementares são: $C_{1}=\alpha_{1} \alpha_{9} \alpha_{6} \alpha_{8}, C_{2}=$ $\alpha_{1} \alpha_{9} \alpha_{3} \alpha_{11}, C_{3}=\alpha_{11} \alpha_{4} \alpha_{10}, C_{4}=\alpha_{4} \alpha_{2} \alpha_{7}$ e $C_{5}=$ $\alpha_{5} \alpha_{12} \alpha_{6}$. Não obtemos nenhuma álgebra de incidência.

Essa extensão trivial tem as seguintes relações do tipo 2: $r 1=\alpha_{8} \alpha_{1} \alpha_{11}, r 2=\alpha_{6} \alpha_{1} \alpha_{5}, r 3=\alpha_{11} \alpha_{6} \alpha_{4}$, $r 4=\alpha_{10} \alpha_{6} \alpha_{1}, r 5=\alpha_{4} \alpha_{10} \alpha_{3}, r 6=\alpha_{9} \alpha_{10} \alpha_{6}, r 7=$ $\alpha_{7} \alpha_{4} \alpha_{10}, r 8=\alpha_{6} \alpha_{4} \alpha_{2}, r 9=\alpha_{9} \alpha_{2}, r 10=\alpha_{7} \alpha_{1}$, $r 11=\alpha_{8} \alpha_{4}$ e $r 12=\alpha_{11} \alpha_{3}$.

Os ciclos elementares dessa extensão trivial são: $C_{1}=\alpha_{3} \alpha_{12} \alpha_{9} \alpha_{10}, C_{2}=\alpha_{10} \alpha_{6} \alpha_{4}, C_{3}=\alpha_{4} \alpha_{2} \alpha_{7}$, $C_{4}=\alpha_{1} \alpha_{5} \alpha_{8}$ e $C_{5}=\alpha_{1} \alpha_{11} \alpha_{6}$. O programa mostra que não tem solução para esse caso.

Essa extensão trivial tem as seguintes relações do tipo 2: $r 1=\alpha_{8} \alpha_{1} \alpha_{11}, r 2=\alpha_{6} \alpha_{1} \alpha_{5}, r 3=\alpha_{11} \alpha_{6} \alpha_{4}$, $r 4=\alpha_{10} \alpha_{6} \alpha_{1}, r 5=\alpha_{4} \alpha_{10} \alpha_{3}, r 6=\alpha_{7} \alpha_{10} \alpha_{6}, r 7=$ $\alpha_{6} \alpha_{4} \alpha_{2}, r 8=\alpha_{9} \alpha_{4} \alpha_{10}, r 9=\alpha_{7} \alpha_{2}, r 10=\alpha_{11} \alpha_{3}$, $r 11=\alpha_{9} \alpha_{1}$ e $r 12=\alpha_{8} \alpha_{4}$.

Os ciclos elementares dessa extensão trivial são: $C_{1}=\alpha_{4} \alpha_{2} \alpha_{12} \alpha_{9}, C_{2}=\alpha_{4} \alpha_{10} \alpha_{6}, C_{3}=\alpha_{10} \alpha_{3} \alpha_{7}$, $C_{4}=\alpha_{6} \alpha_{1} \alpha_{11}$ e $C_{5}=\alpha_{8} \alpha_{1} \alpha_{5}$. Portanto, não obtemos álgebra de incidência através de corte. 
(55)

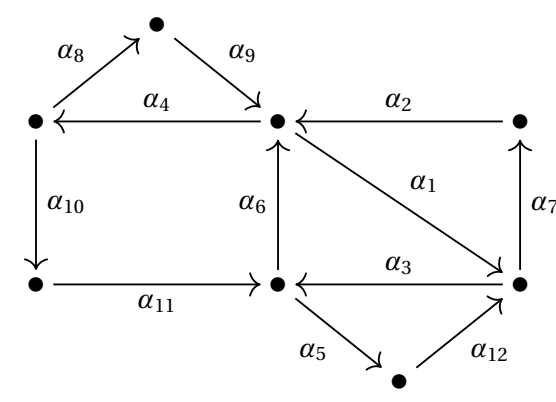

(56)

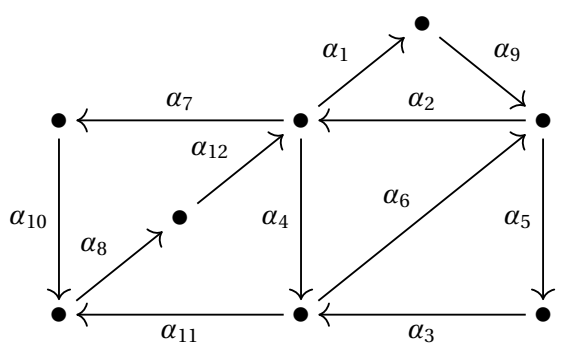

(57)

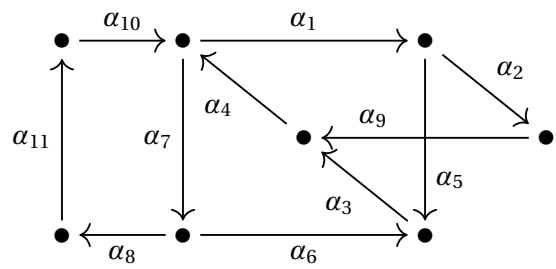

(58)

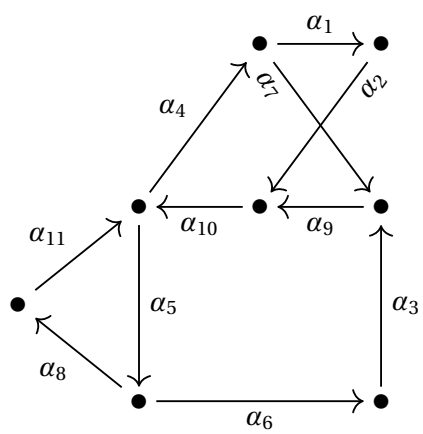

Essa extensão trivial tem as seguintes relações do tipo 2: $r 1=\alpha_{9} \alpha_{4} \alpha_{10}, r 2=\alpha_{6} \alpha_{4} \alpha_{8}, r 3=\alpha_{11} \alpha_{6} \alpha_{1}$, $r 4=\alpha_{3} \alpha_{6} \alpha_{4}, r 5=\alpha_{6} \alpha_{1} \alpha_{7}, r 6=\alpha_{2} \alpha_{1} \alpha_{3}, r 7=$ $\alpha_{12} \alpha_{3} \alpha_{6}, r 8=\alpha_{1} \alpha_{3} \alpha_{5}, r 9=\alpha_{11} \alpha_{5}, r 10=\alpha_{12} \alpha_{7}$, $r 11=\alpha_{2} \alpha_{4}$ e $r 12=\alpha_{9} \alpha_{1}$.

Os ciclos elementares dessa extensão trivial são: $C_{1}=\alpha_{11} \alpha_{6} \alpha_{4} \alpha_{10}, C_{2}=\alpha_{4} \alpha_{8} \alpha_{9}, C_{3}=\alpha_{1} \alpha_{7} \alpha_{2}$, $C_{4}=\alpha_{6} \alpha_{1} \alpha_{3}$ e $C_{5}=\alpha_{3} \alpha_{5} \alpha_{12}$. Logo, o programa não exibe solução para essa extensão trivial.

Essa extensão trivial tem as seguintes relações do tipo 2: $r 1=\alpha_{10} \alpha_{8} \alpha_{12} \alpha_{4}, r 2=\alpha_{11} \alpha_{8} \alpha_{12} \alpha_{7}, r 3=$ $\alpha_{12} \alpha_{4} \alpha_{6}, r 4=\alpha_{2} \alpha_{4} \alpha_{11}, r 5=\alpha_{4} \alpha_{6} \alpha_{5}, r 6=\alpha_{3} \alpha_{6} \alpha_{2}$, $r 7=\alpha_{6} \alpha_{2} \alpha_{1}, r 8=\alpha_{9} \alpha_{2} \alpha_{6}, r 9=\alpha_{3} \alpha_{11}, r 10=$ $\alpha_{12} \alpha_{1}, r 11=\alpha_{2} \alpha_{7}$ e $r 12=\alpha_{9} \alpha_{5}$.

Os ciclos elementares dessa extensão trivial são: $C_{1}=\alpha_{7} \alpha_{10} \alpha_{8} \alpha_{12}, C_{2}=\alpha_{8} \alpha_{12} \alpha_{4} \alpha_{11}, C_{3}=\alpha_{4} \alpha_{6} \alpha_{2}$, $C_{4}=\alpha_{2} \alpha_{1} \alpha_{9}$ e $C_{5}=\alpha_{5} \alpha_{3} \alpha_{6}$. Portanto, não obtemos corte que resulte uma álgebra de incidência.

Essa extensão trivial tem as seguintes relações do tipo 2: $r 1=\alpha_{5} \alpha_{3} \alpha_{4} \alpha_{7}, r 2=\alpha_{6} \alpha_{3} \alpha_{4} \alpha_{1}, r 3=$ $\alpha_{3} \alpha_{4} \alpha_{1} \alpha_{2}, r 4=\alpha_{9} \alpha_{4} \alpha_{1} \alpha_{5}, r 5=\alpha_{9} \alpha_{4} \alpha_{7}, r 6=$ $\alpha_{10} \alpha_{7} \alpha_{6}, r 7=\alpha_{4} \alpha_{7} \alpha_{8}$ e $r 8=\alpha_{10} \alpha_{1}$.

Os ciclos elementares dessa extensão trivial são: $C_{1}=\alpha_{1} \alpha_{2} \alpha_{9} \alpha_{4}, C_{2}=\alpha_{1} \alpha_{5} \alpha_{3} \alpha_{4}, C_{3}=\alpha_{3} \alpha_{4} \alpha_{7} \alpha_{6}$ e $C_{4}=\alpha_{10} \alpha_{7} \alpha_{8} \alpha_{11}$. Portanto, o programa exibe dois cortes que resultam nas álgebras 70 e 71 .

Essa extensão trivial tem as seguintes relações do tipo 2: $r 1=\alpha_{7} \alpha_{9} \alpha_{10} \alpha_{5}, r 2=\alpha_{3} \alpha_{9} \alpha_{10} \alpha_{4}, r 3=$ $\alpha_{2} \alpha_{10} \alpha_{4} \alpha_{7}, r 4=\alpha_{9} \alpha_{10} \alpha_{4} \alpha_{1}, r 5=\alpha_{2} \alpha_{10} \alpha_{5}, r 6=$ $\alpha_{10} \alpha_{5} \alpha_{8}, r 7=\alpha_{11} \alpha_{5} \alpha_{6}$ e $r 8=\alpha_{11} \alpha_{4}$.

Os ciclos elementares são: $C_{1}=\alpha_{6} \alpha_{3} \alpha_{9} \alpha_{10} \alpha_{5}, C_{2}=$ $\alpha_{10} \alpha_{4} \alpha_{1} \alpha_{2}, C_{3}=\alpha_{10} \alpha_{4} \alpha_{7} \alpha_{9}$ e $C_{4}=\alpha_{5} \alpha_{8} \alpha_{11}$. Logo, obtemos as soluções 72 e 73 . 
(59)

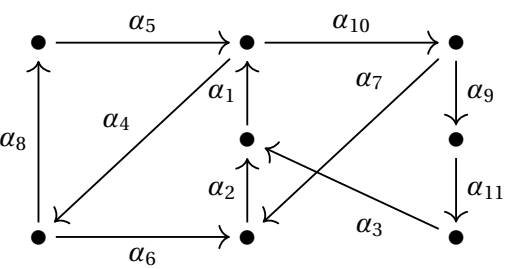

(60)

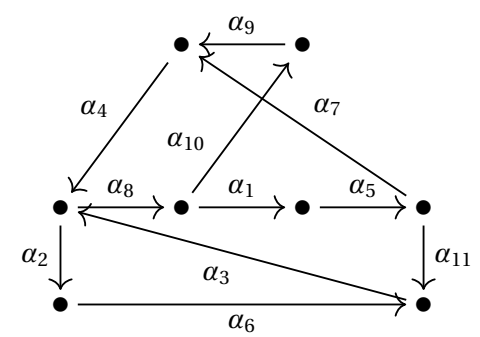

(61)

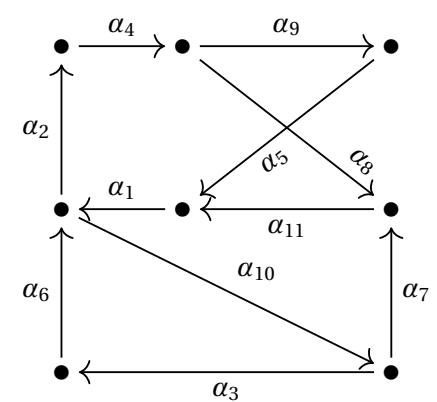

(62)

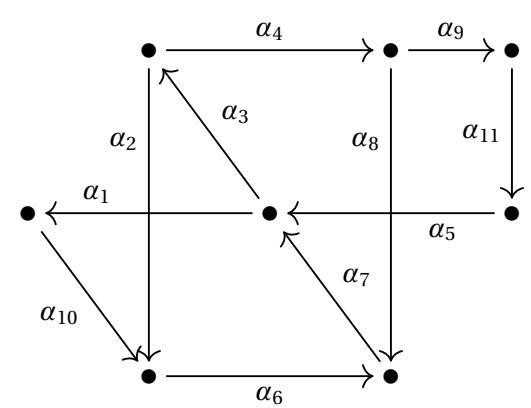

(63)

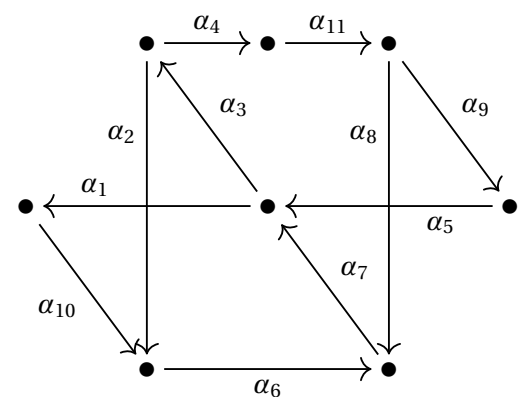

Essa extensão trivial tem as seguintes relações do tipo 2: $r 1=\alpha_{2} \alpha_{1} \alpha_{10} \alpha_{9}, r 2=\alpha_{3} \alpha_{1} \alpha_{10} \alpha_{7}, r 3=$ $\alpha_{7} \alpha_{2} \alpha_{1} \alpha_{4}, r 4=\alpha_{6} \alpha_{2} \alpha_{1} \alpha_{10}, r 5=\alpha_{3} \alpha_{1} \alpha_{4}, r 6=$ $\alpha_{1} \alpha_{4} \alpha_{8}, r 7=\alpha_{5} \alpha_{4} \alpha_{6}$ e $r 8=\alpha_{5} \alpha_{10}$.

Os ciclos elementares são: $C_{1}=\alpha_{1} \alpha_{10} \alpha_{9} \alpha_{11} \alpha_{3}$, $C_{2}=\alpha_{1} \alpha_{10} \alpha_{7} \alpha_{2}, C_{3}=\alpha_{2} \alpha_{1} \alpha_{4} \alpha_{6}$ e $C_{4}=\alpha_{4} \alpha_{8} \alpha_{5}$. O programa exibe dois cortes que originam as soluções 74 e 75.

Essa extensão trivial tem as seguintes relações do tipo 2: $r 1=\alpha_{3} \alpha_{8} \alpha_{1} \alpha_{5} \alpha_{7}, r 2=\alpha_{4} \alpha_{8} \alpha_{1} \alpha_{5} \alpha_{11}, r 3=$ $\alpha_{9} \alpha_{4} \alpha_{8} \alpha_{1}, r 4=\alpha_{7} \alpha_{4} \alpha_{8} \alpha_{10}, r 5=\alpha_{3} \alpha_{8} \alpha_{10}, r 6=$ $\alpha_{11} \alpha_{3} \alpha_{2}, r 7=\alpha_{6} \alpha_{3} \alpha_{8}$ e $r 8=\alpha_{4} \alpha_{2}$.

Os ciclos elementares são: $C_{1}=\alpha_{8} \alpha_{1} \alpha_{5} \alpha_{7} \alpha_{4}, C_{2}=$ $\alpha_{8} \alpha_{1} \alpha_{5} \alpha_{11} \alpha_{3}, C_{3}=\alpha_{8} \alpha_{10} \alpha_{9} \alpha_{4}$ e $C_{4}=\alpha_{3} \alpha_{2} \alpha_{6}$. Logo, temos as álgebras de incidência 76 e 77.

Essa extensão trivial tem as seguintes relações do tipo 2: $r 1=\alpha_{11} \alpha_{1} \alpha_{2} \alpha_{4} \alpha_{9}, r 2=\alpha_{5} \alpha_{1} \alpha_{2} \alpha_{4} \alpha_{8}, r 3=$ $\alpha_{7} \alpha_{11} \alpha_{1} \alpha_{2}, r 4=\alpha_{8} \alpha_{11} \alpha_{1} \alpha_{10}, r 5=\alpha_{5} \alpha_{1} \alpha_{10}, r 6=$ $\alpha_{6} \alpha_{10} \alpha_{7}, r 7=\alpha_{1} \alpha_{10} \alpha_{3}$ e $r 8=\alpha_{6} \alpha_{2}$.

Os ciclos elementares são: $C_{1}=\alpha_{2} \alpha_{4} \alpha_{8} \alpha_{11} \alpha_{1}, C_{2}=$ $\alpha_{10} \alpha_{7} \alpha_{11} \alpha_{1}, C_{3}=\alpha_{2} \alpha_{4} \alpha_{9} \alpha_{5} \alpha_{1}$ e $C_{4}=\alpha_{6} \alpha_{10} \alpha_{3}$. O programa exibe os cortes que originam as álgebras de incidência 78 e 79.

Essa extensão trivial tem as seguintes relações do tipo 2: $r 1=\alpha_{5} \alpha_{3} \alpha_{4} \alpha_{8}, r 2=\alpha_{7} \alpha_{3} \alpha_{4} \alpha_{9}, r 3=$ $\alpha_{6} \alpha_{7} \alpha_{3} \alpha_{4}, r 4=\alpha_{8} \alpha_{7} \alpha_{3} \alpha_{2}, r 5=\alpha_{5} \alpha_{3} \alpha_{2}, r 6=$ $\alpha_{10} \alpha_{6} \alpha_{7} \alpha_{3}, r 7=\alpha_{2} \alpha_{6} \alpha_{7} \alpha_{1}$ e $r 8=\alpha_{5} \alpha_{1}$.

Os ciclos elementares dessa extensão trivial são: $C_{1}=\alpha_{1} \alpha_{10} \alpha_{6} \alpha_{7}, C_{2}=\alpha_{6} \alpha_{7} \alpha_{3} \alpha_{2}, C_{3}=\alpha_{7} \alpha_{3} \alpha_{4} \alpha_{8}$ e $C_{4}=\alpha_{4} \alpha_{9} \alpha_{11} \alpha_{5} \alpha_{3}$. Portanto temos as soluções 80 e 81 .

Essa extensão trivial tem as seguintes relações do tipo 2: $r 1=\alpha_{5} \alpha_{3} \alpha_{4} \alpha_{11} \alpha_{8}, \quad r 2=\alpha_{7} \alpha_{3} \alpha_{4} \alpha_{11} \alpha_{9}$, $r 3=\alpha_{8} \alpha_{7} \alpha_{3} \alpha_{2}, r 4=\alpha_{6} \alpha_{7} \alpha_{3} \alpha_{4}, r 5=\alpha_{5} \alpha_{3} \alpha_{2}$, $r 6=\alpha_{2} \alpha_{6} \alpha_{7} \alpha_{1}, r 7=\alpha_{10} \alpha_{6} \alpha_{7} \alpha_{3}$ e $r 8=\alpha_{5} \alpha_{1}$.

Os ciclos elementares são: $C_{1}=\alpha_{4} \alpha_{11} \alpha_{9} \alpha_{5} \alpha_{3}, C_{2}=$ $\alpha_{4} \alpha_{11} \alpha_{8} \alpha_{7} \alpha_{3}, C_{3}=\alpha_{7} \alpha_{3} \alpha_{2} \alpha_{6}$ e $C_{4}=\alpha_{7} \alpha_{1} \alpha_{10} \alpha_{6}$. Logo, o programa exibe os cortes para originar as álgebras 82 e 83. 
(64)

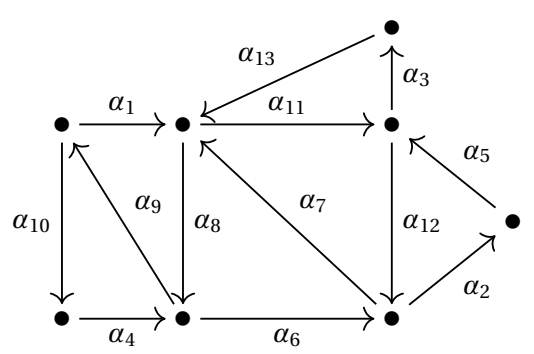

(65)

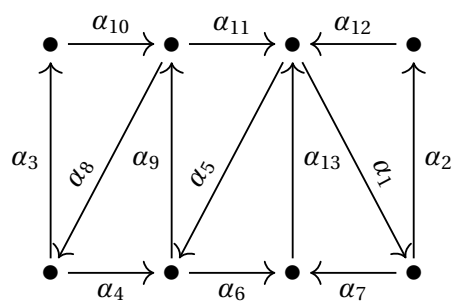

(66)

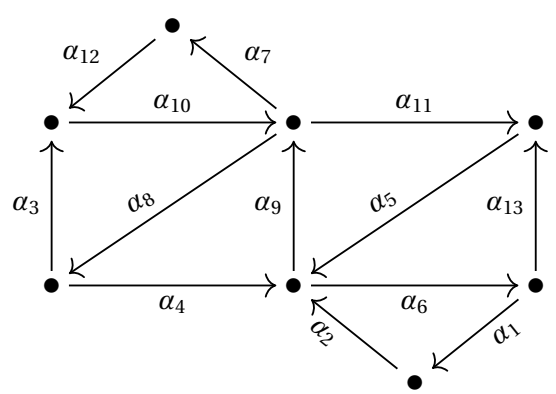

Essa extensão trivial tem as seguintes relações do tipo 2: $r 1=\alpha_{4} \alpha_{9} \alpha_{1}, r 2=\alpha_{8} \alpha_{9} \alpha_{10}, r 3=\alpha_{1} \alpha_{8} \alpha_{6}$, $r 4=\alpha_{7} \alpha_{8} \alpha_{9}, r 5=\alpha_{6} \alpha_{7} \alpha_{11}, r 6=\alpha_{12} \alpha_{7} \alpha_{8}, r 7=$ $\alpha_{11} \alpha_{12} \alpha_{2}, r 8=\alpha_{5} \alpha_{12} \alpha_{7}, r 9=\alpha_{7} \alpha_{11} \alpha_{3}, r 10=$ $\alpha_{13} \alpha_{11} \alpha_{12}, r 11=\alpha_{13} \alpha_{8}, r 12=\alpha_{4} \alpha_{6}, r 13=\alpha_{6} \alpha_{2}$, $r 14=\alpha_{5} \alpha_{3}$ e $r 15=\alpha_{1} \alpha_{11}$.

Essa extensão trivial apresenta os seguintes ciclos elementares: $C_{1}=\alpha_{4} \alpha_{9} \alpha_{10}, C_{2}=\alpha_{9} \alpha_{1} \alpha_{8}, C_{3}=$ $\alpha_{8} \alpha_{6} \alpha_{7}, C_{4}=\alpha_{7} \alpha_{11} \alpha_{12}, C 5=\alpha_{12} \alpha_{2} \alpha_{5}$ e $C 6=$ $\alpha_{3} \alpha_{13} \alpha_{11}$. Portanto, não temos álgebra de incidência para esse caso.

Essa extensão trivial tem as seguintes relações do tipo 2: $r 1=\alpha_{10} \alpha_{8} \alpha_{4}, r 2=\alpha_{9} \alpha_{8} \alpha_{3}, r 3=\alpha_{4} \alpha_{9} \alpha_{11}$, $r 4=\alpha_{5} \alpha_{9} \alpha_{8}, r 5=\alpha_{11} \alpha_{5} \alpha_{6}, r 6=\alpha_{13} \alpha_{5} \alpha_{9}, r 7=$ $\alpha_{6} \alpha_{13} \alpha_{1}, r 8=\alpha_{7} \alpha_{13} \alpha_{5}, r 9=\alpha_{13} \alpha_{1} \alpha_{2}, r 10=$ $\alpha_{12} \alpha_{1} \alpha_{7}, r 11=\alpha_{4} \alpha_{6}, r 12=\alpha_{10} \alpha_{11}, r 13=\alpha_{11} \alpha_{1}$ e $r 14=\alpha_{12} \alpha_{5}$.

Essa extensão trivial apresenta os seguintes ciclos elementares: $C_{1}=\alpha_{3} \alpha_{10} \alpha_{8}, C_{2}=\alpha_{8} \alpha_{4} \alpha_{9}, C_{3}=$ $\alpha_{9} \alpha_{11} \alpha_{5}, C_{4}=\alpha_{5} \alpha_{6} \alpha_{13}, C 5=\alpha_{13} \alpha_{1} \alpha_{7}$ e $C 6=$ $\alpha_{1} \alpha_{2} \alpha_{12}$. Logo, não obtemos solução para essa extensão trivial.

Essa extensão trivial tem as seguintes relações do tipo 2: $r 1=\alpha_{12} \alpha_{10} \alpha_{8}, r 2=\alpha_{3} \alpha_{10} \alpha_{7}, r 3=\alpha_{10} \alpha_{8} \alpha_{4}$, $r 4=\alpha_{9} \alpha_{8} \alpha_{3}, r 5=\alpha_{4} \alpha_{9} \alpha_{11}, r 6=\alpha_{5} \alpha_{9} \alpha_{8}, r 7=$ $\alpha_{11} \alpha_{5} \alpha_{6}, \quad r 8=\alpha_{13} \alpha_{5} \alpha_{9}, \quad r 9=\alpha_{5} \alpha_{6} \alpha_{1}, \quad r 10=$ $\alpha_{2} \alpha_{6} \alpha_{13}, r 11=\alpha_{10} \alpha_{11}, r 12=\alpha_{9} \alpha_{7}, r 13=\alpha_{4} \alpha_{6}$ e $r 14=\alpha_{2} \alpha_{9}$.

Essa extensão trivial apresenta os seguintes ciclos elementares: $C_{1}=\alpha_{7} \alpha_{12} \alpha_{10}, C_{2}=\alpha_{10} \alpha_{8} \alpha_{3}, C_{3}=$ $\alpha_{8} \alpha_{4} \alpha_{9}, C_{4}=\alpha_{9} \alpha_{11} \alpha_{5}, C 5=\alpha_{5} \alpha_{6} \alpha_{13}$ e $C 6=$ $\alpha_{6} \alpha_{1} \alpha_{2}$. O programa exibe a solução 84 . 
(67)

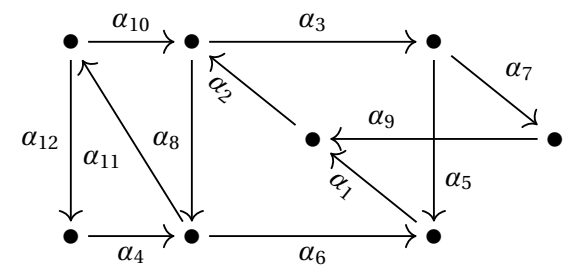

(68)

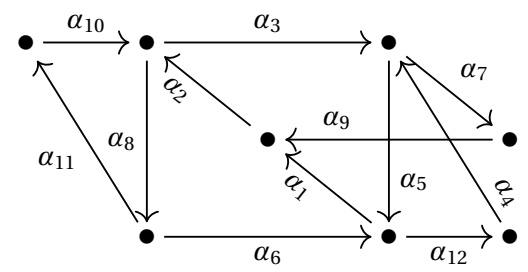

(69)

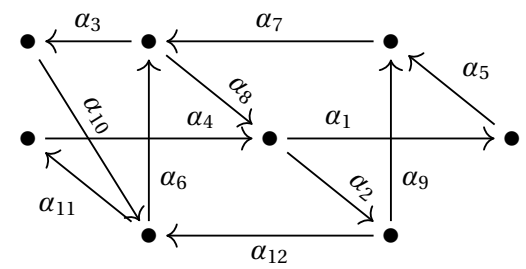

(70)

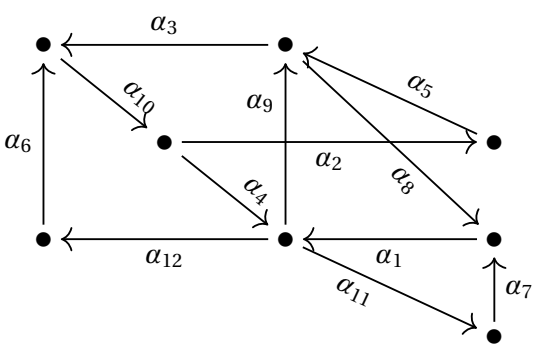

Essa extensão trivial tem as seguintes relações do tipo 2: $r 1=\alpha_{6} \alpha_{1} \alpha_{2} \alpha_{3}, r 2=\alpha_{5} \alpha_{1} \alpha_{2} \alpha_{8}, r 3=$ $\alpha_{1} \alpha_{2} \alpha_{3} \alpha_{7}, r 4=\alpha_{9} \alpha_{2} \alpha_{3} \alpha_{5}, r 5=\alpha_{9} \alpha_{2} \alpha_{8}, r 6=$ $\alpha_{2} \alpha_{8} \alpha_{11}, r 7=\alpha_{10} \alpha_{8} \alpha_{6}, \quad r 8=\alpha_{8} \alpha_{11} \alpha_{12}, \quad r 9=$ $\alpha_{4} \alpha_{11} \alpha_{10}, r 10=\alpha_{4} \alpha_{6}$ e $r 11=\alpha_{10} \alpha_{3}$.

Essa extensão trivial apresenta os seguintes ciclos elementares: $C_{1}=\alpha_{6} \alpha_{1} \alpha_{2} \alpha_{8}, C_{2}=\alpha_{2} \alpha_{3} \alpha_{7} \alpha_{9}$, $C_{3}=\alpha_{1} \alpha_{2} \alpha_{3} \alpha_{5}, C_{4}=\alpha_{10} \alpha_{8} \alpha_{11}$ e $C 5=\alpha_{11} \alpha_{12} \alpha_{4}$. Portanto, obtemos a álgebra de incidência 85 .

As relações do tipo 2 para essa álgebra são: $r 1=$ $\alpha_{6} \alpha_{1} \alpha_{2} \alpha_{3}, r 2=\alpha_{5} \alpha_{1} \alpha_{2} \alpha_{8}, r 3=\alpha_{1} \alpha_{2} \alpha_{3} \alpha_{7}, r 4=$ $\alpha_{9} \alpha_{2} \alpha_{3} \alpha_{5}, \quad r 5=\alpha_{9} \alpha_{2} \alpha_{8}, r 6=\alpha_{2} \alpha_{8} \alpha_{11}, r 7=$ $\alpha_{10} \alpha_{8} \alpha_{6}, r 8=\alpha_{3} \alpha_{5} \alpha_{12}, r 9=\alpha_{4} \alpha_{5} \alpha_{1}, r 10=\alpha_{6} \alpha_{12}$, $r 11=\alpha_{10} \alpha_{3}$ e $r 12=\alpha_{4} \alpha_{7}$.

Essa extensão trivial apresenta os seguintes ciclos elementares: $C_{1}=\alpha_{6} \alpha_{1} \alpha_{2} \alpha_{8}, C_{2}=\alpha_{2} \alpha_{3} \alpha_{7} \alpha_{9}$, $C_{3}=\alpha_{1} \alpha_{2} \alpha_{3} \alpha_{5}, C_{4}=\alpha_{5} \alpha_{12} \alpha_{4}$ e $C 5=\alpha_{10} \alpha_{8} \alpha_{11}$. Portanto, não temos corte que origine uma álgebra de incidência.

Essa extensão trivial tem as seguintes relações do tipo 2: $r 1=\alpha_{5} \alpha_{7} \alpha_{8} \alpha_{2}, r 2=\alpha_{9} \alpha_{7} \alpha_{8} \alpha_{1}, r 3=$ $\alpha_{7} \alpha_{8} \alpha_{2} \alpha_{12}, r 4=\alpha_{6} \alpha_{8} \alpha_{2} \alpha_{9}, r 5=\alpha_{6} \alpha_{8} \alpha_{1}, r 6=$ $\alpha_{8} \alpha_{2} \alpha_{12} \alpha_{11}, r 7=\alpha_{4} \alpha_{2} \alpha_{12} \alpha_{6}, r 8=\alpha_{12} \alpha_{6} \alpha_{3}, r 9=$ $\alpha_{10} \alpha_{6} \alpha_{8}, r 10=\alpha_{4} \alpha_{2} \alpha_{9}, r 11=\alpha_{4} \alpha_{1}, r 12=\alpha_{7} \alpha_{3} \mathrm{e}$ $r 13=\alpha_{10} \alpha_{11}$.

Essa extensão trivial apresenta os seguintes ciclos elementares: $C_{1}=\alpha_{1} \alpha_{5} \alpha_{7} \alpha_{8}, C_{2}=\alpha_{7} \alpha_{8} \alpha_{2} \alpha_{9}, C_{3}=$ $\alpha_{8} \alpha_{2} \alpha_{12} \alpha_{6}, C_{4}=\alpha_{2} \alpha_{12} \alpha_{11} \alpha_{4}$ e $C 5=\alpha_{6} \alpha_{3} \alpha_{10}$. O programa não exibe corte.

Essa extensão trivial tem as seguintes relações do tipo 2: $r 1=\alpha_{6} \alpha_{10} \alpha_{4} \alpha_{9}, r 2=\alpha_{3} \alpha_{10} \alpha_{4} \alpha_{12}, r 3=$ $\alpha_{5} \alpha_{3} \alpha_{10} \alpha_{4}, r 4=\alpha_{9} \alpha_{3} \alpha_{10} \alpha_{2}, r 5=\alpha_{6} \alpha_{10} \alpha_{2}, r 6=$ $\alpha_{4} \alpha_{9} \alpha_{8}, r 7=\alpha_{1} \alpha_{9} \alpha_{3}, r 8=\alpha_{8} \alpha_{1} \alpha_{11}, r 9=\alpha_{7} \alpha_{1} \alpha_{9}$, $r 10=\alpha_{1} \alpha_{12}, r 11=\alpha_{5} \alpha_{8}$ e $r 12=\alpha_{4} \alpha_{11}$.

Essa extensão trivial apresenta os seguintes ciclos elementares: $C_{1}=\alpha_{12} \alpha_{6} \alpha_{10} \alpha_{4}, C_{2}=\alpha_{10} \alpha_{4} \alpha_{9} \alpha_{6}$, $C_{3}=\alpha_{3} \alpha_{10} \alpha_{2} \alpha_{5}, C_{4}=\alpha_{9} \alpha_{8} \alpha_{1}$ e $C 5=\alpha_{1} \alpha_{11} \alpha_{7}$. Logo não obtemos corte. 
(71)

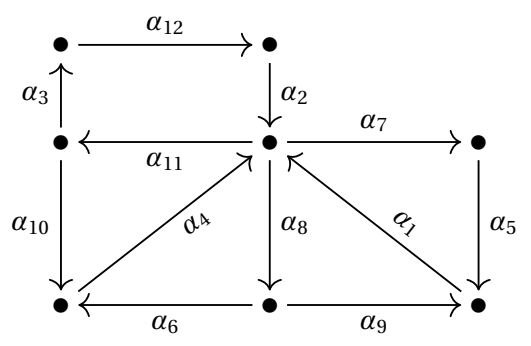

(72)

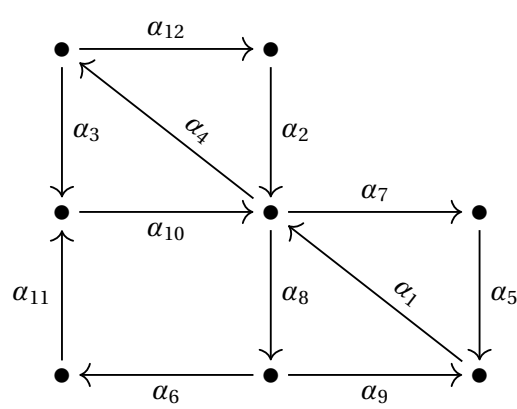

(73)

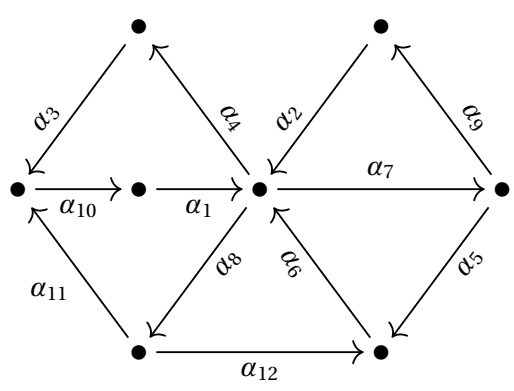

(74)

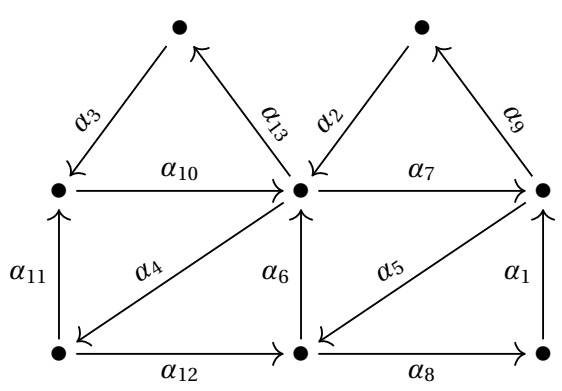

Essa extensão trivial tem as seguintes relações do tipo 2: $r 1=\alpha_{2} \alpha_{11} \alpha_{10}, r 2=\alpha_{4} \alpha_{11} \alpha_{3}, r 3=\alpha_{10} \alpha_{4} \alpha_{8}$, $r 4=\alpha_{6} \alpha_{4} \alpha_{11}, r 5=\alpha_{4} \alpha_{8} \alpha_{9}, r 6=\alpha_{1} \alpha_{8} \alpha_{6}, r 7=$ $\alpha_{9} \alpha_{1} \alpha_{7}, r 8=\alpha_{5} \alpha_{1} \alpha_{8}, r 9=\alpha_{2} \alpha_{7}, r 10=\alpha_{1} \alpha_{11}$, $r 11=\alpha_{4} \alpha_{7}$ e $r 12=\alpha_{2} \alpha_{8}$.

Essa extensão trivial apresenta os seguintes ciclos elementares: $C_{1}=\alpha_{12} \alpha_{2} \alpha_{11} \alpha_{3}, C_{2}=\alpha_{11} \alpha_{10} \alpha_{4}, C_{3}=$ $\alpha_{4} \alpha_{8} \alpha_{6}, C_{4}=\alpha_{8} \alpha_{9} \alpha_{1}$ e $C 5=\alpha_{1} \alpha_{7} \alpha_{5}$. Portanto, temos as álgebras de incidência 86 e 87.

Essa extensão trivial tem as seguintes relações do tipo 2: $r 1=\alpha_{2} \alpha_{4} \alpha_{3}, r 2=\alpha_{10} \alpha_{4} \alpha_{12}, r 3=\alpha_{3} \alpha_{10} \alpha_{8}$, $r 4=\alpha_{11} \alpha_{10} \alpha_{4}, r 5=\alpha_{10} \alpha_{8} \alpha_{9}, r 6=\alpha_{1} \alpha_{8} \alpha_{6}, r 7=$ $\alpha_{9} \alpha_{1} \alpha_{7}, r 8=\alpha_{5} \alpha_{1} \alpha_{8}, r 9=\alpha_{2} \alpha_{7}, r 10=\alpha_{2} \alpha_{8}$, $r 11=\alpha_{1} \alpha_{4}$ e $r 12=\alpha_{10} \alpha_{7}$.

Essa extensão trivial apresenta os seguintes ciclos elementares: $C_{1}=\alpha_{6} \alpha_{11} \alpha_{10} \alpha_{8}, C_{2}=\alpha_{10} \alpha_{4} \alpha_{3}, C_{3}=$ $\alpha_{4} \alpha_{12} \alpha_{2}, C_{4}=\alpha_{8} \alpha_{9} \alpha_{1}$ e $C 5=\alpha_{1} \alpha_{7} \alpha_{5}$. Logo, obtemos as soluções 88 e 89 .

As relações do tipo 2 são: $r 1=\alpha_{3} \alpha_{10} \alpha_{1} \alpha_{8}, r 2=$ $\alpha_{11} \alpha_{10} \alpha_{1} \alpha_{4}, r 3=\alpha_{1} \alpha_{8} \alpha_{12}, r 4=\alpha_{6} \alpha_{8} \alpha_{11}, r 5=$ $\alpha_{12} \alpha_{6} \alpha_{7}, r 6=\alpha_{5} \alpha_{6} \alpha_{8}, r 7=\alpha_{6} \alpha_{7} \alpha_{9}, r 8=\alpha_{2} \alpha_{7} \alpha_{5}$, $r 9=\alpha_{6} \alpha_{4}, r 10=\alpha_{2} \alpha_{8}, r 11=\alpha_{1} \alpha_{7}$ e $r 12=\alpha_{2} \alpha_{4}$.

Essa extensão trivial apresenta os seguintes ciclos elementares: $C_{1}=\alpha_{4} \alpha_{3} \alpha_{10} \alpha_{1}, C_{2}=\alpha_{10} \alpha_{1} \alpha_{8} \alpha_{11}$, $C_{3}=\alpha_{8} \alpha_{12} \alpha_{6}, C_{4}=\alpha_{6} \alpha_{7} \alpha_{5}$ e $C 5=\alpha_{7} \alpha_{9} \alpha_{2}$. O programa exibe os cortes para as álgebras 90 e 91 .

As relações do tipo 2 são: $r 1=\alpha_{3} \alpha_{10} \alpha_{4}, r 2=$ $\alpha_{11} \alpha_{10} \alpha_{13}, r 3=\alpha_{10} \alpha_{4} \alpha_{12}, r 4=\alpha_{6} \alpha_{4} \alpha_{11}, r 5=$ $\alpha_{12} \alpha_{6} \alpha_{7}, r 6=\alpha_{5} \alpha_{6} \alpha_{4}, r 7=\alpha_{6} \alpha_{7} \alpha_{9}, r 8=\alpha_{2} \alpha_{7} \alpha_{5}$, $r 9=\alpha_{7} \alpha_{5} \alpha_{8}, r 10=\alpha_{1} \alpha_{5} \alpha_{6}, r 11=\alpha_{2} \alpha_{13}, r 12=$ $\alpha_{2} \alpha_{4}, r 13=\alpha_{10} \alpha_{7}, r 14=\alpha_{6} \alpha_{13}, r 15=\alpha_{12} \alpha_{8} \mathrm{e}$ $r 16=\alpha_{1} \alpha_{9}$.

Essa extensão trivial apresenta os seguintes ciclos elementares: $C_{1}=\alpha_{13} \alpha_{3} \alpha_{10}, C_{2}=\alpha_{10} \alpha_{4} \alpha_{11}, C_{3}=$ $\alpha_{4} \alpha_{12} \alpha_{6}, C_{4}=\alpha_{6} \alpha_{7} \alpha_{5}, C 5=\alpha_{5} \alpha_{8} \alpha_{1}$ e $C 6=$ $\alpha_{7} \alpha_{9} \alpha_{2}$. O programa não exibe corte. 
(75)

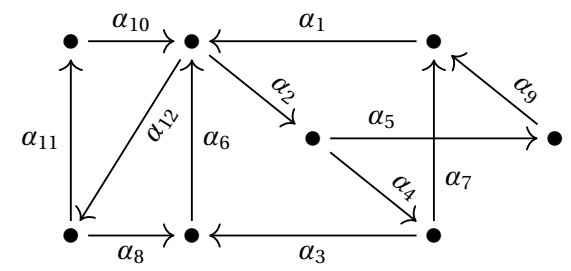

(76)

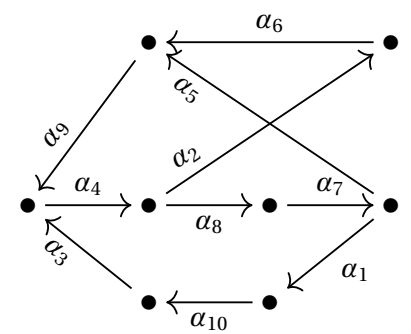

(77)

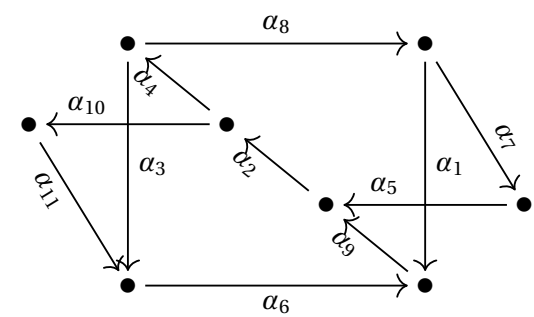

As relações do tipo 2 para essa álgebra são: $r 1=$ $\alpha_{9} \alpha_{1} \alpha_{2} \alpha_{4}, r 2=\alpha_{7} \alpha_{1} \alpha_{2} \alpha_{5}, r 3=\alpha_{1} \alpha_{2} \alpha_{4} \alpha_{3}, r 4=$ $\alpha_{6} \alpha_{2} \alpha_{4} \alpha_{7}, r 5=\alpha_{6} \alpha_{2} \alpha_{5}, r 6=\alpha_{3} \alpha_{6} \alpha_{12}, r 7=$ $\alpha_{8} \alpha_{6} \alpha_{2}, r 8=\alpha_{6} \alpha_{12} \alpha_{11}, r 9=\alpha_{10} \alpha_{12} \alpha_{8}$ e $r 10=$ $\alpha_{10} \alpha_{2}$.

Os ciclos elementares dessa extensão trivial são: $C_{1}=\alpha_{2} \alpha_{5} \alpha_{9} \alpha_{1}, C_{2}=\alpha_{2} \alpha_{4} \alpha_{3} \alpha_{6}, C_{3}=\alpha_{2} \alpha_{4} \alpha_{7} \alpha_{1}$, $C_{4}=\alpha_{12} \alpha_{8} \alpha_{6}$ e $C_{5}=\alpha_{12} \alpha_{11} \alpha_{10}$. Logo obtemos as soluções 92 e 93.

As relações do tipo 2 são: $r 1=\alpha_{9} \alpha_{4} \alpha_{8} \alpha_{7} \alpha_{1}, r 2=$ $\alpha_{3} \alpha_{4} \alpha_{8} \alpha_{7} \alpha_{5}, r 3=\alpha_{6} \alpha_{9} \alpha_{4} \alpha_{8}, r 4=\alpha_{5} \alpha_{9} \alpha_{4} \alpha_{2}$ e $r 5=\alpha_{3} \alpha_{4} \alpha_{2}$.

Os ciclos elementares são: $C_{1}=\alpha_{4} \alpha_{8} \alpha_{7} \alpha_{1} \alpha_{10} \alpha_{3}$, $C_{2}=\alpha_{4} \alpha_{8} \alpha_{7} \alpha_{5} \alpha_{9}$ e $C_{3}=\alpha_{4} \alpha_{2} \alpha_{6} \alpha_{9}$. Nesse caso, obtemos as álgebras 94,95 e na dual da álgebra 5 .

Essa extensão trivial tem as seguintes relações de tipo 2: $r 1=\alpha_{1} \alpha_{9} \alpha_{2} \alpha_{10}, r 2=\alpha_{5} \alpha_{2} \alpha_{10}, r 3=$ $\alpha_{9} \alpha_{2} \alpha_{4} \alpha_{8} \alpha_{7}, r 4=\alpha_{5} \alpha_{2} \alpha_{4} \alpha_{8} \alpha_{1}, r 5=\alpha_{5} \alpha_{2} \alpha_{4} \alpha_{3}$, $r 6=\alpha_{3} \alpha_{6} \alpha_{9} \alpha_{2} \alpha_{10}, \quad r 7=\alpha_{11} \alpha_{6} \alpha_{9} \alpha_{2} \alpha_{4}, \quad r 8=$ $\alpha_{6} \alpha_{9} \alpha_{2} \alpha_{4} \alpha_{8}$ e $r 9=\alpha_{1} \alpha_{9} \alpha_{2} \alpha_{4} \alpha_{3}$.

Essa extensão trivial apresenta os seguintes ciclos elementares: $C_{1}=\alpha_{9} \alpha_{2} \alpha_{4} \alpha_{3} \alpha_{6}, C_{2}=\alpha_{9} \alpha_{2} \alpha_{4} \alpha_{8} \alpha_{1}$, $C_{3}=\alpha_{2} \alpha_{4} \alpha_{8} \alpha_{7} \alpha_{5}$ e $C_{4}=\alpha_{9} \alpha_{2} \alpha_{10} \alpha_{11} \alpha_{6}$. Portanto, temos as álgebras de incidência 96,97 e 98.

Finalmente, o último passo é repetir o processo para a lista enumerada do teorema 3.3.17 de [Fer99]. O corte em cada uma dessas extensões triviais originam álgebras de incidência hereditárias apenas nas álgebras (1), (2), (3), (5), (6), (7), (8), (9), (10), (13), (16), (18), (19), (20), (21), (22), (25), (28), (54), (56), (57), (58), (93), (95) e (97).

Algumas extensões triviais têm um vértice $\square$. No vértice $\square$ é anexado uma extensão trivial da classe de Cartan de tipo $A_{n}$, o número $n$ é igual a quantidade de vértices $\bullet$ que faltam para completar oito $\left(\mathbb{E}_{8}\right)$. O primeiro passo é fazer o vértice $\square$ como um normal $\bullet$, ver as relações do tipo 2 e os ciclos elementares. O outro passo é listar as possíveis extensões triviais que podem ser anexadas no vértice $\square$, completar as informações sobre as relações do tipo 2 e os ciclos elementares da extensão trivial com oito vértices. Conforme a lista abaixo, anexaremos apenas as extensões triviais de tipo $A_{4}$ ou $A_{3}$. Para esses casos, com o intuito de resumir a demonstração, faremos algumas observações: 
- No tipo $A_{4}$, duas opções aparecem para anexar no vértice $\square$,

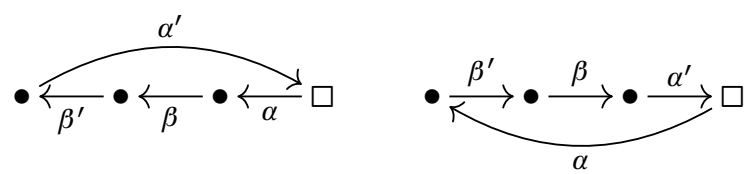

As flechas $\beta^{\prime}$ e $\beta$ não fazem parte das relações de tipo 2, as flechas $\alpha^{\prime}$ têm o mesmo término, as flechas $\alpha$ têm a mesma origem, então colocaremos umas arestas no lugar das flechas $\beta^{\prime}$ e $\beta$ e calcularemos a presença dos cortes que origina as álgebras de incidência na extensão trivial com esse anexo:

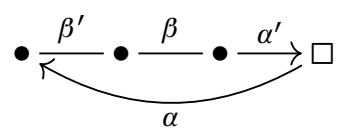

Assim, estamos analisando os dois casos equivalentes e as arestas devem ser orientadas conformes essas opções. Iremos proceder da mesma maneira para os anexos da classe de Cartan de tipo $A_{3}$.

- De forma análoga, teremos esses dois casos equivalentes:
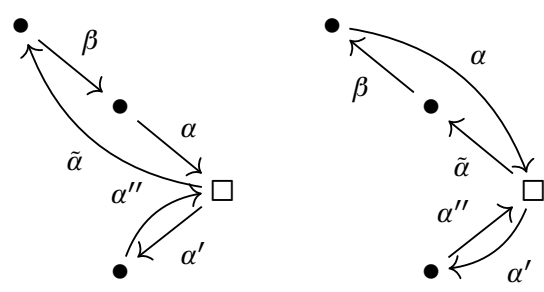

Então colocaremos uma aresta no lugar da flecha $\beta$ e calcularemos a presença dos cortes que origina as álgebras de incidência na extensão trivial com esse anexo:

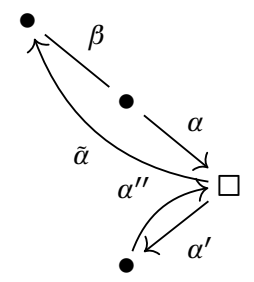

Assim, analisamos os dois casos equivalentes e a aresta deve ser orientada de acordo com as opções.

- E as outras duas opções de extensões triviais da classe de Cartan de tipo $A_{4}$ são:
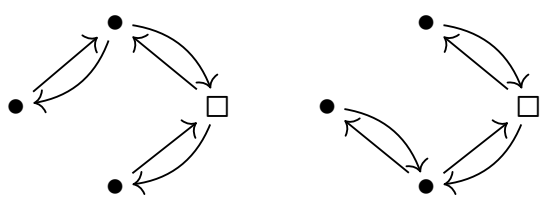
Essas aljavas são equivalentes então iremos calcular apenas com uma delas como anexo. Enquanto, na extensão trivial da classe de Cartan $A_{3}$, teremos apenas uma aljava do tipo acima.

(1)
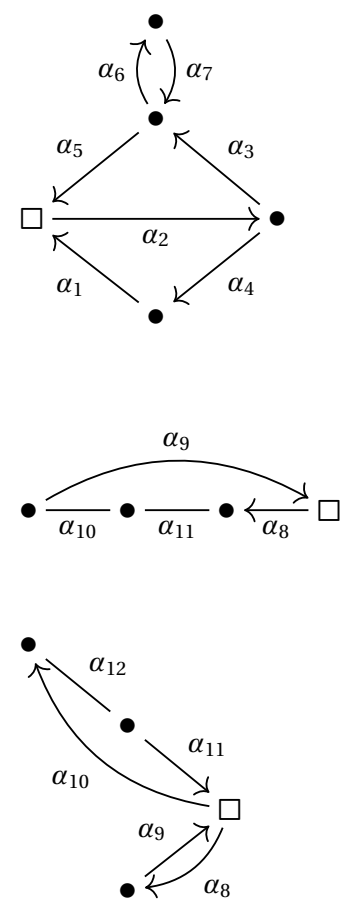

(2)
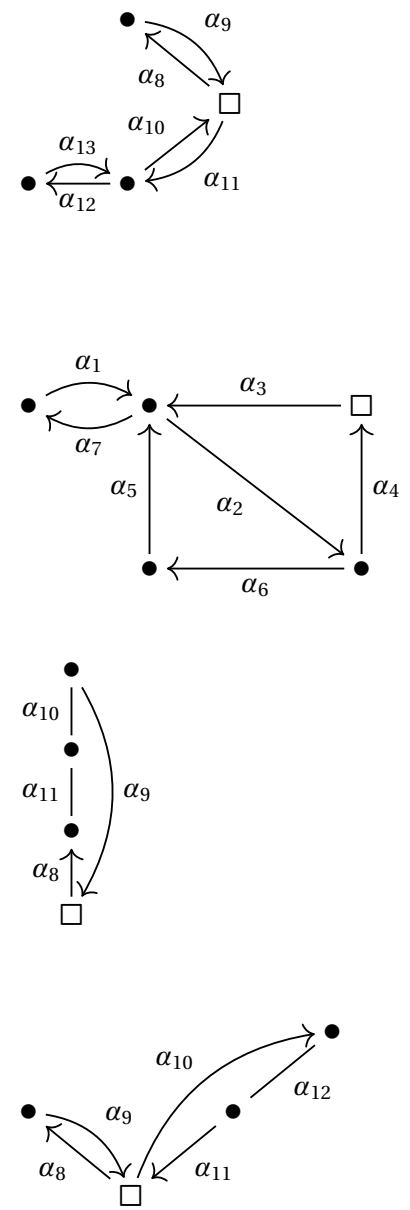

As relações do tipo 2 são: $r 1=\alpha_{5} \alpha_{2} \alpha_{4}, r 2=$ $\alpha_{1} \alpha_{2} \alpha_{3}, r 3=\alpha_{3} \alpha_{6}$ e $r 4=\alpha_{7} \alpha_{5}$.

Os ciclos elementares são: $C_{1}=\alpha_{2} \alpha_{3} \alpha_{5}, C_{2}=$ $\alpha_{2} \alpha_{4} \alpha_{1}$ e $C_{3}=\alpha_{6} \alpha_{7}$.

Completando, temos mais essas relações do tipo 2: $r 5=\alpha_{5} \alpha_{8}, r 6=\alpha_{1} \alpha_{8}$ e $r 7=\alpha_{9} \alpha_{2}$.

E o ciclo elementar é $C_{4}=\alpha_{8} \alpha_{11} \alpha_{10} \alpha_{9}$. O programa exibe apenas um corte que origina uma álgebra de incidência hereditária.

Completando, temos mais essas relações do tipo 2: $r 5=\alpha_{5} \alpha_{8}, r 6=\alpha_{5} \alpha_{10}, r 7=\alpha_{1} \alpha_{8}, r 8=\alpha_{1} \alpha_{10}, r 9=$ $\alpha_{11} \alpha_{2}, r 10=\alpha_{9} \alpha_{2}, r 11=\alpha_{11} \alpha_{8}$ e $r 12=\alpha_{9} \alpha_{10}$.

E os ciclos elementares são $C_{4}=\alpha_{10} \alpha_{12} \alpha_{11}$ e $C_{5}=$ $\alpha_{9} \alpha_{8}$. Portanto, o programa não exibe solução.

Completando, temos mais essas relações do tipo 2: $r 5=\alpha_{5} \alpha_{8}, r 6=\alpha_{5} \alpha_{11}, r 7=\alpha_{1} \alpha_{8}, r 8=\alpha_{1} \alpha_{11}, r 9=$ $\alpha_{10} \alpha_{2}, r 10=\alpha_{9} \alpha_{2}, r 11=\alpha_{13} \alpha_{10}, r 12=\alpha_{11} \alpha_{12}$, $r 13=\alpha_{9} \alpha_{11}$ e $r 14=\alpha_{10} \alpha_{8}$.

E os ciclos elementares são $C_{4}=\alpha_{10} \alpha_{11}, C_{5}=\alpha_{9} \alpha_{8}$ e $C_{6}=\alpha_{12} \alpha_{13}$. Portanto, temos mais uma álgebra hereditária.

As relações do tipo 2 são: $r 1=\alpha_{3} \alpha_{2} \alpha_{6}, r 2=$ $\alpha_{5} \alpha_{2} \alpha_{4}, r 3=\alpha_{3} \alpha_{7}, r 4=\alpha_{5} \alpha_{7}$ e $r 5=\alpha_{1} \alpha_{2}$.

Os ciclos elementares são: $C_{1}=\alpha_{2} \alpha_{4} \alpha_{3}, C_{2}=$ $\alpha_{2} \alpha_{6} \alpha_{5}$ e $C_{3}=\alpha_{7} \alpha_{1}$.

Completando, temos mais essas relações do tipo 2: $r 6=\alpha_{4} \alpha_{8}$ e $r 7=\alpha_{9} \alpha_{3}$.

E o ciclo elementar é $C_{4}=\alpha_{8} \alpha_{11} \alpha_{10} \alpha_{9}$. O programa exibe uma solução hereditária.

Nesse caso, temos mais essas relações do tipo 2: $r 6=\alpha_{4} \alpha_{8}, r 7=\alpha_{4} \alpha_{10}, r 8=\alpha_{9} \alpha_{3}, r 9=\alpha_{11} \alpha_{3}$, $r 10=\alpha_{11} \alpha_{8}$ e $r 11=\alpha_{9} \alpha_{10}$.

E os ciclos elementares são $C_{4}=\alpha_{10} \alpha_{12} \alpha_{11}$ e $C_{5}=$ $\alpha_{9} \alpha_{8}$. Portanto, o programa exibe uma solução hereditária. 


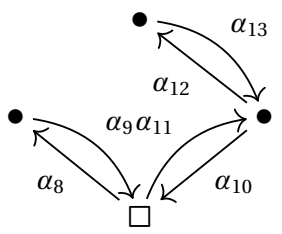

(3)
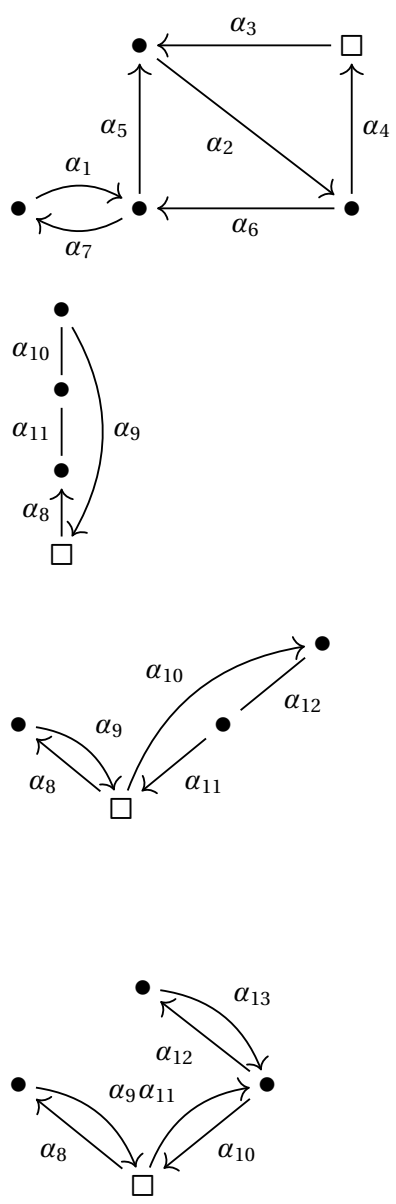

(4)
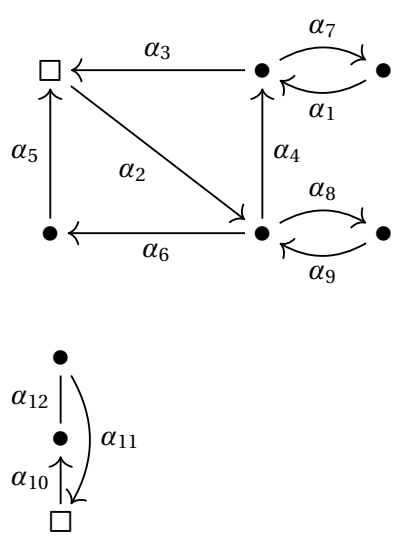

Nesse anexo, temos mais essas relações do tipo 2: $r 6=\alpha_{4} \alpha_{8}, r 7=\alpha_{4} \alpha_{11}, r 8=\alpha_{9} \alpha_{3}, r 9=\alpha_{10} \alpha_{3}$, $r 10=\alpha_{10} \alpha_{8}, r 11=\alpha_{9} \alpha_{11}, r 12=\alpha_{13} \alpha_{10}$ e $r 13=$ $\alpha_{11} \alpha_{12}$.

E os ciclos elementares são $C_{4}=\alpha_{10} \alpha_{11}, C_{5}=\alpha_{9} \alpha_{8}$ e $C_{6}=\alpha_{12} \alpha_{13}$. Portanto, temos mais uma álgebra hereditária.

As relações do tipo 2 são: $r 1=\alpha_{3} \alpha_{2} \alpha_{6}, r 2=$ $\alpha_{5} \alpha_{2} \alpha_{4}, r 3=\alpha_{3} \alpha_{6}, r 4=\alpha_{6} \alpha_{7}$ e $r 5=\alpha_{1} \alpha_{5}$.

Os ciclos elementares são: $C_{1}=\alpha_{2} \alpha_{4} \alpha_{3}, C_{2}=$ $\alpha_{2} \alpha_{6} \alpha_{5}$ e $C_{3}=\alpha_{7} \alpha_{1}$.

Completando, temos mais essas relações do tipo 2: $r 6=\alpha_{4} \alpha_{8}$ e $r 7=\alpha_{9} \alpha_{3}$.

E o ciclo elementar é $C_{4}=\alpha_{8} \alpha_{11} \alpha_{10} \alpha_{9}$. O programa exibe duas soluções hereditárias.

Nesse caso, temos mais essas relações do tipo 2: $r 6=\alpha_{4} \alpha_{8}, r 7=\alpha_{4} \alpha_{10}, r 8=\alpha_{9} \alpha_{3}, r 9=\alpha_{11} \alpha_{3}$, $r 10=\alpha_{11} \alpha_{8}$ e $r 11=\alpha_{9} \alpha_{10}$.

E os ciclos elementares são $C_{4}=\alpha_{10} \alpha_{12} \alpha_{11}$ e $C_{5}=$ $\alpha_{9} \alpha_{8}$. Logo obtemos duas soluções hereditárias.

Nesse anexo, temos mais essas relações do tipo 2: $r 6=\alpha_{4} \alpha_{8}, r 7=\alpha_{4} \alpha_{11}, r 8=\alpha_{9} \alpha_{3}, r 9=\alpha_{10} \alpha_{3}$, $r 10=\alpha_{10} \alpha_{8}, r 11=\alpha_{9} \alpha_{11}, r 12=\alpha_{13} \alpha_{10}$ e $r 13=$ $\alpha_{11} \alpha_{12}$.

E os ciclos elementares são $C_{4}=\alpha_{10} \alpha_{11}, C_{5}=\alpha_{9} \alpha_{8}$ e $C_{6}=\alpha_{12} \alpha_{13}$. Portanto, temos mais duas álgebras hereditárias.

As relações do tipo 2 são: $r 1=\alpha_{3} \alpha_{2} \alpha_{6}, r 2=$ $\alpha_{5} \alpha_{2} \alpha_{4}, r 3=\alpha_{1} \alpha_{3}, r 4=\alpha_{4} \alpha_{7}, r 5=\alpha_{9} \alpha_{6}, r 6=$ $\alpha_{9} \alpha_{4}$ e $r 7=\alpha_{2} \alpha_{8}$.

Os ciclos elementares são: $C_{1}=\alpha_{3} \alpha_{2} \alpha_{4}, C_{2}=$ $\alpha_{2} \alpha_{6} \alpha_{5}, C_{3}=\alpha_{7} \alpha_{1}$ e $C_{4}=\alpha_{8} \alpha_{9}$.

Completando, temos mais essas relações do tipo 2 : $r 8=\alpha_{5} \alpha_{10}, r 9=\alpha_{3} \alpha_{10}$ e $r 10=\alpha_{11} \alpha_{2}$.

E o ciclo elementar é $C_{5}=\alpha_{10} \alpha_{12} \alpha_{11}$. O programa não exibe solução. 

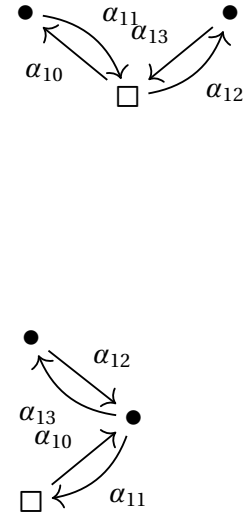

(5)
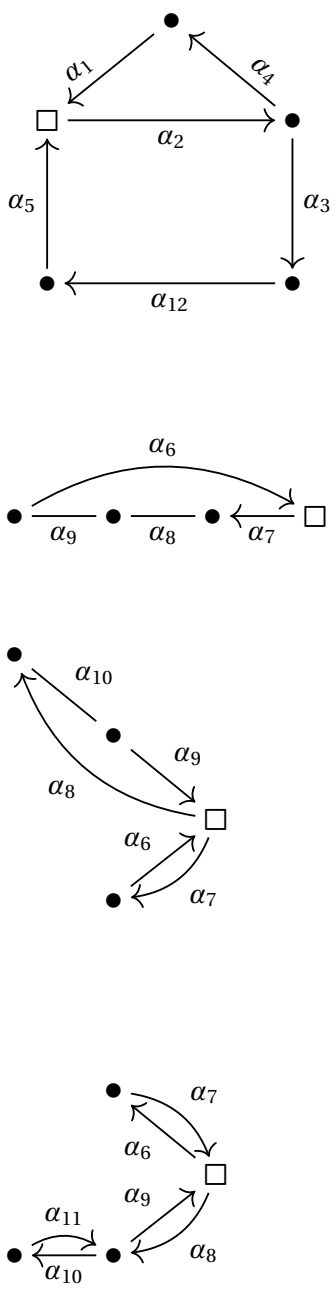

Nesse caso, temos mais essas relações do tipo 2: $r 8=\alpha_{5} \alpha_{10}, r 9=\alpha_{3} \alpha_{10}, r 10=\alpha_{11} \alpha_{2}, r 11=\alpha_{13} \alpha_{2}$, $r 12=\alpha_{5} \alpha_{12}, r 13=\alpha_{3} \alpha_{12}, r 14=\alpha_{13} \alpha_{10}$ e $r 15=$ $\alpha_{11} \alpha_{12}$

E os ciclos elementares são $C_{5}=\alpha_{10} \alpha_{11}$ e $C_{6}=$ $\alpha_{12} \alpha_{13}$. Logo não obtemos soluções.

Nesse anexo, temos mais essas relações do tipo 2: $r 8=\alpha_{5} \alpha_{10}, r 9=\alpha_{3} \alpha_{10}, r 10=\alpha_{11} \alpha_{2}, r 11=\alpha_{12} \alpha_{11}$ e $r 12=\alpha_{10} \alpha_{13}$.

E os ciclos elementares são $C_{5}=\alpha_{10} \alpha_{11}$ e $C_{6}=$ $\alpha_{12} \alpha_{13}$. Portanto, não temos corte que origine uma álgebra de incidência.

As relações do tipo 2 são: $r 1=\alpha_{1} \alpha_{2} \alpha_{3}$ e $r 2=$ $\alpha_{5} \alpha_{2} \alpha_{4}$.

Os ciclos elementares são: $C_{1}=\alpha_{5} \alpha_{2} \alpha_{3} \alpha_{12}$ e $C_{2}=$ $\alpha_{1} \alpha_{2} \alpha_{4}$.

Completando, temos mais essas relações do tipo 2: $r 3=\alpha_{5} \alpha_{7}, r 4=\alpha_{1} \alpha_{7}$ e $r 5=\alpha_{6} \alpha_{2}$.

E o ciclo elementar é $C_{3}=\alpha_{7} \alpha_{8} \alpha_{9} \alpha_{6}$. O programa mostra a solução 99 e uma hereditária.

Completando, temos mais essas relações do tipo 2: $r 3=\alpha_{5} \alpha_{7}, r 4=\alpha_{1} \alpha_{7}, r 5=\alpha_{9} \alpha_{2}, r 6=\alpha_{1} \alpha_{8}, r 7=$ $\alpha_{5} \alpha_{8}, r 8=\alpha_{6} \alpha_{2}, r 9=\alpha_{6} \alpha_{8}$ e $r 10=\alpha_{9} \alpha_{7}$.

E os ciclos elementares são $C_{3}=\alpha_{8} \alpha_{10} \alpha_{9}$ e $C_{4}=$ $\alpha_{6} \alpha_{7}$. Portanto, o programa exibe o corte que origina a álgebra 100 e uma hereditária.

Completando, temos mais essas relações do tipo 2 : $r 3=\alpha_{5} \alpha_{6}, r 4=\alpha_{1} \alpha_{6}, r 5=\alpha_{5} \alpha_{8}, r 6=\alpha_{9} \alpha_{2}, r 7=$ $\alpha_{1} \alpha_{8}, r 8=\alpha_{7} \alpha_{8}, r 9=\alpha_{7} \alpha_{2}, r 10=\alpha_{11} \alpha_{9}, r 11=$ $\alpha_{9} \alpha_{6}$ e $r 12=\alpha_{8} \alpha_{10}$.

E os ciclos elementares são $C_{3}=\alpha_{6} \alpha_{7}, C_{4}=\alpha_{8} \alpha_{9} \mathrm{e}$ $C_{5}=\alpha_{11} \alpha_{10}$. Portanto, temos a álgebra 101 e mais uma álgebra hereditária. 
(6)
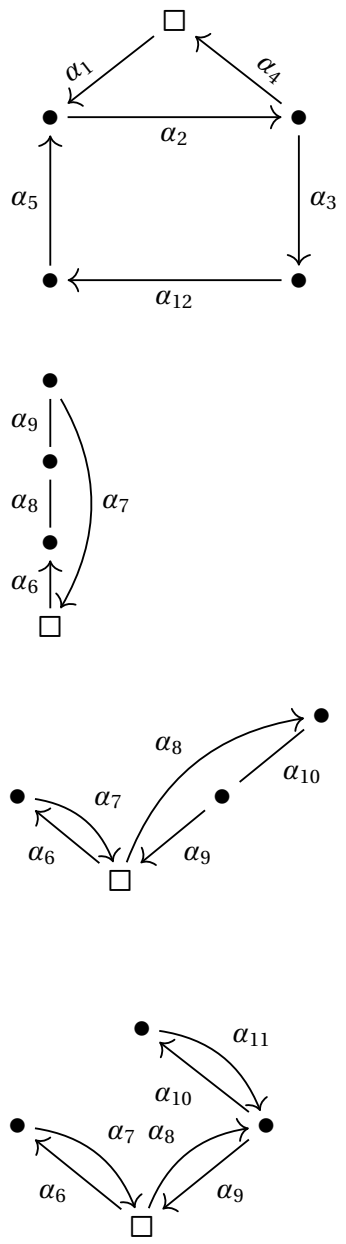

(7)
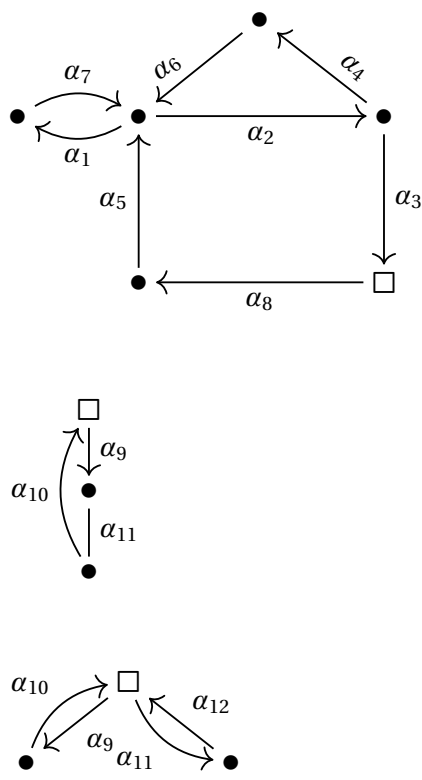

As relações do tipo 2 são: $r 1=\alpha_{1} \alpha_{2} \alpha_{3}$ e $r 2=$ $\alpha_{5} \alpha_{2} \alpha_{4}$.

Os ciclos elementares são: $C_{1}=\alpha_{5} \alpha_{2} \alpha_{3} \alpha_{12}$ e $C_{2}=$ $\alpha_{1} \alpha_{2} \alpha_{4}$.

Completando, temos mais essas relações do tipo 2 : $r 3=\alpha_{4} \alpha_{6}$ e $r 4=\alpha_{7} \alpha_{1}$.

E o ciclo elementar é $C_{4}=\alpha_{6} \alpha_{8} \alpha_{9} \alpha_{7}$. O programa exibe duas soluções hereditárias.

Nesse caso, temos mais essas relações do tipo 2: $r 3=\alpha_{4} \alpha_{6}, r 4=\alpha_{7} \alpha_{1}, r 5=\alpha_{4} \alpha_{8}, r 6=\alpha_{9} \alpha_{1}, r 7=$ $\alpha_{9} \alpha_{6}$ e $r 8=\alpha_{7} \alpha_{8}$.

E os ciclos elementares são $C_{4}=\alpha_{8} \alpha_{10} \alpha_{9}$ e $C_{5}=$ $\alpha_{7} \alpha_{6}$. Logo obtemos duas soluções hereditárias.

Nesse anexo, temos mais essas relações do tipo 2: $r 3=\alpha_{4} \alpha_{6}, r 4=\alpha_{7} \alpha_{1}, r 5=\alpha_{4} \alpha_{8}, r 6=\alpha_{9} \alpha_{1}, r 7=$ $\alpha_{9} \alpha_{6}, r 8=\alpha_{7} \alpha_{8}, r 9=\alpha_{11} \alpha_{9}$ e $r 10=\alpha_{8} \alpha_{10}$.

$\mathrm{E}$ os ciclos elementares são $C_{4}=\alpha_{8} \alpha_{9}, C_{5}=\alpha_{7} \alpha_{6}$ e $C_{6}=\alpha_{10} \alpha_{11}$. Portanto, temos mais duas álgebras hereditárias.

As relações do tipo 2 são: $r 1=\alpha_{6} \alpha_{2} \alpha_{3}, r 2=$ $\alpha_{5} \alpha_{2} \alpha_{4}, r 3=\alpha_{6} \alpha_{1}, r 4=\alpha_{5} \alpha_{1}$ e $r 5=\alpha_{7} \alpha_{2}$.

Os ciclos elementares são: $C_{1}=\alpha_{5} \alpha_{2} \alpha_{3} \alpha_{8}, C_{2}=$ $\alpha_{6} \alpha_{2} \alpha_{4}$ e $C_{3}=\alpha_{1} \alpha_{7}$.

Completando, temos mais essas relações do tipo 2: $r 6=\alpha_{3} \alpha_{9}$ e $r 7=\alpha_{10} \alpha_{8}$.

E o ciclo elementar é $C_{4}=\alpha_{9} \alpha_{11} \alpha_{10}$. O programa não exibe solução.

Nesse caso, temos mais essas relações do tipo 2: $r 6=\alpha_{3} \alpha_{9}, r 7=\alpha_{10} \alpha_{8}, r 8=\alpha_{3} \alpha_{11}, r 9=\alpha_{12} \alpha_{8}$, $r 10=\alpha_{10} \alpha_{11}$ e $r 11=\alpha_{12} \alpha_{9}$.

E os ciclos elementares são $C_{4}=\alpha_{10} \alpha_{9}$ e $C_{5}=$ $\alpha_{11} \alpha_{12}$. Nesse caso, não obtemos soluções. 


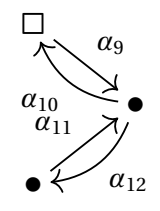

(8)
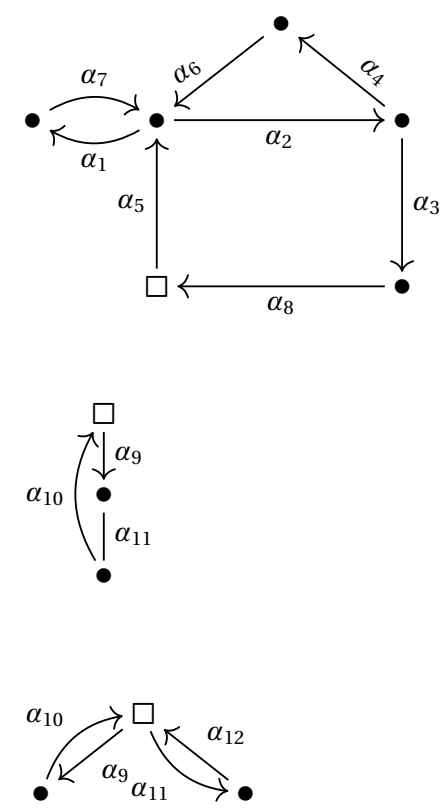

(9)
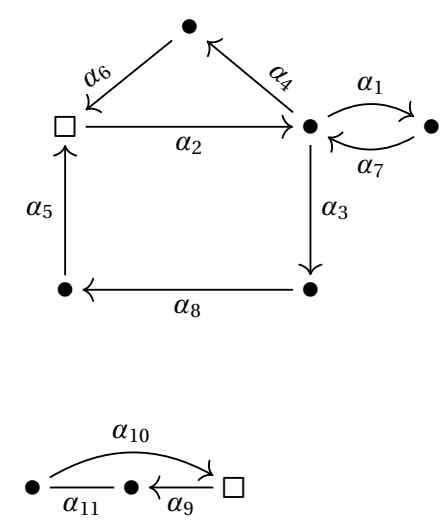

Nesse anexo, temos mais essas relações do tipo 2: $r 6=\alpha_{3} \alpha_{9}, r 7=\alpha_{10} \alpha_{8}, r 8=\alpha_{11} \alpha_{10}$ e $r 9=\alpha_{9} \alpha_{12}$. $\mathrm{E}$ os ciclos elementares são $C_{4}=\alpha_{10} \alpha_{9}$ e $C_{5}=$ $\alpha_{12} \alpha_{11}$. Portanto, não temos corte que origine uma álgebra de incidência.

As relações do tipo 2 são: $r 1=\alpha_{6} \alpha_{2} \alpha_{3}, r 2=$ $\alpha_{5} \alpha_{2} \alpha_{4}, r 3=\alpha_{6} \alpha_{1}, r 4=\alpha_{5} \alpha_{1}$ e $r 5=\alpha_{7} \alpha_{2}$.

Os ciclos elementares são: $C_{1}=\alpha_{5} \alpha_{2} \alpha_{3} \alpha_{8}, C_{2}=$ $\alpha_{6} \alpha_{2} \alpha_{4}$ e $C_{3}=\alpha_{1} \alpha_{7}$.

Completando, temos mais essas relações do tipo 2: $r 6=\alpha_{8} \alpha_{9}$ e $r 7=\alpha_{10} \alpha_{5}$.

E o ciclo elementar é $C_{4}=\alpha_{9} \alpha_{11} \alpha_{10}$. O programa exibe apenas uma solução hereditária.

Nesse caso, temos mais essas relações do tipo 2: $r 6=\alpha_{8} \alpha_{9}, r 7=\alpha_{10} \alpha_{5}, r 8=\alpha_{8} \alpha_{11}, r 9=\alpha_{12} \alpha_{5}$, $r 10=\alpha_{10} \alpha_{11}$ e $r 11=\alpha_{12} \alpha_{9}$.

$\mathrm{E}$ os ciclos elementares são $C_{4}=\alpha_{10} \alpha_{9}$ e $C_{5}=$ $\alpha_{11} \alpha_{12}$. Nesse caso, obtemos uma solução hereditária.

Nesse anexo, temos mais essas relações do tipo 2: $r 6=\alpha_{8} \alpha_{9}, r 7=\alpha_{10} \alpha_{5}, r 8=\alpha_{11} \alpha_{10}$ e $r 9=\alpha_{9} \alpha_{12}$.

$\mathrm{E}$ os ciclos elementares são $C_{4}=\alpha_{10} \alpha_{9}$ e $C_{5}=$ $\alpha_{12} \alpha_{11}$. Logo, temos um corte que origine uma álgebra de incidência hereditária.

As relações do tipo 2 são: $r 1=\alpha_{6} \alpha_{2} \alpha_{3}, r 2=$ $\alpha_{5} \alpha_{2} \alpha_{4}, r 3=\alpha_{7} \alpha_{4}, r 4=\alpha_{7} \alpha_{3}$ e $r 5=\alpha_{2} \alpha_{1}$.

Os ciclos elementares são: $C_{1}=\alpha_{5} \alpha_{2} \alpha_{3} \alpha_{8}, C_{2}=$ $\alpha_{6} \alpha_{2} \alpha_{4}$ e $C_{3}=\alpha_{1} \alpha_{7}$.

Completando, temos mais essas relações do tipo 2: $r 6=\alpha_{6} \alpha_{9}, r 7=\alpha_{10} \alpha_{2}$ e $r 8=\alpha_{5} \alpha_{9}$.

E o ciclo elementar é $C_{4}=\alpha_{9} \alpha_{11} \alpha_{10}$. O programa exibe a solução 102 . 

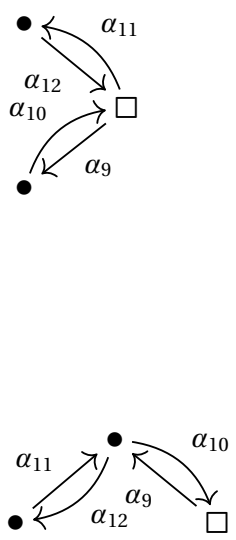

(10)

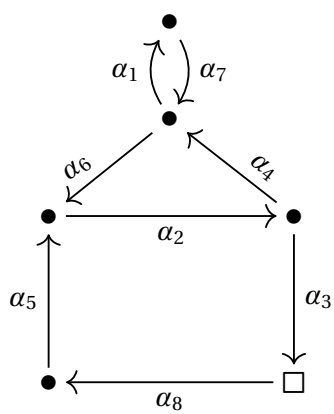

$\alpha_{10}\left(\begin{array}{l}\square \\ \searrow \\ \vdots \\ \vdots\end{array} \alpha_{11}\right.$
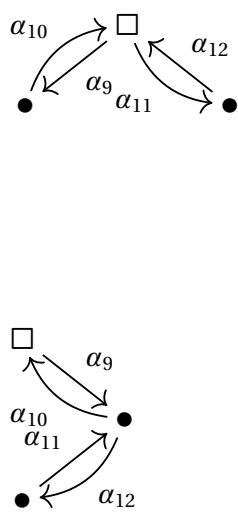

Nesse caso, temos mais essas relações do tipo 2: $r 6=\alpha_{6} \alpha_{9}, r 7=\alpha_{10} \alpha_{2}, r 8=\alpha_{5} \alpha_{9}, r 9=\alpha_{6} \alpha_{11}$, $r 10=\alpha_{12} \alpha_{2}, r 11=\alpha_{5} \alpha_{11}, r 12=\alpha_{10} \alpha_{11}$ e $r 13=$ $\alpha_{12} \alpha_{9}$.

$\mathrm{E}$ os ciclos elementares são $C_{4}=\alpha_{10} \alpha_{9}$ e $C_{5}=$ $\alpha_{11} \alpha_{12}$. Nesse caso, obtemos a solução 103.

Nesse anexo, temos mais essas relações do tipo 2: $r 6=\alpha_{6} \alpha_{9}, r 7=\alpha_{10} \alpha_{2}, r 8=\alpha_{5} \alpha_{9}, r 9=\alpha_{11} \alpha_{10} \mathrm{e}$ $r 10=\alpha_{9} \alpha_{12}$.

E os ciclos elementares são $C_{4}=\alpha_{10} \alpha_{9}$ e $C_{5}=$ $\alpha_{12} \alpha_{11}$. Logo, temos um corte que origine a álgebra 104.

As relações do tipo 2 são: $r 1=\alpha_{6} \alpha_{2} \alpha_{3}, r 2=$ $\alpha_{5} \alpha_{2} \alpha_{4}, r 3=\alpha_{7} \alpha_{6}$ e $r 4=\alpha_{4} \alpha_{1}$.

Os ciclos elementares são: $C_{1}=\alpha_{5} \alpha_{2} \alpha_{3} \alpha_{8}, C_{2}=$ $\alpha_{6} \alpha_{2} \alpha_{4}$ e $C_{3}=\alpha_{1} \alpha_{7}$

Completando, temos mais essas relações do tipo 2: $r 5=\alpha_{3} \alpha_{9}$ e $r 6=\alpha_{10} \alpha_{8}$.

E o ciclo elementar é $C_{4}=\alpha_{9} \alpha_{11} \alpha_{10}$. O programa exibe apenas uma solução hereditária.

Nesse caso, temos mais essas relações do tipo 2: $r 5=\alpha_{3} \alpha_{9}, r 6=\alpha_{10} \alpha_{8}, r 7=\alpha_{3} \alpha_{11}, r 8=\alpha_{12} \alpha_{8}$, $r 9=\alpha_{10} \alpha_{11}$ e $r 10=\alpha_{12} \alpha_{9}$.

$\mathrm{E}$ os ciclos elementares são $C_{4}=\alpha_{10} \alpha_{9}$ e $C_{5}=$ $\alpha_{11} \alpha_{12}$. Nesse caso, obtemos uma solução hereditária.

Nesse anexo, temos mais essas relações do tipo 2: $r 5=\alpha_{3} \alpha_{9}, r 6=\alpha_{10} \alpha_{8}, r 7=\alpha_{11} \alpha_{10}$ e $r 8=\alpha_{9} \alpha_{12}$. $\mathrm{E}$ os ciclos elementares são $C_{4}=\alpha_{10} \alpha_{9}$ e $C_{5}=$ $\alpha_{12} \alpha_{11}$. Logo, temos um corte que origine uma álgebra de incidência hereditária. 
(11)

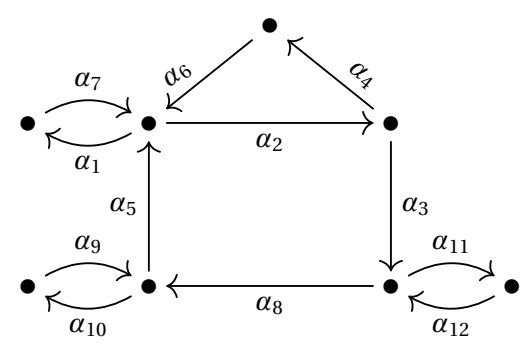

(12)

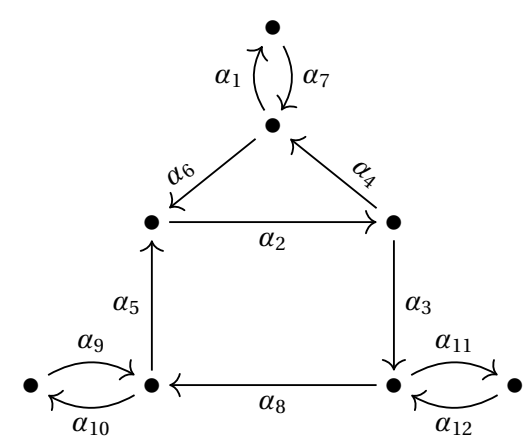

(13)

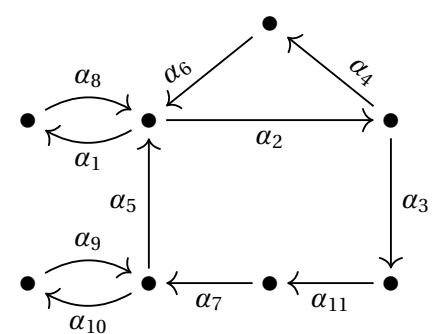

(14)

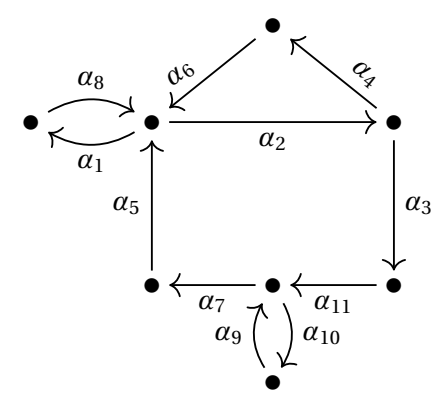

(15)

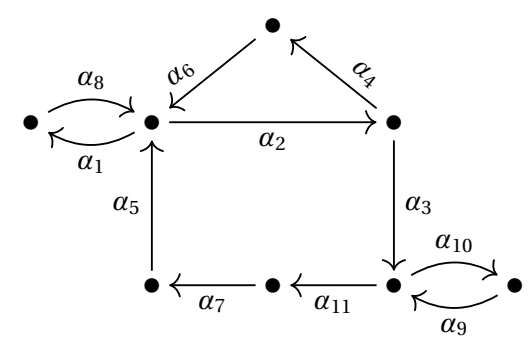

As relações do tipo 2 são: $r 1=\alpha_{6} \alpha_{2} \alpha_{3}, r 2=$ $\alpha_{5} \alpha_{2} \alpha_{4}, r 3=\alpha_{7} \alpha_{2}, r 4=\alpha_{6} \alpha_{1}, r 5=\alpha_{5} \alpha_{1}, r 6=$ $\alpha_{9} \alpha_{5}, r 7=\alpha_{8} \alpha_{10}, r 8=\alpha_{3} \alpha_{11}$ e $r 9=\alpha_{12} \alpha_{8}$.

Os ciclos elementares são: $C_{1}=\alpha_{5} \alpha_{2} \alpha_{3} \alpha_{8}, C_{2}=$ $\alpha_{6} \alpha_{2} \alpha_{4}, C_{3}=\alpha_{1} \alpha_{7}, C_{4}=\alpha_{9} \alpha_{10}$ e $C_{5}=\alpha_{11} \alpha_{12}$. O programa não exibe solução.

As relações do tipo 2 são: $r 1=\alpha_{6} \alpha_{2} \alpha_{3}, r 2=$ $\alpha_{5} \alpha_{2} \alpha_{4}, r 3=\alpha_{4} \alpha_{1}, r 4=\alpha_{7} \alpha_{6}, r 5=\alpha_{9} \alpha_{5}, r 6=$ $\alpha_{8} \alpha_{10}, r 7=\alpha_{3} \alpha_{11}$ e $r 8=\alpha_{12} \alpha_{8}$.

Os ciclos elementares são: $C_{1}=\alpha_{5} \alpha_{2} \alpha_{3} \alpha_{8}, C_{2}=$ $\alpha_{6} \alpha_{2} \alpha_{4}, C_{3}=\alpha_{1} \alpha_{7}, C_{4}=\alpha_{9} \alpha_{10}$ e $C_{5}=\alpha_{11} \alpha_{12}$. Logo, não temos solução.

As relações do tipo 2 são: $r 1=\alpha_{6} \alpha_{2} \alpha_{3}, r 2=$ $\alpha_{5} \alpha_{2} \alpha_{4}, r 3=\alpha_{8} \alpha_{2}, r 4=\alpha_{6} \alpha_{1}, r 5=\alpha_{5} \alpha_{1}, r 6=$ $\alpha_{9} \alpha_{5}$ e $r 7=\alpha_{7} \alpha_{10}$.

Os ciclos elementares são: $C_{1}=\alpha_{5} \alpha_{2} \alpha_{3} \alpha_{11} \alpha_{7}, C_{2}=$ $\alpha_{6} \alpha_{2} \alpha_{4}, C_{3}=\alpha_{1} \alpha_{8}$ e $C_{4}=\alpha_{9} \alpha_{10}$. Portanto, o programa mostra apenas uma solução hereditária.

As relações do tipo 2 são: $r 1=\alpha_{6} \alpha_{2} \alpha_{3}, r 2=$ $\alpha_{5} \alpha_{2} \alpha_{4}, r 3=\alpha_{8} \alpha_{2}, r 4=\alpha_{6} \alpha_{1}, r 5=\alpha_{5} \alpha_{1}, r 6=$ $\alpha_{9} \alpha_{7}$ e $r 7=\alpha_{11} \alpha_{10}$.

Os ciclos elementares são: $C_{1}=\alpha_{5} \alpha_{2} \alpha_{3} \alpha_{11} \alpha_{7}, C_{2}=$ $\alpha_{6} \alpha_{2} \alpha_{4}, C_{3}=\alpha_{1} \alpha_{8}$ e $C_{4}=\alpha_{9} \alpha_{10}$. Nesse caso, o programa não exibe solução.

As relações do tipo 2 são: $r 1=\alpha_{6} \alpha_{2} \alpha_{3}, r 2=$ $\alpha_{5} \alpha_{2} \alpha_{4}, r 3=\alpha_{8} \alpha_{2}, r 4=\alpha_{6} \alpha_{1}, r 5=\alpha_{5} \alpha_{1}, r 6=$ $\alpha_{9} \alpha_{11}$ e $r 7=\alpha_{3} \alpha_{10}$.

Os ciclos elementares são: $C_{1}=\alpha_{5} \alpha_{2} \alpha_{3} \alpha_{11} \alpha_{7}, C_{2}=$ $\alpha_{6} \alpha_{2} \alpha_{4}, C_{3}=\alpha_{1} \alpha_{8}$ e $C_{4}=\alpha_{9} \alpha_{10}$. Logo não temos soluções. 
(16)

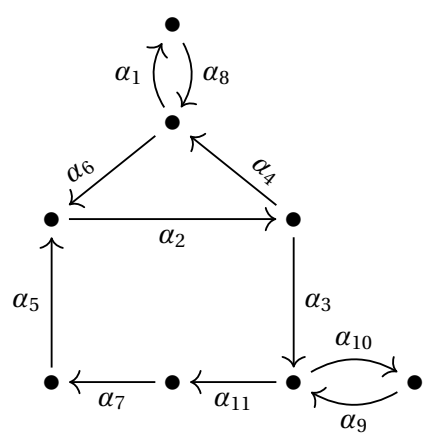

(17)

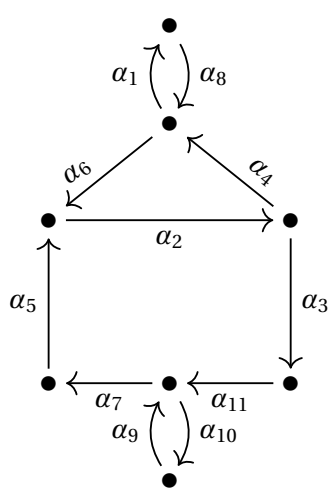

(18)

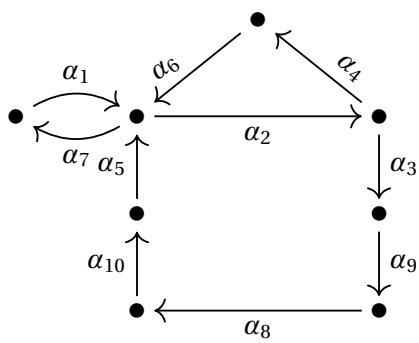

(19)

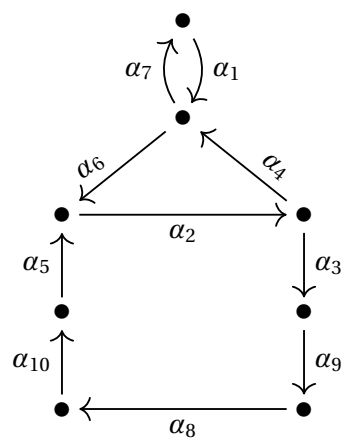

(20)

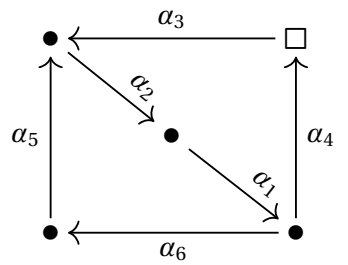

As relações do tipo 2 são: $r 1=\alpha_{6} \alpha_{2} \alpha_{3}, r 2=$ $\alpha_{5} \alpha_{2} \alpha_{4}, r 3=\alpha_{8} \alpha_{6}, r 4=\alpha_{4} \alpha_{1}, r 5=\alpha_{9} \alpha_{11} \mathrm{e}$ $r 6=\alpha_{3} \alpha_{10}$.

Os ciclos elementares são: $C_{1}=\alpha_{5} \alpha_{2} \alpha_{3} \alpha_{11} \alpha_{7}, C_{2}=$ $\alpha_{6} \alpha_{2} \alpha_{4}, C_{3}=\alpha_{1} \alpha_{8}$ e $C_{4}=\alpha_{9} \alpha_{10}$. O programa exibe apenas uma solução hereditária.

As relações do tipo 2 são: $r 1=\alpha_{6} \alpha_{2} \alpha_{3}, r 2=$ $\alpha_{5} \alpha_{2} \alpha_{4}, r 3=\alpha_{8} \alpha_{6}, r 4=\alpha_{4} \alpha_{1}, r 5=\alpha_{9} \alpha_{7} \mathrm{e}$ $r 6=\alpha_{11} \alpha_{10}$.

Os ciclos elementares são: $C_{1}=\alpha_{5} \alpha_{2} \alpha_{3} \alpha_{11} \alpha_{7}, C_{2}=$ $\alpha_{6} \alpha_{2} \alpha_{4}, C_{3}=\alpha_{1} \alpha_{8}$ e $C_{4}=\alpha_{9} \alpha_{10}$. Portanto, o programa não exibe solução.

As relações do tipo 2 são: $r 1=\alpha_{6} \alpha_{2} \alpha_{3}, r 2=$ $\alpha_{5} \alpha_{2} \alpha_{4}, r 3=\alpha_{1} \alpha_{2}, r 4=\alpha_{6} \alpha_{7}$ e $r 5=\alpha_{5} \alpha_{7}$.

Os ciclos elementares são: $C_{1}=\alpha_{5} \alpha_{2} \alpha_{3} \alpha_{9} \alpha_{8} \alpha_{10}$, $C_{2}=\alpha_{6} \alpha_{2} \alpha_{4}$ e $C_{3}=\alpha_{1} \alpha_{7}$. Logo, obtemos a álgebra 105 e uma hereditária.

As relações do tipo 2 são: $r 1=\alpha_{6} \alpha_{2} \alpha_{3}, r 2=$ $\alpha_{5} \alpha_{2} \alpha_{4}, r 3=\alpha_{1} \alpha_{6}$ e $r 4=\alpha_{4} \alpha_{7}$.

Os ciclos elementares são: $C_{1}=\alpha_{5} \alpha_{2} \alpha_{3} \alpha_{9} \alpha_{8} \alpha_{10}$, $C_{2}=\alpha_{6} \alpha_{2} \alpha_{4}$ e $C_{3}=\alpha_{1} \alpha_{7}$. Portanto, o programa exibe apenas soluções hereditárias.

As relações do tipo 2 são: $r 1=\alpha_{5} \alpha_{2} \alpha_{1} \alpha_{4}$ e $r 2=$ $\alpha_{3} \alpha_{2} \alpha_{1} \alpha_{6}$.

Os ciclos elementares são: $C_{1}=\alpha_{3} \alpha_{2} \alpha_{1} \alpha_{4}$ e $C_{2}=$ $\alpha_{5} \alpha_{2} \alpha_{1} \alpha_{6}$. 

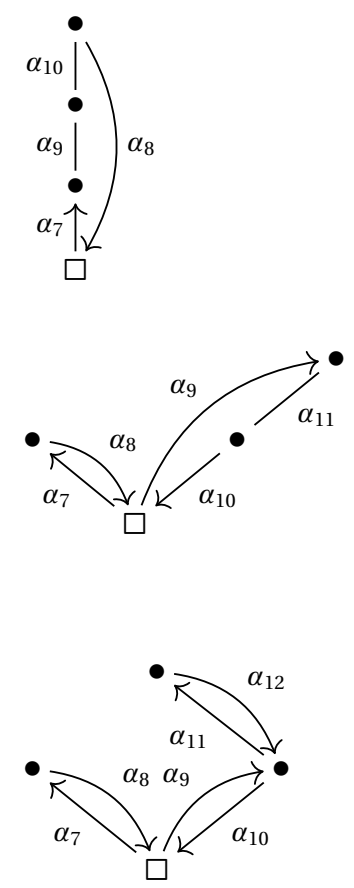

(21)
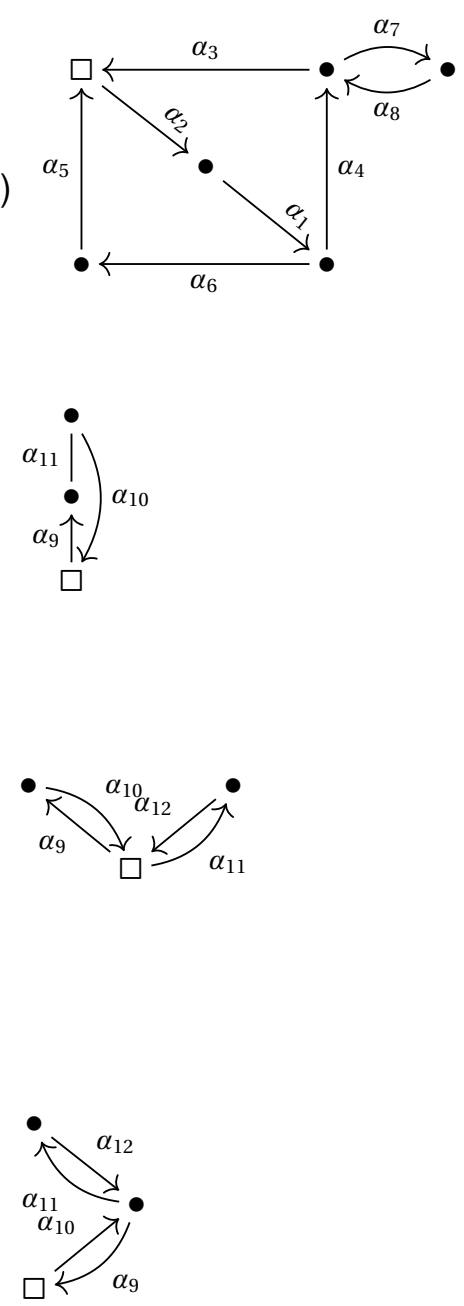

Completando, temos mais essas relações do tipo 2: $r 3=\alpha_{4} \alpha_{7}$ e $r 4=\alpha_{8} \alpha_{3}$.

E o ciclo elementar é $C_{3}=\alpha_{7} \alpha_{9} \alpha_{10} \alpha_{8}$. O programa mostra duas soluções hereditárias.

Nesse caso, temos mais essas relações do tipo 2: $r 3=\alpha_{4} \alpha_{7}, r 4=\alpha_{8} \alpha_{3}, r 5=\alpha_{4} \alpha_{9}, r 6=\alpha_{10} \alpha_{3}$, $r 7=\alpha_{10} \alpha_{7}$ e $r 8=\alpha_{8} \alpha_{9}$.

$\mathrm{E}$ os ciclos elementares são $C_{3}=\alpha_{9} \alpha_{11} \alpha_{10}$ e $C_{4}=$ $\alpha_{8} \alpha_{7}$. Logo obtemos duas soluções hereditárias.

Nesse anexo, temos mais essas relações do tipo 2: $r 3=\alpha_{4} \alpha_{7}, r 4=\alpha_{8} \alpha_{3}, r 5=\alpha_{4} \alpha_{9}, r 6=\alpha_{10} \alpha_{3}$, $r 7=\alpha_{10} \alpha_{7}, r 8=\alpha_{8} \alpha_{9}, r 9=\alpha_{12} \alpha_{10}$ e $r 10=\alpha_{9} \alpha_{11}$. E os ciclos elementares são $C_{3}=\alpha_{9} \alpha_{10}, C_{4}=\alpha_{8} \alpha_{7}$ e $C_{5}=\alpha_{11} \alpha_{12}$. Portanto, temos mais duas álgebras hereditárias.

As relações do tipo 2 são: $r 1=\alpha_{5} \alpha_{2} \alpha_{1} \alpha_{4}, r 2=$ $\alpha_{3} \alpha_{2} \alpha_{1} \alpha_{6}, r 3=\alpha_{4} \alpha_{7}$ e $r 4=\alpha_{8} \alpha_{3}$.

Os ciclos elementares são: $C_{1}=\alpha_{3} \alpha_{2} \alpha_{1} \alpha_{4}, C_{2}=$ $\alpha_{5} \alpha_{2} \alpha_{1} \alpha_{6}$ e $C_{3}=\alpha_{8} \alpha_{7}$.

Completando, temos mais essas relações do tipo 2: $r 5=\alpha_{3} \alpha_{9}, r 6=\alpha_{5} \alpha_{9}$ e $r 7=\alpha_{10} \alpha_{2}$.

E o ciclo elementar é $C_{4}=\alpha_{9} \alpha_{11} \alpha_{10}$. O programa exibe um corte que origina uma álgebra de incidência hereditária.

Nesse caso, temos mais essas relações do tipo $2: r 5=\alpha_{3} \alpha_{9}, r 6=\alpha_{5} \alpha_{9}, r 7=\alpha_{10} \alpha_{2}, r 8=\alpha_{3} \alpha_{11}$, $r 9=\alpha_{5} \alpha_{11}, r 10=\alpha_{12} \alpha_{2}, r 11=\alpha_{12} \alpha_{9}$ e $r 12=$ $\alpha_{10} \alpha_{11}$.

$\mathrm{E}$ os ciclos elementares são $C_{4}=\alpha_{9} \alpha_{10}$ e $C_{5}=$ $\alpha_{11} \alpha_{12}$. Logo obtemos uma solução hereditária.

Nesse anexo, temos mais essas relações do tipo 2: $r 5=\alpha_{3} \alpha_{9}, r 6=\alpha_{5} \alpha_{9}, r 7=\alpha_{10} \alpha_{2}, r 8=\alpha_{10} \alpha_{11} \mathrm{e}$ $r 9=\alpha_{12} \alpha_{9}$.

$\mathrm{E}$ os ciclos elementares são $C_{4}=\alpha_{10} \alpha_{9}$ e $C_{5}=$ $\alpha_{12} \alpha_{11}$. Portanto, temos um corte que origine uma álgebra de incidência hereditária. 
(22)
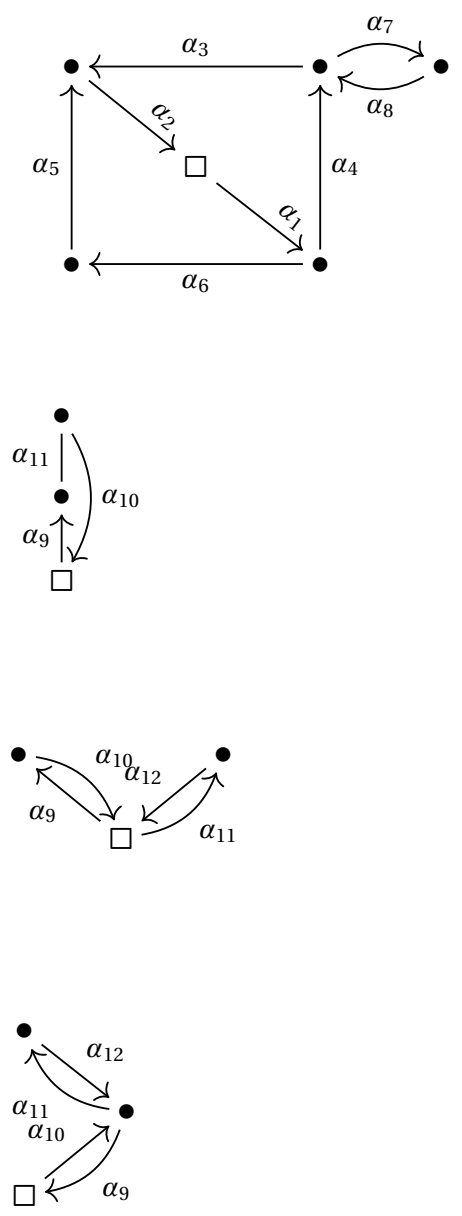

(23)

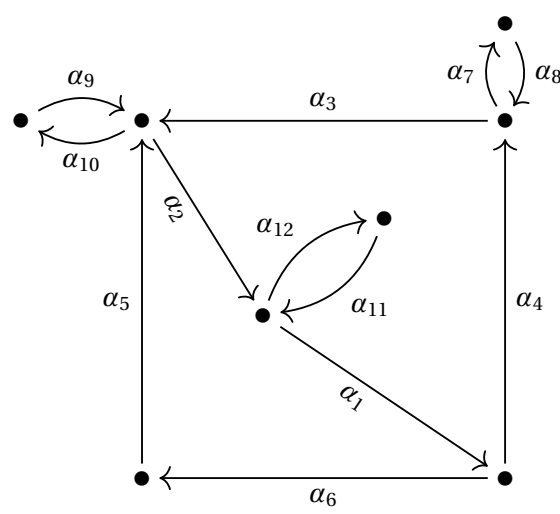

(24)

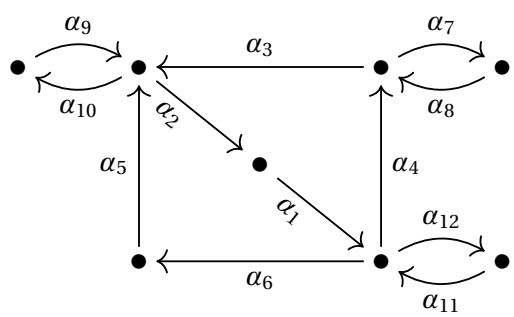

As relações do tipo 2 são: $r 1=\alpha_{5} \alpha_{2} \alpha_{1} \alpha_{4}, r 2=$ $\alpha_{3} \alpha_{2} \alpha_{1} \alpha_{6}, r 3=\alpha_{4} \alpha_{7}$ e $r 4=\alpha_{8} \alpha_{3}$.

Os ciclos elementares são: $C_{1}=\alpha_{3} \alpha_{2} \alpha_{1} \alpha_{4}, C_{2}=$ $\alpha_{5} \alpha_{2} \alpha_{1} \alpha_{6}$ e $C_{3}=\alpha_{8} \alpha_{7}$.

Completando, temos mais essas relações do tipo 2: $r 5=\alpha_{2} \alpha_{9}$ e $r 6=\alpha_{10} \alpha_{1}$.

E o ciclo elementar é $C_{4}=\alpha_{9} \alpha_{11} \alpha_{10}$. O programa não exibe corte que origina uma álgebra de incidência.

Nesse caso, temos mais essas relações do tipo 2: $r 5=\alpha_{2} \alpha_{9}, r 6=\alpha_{10} \alpha_{1}, r 7=\alpha_{2} \alpha_{11}, r 8=\alpha_{12} \alpha_{1}$, $r 9=\alpha_{12} \alpha_{9}$ e $r 10=\alpha_{10} \alpha_{11}$.

$\mathrm{E}$ os ciclos elementares são $C_{4}=\alpha_{9} \alpha_{10}$ e $C_{5}=$ $\alpha_{11} \alpha_{12}$. Logo não obtemos solução.

Nesse anexo, temos mais essas relações do tipo 2: $r 5=\alpha_{2} \alpha_{9}, r 6=\alpha_{10} \alpha_{1}, r 7=\alpha_{10} \alpha_{11}$ e $r 8=\alpha_{12} \alpha_{9}$.

$\mathrm{E}$ os ciclos elementares são $C_{4}=\alpha_{10} \alpha_{9}$ e $C_{5}=$ $\alpha_{12} \alpha_{11}$. Portanto, não temos corte que origine uma álgebra de incidência.

As relações do tipo 2 são: $r 1=\alpha_{5} \alpha_{2} \alpha_{1} \alpha_{4}, r 2=$ $\alpha_{3} \alpha_{2} \alpha_{1} \alpha_{6}, r 3=\alpha_{4} \alpha_{7}, r 4=\alpha_{8} \alpha_{3}, r 5=\alpha_{2} \alpha_{12}, r 6=$ $\alpha_{11} \alpha_{1}, r 7=\alpha_{3} \alpha_{10}, r 8=\alpha_{5} \alpha_{10}$ e $r 9=\alpha_{9} \alpha_{2}$.

Os ciclos elementares são: $C_{1}=\alpha_{3} \alpha_{2} \alpha_{1} \alpha_{4}, C_{2}=$ $\alpha_{5} \alpha_{2} \alpha_{1} \alpha_{6}, C_{3}=\alpha_{8} \alpha_{7}, C_{4}=\alpha_{9} \alpha_{10}$ e $C_{5}=\alpha_{11} \alpha_{12}$. O programa não exibe resposta.

As relações do tipo 2 são: $r 1=\alpha_{5} \alpha_{2} \alpha_{1} \alpha_{4}, r 2=$ $\alpha_{3} \alpha_{2} \alpha_{1} \alpha_{6}, r 3=\alpha_{4} \alpha_{7}, r 4=\alpha_{8} \alpha_{3}, r 5=\alpha_{9} \alpha_{2}, r 6=$ $\alpha_{5} \alpha_{10}, r 7=\alpha_{3} \alpha_{10}, r 8=\alpha_{11} \alpha_{6}, r 9=\alpha_{11} \alpha_{4}$ e $r 10=$ $\alpha_{1} \alpha_{12}$.

Os ciclos elementares são: $C_{1}=\alpha_{3} \alpha_{2} \alpha_{1} \alpha_{4}, C_{2}=$ $\alpha_{5} \alpha_{2} \alpha_{1} \alpha_{6}, C_{3}=\alpha_{8} \alpha_{7}, C_{4}=\alpha_{9} \alpha_{10}$ e $C_{5}=\alpha_{11} \alpha_{12}$. Logo, não temos nenhuma álgebra. 
(25)

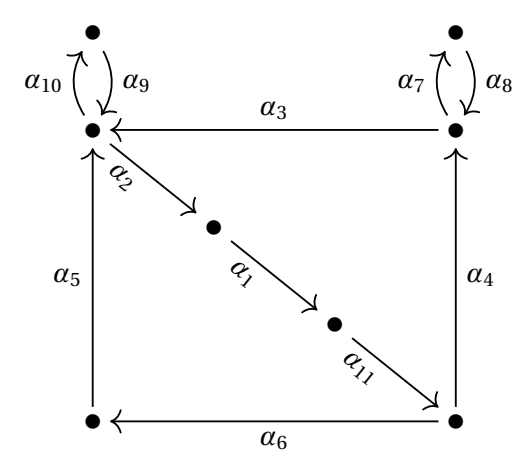

(26)

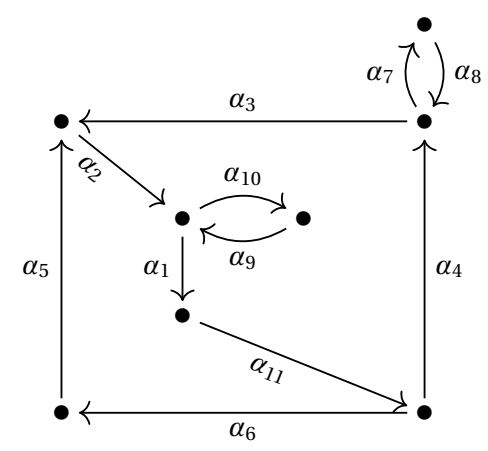

(27)

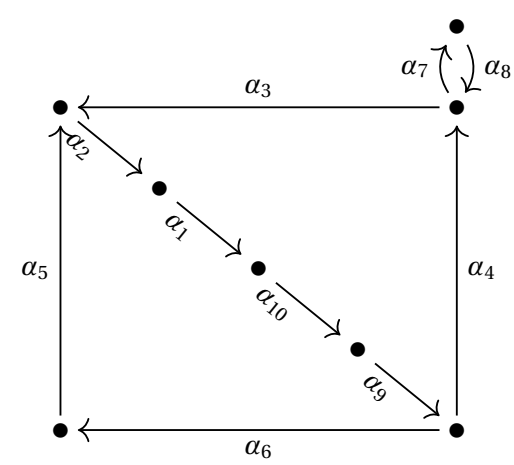

(28)
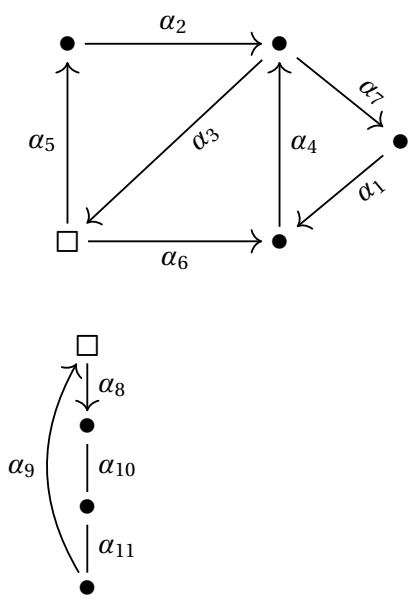

As relações do tipo 2 são: $r 1=\alpha_{5} \alpha_{2} \alpha_{1} \alpha_{11} \alpha_{4}, r 2=$ $\alpha_{3} \alpha_{2} \alpha_{1} \alpha_{11} \alpha_{6}, r 3=\alpha_{4} \alpha_{7}, r 4=\alpha_{8} \alpha_{3}, r 5=\alpha_{9} \alpha_{2}$, $r 6=\alpha_{5} \alpha_{10}$ e $r 7=\alpha_{3} \alpha_{10}$.

Os ciclos elementares são: $C_{1}=\alpha_{3} \alpha_{2} \alpha_{1} \alpha_{11} \alpha_{4}, C_{2}=$ $\alpha_{5} \alpha_{2} \alpha_{1} \alpha_{11} \alpha_{6}, C_{3}=\alpha_{8} \alpha_{7}$ e $C_{4}=\alpha_{9} \alpha_{10}$. Portanto, obtemos apenas um corte que origina uma álgebra de incidência hereditária.

As relações do tipo 2 são: $r 1=\alpha_{5} \alpha_{2} \alpha_{1} \alpha_{11} \alpha_{4}, r 2=$ $\alpha_{3} \alpha_{2} \alpha_{1} \alpha_{11} \alpha_{6}, r 3=\alpha_{4} \alpha_{7}, r 4=\alpha_{8} \alpha_{3}, r 5=\alpha_{9} \alpha_{1} \mathrm{e}$ $r 6=\alpha_{2} \alpha_{10}$.

Os ciclos elementares são: $C_{1}=\alpha_{3} \alpha_{2} \alpha_{1} \alpha_{11} \alpha_{4}, C_{2}=$ $\alpha_{5} \alpha_{2} \alpha_{1} \alpha_{11} \alpha_{6}, C_{3}=\alpha_{8} \alpha_{7}$ e $C_{4}=\alpha_{9} \alpha_{10}$. Portanto, não temos solução para esse caso.

As relações do tipo 2 são: $r 1=\alpha_{5} \alpha_{2} \alpha_{1} \alpha_{10} \alpha_{9} \alpha_{4}$, $r 2=\alpha_{3} \alpha_{2} \alpha_{1} \alpha_{10} \alpha_{9} \alpha_{6}, r 3=\alpha_{4} \alpha_{7}$ e $r 4=\alpha_{8} \alpha_{3}$.

Os ciclos elementares são: $C_{1}=\alpha_{3} \alpha_{2} \alpha_{1} \alpha_{10} \alpha_{9} \alpha_{4}$, $C_{2}=\alpha_{5} \alpha_{2} \alpha_{1} \alpha_{10} \alpha_{9} \alpha_{6}$ e $C_{3}=\alpha_{8} \alpha_{7}$. O programa exibe apenas os cortes que resultam nas hereditárias.

As relações do tipo 2 são: $r 1=\alpha_{2} \alpha_{3} \alpha_{6}, r 2=$ $\alpha_{4} \alpha_{3} \alpha_{5}, r 3=\alpha_{6} \alpha_{4} \alpha_{7}, r 4=\alpha_{1} \alpha_{4} \alpha_{3}$ e $r 5=\alpha_{2} \alpha_{7}$.

Os ciclos elementares são: $C_{1}=\alpha_{2} \alpha_{3} \alpha_{5}, C_{2}=$ $\alpha_{3} \alpha_{6} \alpha_{4}$ e $C_{3}=\alpha_{4} \alpha_{7} \alpha_{1}$.

Completando, temos mais essas relações do tipo 2: $r 6=\alpha_{3} \alpha_{8}, r 7=\alpha_{9} \alpha_{5}$ e $r 8=\alpha_{9} \alpha_{6}$.

E o ciclo elementar é $C_{4}=\alpha_{8} \alpha_{10} \alpha_{11} \alpha_{9}$. O programa exibe a solução 106. 

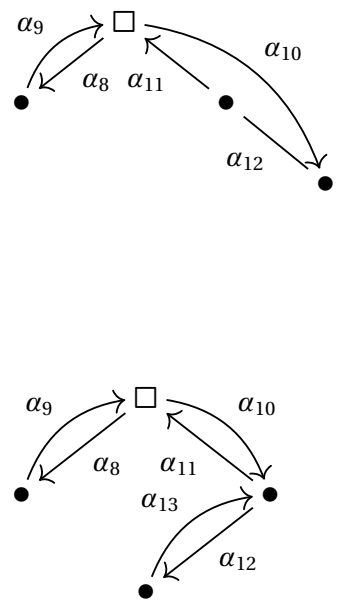

(29)
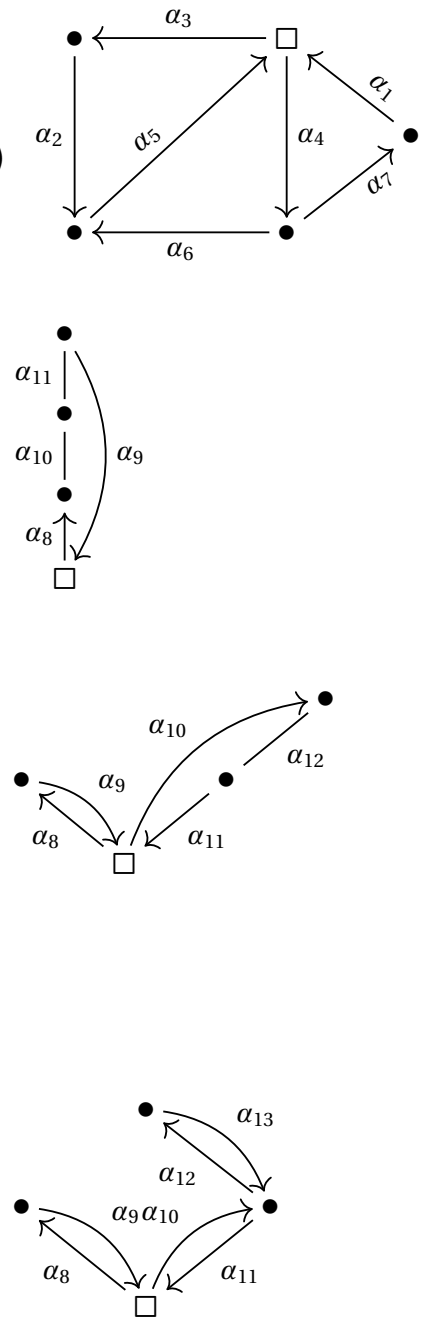

Nesse caso, temos mais essas relações do tipo 2: $r 6=\alpha_{3} \alpha_{8}, r 7=\alpha_{9} \alpha_{5}, r 8=\alpha_{9} \alpha_{6}, r 9=\alpha_{3} \alpha_{10}$, $r 10=\alpha_{11} \alpha_{5}, r 11=\alpha_{11} \alpha_{6}, r 12=\alpha_{11} \alpha_{8}$ e $r 13=$ $\alpha_{9} \alpha_{10}$.

E os ciclos elementares são $C_{4}=\alpha_{10} \alpha_{12} \alpha_{11}$ e $C_{5}=$ $\alpha_{9} \alpha_{8}$. Logo obtemos a álgebra 107.

Nesse anexo, temos mais essas relações do tipo 2: $r 6=\alpha_{3} \alpha_{8}, r 7=\alpha_{9} \alpha_{5}, r 8=\alpha_{9} \alpha_{6}, r 9=\alpha_{3} \alpha_{10}$, $r 10=\alpha_{11} \alpha_{5}, r 11=\alpha_{11} \alpha_{6}, r 12=\alpha_{11} \alpha_{8}, r 13=$ $\alpha_{9} \alpha_{10}, r 14=\alpha_{10} \alpha_{12}$ e $r 15=\alpha_{13} \alpha_{11}$.

E os ciclos elementares são $C_{4}=\alpha_{10} \alpha_{11}, C_{5}=\alpha_{9} \alpha_{8}$ e $C_{6}=\alpha_{12} \alpha_{13}$. Portanto, temos a álgebra de incidência 108.

As relações do tipo 2 são: $r 1=\alpha_{2} \alpha_{5} \alpha_{4}, r 2=$ $\alpha_{6} \alpha_{5} \alpha_{3}, r 3=\alpha_{5} \alpha_{4} \alpha_{7}, r 4=\alpha_{1} \alpha_{4} \alpha_{6}$ e $r 5=\alpha_{1} \alpha_{3}$.

Os ciclos elementares são: $C_{1}=\alpha_{2} \alpha_{5} \alpha_{3}, C_{2}=$ $\alpha_{6} \alpha_{5} \alpha_{4}$ e $C_{3}=\alpha_{4} \alpha_{7} \alpha_{1}$.

Completando, temos mais essas relações do tipo 2: $r 6=\alpha_{5} \alpha_{8}, r 7=\alpha_{1} \alpha_{8}, r 8=\alpha_{9} \alpha_{3}$ e $r 9=\alpha_{9} \alpha_{4}$.

E o ciclo elementar é $C_{4}=\alpha_{8} \alpha_{10} \alpha_{11} \alpha_{9}$. Portanto, temos as álgebras 109 e 110.

Nesse caso, temos mais essas relações do tipo 2: $r 6=\alpha_{5} \alpha_{8}, r 7=\alpha_{1} \alpha_{8}, r 8=\alpha_{9} \alpha_{3}, r 9=\alpha_{9} \alpha_{4}, r 10=$ $\alpha_{5} \alpha_{10}, r 11=\alpha_{1} \alpha_{10}, r 12=\alpha_{11} \alpha_{3}, r 13=\alpha_{11} \alpha_{4}$, $r 14=\alpha_{11} \alpha_{8}$ e $r 15=\alpha_{9} \alpha_{10}$.

E os ciclos elementares são $C_{4}=\alpha_{10} \alpha_{12} \alpha_{11}$ e $C_{5}=$ $\alpha_{9} \alpha_{8}$. Logo obtemos as soluções 111 e 112 .

Nesse anexo, temos mais essas relações do tipo 2: $r 6=\alpha_{5} \alpha_{8}, r 7=\alpha_{1} \alpha_{8}, r 8=\alpha_{9} \alpha_{3}, r 9=\alpha_{9} \alpha_{4}, r 10=$ $\alpha_{5} \alpha_{10}, r 11=\alpha_{1} \alpha_{10}, r 12=\alpha_{11} \alpha_{3}, r 13=\alpha_{11} \alpha_{4}$, $r 14=\alpha_{11} \alpha_{8}, r 15=\alpha_{9} \alpha_{10}, r 16=\alpha_{13} \alpha_{11}$ e $r 17=$ $\alpha_{10} \alpha_{12}$.

E os ciclos elementares são $C_{4}=\alpha_{10} \alpha_{11}, C_{5}=\alpha_{9} \alpha_{8}$ e $C_{6}=\alpha_{12} \alpha_{13}$. Portanto, o programa mostra as soluções 113 e 114. 
(30)
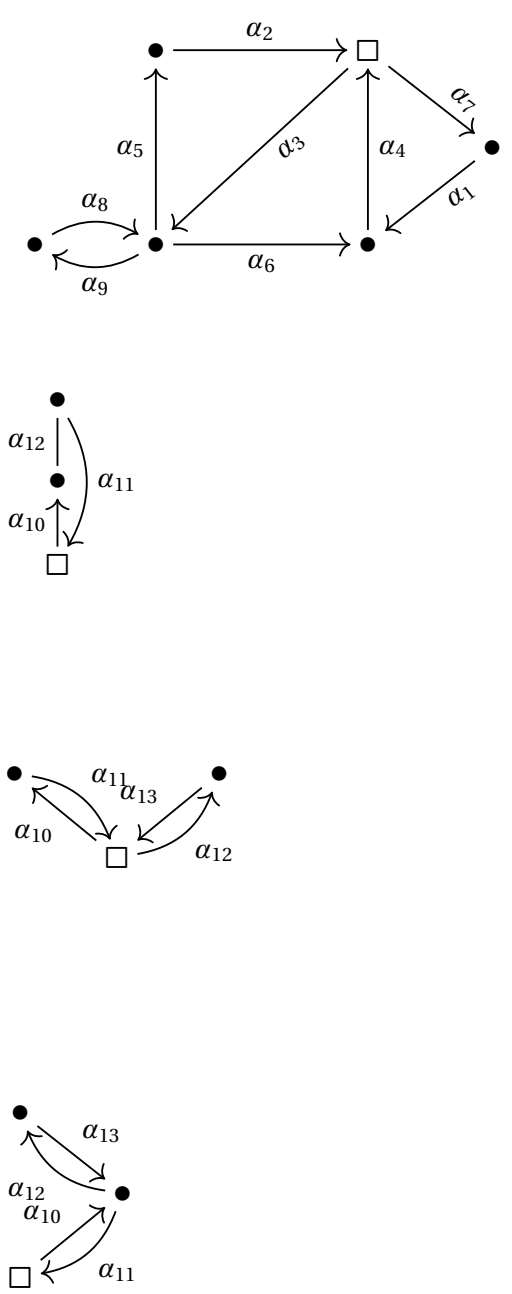

(31)
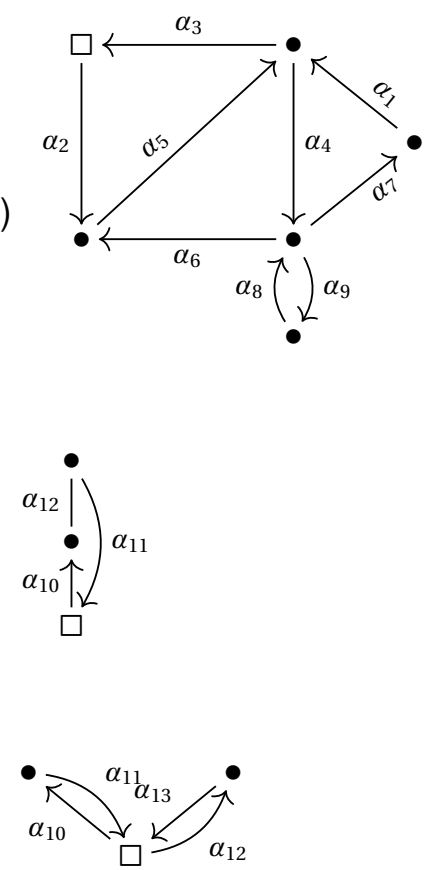

As relações do tipo 2 são: $r 1=\alpha_{2} \alpha_{3} \alpha_{6}, r 2=$ $\alpha_{4} \alpha_{3} \alpha_{5}, r 3=\alpha_{6} \alpha_{4} \alpha_{7}, r 4=\alpha_{1} \alpha_{4} \alpha_{3}, r 5=\alpha_{2} \alpha_{7}$, $r 6=\alpha_{8} \alpha_{6}, r 7=\alpha_{8} \alpha_{5}$ e $r 8=\alpha_{3} \alpha_{9}$.

Os ciclos elementares são: $C_{1}=\alpha_{2} \alpha_{3} \alpha_{5}, C_{2}=$ $\alpha_{3} \alpha_{6} \alpha_{4}, C_{3}=\alpha_{4} \alpha_{7} \alpha_{1}$ e $C_{4}=\alpha_{8} \alpha_{9}$.

Completando, temos mais essas relações do tipo 2: $r 9=\alpha_{4} \alpha_{10}, r 10=\alpha_{2} \alpha_{10}, r 11=\alpha_{11} \alpha_{3}$ e $r 12=$ $\alpha_{11} \alpha_{7}$.

E o ciclo elementar é $C_{5}=\alpha_{10} \alpha_{12} \alpha_{11}$. O programa exibe a álgebra 115.

Nesse caso, temos mais essas relações do tipo 2: $r 9=\alpha_{4} \alpha_{10}, r 10=\alpha_{2} \alpha_{10}, r 11=\alpha_{11} \alpha_{3}, r 12=$ $\alpha_{11} \alpha_{7}, r 13=\alpha_{4} \alpha_{12}, r 14=\alpha_{2} \alpha_{12}, r 15=\alpha_{13} \alpha_{3}$, $r 16=\alpha_{13} \alpha_{7}, r 17=\alpha_{13} \alpha_{10}$ e $r 18=\alpha_{11} \alpha_{12}$.

$\mathrm{E}$ os ciclos elementares são $C_{5}=\alpha_{10} \alpha_{11}$ e $C_{6}=$ $\alpha_{12} \alpha_{13}$. Logo obtemos a solução 116.

Nesse anexo, temos mais essas relações do tipo 2: $r 9=\alpha_{4} \alpha_{10}, r 10=\alpha_{2} \alpha_{10}, r 11=\alpha_{11} \alpha_{3}, r 12=$ $\alpha_{11} \alpha_{7}, r 13=\alpha_{10} \alpha_{12}$ e $r 14=\alpha_{13} \alpha_{11}$.

$\mathrm{E}$ os ciclos elementares são $C_{5}=\alpha_{11} \alpha_{10}$ e $C_{6}=$ $\alpha_{13} \alpha_{12}$. Portanto, temos um corte que origina a álgebra de incidência 117.

As relações do tipo 2 são: $r 1=\alpha_{2} \alpha_{5} \alpha_{4}, r 2=$ $\alpha_{6} \alpha_{5} \alpha_{3}, r 3=\alpha_{5} \alpha_{4} \alpha_{7}, r 4=\alpha_{1} \alpha_{4} \alpha_{6}, r 5=\alpha_{1} \alpha_{3}$, $r 6=\alpha_{8} \alpha_{7}, r 7=\alpha_{8} \alpha_{6}$ e $r 8=\alpha_{4} \alpha_{9}$.

Os ciclos elementares são: $C_{1}=\alpha_{2} \alpha_{5} \alpha_{3}, C_{2}=$ $\alpha_{6} \alpha_{5} \alpha_{4}, C_{3}=\alpha_{4} \alpha_{7} \alpha_{1}$ e $C_{4}=\alpha_{8} \alpha_{9}$.

Completando, temos mais essas relações do tipo 2: $r 9=\alpha_{3} \alpha_{10}$ e $r 10=\alpha_{11} \alpha_{2}$.

E o ciclo elementar é $C_{5}=\alpha_{10} \alpha_{12} \alpha_{11}$. O programa exibe a álgebra 118.

Nesse caso, temos mais essas relações do tipo 2: $r 9=\alpha_{3} \alpha_{10}, r 10=\alpha_{11} \alpha_{2}, r 11=\alpha_{3} \alpha_{12}, r 12=$ $\alpha_{13} \alpha_{2}, r 13=\alpha_{13} \alpha_{10}$ e $r 14=\alpha_{11} \alpha_{12}$.

$\mathrm{E}$ os ciclos elementares são $C_{5}=\alpha_{10} \alpha_{11}$ e $C_{6}=$ $\alpha_{12} \alpha_{13}$. Logo obtemos a solução 119 . 


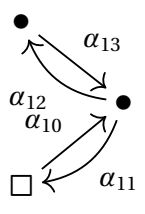

(32)

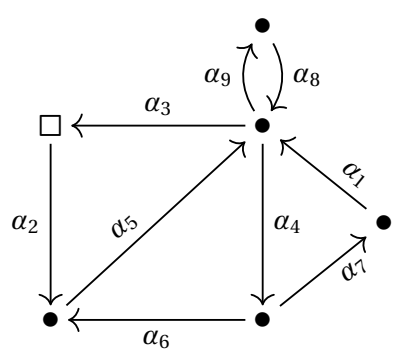

$\underset{\alpha_{10} \uparrow}{\alpha_{12}} \stackrel{\bullet}{\bullet} \alpha_{11}$
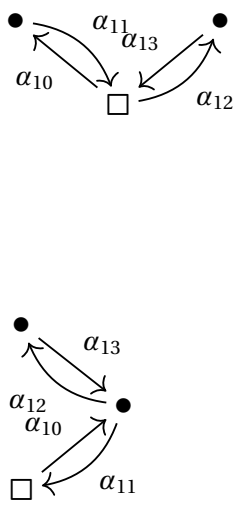

(33)
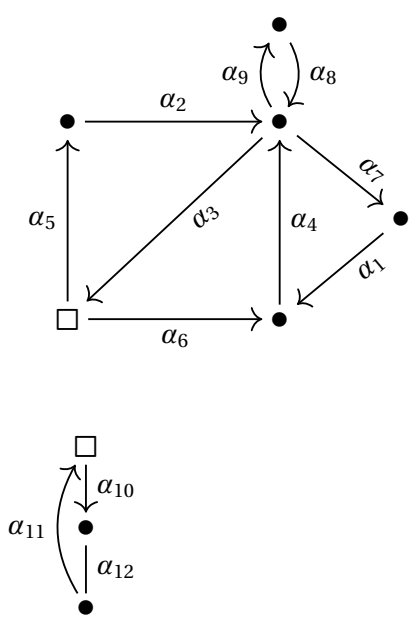

Nesse anexo, temos mais essas relações do tipo 2: $r 9=\alpha_{3} \alpha_{10}, r 10=\alpha_{11} \alpha_{2}, r 11=\alpha_{10} \alpha_{12}$ e $r 12=$ $\alpha_{13} \alpha_{11}$.

$\mathrm{E}$ os ciclos elementares são $C_{5}=\alpha_{11} \alpha_{10}$ e $C_{6}=$ $\alpha_{13} \alpha_{12}$. Portanto, temos um corte que origina a álgebra de incidência 120 .

As relações do tipo 2 são: $r 1=\alpha_{2} \alpha_{5} \alpha_{4}, r 2=$ $\alpha_{6} \alpha_{5} \alpha_{3}, r 3=\alpha_{5} \alpha_{4} \alpha_{7}, r 4=\alpha_{1} \alpha_{4} \alpha_{6}, r 5=\alpha_{1} \alpha_{3}$, $r 6=\alpha_{1} \alpha_{9}, r 7=\alpha_{5} \alpha_{9}, r 8=\alpha_{8} \alpha_{4}$ e $r 9=\alpha_{8} \alpha_{3}$.

Os ciclos elementares são: $C_{1}=\alpha_{2} \alpha_{5} \alpha_{3}, C_{2}=$ $\alpha_{6} \alpha_{5} \alpha_{4}, C_{3}=\alpha_{4} \alpha_{7} \alpha_{1}$ e $C_{4}=\alpha_{8} \alpha_{9}$.

Completando, temos mais essas relações do tipo 2: $r 9=\alpha_{3} \alpha_{10}$ e $r 10=\alpha_{11} \alpha_{2}$.

E o ciclo elementar é $C_{5}=\alpha_{10} \alpha_{12} \alpha_{11}$. O programa mostra o corte para a álgebra 121.

Nesse caso, temos mais essas relações do tipo 2: $r 9=\alpha_{3} \alpha_{10}, r 10=\alpha_{11} \alpha_{2}, r 11=\alpha_{3} \alpha_{12}, r 12=$ $\alpha_{13} \alpha_{2}, r 13=\alpha_{13} \alpha_{10}$ e $r 14=\alpha_{11} \alpha_{12}$.

$\mathrm{E}$ os ciclos elementares são $C_{5}=\alpha_{10} \alpha_{11}$ e $C_{6}=$ $\alpha_{12} \alpha_{13}$. Nesse caso, obtemos a solução 122 .

Nesse anexo, temos mais essas relações do tipo 2: $r 9=\alpha_{3} \alpha_{10}, r 10=\alpha_{11} \alpha_{2}, r 11=\alpha_{10} \alpha_{12}$ e $r 12=$ $\alpha_{13} \alpha_{11}$.

$\mathrm{E}$ os ciclos elementares são $C_{5}=\alpha_{11} \alpha_{10}$ e $C_{6}=$ $\alpha_{13} \alpha_{12}$. Portanto, extraímos a álgebra de incidência 123.

As relações do tipo 2 são: $r 1=\alpha_{2} \alpha_{3} \alpha_{6}, r 2=$ $\alpha_{4} \alpha_{3} \alpha_{5}, r 3=\alpha_{6} \alpha_{4} \alpha_{7}, r 4=\alpha_{1} \alpha_{4} \alpha_{3}, r 5=\alpha_{2} \alpha_{7}$, $r 6=\alpha_{8} \alpha_{7}, r 7=\alpha_{8} \alpha_{3}, r 8=\alpha_{4} \alpha_{9}$ e $r 9=\alpha_{2} \alpha_{9}$.

Os ciclos elementares são: $C_{1}=\alpha_{2} \alpha_{3} \alpha_{5}, C_{2}=$ $\alpha_{3} \alpha_{6} \alpha_{4}, C_{3}=\alpha_{4} \alpha_{7} \alpha_{1}$ e $C_{4}=\alpha_{8} \alpha_{9}$.

Completando, temos mais essas relações do tipo 2: $r 10=\alpha_{3} \alpha_{10}, r 11=\alpha_{11} \alpha_{5}$ e $r 12=\alpha_{11} \alpha_{6}$.

E o ciclo elementar é $C_{5}=\alpha_{10} \alpha_{12} \alpha_{11}$. Logo, com essas informações, temos a álgebra 124. 

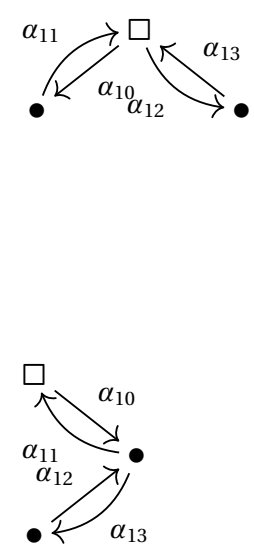

(34)
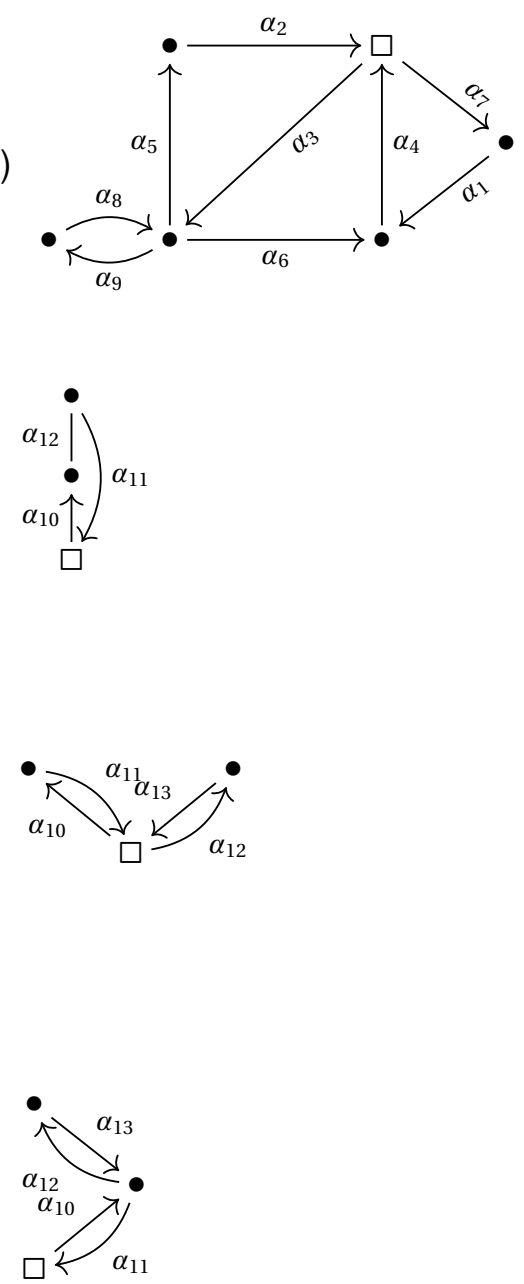

Nesse caso, temos mais essas relações do tipo 2: $r 10=\alpha_{3} \alpha_{10}, r 11=\alpha_{11} \alpha_{5}, r 12=\alpha_{11} \alpha_{6}, r 13=$ $\alpha_{3} \alpha_{12}, r 14=\alpha_{13} \alpha_{5}, r 15=\alpha_{13} \alpha_{6}, r 16=\alpha_{11} \alpha_{12} \mathrm{e}$ $r 17=\alpha_{13} \alpha_{10}$.

E os ciclos elementares são $C_{5}=\alpha_{11} \alpha_{10}$ e $C_{6}=$ $\alpha_{12} \alpha_{13}$. Nesse caso, obtemos a solução 125 .

Nesse anexo, temos mais essas relações do tipo 2: $r 10=\alpha_{3} \alpha_{10}, r 11=\alpha_{11} \alpha_{5}, r 12=\alpha_{11} \alpha_{6}, r 13=$ $\alpha_{12} \alpha_{11}$ e $r 14=\alpha_{10} \alpha_{13}$.

$\mathrm{E}$ os ciclos elementares são $C_{5}=\alpha_{11} \alpha_{10}$ e $C_{6}=$ $\alpha_{13} \alpha_{12}$. Logo, temos um corte que origina a álgebra 126.

As relações do tipo 2 são: $r 1=\alpha_{2} \alpha_{3} \alpha_{6}, r 2=$ $\alpha_{4} \alpha_{3} \alpha_{5}, r 3=\alpha_{6} \alpha_{4} \alpha_{7}, r 4=\alpha_{1} \alpha_{4} \alpha_{3}, r 5=\alpha_{2} \alpha_{7}$, $r 6=\alpha_{8} \alpha_{5}, r 7=\alpha_{8} \alpha_{6}$ e $r 8=\alpha_{3} \alpha_{9}$.

Os ciclos elementares são: $C_{1}=\alpha_{2} \alpha_{3} \alpha_{5}, C_{2}=$ $\alpha_{3} \alpha_{6} \alpha_{4}, C_{3}=\alpha_{4} \alpha_{7} \alpha_{1}$ e $C_{4}=\alpha_{8} \alpha_{9}$.

Completando, temos mais essas relações do tipo 2 : $r 9=\alpha_{2} \alpha_{10}, r 10=\alpha_{4} \alpha_{10}, r 11=\alpha_{11} \alpha_{3}$ e $r 12=$ $\alpha_{11} \alpha_{7}$.

E o ciclo elementar é $C_{5}=\alpha_{10} \alpha_{12} \alpha_{11}$. O programa mostra o corte para a álgebra 127 .

Nesse caso, temos mais essas relações do tipo 2: $r 9=\alpha_{2} \alpha_{10}, r 10=\alpha_{4} \alpha_{10}, r 11=\alpha_{11} \alpha_{3}, r 12=$ $\alpha_{11} \alpha_{7}, r 13=\alpha_{2} \alpha_{12}, r 14=\alpha_{4} \alpha_{12}, r 15=\alpha_{13} \alpha_{3}$, $r 16=\alpha_{13} \alpha_{7}, r 17=\alpha_{13} \alpha_{10}$ e $r 18=\alpha_{11} \alpha_{12}$.

$\mathrm{E}$ os ciclos elementares são $C_{5}=\alpha_{10} \alpha_{11}$ e $C_{6}=$ $\alpha_{12} \alpha_{13}$. Nesse caso, obtemos a solução 128.

Nesse anexo, temos mais essas relações do tipo 2: $r 9=\alpha_{2} \alpha_{10}, r 10=\alpha_{4} \alpha_{10}, r 11=\alpha_{11} \alpha_{3}, r 12=$ $\alpha_{11} \alpha_{7}, r 13=\alpha_{10} \alpha_{12}$ e $r 14=\alpha_{13} \alpha_{11}$.

$\mathrm{E}$ os ciclos elementares são $C_{5}=\alpha_{11} \alpha_{10}$ e $C_{6}=$ $\alpha_{13} \alpha_{12}$. Portanto, extraímos a álgebra de incidência 129. 
(35)

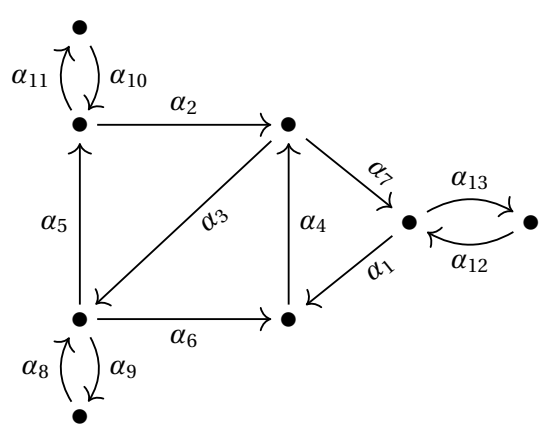

(36)

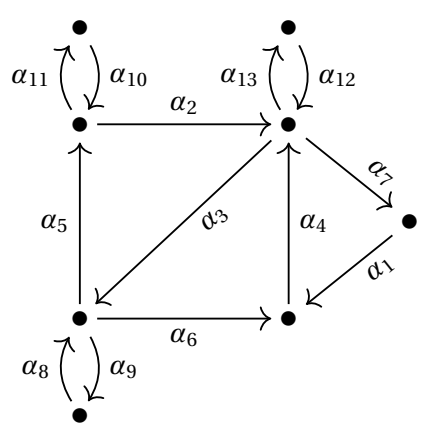

(37)

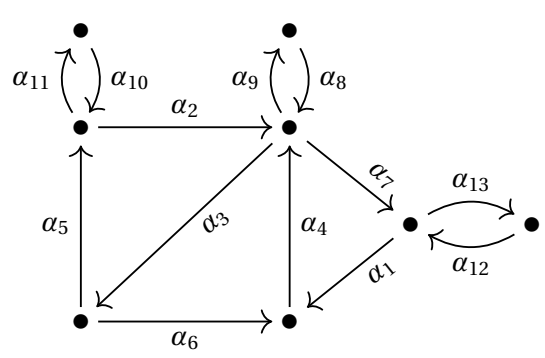

(38)

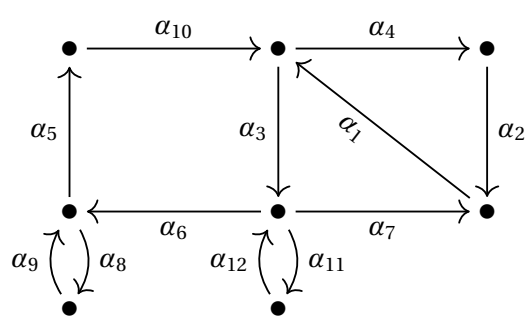

(39)

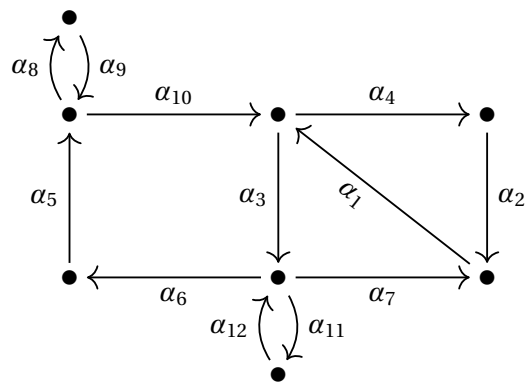

As relações do tipo 2 são: $r 1=\alpha_{2} \alpha_{3} \alpha_{6}, r 2=$ $\alpha_{4} \alpha_{3} \alpha_{5}, r 3=\alpha_{6} \alpha_{4} \alpha_{7}, r 4=\alpha_{1} \alpha_{4} \alpha_{3}, r 5=\alpha_{2} \alpha_{7}$, $r 6=\alpha_{8} \alpha_{5}, r 7=\alpha_{8} \alpha_{6}, r 8=\alpha_{3} \alpha_{9}, r 9=\alpha_{5} \alpha_{11}$, $r 10=\alpha_{10} \alpha_{2}, r 11=\alpha_{7} \alpha_{13}$ e $r 12=\alpha_{12} \alpha_{1}$.

Os ciclos elementares são: $C_{1}=\alpha_{2} \alpha_{3} \alpha_{5}, C_{2}=$ $\alpha_{3} \alpha_{6} \alpha_{4}, C_{3}=\alpha_{4} \alpha_{7} \alpha_{1}, C_{4}=\alpha_{8} \alpha_{9}, C_{5}=\alpha_{10} \alpha_{11} \mathrm{e}$ $C_{6}=\alpha_{12} \alpha_{13}$. Portanto, o programa não exibe resposta.

As relações do tipo 2 são: $r 1=\alpha_{2} \alpha_{3} \alpha_{6}, r 2=$ $\alpha_{4} \alpha_{3} \alpha_{5}, r 3=\alpha_{6} \alpha_{4} \alpha_{7}, r 4=\alpha_{1} \alpha_{4} \alpha_{3}, r 5=\alpha_{2} \alpha_{7}$, $r 6=\alpha_{8} \alpha_{5}, r 7=\alpha_{8} \alpha_{6}, r 8=\alpha_{3} \alpha_{9}, r 9=\alpha_{5} \alpha_{11}$, $r 10=\alpha_{10} \alpha_{2}, r 11=\alpha_{2} \alpha_{13}, r 12=\alpha_{4} \alpha_{13}$ e $r 13=$ $\alpha_{12} \alpha_{3}$.

Os ciclos elementares são: $C_{1}=\alpha_{2} \alpha_{3} \alpha_{5}, C_{2}=$ $\alpha_{3} \alpha_{6} \alpha_{4}, C_{3}=\alpha_{4} \alpha_{7} \alpha_{1}, C_{4}=\alpha_{8} \alpha_{9}, C_{5}=\alpha_{10} \alpha_{11} \mathrm{e}$ $C_{6}=\alpha_{12} \alpha_{13}$. Logo, não obtemos solução.

As relações do tipo 2 são: $r 1=\alpha_{2} \alpha_{3} \alpha_{6}, r 2=$ $\alpha_{4} \alpha_{3} \alpha_{5}, r 3=\alpha_{6} \alpha_{4} \alpha_{7}, r 4=\alpha_{1} \alpha_{4} \alpha_{3}, r 5=\alpha_{2} \alpha_{7}$, $r 6=\alpha_{8} \alpha_{7}, r 7=\alpha_{8} \alpha_{3}, r 8=\alpha_{2} \alpha_{9}, r 9=\alpha_{4} \alpha_{9}, r 10=$ $\alpha_{5} \alpha_{11}, r 11=\alpha_{10} \alpha_{2}, r 12=\alpha_{7} \alpha_{13}$ e $r 13=\alpha_{12} \alpha_{1}$.

Os ciclos elementares são: $C_{1}=\alpha_{2} \alpha_{3} \alpha_{5}, C_{2}=$ $\alpha_{3} \alpha_{6} \alpha_{4}, C_{3}=\alpha_{4} \alpha_{7} \alpha_{1}, C_{4}=\alpha_{8} \alpha_{9}, C_{5}=\alpha_{10} \alpha_{11} \mathrm{e}$ $C_{6}=\alpha_{12} \alpha_{13}$. O programa não exibe solução.

As relações do tipo 2 são: $r 1=\alpha_{10} \alpha_{3} \alpha_{7}, r 2=$ $\alpha_{1} \alpha_{3} \alpha_{6}, r 3=\alpha_{7} \alpha_{1} \alpha_{4}, r 4=\alpha_{2} \alpha_{1} \alpha_{3}, r 5=\alpha_{3} \alpha_{11}$, $r 6=\alpha_{12} \alpha_{6}, r 7=\alpha_{12} \alpha_{7}, r 8=\alpha_{9} \alpha_{5}, r 9=\alpha_{6} \alpha_{8} \mathrm{e}$ $r 10=\alpha_{10} \alpha_{4}$.

Os ciclos elementares são: $C_{1}=\alpha_{5} \alpha_{10} \alpha_{3} \alpha_{6}, C_{2}=$ $\alpha_{3} \alpha_{7} \alpha_{1}, C_{3}=\alpha_{1} \alpha_{4} \alpha_{2}, C_{4}=\alpha_{12} \alpha_{11}$ e $C_{5}=\alpha_{9} \alpha_{8}$. Nesse caso, não tem álgebra de incidência.

As relações do tipo 2 são: $r 1=\alpha_{10} \alpha_{3} \alpha_{7}, r 2=$ $\alpha_{1} \alpha_{3} \alpha_{6}, r 3=\alpha_{7} \alpha_{1} \alpha_{4}, r 4=\alpha_{2} \alpha_{1} \alpha_{3}, r 5=\alpha_{3} \alpha_{11}$, $r 6=\alpha_{12} \alpha_{6}, r 7=\alpha_{12} \alpha_{7}, r 8=\alpha_{5} \alpha_{8}, r 9=\alpha_{9} \alpha_{10} \mathrm{e}$ $r 10=\alpha_{10} \alpha_{4}$.

Os ciclos elementares são: $C_{1}=\alpha_{5} \alpha_{10} \alpha_{3} \alpha_{6}, C_{2}=$ $\alpha_{3} \alpha_{7} \alpha_{1}, C_{3}=\alpha_{1} \alpha_{4} \alpha_{2}, C_{4}=\alpha_{12} \alpha_{11}$ e $C_{5}=\alpha_{9} \alpha_{8}$. Essa extensão trivial não tem corte que resulte em uma álgebra de incidência. 
(40)

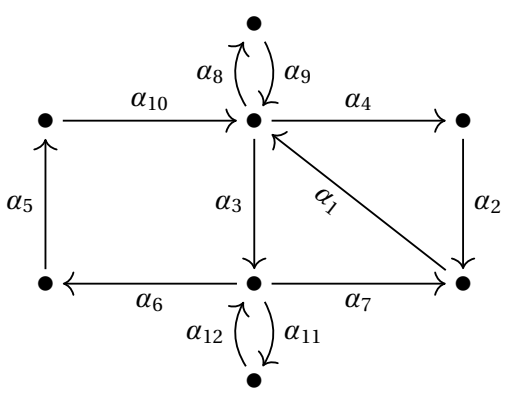

(41)

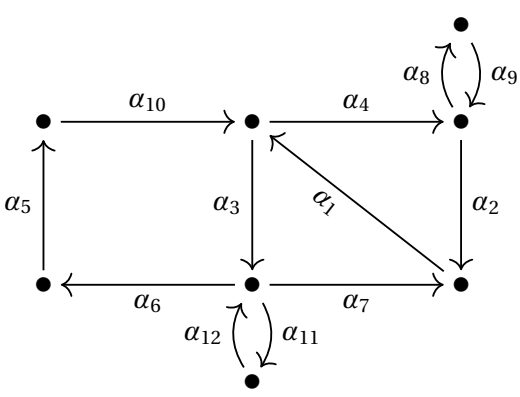

(42)

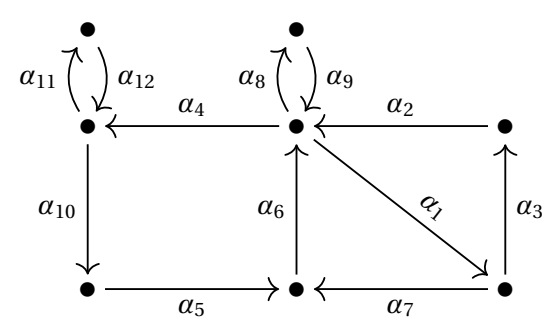

(43)

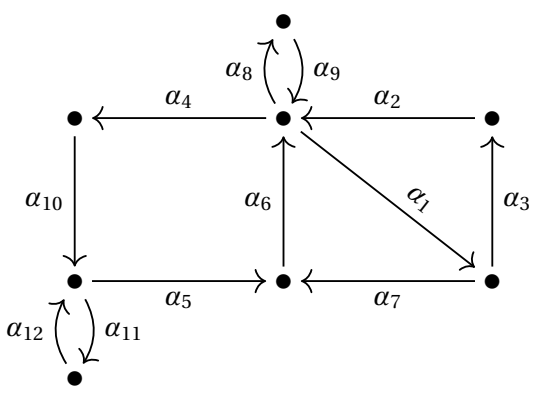

(44)

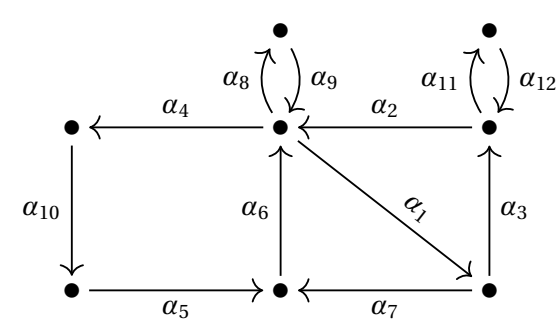

As relações do tipo 2 são: $r 1=\alpha_{10} \alpha_{3} \alpha_{7}, r 2=$ $\alpha_{1} \alpha_{3} \alpha_{6}, r 3=\alpha_{7} \alpha_{1} \alpha_{4}, r 4=\alpha_{2} \alpha_{1} \alpha_{3}, r 5=\alpha_{3} \alpha_{11}$, $r 6=\alpha_{12} \alpha_{6}, r 7=\alpha_{12} \alpha_{7}, r 8=\alpha_{10} \alpha_{8}, r 9=\alpha_{1} \alpha_{8}$, $r 10=\alpha_{9} \alpha_{3}, r 11=\alpha_{9} \alpha_{4}$ e $r 12=\alpha_{10} \alpha_{4}$.

Os ciclos elementares são: $C_{1}=\alpha_{5} \alpha_{10} \alpha_{3} \alpha_{6}, C_{2}=$ $\alpha_{3} \alpha_{7} \alpha_{1}, C_{3}=\alpha_{1} \alpha_{4} \alpha_{2}, C_{4}=\alpha_{12} \alpha_{11}$ e $C_{5}=\alpha_{9} \alpha_{8}$. Essa extensão trivial tem apenas um corte que resulta na álgebra 130 .

As relações do tipo 2 são: $r 1=\alpha_{10} \alpha_{3} \alpha_{7}, r 2=$ $\alpha_{1} \alpha_{3} \alpha_{6}, r 3=\alpha_{7} \alpha_{1} \alpha_{4}, r 4=\alpha_{2} \alpha_{1} \alpha_{3}, r 5=\alpha_{3} \alpha_{11}$, $r 6=\alpha_{12} \alpha_{6}, r 7=\alpha_{12} \alpha_{7}, r 8=\alpha_{4} \alpha_{8}, r 9=\alpha_{9} \alpha_{2}$ e $r 10=\alpha_{10} \alpha_{4}$.

Os ciclos elementares são: $C_{1}=\alpha_{5} \alpha_{10} \alpha_{3} \alpha_{6}, C_{2}=$ $\alpha_{3} \alpha_{7} \alpha_{1}, C_{3}=\alpha_{1} \alpha_{4} \alpha_{2}, C_{4}=\alpha_{12} \alpha_{11}$ e $C_{5}=\alpha_{9} \alpha_{8}$. Nesse caso, temos a álgebra de incidência 131.

As relações do tipo 2 são: $r 1=\alpha_{5} \alpha_{6} \alpha_{1}, r 2=$ $\alpha_{7} \alpha_{6} \alpha_{4}, r 3=\alpha_{6} \alpha_{1} \alpha_{3}, r 4=\alpha_{2} \alpha_{1} \alpha_{7}, r 5=\alpha_{2} \alpha_{4}$, $r 6=\alpha_{6} \alpha_{8}, r 7=\alpha_{2} \alpha_{8}, r 8=\alpha_{9} \alpha_{1}, r 9=\alpha_{9} \alpha_{4}$, $r 10=\alpha_{4} \alpha_{11}$ e $r 11=\alpha_{12} \alpha_{10}$.

Os ciclos elementares são: $C_{1}=\alpha_{10} \alpha_{5} \alpha_{6} \alpha_{4}, C_{2}=$ $\alpha_{6} \alpha_{1} \alpha_{7}, C_{3}=\alpha_{1} \alpha_{3} \alpha_{2}, C_{4}=\alpha_{12} \alpha_{11}$ e $C_{5}=\alpha_{9} \alpha_{8}$. Portanto, obtemos a álgebra de incidência 132.

As relações do tipo 2 são: $r 1=\alpha_{5} \alpha_{6} \alpha_{1}, r 2=$ $\alpha_{7} \alpha_{6} \alpha_{4}, r 3=\alpha_{6} \alpha_{1} \alpha_{3}, r 4=\alpha_{2} \alpha_{1} \alpha_{7}, r 5=\alpha_{2} \alpha_{4}$, $r 6=\alpha_{6} \alpha_{8}, r 7=\alpha_{2} \alpha_{8}, r 8=\alpha_{9} \alpha_{1}, r 9=\alpha_{9} \alpha_{4}$, $r 10=\alpha_{10} \alpha_{11}$ e $r 11=\alpha_{12} \alpha_{5}$.

Os ciclos elementares são: $C_{1}=\alpha_{10} \alpha_{5} \alpha_{6} \alpha_{4}, C_{2}=$ $\alpha_{6} \alpha_{1} \alpha_{7}, C_{3}=\alpha_{1} \alpha_{3} \alpha_{2}, C_{4}=\alpha_{12} \alpha_{11}$ e $C_{5}=\alpha_{9} \alpha_{8}$. Logo, o programa não exibe solução.

As relações do tipo 2 são: $r 1=\alpha_{5} \alpha_{6} \alpha_{1}, r 2=$ $\alpha_{7} \alpha_{6} \alpha_{4}, r 3=\alpha_{6} \alpha_{1} \alpha_{3}, r 4=\alpha_{2} \alpha_{1} \alpha_{7}, r 5=\alpha_{2} \alpha_{4}$, $r 6=\alpha_{6} \alpha_{8}, r 7=\alpha_{2} \alpha_{8}, r 8=\alpha_{9} \alpha_{1}, r 9=\alpha_{9} \alpha_{4}$, $r 10=\alpha_{3} \alpha_{11}$ e $r 11=\alpha_{12} \alpha_{2}$.

Os ciclos elementares são: $C_{1}=\alpha_{10} \alpha_{5} \alpha_{6} \alpha_{4}, C_{2}=$ $\alpha_{6} \alpha_{1} \alpha_{7}, C_{3}=\alpha_{1} \alpha_{3} \alpha_{2}, C_{4}=\alpha_{12} \alpha_{11}$ e $C_{5}=\alpha_{9} \alpha_{8}$. Portanto, o programa exibe a solução 133. 
(45)

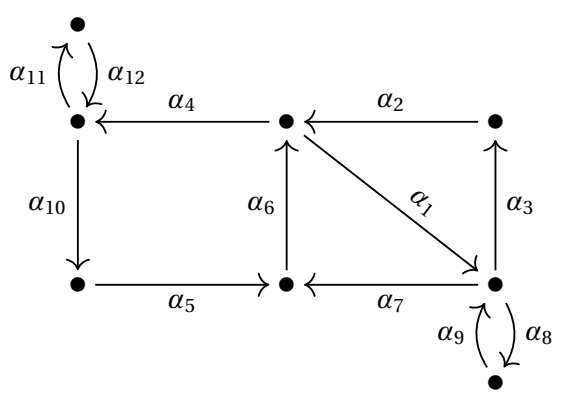

(46)

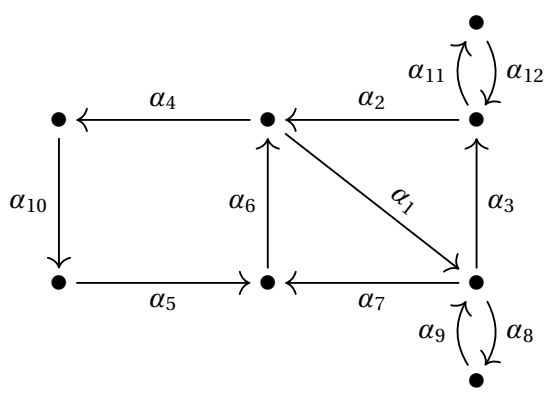

(47)

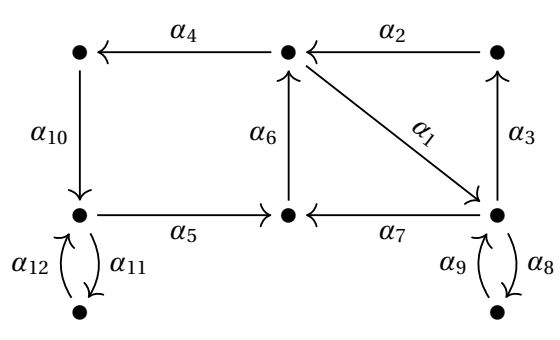

(48)

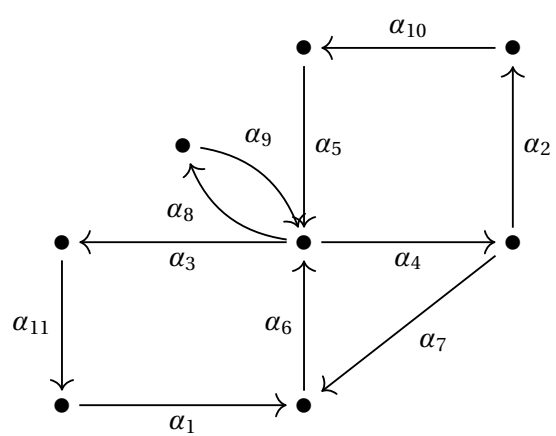

As relações do tipo 2 são: $r 1=\alpha_{5} \alpha_{6} \alpha_{1}, r 2=$ $\alpha_{7} \alpha_{6} \alpha_{4}, r 3=\alpha_{6} \alpha_{1} \alpha_{3}, r 4=\alpha_{2} \alpha_{1} \alpha_{7}, r 5=\alpha_{2} \alpha_{4}$, $r 6=\alpha_{1} \alpha_{8}, r 7=\alpha_{9} \alpha_{7}, r 8=\alpha_{9} \alpha_{3}, r 9=\alpha_{4} \alpha_{11} \mathrm{e}$ $r 10=\alpha_{12} \alpha_{10}$.

Os ciclos elementares são: $C_{1}=\alpha_{10} \alpha_{5} \alpha_{6} \alpha_{4}, C_{2}=$ $\alpha_{6} \alpha_{1} \alpha_{7}, C_{3}=\alpha_{1} \alpha_{3} \alpha_{2}, C_{4}=\alpha_{12} \alpha_{11}$ e $C_{5}=\alpha_{9} \alpha_{8}$. Logo, nesse caso, obtemos a álgebra de incidência 134.

As relações do tipo 2 são: $r 1=\alpha_{5} \alpha_{6} \alpha_{1}, r 2=$ $\alpha_{7} \alpha_{6} \alpha_{4}, r 3=\alpha_{6} \alpha_{1} \alpha_{3}, r 4=\alpha_{2} \alpha_{1} \alpha_{7}, r 5=\alpha_{2} \alpha_{4}$, $r 6=\alpha_{1} \alpha_{8}, r 7=\alpha_{9} \alpha_{7}, r 8=\alpha_{9} \alpha_{3}, r 9=\alpha_{3} \alpha_{11} \mathrm{e}$ $r 10=\alpha_{12} \alpha_{2}$.

Os ciclos elementares são: $C_{1}=\alpha_{10} \alpha_{5} \alpha_{6} \alpha_{4}, C_{2}=$ $\alpha_{6} \alpha_{1} \alpha_{7}, C_{3}=\alpha_{1} \alpha_{3} \alpha_{2}, C_{4}=\alpha_{12} \alpha_{11}$ e $C_{5}=\alpha_{9} \alpha_{8}$. O programa não mostra solução.

As relações do tipo 2 são: $r 1=\alpha_{5} \alpha_{6} \alpha_{1}, r 2=$ $\alpha_{7} \alpha_{6} \alpha_{4}, r 3=\alpha_{6} \alpha_{1} \alpha_{3}, r 4=\alpha_{2} \alpha_{1} \alpha_{7}, r 5=\alpha_{2} \alpha_{4}$, $r 6=\alpha_{1} \alpha_{8}, r 7=\alpha_{9} \alpha_{7}, r 8=\alpha_{9} \alpha_{3}, r 9=\alpha_{10} \alpha_{11} \mathrm{e}$ $r 10=\alpha_{12} \alpha_{5}$.

Os ciclos elementares são: $C_{1}=\alpha_{10} \alpha_{5} \alpha_{6} \alpha_{4}, C_{2}=$ $\alpha_{6} \alpha_{1} \alpha_{7}, C_{3}=\alpha_{1} \alpha_{3} \alpha_{2}, C_{4}=\alpha_{12} \alpha_{11}$ e $C_{5}=\alpha_{9} \alpha_{8}$. Não tem corte nessa extensão trivial que resulte numa álgebra de incidência.

Essa extensão trivial tem as seguintes relações do tipo 2: $r 1=\alpha_{1} \alpha_{6} \alpha_{4}, r 2=\alpha_{7} \alpha_{6} \alpha_{3}, r 3=\alpha_{5} \alpha_{4} \alpha_{7}$, $r 4=\alpha_{6} \alpha_{4} \alpha_{2}, r 5=\alpha_{5} \alpha_{3}, r 6=\alpha_{6} \alpha_{8}, r 7=\alpha_{5} \alpha_{8}$, $r 8=\alpha_{9} \alpha_{3}$ e $r 9=\alpha_{9} \alpha_{4}$.

Os ciclos elementares dessa extensão trivial são: $C_{1}=\alpha_{3} \alpha_{11} \alpha_{1} \alpha_{6}, C_{2}=\alpha_{5} \alpha_{4} \alpha_{2} \alpha_{10}, C_{3}=\alpha_{4} \alpha_{7} \alpha_{6} \mathrm{e}$ $C_{4}=\alpha_{9} \alpha_{8}$. Portanto, temos as álgebras de incidência 135 e 136. 
(49)

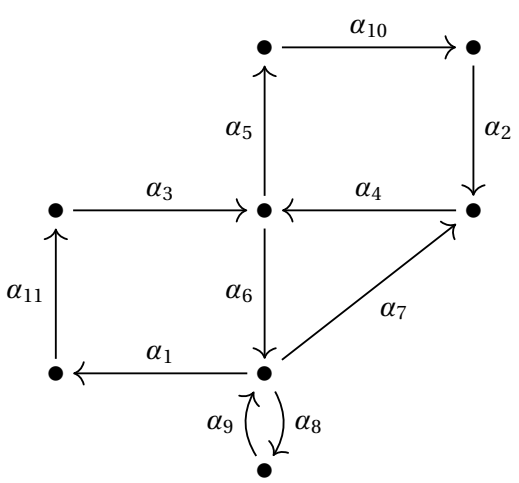

(50)

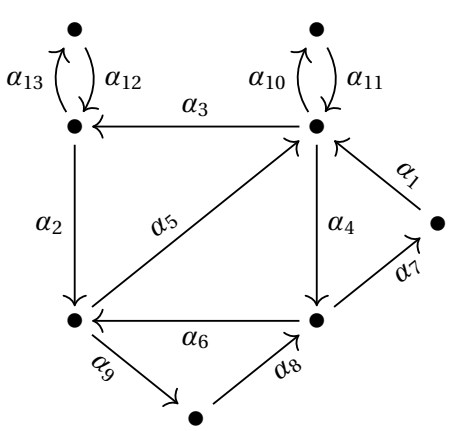

(51)

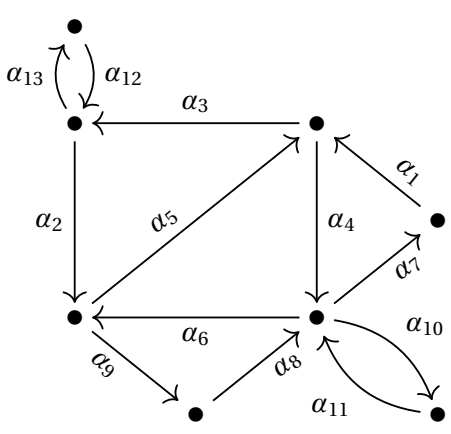

(52)

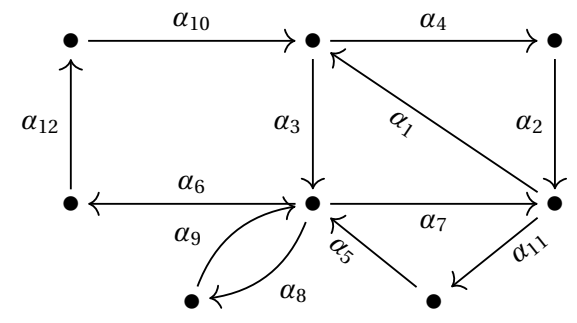

Essa extensão trivial tem as seguintes relações do tipo 2: $r 1=\alpha_{4} \alpha_{6} \alpha_{1}, r 2=\alpha_{3} \alpha_{6} \alpha_{7}, r 3=\alpha_{7} \alpha_{4} \alpha_{5}$, $r 4=\alpha_{2} \alpha_{4} \alpha_{6}, r 5=\alpha_{3} \alpha_{5}, r 6=\alpha_{6} \alpha_{8}, r 7=\alpha_{9} \alpha_{1} \mathrm{e}$ $r 8=\alpha_{9} \alpha_{7}$.

Os ciclos elementares dessa extensão trivial são: $C_{1}=\alpha_{3} \alpha_{6} \alpha_{1} \alpha_{11}, C_{2}=\alpha_{5} \alpha_{10} \alpha_{2} \alpha_{4}, C_{3}=\alpha_{4} \alpha_{6} \alpha_{7} \mathrm{e}$ $C_{4}=\alpha_{9} \alpha_{8}$. O programa mostra a solução 137 .

As relações do tipo 2 são: $r 1=\alpha_{2} \alpha_{5} \alpha_{4}, r 2=$ $\alpha_{6} \alpha_{5} \alpha_{3}, r 3=\alpha_{5} \alpha_{4} \alpha_{7}, r 4=\alpha_{1} \alpha_{4} \alpha_{6}, r 5=\alpha_{4} \alpha_{6} \alpha_{9}$, $r 6=\alpha_{8} \alpha_{6} \alpha_{5}, r 7=\alpha_{2} \alpha_{9}, r 8=\alpha_{1} \alpha_{3}, r 9=\alpha_{8} \alpha_{7}$, $r 10=\alpha_{11} \alpha_{4}, r 11=\alpha_{11} \alpha_{3}, r 12=\alpha_{1} \alpha_{10}, r 13=$ $\alpha_{5} \alpha_{10}, r 14=\alpha_{12} \alpha_{2}$ e $r 15=\alpha_{3} \alpha_{13}$.

Os ciclos elementares são: $C_{1}=\alpha_{2} \alpha_{5} \alpha_{3}, C_{2}=$ $\alpha_{6} \alpha_{5} \alpha_{4}, C_{3}=\alpha_{4} \alpha_{7} \alpha_{1}, C_{4}=\alpha_{6} \alpha_{8} \alpha_{9}, C_{5}=\alpha_{10} \alpha_{11}$ e $C_{6}=\alpha_{12} \alpha_{13}$. O programa não exibe solução.

As relações do tipo 2 são: $r 1=\alpha_{2} \alpha_{5} \alpha_{4}, r 2=$ $\alpha_{6} \alpha_{5} \alpha_{3}, r 3=\alpha_{5} \alpha_{4} \alpha_{7}, r 4=\alpha_{1} \alpha_{4} \alpha_{6}, r 5=\alpha_{4} \alpha_{6} \alpha_{9}$, $r 6=\alpha_{8} \alpha_{6} \alpha_{5}, r 7=\alpha_{2} \alpha_{9}, r 8=\alpha_{1} \alpha_{3}, r 9=\alpha_{8} \alpha_{7}$, $r 10=\alpha_{11} \alpha_{6}, r 11=\alpha_{11} \alpha_{7}, r 12=\alpha_{4} \alpha_{10}, r 13=$ $\alpha_{8} \alpha_{10}, r 14=\alpha_{12} \alpha_{2}$ e $r 15=\alpha_{3} \alpha_{13}$.

Os ciclos elementares são: $C_{1}=\alpha_{2} \alpha_{5} \alpha_{3}, C_{2}=$ $\alpha_{6} \alpha_{5} \alpha_{4}, C_{3}=\alpha_{4} \alpha_{7} \alpha_{1}, C_{4}=\alpha_{6} \alpha_{8} \alpha_{9}, C_{5}=\alpha_{10} \alpha_{11}$ e $C_{6}=\alpha_{12} \alpha_{13}$. Portanto, nesse caso, não temos álgebra de incidência.

As relações do tipo 2 são: $r 1=\alpha_{10} \alpha_{3} \alpha_{7}, r 2=$ $\alpha_{1} \alpha_{3} \alpha_{6}, r 3=\alpha_{7} \alpha_{1} \alpha_{4}, r 4=\alpha_{2} \alpha_{1} \alpha_{3}, r 5=\alpha_{3} \alpha_{7} \alpha_{11}$, $r 6=\alpha_{5} \alpha_{7} \alpha_{1}, r 7=\alpha_{10} \alpha_{4}, r 8=\alpha_{2} \alpha_{11}, r 9=\alpha_{5} \alpha_{6}$, $r 10=\alpha_{9} \alpha_{7}, r 11=\alpha_{9} \alpha_{6}, r 12=\alpha_{3} \alpha_{8}$ e $r 13=\alpha_{5} \alpha_{8}$.

Os ciclos elementares são: $C_{1}=\alpha_{6} \alpha_{12} \alpha_{10} \alpha_{3}, C_{2}=$ $\alpha_{3} \alpha_{7} \alpha_{1}, C_{3}=\alpha_{1} \alpha_{4} \alpha_{2}, C_{4}=\alpha_{7} \alpha_{11} \alpha_{5}$ e $C_{5}=\alpha_{9} \alpha_{8}$. Essa extensão trivial não tem corte que resulte em uma álgebra de incidência. 
(53)

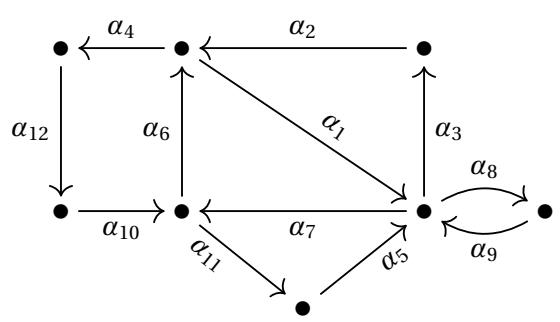

(54)

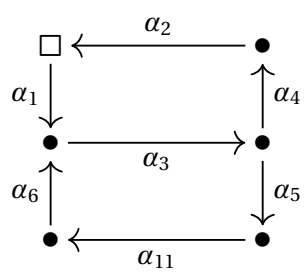

$\underset{\alpha_{7} \uparrow \grave{i}}{\alpha_{9} \mid} \alpha_{8}$
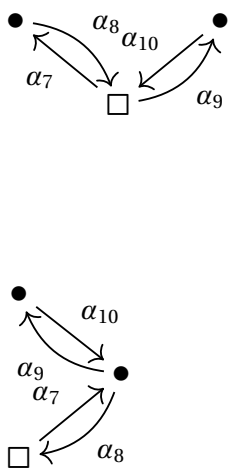

(55)

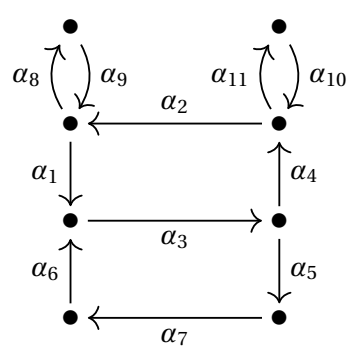

(56)

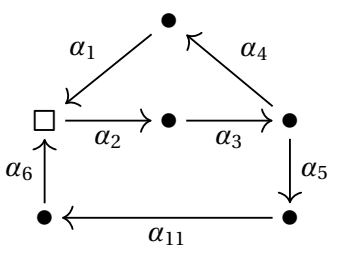

As relações do tipo 2 são: $r 1=\alpha_{10} \alpha_{6} \alpha_{1}, r 2=$ $\alpha_{7} \alpha_{6} \alpha_{4}, r 3=\alpha_{6} \alpha_{1} \alpha_{3}, r 4=\alpha_{2} \alpha_{1} \alpha_{7}, r 5=\alpha_{1} \alpha_{7} \alpha_{11}$, $r 6=\alpha_{5} \alpha_{7} \alpha_{6}, r 7=\alpha_{5} \alpha_{3}, r 8=\alpha_{2} \alpha_{4}, r 9=\alpha_{10} \alpha_{11}$, $r 10=\alpha_{1} \alpha_{8}, r 11=\alpha_{5} \alpha_{8}, r 12=\alpha_{9} \alpha_{3}$ e $r 13=\alpha_{9} \alpha_{7}$. Os ciclos elementares são: $C_{1}=\alpha_{4} \alpha_{12} \alpha_{10} \alpha_{6}, C_{2}=$ $\alpha_{6} \alpha_{1} \alpha_{7}, C_{3}=\alpha_{1} \alpha_{3} \alpha_{2}, C_{4}=\alpha_{7} \alpha_{11} \alpha_{5}$ e $C_{5}=\alpha_{9} \alpha_{8}$. Não tem corte nessa extensão trivial que resulte numa álgebra de incidência.

As relações do tipo 2 são: $r 1=\alpha_{1} \alpha_{3} \alpha_{5}$ e $r 2=$ $\alpha_{6} \alpha_{3} \alpha_{4}$.

Os ciclos elementares são: $C_{1}=\alpha_{1} \alpha_{3} \alpha_{4} \alpha_{2}$ e $C_{2}=$ $\alpha_{3} \alpha_{5} \alpha_{11} \alpha_{6}$.

Completando, temos mais essas relações do tipo 2: $r 3=\alpha_{2} \alpha_{7}$ e $r 4=\alpha_{8} \alpha_{1}$.

E o ciclo elementar é $C_{3}=\alpha_{7} \alpha_{9} \alpha_{8}$. O programa mostra um corte para uma álgebra hereditária.

Nesse caso, temos mais essas relações do tipo 2: $r 3=\alpha_{2} \alpha_{7}, r 4=\alpha_{8} \alpha_{1}, r 5=\alpha_{2} \alpha_{9}, r 6=\alpha_{10} \alpha_{1}$, $r 7=\alpha_{10} \alpha_{7}$ e $r 8=\alpha_{8} \alpha_{9}$.

E os ciclos elementares são $C_{3}=\alpha_{7} \alpha_{8}$ e $C_{4}=\alpha_{9} \alpha_{10}$. Nesse caso, obtemos uma solução hereditária.

Nesse anexo, temos mais essas relações do tipo 2: $r 3=\alpha_{2} \alpha_{7}, r 4=\alpha_{8} \alpha_{1}, r 5=\alpha_{7} \alpha_{9}$ e $r 6=\alpha_{10} \alpha_{8}$.

E os ciclos elementares são $C_{3}=\alpha_{8} \alpha_{7}$ e $C_{4}=\alpha_{10} \alpha_{9}$. Portanto, extraímos uma álgebra de incidência hereditária.

As relações do tipo 2 são: $r 1=\alpha_{1} \alpha_{3} \alpha_{5}, r 2=$ $\alpha_{6} \alpha_{3} \alpha_{4}, r 3=\alpha_{9} \alpha_{1}, r 4=\alpha_{2} \alpha_{8}, r 5=\alpha_{4} \alpha_{11} \mathrm{e}$ $r 6=\alpha_{10} \alpha_{2}$.

Os ciclos elementares são: $C_{1}=\alpha_{1} \alpha_{3} \alpha_{4} \alpha_{2}, C_{2}=$ $\alpha_{3} \alpha_{5} \alpha_{7} \alpha_{6}, C_{3}=\alpha_{10} \alpha_{11} \alpha_{5}$ e $C_{4}=\alpha_{9} \alpha_{8}$. Logo, o programa não exibe solução.

As relações do tipo 2 são: $r 1=\alpha_{1} \alpha_{2} \alpha_{3} \alpha_{5}$ e $r 2=$ $\alpha_{6} \alpha_{2} \alpha_{3} \alpha_{4}$.

Os ciclos elementares são: $C_{1}=\alpha_{1} \alpha_{2} \alpha_{3} \alpha_{4}$ e $C_{2}=$ $\alpha_{2} \alpha_{3} \alpha_{5} \alpha_{11} \alpha_{6}$. 

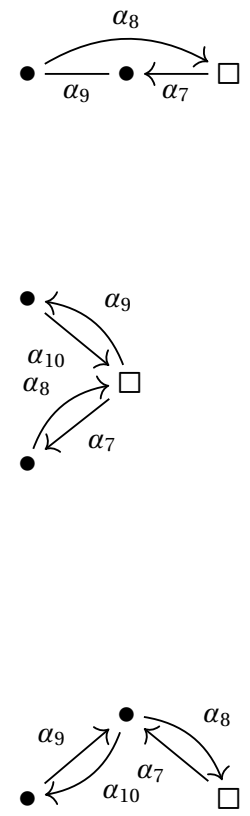

(57)
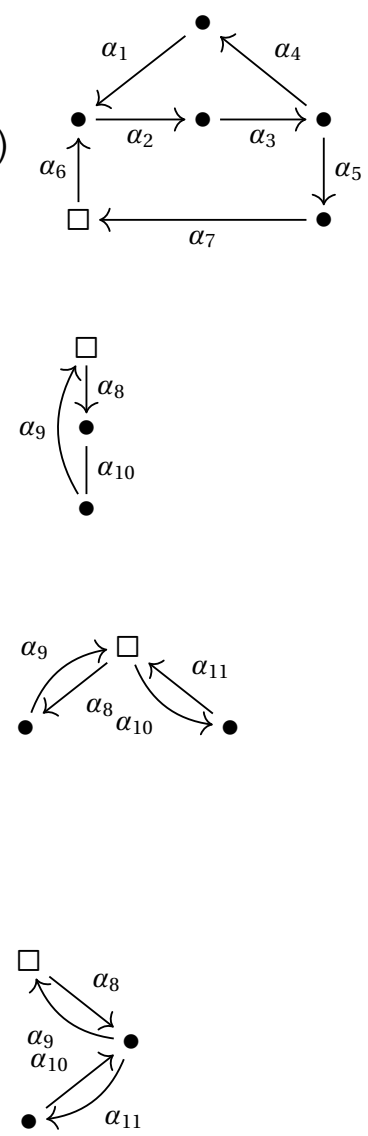

Completando, temos mais essas relações do tipo 2: $r 3=\alpha_{6} \alpha_{7}, r 4=\alpha_{1} \alpha_{7}$ e $r 5=\alpha_{8} \alpha_{2}$.

E o ciclo elementar é $C_{3}=\alpha_{7} \alpha_{9} \alpha_{8}$. O programa exibe a solução 138 e mais uma hereditária.

Nesse caso, temos mais essas relações do tipo 2: $r 3=\alpha_{6} \alpha_{7}, r 4=\alpha_{1} \alpha_{7}, r 5=\alpha_{8} \alpha_{2}, r 6=\alpha_{6} \alpha_{9}, r 7=$ $\alpha_{1} \alpha_{9}, r 8=\alpha_{10} \alpha_{2}, r 9=\alpha_{8} \alpha_{9}$ e $r 10=\alpha_{10} \alpha_{7}$.

E os ciclos elementares são $C_{3}=\alpha_{8} \alpha_{7}$ e $C_{4}=\alpha_{9} \alpha_{10}$. Nesse caso, obtemos a solução 139 e mais uma hereditária.

Nesse anexo, temos mais essas relações do tipo $2: r 3=\alpha_{6} \alpha_{7}, r 4=\alpha_{1} \alpha_{7}, r 5=\alpha_{8} \alpha_{2}, r 6=\alpha_{9} \alpha_{8} \mathrm{e}$ $r 7=\alpha_{7} \alpha_{10}$.

E os ciclos elementares são $C_{3}=\alpha_{8} \alpha_{7}$ e $C_{4}=\alpha_{10} \alpha_{9}$. Logo, temos um corte que origina a álgebra $140 \mathrm{e}$ outro corte que origina uma hereditária.

As relações do tipo 2 são: $r 1=\alpha_{1} \alpha_{2} \alpha_{3} \alpha_{5}$ e $r 2=$ $\alpha_{6} \alpha_{2} \alpha_{3} \alpha_{4}$.

Os ciclos elementares são: $C_{1}=\alpha_{1} \alpha_{2} \alpha_{3} \alpha_{4}$ e $C_{2}=$ $\alpha_{2} \alpha_{3} \alpha_{5} \alpha_{7} \alpha_{6}$.

Completando, temos mais essas relações do tipo 2: $r 3=\alpha_{7} \alpha_{8}$ e $r 4=\alpha_{9} \alpha_{6}$.

E o ciclo elementar é $C_{3}=\alpha_{8} \alpha_{10} \alpha_{9}$. O programa exibe uma solução hereditária.

Nesse caso, temos mais essas relações do tipo 2: $r 3=\alpha_{7} \alpha_{8}, r 4=\alpha_{9} \alpha_{6}, r 3=\alpha_{7} \alpha_{10}, r 4=\alpha_{11} \alpha_{6}$, $r 7=\alpha_{9} \alpha_{10}$ e $r 8=\alpha_{11} \alpha_{8}$.

$\mathrm{E}$ os ciclos elementares são $C_{3}=\alpha_{9} \alpha_{8}$ e $C_{4}=$ $\alpha_{10} \alpha_{11}$. Nesse caso, obtemos uma solução hereditária.

Nesse anexo, temos mais essas relações do tipo 2: $r 3=\alpha_{7} \alpha_{8}, r 4=\alpha_{9} \alpha_{6}, r 5=\alpha_{10} \alpha_{9}$ e $r 6=\alpha_{8} \alpha_{11}$.

$\mathrm{E}$ os ciclos elementares são $C_{3}=\alpha_{9} \alpha_{8}$ e $C_{4}=$ $\alpha_{11} \alpha_{10}$. Logo, temos um corte que origina uma álgebra de incidência hereditária. 
(58)
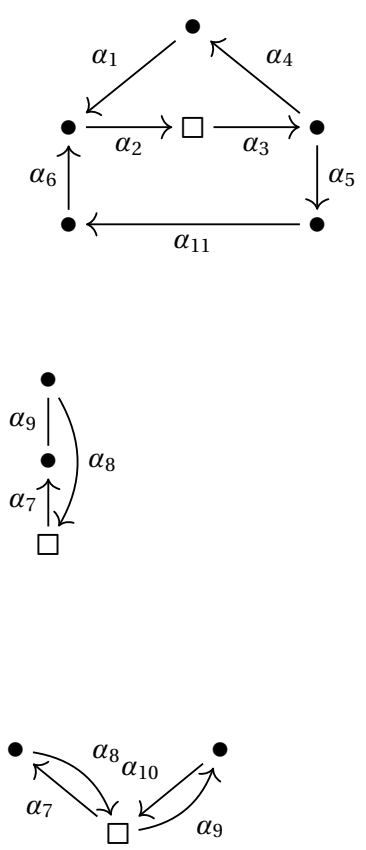

(59)

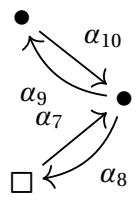

(60)
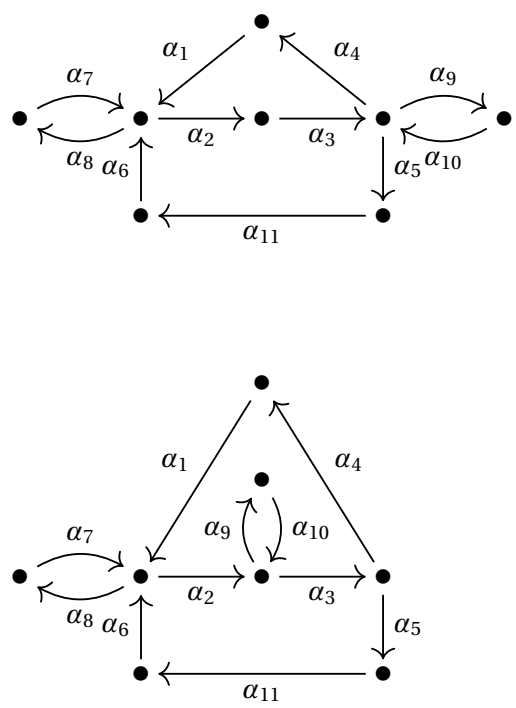

As relações do tipo 2 são: $r 1=\alpha_{1} \alpha_{2} \alpha_{3} \alpha_{5}$ e $r 2=$ $\alpha_{6} \alpha_{2} \alpha_{3} \alpha_{4}$.

Os ciclos elementares são: $C_{1}=\alpha_{1} \alpha_{2} \alpha_{3} \alpha_{4}$ e $C_{2}=$ $\alpha_{2} \alpha_{3} \alpha_{5} \alpha_{11} \alpha_{6}$.

Completando, temos mais essas relações do tipo 2: $r 3=\alpha_{2} \alpha_{7}$ e $r 4=\alpha_{8} \alpha_{3}$.

E o ciclo elementar é $C_{3}=\alpha_{7} \alpha_{9} \alpha_{8}$. O programa mostra dois cortes que originam as álgebras 141 e a sua álgebra dual.

Nesse caso, temos mais essas relações do tipo 2: $r 3=\alpha_{2} \alpha_{7}, r 4=\alpha_{8} \alpha_{3}, r 5=\alpha_{2} \alpha_{9}, r 6=\alpha_{10} \alpha_{3}$, $r 7=\alpha_{10} \alpha_{7}$ e $r 8=\alpha_{8} \alpha_{9}$.

E os ciclos elementares são $C_{3}=\alpha_{7} \alpha_{8}$ e $C_{4}=\alpha_{9} \alpha_{10}$. Nesse caso, obtemos a álgebra 142 e a sua álgebra dual.

Nesse anexo, temos mais essas relações do tipo 2: $r 3=\alpha_{2} \alpha_{7}, r 4=\alpha_{8} \alpha_{3}, r 5=\alpha_{7} \alpha_{9}$ e $r 6=\alpha_{10} \alpha_{8}$.

E os ciclos elementares são $C_{3}=\alpha_{8} \alpha_{7}$ e $C_{4}=\alpha_{10} \alpha_{9}$. Portanto, extraímos a álgebra 143 e a sua álgebra dual.

As relações do tipo 2 são: $r 1=\alpha_{1} \alpha_{2} \alpha_{3} \alpha_{5}, r 2=$ $\alpha_{6} \alpha_{2} \alpha_{3} \alpha_{4}, r 3=\alpha_{7} \alpha_{2}, r 4=\alpha_{1} \alpha_{8}, r 5=\alpha_{6} \alpha_{8}, r 6=$ $\alpha_{3} \alpha_{9}, r 7=\alpha_{10} \alpha_{4}$ e $r 8=\alpha_{10} \alpha_{5}$.

Os ciclos elementares são: $C_{1}=\alpha_{1} \alpha_{2} \alpha_{3} \alpha_{4}, C_{2}=$ $\alpha_{2} \alpha_{3} \alpha_{5} \alpha_{11} \alpha_{6}, C_{3}=\alpha_{10} \alpha_{9}$ e $C_{4}=\alpha_{7} \alpha_{8}$. Portanto, nesse caso, não temos solução.

As relações do tipo 2 são: $r 1=\alpha_{1} \alpha_{2} \alpha_{3} \alpha_{5}, r 2=$ $\alpha_{6} \alpha_{2} \alpha_{3} \alpha_{4}, r 3=\alpha_{7} \alpha_{2}, r 4=\alpha_{1} \alpha_{8}, r 5=\alpha_{6} \alpha_{8}, r 6=$ $\alpha_{2} \alpha_{9}$ e $r 7=\alpha_{10} \alpha_{3}$.

Os ciclos elementares são: $C_{1}=\alpha_{1} \alpha_{2} \alpha_{3} \alpha_{4}, C_{2}=$ $\alpha_{2} \alpha_{3} \alpha_{5} \alpha_{11} \alpha_{6}, C_{3}=\alpha_{10} \alpha_{9}$ e $C_{4}=\alpha_{7} \alpha_{8}$. Logo, o programa mostra apenas a solução 144 . 
(61)

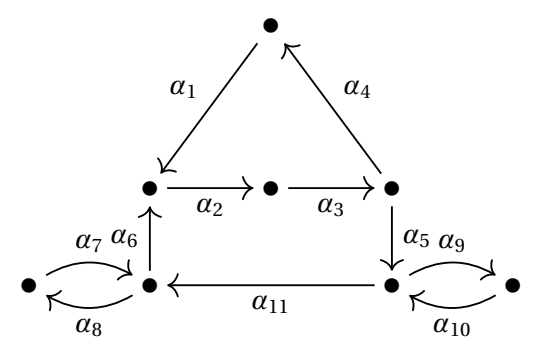

(62)
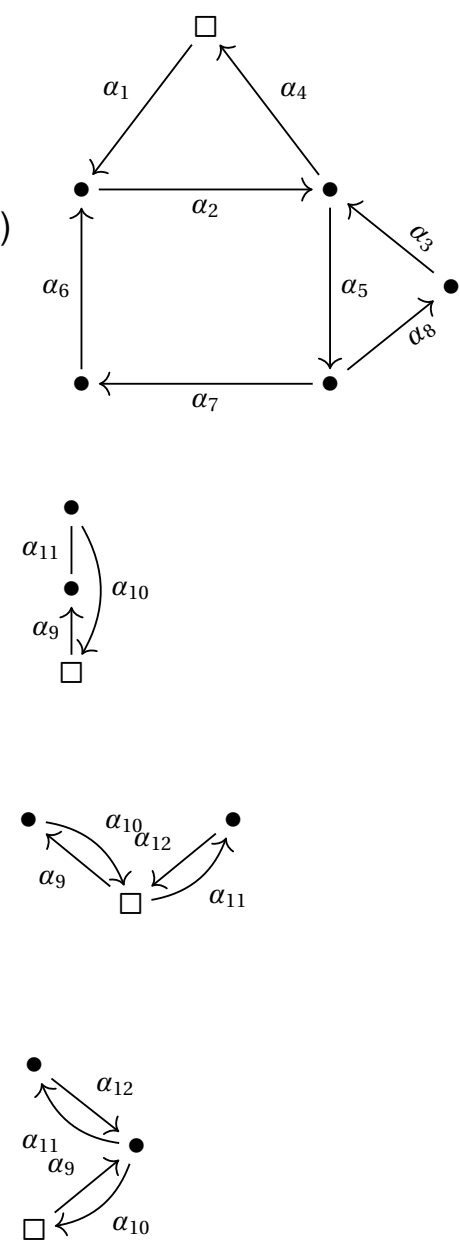

(63)
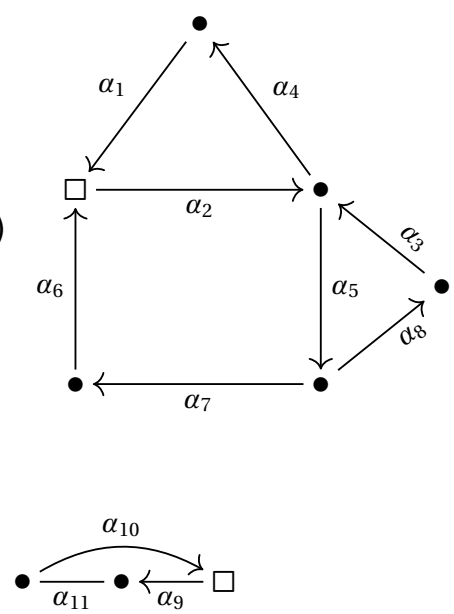

As relações do tipo 2 são: $r 1=\alpha_{1} \alpha_{2} \alpha_{3} \alpha_{5}, r 2=$ $\alpha_{6} \alpha_{2} \alpha_{3} \alpha_{4}, r 3=\alpha_{7} \alpha_{6}, r 4=\alpha_{11} \alpha_{8}, r 5=\alpha_{5} \alpha_{9} \mathrm{e}$ $r 7=\alpha_{10} \alpha_{11}$.

Os ciclos elementares são: $C_{1}=\alpha_{1} \alpha_{2} \alpha_{3} \alpha_{4}, C_{2}=$ $\alpha_{2} \alpha_{3} \alpha_{5} \alpha_{11} \alpha_{6}, C_{3}=\alpha_{10} \alpha_{9}$ e $C_{4}=\alpha_{7} \alpha_{8}$. Essa extensão trivial não tem corte que origine uma álgebra de incidência.

As relações do tipo 2 são: $r 1=\alpha_{1} \alpha_{2} \alpha_{5}, r 2=$ $\alpha_{6} \alpha_{2} \alpha_{4}, r 3=\alpha_{2} \alpha_{5} \alpha_{8}, r 4=\alpha_{3} \alpha_{5} \alpha_{7}$ e $r 5=\alpha_{3} \alpha_{4}$.

Os ciclos elementares são: $C_{1}=\alpha_{1} \alpha_{2} \alpha_{4}, C_{2}=$ $\alpha_{2} \alpha_{5} \alpha_{7} \alpha_{6}$ e $C_{3}=\alpha_{5} \alpha_{8} \alpha_{3}$.

Completando, temos mais essas relações do tipo 2: $r 6=\alpha_{4} \alpha_{9}$ e $r 7=\alpha_{10} \alpha_{1}$.

E o ciclo elementar é $C_{4}=\alpha_{9} \alpha_{11} \alpha_{10}$. O programa mostra um corte que origina a álgebra 145.

Nesse caso, temos mais essas relações do tipo 2: $r 6=\alpha_{4} \alpha_{9}, r 7=\alpha_{10} \alpha_{1}, r 8=\alpha_{4} \alpha_{11}, r 9=\alpha_{12} \alpha_{1}$, $r 10=\alpha_{12} \alpha_{9}$ e $r 11=\alpha_{10} \alpha_{11}$.

$\mathrm{E}$ os ciclos elementares são $C_{4}=\alpha_{9} \alpha_{10}$ e $C_{5}=$ $\alpha_{11} \alpha_{12}$. Nesse caso, obtemos a solução 146.

Nesse anexo, temos mais essas relações do tipo 2: $r 6=\alpha_{4} \alpha_{9}, r 7=\alpha_{10} \alpha_{1}, r 8=\alpha_{9} \alpha_{11}$ e $r 9=\alpha_{12} \alpha_{10}$. $\mathrm{E}$ os ciclos elementares são $C_{4}=\alpha_{10} \alpha_{9}$ e $C_{5}=$ $\alpha_{12} \alpha_{11}$. Portanto, extraímos a álgebra 147.

As relações do tipo 2 são: $r 1=\alpha_{1} \alpha_{2} \alpha_{5}, r 2=$ $\alpha_{6} \alpha_{2} \alpha_{4}, r 3=\alpha_{2} \alpha_{5} \alpha_{8}, r 4=\alpha_{3} \alpha_{5} \alpha_{7}$ e $r 5=\alpha_{3} \alpha_{4}$.

Os ciclos elementares são: $C_{1}=\alpha_{1} \alpha_{2} \alpha_{4}, C_{2}=$ $\alpha_{2} \alpha_{5} \alpha_{7} \alpha_{6}$ e $C_{3}=\alpha_{5} \alpha_{8} \alpha_{3}$.

Completando, temos mais essas relações do tipo 2 : $r 6=\alpha_{6} \alpha_{9}, r 7=\alpha_{1} \alpha_{9}$ e $r 8=\alpha_{10} \alpha_{2}$.

E o ciclo elementar é $C_{4}=\alpha_{9} \alpha_{11} \alpha_{10}$. O programa exibe a solução 148 . 

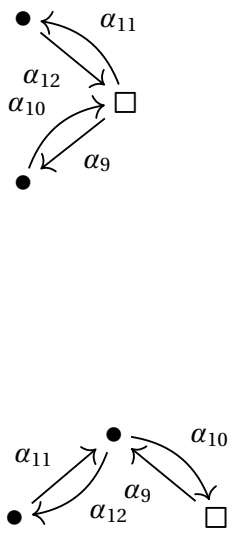

(64)

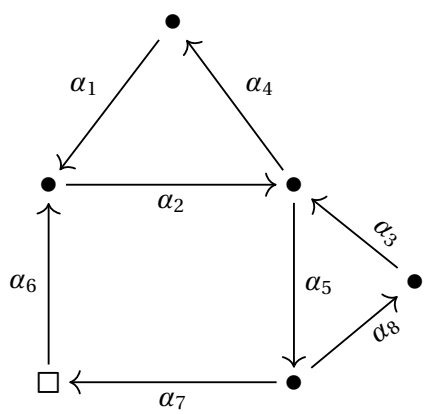

$\alpha_{10}\left(\begin{array}{l}\square \\ \searrow \\ \vdots \\ \vdots\end{array} \alpha_{11}\right.$
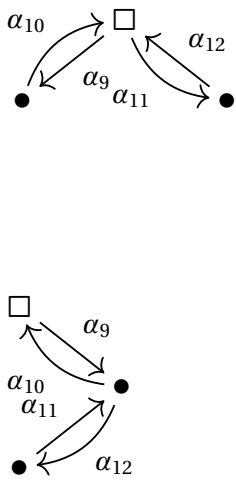

Nesse caso, temos mais essas relações do tipo 2: $r 6=\alpha_{6} \alpha_{9}, r 7=\alpha_{1} \alpha_{9}, r 8=\alpha_{10} \alpha_{2}, r 9=\alpha_{6} \alpha_{11}$, $r 10=\alpha_{1} \alpha_{11}, r 11=\alpha_{12} \alpha_{2}, r 12=\alpha_{10} \alpha_{11}$ e $r 13=$ $\alpha_{12} \alpha_{9}$.

$\mathrm{E}$ os ciclos elementares são $C_{4}=\alpha_{10} \alpha_{9}$ e $C_{5}=$ $\alpha_{11} \alpha_{12}$. Nesse caso, obtemos a solução 149.

Nesse anexo, temos mais essas relações do tipo 2: $r 6=\alpha_{6} \alpha_{9}, r 7=\alpha_{1} \alpha_{9}, r 8=\alpha_{10} \alpha_{2}, r 9=\alpha_{11} \alpha_{10} \mathrm{e}$ $r 10=\alpha_{9} \alpha_{12}$.

$\mathrm{E}$ os ciclos elementares são $C_{4}=\alpha_{10} \alpha_{9}$ e $C_{5}=$ $\alpha_{12} \alpha_{11}$. Logo, temos um corte que origina a álgebra 150.

As relações do tipo 2 são: $r 1=\alpha_{1} \alpha_{2} \alpha_{5}, r 2=$ $\alpha_{6} \alpha_{2} \alpha_{4}, r 3=\alpha_{2} \alpha_{5} \alpha_{8}, r 4=\alpha_{3} \alpha_{5} \alpha_{7}$ e $r 5=\alpha_{3} \alpha_{4}$.

Os ciclos elementares são: $C_{1}=\alpha_{1} \alpha_{2} \alpha_{4}, C_{2}=$ $\alpha_{2} \alpha_{5} \alpha_{7} \alpha_{6}$ e $C_{3}=\alpha_{5} \alpha_{8} \alpha_{3}$.

Completando, temos mais essas relações do tipo 2 : $r 6=\alpha_{7} \alpha_{9}$ e $r 7=\alpha_{10} \alpha_{6}$.

E o ciclo elementar é $C_{4}=\alpha_{9} \alpha_{11} \alpha_{10}$. O programa não exibe solução.

Nesse caso, temos mais essas relações do tipo 2: $r 6=\alpha_{7} \alpha_{9}, r 7=\alpha_{10} \alpha_{6}, r 8=\alpha_{7} \alpha_{11}, r 9=\alpha_{12} \alpha_{6}$, $r 10=\alpha_{10} \alpha_{11}$ e $r 11=\alpha_{12} \alpha_{9}$.

$\mathrm{E}$ os ciclos elementares são $C_{4}=\alpha_{10} \alpha_{9}$ e $C_{5}=$ $\alpha_{11} \alpha_{12}$. Nesse caso, não obtemos solução.

Nesse anexo, temos mais essas relações do tipo 2: $r 6=\alpha_{7} \alpha_{9}, r 7=\alpha_{10} \alpha_{6}, r 8=\alpha_{11} \alpha_{10}$ e $r 9=\alpha_{9} \alpha_{12}$. $\mathrm{E}$ os ciclos elementares são $C_{4}=\alpha_{10} \alpha_{9}$ e $C_{5}=$ $\alpha_{12} \alpha_{11}$. Logo, não temos um corte que origina uma álgebra de incidência. 
(65)

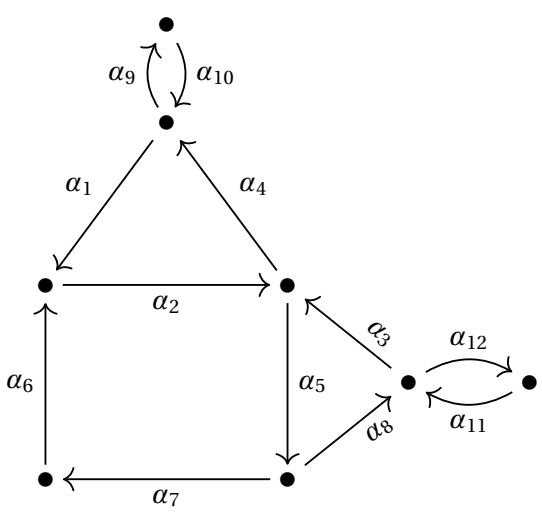

(66)

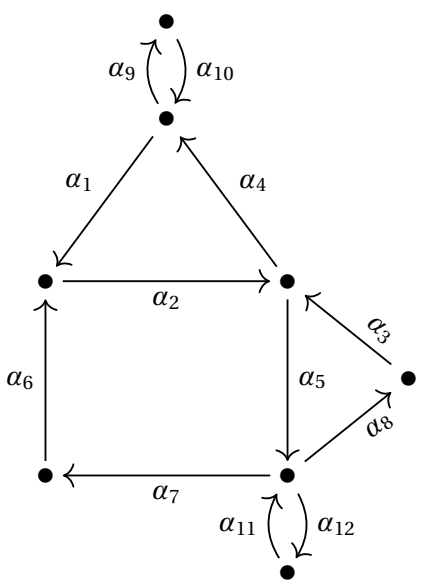

(67)

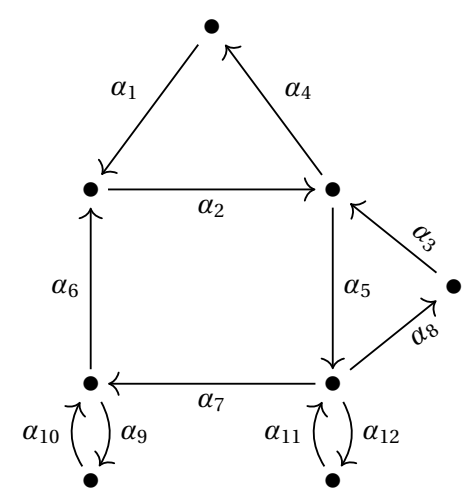

(68)

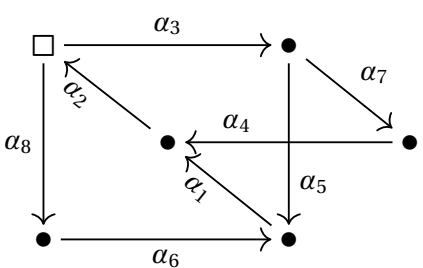

As relações do tipo 2 são: $r 1=\alpha_{1} \alpha_{2} \alpha_{5}, r 2=$ $\alpha_{6} \alpha_{2} \alpha_{4}, r 3=\alpha_{2} \alpha_{5} \alpha_{8}, r 4=\alpha_{3} \alpha_{5} \alpha_{7}, r 5=\alpha_{3} \alpha_{4}$, $r 6=\alpha_{10} \alpha_{1}, r 7=\alpha_{4} \alpha_{9}, r 8=\alpha_{11} \alpha_{3}$ e $r 9=\alpha_{8} \alpha_{12}$.

Os ciclos elementares são: $C_{1}=\alpha_{1} \alpha_{2} \alpha_{4}, C_{2}=$ $\alpha_{2} \alpha_{5} \alpha_{7} \alpha_{6}, C_{3}=\alpha_{5} \alpha_{8} \alpha_{3}, C_{4}=\alpha_{9} \alpha_{10}$ e $C_{5}=\alpha_{11} \alpha_{12}$. O programa não exibe nenhuma solução.

As relações do tipo 2 são: $r 1=\alpha_{1} \alpha_{2} \alpha_{5}, r 2=$ $\alpha_{6} \alpha_{2} \alpha_{4}, r 3=\alpha_{2} \alpha_{5} \alpha_{8}, r 4=\alpha_{3} \alpha_{5} \alpha_{7}, r 5=\alpha_{3} \alpha_{4}$, $r 6=\alpha_{10} \alpha_{1}, r 7=\alpha_{4} \alpha_{9}, r 8=\alpha_{11} \alpha_{8}, r 9=\alpha_{11} \alpha_{7} \mathrm{e}$ $r 10=\alpha_{5} \alpha_{12}$.

Os ciclos elementares são: $C_{1}=\alpha_{1} \alpha_{2} \alpha_{4}, C_{2}=$ $\alpha_{2} \alpha_{5} \alpha_{7} \alpha_{6}, C_{3}=\alpha_{5} \alpha_{8} \alpha_{3}, C_{4}=\alpha_{9} \alpha_{10}$ e $C_{5}=\alpha_{11} \alpha_{12}$. O programa mostra a solução 151 .

As relações do tipo 2 são: $r 1=\alpha_{1} \alpha_{2} \alpha_{5}, r 2=$ $\alpha_{6} \alpha_{2} \alpha_{4}, r 3=\alpha_{2} \alpha_{5} \alpha_{8}, r 4=\alpha_{3} \alpha_{5} \alpha_{7}, r 5=\alpha_{3} \alpha_{4}$, $r 6=\alpha_{10} \alpha_{6}, r 7=\alpha_{7} \alpha_{9}, r 8=\alpha_{11} \alpha_{8}, r 9=\alpha_{11} \alpha_{7} \mathrm{e}$ $r 10=\alpha_{5} \alpha_{12}$.

Os ciclos elementares são: $C_{1}=\alpha_{1} \alpha_{2} \alpha_{4}, C_{2}=$ $\alpha_{2} \alpha_{5} \alpha_{7} \alpha_{6}, C_{3}=\alpha_{5} \alpha_{8} \alpha_{3}, C_{4}=\alpha_{9} \alpha_{10}$ e $C_{5}=\alpha_{11} \alpha_{12}$. Portanto, não obtemos nenhuma álgebra de incidência.

Essa extensão trivial tem as seguintes relações do tipo 2: $r 1=\alpha_{6} \alpha_{1} \alpha_{2} \alpha_{3}, r 2=\alpha_{5} \alpha_{1} \alpha_{2} \alpha_{8}, r 3=$ $\alpha_{1} \alpha_{2} \alpha_{3} \alpha_{7}, r 4=\alpha_{4} \alpha_{2} \alpha_{3} \alpha_{5}$ e $r 5=\alpha_{4} \alpha_{2} \alpha_{8}$.

Essa extensão trivial apresenta os seguintes ciclos elementares: $C_{1}=\alpha_{6} \alpha_{1} \alpha_{2} \alpha_{8}, C_{2}=\alpha_{2} \alpha_{3} \alpha_{7} \alpha_{4}$ e $C_{3}=$ $\alpha_{1} \alpha_{2} \alpha_{3} \alpha_{5}$. 

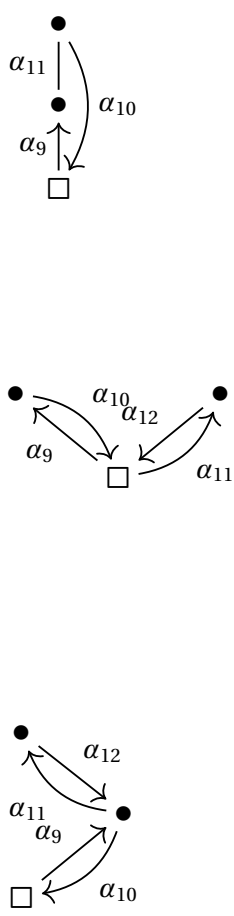

(69)
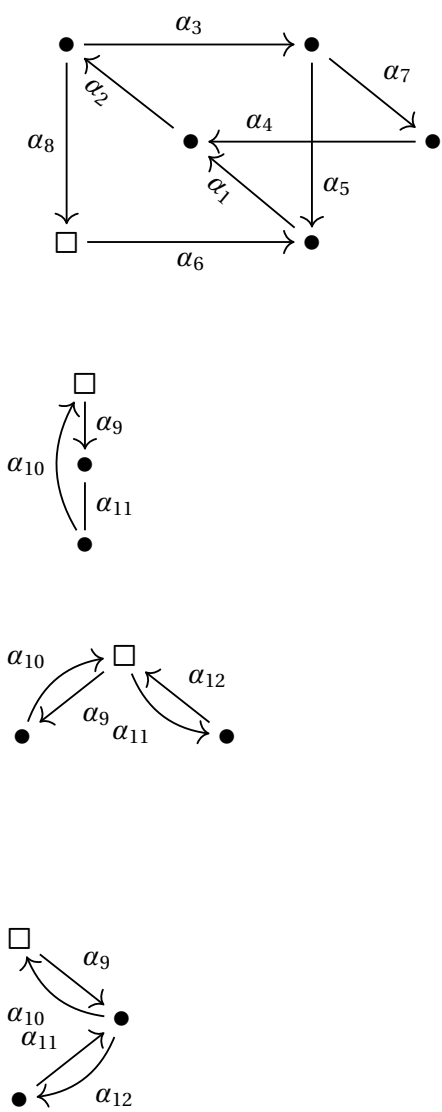

Completando, temos mais essas relações do tipo 2: $r 6=\alpha_{2} \alpha_{9}, r 7=\alpha_{10} \alpha_{3}$ e $r 8=\alpha_{10} \alpha_{8}$.

E o ciclo elementar é $C_{4}=\alpha_{9} \alpha_{11} \alpha_{10}$. O programa mostra dois corte, assim originando as álgebras 152 e 153.

Nesse caso, temos mais essas relações do tipo 2: $r 6=\alpha_{2} \alpha_{9}, r 7=\alpha_{10} \alpha_{3}, r 8=\alpha_{10} \alpha_{8}, r 9=\alpha_{2} \alpha_{11}$, $r 10=\alpha_{12} \alpha_{3}, r 11=\alpha_{12} \alpha_{8}, r 12=\alpha_{12} \alpha_{9}$ e $r 13=$ $\alpha_{10} \alpha_{11}$.

E os ciclos elementares são $C_{4}=\alpha_{9} \alpha_{10}$ e $C_{5}=$ $\alpha_{11} \alpha_{12}$. Portanto, obtemos as soluções 154 e 155.

Nesse anexo, temos mais essas relações do tipo 2: $r 6=\alpha_{2} \alpha_{9}, r 7=\alpha_{10} \alpha_{3}, r 8=\alpha_{10} \alpha_{8}, r 9=\alpha_{9} \alpha_{11} \mathrm{e}$ $r 10=\alpha_{12} \alpha_{10}$.

$\mathrm{E}$ os ciclos elementares são $C_{4}=\alpha_{10} \alpha_{9}$ e $C_{5}=$ $\alpha_{12} \alpha_{11}$. Logo, temos dois cortes que originam as álgebras de incidência 156 e 157.

Essa extensão trivial tem as seguintes relações do tipo 2: $r 1=\alpha_{6} \alpha_{1} \alpha_{2} \alpha_{3}, r 2=\alpha_{5} \alpha_{1} \alpha_{2} \alpha_{8}, r 3=$ $\alpha_{1} \alpha_{2} \alpha_{3} \alpha_{7}, r 4=\alpha_{4} \alpha_{2} \alpha_{3} \alpha_{5}$ e $r 5=\alpha_{4} \alpha_{2} \alpha_{8}$.

Essa extensão trivial apresenta os seguintes ciclos elementares: $C_{1}=\alpha_{6} \alpha_{1} \alpha_{2} \alpha_{8}, C_{2}=\alpha_{2} \alpha_{3} \alpha_{7} \alpha_{4}$ e $C_{3}=$ $\alpha_{1} \alpha_{2} \alpha_{3} \alpha_{5}$.

Completando, temos mais essas relações do tipo 2: $r 6=\alpha_{8} \alpha_{9}$ e $r 7=\alpha_{10} \alpha_{6}$.

E o ciclo elementar é $C_{4}=\alpha_{9} \alpha_{11} \alpha_{10}$. O programa exibe a solução 158.

Nesse caso, temos mais essas relações do tipo 2: $r 6=\alpha_{8} \alpha_{9}, r 7=\alpha_{10} \alpha_{6}, r 8=\alpha_{8} \alpha_{11}, r 9=\alpha_{12} \alpha_{6}$, $r 10=\alpha_{10} \alpha_{11}$ e $r 11=\alpha_{12} \alpha_{9}$.

$\mathrm{E}$ os ciclos elementares são $C_{4}=\alpha_{10} \alpha_{9}$ e $C_{5}=$ $\alpha_{11} \alpha_{12}$. Nesse caso, obtemos a álgebra 159 .

Nesse anexo, temos mais essas relações do tipo 2: $r 6=\alpha_{8} \alpha_{9}, r 7=\alpha_{10} \alpha_{6}, r 8=\alpha_{11} \alpha_{10}$ e $r 9=\alpha_{9} \alpha_{12}$. $\mathrm{E}$ os ciclos elementares são $C_{4}=\alpha_{10} \alpha_{9}$ e $C_{5}=$ $\alpha_{12} \alpha_{11}$. Logo, temos um corte que origina a álgebra 160. 
(70)
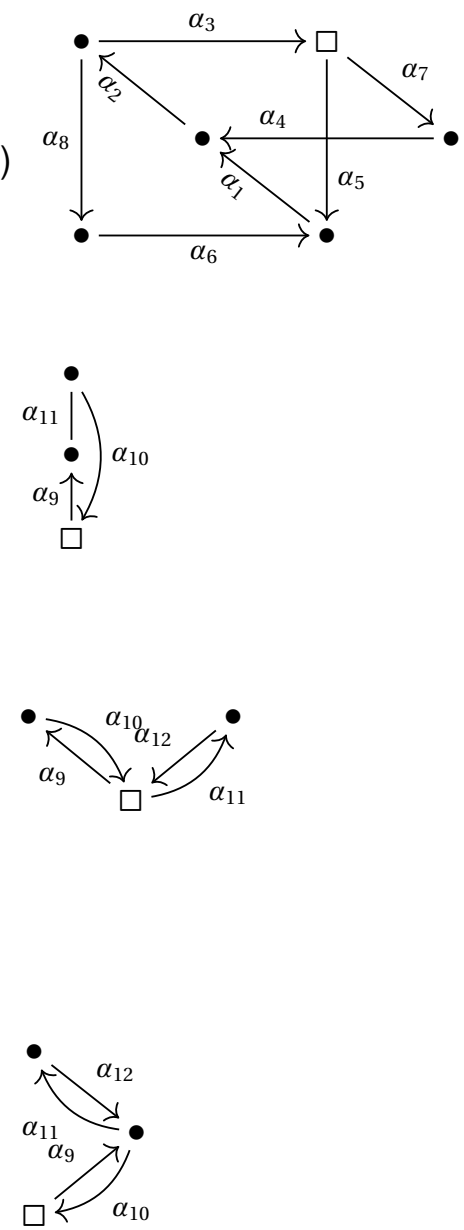

(71)

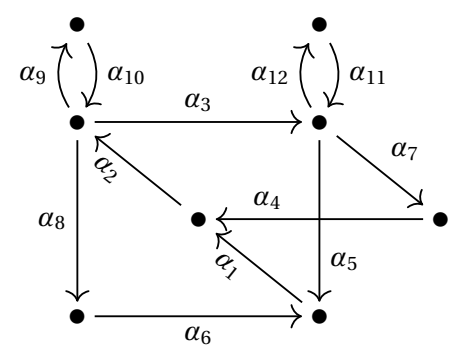

Essa extensão trivial tem as seguintes relações do tipo 2: $r 1=\alpha_{6} \alpha_{1} \alpha_{2} \alpha_{3}, r 2=\alpha_{5} \alpha_{1} \alpha_{2} \alpha_{8}, r 3=$ $\alpha_{1} \alpha_{2} \alpha_{3} \alpha_{7}, r 4=\alpha_{4} \alpha_{2} \alpha_{3} \alpha_{5}$ e $r 5=\alpha_{4} \alpha_{2} \alpha_{8}$.

Essa extensão trivial apresenta os seguintes ciclos elementares: $C_{1}=\alpha_{6} \alpha_{1} \alpha_{2} \alpha_{8}, C_{2}=\alpha_{2} \alpha_{3} \alpha_{7} \alpha_{4}$ e $C_{3}=$ $\alpha_{1} \alpha_{2} \alpha_{3} \alpha_{5}$.

Completando, temos mais essas relações do tipo 2: $r 6=\alpha_{3} \alpha_{9}, r 7=\alpha_{10} \alpha_{7}$ e $r 8=\alpha_{10} \alpha_{5}$.

E o ciclo elementar é $C_{4}=\alpha_{9} \alpha_{11} \alpha_{10}$. O programa mostra um corte, assim originando a álgebra 161.

Nesse caso, temos mais essas relações do tipo 2: $r 6=\alpha_{3} \alpha_{9}, r 7=\alpha_{10} \alpha_{7}, r 8=\alpha_{10} \alpha_{5}, r 9=\alpha_{3} \alpha_{11}$, $r 10=\alpha_{12} \alpha_{7}, r 11=\alpha_{12} \alpha_{5}, r 12=\alpha_{12} \alpha_{9}$ e $r 13=$ $\alpha_{10} \alpha_{11}$.

$\mathrm{E}$ os ciclos elementares são $C_{4}=\alpha_{9} \alpha_{10}$ e $C_{5}=$ $\alpha_{11} \alpha_{12}$. Portanto, obtemos a solução 162.

Nesse anexo, temos mais essas relações do tipo 2: $r 6=\alpha_{3} \alpha_{9}, r 7=\alpha_{10} \alpha_{7}, r 8=\alpha_{10} \alpha_{5}, r 9=\alpha_{9} \alpha_{11} \mathrm{e}$ $r 10=\alpha_{12} \alpha_{10}$.

$\mathrm{E}$ os ciclos elementares são $C_{4}=\alpha_{10} \alpha_{9}$ e $C_{5}=$ $\alpha_{12} \alpha_{11}$. Logo, temos um corte que origina a álgebra de incidência 163.

Essa extensão trivial tem as seguintes relações do tipo 2: $r 1=\alpha_{6} \alpha_{1} \alpha_{2} \alpha_{3}, r 2=\alpha_{5} \alpha_{1} \alpha_{2} \alpha_{8}, r 3=$ $\alpha_{1} \alpha_{2} \alpha_{3} \alpha_{7}, r 4=\alpha_{4} \alpha_{2} \alpha_{3} \alpha_{5}, r 5=\alpha_{4} \alpha_{2} \alpha_{8}, r 6=$ $\alpha_{2} \alpha_{9}, r 7=\alpha_{10} \alpha_{3}, r 8=\alpha_{10} \alpha_{8}, r 9=\alpha_{11} \alpha_{7}, r 10=$ $\alpha_{11} \alpha_{5}$ e $r 11=\alpha_{3} \alpha_{12}$.

Essa extensão trivial apresenta os seguintes ciclos elementares: $C_{1}=\alpha_{6} \alpha_{1} \alpha_{2} \alpha_{8}, C_{2}=\alpha_{2} \alpha_{3} \alpha_{7} \alpha_{4}, C_{3}=$ $\alpha_{1} \alpha_{2} \alpha_{3} \alpha_{5}, C_{4}=\alpha_{10} \alpha_{9}$ e $C_{5}=\alpha_{11} \alpha_{12}$. O programa exibe a álgebra 164 . 
(72)

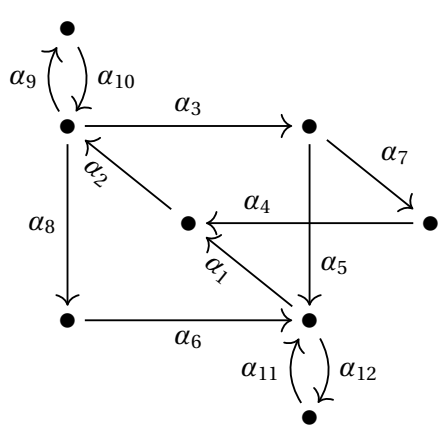

(73)

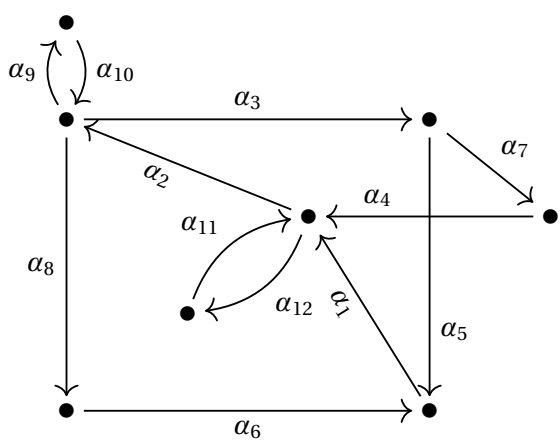

(74)

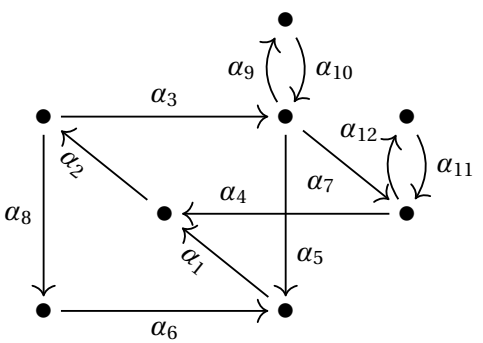

(75)

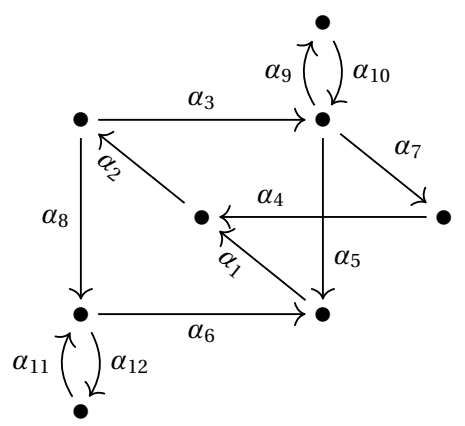

Essa extensão trivial tem as seguintes relações do tipo 2: $r 1=\alpha_{6} \alpha_{1} \alpha_{2} \alpha_{3}, r 2=\alpha_{5} \alpha_{1} \alpha_{2} \alpha_{8}, r 3=$ $\alpha_{1} \alpha_{2} \alpha_{3} \alpha_{7}, r 4=\alpha_{4} \alpha_{2} \alpha_{3} \alpha_{5}, r 5=\alpha_{4} \alpha_{2} \alpha_{8}, r 6=$ $\alpha_{2} \alpha_{9}, r 7=\alpha_{10} \alpha_{3}, r 8=\alpha_{10} \alpha_{8}, r 9=\alpha_{11} \alpha_{1}, r 10=$ $\alpha_{5} \alpha_{12}$ e $r 11=\alpha_{6} \alpha_{12}$.

Essa extensão trivial apresenta os seguintes ciclos elementares: $C_{1}=\alpha_{6} \alpha_{1} \alpha_{2} \alpha_{8}, C_{2}=\alpha_{2} \alpha_{3} \alpha_{7} \alpha_{4}, C_{3}=$ $\alpha_{1} \alpha_{2} \alpha_{3} \alpha_{5}, C_{4}=\alpha_{10} \alpha_{9}$ e $C_{5}=\alpha_{11} \alpha_{12}$. Nessa álgebra, não obtemos nenhuma álgebra de incidência através do corte.

Essa extensão trivial tem as seguintes relações do tipo 2: $r 1=\alpha_{6} \alpha_{1} \alpha_{2} \alpha_{3}, r 2=\alpha_{5} \alpha_{1} \alpha_{2} \alpha_{8}, r 3=$ $\alpha_{1} \alpha_{2} \alpha_{3} \alpha_{7}, r 4=\alpha_{4} \alpha_{2} \alpha_{3} \alpha_{5}, r 5=\alpha_{4} \alpha_{2} \alpha_{8}, r 6=$ $\alpha_{2} \alpha_{9}, r 7=\alpha_{10} \alpha_{3}, r 8=\alpha_{10} \alpha_{8}, r 9=\alpha_{11} \alpha_{2}, r 10=$ $\alpha_{4} \alpha_{12}$ e $r 11=\alpha_{1} \alpha_{12}$.

Essa extensão trivial apresenta os seguintes ciclos elementares: $C_{1}=\alpha_{6} \alpha_{1} \alpha_{2} \alpha_{8}, C_{2}=\alpha_{2} \alpha_{3} \alpha_{7} \alpha_{4}, C_{3}=$ $\alpha_{1} \alpha_{2} \alpha_{3} \alpha_{5}, C_{4}=\alpha_{10} \alpha_{9}$ e $C_{5}=\alpha_{11} \alpha_{12}$. Logo, obtemos a álgebra 165.

Essa extensão trivial tem as seguintes relações do tipo 2: $r 1=\alpha_{6} \alpha_{1} \alpha_{2} \alpha_{3}, r 2=\alpha_{5} \alpha_{1} \alpha_{2} \alpha_{8}, r 3=$ $\alpha_{1} \alpha_{2} \alpha_{3} \alpha_{7}, r 4=\alpha_{4} \alpha_{2} \alpha_{3} \alpha_{5}, r 5=\alpha_{4} \alpha_{2} \alpha_{8}, r 6=$ $\alpha_{3} \alpha_{9}, r 7=\alpha_{10} \alpha_{7}, r 8=\alpha_{10} \alpha_{5}, r 9=\alpha_{11} \alpha_{4}$ e $r 10=$ $\alpha_{7} \alpha_{12}$.

Essa extensão trivial apresenta os seguintes ciclos elementares: $C_{1}=\alpha_{6} \alpha_{1} \alpha_{2} \alpha_{8}, C_{2}=\alpha_{2} \alpha_{3} \alpha_{7} \alpha_{4}, C_{3}=$ $\alpha_{1} \alpha_{2} \alpha_{3} \alpha_{5}, C_{4}=\alpha_{10} \alpha_{9}$ e $C_{5}=\alpha_{11} \alpha_{12}$. O programa não exibe resposta para esse caso.

Essa extensão trivial tem as seguintes relações do tipo 2: $r 1=\alpha_{6} \alpha_{1} \alpha_{2} \alpha_{3}, r 2=\alpha_{5} \alpha_{1} \alpha_{2} \alpha_{8}, r 3=$ $\alpha_{1} \alpha_{2} \alpha_{3} \alpha_{7}, r 4=\alpha_{4} \alpha_{2} \alpha_{3} \alpha_{5}, r 5=\alpha_{4} \alpha_{2} \alpha_{8}, r 6=$ $\alpha_{3} \alpha_{9}, r 7=\alpha_{10} \alpha_{7}, r 8=\alpha_{10} \alpha_{5}, r 9=\alpha_{11} \alpha_{4}$ e $r 10=$ $\alpha_{7} \alpha_{12}$.

Essa extensão trivial apresenta os seguintes ciclos elementares: $C_{1}=\alpha_{6} \alpha_{1} \alpha_{2} \alpha_{8}, C_{2}=\alpha_{2} \alpha_{3} \alpha_{7} \alpha_{4}, C_{3}=$ $\alpha_{1} \alpha_{2} \alpha_{3} \alpha_{5}, C_{4}=\alpha_{10} \alpha_{9}$ e $C_{5}=\alpha_{11} \alpha_{12}$. Portanto, através do corte, temos a álgebra 166. 
(76)
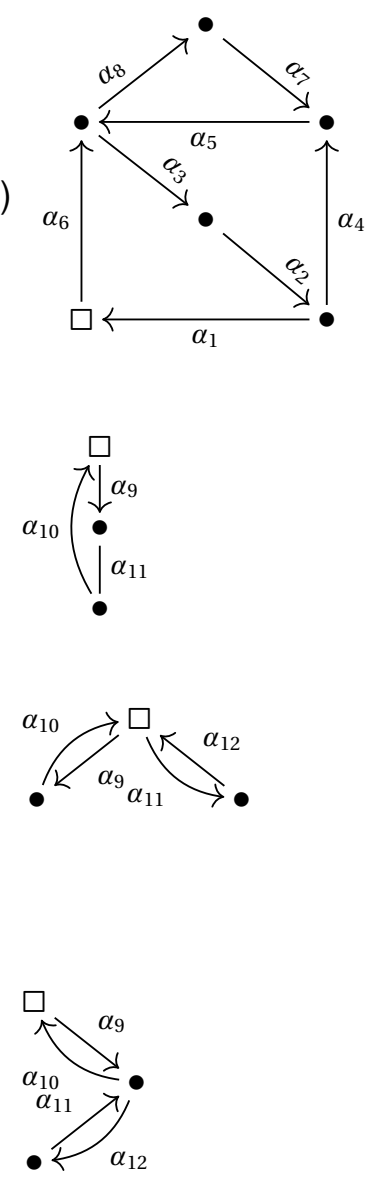

(77)
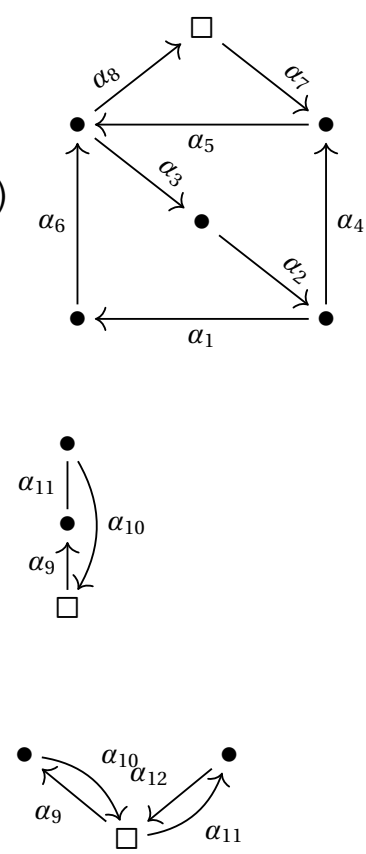

Essa extensão trivial tem as seguintes relações do tipo 2: $r 1=\alpha_{6} \alpha_{3} \alpha_{2} \alpha_{4}, r 2=\alpha_{5} \alpha_{3} \alpha_{2} \alpha_{1}, r 3=$ $\alpha_{4} \alpha_{5} \alpha_{8}, r 4=\alpha_{7} \alpha_{5} \alpha_{3}$ e $r 5=\alpha_{6} \alpha_{8}$.

Essa extensão trivial apresenta os seguintes ciclos elementares: $C_{1}=\alpha_{1} \alpha_{6} \alpha_{3} \alpha_{2}, C_{2}=\alpha_{3} \alpha_{2} \alpha_{4} \alpha_{5}$ e $C_{3}=$ $\alpha_{5} \alpha_{8} \alpha_{7}$.

Completando, temos mais essas relações do tipo 2 : $r 6=\alpha_{1} \alpha_{9}$ e $r 7=\alpha_{10} \alpha_{6}$.

E o ciclo elementar é $C_{4}=\alpha_{9} \alpha_{11} \alpha_{10}$. O programa exibe a solução 167.

Nesse caso, temos mais essas relações do tipo 2: $r 6=\alpha_{1} \alpha_{9}, r 7=\alpha_{10} \alpha_{6}, r 8=\alpha_{1} \alpha_{11}, r 9=\alpha_{12} \alpha_{6}$, $r 10=\alpha_{10} \alpha_{11}$ e $r 11=\alpha_{12} \alpha_{9}$.

$\mathrm{E}$ os ciclos elementares são $C_{4}=\alpha_{10} \alpha_{9}$ e $C_{5}=$ $\alpha_{11} \alpha_{12}$. Nesse caso, obtemos a álgebra 168.

Nesse anexo, temos mais essas relações do tipo 2: $r 6=\alpha_{1} \alpha_{9}, r 7=\alpha_{10} \alpha_{6}, r 8=\alpha_{11} \alpha_{10}$ e $r 9=\alpha_{9} \alpha_{12}$. $\mathrm{E}$ os ciclos elementares são $C_{4}=\alpha_{10} \alpha_{9}$ e $C_{5}=$ $\alpha_{12} \alpha_{11}$. Logo, temos um corte que origina a álgebra 169.

Essa extensão trivial tem as seguintes relações do tipo 2: $r 1=\alpha_{6} \alpha_{3} \alpha_{2} \alpha_{4}, r 2=\alpha_{5} \alpha_{3} \alpha_{2} \alpha_{1}, r 3=$ $\alpha_{4} \alpha_{5} \alpha_{8}, r 4=\alpha_{7} \alpha_{5} \alpha_{3}$ e $r 5=\alpha_{6} \alpha_{8}$.

Essa extensão trivial apresenta os seguintes ciclos elementares: $C_{1}=\alpha_{1} \alpha_{6} \alpha_{3} \alpha_{2}, C_{2}=\alpha_{3} \alpha_{2} \alpha_{4} \alpha_{5}$ e $C_{3}=$ $\alpha_{5} \alpha_{8} \alpha_{7}$.

Completando, temos mais essas relações do tipo 2 : $r 6=\alpha_{8} \alpha_{9}$ e $r 7=\alpha_{10} \alpha_{7}$.

E o ciclo elementar é $C_{4}=\alpha_{9} \alpha_{11} \alpha_{10}$. O programa mostra um corte, assim originando a álgebra 170 .

Nesse caso, temos mais essas relações do tipo 2: $r 6=\alpha_{8} \alpha_{9}, r 7=\alpha_{10} \alpha_{7}, r 8=\alpha_{8} \alpha_{11}, r 9=\alpha_{12} \alpha_{7}$, $r 10=\alpha_{12} \alpha_{9}$ e $r 11=\alpha_{10} \alpha_{11}$.

$\mathrm{E}$ os ciclos elementares são $C_{4}=\alpha_{9} \alpha_{10}$ e $C_{5}=$ $\alpha_{11} \alpha_{12}$. Portanto, obtemos a solução 171 . 


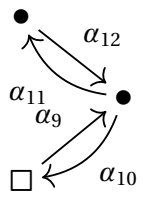

(78)
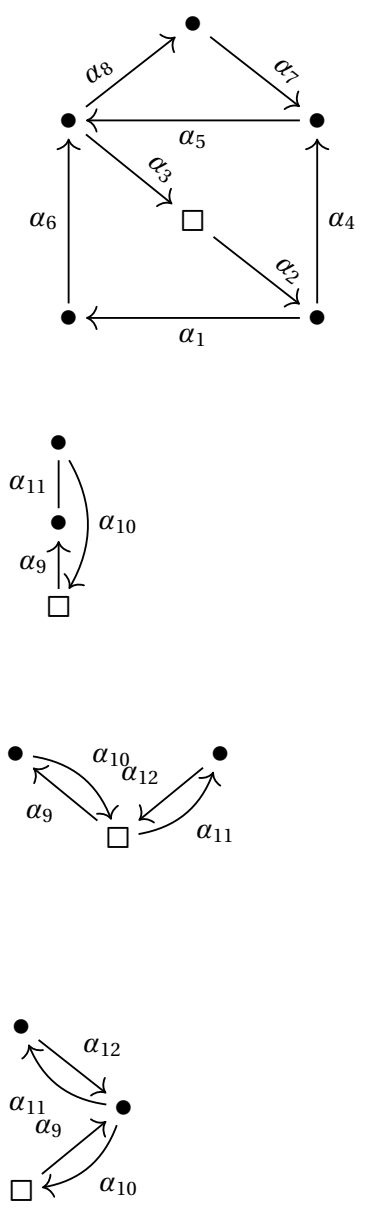

(79)
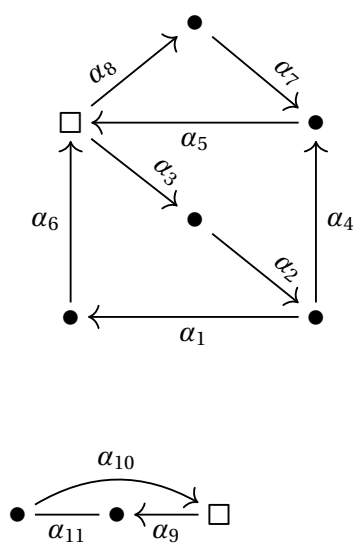

Nesse anexo, temos mais essas relações do tipo 2: $r 6=\alpha_{8} \alpha_{9}, r 7=\alpha_{10} \alpha_{7}, r 8=\alpha_{9} \alpha_{11}$ e $r 9=\alpha_{12} \alpha_{10}$. $\mathrm{E}$ os ciclos elementares são $C_{4}=\alpha_{10} \alpha_{9}$ e $C_{5}=$ $\alpha_{12} \alpha_{11}$. Logo, temos um corte que origina a álgebra de incidência 172.

Essa extensão trivial tem as seguintes relações do tipo 2: $r 1=\alpha_{6} \alpha_{3} \alpha_{2} \alpha_{4}, r 2=\alpha_{5} \alpha_{3} \alpha_{2} \alpha_{1}, r 3=$ $\alpha_{4} \alpha_{5} \alpha_{8}, r 4=\alpha_{7} \alpha_{5} \alpha_{3}$ e $r 5=\alpha_{6} \alpha_{8}$.

Essa extensão trivial apresenta os seguintes ciclos elementares: $C_{1}=\alpha_{1} \alpha_{6} \alpha_{3} \alpha_{2}, C_{2}=\alpha_{3} \alpha_{2} \alpha_{4} \alpha_{5}$ e $C_{3}=$ $\alpha_{5} \alpha_{8} \alpha_{7}$.

Completando, temos mais essas relações do tipo 2: $r 6=\alpha_{3} \alpha_{9}$ e $r 7=\alpha_{10} \alpha_{2}$.

E o ciclo elementar é $C_{4}=\alpha_{9} \alpha_{11} \alpha_{10}$. O programa mostra um corte, assim originando a álgebra 173.

Nesse caso, temos mais essas relações do tipo 2: $r 6=\alpha_{3} \alpha_{9}, r 7=\alpha_{10} \alpha_{2}, r 8=\alpha_{3} \alpha_{11}, r 9=\alpha_{12} \alpha_{2}$, $r 10=\alpha_{12} \alpha_{9}$ e $r 11=\alpha_{10} \alpha_{11}$.

$\mathrm{E}$ os ciclos elementares são $C_{4}=\alpha_{9} \alpha_{10}$ e $C_{5}=$ $\alpha_{11} \alpha_{12}$. Portanto, obtemos a solução 174 .

Nesse anexo, temos mais essas relações do tipo 2: $r 6=\alpha_{3} \alpha_{9}, r 7=\alpha_{10} \alpha_{2}, r 8=\alpha_{9} \alpha_{11}$ e $r 9=\alpha_{12} \alpha_{10}$. $\mathrm{E}$ os ciclos elementares são $C_{4}=\alpha_{10} \alpha_{9}$ e $C_{5}=$ $\alpha_{12} \alpha_{11}$. Logo, temos um corte que origina a álgebra de incidência 175.

Essa extensão trivial tem as seguintes relações do tipo 2: $r 1=\alpha_{6} \alpha_{3} \alpha_{2} \alpha_{4}, r 2=\alpha_{5} \alpha_{3} \alpha_{2} \alpha_{1}, r 3=$ $\alpha_{4} \alpha_{5} \alpha_{8}, r 4=\alpha_{7} \alpha_{5} \alpha_{3}$ e $r 5=\alpha_{6} \alpha_{8}$.

Essa extensão trivial apresenta os seguintes ciclos elementares: $C_{1}=\alpha_{1} \alpha_{6} \alpha_{3} \alpha_{2}, C_{2}=\alpha_{3} \alpha_{2} \alpha_{4} \alpha_{5}$ e $C_{3}=$ $\alpha_{5} \alpha_{8} \alpha_{7}$.

Completando, temos mais essas relações do tipo 2 : $r 6=\alpha_{5} \alpha_{9}, r 7=\alpha_{6} \alpha_{9}, r 8=\alpha_{10} \alpha_{3}$ e $r 9=\alpha_{10} \alpha_{8}$.

E o ciclo elementar é $C_{4}=\alpha_{9} \alpha_{11} \alpha_{10}$. O programa exibe as soluções 176 e 177. 

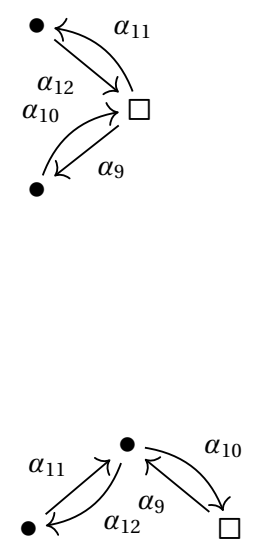

(80)
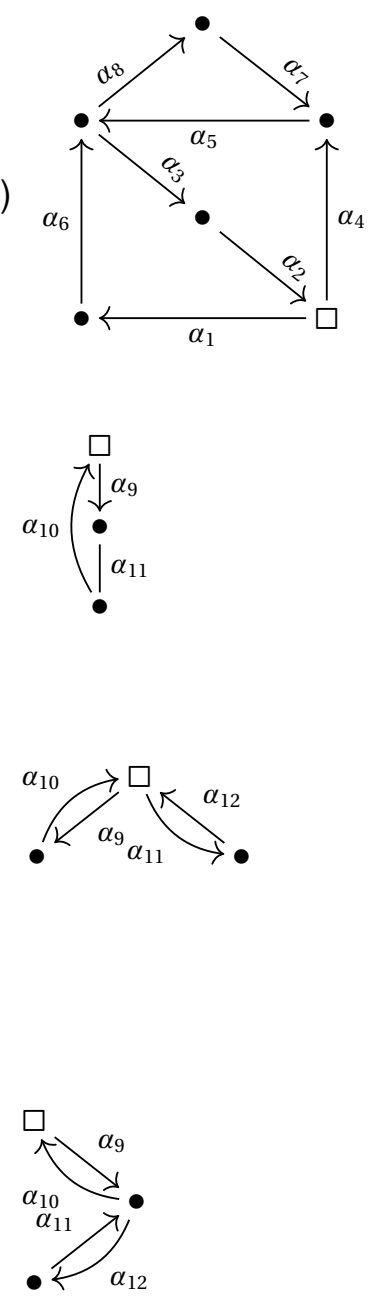

Nesse caso, temos mais essas relações do tipo 2: $r 6=\alpha_{6} \alpha_{9}, r 7=\alpha_{5} \alpha_{9}, r 8=\alpha_{10} \alpha_{3}, r 9=\alpha_{10} \alpha_{8}$, $r 10=\alpha_{6} \alpha_{11}, r 11=\alpha_{5} \alpha_{11}, r 12=\alpha_{12} \alpha_{3}, r 13=$ $\alpha_{12} \alpha_{8}, r 14=\alpha_{10} \alpha_{11}$ e $r 15=\alpha_{12} \alpha_{9}$.

$\mathrm{E}$ os ciclos elementares são $C_{4}=\alpha_{10} \alpha_{9}$ e $C_{5}=$ $\alpha_{11} \alpha_{12}$. Nesse caso, obtemos as soluções 178 e 179. Nesse anexo, temos mais essas relações do tipo 2: $r 6=\alpha_{6} \alpha_{9}, r 7=\alpha_{5} \alpha_{9}, r 8=\alpha_{10} \alpha_{3}, r 9=\alpha_{10} \alpha_{8}$, $r 10=\alpha_{11} \alpha_{10}$ e $r 11=\alpha_{9} \alpha_{12}$.

$\mathrm{E}$ os ciclos elementares são $C_{4}=\alpha_{10} \alpha_{9}$ e $C_{5}=$ $\alpha_{12} \alpha_{11}$. Logo, temos dois cortes que originam as álgebras 180 e 181 .

Essa extensão trivial tem as seguintes relações do tipo 2: $r 1=\alpha_{6} \alpha_{3} \alpha_{2} \alpha_{4}, r 2=\alpha_{5} \alpha_{3} \alpha_{2} \alpha_{1}, r 3=$ $\alpha_{4} \alpha_{5} \alpha_{8}, r 4=\alpha_{7} \alpha_{5} \alpha_{3}$ e $r 5=\alpha_{6} \alpha_{8}$.

Essa extensão trivial apresenta os seguintes ciclos elementares: $C_{1}=\alpha_{1} \alpha_{6} \alpha_{3} \alpha_{2}, C_{2}=\alpha_{3} \alpha_{2} \alpha_{4} \alpha_{5}$ e $C_{3}=$ $\alpha_{5} \alpha_{8} \alpha_{7}$.

Completando, temos mais essas relações do tipo 2: $r 6=\alpha_{2} \alpha_{9}, r 7=\alpha_{10} \alpha_{4}$ e $r 8=\alpha_{10} \alpha_{1}$.

E o ciclo elementar é $C_{4}=\alpha_{9} \alpha_{11} \alpha_{10}$. O programa não exibe solução.

Nesse caso, temos mais essas relações do tipo 2: $r 6=\alpha_{2} \alpha_{9}, r 7=\alpha_{10} \alpha_{4}, r 8=\alpha_{10} \alpha_{1}, r 9=\alpha_{2} \alpha_{11}$, $r 10=\alpha_{12} \alpha_{4}, r 11=\alpha_{12} \alpha_{1}, r 12=\alpha_{10} \alpha_{11}$ e $r 13=$ $\alpha_{12} \alpha_{9}$.

$\mathrm{E}$ os ciclos elementares são $C_{4}=\alpha_{10} \alpha_{9}$ e $C_{5}=$ $\alpha_{11} \alpha_{12}$. Nesse caso, obtemos a mesma resposta anterior.

Nesse anexo, temos mais essas relações do tipo 2: $r 6=\alpha_{2} \alpha_{9}, r 7=\alpha_{10} \alpha_{4}, r 8=\alpha_{10} \alpha_{1}, r 9=\alpha_{11} \alpha_{10} \mathrm{e}$ $r 10=\alpha_{9} \alpha_{12}$.

$\mathrm{E}$ os ciclos elementares são $C_{4}=\alpha_{10} \alpha_{9}$ e $C_{5}=$ $\alpha_{12} \alpha_{11}$. Logo, continuamos sem solução. 
(81)

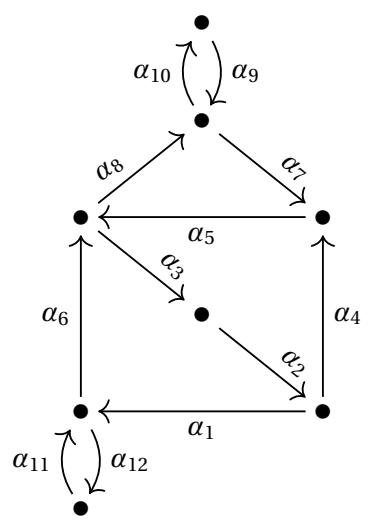

(82)

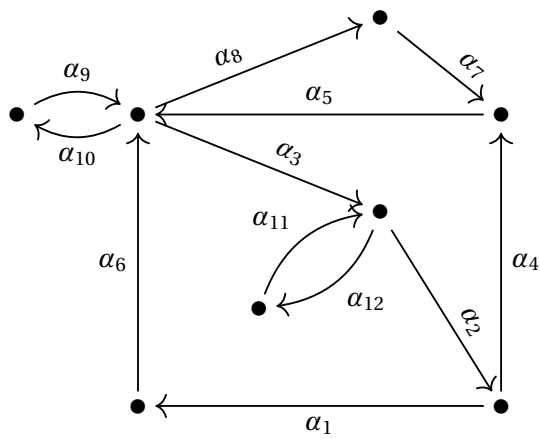

(83)

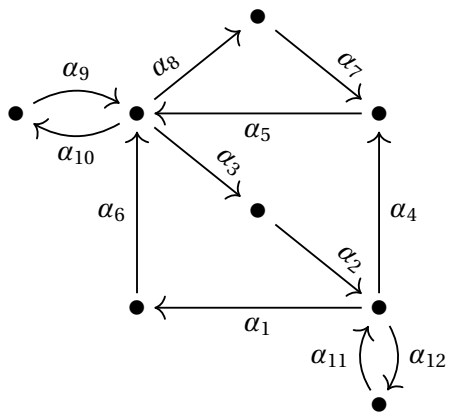

(84)

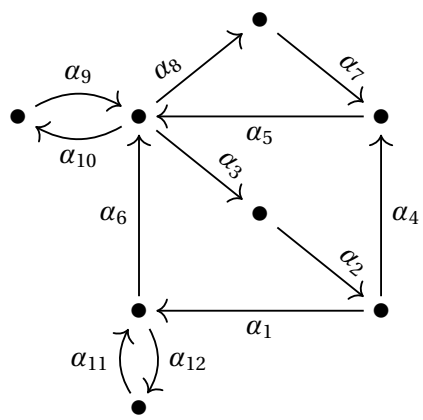

Essa extensão trivial tem as seguintes relações do tipo 2: $r 1=\alpha_{6} \alpha_{3} \alpha_{2} \alpha_{4}, r 2=\alpha_{5} \alpha_{3} \alpha_{2} \alpha_{1}, r 3=$ $\alpha_{4} \alpha_{5} \alpha_{8}, r 4=\alpha_{7} \alpha_{5} \alpha_{3}, r 5=\alpha_{6} \alpha_{8}, r 6=\alpha_{9} \alpha_{7}$, $r 7=\alpha_{8} \alpha_{10}, r 8=\alpha_{11} \alpha_{6}$ e $r 9=\alpha_{1} \alpha_{12}$.

Essa extensão trivial apresenta os seguintes ciclos elementares: $C_{1}=\alpha_{1} \alpha_{6} \alpha_{3} \alpha_{2}, C_{2}=\alpha_{3} \alpha_{2} \alpha_{4} \alpha_{5}, C_{3}=$ $\alpha_{5} \alpha_{8} \alpha_{7}, C_{4}=\alpha_{9} \alpha_{10}$ e $C_{5}=\alpha_{11} \alpha_{12}$. O programa não exibe nenhuma solução.

Essa extensão trivial tem as seguintes relações do tipo 2: $r 1=\alpha_{6} \alpha_{3} \alpha_{2} \alpha_{4}, r 2=\alpha_{5} \alpha_{3} \alpha_{2} \alpha_{1}, r 3=$ $\alpha_{4} \alpha_{5} \alpha_{8}, r 4=\alpha_{7} \alpha_{5} \alpha_{3}, r 5=\alpha_{6} \alpha_{8}, r 6=\alpha_{9} \alpha_{8}$, $r 7=\alpha_{9} \alpha_{3}, r 8=\alpha_{5} \alpha_{10}, r 9=\alpha_{6} \alpha_{10}, r 10=\alpha_{11} \alpha_{2}$ e $r 11=\alpha_{3} \alpha_{12}$.

Essa extensão trivial apresenta os seguintes ciclos elementares: $C_{1}=\alpha_{1} \alpha_{6} \alpha_{3} \alpha_{2}, C_{2}=\alpha_{3} \alpha_{2} \alpha_{4} \alpha_{5}, C_{3}=$ $\alpha_{5} \alpha_{8} \alpha_{7}, C_{4}=\alpha_{9} \alpha_{10}$ e $C_{5}=\alpha_{11} \alpha_{12}$. Portanto, obtemos a álgebra 182 .

Essa extensão trivial tem as seguintes relações do tipo 2: $r 1=\alpha_{6} \alpha_{3} \alpha_{2} \alpha_{4}, r 2=\alpha_{5} \alpha_{3} \alpha_{2} \alpha_{1}, r 3=$ $\alpha_{4} \alpha_{5} \alpha_{8}, r 4=\alpha_{7} \alpha_{5} \alpha_{3}, r 5=\alpha_{6} \alpha_{8}, r 6=\alpha_{9} \alpha_{8}$, $r 7=\alpha_{9} \alpha_{3}, r 8=\alpha_{5} \alpha_{10}, r 9=\alpha_{6} \alpha_{10}, r 10=\alpha_{11} \alpha_{1}$, $r 11=\alpha_{11} \alpha_{4}$ e $r 12=\alpha_{2} \alpha_{12}$.

Essa extensão trivial apresenta os seguintes ciclos elementares: $C_{1}=\alpha_{1} \alpha_{6} \alpha_{3} \alpha_{2}, C_{2}=\alpha_{3} \alpha_{2} \alpha_{4} \alpha_{5}, C_{3}=$ $\alpha_{5} \alpha_{8} \alpha_{7}, C_{4}=\alpha_{9} \alpha_{10}$ e $C_{5}=\alpha_{11} \alpha_{12}$. Não temos solução para esse caso.

Essa extensão trivial tem as seguintes relações do tipo 2: $r 1=\alpha_{6} \alpha_{3} \alpha_{2} \alpha_{4}, r 2=\alpha_{5} \alpha_{3} \alpha_{2} \alpha_{1}, r 3=$ $\alpha_{4} \alpha_{5} \alpha_{8}, r 4=\alpha_{7} \alpha_{5} \alpha_{3}, r 5=\alpha_{6} \alpha_{8}, r 6=\alpha_{9} \alpha_{8}$, $r 7=\alpha_{9} \alpha_{3}, r 8=\alpha_{5} \alpha_{10}, r 9=\alpha_{6} \alpha_{10}, r 10=\alpha_{11} \alpha_{6}$ e $r 11=\alpha_{1} \alpha_{12}$.

Essa extensão trivial apresenta os seguintes ciclos elementares: $C_{1}=\alpha_{1} \alpha_{6} \alpha_{3} \alpha_{2}, C_{2}=\alpha_{3} \alpha_{2} \alpha_{4} \alpha_{5}, C_{3}=$ $\alpha_{5} \alpha_{8} \alpha_{7}, C_{4}=\alpha_{9} \alpha_{10}$ e $C_{5}=\alpha_{11} \alpha_{12}$. O programa exibe a solução 183 . 


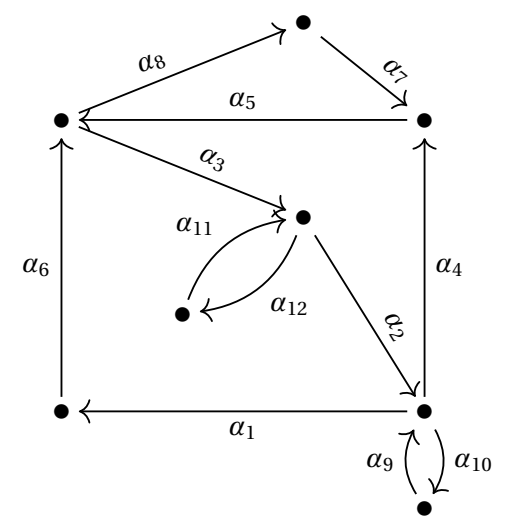

(86)
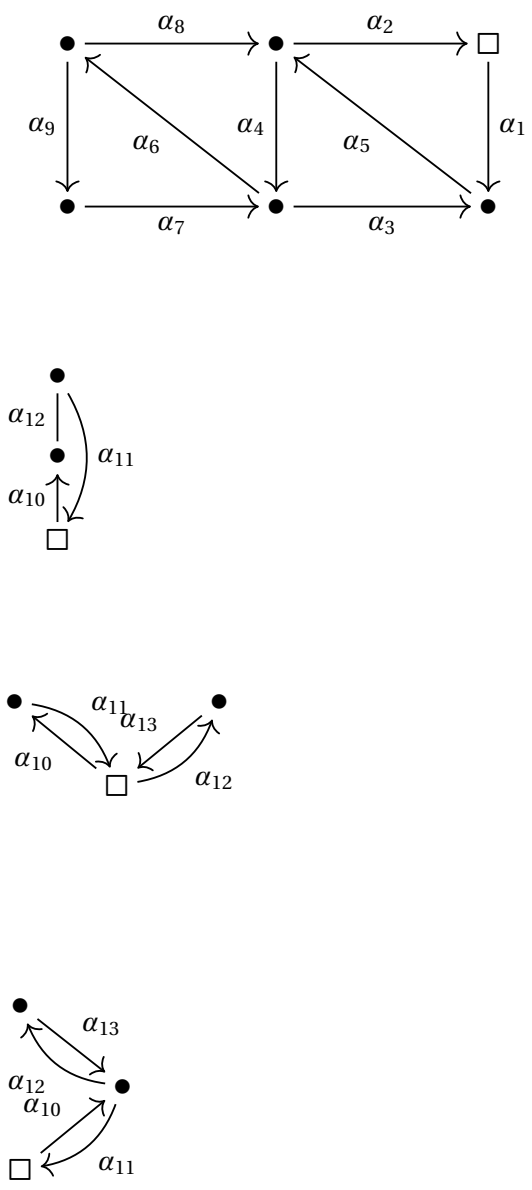

(87)

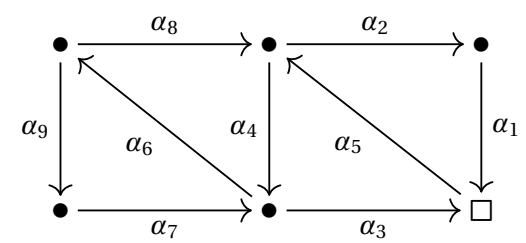

Essa extensão trivial tem as seguintes relações do tipo 2: $r 1=\alpha_{6} \alpha_{3} \alpha_{2} \alpha_{4}, r 2=\alpha_{5} \alpha_{3} \alpha_{2} \alpha_{1}, r 3=$ $\alpha_{4} \alpha_{5} \alpha_{8}, r 4=\alpha_{7} \alpha_{5} \alpha_{3}, r 5=\alpha_{6} \alpha_{8}, r 6=\alpha_{9} \alpha_{1}$, $r 7=\alpha_{9} \alpha_{4}, r 8=\alpha_{2} \alpha_{10}, r 9=\alpha_{11} \alpha_{2}$ e $r 10=\alpha_{3} \alpha_{12}$.

Essa extensão trivial apresenta os seguintes ciclos elementares: $C_{1}=\alpha_{1} \alpha_{6} \alpha_{3} \alpha_{2}, C_{2}=\alpha_{3} \alpha_{2} \alpha_{4} \alpha_{5}, C_{3}=$ $\alpha_{5} \alpha_{8} \alpha_{7}, C_{4}=\alpha_{9} \alpha_{10}$ e $C_{5}=\alpha_{11} \alpha_{12}$. Logo, não temos corte para gerar uma álgebra de incidência.

Essa extensão trivial tem as seguintes relações do tipo 2: $r 1=\alpha_{1} \alpha_{5} \alpha_{4}, r 2=\alpha_{3} \alpha_{5} \alpha_{2}, r 3=\alpha_{5} \alpha_{4} \alpha_{6}$, $r 4=\alpha_{8} \alpha_{4} \alpha_{3}, r 5=\alpha_{4} \alpha_{6} \alpha_{9}, r 6=\alpha_{7} \alpha_{6} \alpha_{8}, r 7=\alpha_{8} \alpha_{2}$ e $r 8=\alpha_{7} \alpha_{3}$.

Os ciclos elementares são: $C_{1}=\alpha_{2} \alpha_{1} \alpha_{5}, C_{2}=$ $\alpha_{3} \alpha_{5} \alpha_{4}, C_{3}=\alpha_{8} \alpha_{4} \alpha_{6}$ e $C_{4}=\alpha_{7} \alpha_{6} \alpha_{9}$.

Completando, temos mais essas relações do tipo 2 : $r 9=\alpha_{2} \alpha_{10}$ e $r 10=\alpha_{11} \alpha_{1}$.

E o ciclo elementar é $C_{5}=\alpha_{10} \alpha_{12} \alpha_{11}$. O programa mostra um corte, assim originando a álgebra 184.

Nesse caso, temos mais essas relações do tipo 2: $r 9=\alpha_{2} \alpha_{10}, r 10=\alpha_{11} \alpha_{1}, r 11=\alpha_{2} \alpha_{12}, r 12=$ $\alpha_{13} \alpha_{1}, r 13=\alpha_{13} \alpha_{10}$ e $r 14=\alpha_{11} \alpha_{12}$.

$\mathrm{E}$ os ciclos elementares são $C_{5}=\alpha_{10} \alpha_{11}$ e $C_{6}=$ $\alpha_{12} \alpha_{13}$. Portanto, obtemos a solução 185.

Nesse anexo, temos mais essas relações do tipo 2: $r 9=\alpha_{2} \alpha_{10}, r 10=\alpha_{11} \alpha_{1}, r 11=\alpha_{10} \alpha_{12}$ e $r 12=$ $\alpha_{13} \alpha_{11}$.

$\mathrm{E}$ os ciclos elementares são $C_{5}=\alpha_{11} \alpha_{10}$ e $C_{6}=$ $\alpha_{13} \alpha_{12}$. Logo, temos um corte que origina a álgebra de incidência 186.

Essa extensão trivial tem as seguintes relações do tipo 2: $r 1=\alpha_{1} \alpha_{5} \alpha_{4}, r 2=\alpha_{3} \alpha_{5} \alpha_{2}, r 3=\alpha_{5} \alpha_{4} \alpha_{6}$, $r 4=\alpha_{8} \alpha_{4} \alpha_{3}, r 5=\alpha_{4} \alpha_{6} \alpha_{9}, r 6=\alpha_{7} \alpha_{6} \alpha_{8}, r 7=\alpha_{8} \alpha_{2}$ e $r 8=\alpha_{7} \alpha_{3}$.

Os ciclos elementares são: $C_{1}=\alpha_{2} \alpha_{1} \alpha_{5}, C_{2}=$ $\alpha_{3} \alpha_{5} \alpha_{4}, C_{3}=\alpha_{8} \alpha_{4} \alpha_{6}$ e $C_{4}=\alpha_{7} \alpha_{6} \alpha_{9}$. 

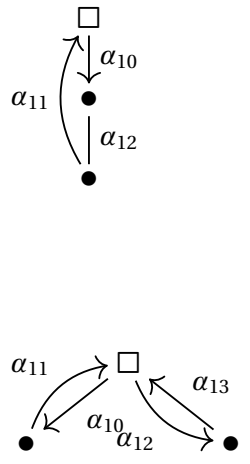

(88)

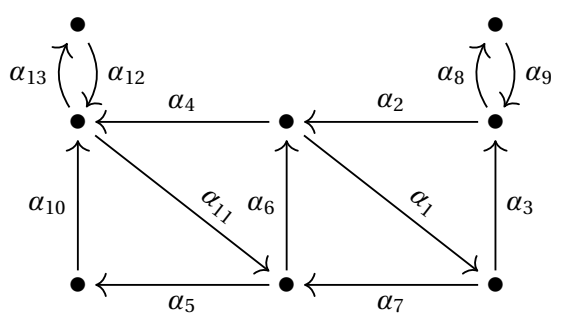

(89)

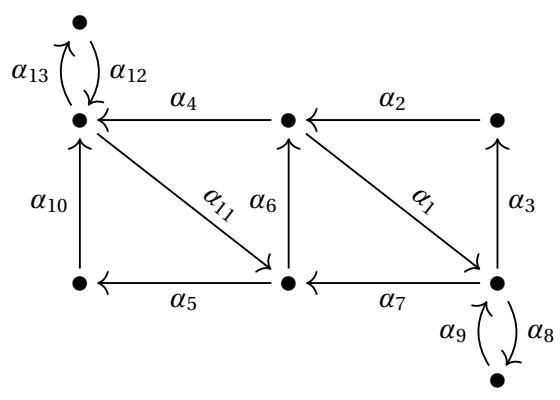

Completando, temos mais essas relações do tipo 2: $r 9=\alpha_{1} \alpha_{10}, r 10=\alpha_{3} \alpha_{10}$ e $r 11=\alpha_{11} \alpha_{5}$.

E o ciclo elementar é $C_{5}=\alpha_{10} \alpha_{12} \alpha_{11}$. O programa não exibe solução para esse caso.

Nesse caso, temos mais essas relações do tipo 2: $r 9=\alpha_{1} \alpha_{10}, r 10=\alpha_{3} \alpha_{10}, r 11=\alpha_{11} \alpha_{5}, r 12=$ $\alpha_{1} \alpha_{12}, r 13=\alpha_{3} \alpha_{12}, r 14=\alpha_{13} \alpha_{5}, r 15=\alpha_{11} \alpha_{12}$ e $r 16=\alpha_{13} \alpha_{10}$.

E os ciclos elementares são $C_{5}=\alpha_{11} \alpha_{10}$ e $C_{6}=$ $\alpha_{12} \alpha_{13}$. Portanto, não obtemos álgebra de incidência.

Nesse anexo, temos mais essas relações do tipo 2: $r 9=\alpha_{1} \alpha_{10}, r 10=\alpha_{3} \alpha_{10}, r 11=\alpha_{11} \alpha_{5}, r 12=$ $\alpha_{12} \alpha_{11}$ e $r 13=\alpha_{10} \alpha_{13}$.

E os ciclos elementares são $C_{5}=\alpha_{11} \alpha_{10}$ e $C_{6}=$ $\alpha_{12} \alpha_{13}$. Logo, não temos corte que origina uma álgebra de incidência.

As relações do tipo 2 são: $r 1=\alpha_{11} \alpha_{6} \alpha_{1}, r 2=$ $\alpha_{7} \alpha_{6} \alpha_{4}, r 3=\alpha_{6} \alpha_{1} \alpha_{3}, r 4=\alpha_{2} \alpha_{1} \alpha_{7}, r 5=\alpha_{2} \alpha_{4}$, $r 6=\alpha_{7} \alpha_{5}, r 7=\alpha_{9} \alpha_{2}, r 8=\alpha_{3} \alpha_{8}, r 9=\alpha_{4} \alpha_{13}$, $r 10=\alpha_{12} \alpha_{11}, r 11=\alpha_{10} \alpha_{13}, r 12=\alpha_{4} \alpha_{11} \alpha_{5} \mathrm{e}$ $r 13=\alpha_{10} \alpha_{11} \alpha_{6}$.

Os ciclos elementares são: $C_{1}=\alpha_{5} \alpha_{10} \alpha_{11}, C_{2}=$ $\alpha_{6} \alpha_{1} \alpha_{7}, C_{3}=\alpha_{1} \alpha_{3} \alpha_{2}, C_{4}=\alpha_{12} \alpha_{13}, C_{5}=\alpha_{9} \alpha_{8} \mathrm{e}$ $C_{6}=\alpha_{11} \alpha_{6} \alpha_{4}$. Logo, nesse caso, não obtemos nenhuma álgebra de incidência.

As relações do tipo 2 são: $r 1=\alpha_{11} \alpha_{6} \alpha_{1}, r 2=$ $\alpha_{7} \alpha_{6} \alpha_{4}, r 3=\alpha_{6} \alpha_{1} \alpha_{3}, r 4=\alpha_{2} \alpha_{1} \alpha_{7}, r 5=\alpha_{2} \alpha_{4}$, $r 6=\alpha_{7} \alpha_{5}, r 7=\alpha_{9} \alpha_{7}, r 8=\alpha_{1} \alpha_{8}, r 9=\alpha_{4} \alpha_{13}$, $r 10=\alpha_{12} \alpha_{11}, r 11=\alpha_{10} \alpha_{13}, r 12=\alpha_{4} \alpha_{11} \alpha_{5}$, $r 13=\alpha_{10} \alpha_{11} \alpha_{6}$ e $r 14=\alpha_{9} \alpha_{3}$.

Os ciclos elementares são: $C_{1}=\alpha_{5} \alpha_{10} \alpha_{11}, C_{2}=$ $\alpha_{6} \alpha_{1} \alpha_{7}, C_{3}=\alpha_{1} \alpha_{3} \alpha_{2}, C_{4}=\alpha_{12} \alpha_{13}, C_{5}=\alpha_{9} \alpha_{8} \mathrm{e}$ $C_{6}=\alpha_{11} \alpha_{6} \alpha_{4}$. O programa não exibe nenhuma solução para esse caso. 
(90)
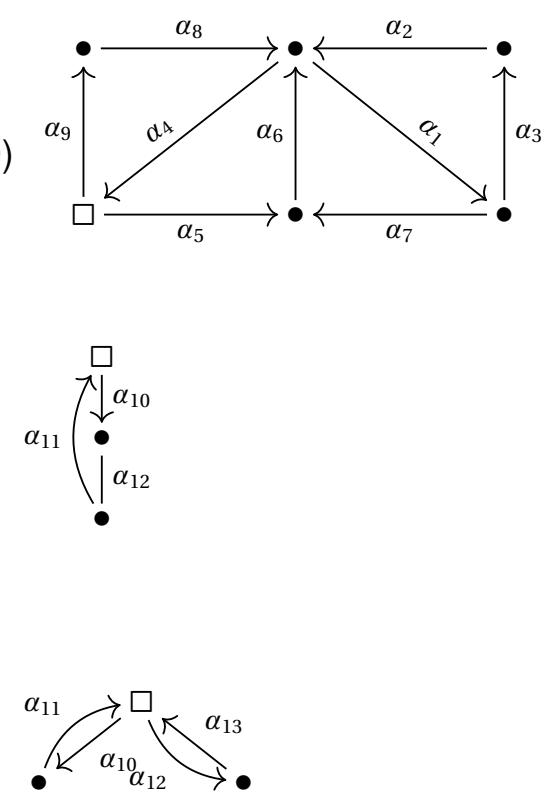

(91)
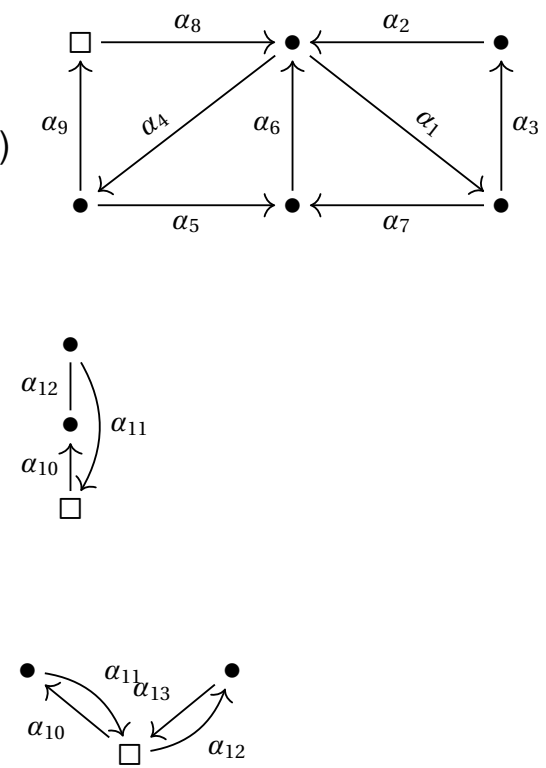

As relações do tipo 2 são: $r 1=\alpha_{5} \alpha_{6} \alpha_{1}, r 2=$ $\alpha_{7} \alpha_{6} \alpha_{4}, r 3=\alpha_{6} \alpha_{1} \alpha_{3}, r 4=\alpha_{2} \alpha_{1} \alpha_{7}, r 5=\alpha_{2} \alpha_{4}$, $r 6=\alpha_{8} \alpha_{1}, r 7=\alpha_{6} \alpha_{4} \alpha_{9}$ e $r 8=\alpha_{8} \alpha_{4} \alpha_{5}$.

Os ciclos elementares são: $C_{1}=\alpha_{5} \alpha_{6} \alpha_{4}, C_{2}=$ $\alpha_{6} \alpha_{1} \alpha_{7}, C_{3}=\alpha_{1} \alpha_{3} \alpha_{2}$ e $C_{4}=\alpha_{8} \alpha_{4} \alpha_{9}$.

Completando, temos mais essas relações do tipo 2: $r 9=\alpha_{4} \alpha_{10}, r 10=\alpha_{11} \alpha_{9}$ e $r 11=\alpha_{11} \alpha_{5}$.

E o ciclo elementar é $C_{5}=\alpha_{10} \alpha_{12} \alpha_{11}$. O programa mostra a solução 187.

Nesse caso, temos mais essas relações do tipo 2: $r 9=\alpha_{4} \alpha_{10}, r 10=\alpha_{11} \alpha_{9}, r 11=\alpha_{11} \alpha_{5}, r 12=$ $\alpha_{4} \alpha_{12}, r 13=\alpha_{13} \alpha_{9}, r 14=\alpha_{13} \alpha_{5}, r 15=\alpha_{11} \alpha_{12}$ e $r 16=\alpha_{13} \alpha_{10}$.

$\mathrm{E}$ os ciclos elementares são $C_{5}=\alpha_{11} \alpha_{10}$ e $C_{6}=$ $\alpha_{12} \alpha_{13}$. Portanto, obtemos a álgebra de incidência 188.

Nesse anexo, temos mais essas relações do tipo 2: $r 9=\alpha_{4} \alpha_{10}, r 10=\alpha_{11} \alpha_{9}, r 11=\alpha_{11} \alpha_{5}, r 12=$ $\alpha_{12} \alpha_{11}$ e $r 13=\alpha_{10} \alpha_{13}$.

$\mathrm{E}$ os ciclos elementares são $C_{5}=\alpha_{11} \alpha_{10}$ e $C_{6}=$ $\alpha_{12} \alpha_{13}$. O programa exibe um corte que origina a álgebra 189.

As relações do tipo 2 são: $r 1=\alpha_{5} \alpha_{6} \alpha_{1}, r 2=$ $\alpha_{7} \alpha_{6} \alpha_{4}, r 3=\alpha_{6} \alpha_{1} \alpha_{3}, r 4=\alpha_{2} \alpha_{1} \alpha_{7}, r 5=\alpha_{2} \alpha_{4}$, $r 6=\alpha_{8} \alpha_{1}, r 7=\alpha_{6} \alpha_{4} \alpha_{9}$ e $r 8=\alpha_{8} \alpha_{4} \alpha_{5}$.

Os ciclos elementares são: $C_{1}=\alpha_{5} \alpha_{6} \alpha_{4}, C_{2}=$ $\alpha_{6} \alpha_{1} \alpha_{7}, C_{3}=\alpha_{1} \alpha_{3} \alpha_{2}$ e $C_{4}=\alpha_{8} \alpha_{4} \alpha_{9}$.

Completando, temos mais essas relações do tipo 2 : $r 9=\alpha_{9} \alpha_{10}$ e $r 10=\alpha_{11} \alpha_{8}$.

E o ciclo elementar é $C_{5}=\alpha_{10} \alpha_{12} \alpha_{11}$. O programa mostra um corte, assim originando a álgebra 190.

Nesse caso, temos mais essas relações do tipo 2: $r 9=\alpha_{9} \alpha_{10}, r 10=\alpha_{11} \alpha_{8}, r 11=\alpha_{9} \alpha_{12}, r 12=$ $\alpha_{13} \alpha_{8}, r 13=\alpha_{13} \alpha_{10}$ e $r 14=\alpha_{11} \alpha_{12}$.

$\mathrm{E}$ os ciclos elementares são $C_{5}=\alpha_{10} \alpha_{11}$ e $C_{6}=$ $\alpha_{12} \alpha_{13}$. Portanto, obtemos a solução 191. 


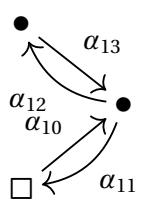

(92)

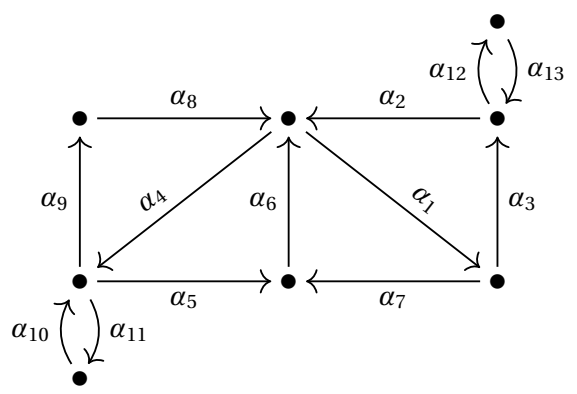

(93)

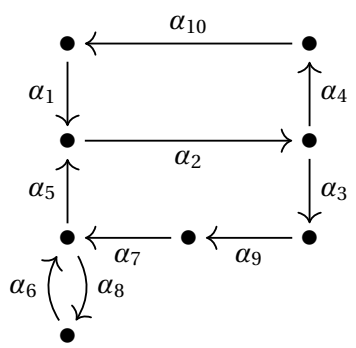

(94)

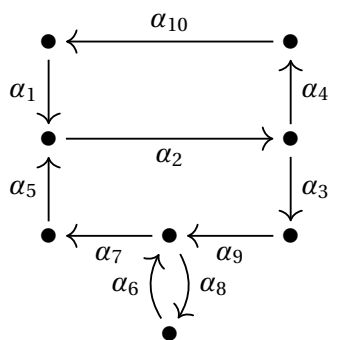

(95)

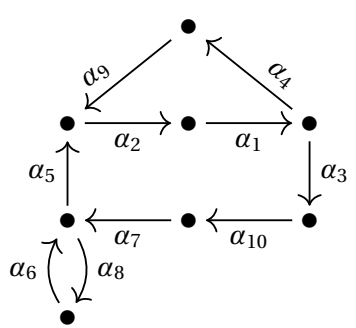

(96)

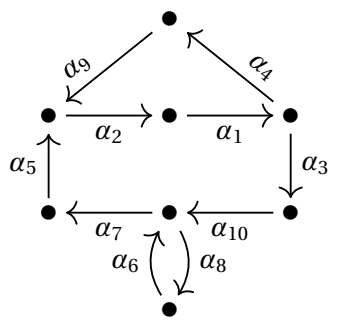

Nesse anexo, temos mais essas relações do tipo 2: $r 9=\alpha_{9} \alpha_{10}, r 10=\alpha_{11} \alpha_{8}, r 11=\alpha_{10} \alpha_{12}$ e $r 12=$ $\alpha_{13} \alpha_{11}$.

E os ciclos elementares são $C_{5}=\alpha_{11} \alpha_{10}$ e $C_{6}=$ $\alpha_{13} \alpha_{12}$. Logo, temos um corte que origina a álgebra de incidência 192.

As relações do tipo 2 são: $r 1=\alpha_{5} \alpha_{6} \alpha_{1}, r 2=$ $\alpha_{7} \alpha_{6} \alpha_{4}, r 3=\alpha_{6} \alpha_{1} \alpha_{3}, r 4=\alpha_{2} \alpha_{1} \alpha_{7}, r 5=\alpha_{2} \alpha_{4}$, $r 6=\alpha_{8} \alpha_{1}, r 7=\alpha_{6} \alpha_{4} \alpha_{9}, r 8=\alpha_{8} \alpha_{4} \alpha_{5}, r 9=\alpha_{10} \alpha_{9}$, $r 10=\alpha_{10} \alpha_{5}, r 11=\alpha_{4} \alpha_{11}, r 12=\alpha_{13} \alpha_{2}$ e $r 13=$ $\alpha_{3} \alpha_{12}$.

Os ciclos elementares são: $C_{1}=\alpha_{5} \alpha_{6} \alpha_{4}, C_{2}=$ $\alpha_{6} \alpha_{1} \alpha_{7}, C_{3}=\alpha_{1} \alpha_{3} \alpha_{2}, C_{4}=\alpha_{8} \alpha_{4} \alpha_{9}, C_{5}=\alpha_{10} \alpha_{11}$ e $C_{6}=\alpha_{12} \alpha_{13}$. Nesse caso, não temos solução.

As relações do tipo 2 são: $r 1=\alpha_{1} \alpha_{2} \alpha_{3}, r 2=$ $\alpha_{5} \alpha_{2} \alpha_{4}, r 3=\alpha_{6} \alpha_{5}$ e $r 4=\alpha_{7} \alpha_{8}$.

Os ciclos elementares são: $C_{1}=\alpha_{5} \alpha_{2} \alpha_{3} \alpha_{9} \alpha_{7}, C_{2}=$ $\alpha_{1} \alpha_{2} \alpha_{4} \alpha_{10}$ e $C_{3}=\alpha_{6} \alpha_{8}$. Portanto, o programa mostra apenas uma solução hereditária.

As relações do tipo 2 são: $r 1=\alpha_{1} \alpha_{2} \alpha_{3}, r 2=$ $\alpha_{5} \alpha_{2} \alpha_{4}, r 3=\alpha_{6} \alpha_{7}$ e $r 4=\alpha_{9} \alpha_{8}$.

Os ciclos elementares são: $C_{1}=\alpha_{5} \alpha_{2} \alpha_{3} \alpha_{9} \alpha_{7}, C_{2}=$ $\alpha_{1} \alpha_{2} \alpha_{4} \alpha_{10}$ e $C_{3}=\alpha_{6} \alpha_{8}$. Para esse caso, não temos solução.

As relações do tipo 2 são: $r 1=\alpha_{9} \alpha_{2} \alpha_{1} \alpha_{3}, r 2=$ $\alpha_{5} \alpha_{2} \alpha_{1} \alpha_{4}, r 3=\alpha_{6} \alpha_{5}$ e $r 4=\alpha_{7} \alpha_{8}$.

Os ciclos elementares são: $C_{1}=\alpha_{5} \alpha_{2} \alpha_{1} \alpha_{3} \alpha_{10} \alpha_{7}$, $C_{2}=\alpha_{9} \alpha_{2} \alpha_{1} \alpha_{4}$ e $C_{3}=\alpha_{6} \alpha_{8}$. Para esse caso, temos apenas solução hereditária.

As relações do tipo 2 são: $r 1=\alpha_{9} \alpha_{2} \alpha_{1} \alpha_{3}, r 2=$ $\alpha_{5} \alpha_{2} \alpha_{1} \alpha_{4}, r 3=\alpha_{6} \alpha_{7}$ e $r 4=\alpha_{10} \alpha_{8}$.

Os ciclos elementares são: $C_{1}=\alpha_{5} \alpha_{2} \alpha_{1} \alpha_{3} \alpha_{10} \alpha_{7}$, $C_{2}=\alpha_{9} \alpha_{2} \alpha_{1} \alpha_{4}$ e $C_{3}=\alpha_{6} \alpha_{8}$. O programa não exibe nenhuma solução. 
(97)

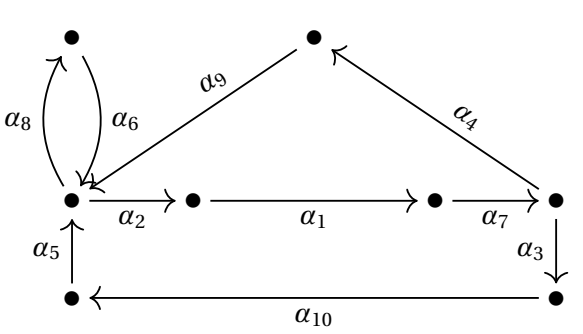

(98)

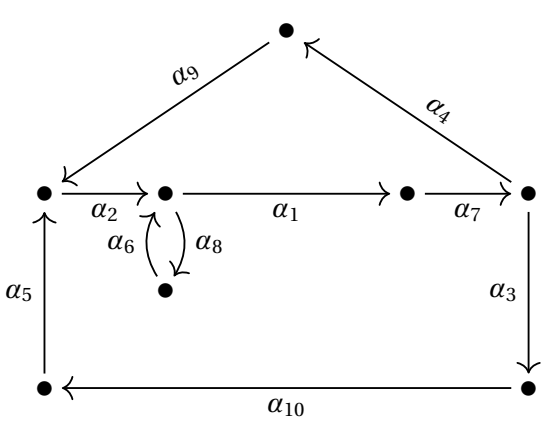

(99)

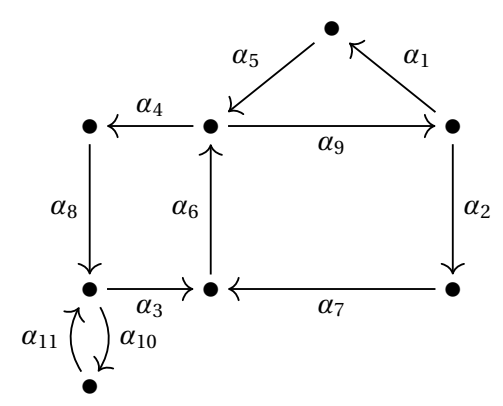

(100)

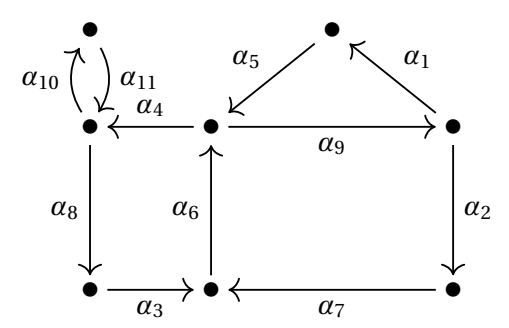

(101)

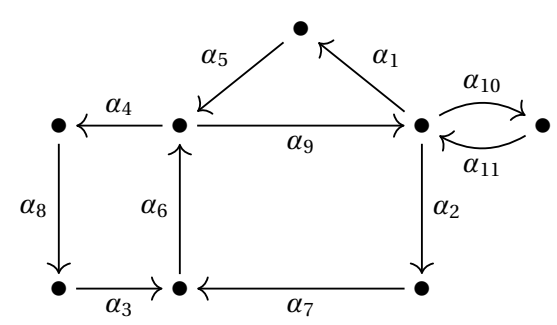

As relações do tipo 2 são: $r 1=\alpha_{9} \alpha_{2} \alpha_{1} \alpha_{7} \alpha_{3}, r 2=$ $\alpha_{5} \alpha_{2} \alpha_{1} \alpha_{7} \alpha_{4}, r 3=\alpha_{6} \alpha_{2}, r 4=\alpha_{9} \alpha_{8}$ e $r 5=\alpha_{5} \alpha_{8}$.

Os ciclos elementares são: $C_{1}=\alpha_{5} \alpha_{2} \alpha_{1} \alpha_{7} \alpha_{3} \alpha_{10}$, $C_{2}=\alpha_{9} \alpha_{2} \alpha_{1} \alpha_{7} \alpha_{4}$ e $C_{3}=\alpha_{6} \alpha_{8}$. O programa mostra duas soluções: uma hereditária e a álgebra 193.

As relações do tipo 2 são: $r 1=\alpha_{9} \alpha_{2} \alpha_{1} \alpha_{7} \alpha_{3}, r 2=$ $\alpha_{5} \alpha_{2} \alpha_{1} \alpha_{7} \alpha_{4}, r 3=\alpha_{6} \alpha_{1}$ e $r 4=\alpha_{2} \alpha_{8}$.

Os ciclos elementares são: $C_{1}=\alpha_{5} \alpha_{2} \alpha_{1} \alpha_{7} \alpha_{3} \alpha_{10}$, $C_{2}=\alpha_{9} \alpha_{2} \alpha_{1} \alpha_{7} \alpha_{4}$ e $C_{3}=\alpha_{6} \alpha_{8}$. Portanto temos as álgebras 194 e 195 .

Essa extensão trivial tem as seguintes relações do tipo 2: $r 1=\alpha_{8} \alpha_{10}, r 2=\alpha_{11} \alpha_{3}, r 3=\alpha_{3} \alpha_{6} \alpha_{9}, r 4=$ $\alpha_{7} \alpha_{6} \alpha_{4}, r 5=\alpha_{6} \alpha_{9} \alpha_{1}, r 6=\alpha_{5} \alpha_{9} \alpha_{2}$ e $r 7=\alpha_{5} \alpha_{4}$.

Os ciclos elementares são: $C_{1}=\alpha_{3} \alpha_{6} \alpha_{4} \alpha_{8}, C_{2}=$ $\alpha_{10} \alpha_{11}, C_{3}=\alpha_{6} \alpha_{9} \alpha_{2} \alpha_{7}$ e $C_{4}=\alpha_{5} \alpha_{9} \alpha_{1}$. Assim, o programa não mostra nenhuma solução.

Essa extensão trivial tem as seguintes relações do tipo 2: $r 1=\alpha_{4} \alpha_{10}, r 2=\alpha_{11} \alpha_{8}, r 3=\alpha_{3} \alpha_{6} \alpha_{9}, r 4=$ $\alpha_{7} \alpha_{6} \alpha_{4}, r 5=\alpha_{6} \alpha_{9} \alpha_{1}, r 6=\alpha_{5} \alpha_{9} \alpha_{2}$ e $r 7=\alpha_{5} \alpha_{4}$.

Os ciclos elementares são: $C_{1}=\alpha_{3} \alpha_{6} \alpha_{4} \alpha_{8}, C_{2}=$ $\alpha_{10} \alpha_{11}, C_{3}=\alpha_{6} \alpha_{9} \alpha_{2} \alpha_{7}$ e $C_{4}=\alpha_{5} \alpha_{9} \alpha_{1}$. Portanto, obtemos a álgebra 196.

Essa extensão trivial tem as seguintes relações do tipo 2: $r 1=\alpha_{9} \alpha_{10}, r 2=\alpha_{11} \alpha_{1}, r 3=\alpha_{3} \alpha_{6} \alpha_{9}, r 4=$ $\alpha_{7} \alpha_{6} \alpha_{4}, r 5=\alpha_{6} \alpha_{9} \alpha_{1}, r 6=\alpha_{5} \alpha_{9} \alpha_{2}, r 7=\alpha_{5} \alpha_{4} \mathrm{e}$ $r 8=\alpha_{11} \alpha_{2}$.

Os ciclos elementares são: $C_{1}=\alpha_{3} \alpha_{6} \alpha_{4} \alpha_{8}, C_{2}=$ $\alpha_{10} \alpha_{11}, C_{3}=\alpha_{6} \alpha_{9} \alpha_{2} \alpha_{7}$ e $C_{4}=\alpha_{5} \alpha_{9} \alpha_{1}$. Portanto, obtemos a álgebra 197. 
(102)

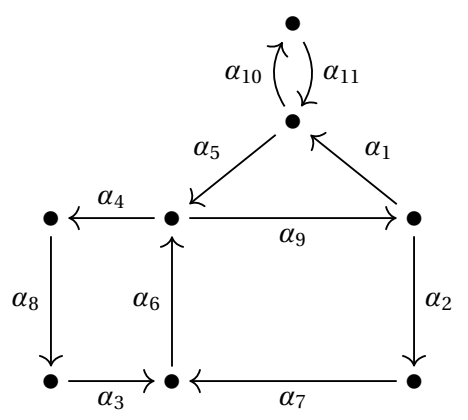

(103)

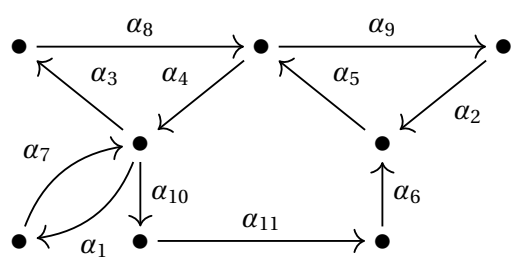

(104)

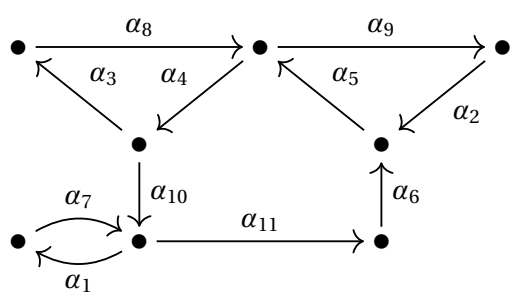

(105)

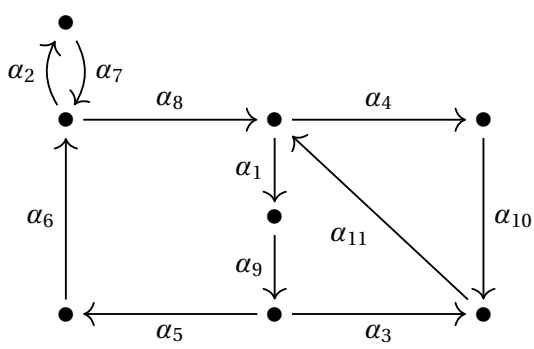

(106)

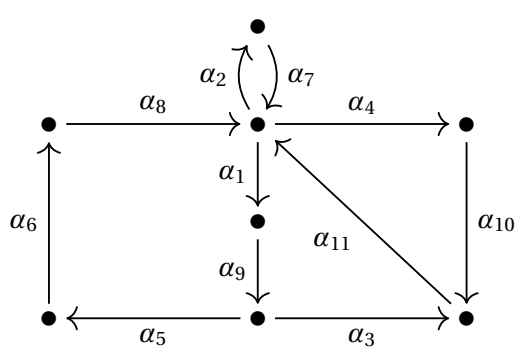

Essa extensão trivial tem as seguintes relações do tipo 2: $r 1=\alpha_{1} \alpha_{10}, r 2=\alpha_{11} \alpha_{5}, r 3=\alpha_{3} \alpha_{6} \alpha_{9}, r 4=$ $\alpha_{7} \alpha_{6} \alpha_{4}, r 5=\alpha_{6} \alpha_{9} \alpha_{1}, r 6=\alpha_{5} \alpha_{9} \alpha_{2}$ e $r 7=\alpha_{5} \alpha_{4}$.

Os ciclos elementares são: $C_{1}=\alpha_{3} \alpha_{6} \alpha_{4} \alpha_{8}, C_{2}=$ $\alpha_{10} \alpha_{11}, C_{3}=\alpha_{6} \alpha_{9} \alpha_{2} \alpha_{7}$ e $C_{4}=\alpha_{5} \alpha_{9} \alpha_{1}$. Logo, o programa nos mostra a solução 198.

Essa extensão trivial tem as seguintes relações do tipo 2: $r 1=\alpha_{6} \alpha_{5} \alpha_{9}, r 2=\alpha_{2} \alpha_{5} \alpha_{4}, r 3=\alpha_{7} \alpha_{3}, r 4=$ $\alpha_{4} \alpha_{1}, r 5=\alpha_{5} \alpha_{4} \alpha_{3}, r 6=\alpha_{8} \alpha_{4} \alpha_{10}, r 7=\alpha_{8} \alpha_{9} \mathrm{e}$ $r 8=\alpha_{7} \alpha_{10}$.

Os ciclos elementares são: $C_{1}=\alpha_{4} \alpha_{10} \alpha_{11} \alpha_{6} \alpha_{5}$, $C_{2}=\alpha_{5} \alpha_{9} \alpha_{2}, C_{3}=\alpha_{7} \alpha_{1}$ e $C_{4}=\alpha_{4} \alpha_{3} \alpha_{8}$. Portanto, obtemos a álgebra de incidência 199.

Essa extensão trivial tem as seguintes relações do tipo 2: $r 1=\alpha_{6} \alpha_{5} \alpha_{9}, r 2=\alpha_{2} \alpha_{5} \alpha_{4}, r 3=\alpha_{7} \alpha_{11}, r 4=$ $\alpha_{10} \alpha_{1}, r 5=\alpha_{5} \alpha_{4} \alpha_{3}, r 6=\alpha_{8} \alpha_{4} \alpha_{10}$ e $r 7=\alpha_{8} \alpha_{9}$.

Os ciclos elementares são: $C_{1}=\alpha_{4} \alpha_{10} \alpha_{11} \alpha_{6} \alpha_{5}, C_{2}=$ $\alpha_{5} \alpha_{9} \alpha_{2}, C_{3}=\alpha_{7} \alpha_{1}$ e $C_{4}=\alpha_{4} \alpha_{3} \alpha_{8}$. O programa não exibe solução.

As relações do tipo 2 são: $r 1=\alpha_{8} \alpha_{1} \alpha_{9} \alpha_{3}, r 2=$ $\alpha_{11} \alpha_{1} \alpha_{9} \alpha_{5}, r 3=\alpha_{6} \alpha_{2}, r 4=\alpha_{7} \alpha_{8}, r 5=\alpha_{3} \alpha_{11} \alpha_{4}$, $r 6=\alpha_{10} \alpha_{11} \alpha_{1}$ e $r 7=\alpha_{8} \alpha_{4}$.

Os ciclos elementares são: $C_{1}=\alpha_{1} \alpha_{9} \alpha_{5} \alpha_{6} \alpha_{8}, C_{2}=$ $\alpha_{1} \alpha_{9} \alpha_{3} \alpha_{11}, C_{3}=\alpha_{11} \alpha_{4} \alpha_{10}$ e $C_{4}=\alpha_{2} \alpha_{7}$. Portanto, obtemos a álgebra de incidência 200 .

As relações do tipo 2 são: $r 1=\alpha_{8} \alpha_{1} \alpha_{9} \alpha_{3}, r 2=$ $\alpha_{11} \alpha_{1} \alpha_{9} \alpha_{5}, r 3=\alpha_{6} \alpha_{2}, r 4=\alpha_{7} \alpha_{8}, r 5=\alpha_{3} \alpha_{11} \alpha_{4}$, $r 6=\alpha_{10} \alpha_{11} \alpha_{1}$ e $r 7=\alpha_{8} \alpha_{4}$.

Os ciclos elementares são: $C_{1}=\alpha_{1} \alpha_{9} \alpha_{5} \alpha_{6} \alpha_{8}, C_{2}=$ $\alpha_{1} \alpha_{9} \alpha_{3} \alpha_{11}, C_{3}=\alpha_{11} \alpha_{4} \alpha_{10}$ e $C_{4}=\alpha_{2} \alpha_{7}$. Logo, o programa exibe as soluções 201 e 202. 
(107)

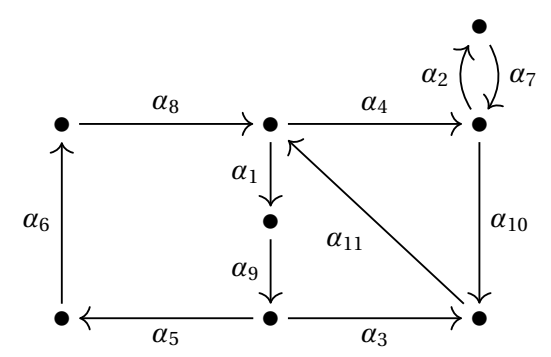

(108)

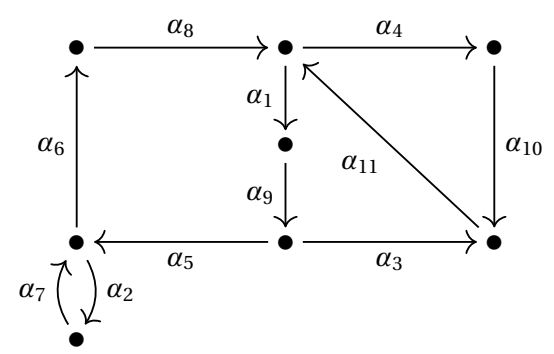

(109)

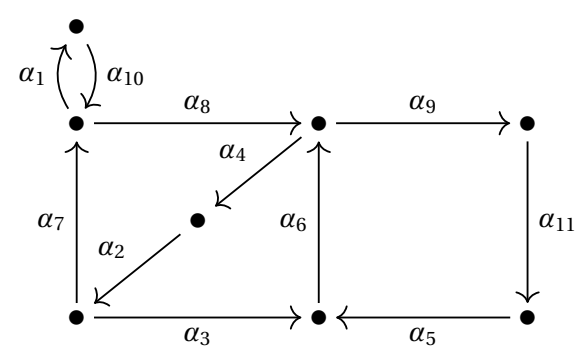

(110)

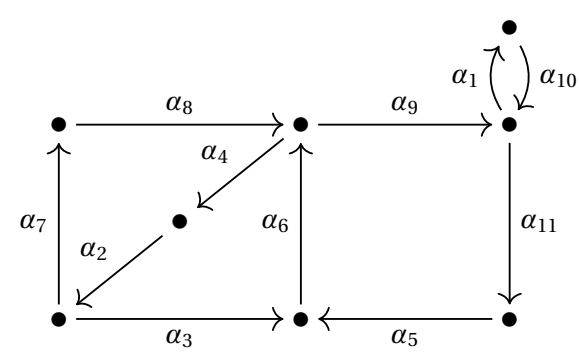

(111)

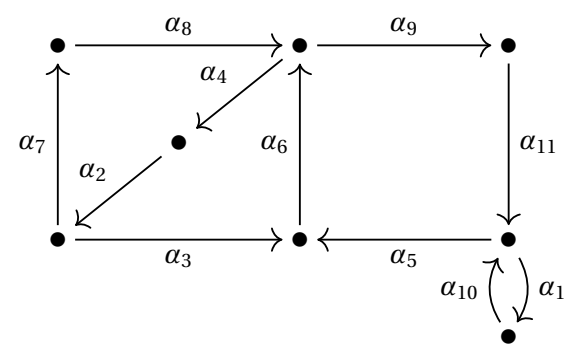

As relações do tipo 2 são: $r 1=\alpha_{8} \alpha_{1} \alpha_{9} \alpha_{3}, r 2=$ $\alpha_{11} \alpha_{1} \alpha_{9} \alpha_{5}, r 3=\alpha_{4} \alpha_{2}, r 4=\alpha_{7} \alpha_{10}, r 5=\alpha_{3} \alpha_{11} \alpha_{4}$, $r 6=\alpha_{10} \alpha_{11} \alpha_{1}$ e $r 7=\alpha_{8} \alpha_{4}$.

Os ciclos elementares são: $C_{1}=\alpha_{1} \alpha_{9} \alpha_{5} \alpha_{6} \alpha_{8}, C_{2}=$ $\alpha_{1} \alpha_{9} \alpha_{3} \alpha_{11}, C_{3}=\alpha_{11} \alpha_{4} \alpha_{10}$ e $C_{4}=\alpha_{2} \alpha_{7}$. Logo, o programa exibe a solução 203.

As relações do tipo 2 são: $r 1=\alpha_{8} \alpha_{1} \alpha_{9} \alpha_{3}, r 2=$ $\alpha_{11} \alpha_{1} \alpha_{9} \alpha_{5}, r 3=\alpha_{5} \alpha_{2}, r 4=\alpha_{7} \alpha_{6}, r 5=\alpha_{3} \alpha_{11} \alpha_{4}$, $r 6=\alpha_{10} \alpha_{11} \alpha_{1}$ e $r 7=\alpha_{8} \alpha_{4}$.

Os ciclos elementares são: $C_{1}=\alpha_{1} \alpha_{9} \alpha_{5} \alpha_{6} \alpha_{8}, C_{2}=$ $\alpha_{1} \alpha_{9} \alpha_{3} \alpha_{11}, C_{3}=\alpha_{11} \alpha_{4} \alpha_{10}$ e $C_{4}=\alpha_{2} \alpha_{7}$. Não obtemos nenhuma álgebra de incidência.

Essa extensão trivial tem as seguintes relações do tipo 2: $r 1=\alpha_{8} \alpha_{4} \alpha_{2} \alpha_{3}, r 2=\alpha_{6} \alpha_{4} \alpha_{2} \alpha_{7}, r 3=$ $\alpha_{3} \alpha_{6} \alpha_{9}, r 4=\alpha_{5} \alpha_{6} \alpha_{4}, r 5=\alpha_{7} \alpha_{1}, r 6=\alpha_{10} \alpha_{8} \mathrm{e}$ $r 7=\alpha_{8} \alpha_{9}$.

Os ciclos elementares são: $C_{1}=\alpha_{4} \alpha_{2} \alpha_{7} \alpha_{8}, C_{2}=$ $\alpha_{5} \alpha_{6} \alpha_{9} \alpha_{11}, C_{3}=\alpha_{4} \alpha_{2} \alpha_{3} \alpha_{6}$ e $C_{4}=\alpha_{1} \alpha_{10}$. Portanto, obtemos a álgebra de incidência 204.

Essa extensão trivial tem as seguintes relações do tipo 2: $r 1=\alpha_{8} \alpha_{4} \alpha_{2} \alpha_{3}, r 2=\alpha_{6} \alpha_{4} \alpha_{2} \alpha_{7}, r 3=$ $\alpha_{3} \alpha_{6} \alpha_{9}, r 4=\alpha_{5} \alpha_{6} \alpha_{4}, r 5=\alpha_{9} \alpha_{1}, r 6=\alpha_{10} \alpha_{11} \mathrm{e}$ $r 7=\alpha_{8} \alpha_{9}$.

Os ciclos elementares são: $C_{1}=\alpha_{4} \alpha_{2} \alpha_{7} \alpha_{8}, C_{2}=$ $\alpha_{5} \alpha_{6} \alpha_{9} \alpha_{11}, C_{3}=\alpha_{4} \alpha_{2} \alpha_{3} \alpha_{6}$ e $C_{4}=\alpha_{1} \alpha_{10}$. Logo, através do corte, temos a álgebra de incidência 205.

Essa extensão trivial tem as seguintes relações do tipo 2: $r 1=\alpha_{8} \alpha_{4} \alpha_{2} \alpha_{3}, r 2=\alpha_{6} \alpha_{4} \alpha_{2} \alpha_{7}, r 3=$ $\alpha_{3} \alpha_{6} \alpha_{9}, r 4=\alpha_{5} \alpha_{6} \alpha_{4}, r 5=\alpha_{11} \alpha_{1}, r 6=\alpha_{10} \alpha_{5} \mathrm{e}$ $r 7=\alpha_{8} \alpha_{9}$.

Os ciclos elementares são: $C_{1}=\alpha_{4} \alpha_{2} \alpha_{7} \alpha_{8}, C_{2}=$ $\alpha_{5} \alpha_{6} \alpha_{9} \alpha_{11}, C_{3}=\alpha_{4} \alpha_{2} \alpha_{3} \alpha_{6}$ e $C_{4}=\alpha_{1} \alpha_{10}$. O programa exibe nenhuma solução. 
(112)

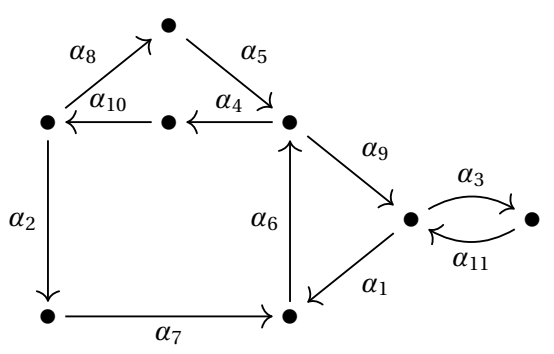

(113)

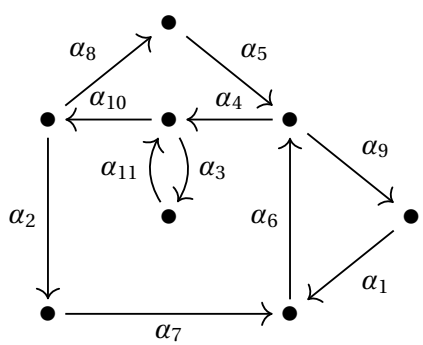

(114)

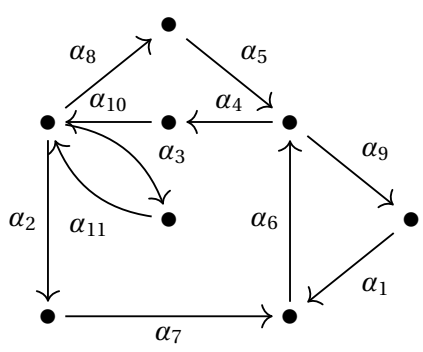

(115)

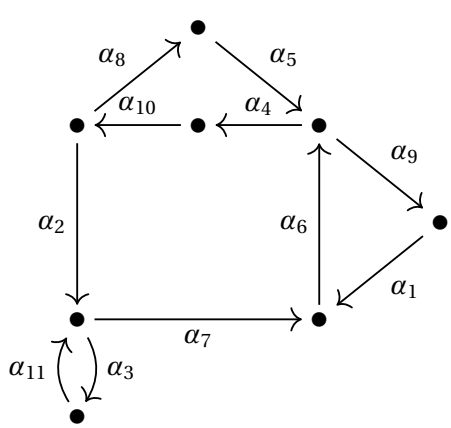

(116)

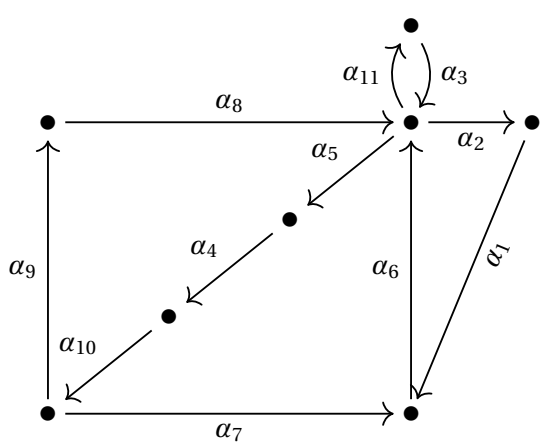

Essa extensão trivial tem as seguintes relações do tipo 2: $r 1=\alpha_{7} \alpha_{6} \alpha_{9}, r 2=\alpha_{1} \alpha_{6} \alpha_{4}, r 3=\alpha_{6} \alpha_{4} \alpha_{10} \alpha_{8}$, $r 4=\alpha_{5} \alpha_{4} \alpha_{10} \alpha_{2}, r 5=\alpha_{9} \alpha_{3}, r 6=\alpha_{11} \alpha_{1}$ e $r 7=$ $\alpha_{5} \alpha_{9}$.

Os ciclos elementares são: $C_{1}=\alpha_{4} \alpha_{10} \alpha_{2} \alpha_{7} \alpha_{6}, C_{2}=$ $\alpha_{4} \alpha_{10} \alpha_{8} \alpha_{5}, C_{3}=\alpha_{3} \alpha_{11}$ e $C_{4}=\alpha_{9} \alpha_{1} \alpha_{6}$. Com isso, temos a álgebra de incidência 206.

Essa extensão trivial tem as seguintes relações do tipo 2: $r 1=\alpha_{7} \alpha_{6} \alpha_{9}, r 2=\alpha_{1} \alpha_{6} \alpha_{4}, r 3=\alpha_{6} \alpha_{4} \alpha_{10} \alpha_{8}$, $r 4=\alpha_{5} \alpha_{4} \alpha_{10} \alpha_{2}, r 5=\alpha_{4} \alpha_{3}, r 6=\alpha_{11} \alpha_{10}$ e $r 7=$ $\alpha_{5} \alpha_{9}$.

Os ciclos elementares são: $C_{1}=\alpha_{4} \alpha_{10} \alpha_{2} \alpha_{7} \alpha_{6}, C_{2}=$ $\alpha_{4} \alpha_{10} \alpha_{8} \alpha_{5}, C_{3}=\alpha_{3} \alpha_{11}$ e $C_{4}=\alpha_{9} \alpha_{1} \alpha_{6}$. O programa exibe a solução 207 .

Essa extensão trivial tem as seguintes relações do tipo 2: $r 1=\alpha_{7} \alpha_{6} \alpha_{9}, r 2=\alpha_{1} \alpha_{6} \alpha_{4}, r 3=\alpha_{6} \alpha_{4} \alpha_{10} \alpha_{8}$, $r 4=\alpha_{5} \alpha_{4} \alpha_{10} \alpha_{2}, r 5=\alpha_{10} \alpha_{3}, r 6=\alpha_{11} \alpha_{2}, r 7=\alpha_{5} \alpha_{9}$ e $r 8=\alpha_{11} \alpha_{8}$.

Os ciclos elementares são: $C_{1}=\alpha_{4} \alpha_{10} \alpha_{2} \alpha_{7} \alpha_{6}, C_{2}=$ $\alpha_{4} \alpha_{10} \alpha_{8} \alpha_{5}, C_{3}=\alpha_{3} \alpha_{11}$ e $C_{4}=\alpha_{9} \alpha_{1} \alpha_{6}$. O programa não exibe solução.

Essa extensão trivial tem as seguintes relações do tipo 2: $r 1=\alpha_{7} \alpha_{6} \alpha_{9}, r 2=\alpha_{1} \alpha_{6} \alpha_{4}, r 3=\alpha_{6} \alpha_{4} \alpha_{10} \alpha_{8}$, $r 4=\alpha_{5} \alpha_{4} \alpha_{10} \alpha_{2}, r 5=\alpha_{2} \alpha_{3}, r 6=\alpha_{11} \alpha_{7}$ e $r 7=$ $\alpha_{5} \alpha_{9}$.

Os ciclos elementares são: $C_{1}=\alpha_{4} \alpha_{10} \alpha_{2} \alpha_{7} \alpha_{6}, C_{2}=$ $\alpha_{4} \alpha_{10} \alpha_{8} \alpha_{5}, C_{3}=\alpha_{3} \alpha_{11}$ e $C_{4}=\alpha_{9} \alpha_{1} \alpha_{6}$. Portanto, para esse caso, não temos corte que gera uma álgebra de incidência.

Essa extensão trivial tem as seguintes relações do tipo 2: $r 1=\alpha_{6} \alpha_{5} \alpha_{4} \alpha_{10} \alpha_{9}, r 2=\alpha_{8} \alpha_{5} \alpha_{4} \alpha_{10} \alpha_{7}, r 3=$ $\alpha_{7} \alpha_{6} \alpha_{2}, r 4=\alpha_{1} \alpha_{6} \alpha_{5}, r 5=\alpha_{3} \alpha_{5}, r 6=\alpha_{3} \alpha_{2}, r 7=$ $\alpha_{8} \alpha_{11}, r 8=\alpha_{8} \alpha_{2}$ e $r 7=\alpha_{6} \alpha_{11}$.

Os ciclos elementares são: $C_{1}=\alpha_{8} \alpha_{5} \alpha_{4} \alpha_{10} \alpha_{9}, C_{2}=$ $\alpha_{5} \alpha_{4} \alpha_{10} \alpha_{7} \alpha_{6}, C_{3}=\alpha_{6} \alpha_{2} \alpha_{1}$ e $C_{4}=\alpha_{11} \alpha_{3}$. Logo obtemos as soluções 208 e 209. 


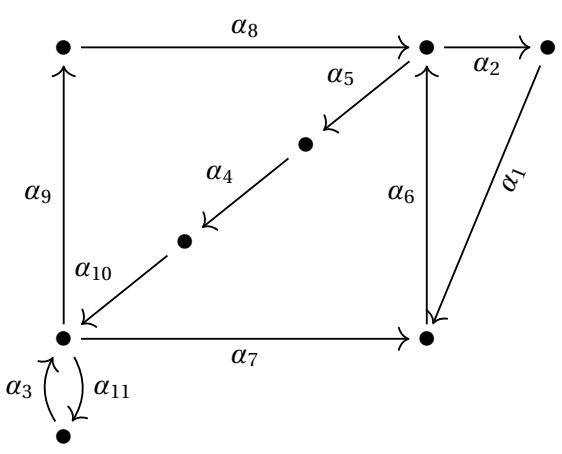

Essa extensão trivial tem as seguintes relações do tipo 2: $r 1=\alpha_{6} \alpha_{5} \alpha_{4} \alpha_{10} \alpha_{9}, r 2=\alpha_{8} \alpha_{5} \alpha_{4} \alpha_{10} \alpha_{7}, r 3=$ $\alpha_{7} \alpha_{6} \alpha_{2}, r 4=\alpha_{1} \alpha_{6} \alpha_{5}, r 5=\alpha_{3} \alpha_{9}, r 6=\alpha_{3} \alpha_{7}, r 7=$ $\alpha_{10} \alpha_{11}$ e $r 8=\alpha_{8} \alpha_{2}$.

Os ciclos elementares são: $C_{1}=\alpha_{8} \alpha_{5} \alpha_{4} \alpha_{10} \alpha_{9}, C_{2}=$ $\alpha_{5} \alpha_{4} \alpha_{10} \alpha_{7} \alpha_{6}, C_{3}=\alpha_{6} \alpha_{2} \alpha_{1}$ e $C_{4}=\alpha_{11} \alpha_{3}$. Nesse caso, não temos solução.

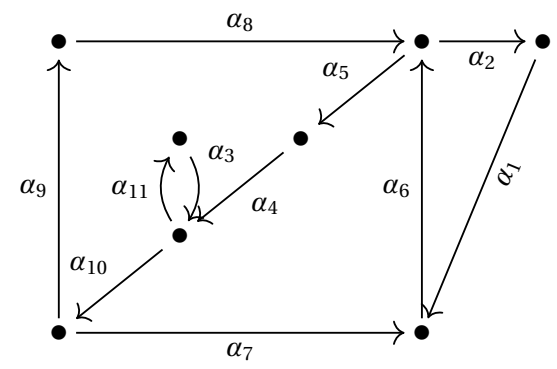

(119)

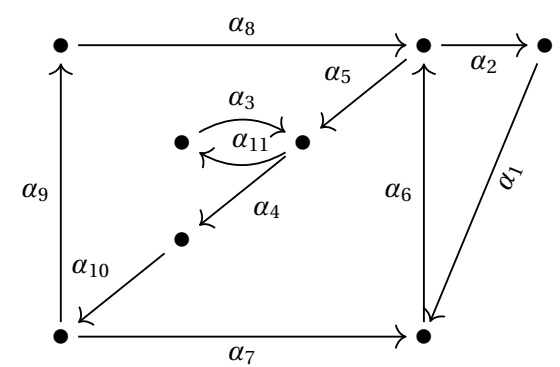

(120)

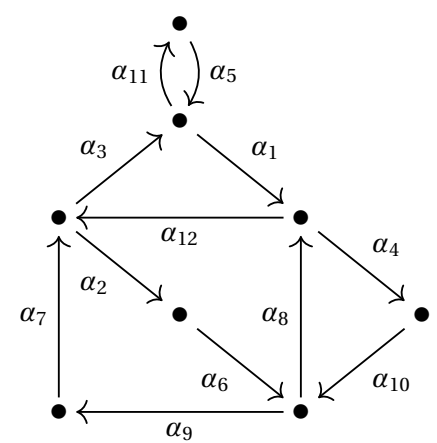

(121)

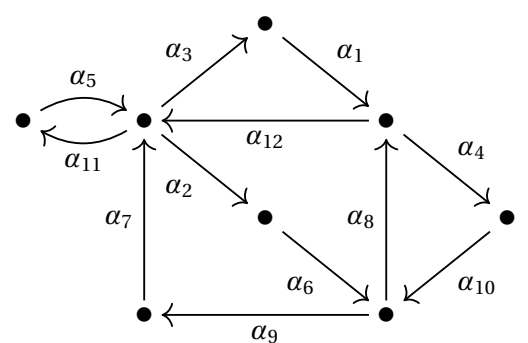

As relações do tipo 2 são: $r 1=\alpha_{12} \alpha_{2} \alpha_{6} \alpha_{9}, r 2=$ $\alpha_{7} \alpha_{2} \alpha_{6} \alpha_{8}, r 3=\alpha_{6} \alpha_{8} \alpha_{4}, r 4=\alpha_{10} \alpha_{8} \alpha_{12}, r 5=$ $\alpha_{8} \alpha_{12} \alpha_{3}, r 6=\alpha_{1} \alpha_{12} \alpha_{2}, r 7=\alpha_{10} \alpha_{9}, r 8=\alpha_{1} \alpha_{4}$, $r 9=\alpha_{7} \alpha_{3}, r 10=\alpha_{5} \alpha_{3}, r 11=\alpha_{12} \alpha_{11}, r 12=\alpha_{5} \alpha_{2} \mathrm{e}$ $r 13=\alpha_{7} \alpha_{11}$.

Os ciclos elementares são: $C_{1}=\alpha_{12} \alpha_{3} \alpha_{1}, C_{2}=$ $\alpha_{2} \alpha_{6} \alpha_{9} \alpha_{7}, C_{3}=\alpha_{8} \alpha_{4} \alpha_{10}, C_{4}=\alpha_{8} \alpha_{12} \alpha_{2} \alpha_{6}$ e $C_{5}=$ $\alpha_{11} \alpha_{5}$. Também, o programa não exibe solução. 
(122)

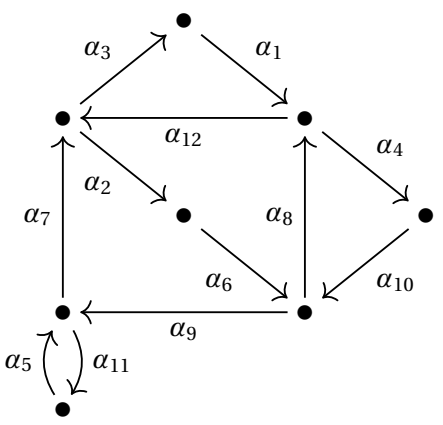

(123)

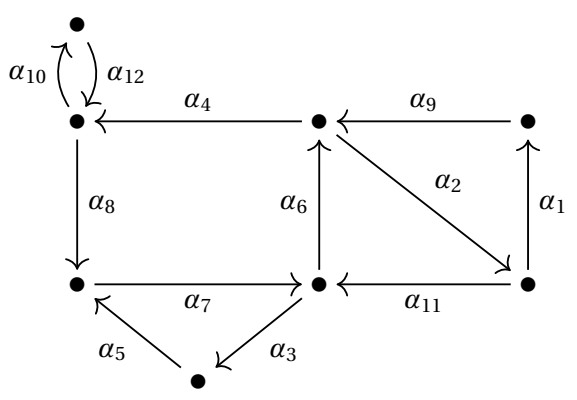

(124)

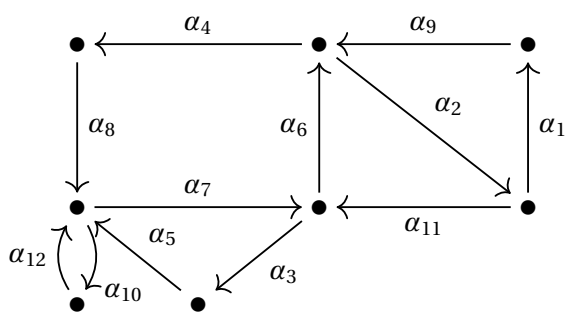

(125)

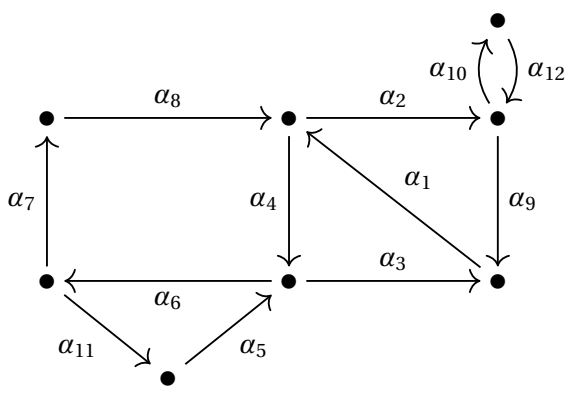

As relações do tipo 2 são: $r 1=\alpha_{12} \alpha_{2} \alpha_{6} \alpha_{9}, r 2=$ $\alpha_{7} \alpha_{2} \alpha_{6} \alpha_{8}, r 3=\alpha_{6} \alpha_{8} \alpha_{4}, r 4=\alpha_{10} \alpha_{8} \alpha_{12}, r 5=$ $\alpha_{8} \alpha_{12} \alpha_{3}, r 6=\alpha_{1} \alpha_{12} \alpha_{2}, r 7=\alpha_{10} \alpha_{9}, r 8=\alpha_{1} \alpha_{4}$, $r 9=\alpha_{7} \alpha_{3}, r 10=\alpha_{5} \alpha_{7}$ e $r 11=\alpha_{9} \alpha_{11}$.

Os ciclos elementares são: $C_{1}=\alpha_{12} \alpha_{3} \alpha_{1}, C_{2}=$ $\alpha_{2} \alpha_{6} \alpha_{9} \alpha_{7}, C_{3}=\alpha_{8} \alpha_{4} \alpha_{10}, C_{4}=\alpha_{8} \alpha_{12} \alpha_{2} \alpha_{6}$ e $C_{5}=$ $\alpha_{11} \alpha_{5}$. Novamente, não temos solução.

Essa extensão trivial tem as seguintes relações do tipo 2: $r 1=\alpha_{9} \alpha_{2} \alpha_{11}, r 2=\alpha_{6} \alpha_{2} \alpha_{1}, r 3=\alpha_{11} \alpha_{6} \alpha_{4}$, $r 4=\alpha_{7} \alpha_{6} \alpha_{2}, r 5=\alpha_{8} \alpha_{7} \alpha_{3}, r 6=\alpha_{5} \alpha_{7} \alpha_{6}, r 7=$ $\alpha_{9} \alpha_{4}, r 8=\alpha_{11} \alpha_{3}, r 9=\alpha_{12} \alpha_{8}$ e $r 10=\alpha_{4} \alpha_{10}$.

Os ciclos elementares são: $C_{1}=\alpha_{4} \alpha_{8} \alpha_{7} \alpha_{6}, C_{2}=$ $\alpha_{2} \alpha_{1} \alpha_{9}, C_{3}=\alpha_{2} \alpha_{11} \alpha_{6}, C_{4}=\alpha_{7} \alpha_{3} \alpha_{5}$ e $C_{5}=\alpha_{10} \alpha_{12}$. Portanto, não obtemos álgebra de incidência.

Essa extensão trivial tem as seguintes relações do tipo 2: $r 1=\alpha_{9} \alpha_{2} \alpha_{11}, r 2=\alpha_{6} \alpha_{2} \alpha_{1}, r 3=\alpha_{11} \alpha_{6} \alpha_{4}$, $r 4=\alpha_{7} \alpha_{6} \alpha_{2}, r 5=\alpha_{8} \alpha_{7} \alpha_{3}, r 6=\alpha_{5} \alpha_{7} \alpha_{6}, r 7=$ $\alpha_{9} \alpha_{4}, r 8=\alpha_{11} \alpha_{3}, r 9=\alpha_{12} \alpha_{8}$ e $r 10=\alpha_{4} \alpha_{10}$.

Os ciclos elementares são: $C_{1}=\alpha_{4} \alpha_{8} \alpha_{7} \alpha_{6}, C_{2}=$ $\alpha_{2} \alpha_{1} \alpha_{9}, C_{3}=\alpha_{2} \alpha_{11} \alpha_{6}, C_{4}=\alpha_{7} \alpha_{3} \alpha_{5}$ e $C_{5}=\alpha_{10} \alpha_{12}$. Portanto, não obtemos álgebra de incidência.

Essa extensão trivial tem as seguintes relações do tipo 2: $r 1=\alpha_{9} \alpha_{1} \alpha_{4}, r 2=\alpha_{3} \alpha_{1} \alpha_{2}, r 3=\alpha_{1} \alpha_{4} \alpha_{6}$, $r 4=\alpha_{8} \alpha_{4} \alpha_{3}, r 5=\alpha_{4} \alpha_{6} \alpha_{11}, r 6=\alpha_{5} \alpha_{6} \alpha_{7}, r 7=$ $\alpha_{8} \alpha_{2}, r 8=\alpha_{5} \alpha_{3}, r 9=\alpha_{12} \alpha_{9}$ e $r 10=\alpha_{2} \alpha_{10}$.

Os ciclos elementares são: $C_{1}=\alpha_{4} \alpha_{6} \alpha_{7} \alpha_{8}, C_{2}=$ $\alpha_{2} \alpha_{9} \alpha_{1}, C_{3}=\alpha_{6} \alpha_{11} \alpha_{5}, C_{4}=\alpha_{1} \alpha_{4} \alpha_{3}$ e $C_{5}=\alpha_{10} \alpha_{12}$. O programa mostra a solução 211 . 
(126)

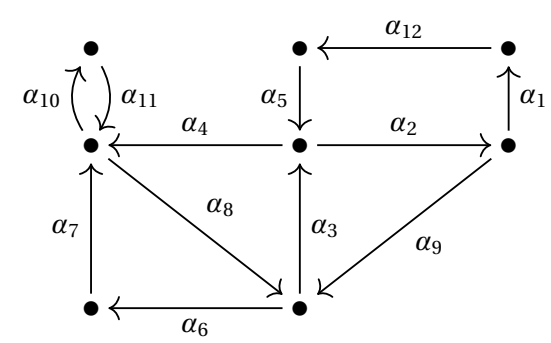

(127)

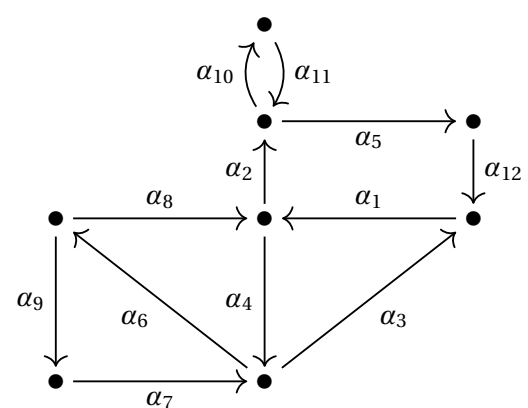

(128)

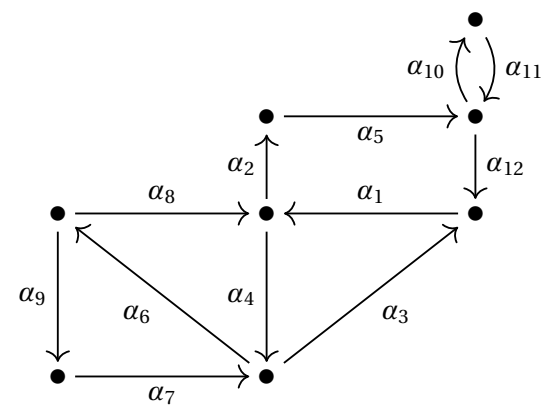

(129)

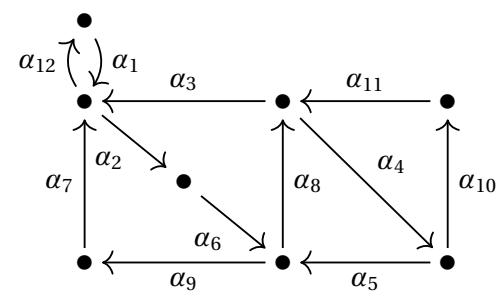

Essa extensão trivial tem as seguintes relações do tipo 2: $r 1=\alpha_{7} \alpha_{8} \alpha_{3}, r 2=\alpha_{4} \alpha_{8} \alpha_{6}, r 3=\alpha_{8} \alpha_{3} \alpha_{2}$, $r 4=\alpha_{9} \alpha_{3} \alpha_{4}, r 5=\alpha_{5} \alpha_{2} \alpha_{9}, r 6=\alpha_{3} \alpha_{2} \alpha_{1}, r 7=$ $\alpha_{5} \alpha_{4}, r 8=\alpha_{9} \alpha_{6}, r 9=\alpha_{4} \alpha_{10}, r 10=\alpha_{7} \alpha_{10}$ e $r 11=$ $\alpha_{11} \alpha_{8}$.

Os ciclos elementares são: $C_{1}=\alpha_{2} \alpha_{1} \alpha_{12} \alpha_{5}, C_{2}=$ $\alpha_{2} \alpha_{9} \alpha_{3}, C_{3}=\alpha_{3} \alpha_{4} \alpha_{8}, C_{4}=\alpha_{8} \alpha_{6} \alpha_{7}$ e $C_{5}=\alpha_{10} \alpha_{11}$. Portanto, não temos corte que gere uma álgebra de incidência.

Essa extensão trivial tem as seguintes relações do tipo 2: $r 1=\alpha_{7} \alpha_{6} \alpha_{8}, r 2=\alpha_{4} \alpha_{6} \alpha_{9}, r 3=\alpha_{8} \alpha_{4} \alpha_{3}$, $r 4=\alpha_{1} \alpha_{4} \alpha_{6}, r 5=\alpha_{3} \alpha_{1} \alpha_{2}, r 6=\alpha_{12} \alpha_{1} \alpha_{4}, r 7=$ $\alpha_{8} \alpha_{2}, r 8=\alpha_{7} \alpha_{3}, r 9=\alpha_{2} \alpha_{10}$ e $r 10=\alpha_{11} \alpha_{5}$.

Os ciclos elementares são: $C_{1}=\alpha_{2} \alpha_{5} \alpha_{12} \alpha_{1}, C_{2}=$ $\alpha_{1} \alpha_{4} \alpha_{3}, C_{3}=\alpha_{10} \alpha_{11}, C_{4}=\alpha_{8} \alpha_{4} \alpha_{6}$ e $C_{5}=\alpha_{6} \alpha_{9} \alpha_{7}$. Portanto, temos apenas a álgebra 212.

Essa extensão trivial tem as seguintes relações do tipo 2: $r 1=\alpha_{7} \alpha_{6} \alpha_{8}, r 2=\alpha_{4} \alpha_{6} \alpha_{9}, r 3=\alpha_{8} \alpha_{4} \alpha_{3}$, $r 4=\alpha_{1} \alpha_{4} \alpha_{6}, r 5=\alpha_{3} \alpha_{1} \alpha_{2}, r 6=\alpha_{12} \alpha_{1} \alpha_{4}, r 7=$ $\alpha_{8} \alpha_{2}, r 8=\alpha_{7} \alpha_{3}, r 9=\alpha_{5} \alpha_{10}$ e $r 10=\alpha_{11} \alpha_{12}$.

Os ciclos elementares são: $C_{1}=\alpha_{2} \alpha_{5} \alpha_{12} \alpha_{1}, C_{2}=$ $\alpha_{1} \alpha_{4} \alpha_{3}, C_{3}=\alpha_{10} \alpha_{11}, C_{4}=\alpha_{8} \alpha_{4} \alpha_{6}$ e $C_{5}=\alpha_{6} \alpha_{9} \alpha_{7}$. O programa não exibe corte.

As relações do tipo 2 dessa extensão trivial são: $r 1=$ $\alpha_{11} \alpha_{4} \alpha_{5}, r 2=\alpha_{8} \alpha_{4} \alpha_{10}, r 3=\alpha_{5} \alpha_{8} \alpha_{3}, r 4=\alpha_{6} \alpha_{8} \alpha_{4}$, $r 5=\alpha_{3} \alpha_{2} \alpha_{6} \alpha_{9}, r 6=\alpha_{7} \alpha_{2} \alpha_{6} \alpha_{8}, r 7=\alpha_{11} \alpha_{3}, r 8=$ $\alpha_{5} \alpha_{9}, r 9=\alpha_{1} \alpha_{2}, r 10=\alpha_{3} \alpha_{12}$ e $r 11=\alpha_{7} \alpha_{12}$.

Os ciclos elementares são: $C_{1}=\alpha_{4} \alpha_{10} \alpha_{11}, C_{2}=$ $\alpha_{4} \alpha_{5} \alpha_{8}, C_{3}=\alpha_{3} \alpha_{2} \alpha_{6} \alpha_{8}, C_{4}=\alpha_{2} \alpha_{6} \alpha_{9} \alpha_{7}$ e $C_{5}=$ $\alpha_{12} \alpha_{1}$. O programa não exibe um corte. 
(130)

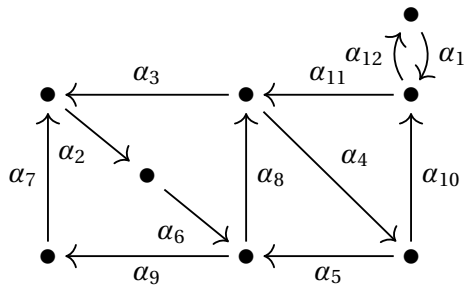

(131)

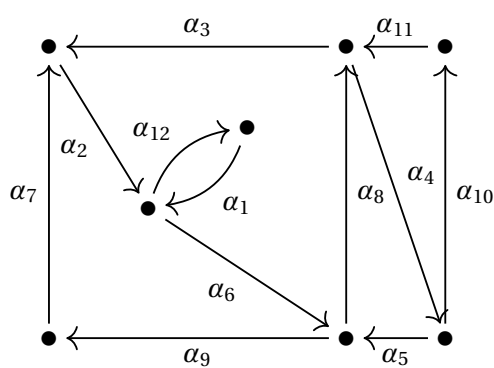

(132)

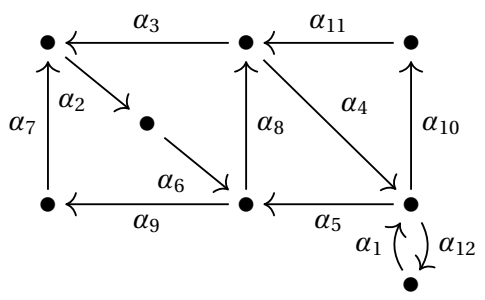

(133)

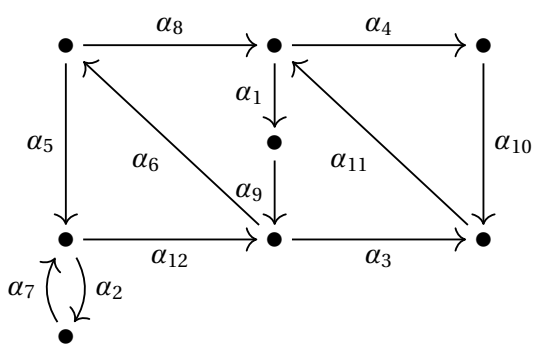

(134)

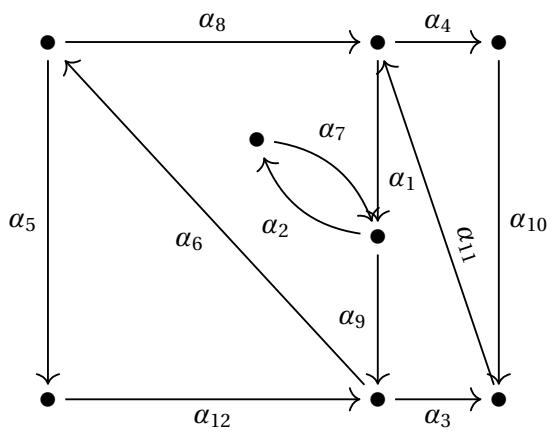

As relações do tipo 2 dessa extensão trivial são: $r 1=$ $\alpha_{11} \alpha_{4} \alpha_{5}, r 2=\alpha_{8} \alpha_{4} \alpha_{10}, r 3=\alpha_{5} \alpha_{8} \alpha_{3}, r 4=\alpha_{6} \alpha_{8} \alpha_{4}$, $r 5=\alpha_{3} \alpha_{2} \alpha_{6} \alpha_{9}, r 6=\alpha_{7} \alpha_{2} \alpha_{6} \alpha_{8}, r 7=\alpha_{11} \alpha_{3}, r 8=$ $\alpha_{5} \alpha_{9}, r 9=\alpha_{1} \alpha_{11}$ e $r 10=\alpha_{10} \alpha_{12}$.

Os ciclos elementares são: $C_{1}=\alpha_{4} \alpha_{10} \alpha_{11}, C_{2}=$ $\alpha_{4} \alpha_{5} \alpha_{8}, C_{3}=\alpha_{3} \alpha_{2} \alpha_{6} \alpha_{8}, C_{4}=\alpha_{2} \alpha_{6} \alpha_{9} \alpha_{7}$ e $C_{5}=$ $\alpha_{12} \alpha_{1}$. O programa exibe apenas o corte que origina a solução 213 .

As relações do tipo 2 dessa extensão trivial são: $r 1=$ $\alpha_{11} \alpha_{4} \alpha_{5}, r 2=\alpha_{8} \alpha_{4} \alpha_{10}, r 3=\alpha_{5} \alpha_{8} \alpha_{3}, r 4=\alpha_{6} \alpha_{8} \alpha_{4}$, $r 5=\alpha_{3} \alpha_{2} \alpha_{6} \alpha_{9}, r 6=\alpha_{7} \alpha_{2} \alpha_{6} \alpha_{8}, r 7=\alpha_{11} \alpha_{3}, r 8=$ $\alpha_{5} \alpha_{9}, r 9=\alpha_{1} \alpha_{6}$ e $r 10=\alpha_{2} \alpha_{12}$.

Os ciclos elementares são: $C_{1}=\alpha_{4} \alpha_{10} \alpha_{11}, C_{2}=$ $\alpha_{4} \alpha_{5} \alpha_{8}, C_{3}=\alpha_{3} \alpha_{2} \alpha_{6} \alpha_{8}, C_{4}=\alpha_{2} \alpha_{6} \alpha_{9} \alpha_{7}$ e $C_{5}=$ $\alpha_{12} \alpha_{1}$. O programa não mostra corte.

As relações do tipo 2 dessa extensão trivial são: $r 1=$ $\alpha_{11} \alpha_{4} \alpha_{5}, r 2=\alpha_{8} \alpha_{4} \alpha_{10}, r 3=\alpha_{5} \alpha_{8} \alpha_{3}, r 4=\alpha_{6} \alpha_{8} \alpha_{4}$, $r 5=\alpha_{3} \alpha_{2} \alpha_{6} \alpha_{9}, r 6=\alpha_{7} \alpha_{2} \alpha_{6} \alpha_{8}, r 7=\alpha_{11} \alpha_{3}, r 8=$ $\alpha_{5} \alpha_{9}, r 9=\alpha_{1} \alpha_{10}, r 10=\alpha_{1} \alpha_{5}$ e $r 11=\alpha_{4} \alpha_{12}$.

Os ciclos elementares são: $C_{1}=\alpha_{4} \alpha_{10} \alpha_{11}, C_{2}=$ $\alpha_{4} \alpha_{5} \alpha_{8}, C_{3}=\alpha_{3} \alpha_{2} \alpha_{6} \alpha_{8}, C_{4}=\alpha_{2} \alpha_{6} \alpha_{9} \alpha_{7}$ e $C_{5}=$ $\alpha_{12} \alpha_{1}$. O programa não exibe solução.

As relações do tipo 2 são: $r 1=\alpha_{8} \alpha_{1} \alpha_{9} \alpha_{3}, r 2=$ $\alpha_{11} \alpha_{1} \alpha_{9} \alpha_{6}, r 3=\alpha_{9} \alpha_{6} \alpha_{5}, r 4=\alpha_{12} \alpha_{6} \alpha_{8}, r 5=$ $\alpha_{3} \alpha_{11} \alpha_{4}, r 6=\alpha_{10} \alpha_{11} \alpha_{1}, r 7=\alpha_{7} \alpha_{12}, r 8=\alpha_{5} \alpha_{2}$, $r 9=\alpha_{8} \alpha_{4}$ e $r 10=\alpha_{12} \alpha_{3}$.

Os ciclos elementares são: $C_{1}=\alpha_{1} \alpha_{9} \alpha_{6} \alpha_{8}, C_{2}=$ $\alpha_{1} \alpha_{9} \alpha_{3} \alpha_{11}, C_{3}=\alpha_{11} \alpha_{4} \alpha_{10}, C_{4}=\alpha_{7} \alpha_{12}$ e $C_{5}=$ $\alpha_{5} \alpha_{12} \alpha_{6}$. Não obtemos álgebra de incidência.

As relações do tipo 2 são: $r 1=\alpha_{8} \alpha_{1} \alpha_{9} \alpha_{3}, r 2=$ $\alpha_{11} \alpha_{1} \alpha_{9} \alpha_{6}, r 3=\alpha_{9} \alpha_{6} \alpha_{5}, r 4=\alpha_{12} \alpha_{6} \alpha_{8}, r 5=$ $\alpha_{3} \alpha_{11} \alpha_{4}, r 6=\alpha_{10} \alpha_{11} \alpha_{1}, r 7=\alpha_{7} \alpha_{9}, r 8=\alpha_{1} \alpha_{2}$, $r 9=\alpha_{8} \alpha_{4}$ e $r 10=\alpha_{12} \alpha_{3}$.

Os ciclos elementares são: $C_{1}=\alpha_{1} \alpha_{9} \alpha_{6} \alpha_{8}, C_{2}=$ $\alpha_{1} \alpha_{9} \alpha_{3} \alpha_{11}, C_{3}=\alpha_{11} \alpha_{4} \alpha_{10}, C_{4}=\alpha_{7} \alpha_{12}$ e $C_{5}=$ $\alpha_{5} \alpha_{12} \alpha_{6}$. O programa não mostra corte que gera uma álgebra de incidência. 
(135)

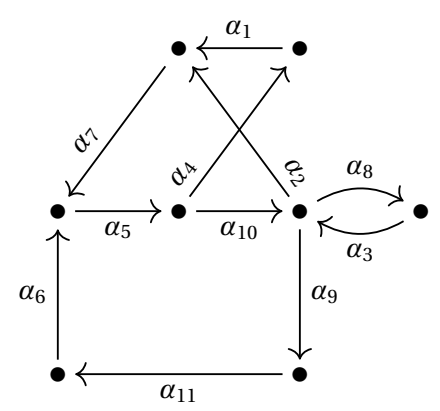

(136)

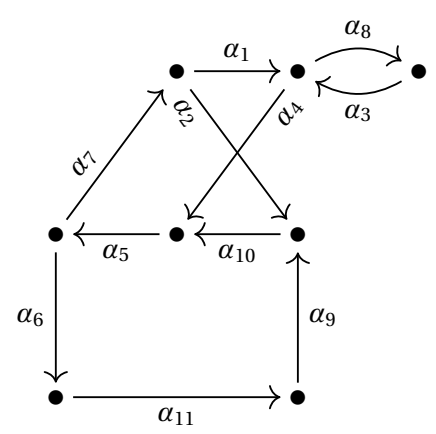

(137)

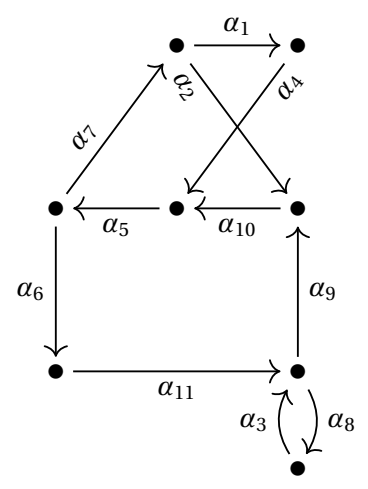

(138)

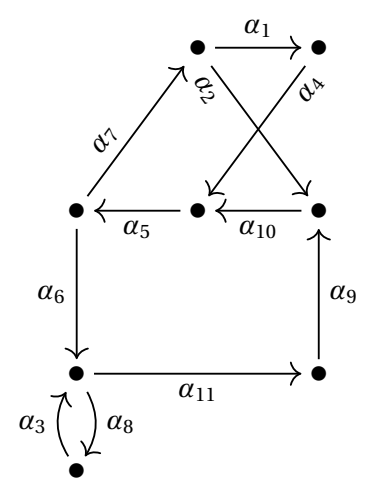

Essa extensão trivial tem as seguintes relações do tipo 2: $r 1=\alpha_{7} \alpha_{5} \alpha_{10} \alpha_{9}, r 2=\alpha_{6} \alpha_{5} \alpha_{10} \alpha_{2}, r 3=$ $\alpha_{1} \alpha_{7} \alpha_{5} \alpha_{10}, r 4=\alpha_{2} \alpha_{7} \alpha_{5} \alpha_{4}, r 5=\alpha_{6} \alpha_{5} \alpha_{4}, r 6=$ $\alpha_{3} \alpha_{2}, r 7=\alpha_{3} \alpha_{9}$ e $r 8=\alpha_{10} \alpha_{8}$.

Os ciclos elementares são: $C_{1}=\alpha_{6} \alpha_{5} \alpha_{10} \alpha_{9} \alpha_{11}$, $C_{2}=\alpha_{10} \alpha_{2} \alpha_{7} \alpha_{5}, C_{3}=\alpha_{4} \alpha_{1} \alpha_{7} \alpha_{5}$ e $C_{4}=\alpha_{3} \alpha_{8}$. Logo, obtemos a solução 214 .

Essa extensão trivial tem as seguintes relações do tipo 2: $r 1=\alpha_{9} \alpha_{10} \alpha_{5} \alpha_{7}, r 2=\alpha_{2} \alpha_{10} \alpha_{5} \alpha_{6}, r 3=$ $\alpha_{10} \alpha_{5} \alpha_{7} \alpha_{1}, r 4=\alpha_{4} \alpha_{5} \alpha_{7} \alpha_{2}, r 5=\alpha_{4} \alpha_{5} \alpha_{6}, r 6=$ $\alpha_{3} \alpha_{4}$ e $r 7=\alpha_{1} \alpha_{8}$.

Os ciclos elementares são: $C_{1}=\alpha_{9} \alpha_{10} \alpha_{5} \alpha_{6} \alpha_{11}, C_{2}=$ $\alpha_{10} \alpha_{5} \alpha_{7} \alpha_{2}, C_{3}=\alpha_{4} \alpha_{5} \alpha_{7} \alpha_{1}$ e $C_{4}=\alpha_{3} \alpha_{8}$. Portanto, temos a solução 215 .

Essa extensão trivial tem as seguintes relações do tipo 2: $r 1=\alpha_{9} \alpha_{10} \alpha_{5} \alpha_{7}, r 2=\alpha_{2} \alpha_{10} \alpha_{5} \alpha_{6}, r 3=$ $\alpha_{10} \alpha_{5} \alpha_{7} \alpha_{1}, r 4=\alpha_{4} \alpha_{5} \alpha_{7} \alpha_{2}, r 5=\alpha_{4} \alpha_{5} \alpha_{6}, r 6=$ $\alpha_{3} \alpha_{9}$ e $r 7=\alpha_{11} \alpha_{8}$.

Os ciclos elementares são: $C_{1}=\alpha_{9} \alpha_{10} \alpha_{5} \alpha_{6} \alpha_{11}, C_{2}=$ $\alpha_{10} \alpha_{5} \alpha_{7} \alpha_{2}, C_{3}=\alpha_{4} \alpha_{5} \alpha_{7} \alpha_{1}$ e $C_{4}=\alpha_{3} \alpha_{8}$. Portanto, não temos solução.

Essa extensão trivial tem as seguintes relações do tipo 2: $r 1=\alpha_{9} \alpha_{10} \alpha_{5} \alpha_{7}, r 2=\alpha_{2} \alpha_{10} \alpha_{5} \alpha_{6}, r 3=$ $\alpha_{10} \alpha_{5} \alpha_{7} \alpha_{1}, r 4=\alpha_{4} \alpha_{5} \alpha_{7} \alpha_{2}, r 5=\alpha_{4} \alpha_{5} \alpha_{6}, r 6=$ $\alpha_{3} \alpha_{11}$ e $r 7=\alpha_{6} \alpha_{8}$.

Os ciclos elementares são: $C_{1}=\alpha_{9} \alpha_{10} \alpha_{5} \alpha_{6} \alpha_{11}$, $C_{2}=\alpha_{10} \alpha_{5} \alpha_{7} \alpha_{2}, C_{3}=\alpha_{4} \alpha_{5} \alpha_{7} \alpha_{1}$ e $C_{4}=\alpha_{3} \alpha_{8}$. O programa mostra a solução 216 . 
(139)

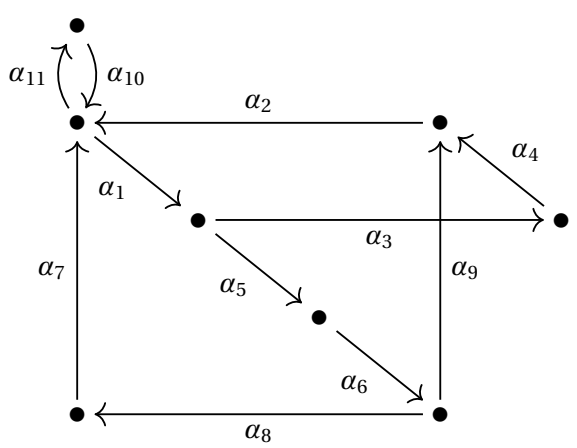

(140)

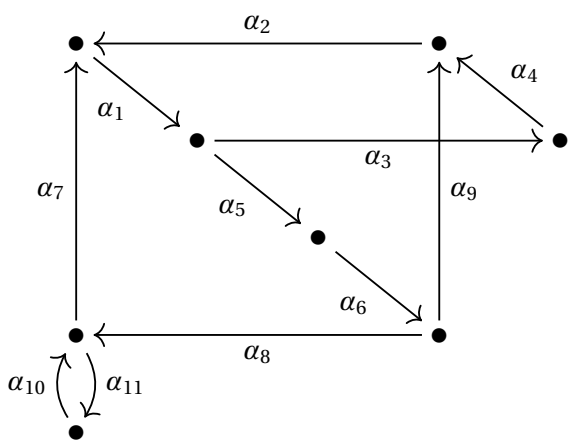

(141)

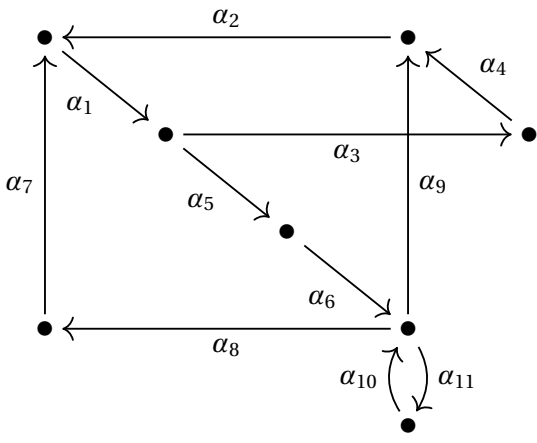

(142)

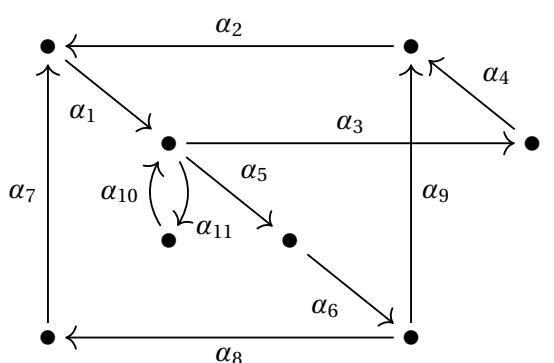

As relações do tipo 2 dessa extensão trivial são: $r 1=\alpha_{4} \alpha_{2} \alpha_{1} \alpha_{5}, r 2=\alpha_{9} \alpha_{2} \alpha_{1} \alpha_{3}, r 3=\alpha_{7} \alpha_{1} \alpha_{3}$, $r 4=\alpha_{2} \alpha_{1} \alpha_{5} \alpha_{6} \alpha_{8}, r 5=\alpha_{7} \alpha_{1} \alpha_{5} \alpha_{6} \alpha_{9}, r 6=\alpha_{10} \alpha_{1}$, $r 7=\alpha_{7} \alpha_{11}$ e $r 8=\alpha_{2} \alpha_{11}$.

Os ciclos elementares são: $C_{1}=\alpha_{2} \alpha_{1} \alpha_{5} \alpha_{6} \alpha_{9}, C_{2}=$ $\alpha_{1} \alpha_{5} \alpha_{6} \alpha_{8} \alpha_{7}, C_{3}=\alpha_{2} \alpha_{1} \alpha_{3} \alpha_{4}$ e $C_{4}=\alpha_{10} \alpha_{11}$. Portanto obtemos as soluções 217 e 218 .

As relações do tipo 2 dessa extensão trivial são: $r 1=\alpha_{4} \alpha_{2} \alpha_{1} \alpha_{5}, r 2=\alpha_{9} \alpha_{2} \alpha_{1} \alpha_{3}, r 3=\alpha_{7} \alpha_{1} \alpha_{3}$, $r 4=\alpha_{2} \alpha_{1} \alpha_{5} \alpha_{6} \alpha_{8}, r 5=\alpha_{7} \alpha_{1} \alpha_{5} \alpha_{6} \alpha_{9}, r 6=\alpha_{10} \alpha_{7}$ e $r 7=\alpha_{8} \alpha_{11}$.

Os ciclos elementares são: $C_{1}=\alpha_{2} \alpha_{1} \alpha_{5} \alpha_{6} \alpha_{9}, C_{2}=$ $\alpha_{1} \alpha_{5} \alpha_{6} \alpha_{8} \alpha_{7}, C_{3}=\alpha_{2} \alpha_{1} \alpha_{3} \alpha_{4}$ e $C_{4}=\alpha_{10} \alpha_{11}$. Para essa extensão trivial, temos apenas a álgebra 219.

As relações do tipo 2 dessa extensão trivial são: $r 1=\alpha_{4} \alpha_{2} \alpha_{1} \alpha_{5}, r 2=\alpha_{9} \alpha_{2} \alpha_{1} \alpha_{3}, r 3=\alpha_{7} \alpha_{1} \alpha_{3}$, $r 4=\alpha_{2} \alpha_{1} \alpha_{5} \alpha_{6} \alpha_{8}, r 5=\alpha_{7} \alpha_{1} \alpha_{5} \alpha_{6} \alpha_{9}, r 6=\alpha_{10} \alpha_{8}$, $r 7=\alpha_{10} \alpha_{9}$ e $r 8=\alpha_{6} \alpha_{11}$.

Os ciclos elementares são: $C_{1}=\alpha_{2} \alpha_{1} \alpha_{5} \alpha_{6} \alpha_{9}, C_{2}=$ $\alpha_{1} \alpha_{5} \alpha_{6} \alpha_{8} \alpha_{7}, C_{3}=\alpha_{2} \alpha_{1} \alpha_{3} \alpha_{4}$ e $C_{4}=\alpha_{10} \alpha_{11}$. Nesse caso, não temos solução.

As relações do tipo 2 dessa extensão trivial são: $r 1=\alpha_{4} \alpha_{2} \alpha_{1} \alpha_{5}, r 2=\alpha_{9} \alpha_{2} \alpha_{1} \alpha_{3}, r 3=\alpha_{7} \alpha_{1} \alpha_{3}$, $r 4=\alpha_{2} \alpha_{1} \alpha_{5} \alpha_{6} \alpha_{8}, r 5=\alpha_{7} \alpha_{1} \alpha_{5} \alpha_{6} \alpha_{9}, r 6=\alpha_{10} \alpha_{5}$, $r 7=\alpha_{10} \alpha_{3}$ e $r 8=\alpha_{1} \alpha_{11}$.

Os ciclos elementares são: $C_{1}=\alpha_{2} \alpha_{1} \alpha_{5} \alpha_{6} \alpha_{9}, C_{2}=$ $\alpha_{1} \alpha_{5} \alpha_{6} \alpha_{8} \alpha_{7}, C_{3}=\alpha_{2} \alpha_{1} \alpha_{3} \alpha_{4}$ e $C_{4}=\alpha_{10} \alpha_{11}$. O programa mostra as soluções 220 e 221 . 
(143)

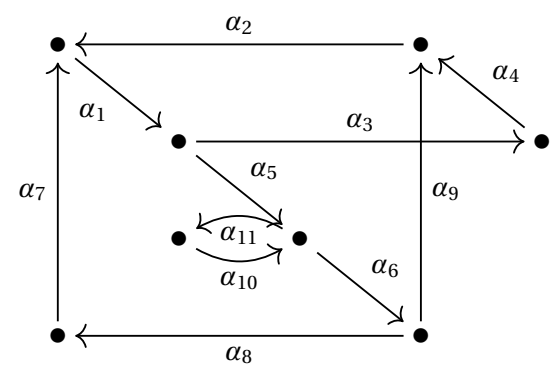

(144)

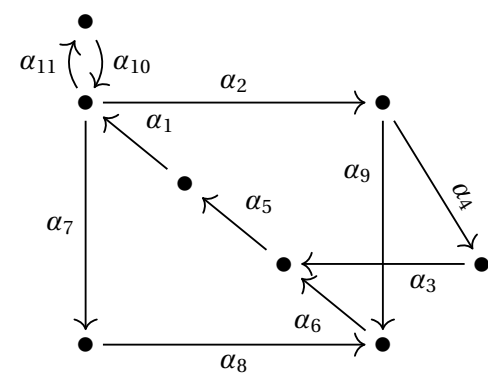

(145)

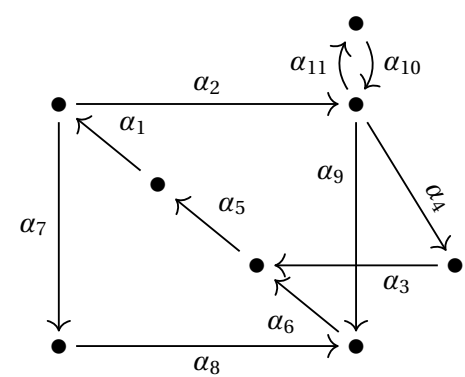

(146)

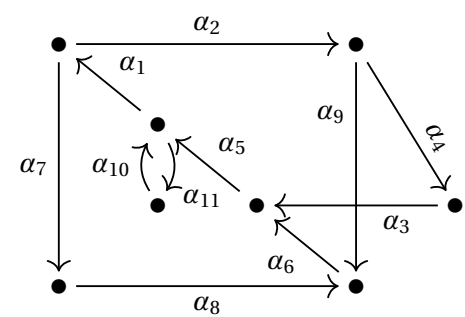

As relações do tipo 2 dessa extensão trivial são: $r 1=\alpha_{4} \alpha_{2} \alpha_{1} \alpha_{5}, r 2=\alpha_{9} \alpha_{2} \alpha_{1} \alpha_{3}, r 3=\alpha_{7} \alpha_{1} \alpha_{3}$, $r 4=\alpha_{2} \alpha_{1} \alpha_{5} \alpha_{6} \alpha_{8}, r 5=\alpha_{7} \alpha_{1} \alpha_{5} \alpha_{6} \alpha_{9}, r 6=\alpha_{10} \alpha_{6}$ e $r 7=\alpha_{5} \alpha_{11}$.

Os ciclos elementares são: $C_{1}=\alpha_{2} \alpha_{1} \alpha_{5} \alpha_{6} \alpha_{9}, C_{2}=$ $\alpha_{1} \alpha_{5} \alpha_{6} \alpha_{8} \alpha_{7}, C_{3}=\alpha_{2} \alpha_{1} \alpha_{3} \alpha_{4}$ e $C_{4}=\alpha_{10} \alpha_{11}$. O programa exibe o corte que gera a álgebra de incidência 222.

As relações do tipo 2 dessa extensão trivial são: $r 1=$ $\alpha_{3} \alpha_{5} \alpha_{1} \alpha_{2} \alpha_{9}, r 2=\alpha_{6} \alpha_{5} \alpha_{1} \alpha_{2} \alpha_{4}, r 3=\alpha_{3} \alpha_{5} \alpha_{1} \alpha_{7}$, $r 4=\alpha_{8} \alpha_{6} \alpha_{5} \alpha_{1} \alpha_{2}, r 5=\alpha_{3} \alpha_{6} \alpha_{5} \alpha_{1} \alpha_{7}, r 6=\alpha_{10} \alpha_{7}$, $r 7=\alpha_{10} \alpha_{2}$ e $r 8=\alpha_{1} \alpha_{11}$.

Os ciclos elementares são: $C_{1}=\alpha_{2} \alpha_{9} \alpha_{6} \alpha_{5} \alpha_{1}, C_{2}=$ $\alpha_{1} \alpha_{7} \alpha_{8} \alpha_{6} \alpha_{5}, C_{3}=\alpha_{2} \alpha_{4} \alpha_{3} \alpha_{5} \alpha_{1}$ e $C_{4}=\alpha_{10} \alpha_{11}$. Portanto obtemos as soluções 223 e 224 .

As relações do tipo 2 dessa extensão trivial são: $r 1=$ $\alpha_{3} \alpha_{5} \alpha_{1} \alpha_{2} \alpha_{9}, r 2=\alpha_{6} \alpha_{5} \alpha_{1} \alpha_{2} \alpha_{4}, r 3=\alpha_{3} \alpha_{5} \alpha_{1} \alpha_{7}$, $r 4=\alpha_{8} \alpha_{6} \alpha_{5} \alpha_{1} \alpha_{2}, r 5=\alpha_{3} \alpha_{6} \alpha_{5} \alpha_{1} \alpha_{7}, r 6=\alpha_{10} \alpha_{9}$, $r 7=\alpha_{10} \alpha_{4}$ e $r 8=\alpha_{2} \alpha_{11}$.

Os ciclos elementares são: $C_{1}=\alpha_{2} \alpha_{9} \alpha_{6} \alpha_{5} \alpha_{1}, C_{2}=$ $\alpha_{1} \alpha_{7} \alpha_{8} \alpha_{6} \alpha_{5}, C_{3}=\alpha_{2} \alpha_{4} \alpha_{3} \alpha_{5} \alpha_{1}$ e $C_{4}=\alpha_{10} \alpha_{11}$. Nesse caso, obtemos a álgebra de incidência 225.

As relações do tipo 2 dessa extensão trivial são: $r 1=$ $\alpha_{3} \alpha_{5} \alpha_{1} \alpha_{2} \alpha_{9}, r 2=\alpha_{6} \alpha_{5} \alpha_{1} \alpha_{2} \alpha_{4}, r 3=\alpha_{3} \alpha_{5} \alpha_{1} \alpha_{7}$, $r 4=\alpha_{8} \alpha_{6} \alpha_{5} \alpha_{1} \alpha_{2}, r 5=\alpha_{3} \alpha_{6} \alpha_{5} \alpha_{1} \alpha_{7}, r 6=\alpha_{10} \alpha_{1} \mathrm{e}$ $r 7=\alpha_{5} \alpha_{11}$.

Os ciclos elementares são: $C_{1}=\alpha_{2} \alpha_{9} \alpha_{6} \alpha_{5} \alpha_{1}, C_{2}=$ $\alpha_{1} \alpha_{7} \alpha_{8} \alpha_{6} \alpha_{5}, C_{3}=\alpha_{2} \alpha_{4} \alpha_{3} \alpha_{5} \alpha_{1}$ e $C_{4}=\alpha_{10} \alpha_{11}$. Assim, temos a Phia 226 e a sua álgebra dual. 
(147)

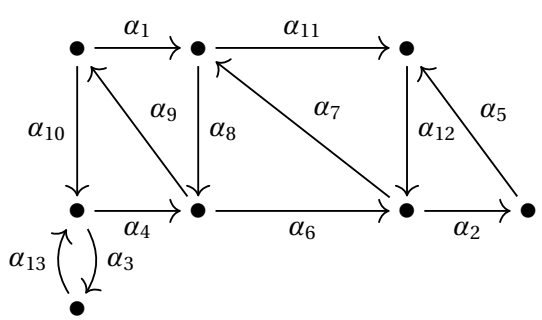

(148)

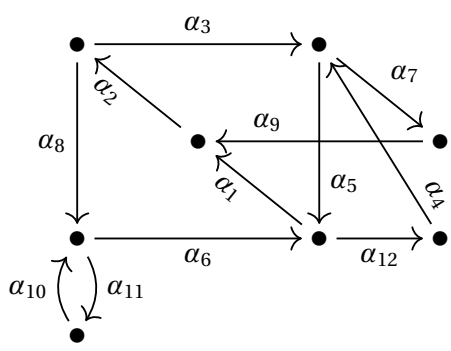

(149)

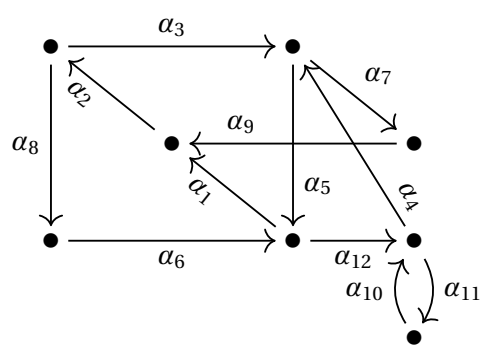

(150)

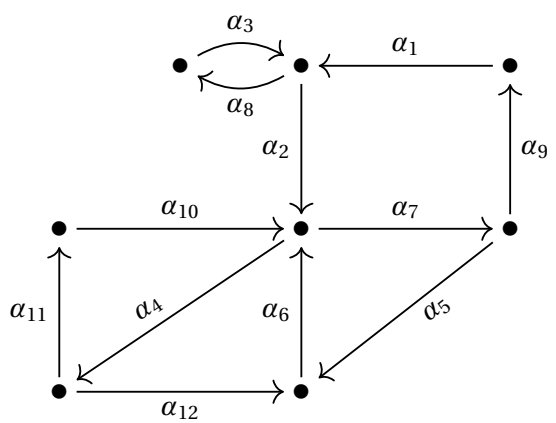

Essa extensão trivial tem as seguintes relações do tipo 2: $r 1=\alpha_{4} \alpha_{9} \alpha_{1}, r 2=\alpha_{8} \alpha_{9} \alpha_{10}, r 3=\alpha_{1} \alpha_{8} \alpha_{6}$, $r 4=\alpha_{7} \alpha_{8} \alpha_{9}, r 5=\alpha_{6} \alpha_{7} \alpha_{11}, r 6=\alpha_{12} \alpha_{7} \alpha_{8}, r 7=$ $\alpha_{11} \alpha_{12} \alpha_{2}, r 8=\alpha_{5} \alpha_{12} \alpha_{7}, r 9=\alpha_{13} \alpha_{4}, r 10=\alpha_{10} \alpha_{3}$, $r 11=\alpha_{4} \alpha_{6}, r 12=\alpha_{6} \alpha_{2}$ e $r 13=\alpha_{1} \alpha_{11}$.

Essa extensão trivial apresenta os seguintes ciclos elementares: $C_{1}=\alpha_{4} \alpha_{9} \alpha_{10}, C_{2}=\alpha_{9} \alpha_{1} \alpha_{8}, C_{3}=$ $\alpha_{8} \alpha_{6} \alpha_{7}, C_{4}=\alpha_{7} \alpha_{11} \alpha_{12}, C 5=\alpha_{12} \alpha_{2} \alpha_{5}$ e $C 6=$ $\alpha_{3} \alpha_{13}$. Portanto, não temos corte que gere uma álgebra de incidência.

As relações do tipo 2 para essa álgebra são: $r 1=$ $\alpha_{6} \alpha_{1} \alpha_{2} \alpha_{3}, r 2=\alpha_{5} \alpha_{1} \alpha_{2} \alpha_{8}, r 3=\alpha_{1} \alpha_{2} \alpha_{3} \alpha_{7}, r 4=$ $\alpha_{9} \alpha_{2} \alpha_{3} \alpha_{5}, r 5=\alpha_{9} \alpha_{2} \alpha_{8}, r 6=\alpha_{8} \alpha_{11}, r 7=\alpha_{10} \alpha_{6}$, $r 8=\alpha_{3} \alpha_{5} \alpha_{12}, r 9=\alpha_{4} \alpha_{5} \alpha_{1}, r 10=\alpha_{6} \alpha_{12}$ e $r 11=$ $\alpha_{4} \alpha_{7}$.

Essa extensão trivial apresenta os seguintes ciclos elementares: $C_{1}=\alpha_{6} \alpha_{1} \alpha_{2} \alpha_{8}, C_{2}=\alpha_{2} \alpha_{3} \alpha_{7} \alpha_{9}, C_{3}=$ $\alpha_{1} \alpha_{2} \alpha_{3} \alpha_{5}, C_{4}=\alpha_{5} \alpha_{12} \alpha_{4}$ e $C 5=\alpha_{10} \alpha_{11}$. Portanto, não temos corte que origine uma álgebra de incidência.

As relações do tipo 2 para essa álgebra são: $r 1=$ $\alpha_{6} \alpha_{1} \alpha_{2} \alpha_{3}, r 2=\alpha_{5} \alpha_{1} \alpha_{2} \alpha_{8}, r 3=\alpha_{1} \alpha_{2} \alpha_{3} \alpha_{7}, r 4=$ $\alpha_{9} \alpha_{2} \alpha_{3} \alpha_{5}, r 5=\alpha_{9} \alpha_{2} \alpha_{8}, r 6=\alpha_{8} \alpha_{11}, r 7=\alpha_{10} \alpha_{6}$, $r 8=\alpha_{3} \alpha_{5} \alpha_{12}, r 9=\alpha_{4} \alpha_{5} \alpha_{1}, r 10=\alpha_{6} \alpha_{12}$ e $r 11=$ $\alpha_{4} \alpha_{7}$.

Essa extensão trivial apresenta os seguintes ciclos elementares: $C_{1}=\alpha_{6} \alpha_{1} \alpha_{2} \alpha_{8}, C_{2}=\alpha_{2} \alpha_{3} \alpha_{7} \alpha_{9}, C_{3}=$ $\alpha_{1} \alpha_{2} \alpha_{3} \alpha_{5}, C_{4}=\alpha_{5} \alpha_{12} \alpha_{4}$ e $C 5=\alpha_{10} \alpha_{11}$. Logo não obtemos corte.

As relações do tipo 2 são: $r 1=\alpha_{3} \alpha_{2}, r 2=\alpha_{1} \alpha_{8}$, $r 3=\alpha_{10} \alpha_{4} \alpha_{12}, r 4=\alpha_{6} \alpha_{4} \alpha_{11}, r 5=\alpha_{12} \alpha_{6} \alpha_{7}, r 6=$ $\alpha_{5} \alpha_{6} \alpha_{4}, r 7=\alpha_{6} \alpha_{7} \alpha_{9}, r 8=\alpha_{2} \alpha_{7} \alpha_{5}, r 9=\alpha_{2} \alpha_{4} \mathrm{e}$ $r 10=\alpha_{10} \alpha_{7}$.

Essa extensão trivial apresenta os seguintes ciclos elementares: $C_{1}=\alpha_{8} \alpha_{3}, C_{2}=\alpha_{10} \alpha_{4} \alpha_{11}, C_{3}=$ $\alpha_{4} \alpha_{12} \alpha_{6}, C_{4}=\alpha_{6} \alpha_{7} \alpha_{5}$ e $C 5=\alpha_{7} \alpha_{9} \alpha_{1} \alpha_{2}$. O programa exibe um corte que origina a solução 227 . 


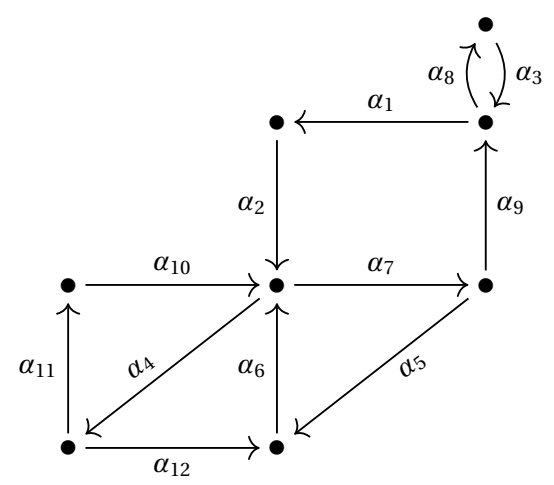

As relações do tipo 2 são: $r 1=\alpha_{3} \alpha_{1}, r 2=\alpha_{9} \alpha_{8}$, $r 3=\alpha_{10} \alpha_{4} \alpha_{12}, r 4=\alpha_{6} \alpha_{4} \alpha_{11}, r 5=\alpha_{12} \alpha_{6} \alpha_{7}, r 6=$ $\alpha_{5} \alpha_{6} \alpha_{4}, r 7=\alpha_{6} \alpha_{7} \alpha_{9}, r 8=\alpha_{2} \alpha_{7} \alpha_{5}, r 9=\alpha_{2} \alpha_{4} \mathrm{e}$ $r 10=\alpha_{10} \alpha_{7}$.

Essa extensão trivial apresenta os seguintes ciclos elementares: $C_{1}=\alpha_{8} \alpha_{3}, C_{2}=\alpha_{10} \alpha_{4} \alpha_{11}, C_{3}=$ $\alpha_{4} \alpha_{12} \alpha_{6}, C_{4}=\alpha_{6} \alpha_{7} \alpha_{5}$ e $C 5=\alpha_{7} \alpha_{9} \alpha_{1} \alpha_{2}$. O programa não exibe corte.

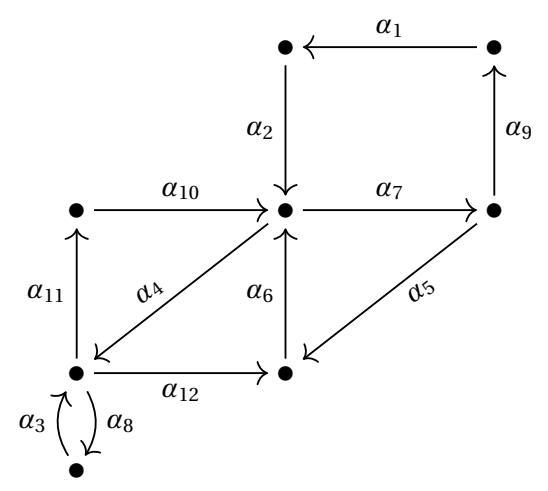

As relações do tipo 2 são: $r 1=\alpha_{3} \alpha_{11}, r 2=\alpha_{4} \alpha_{8}$, $r 3=\alpha_{10} \alpha_{4} \alpha_{12}, r 4=\alpha_{6} \alpha_{4} \alpha_{11}, r 5=\alpha_{12} \alpha_{6} \alpha_{7}, r 6=$ $\alpha_{5} \alpha_{6} \alpha_{4}, r 7=\alpha_{6} \alpha_{7} \alpha_{9}, r 8=\alpha_{2} \alpha_{7} \alpha_{5}, r 9=\alpha_{2} \alpha_{4}$, $r 10=\alpha_{10} \alpha_{7}$ e $r 11=\alpha_{3} \alpha_{12}$.

Essa extensão trivial apresenta os seguintes ciclos elementares: $C_{1}=\alpha_{8} \alpha_{3}, C_{2}=\alpha_{10} \alpha_{4} \alpha_{11}, C_{3}=$ $\alpha_{4} \alpha_{12} \alpha_{6}, C_{4}=\alpha_{6} \alpha_{7} \alpha_{5}$ e $C 5=\alpha_{7} \alpha_{9} \alpha_{1} \alpha_{2}$. Assim, o programa exibe a solução 228 .

As relações do tipo 2 são: $r 1=\alpha_{3} \alpha_{10}, r 2=\alpha_{11} \alpha_{8}$, $r 3=\alpha_{10} \alpha_{4} \alpha_{12}, r 4=\alpha_{6} \alpha_{4} \alpha_{11}, r 5=\alpha_{12} \alpha_{6} \alpha_{7}, r 6=$ $\alpha_{2} \alpha_{6} \alpha_{4}, r 7=\alpha_{6} \alpha_{7} \alpha_{9}, r 8=\alpha_{1} \alpha_{7} \alpha_{5}, r 9=\alpha_{1} \alpha_{4} \mathrm{e}$ $r 10=\alpha_{10} \alpha_{7}$.

(153)

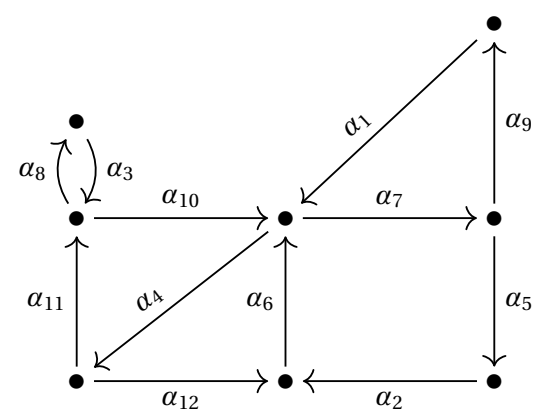

(154)

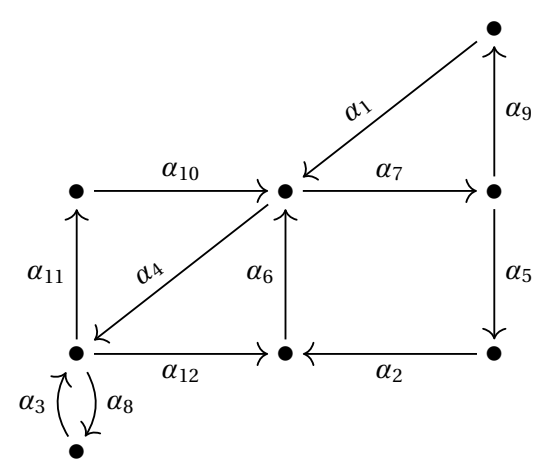

Essa extensão trivial apresenta os seguintes ciclos elementares: $C_{1}=\alpha_{8} \alpha_{3}, C_{2}=\alpha_{10} \alpha_{4} \alpha_{11}, C_{3}=$ $\alpha_{4} \alpha_{12} \alpha_{6}, C_{4}=\alpha_{6} \alpha_{7} \alpha_{5} \alpha_{2}$ e $C 5=\alpha_{7} \alpha_{9} \alpha_{1}$. Nesse caso, o programa exibe a solução 229 .

As relações do tipo 2 são: $r 1=\alpha_{3} \alpha_{11}, r 2=\alpha_{4} \alpha_{8}$, $r 3=\alpha_{10} \alpha_{4} \alpha_{12}, r 4=\alpha_{6} \alpha_{4} \alpha_{11}, r 5=\alpha_{12} \alpha_{6} \alpha_{7}, r 6=$ $\alpha_{2} \alpha_{6} \alpha_{4}, r 7=\alpha_{6} \alpha_{7} \alpha_{9}, r 8=\alpha_{1} \alpha_{7} \alpha_{5}, r 9=\alpha_{1} \alpha_{4}$, $r 10=\alpha_{10} \alpha_{7}$ e $r 11=\alpha_{3} \alpha_{12}$.

Essa extensão trivial apresenta os seguintes ciclos elementares: $C_{1}=\alpha_{8} \alpha_{3}, C_{2}=\alpha_{10} \alpha_{4} \alpha_{11}, C_{3}=$ $\alpha_{4} \alpha_{12} \alpha_{6}, C_{4}=\alpha_{6} \alpha_{7} \alpha_{5} \alpha_{2}$ e $C 5=\alpha_{7} \alpha_{9} \alpha_{1}$. Portanto, obtemos a álgebra 230 . 
(155)

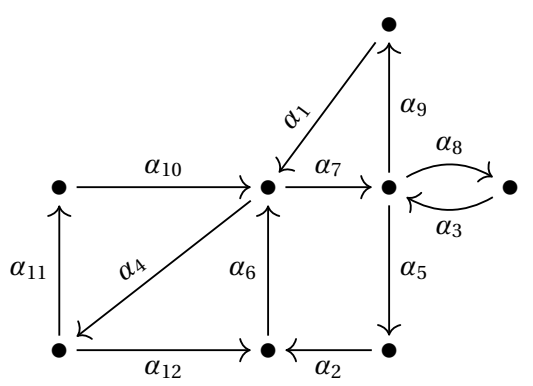

(156)

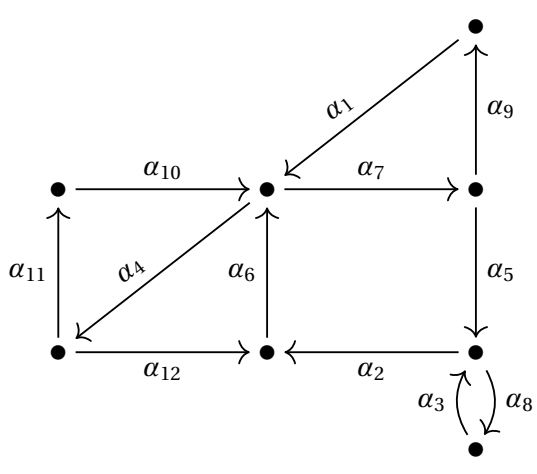

(157)

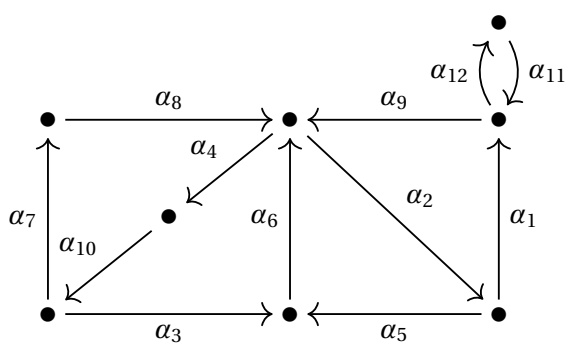

(158)

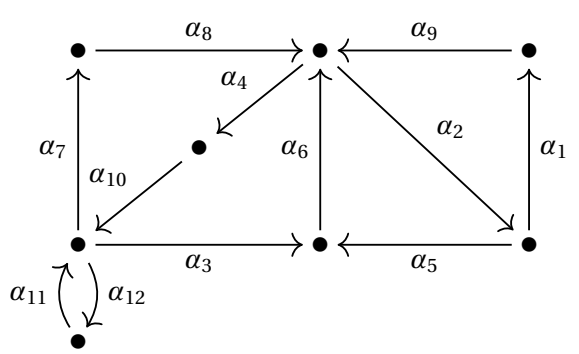

As relações do tipo 2 são: $r 1=\alpha_{3} \alpha_{9}, r 2=\alpha_{7} \alpha_{8}$, $r 3=\alpha_{10} \alpha_{4} \alpha_{12}, r 4=\alpha_{6} \alpha_{4} \alpha_{11}, r 5=\alpha_{12} \alpha_{6} \alpha_{7}, r 6=$ $\alpha_{2} \alpha_{6} \alpha_{4}, r 7=\alpha_{6} \alpha_{7} \alpha_{9}, r 8=\alpha_{1} \alpha_{7} \alpha_{5}, r 9=\alpha_{1} \alpha_{4}$, $r 10=\alpha_{10} \alpha_{7}$ e $r 11=\alpha_{3} \alpha_{5}$.

Essa extensão trivial apresenta os seguintes ciclos elementares: $C_{1}=\alpha_{8} \alpha_{3}, C_{2}=\alpha_{10} \alpha_{4} \alpha_{11}, C_{3}=$ $\alpha_{4} \alpha_{12} \alpha_{6}, C_{4}=\alpha_{6} \alpha_{7} \alpha_{5} \alpha_{2}$ e $C 5=\alpha_{7} \alpha_{9} \alpha_{1}$. Logo, temos o corte que origina a álgebra 231.

As relações do tipo 2 são: $r 1=\alpha_{3} \alpha_{2}, r 2=\alpha_{5} \alpha_{8}$, $r 3=\alpha_{10} \alpha_{4} \alpha_{12}, r 4=\alpha_{6} \alpha_{4} \alpha_{11}, r 5=\alpha_{12} \alpha_{6} \alpha_{7}, r 6=$ $\alpha_{2} \alpha_{6} \alpha_{4}, r 7=\alpha_{6} \alpha_{7} \alpha_{9}, r 8=\alpha_{1} \alpha_{7} \alpha_{5}, r 9=\alpha_{1} \alpha_{4} \mathrm{e}$ $r 10=\alpha_{10} \alpha_{7}$.

Essa extensão trivial apresenta os seguintes ciclos elementares: $C_{1}=\alpha_{8} \alpha_{3}, C_{2}=\alpha_{10} \alpha_{4} \alpha_{11}, C_{3}=$ $\alpha_{4} \alpha_{12} \alpha_{6}, C_{4}=\alpha_{6} \alpha_{7} \alpha_{5} \alpha_{2}$ e $C 5=\alpha_{7} \alpha_{9} \alpha_{1}$. Nesse caso, não obtemos solução.

Essa extensão trivial tem as seguintes relações do tipo 2: $r 1=\alpha_{8} \alpha_{4} \alpha_{10} \alpha_{3}, r 2=\alpha_{6} \alpha_{4} \alpha_{10} \alpha_{7}, r 3=$ $\alpha_{3} \alpha_{6} \alpha_{2}, r 4=\alpha_{5} \alpha_{6} \alpha_{4}, r 5=\alpha_{6} \alpha_{2} \alpha_{1}, r 6=\alpha_{9} \alpha_{2} \alpha_{5}$, $r 7=\alpha_{8} \alpha_{2}, r 8=\alpha_{9} \alpha_{4}, r 9=\alpha_{1} \alpha_{12}$ e $r 10=\alpha_{11} \alpha_{9}$.

Os ciclos elementares são: $C_{1}=\alpha_{4} \alpha_{10} \alpha_{7} \alpha_{8}, C_{2}=$ $\alpha_{9} \alpha_{2} \alpha_{1}, C_{3}=\alpha_{4} \alpha_{10} \alpha_{3} \alpha_{6}, C_{4}=\alpha_{6} \alpha_{2} \alpha_{5}$ e $C 5=$ $\alpha_{12} \alpha_{11}$. Portanto, obtemos a álgebra de incidência 232.

Essa extensão trivial tem as seguintes relações do tipo 2: $r 1=\alpha_{8} \alpha_{4} \alpha_{10} \alpha_{3}, r 2=\alpha_{6} \alpha_{4} \alpha_{10} \alpha_{7}, r 3=$ $\alpha_{3} \alpha_{6} \alpha_{2}, r 4=\alpha_{5} \alpha_{6} \alpha_{4}, r 5=\alpha_{6} \alpha_{2} \alpha_{1}, r 6=\alpha_{9} \alpha_{2} \alpha_{5}$, $r 7=\alpha_{8} \alpha_{2}, r 8=\alpha_{9} \alpha_{4}, r 9=\alpha_{10} \alpha_{12}, r 10=\alpha_{11} \alpha_{7} \mathrm{e}$ $r 11=\alpha_{11} \alpha_{3}$.

Os ciclos elementares são: $C_{1}=\alpha_{4} \alpha_{10} \alpha_{7} \alpha_{8}, C_{2}=$ $\alpha_{9} \alpha_{2} \alpha_{1}, C_{3}=\alpha_{4} \alpha_{10} \alpha_{3} \alpha_{6}, C_{4}=\alpha_{6} \alpha_{2} \alpha_{5}$ e $C 5=$ $\alpha_{12} \alpha_{11}$. Portanto, não temos solução. 
(159)

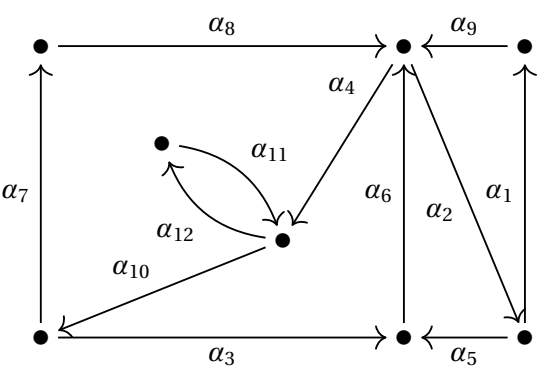

(160)

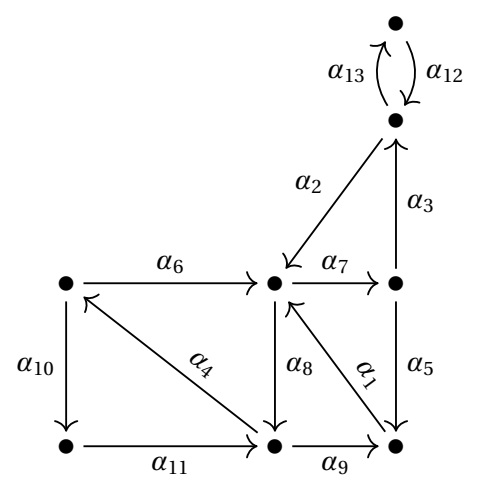

(161)

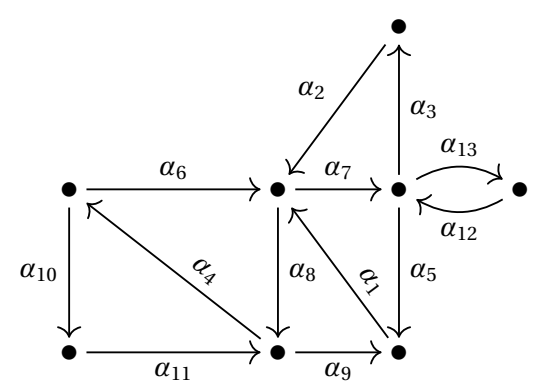

(162)

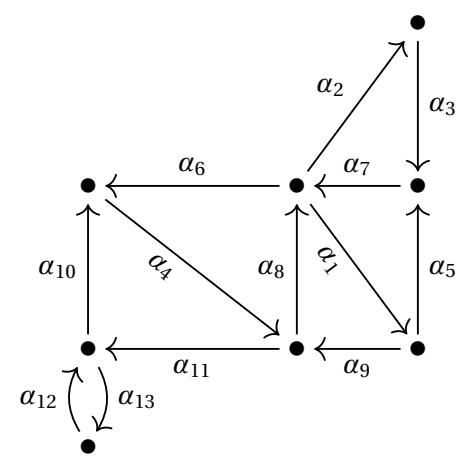

Essa extensão trivial tem as seguintes relações do tipo 2: $r 1=\alpha_{8} \alpha_{4} \alpha_{10} \alpha_{3}, r 2=\alpha_{6} \alpha_{4} \alpha_{10} \alpha_{7}, r 3=$ $\alpha_{3} \alpha_{6} \alpha_{2}, r 4=\alpha_{5} \alpha_{6} \alpha_{4}, r 5=\alpha_{6} \alpha_{2} \alpha_{1}, r 6=\alpha_{9} \alpha_{2} \alpha_{5}$, $r 7=\alpha_{8} \alpha_{2}, r 8=\alpha_{9} \alpha_{4}, r 9=\alpha_{4} \alpha_{12}$ e $r 10=\alpha_{11} \alpha_{10}$.

Os ciclos elementares são: $C_{1}=\alpha_{4} \alpha_{10} \alpha_{7} \alpha_{8}, C_{2}=$ $\alpha_{9} \alpha_{2} \alpha_{1}, C_{3}=\alpha_{4} \alpha_{10} \alpha_{3} \alpha_{6}, C_{4}=\alpha_{6} \alpha_{2} \alpha_{5}$ e $C 5=$ $\alpha_{12} \alpha_{11}$. O programa mostra o corte que origina a álgebra 233.

Essa extensão trivial tem as seguintes relações do tipo 2: $r 1=\alpha_{11} \alpha_{4} \alpha_{6}, r 2=\alpha_{8} \alpha_{4} \alpha_{10}, r 3=\alpha_{6} \alpha_{8} \alpha_{9}$, $r 4=\alpha_{1} \alpha_{8} \alpha_{4}, r 5=\alpha_{9} \alpha_{1} \alpha_{7}, r 6=\alpha_{5} \alpha_{1} \alpha_{8}, r 7=$ $\alpha_{2} \alpha_{7} \alpha_{5}, r 8=\alpha_{1} \alpha_{7} \alpha_{3}, r 9=\alpha_{2} \alpha_{8}, r 10=\alpha_{6} \alpha_{7}$, $r 11=\alpha_{11} \alpha_{9}, r 12=\alpha_{3} \alpha_{13}$ e $r 13=\alpha_{12} \alpha_{2}$.

Essa extensão trivial apresenta os seguintes ciclos elementares: $C_{1}=\alpha_{6} \alpha_{8} \alpha_{4}, C_{2}=\alpha_{10} \alpha_{11} \alpha_{4}, C_{3}=$ $\alpha_{7} \alpha_{3} \alpha_{2}, C_{4}=\alpha_{8} \alpha_{9} \alpha_{1}, C 5=\alpha_{1} \alpha_{7} \alpha_{5}$ e $C 6=\alpha_{12} \alpha_{13}$. Logo, não obtemos solução.

Essa extensão trivial tem as seguintes relações do tipo 2: $r 1=\alpha_{11} \alpha_{4} \alpha_{6}, r 2=\alpha_{8} \alpha_{4} \alpha_{10}, r 3=\alpha_{6} \alpha_{8} \alpha_{9}$, $r 4=\alpha_{1} \alpha_{8} \alpha_{4}, r 5=\alpha_{9} \alpha_{1} \alpha_{7}, r 6=\alpha_{5} \alpha_{1} \alpha_{8}, r 7=$ $\alpha_{2} \alpha_{7} \alpha_{5}, r 8=\alpha_{1} \alpha_{7} \alpha_{3}, r 9=\alpha_{2} \alpha_{8}, r 10=\alpha_{6} \alpha_{7}$, $r 11=\alpha_{11} \alpha_{9}, r 12=\alpha_{7} \alpha_{13}, r 13=\alpha_{12} \alpha_{5}$ e $r 14=$ $\alpha_{12} \alpha_{3}$.

Essa extensão trivial apresenta os seguintes ciclos elementares: $C_{1}=\alpha_{6} \alpha_{8} \alpha_{4}, C_{2}=\alpha_{10} \alpha_{11} \alpha_{4}, C_{3}=$ $\alpha_{7} \alpha_{3} \alpha_{2}, C_{4}=\alpha_{8} \alpha_{9} \alpha_{1}, C 5=\alpha_{1} \alpha_{7} \alpha_{5}$ e $C 6=\alpha_{12} \alpha_{13}$. O programa mostra a solução 234 .

Essa extensão trivial tem as seguintes relações do tipo 2: $r 1=\alpha_{10} \alpha_{4} \alpha_{8}, r 2=\alpha_{6} \alpha_{4} \alpha_{11}, r 3=\alpha_{9} \alpha_{8} \alpha_{6}$, $r 4=\alpha_{4} \alpha_{8} \alpha_{1}, r 5=\alpha_{7} \alpha_{1} \alpha_{9}, r 6=\alpha_{8} \alpha_{1} \alpha_{5}, r 7=$ $\alpha_{3} \alpha_{7} \alpha_{1}, r 8=\alpha_{5} \alpha_{7} \alpha_{2}, r 9=\alpha_{8} \alpha_{2}, r 10=\alpha_{7} \alpha_{6}$, $r 11=\alpha_{9} \alpha_{11}, r 12=\alpha_{11} \alpha_{13}$ e $r 13=\alpha_{12} \alpha_{10}$.

Essa extensão trivial apresenta os seguintes ciclos elementares: $C_{1}=\alpha_{6} \alpha_{4} \alpha_{8}, C_{2}=\alpha_{10} \alpha_{4} \alpha_{11}, C_{3}=$ $\alpha_{7} \alpha_{2} \alpha_{3}, C_{4}=\alpha_{8} \alpha_{1} \alpha_{9}, C 5=\alpha_{1} \alpha_{5} \alpha_{7}$ e $C 6=\alpha_{12} \alpha_{13}$.

Portanto, obtemos a solução 235. 
(163)

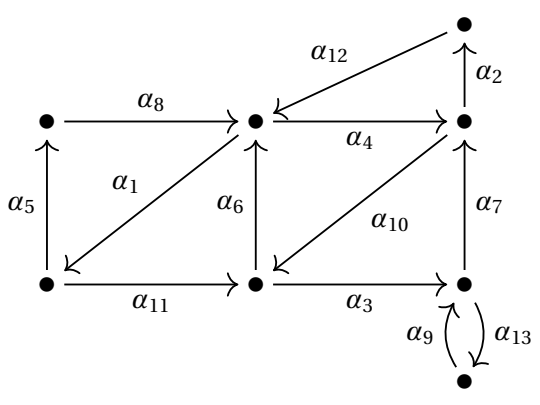

(164)

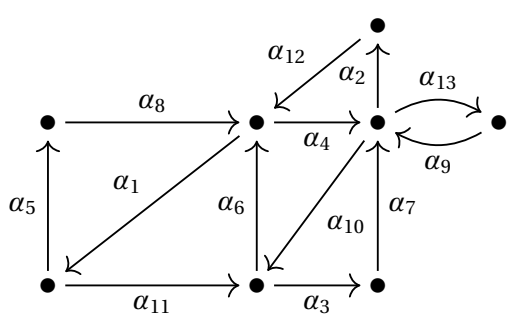

(165)

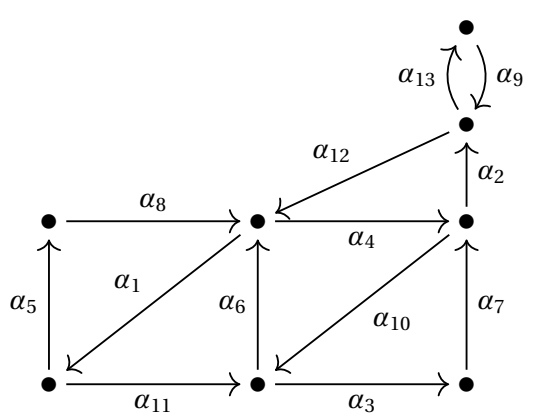

Essa extensão trivial tem as seguintes relações do tipo 2: $r 1=\alpha_{8} \alpha_{1} \alpha_{11}, r 2=\alpha_{6} \alpha_{1} \alpha_{5}, r 3=\alpha_{11} \alpha_{6} \alpha_{4}$, $r 4=\alpha_{10} \alpha_{6} \alpha_{1}, r 5=\alpha_{4} \alpha_{10} \alpha_{3}, r 6=\alpha_{7} \alpha_{10} \alpha_{6}, r 7=$ $\alpha_{6} \alpha_{4} \alpha_{2}, r 8=\alpha_{12} \alpha_{4} \alpha_{10}, r 9=\alpha_{7} \alpha_{2}, r 10=\alpha_{11} \alpha_{3}$, $r 11=\alpha_{12} \alpha_{1}, r 12=\alpha_{8} \alpha_{4}, r 13=\alpha_{9} \alpha_{7}$ e $r 14=$ $\alpha_{3} \alpha_{13}$.

Os ciclos elementares dessa extensão trivial são: $C_{1}=\alpha_{4} \alpha_{2} \alpha_{12}, C_{2}=\alpha_{4} \alpha_{10} \alpha_{6}, C_{3}=\alpha_{10} \alpha_{3} \alpha_{7}, C_{4}=$ $\alpha_{6} \alpha_{1} \alpha_{11}, C_{5}=\alpha_{8} \alpha_{1} \alpha_{5}$ e $C_{6}=\alpha_{9} \alpha_{13}$. Portanto, não obtemos álgebra de incidência através do corte.

Essa extensão trivial tem as seguintes relações do tipo 2: $r 1=\alpha_{8} \alpha_{1} \alpha_{11}, r 2=\alpha_{6} \alpha_{1} \alpha_{5}, r 3=\alpha_{11} \alpha_{6} \alpha_{4}$, $r 4=\alpha_{10} \alpha_{6} \alpha_{1}, r 5=\alpha_{4} \alpha_{10} \alpha_{3}, r 6=\alpha_{7} \alpha_{10} \alpha_{6}, r 7=$ $\alpha_{6} \alpha_{4} \alpha_{2}, r 8=\alpha_{12} \alpha_{4} \alpha_{10}, r 9=\alpha_{7} \alpha_{2}, r 10=\alpha_{11} \alpha_{3}$, $r 11=\alpha_{12} \alpha_{1}, r 12=\alpha_{8} \alpha_{4}, r 13=\alpha_{9} \alpha_{2}, r 14=\alpha_{4} \alpha_{13}$, $r 15=\alpha_{9} \alpha_{10}$ e $r 16=\alpha_{7} \alpha_{13}$.

Os ciclos elementares dessa extensão trivial são: $C_{1}=\alpha_{4} \alpha_{2} \alpha_{12}, C_{2}=\alpha_{4} \alpha_{10} \alpha_{6}, C_{3}=\alpha_{10} \alpha_{3} \alpha_{7}, C_{4}=$ $\alpha_{6} \alpha_{1} \alpha_{11}, C_{5}=\alpha_{8} \alpha_{1} \alpha_{5}$ e $C_{6}=\alpha_{9} \alpha_{13}$. O programa não exibe solução.

Essa extensão trivial tem as seguintes relações do tipo 2: $r 1=\alpha_{8} \alpha_{1} \alpha_{11}, r 2=\alpha_{6} \alpha_{1} \alpha_{5}, r 3=\alpha_{11} \alpha_{6} \alpha_{4}$, $r 4=\alpha_{10} \alpha_{6} \alpha_{1}, r 5=\alpha_{4} \alpha_{10} \alpha_{3}, r 6=\alpha_{7} \alpha_{10} \alpha_{6}, r 7=$ $\alpha_{6} \alpha_{4} \alpha_{2}, r 8=\alpha_{12} \alpha_{4} \alpha_{10}, r 9=\alpha_{7} \alpha_{2}, r 10=\alpha_{11} \alpha_{3}$, $r 11=\alpha_{12} \alpha_{1}, r 12=\alpha_{8} \alpha_{4}, r 13=\alpha_{9} \alpha_{12}$ e $r 14=$ $\alpha_{2} \alpha_{13}$.

Os ciclos elementares dessa extensão trivial são: $C_{1}=\alpha_{4} \alpha_{2} \alpha_{12}, C_{2}=\alpha_{4} \alpha_{10} \alpha_{6}, C_{3}=\alpha_{10} \alpha_{3} \alpha_{7}, C_{4}=$ $\alpha_{6} \alpha_{1} \alpha_{11}, C_{5}=\alpha_{8} \alpha_{1} \alpha_{5}$ e $C_{6}=\alpha_{9} \alpha_{13}$. Logo, não obtemos solução. 
(166)

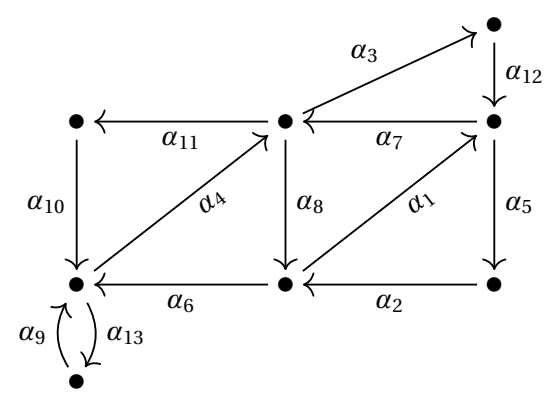

(167)

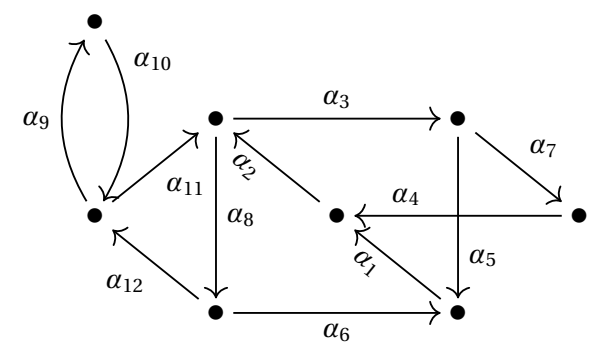

(168)

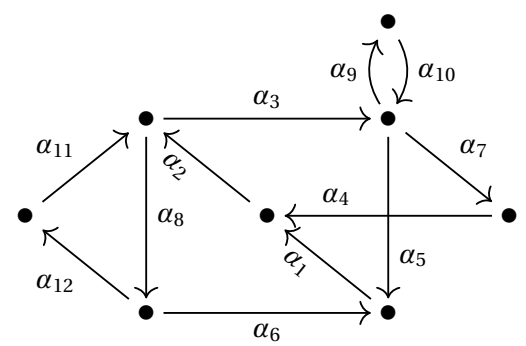

(169)

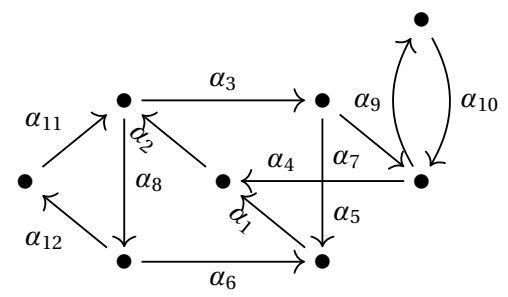

Essa extensão trivial tem as seguintes relações do tipo 2: $r 1=\alpha_{10} \alpha_{4} \alpha_{8}, r 2=\alpha_{6} \alpha_{4} \alpha_{11}, r 3=\alpha_{4} \alpha_{8} \alpha_{1}$, $r 4=\alpha_{7} \alpha_{8} \alpha_{6}, r 5=\alpha_{8} \alpha_{1} \alpha_{5}, r 6=\alpha_{2} \alpha_{1} \alpha_{7}, r 7=$ $\alpha_{1} \alpha_{7} \alpha_{3}, r 8=\alpha_{12} \alpha_{7} \alpha_{8}, r 9=\alpha_{7} \alpha_{11}, r 10=\alpha_{4} \alpha_{3}$, $r 11=\alpha_{12} \alpha_{5}, r 12=\alpha_{2} \alpha_{6}, r 13=\alpha_{9} \alpha_{4}, r 14=\alpha_{10} \alpha_{13}$ e $r 15=\alpha_{6} \alpha_{13}$.

Os ciclos elementares dessa extensão trivial são: $C_{1}=\alpha_{4} \alpha_{8} \alpha_{6}, C_{2}=\alpha_{4} \alpha_{11} \alpha_{10}, C_{3}=\alpha_{12} \alpha_{7} \alpha_{3}, C_{4}=$ $\alpha_{8} \alpha_{1} \alpha_{7}, C_{5}=\alpha_{2} \alpha_{1} \alpha_{5}$ e $C_{6}=\alpha_{9} \alpha_{13}$. Também, não obtemos solução.

Essa extensão trivial tem as seguintes relações do tipo 2: $r 1=\alpha_{6} \alpha_{1} \alpha_{2} \alpha_{3}, r 2=\alpha_{5} \alpha_{1} \alpha_{2} \alpha_{8}, r 3=$ $\alpha_{1} \alpha_{2} \alpha_{3} \alpha_{7}, r 4=\alpha_{4} \alpha_{2} \alpha_{3} \alpha_{5}, r 5=\alpha_{4} \alpha_{2} \alpha_{8}, r 6=$ $\alpha_{12} \alpha_{9}, r 7=\alpha_{10} \alpha_{11}, r 8=\alpha_{2} \alpha_{8} \alpha_{12}, r 9=\alpha_{11} \alpha_{3} \mathrm{e}$ $r 10=\alpha_{11} \alpha_{8} \alpha_{6}$.

Essa extensão trivial apresenta os seguintes ciclos elementares: $C_{1}=\alpha_{6} \alpha_{1} \alpha_{2} \alpha_{8}, C_{2}=\alpha_{2} \alpha_{3} \alpha_{7} \alpha_{4}, C_{3}=$ $\alpha_{1} \alpha_{2} \alpha_{3} \alpha_{5}, C_{4}=\alpha_{10} \alpha_{9}$ e $C_{5}=\alpha_{11} \alpha_{8} \alpha_{12}$. Nessa álgebra, obtemos a álgebra de incidência 236.

Essa extensão trivial tem as seguintes relações do tipo 2: $r 1=\alpha_{6} \alpha_{1} \alpha_{2} \alpha_{3}, r 2=\alpha_{5} \alpha_{1} \alpha_{2} \alpha_{8}, r 3=$ $\alpha_{1} \alpha_{2} \alpha_{3} \alpha_{7}, r 4=\alpha_{4} \alpha_{2} \alpha_{3} \alpha_{5}, r 5=\alpha_{4} \alpha_{2} \alpha_{8}, r 6=$ $\alpha_{3} \alpha_{9}, r 7=\alpha_{10} \alpha_{7}, r 8=\alpha_{2} \alpha_{8} \alpha_{12}, r 9=\alpha_{11} \alpha_{3}$, $r 10=\alpha_{11} \alpha_{8} \alpha_{6}$ e $r 11=\alpha_{10} \alpha_{5}$.

Essa extensão trivial apresenta os seguintes ciclos elementares: $C_{1}=\alpha_{6} \alpha_{1} \alpha_{2} \alpha_{8}, C_{2}=\alpha_{2} \alpha_{3} \alpha_{7} \alpha_{4}, C_{3}=$ $\alpha_{1} \alpha_{2} \alpha_{3} \alpha_{5}, C_{4}=\alpha_{10} \alpha_{9}$ e $C_{5}=\alpha_{11} \alpha_{8} \alpha_{12}$. Portanto obtemos a solução 237 .

Essa extensão trivial tem as seguintes relações do tipo 2: $r 1=\alpha_{6} \alpha_{1} \alpha_{2} \alpha_{3}, r 2=\alpha_{5} \alpha_{1} \alpha_{2} \alpha_{8}, r 3=$ $\alpha_{1} \alpha_{2} \alpha_{3} \alpha_{7}, r 4=\alpha_{4} \alpha_{2} \alpha_{3} \alpha_{5}, r 5=\alpha_{4} \alpha_{2} \alpha_{8}, r 6=$ $\alpha_{7} \alpha_{9}, r 7=\alpha_{10} \alpha_{4}, r 8=\alpha_{2} \alpha_{8} \alpha_{12}, r 9=\alpha_{11} \alpha_{3} \mathrm{e}$ $r 10=\alpha_{11} \alpha_{8} \alpha_{6}$.

Essa extensão trivial apresenta os seguintes ciclos elementares: $C_{1}=\alpha_{6} \alpha_{1} \alpha_{2} \alpha_{8}, C_{2}=\alpha_{2} \alpha_{3} \alpha_{7} \alpha_{4}, C_{3}=$ $\alpha_{1} \alpha_{2} \alpha_{3} \alpha_{5}, C_{4}=\alpha_{10} \alpha_{9}$ e $C_{5}=\alpha_{11} \alpha_{8} \alpha_{12}$. Nessa álgebra, não obtemos nenhuma álgebra de incidência através do corte. 
(170)

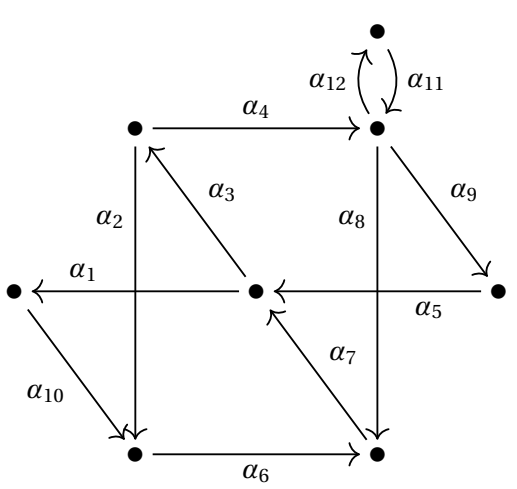

(171)

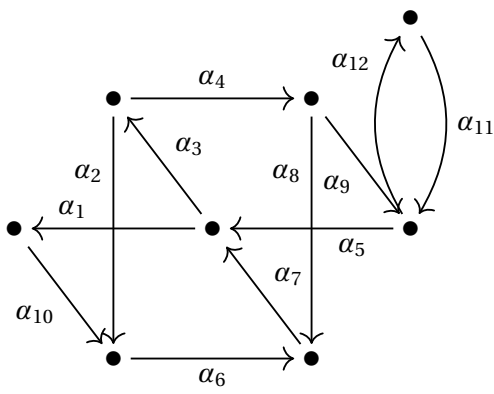

(172)

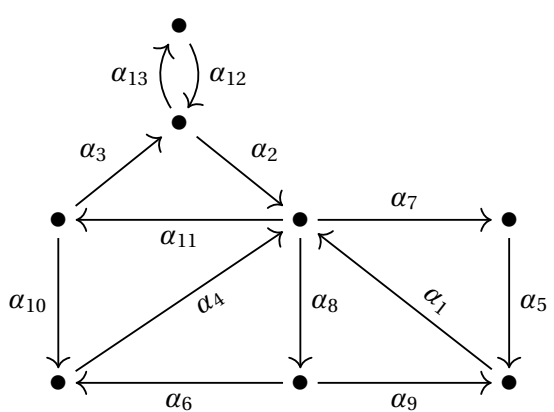

(173)

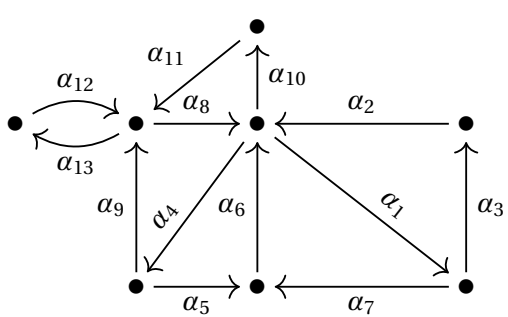

Essa extensão trivial tem as seguintes relações do tipo 2: $r 1=\alpha_{5} \alpha_{3} \alpha_{4} \alpha_{8}, r 2=\alpha_{7} \alpha_{3} \alpha_{4} \alpha_{9}, r 3=$ $\alpha_{8} \alpha_{7} \alpha_{3} \alpha_{2}, r 4=\alpha_{6} \alpha_{7} \alpha_{3} \alpha_{4}, r 5=\alpha_{5} \alpha_{3} \alpha_{2}, r 6=$ $\alpha_{2} \alpha_{6} \alpha_{7} \alpha_{1}, r 7=\alpha_{10} \alpha_{6} \alpha_{7} \alpha_{3}, r 8=\alpha_{5} \alpha_{1}, r 9=\alpha_{11} \alpha_{9}$, $r 10=\alpha_{11} \alpha_{8}$ e $r 11=\alpha_{4} \alpha_{12}$.

Os ciclos elementares são: $C_{1}=\alpha_{4} \alpha_{9} \alpha_{5} \alpha_{3}, C_{2}=$ $\alpha_{4} \alpha_{8} \alpha_{7} \alpha_{3}, C_{3}=\alpha_{7} \alpha_{3} \alpha_{2} \alpha_{6}, C_{4}=\alpha_{7} \alpha_{1} \alpha_{10} \alpha_{6}$ e $C_{5}=$ $\alpha_{12} \alpha_{11}$. Logo, o programa não exibe corte.

Essa extensão trivial tem as seguintes relações do tipo 2: $r 1=\alpha_{5} \alpha_{3} \alpha_{4} \alpha_{8}, r 2=\alpha_{7} \alpha_{3} \alpha_{4} \alpha_{9}, r 3=$ $\alpha_{8} \alpha_{7} \alpha_{3} \alpha_{2}, r 4=\alpha_{6} \alpha_{7} \alpha_{3} \alpha_{4}, r 5=\alpha_{5} \alpha_{3} \alpha_{2}, r 6=$ $\alpha_{2} \alpha_{6} \alpha_{7} \alpha_{1}, r 7=\alpha_{10} \alpha_{6} \alpha_{7} \alpha_{3}, r 8=\alpha_{5} \alpha_{1}, r 9=\alpha_{11} \alpha_{9}$, $r 10=\alpha_{11} \alpha_{8}$ e $r 11=\alpha_{4} \alpha_{12}$.

Os ciclos elementares são: $C_{1}=\alpha_{4} \alpha_{9} \alpha_{5} \alpha_{3}, C_{2}=$ $\alpha_{4} \alpha_{8} \alpha_{7} \alpha_{3}, C_{3}=\alpha_{7} \alpha_{3} \alpha_{2} \alpha_{6}, C_{4}=\alpha_{7} \alpha_{1} \alpha_{10} \alpha_{6}$ e $C_{5}=$ $\alpha_{12} \alpha_{11}$. Logo, o programa exibe a solução 238.

Essa extensão trivial tem as seguintes relações do tipo 2: $r 1=\alpha_{2} \alpha_{11} \alpha_{10}, r 2=\alpha_{4} \alpha_{11} \alpha_{3}, r 3=\alpha_{10} \alpha_{4} \alpha_{8}$, $r 4=\alpha_{6} \alpha_{4} \alpha_{11}, r 5=\alpha_{4} \alpha_{8} \alpha_{9}, r 6=\alpha_{1} \alpha_{8} \alpha_{6}, r 7=$ $\alpha_{9} \alpha_{1} \alpha_{7}, r 8=\alpha_{5} \alpha_{1} \alpha_{8}, r 9=\alpha_{2} \alpha_{7}, r 10=\alpha_{1} \alpha_{11}$, $r 11=\alpha_{4} \alpha_{7}, r 12=\alpha_{2} \alpha_{8}, r 13=\alpha_{3} \alpha_{13}$ e $r 14=$ $\alpha_{12} \alpha_{2}$.

Essa extensão trivial apresenta os seguintes ciclos elementares: $C_{1}=\alpha_{2} \alpha_{11} \alpha_{3}, C_{2}=\alpha_{11} \alpha_{10} \alpha_{4}, C_{3}=$ $\alpha_{4} \alpha_{8} \alpha_{6}, C_{4}=\alpha_{8} \alpha_{9} \alpha_{1}, C 5=\alpha_{1} \alpha_{7} \alpha_{5}$ e $C 6=\alpha_{13} \alpha_{12}$. Portanto, temos a álgebras de incidência 239.

As relações do tipo 2 são: $r 1=\alpha_{5} \alpha_{6} \alpha_{1}, r 2=$ $\alpha_{7} \alpha_{6} \alpha_{4}, r 3=\alpha_{6} \alpha_{1} \alpha_{3}, r 4=\alpha_{2} \alpha_{1} \alpha_{7}, r 5=\alpha_{2} \alpha_{4}$, $r 6=\alpha_{8} \alpha_{1}, r 7=\alpha_{6} \alpha_{4} \alpha_{9}, r 8=\alpha_{8} \alpha_{4} \alpha_{5}, r 9=$ $\alpha_{11} \alpha_{8} \alpha_{4}, r 10=\alpha_{9} \alpha_{8} \alpha_{10}, r 11=\alpha_{6} \alpha_{10}, r 12=\alpha_{12} \alpha_{8}$, $r 13=\alpha_{9} \alpha_{13}$ e $r 14=\alpha_{11} \alpha_{13}$.

Os ciclos elementares são: $C_{1}=\alpha_{5} \alpha_{6} \alpha_{4}, C_{2}=$ $\alpha_{6} \alpha_{1} \alpha_{7}, C_{3}=\alpha_{1} \alpha_{3} \alpha_{2}, C_{4}=\alpha_{8} \alpha_{4} \alpha_{9}, C_{5}=\alpha_{8} \alpha_{10} \alpha_{11}$ e $C_{6}=\alpha_{12} \alpha_{13}$. Logo, obtemos o corte que gera a solução 240 . 
(174)

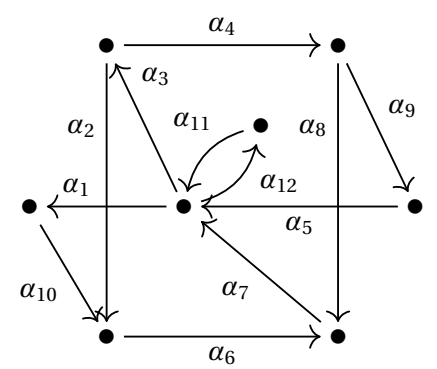

Essa extensão trivial tem as seguintes relações do tipo 2: $r 1=\alpha_{5} \alpha_{3} \alpha_{4} \alpha_{8}, r 2=\alpha_{7} \alpha_{3} \alpha_{4} \alpha_{9}, r 3=$ $\alpha_{8} \alpha_{7} \alpha_{3} \alpha_{2}, r 4=\alpha_{6} \alpha_{7} \alpha_{3} \alpha_{4}, r 5=\alpha_{5} \alpha_{3} \alpha_{2}, r 6=$ $\alpha_{2} \alpha_{6} \alpha_{7} \alpha_{1}, r 7=\alpha_{10} \alpha_{6} \alpha_{7} \alpha_{3}, r 8=\alpha_{5} \alpha_{1}, r 9=\alpha_{11} \alpha_{9}$, $r 10=\alpha_{11} \alpha_{8}$ e $r 11=\alpha_{4} \alpha_{12}$.

Os ciclos elementares são: $C_{1}=\alpha_{4} \alpha_{9} \alpha_{5} \alpha_{3}, C_{2}=$ $\alpha_{4} \alpha_{8} \alpha_{7} \alpha_{3}, C_{3}=\alpha_{7} \alpha_{3} \alpha_{2} \alpha_{6}, C_{4}=\alpha_{7} \alpha_{1} \alpha_{10} \alpha_{6}$ e $C_{5}=$ $\alpha_{12} \alpha_{11}$. Logo, o programa exibe os cortes para originar as álgebras 241 e 242.

\subsection{Os tipos $\widetilde{\mathbb{D}}_{m}, \widetilde{\mathbb{E}}_{6}, \widetilde{\mathbb{E}}_{7}, \widetilde{\mathbb{E}}_{8}$}

As Phias de tipo euclidiano são derivadamente equivalentes à categoria dos feixes coerentes sobre certas retas projetivas com peso. Têm uma ligação com as álgebras canônicas, conforme na introdução, explicamos que a categoria dos feixes coerentes é equivalente à categoria de módulos sobre as álgebras canônicas. As álgebras canônicas $C(2,2, m-2)$, com $m \geq 4, C(2,3,3)$, $C(2,3,4)$ e $C(2,3,5)$, são as chamadas álgebras canônicas de tipo euclidiano $\widetilde{\mathbb{D}}_{m}, \widetilde{\mathbb{E}}_{6}, \widetilde{\mathbb{E}}_{7}, \widetilde{\mathbb{E}}_{8}$, respectivamente, e também chamadas de álgebras disfarçadas de tipo euclidiano.

\section{Definição 5.26 (Álgebra disfarçada de tipo euclidiano)}

Seja $B$ uma álgebra da forma $\operatorname{End}_{K Q} T$, em que $T$ é um módulo inclinante posprojetivo sobre a álgebra $K Q$, com $\bar{Q} \in\left\{\widetilde{\mathbb{A}}_{n}, \widetilde{\mathbb{D}}_{m}, \widetilde{\mathbb{E}}_{6}, \widetilde{\mathbb{E}}_{7}, \widetilde{\mathbb{E}}_{8}\right\}$. Chamamos $B$ de uma álgebra disfarçada de tipo euclidiano.

Por definição, as álgebras canônicas de tipo euclidiano são inclinadas iteradas de tipo $\widetilde{\mathbb{D}}_{m}, \widetilde{\mathbb{E}}_{6}$, $\widetilde{\mathbb{E}}_{7}, \widetilde{\mathbb{E}}_{8}$, respectivamente. Pelo teorema 1.14 , temos que $C(2,2, m-2)$, com $m \geq 4, C(2,3,3)$, $C(2,3,4)$ e $C(2,3,5)$, são hereditárias por partes de tipo $\widetilde{\mathbb{D}}_{m}, \widetilde{\mathbb{E}}_{6}, \widetilde{\mathbb{E}}_{7}, \widetilde{\mathbb{E}}_{8}$, respectivamente.

Outra maneira de mostrar a equivalência derivada entre as álgebras de tipo euclidiano e os feixes coerentes sobre certas retas projetivas com peso é através do teorema de Hubner [H8̈9]. Nesse teorema, é apresentado o objeto inclinante $T$ da categoria Coh $\mathbb{X}$, com $\mathbb{X}$ específico, cujo End $T$ é isomorfo à álgebra de caminhos de uma aljava euclidiana.

Não temos uma descrição completa das Phias de tipo euclidiano como no caso Dynkin, mas conseguimos identificar algumas famílias que mostraremos nas próximas seções. 


\subsection{1 Álgebras minimais de tipo de representação infinito}

Happel e Vossieck caracterizaram as álgebras minimais de tipo representação infinito com componente posprojetiva no trabalho [HV83]. Independemente, Bongartz com o trabalho "Critical simply connected algebras" [Bon84] chegou no mesmo resultado. Essa é a base que usaremos para identificar a família de Phias minimais de tipo de representação infinito com componente posprojetiva. Relembraremos duas definições:

\section{Definição 5.27 (álgebra minimal de tipo de representação infinito)}

Seja A uma álgebra de tipo de representação infinito. Chamaremos de minimal se as álgebras quocientes $A / A e$ A são de tipo de representação finito, para qualquer idempotente não nulo $e \neq 1$ de $A$.

\section{Definição 5.28 (componente posprojetiva)}

Seja A uma álgebra, denotaremos a aljava de Auslander-Reiten de $A$ por $\Gamma(\bmod A)$. Uma componente conexa $C$ de $\Gamma(\bmod A)$ é chamado de posprojetivo caso não existe nenhum ciclo orientado em $C$ e qualquer módulo de $C$ é da forma $\tau^{-t} P$ para algum número natural $t$ e um projetivo indecomponível $P$, em que $\tau$ é a translação de Auslander-Reiten.

A razão para estudar o artigo [HV83] do Happel e Vossieck é o seguinte teorema:

Teorema 5.29 (Happel-Vossieck). Dada uma álgebra A, as condições abaixo são equivalentes:

1. A álgebra A é minimal de tipo de representação infinito e tem uma componente posprojetiva.

2. A álgebra A é a álgebra de caminho da aljava de Kronecker generalizada

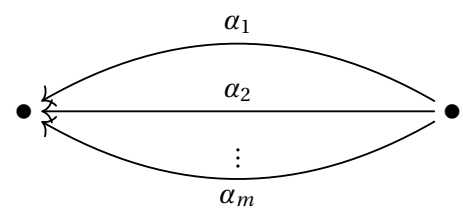

com $m \geq 2$ flechas $\alpha_{1}, \ldots, \alpha_{m}$, ou A é uma álgebra disfarçada de tipo euclidiano.

Assim, seja $A$ uma álgebra de incidência que é minimal de tipo de representação infinito e tem uma componente posprojetiva, se e somente, se $A$ é Phia de tipo euclidiano. Portanto, precisamos identificar as álgebras de incidência na classificação do Happel, Vossieck e Bongartz. Eles fizeram uma lista de 149 grafos, chamados de frames. A descrição completa das aljavas limitadas $\left(Q_{B}, I_{B}\right)$ em que $B \cong K Q_{B} / I_{B}$ é uma álgebra disfarçada de tipo euclidiano é dado por três tipos de operações admissíveis nos frames. Vejamos as operações admissíveis:

(Op1) Trocar qualquer aresta não orientada do frame por uma aresta orientada.

(Op2) Dado um frame da forma 


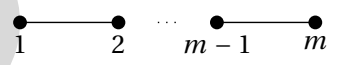

trocar a subaljava

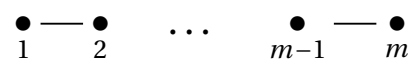

por qualquer subaljava conexa e plena com $m$ vértices da seguinte árvore

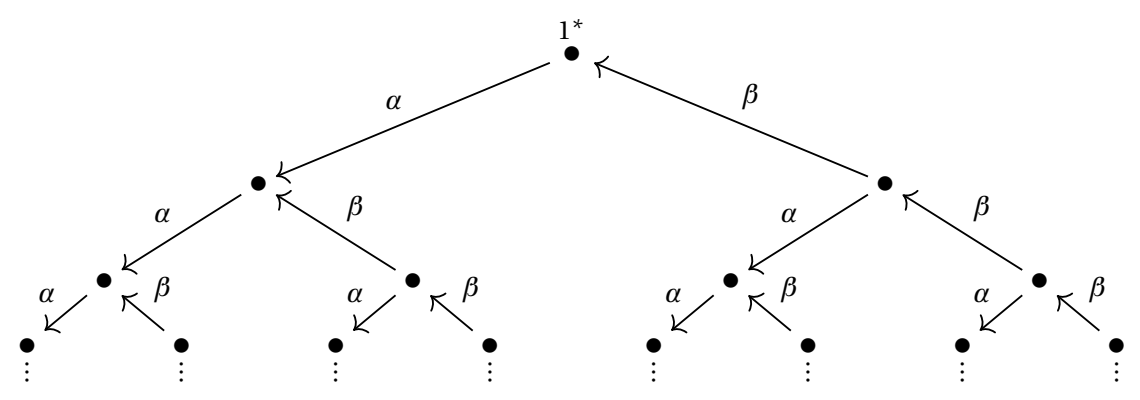

e limitada por todas as possíveis relações $\beta \alpha$, contendo o vértice $1^{*}$, pela identificação dos vértices 1 e $1^{*}$.

(Op3) Construir a sua álgebra oposta.

Agora, vamos resumir as operações admissíveis para o caso das álgebras de incidência. Podemos ver acima que precisamos reescrever apenas a operação admissível dois:

(Op2) Dado um frame da forma

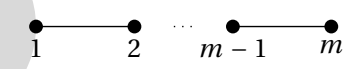

trocar a subaljava

$$
\stackrel{\bullet}{i} \underset{2}{\bullet} \quad \cdots \quad \stackrel{\bullet-1}{m}-\stackrel{\bullet}{m}
$$

por qualquer subaljava abaixo

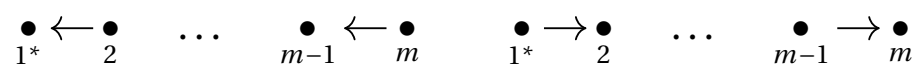

pela identificação dos vértices 1 e $1^{*}$. 
Observamos que a operação admissível dois para o caso das álgebras de incidência recai em alguns casos de operações admissíveis um. Ou seja, vamos admitir que precisamos apenas das operações admissíveis um e três nos frames para as álgebras de incidência.

Exemplo 5.30. Vejamos um exemplo com o frame de tipo $\widetilde{\mathbb{E}}_{7}$.

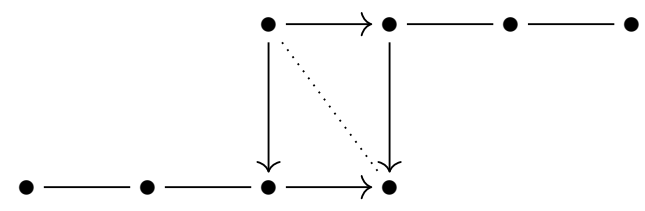

Podemos construir 16 aljavas limitadas não isomorfas aplicando a operação admissível um. Dentro dessas, temos a álgebra abaixo:

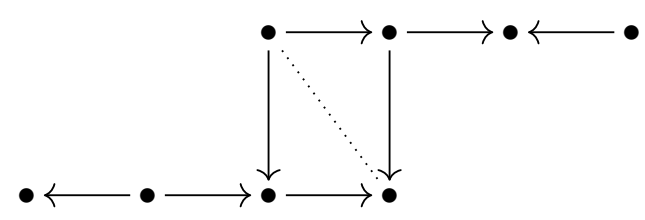

Usando a operação admissível dois, temos 4 aljavas não isomorfas. Reparamos que essas aljavas já estão entre as 16 feitas acima. O que resta é usar a operação admissível três, nas 16 aljavas.

Totalizamos 32 álgebras não isomorfas que são disfarçadas de tipo $\widetilde{\mathbb{E}}_{7}$.

O capítulo XIV do livro "Elements of the representation theory of associative algebras" [SS07a] mostra a lista enumerada de todos os frames do artigo "Minimal algebras of inifinite representation type with preprojective component" [HV83]. Usaremos essa listagem para escrever a série de resultados para as álgebras disfarçadas de tipo $\widetilde{\mathbb{D}}_{m}, \widetilde{\mathbb{E}}_{6}, \widetilde{\mathbb{E}}_{7}$ e $\widetilde{\mathbb{E}}_{8}$, em conjunto com o trabalho [HV83] para corrigir as pequenas falhas do livro.

Teorema 5.31. Seja $K \Delta=K Q / I$ uma álgebra disfarçada de tipo $\widetilde{\mathbb{D}}_{m}$, em que $I$ é não nulo. Então $K \Delta$ é isomorfa a um dos frames abaixo aplicado com as operação(ões) admissível(is).
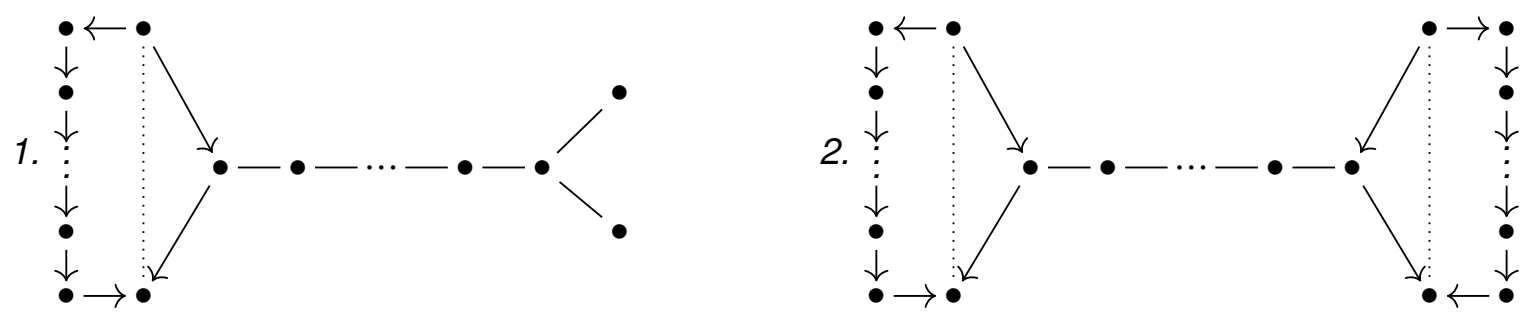

Demonstração $A$ tabela dos frames das álgebras disfarçadas de tipo $\widetilde{\mathbb{D}}_{m}$ tem quatro frames nomeados como $\mathcal{F} r 2, \mathcal{F} r 3, \mathcal{F} r 4$ e $\mathcal{F} r 5$. As únicas que são álgebras de incidência com relações de comutatividade são: $\mathcal{F} r 4$ e $\mathcal{F} r 5$.

Teorema 5.32. Seja $K \Delta=K Q / I$ uma álgebra disfarçada de tipo $\widetilde{\mathbb{E}}_{6}$, em que $I$ é não nulo. Então $K \Delta$ é isomorfa a um dos frames abaixo aplicado com as operação(ões) admissível(is). 

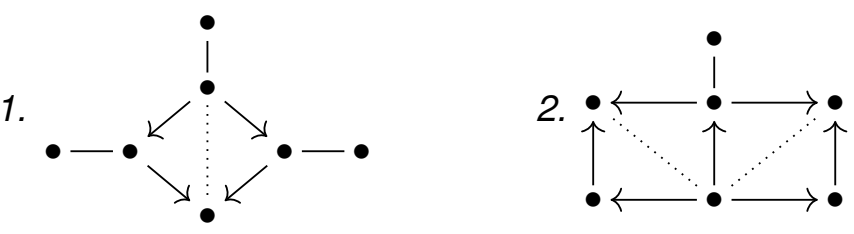

3.

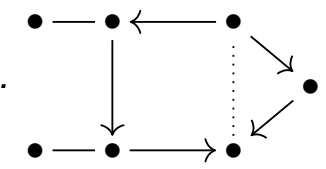

Demonstração A lista dos frames tem cinco frames: $\mathcal{F} r 6, \mathcal{F} r 7, \mathcal{F} r 8, \mathcal{F} r 9$ e $\mathcal{F} r 10$. As únicas que são álgebras de incidência não hereditárias são: $\mathcal{F} r 7, \mathcal{F} r 8$ e $\mathcal{F} r 9$.

Teorema 5.33. Seja $K \Delta=K Q / I$ uma álgebra disfarçada de tipo $\widetilde{\mathbb{E}}_{7}$, em que I é não nulo. Então $K \Delta$ é isomorfa a um dos frames abaixo aplicado com as operação(ões) admissível(is).

1.

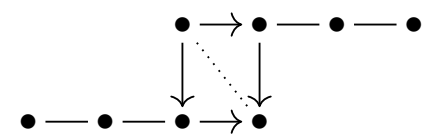

3.

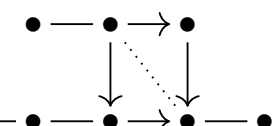

5 .

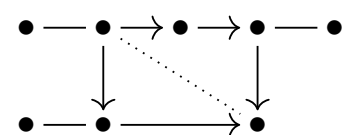

7.

$\bullet-\bullet \leftarrow \bullet \rightarrow \bullet-\bullet$

9.

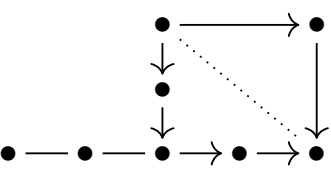

11.

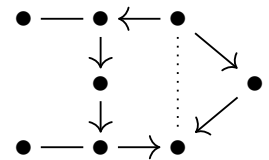

13.

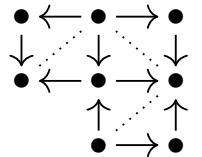

15.

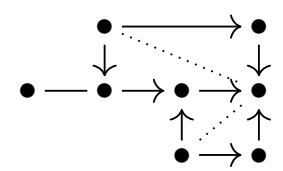

2.

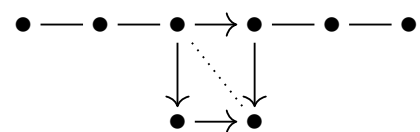

4.

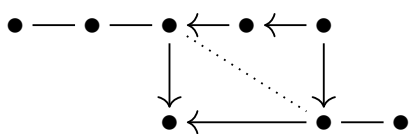

6.

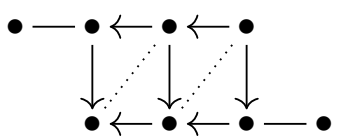

8.<smiles>CC12C3C4C5C3C1C5C42</smiles>

10.

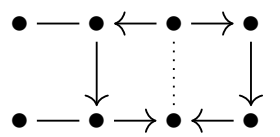

12.

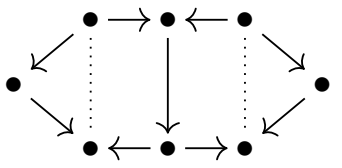

14.

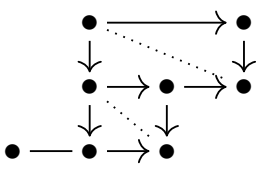

16.

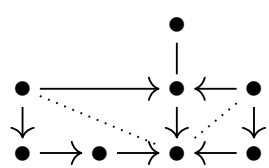

Demonstração A listagem tem 22 frames, do $\mathcal{F} r 11$ ao $\mathcal{F} r 32$. As Phias com $I$ não nulo são: $\mathcal{F} r 12, \mathcal{F} r 13, \mathcal{F} r 14, \mathcal{F} r 16, \mathcal{F} r 17, \mathcal{F} r 18, \mathcal{F} r 19, \mathcal{F} r 20, \mathcal{F} r 22, \mathcal{F} r 25, \mathcal{F} r 26$ (corrigido pelo artigo [HV83]), $\mathcal{F} r 27, \mathcal{F} r 29, \mathcal{F} r 30, \mathcal{F} r 31$ e $\mathcal{F} r 32$ (corrigido pelo artigo [HV83]).

Teorema 5.34. Seja $K \Delta=K Q / I$ uma álgebra disfarçada de tipo $\widetilde{\mathbb{E}}_{8}$, em que $I$ é não nulo. Então $K \Delta$ é isomorfa a um dos frames abaixo aplicado com as operação(ões) admissível(is).

1.

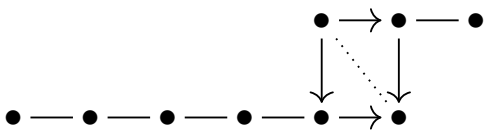

2.

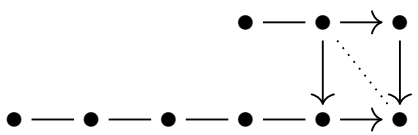


3.<smiles>CCCC[13C]1CC1(C)CC</smiles>

5.

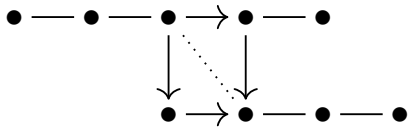

7.

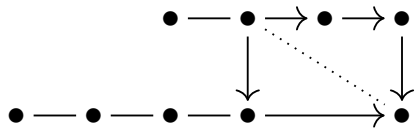

9.

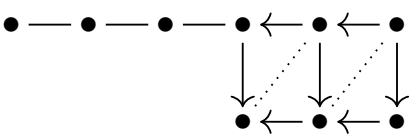

11.

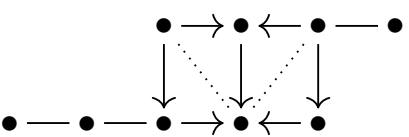

13.

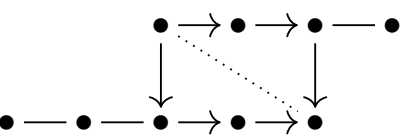

15.

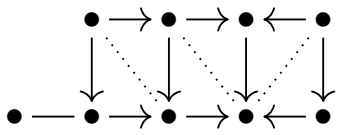

17.

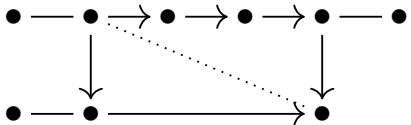

19.

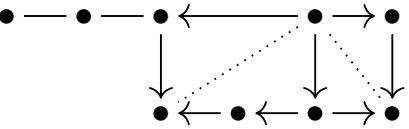

21.

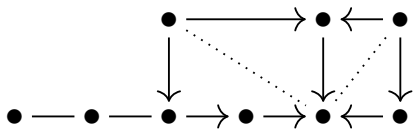

23.

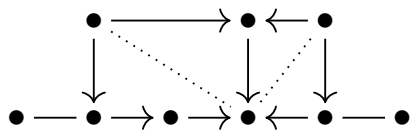

25.

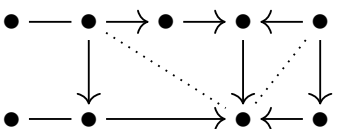

27.

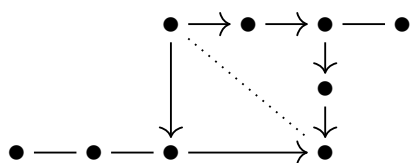

4.

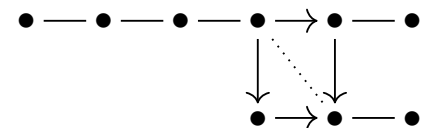

6.

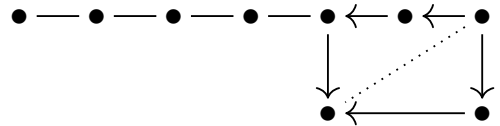

8.

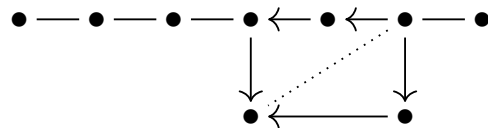

10.

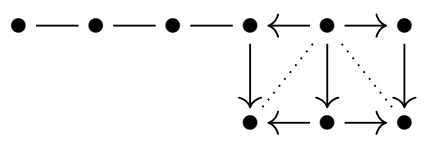

12.

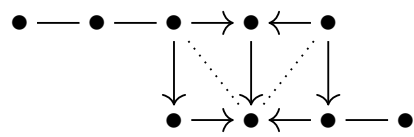

14.

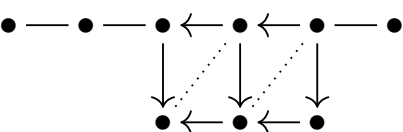

16.

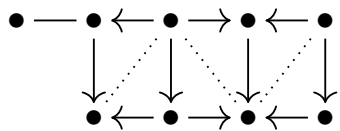

18.

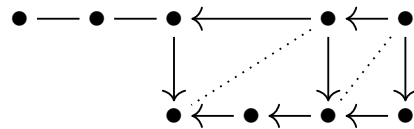

20.

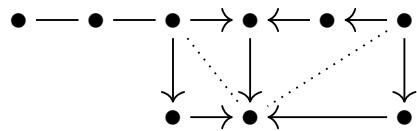

22.

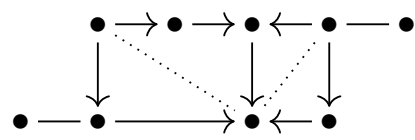

24.

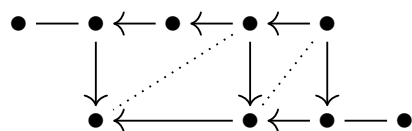

26.

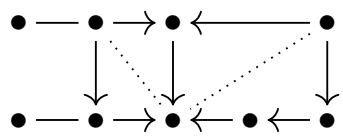

28.

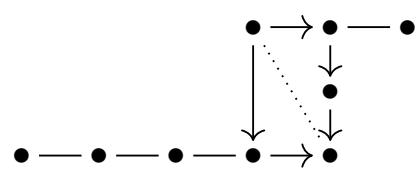


29.

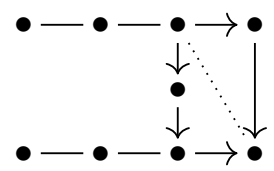

31.

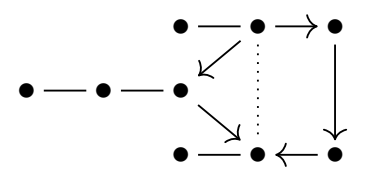

33.<smiles>CC1(C)CC2CCC1C2</smiles>

35.

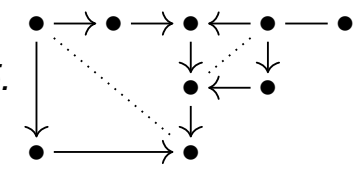

37.

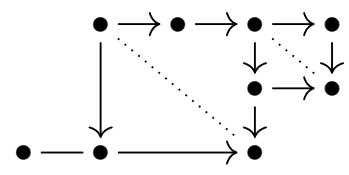

39.

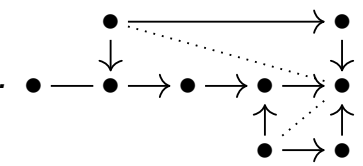

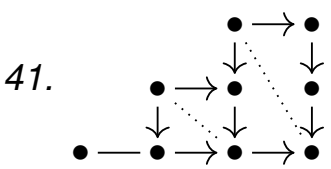

43.

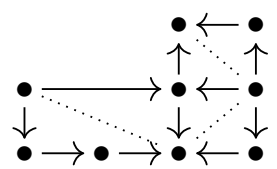

45.

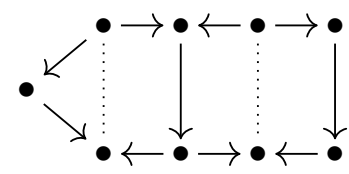

47.

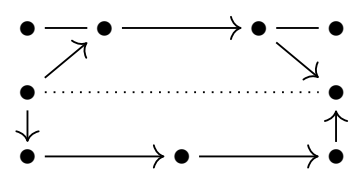

49.

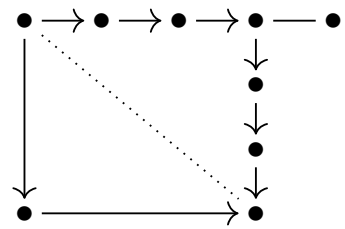

30.

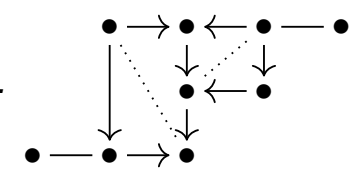

32.

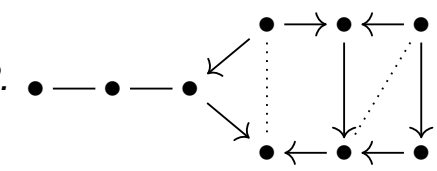

34.

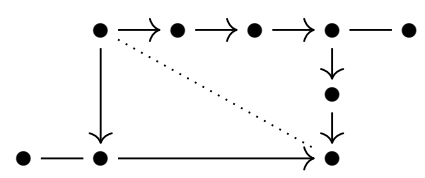

38.

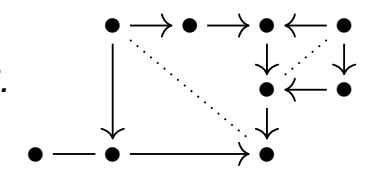

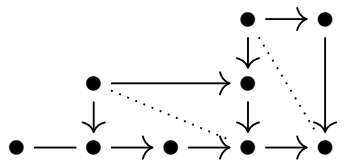

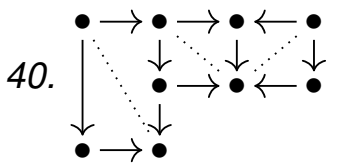

42.

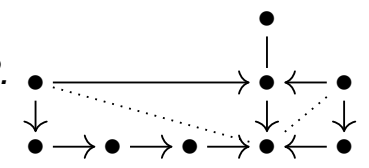

44.

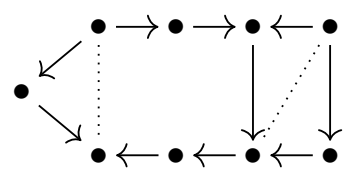

46.

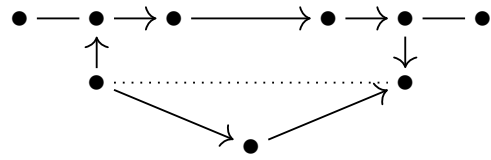

48.

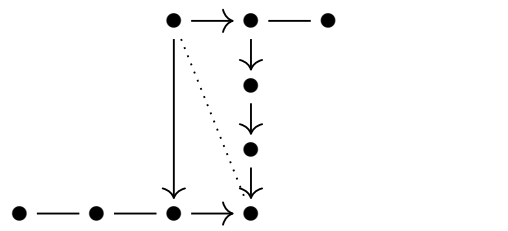

50.

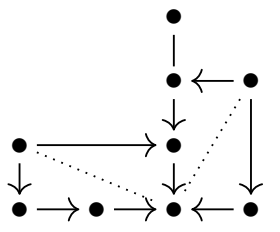


51.

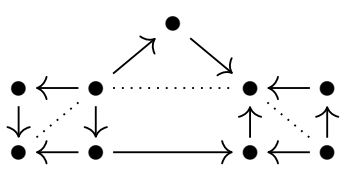

Demonstração Happel, Vossieck e Bongartz mostraram 117 frames, do $\mathcal{F} r 33$ ao $\mathcal{F} r 149$. As Phias com relações de comutatividade são: $\mathcal{F} r 34, \mathcal{F} r 35, \mathcal{F} r 36, \mathcal{F} r 37, \mathcal{F} r 38, \mathcal{F} r 41, \mathcal{F} r 51$, $\mathcal{F} r 52$ (corrigido pelo artigo [HV83]), $\mathcal{F} r 53, \mathcal{F} r 54, \mathcal{F} r 55, \mathcal{F} r 56, \mathcal{F} r 58, \mathcal{F} r 59, \mathcal{F} r 61, \mathcal{F} r 62$, $\mathcal{F} r 63, \mathcal{F} r 64, \mathcal{F} r 65, \mathcal{F} r 67, \mathcal{F} r 69, \mathcal{F} r 70, \mathcal{F} r 71, \mathcal{F} r 72, \mathcal{F} r 73, \mathcal{F} r 74, \mathcal{F} r 75, \mathcal{F} r 76, \mathcal{F} r 77, \mathcal{F} r 90$, $\mathcal{F} r$ 92, $\mathcal{F} r$ 93, $\mathcal{F} r$ 95, $\mathcal{F} r$ 97, $\mathcal{F} r$ 107, $\mathcal{F} r$ 108, $\mathcal{F} r$ 109, $\mathcal{F} r 111$ (corrigido pelo artigo [HV83]), $\mathcal{F} r$ 112, $\mathcal{F} r 113, \mathcal{F} r 114, \mathcal{F} r 115, \mathcal{F} r 116, \mathcal{F} r 117, \mathcal{F} r 118, \mathcal{F} r 127, \mathcal{F} r 128, \mathcal{F} r 133, \mathcal{F} r 143, \mathcal{F} r 146$ e $\mathcal{F} r 148$.

\subsubsection{Família de Phias Assem-Nehring-Skowroński}

No Tsukuba Journal of Mathematics [ANS89], Assem, Nehring e Skowroński publicaram o trabalho intitulado "Domestic trivial extensions of simply connected algebras". O principal resultado deste artigo desempenha um papel importante para a caracterização de uma família de Phias de tipo euclidiano $\widetilde{\mathbb{D}}_{m}, \widetilde{\mathbb{E}}_{6}, \widetilde{\mathbb{E}}_{7}, \widetilde{\mathbb{E}}_{8}$. Chamaremos essa família de ANS, ou seja, família de Phias AssemNehring-Skowroński.

Veremos a seguir que o teorema necessita que as álgebras sejam simplesmente conexas. Trabalharemos com as álgebras disfarçadas de tipo euclidiano $\widetilde{\mathbb{D}}_{m}, \widetilde{\mathbb{E}}_{6}, \widetilde{\mathbb{E}}_{7}$ ou $\widetilde{\mathbb{E}}_{8}$, implicando que são álgebras inclinadas mansas com a primeira cohomologia de Hochschild zero. Assim, pelo trabalho [AMD01], são simplesmente conexas satisfazendo a hipótese desejada.

Teorema 5.35 (Assem-Nehring-Skowroński). Seja A uma álgebra sobre um corpo algebricamente fechado $K$ de dimensão finita, básica e conexa. Se $A$ é simplesmente conexa, então as seguintes condições são equivalentes:

a) Existe uma algebra $B$ de tipo de representação infinito e inclinada de tipo euclidiano $\widetilde{\mathbb{D}}_{m}, \widetilde{\mathbb{E}}_{6}$, $\widetilde{\mathbb{E}}_{7}$ ou $\widetilde{\mathbb{E}}_{8}$ tal que $T(A) \simeq T(B)$.

b) A é inclinada iterada de tipo euclidiano $\widetilde{\mathbb{D}}_{m}, \widetilde{\mathbb{E}}_{6}, \widetilde{\mathbb{E}}_{7}$ ou $\widetilde{\mathbb{E}}_{8}$.

Como explicado anteriormente, cada álgebra $A$ disfarçada de tipo euclidiano satisfaz a condição b. Portanto, a dificuldade é obter as álgebras $B$ tais que $T(B) \simeq T(A)$. As Phias $B$ que farão parte da família ANS.

De acordo com a teoria da extensão trivial envolvida nesse texto, a extensão trivial é para as álgebras schurian. Assim, da lista do Happel e Vossieck [HV83] exploraremos apenas as álgebras schurian. Por sua vez, observamos que as álgebras $A$ schurian e disfarçadas de tipo euclidiano encaixam perfeitamente na estratégia utilizada na descrição completa das Phias de tipo Dynkin. A 
diferença principal é que não temos uma lista das extensões triviais de $A$. Portanto, a maquinaria para achar a Phia $B$ terá mais uma etapa do que foi feito no capítulo anterior. Descreveremos as aljavas das extensões triviais de $A$. Uma observação importante é que não estudaremos as extensões triviais de $A^{o p}$, pois a extensão trivial de $A^{o p}$ é isomorfa a $T(A)^{o p}$.

Lema 5.36. Seja A uma álgebra de dimensão finita. Denotamos a extensão trivial de $A$ por $T(A)$. Portanto, temos a seguinte relação entre álgebras:

$$
T(A)^{o p} \cong T\left(A^{o p}\right) .
$$

Demonstração Consideramos o morfismo

$$
\begin{gathered}
\Phi: T(A)^{o p} \longrightarrow T\left(A^{o p}\right) \\
(a, f) \longmapsto(a, f)
\end{gathered}
$$

Seja $(a, f) *(b, g)=(b, g) \cdot(a, f)$ a operação oposta em $T(A)^{o p}$. Similarmente, deixaremos indicado a operação oposta em $A^{o p}$.

Vamos provar que $\Phi$ é um morfismo de álgebras. Faremos as contas para a operação produto de $\Phi$ que é a mais complicada de visualizar a validade no morfismo de álgebras.

Dados $(a, f),(b, g) \in T(A)^{o p}$, o produto $(a, f) *(b, g)$ é igual a $(b a, g a+b f)$. Assim, $\Phi((a, f) *(b, g))$ é igual a $(b * a, g * a+b * f)$.

Por outro lado, $\Phi((a, f)) \Phi(b, g))=(a, f)(b, g)$ e, em seguida, $(a, f)(b, g)=(a b, a g+$ $f b)$. Sabendo da operação oposta de $A^{o p}$, chegamos na conclusão que $\Phi((a, f) *(b, g))=$ $\Phi((a, f)) \Phi(b, g))$. Consequentemente, podemos afirmar que $T(A)^{o p} \cong T\left(A^{o p}\right)$.

Sendo assim, para cada álgebra schurian $A$ inclinada disfarçada de tipo euclidiano $\widetilde{\mathbb{D}}_{m}, \widetilde{\mathbb{E}}_{6}, \widetilde{\mathbb{E}}_{7}$ ou $\widetilde{\mathbb{E}}_{8}$ faremos:

- descrever a aljava da extensão trivial de $A$;

- mostrar todos os ciclos elementares de $Q_{T(A)}$;

- calcular todas as relações de tipo 2 da presentação de $T(A)$;

- identificar quantas flechas estão envolvidas nas relações do tipo 2;

- utilizar o programa;

- caso exista uma solução não hereditária, então precisamos analisar se o corte origina uma Phia $B$ que não é uma álgebra disfarçada de tipo euclidiano.

Esse vai ser o roteiro da prova dos teoremas de caracterização da família de Phias ANS. 
Começaremos o trabalho da descrição dos elementos da família de Phias ANS de tipo $\widetilde{\mathbb{D}}_{m}$. Primeiro, iremos iniciar com os integrantes de tipo $\widetilde{\mathbb{D}}_{4}$.

Teorema 5.37. As álgebras associadas com as aljavas com relações abaixo:

1.

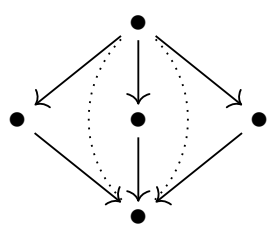

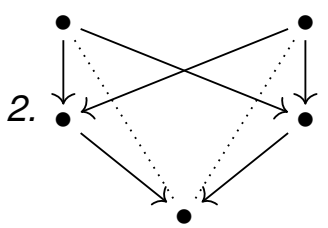

3.

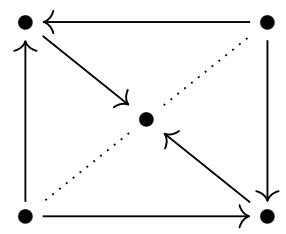

são Phias da família ANS de tipo $\widetilde{\mathbb{D}}_{4}$.

Demonstração Dado uma álgebra vindo de uma operação admissível sobre um frame, usaremos a extensão trivial dessa álgebra e extraímos as informações necessárias para aplicar no programa. Faremos esse procedimento para cada operação admissível em um frame, e para cada frame. Como estamos interessado apenas no tipo $\widetilde{\mathbb{D}}_{4}$, o único frame que se encaixa é o $\mathcal{F} r 2$.

O primeiro detalhe é que a teoria da extensão trivial da seção $\mathbb{E}_{7}$ é para as álgebras schurian, não iremos aplicar nos frames não schurian. No caso $\widetilde{\mathbb{D}}_{m}$, tem o frame $\mathcal{F} r 3$.

O segundo detalhe é que não estamos interessados nas álgebras que suas extensões triviais têm somente corte que origina Phia hereditária. Isso vai ser analisado com mais cuidado no frame $\mathcal{F} r 2$.

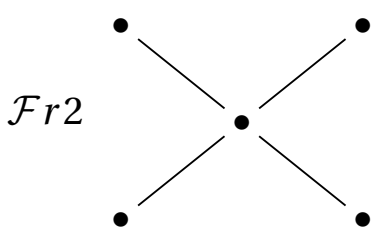

$\widetilde{\mathbb{D}}_{4}-\mathcal{F} r 2.1$

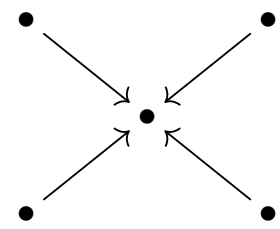

A aljava adjacente euclidiana $\widetilde{\mathbb{D}}_{4}$ tem $2^{4}$ de possibilidades de operações admissíveis um, ou seja, temos $2^{4}$ grafos euclidianos $\widetilde{\mathbb{D}}_{4}$. Agora, o nosso objetivo é estudar a extensão trivial de cada grafo e ver se existe algum corte gerando uma Phia não hereditária. Para isso, a extensão trivial precisa ter no mínimo dois ciclos elementares que tenha pelo menos uma flecha em comum conforme o lema 5.22.
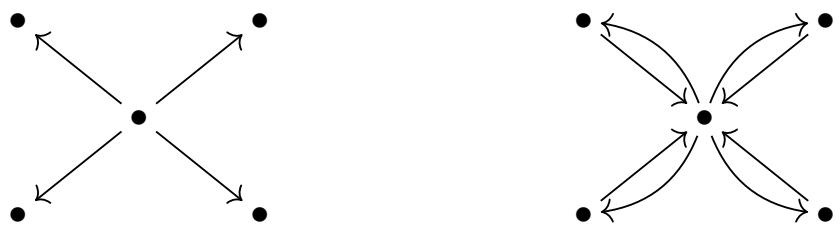

Observamos que não tem ciclos elementares com intersecção de no mínimo uma flecha. Então não precisamos continuar com essa álgebra.

$\widetilde{\mathbb{D}}_{4}-\mathcal{F} r 2.2$
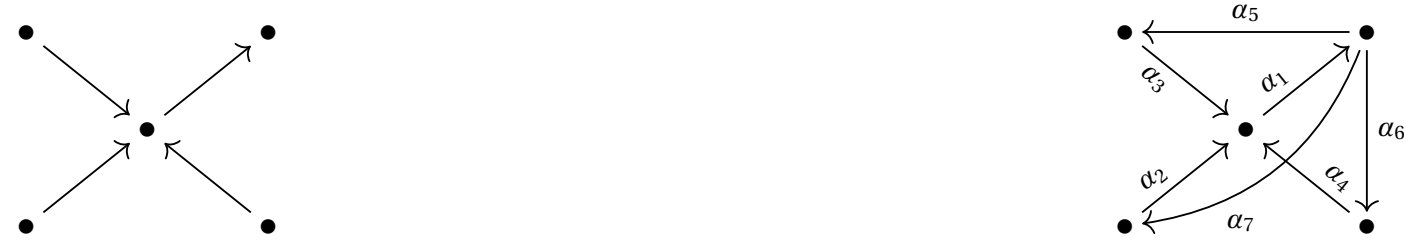
Pela simetria, existem quatro aljavas nessa classe. Vejamos as relações do tipo 2: $r 1=\alpha_{3} \alpha_{1} \alpha_{6}, r 2=\alpha_{3} \alpha_{1} \alpha_{7}, r 3=\alpha_{4} \alpha_{1} \alpha_{7}, r 4=\alpha_{4} \alpha_{1} \alpha_{5}, r 5=\alpha_{2} \alpha_{1} \alpha_{5}$ e $r 6=\alpha_{2} \alpha_{1} \alpha_{6}$. Os ciclos elementares são: $C_{1}=\alpha_{1} \alpha_{5} \alpha_{3}, C_{2}=\alpha_{1} \alpha_{6} \alpha_{4}$ e $C_{3}=\alpha_{1} \alpha_{7} \alpha_{2}$. Logo, o programa nos mostra o corte que gera a álgebra 1 do teorema.
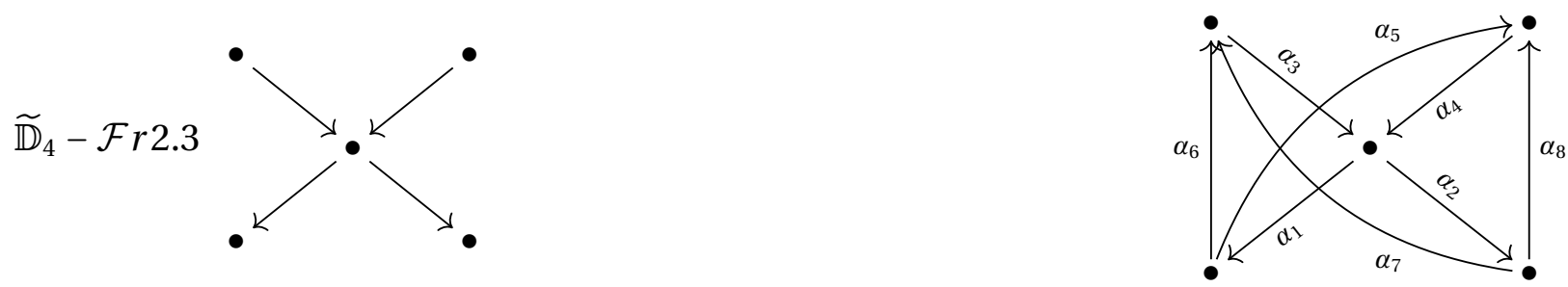

Igual a análise anterior, e mais ainda, essa extensão trivial tem quatro ciclos elementares. Existe um vértice que está presente nesses quatro ciclos e tem quatro flechas. Portanto, pelo lema 5.23, conseguimos obter as álgebras 2 e a dual dela.
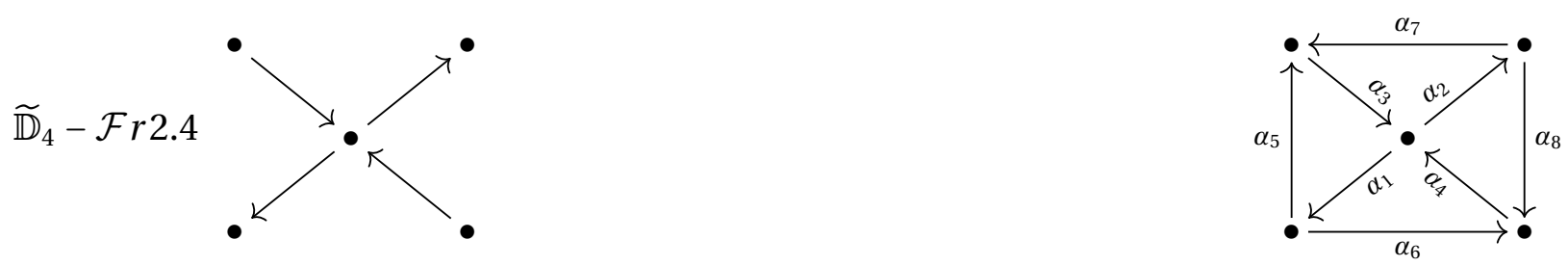

A classe por simetria dessa aljava tem dois representantes. Fazendo a sua extensão trivial, observamos que satisfaz as hipóteses do lema 5.23. Portanto gerando as soluções 3 e a dual dela.

$\widetilde{\mathbb{D}}_{4}-\mathcal{F} r 2.5$
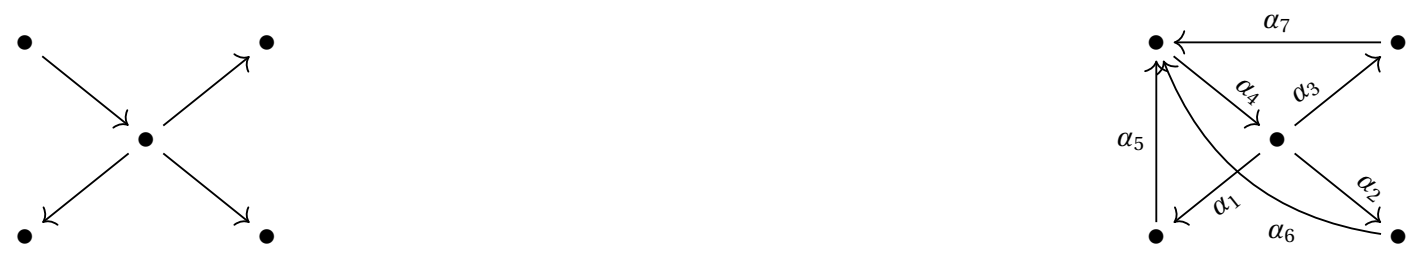

Por último, temos quatro representantes dessa aljava. As relações do tipo 2 são: $r 1=$ $\alpha_{7} \alpha_{4} \alpha_{2}, r 2=\alpha_{7} \alpha_{4} \alpha_{1}, r 3=\alpha_{5} \alpha_{4} \alpha_{3}, r 4=\alpha_{5} \alpha_{4} \alpha_{2}, r 5=\alpha_{6} \alpha_{4} \alpha_{3}$ e $r 6=\alpha_{6} \alpha_{4} \alpha_{1}$. Os ciclos elementares são: $C_{1}=\alpha_{7} \alpha_{4} \alpha_{3}, C_{2}=\alpha_{4} \alpha_{2} \alpha_{6}$ e $C_{3}=\alpha_{4} \alpha_{1} \alpha_{5}$. Nesse caso, temos a álgebra dual da 1.

De uma maneira geral, é difícil descrever as Phias desejadas conforme o tipo $\widetilde{\mathbb{D}}_{m}$. Assim, começamos com o tipo $\widetilde{\mathbb{D}}_{4}$ e o próximo é do tipo $\widetilde{\mathbb{D}}_{5}$.

Teorema 5.38. As álgebras associadas com as aljavas com relações abaixo: 
4.
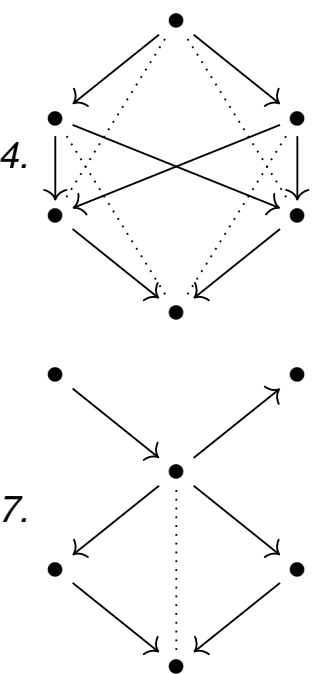

5 .

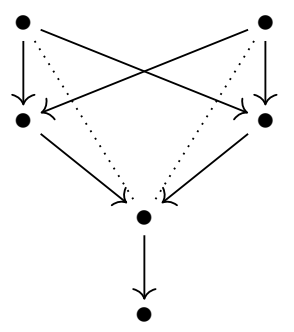

8.

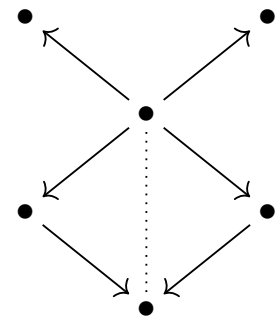

6.

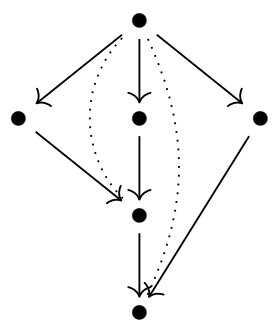

são Phias da família ANS de tipo $\widetilde{\mathbb{D}}_{5}$.

Demonstração Seguindo a mesma demonstração do teorema anterior, só que agora estamos interessados no tipo $\widetilde{\mathbb{D}}_{5}$. O único frame que se encaixa é o $\mathcal{F} r 2$.

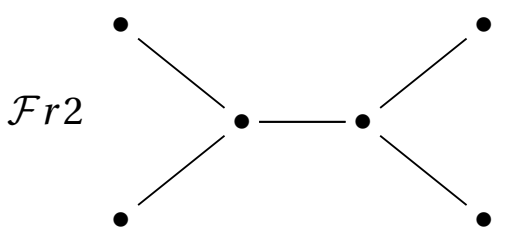

$\widetilde{\mathbb{D}}_{5}-\mathcal{F} r 2.1$

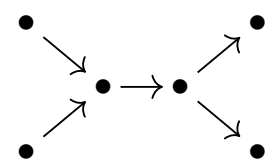

A aljava adjacente euclidiana $\widetilde{\mathbb{D}}_{5}$ tem $2^{5}$ de possibilidades de operações admissíveis um, ou seja, temos $2^{5}$ grafos euclidianos $\widetilde{\mathbb{D}}_{5}$ contando as simétricas e as duais.

As relações do tipo 2 são: $r 1=\alpha_{6} \alpha_{4} \alpha_{3} \alpha_{2}, r 2=\alpha_{4} \alpha_{3} \alpha_{1} \alpha_{9}, r 3=\alpha_{5} \alpha_{3} \alpha_{1} \alpha_{6}, r 4=$ $\alpha_{9} \alpha_{5} \alpha_{3} \alpha_{2}, r 5=\alpha_{5} \alpha_{3} \alpha_{2} \alpha_{8}, r 6=\alpha_{7} \alpha_{5} \alpha_{3} \alpha_{1}, r 7=\alpha_{8} \alpha_{4} \alpha_{3} \alpha_{1}$ e $r 8=\alpha_{4} \alpha_{3} \alpha_{2} \alpha_{7}$. Os ciclos elementares são: $C_{1}=\alpha_{1} \alpha_{6} \alpha_{4} \alpha_{3}, C_{2}=\alpha_{1} \alpha_{9} \alpha_{5} \alpha_{3}, C_{3}=\alpha_{2} \alpha_{7} \alpha_{5} \alpha_{3}$ e $C_{4}=$ $\alpha_{2} \alpha_{8} \alpha_{4} \alpha_{3}$. Com essas informações, o programa nos mostra as soluções 4,5 e o dual da álgebra 5 .
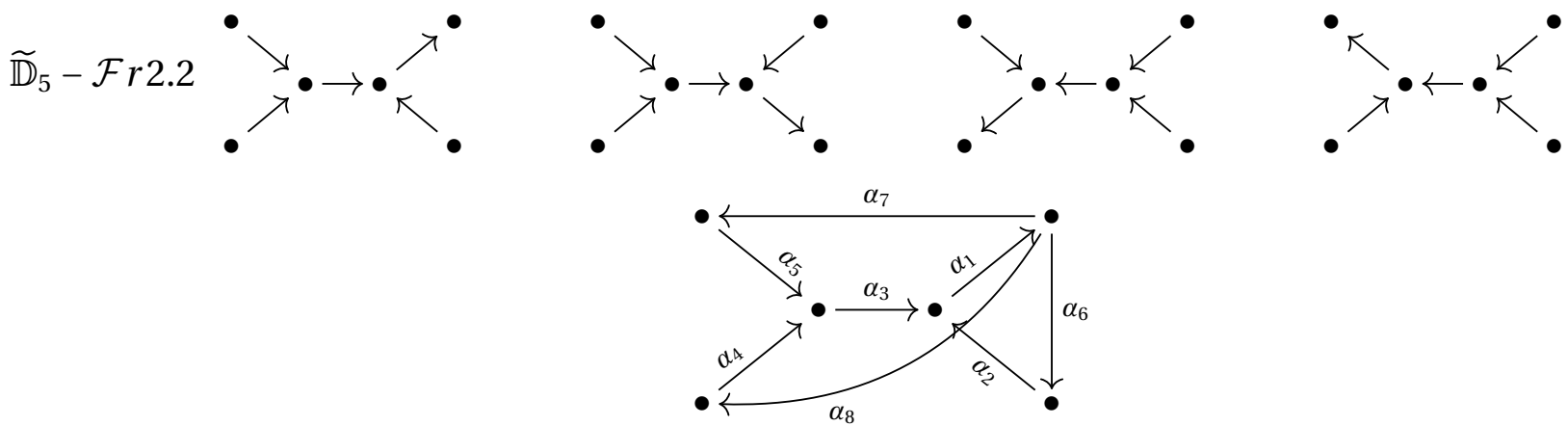

As relações do tipo 2 são: $r 1=\alpha_{3} \alpha_{1} \alpha_{6}, r 2=\alpha_{5} \alpha_{3} \alpha_{1} \alpha_{8}, r 3=\alpha_{2} \alpha_{1} \alpha_{7}, r 4=\alpha_{2} \alpha_{1} \alpha_{8}$ 
e $r 5=\alpha_{4} \alpha_{3} \alpha_{1} \alpha_{7}$. Os ciclos elementares são: $C_{1}=\alpha_{1} \alpha_{7} \alpha_{5} \alpha_{3}, C_{2}=\alpha_{1} \alpha_{6} \alpha_{2}$ e $C_{3}=$ $\alpha_{1} \alpha_{8} \alpha_{4} \alpha_{3}$. Com essas informações, o programa nos mostra as soluções 6 e 7 .

$\widetilde{\mathbb{D}}_{5}-\mathcal{F} r 2.3$
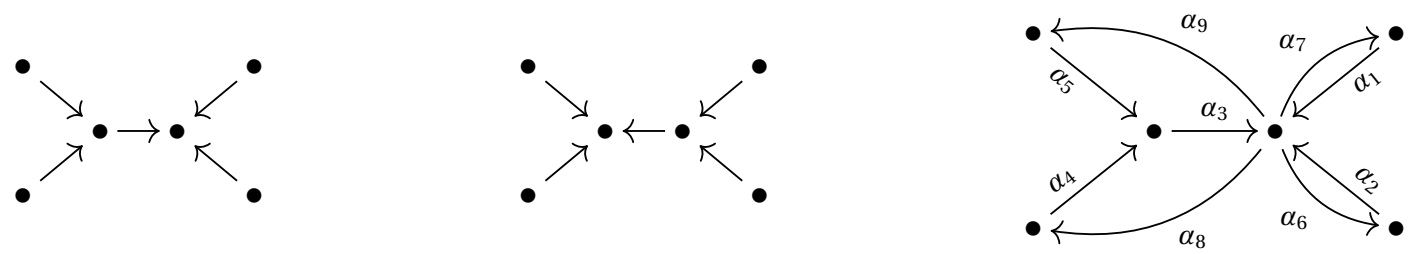

As relações do tipo 2 são: $r 1=\alpha_{5} \alpha_{3} \alpha_{8}, r 2=\alpha_{4} \alpha_{3} \alpha_{9}, r 3=\alpha_{3} \alpha_{7}, r 4=\alpha_{1} \alpha_{9}$, $r 5=\alpha_{1} \alpha_{8}, r 6=\alpha_{3} \alpha_{6}, r 7=\alpha_{2} \alpha_{9}, r 8=\alpha_{2} \alpha_{8}, r 9=\alpha_{1} \alpha_{6}$ e $r 10=\alpha_{2} \alpha_{7}$. Os ciclos elementares são: $C_{1}=\alpha_{3} \alpha_{9} \alpha_{5}, C_{2}=\alpha_{3} \alpha_{8} \alpha_{4}, C_{3}=\alpha_{7} \alpha_{1}$ e $C_{4}=\alpha_{2} \alpha_{6}$. Com isso, existe um corte que origina a Phia 8.

$\widetilde{\mathbb{D}}_{5}-\mathcal{F} r 2.4$
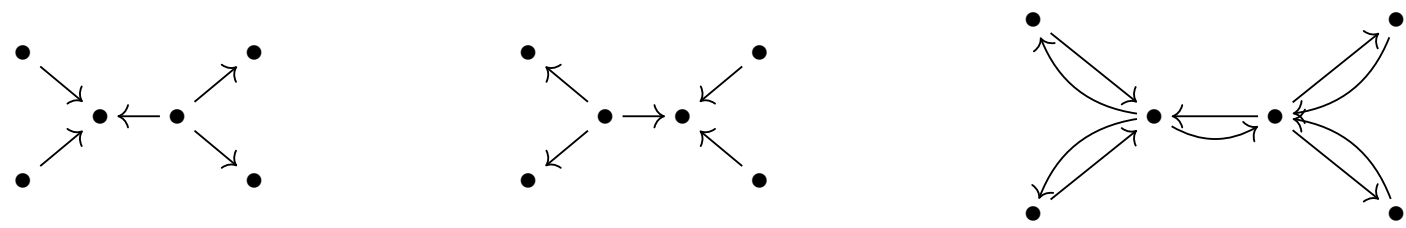

Notamos que não tem ciclos elementares com intersecção de no mínimo uma flecha. Assim, esta álgebra é discarda para o nosso estudo.

$\widetilde{\mathbb{D}}_{5}-\mathcal{F} r 2.5$
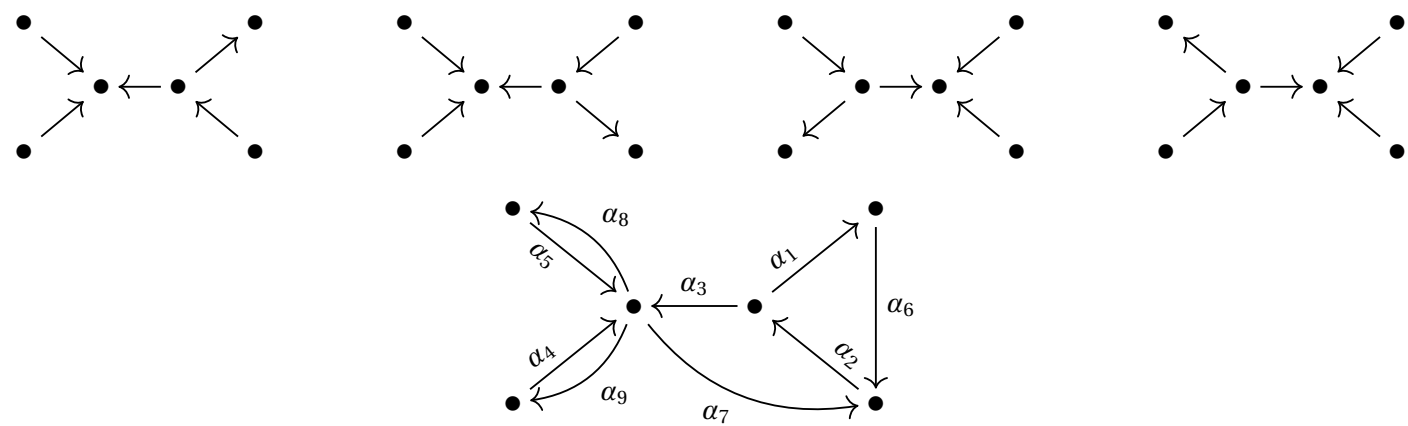

As relações do tipo 2 são: $r 1=\alpha_{7} \alpha_{2} \alpha_{1}, r 2=\alpha_{6} \alpha_{2} \alpha_{3}, r 3=\alpha_{3} \alpha_{8}, r 4=\alpha_{3} \alpha_{9}, r 5=$ $\alpha_{4} \alpha_{7}, r 6=\alpha_{5} \alpha_{7}, r 7=\alpha_{4} \alpha_{8}$ e $r 8=\alpha_{5} \alpha_{9}$. Os ciclos elementares são: $C_{1}=\alpha_{3} \alpha_{7} \alpha_{2}$, $C_{2}=\alpha_{1} \alpha_{6} \alpha_{2}, C_{3}=\alpha_{5} \alpha_{8}$ e $C_{4}=\alpha_{4} \alpha_{9}$. Essa extensão trivial não tem cortes que originam Phias não hereditárias.
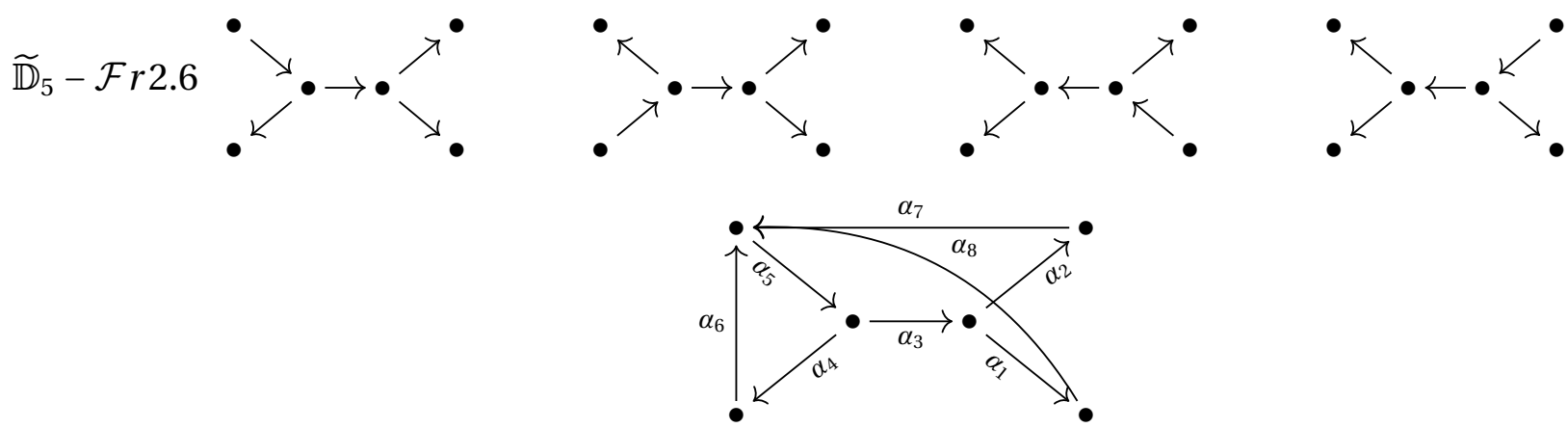

As relações do tipo 2 são: $r 1=\alpha_{7} \alpha_{5} \alpha_{3} \alpha_{1}, r 2=\alpha_{8} \alpha_{5} \alpha_{3} \alpha_{2}, r 3=\alpha_{7} \alpha_{5} \alpha_{4}, r 4=\alpha_{8} \alpha_{5} \alpha_{4}$ 
e $r 5=\alpha_{6} \alpha_{5} \alpha_{3}$. Os ciclos elementares são: $C_{1}=\alpha_{5} \alpha_{3} \alpha_{2} \alpha_{7}, C_{2}=\alpha_{5} \alpha_{3} \alpha_{1} \alpha_{8}$ e $C_{3}=$ $\alpha_{5} \alpha_{4} \alpha_{6}$. Portanto mostramos as álgebrais duais das soluções 6 e 7 .

$\widetilde{\mathbb{D}}_{5}-\mathcal{F} r 2.7$

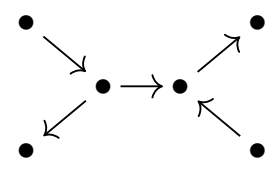

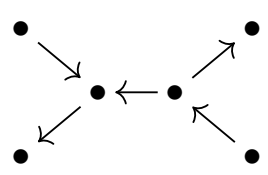
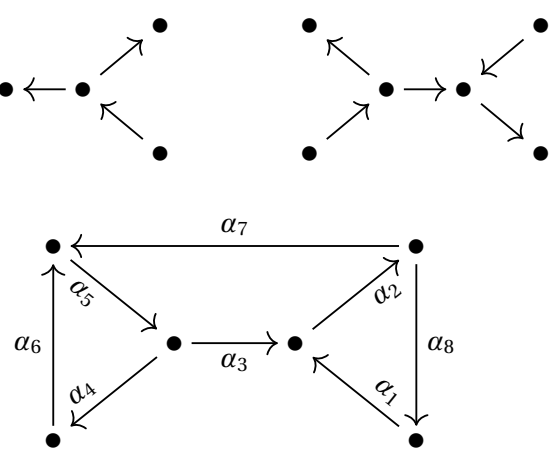

As relações do tipo 2 são: $r 1=\alpha_{3} \alpha_{2} \alpha_{8}, r 2=\alpha_{1} \alpha_{2} \alpha_{7}, r 3=\alpha_{7} \alpha_{5} \alpha_{4}$ e $r 4=\alpha_{6} \alpha_{5} \alpha_{3}$. Os ciclos elementares são: $C_{1}=\alpha_{5} \alpha_{3} \alpha_{2} \alpha_{7}, C_{2}=\alpha_{5} \alpha_{4} \alpha_{6}$ e $C_{3}=\alpha_{1} \alpha_{2} \alpha_{8}$. O programa não exibe solução.

$\widetilde{\mathbb{D}}_{5}-\mathcal{F} r 2.8$
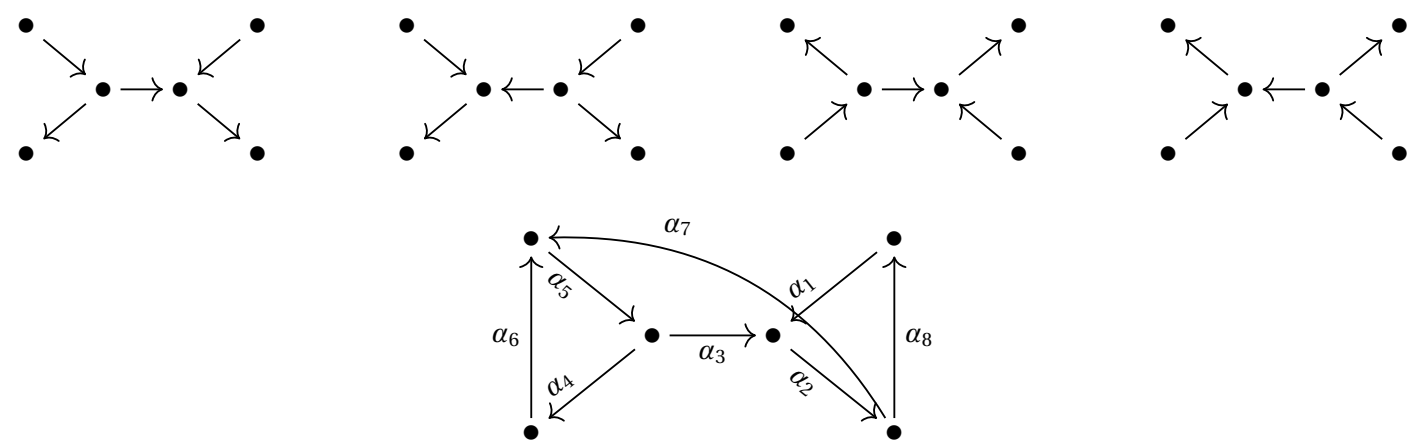

As relações do tipo 2 são: $r 1=\alpha_{7} \alpha_{5} \alpha_{4}, r 2=\alpha_{6} \alpha_{5} \alpha_{3}, r 3=\alpha_{3} \alpha_{2} \alpha_{8}$ e $r 4=\alpha_{1} \alpha_{2} \alpha_{7}$. Os ciclos elementares são: $C_{1}=\alpha_{5} \alpha_{3} \alpha_{2} \alpha_{7}, C_{2}=\alpha_{1} \alpha_{2} \alpha_{8}$ e $C_{3}=\alpha_{5} \alpha_{4} \alpha_{6}$. Novamente, o programa não exibe solução.

$\widetilde{\mathbb{D}}_{5}-\mathcal{F} r 2.9$
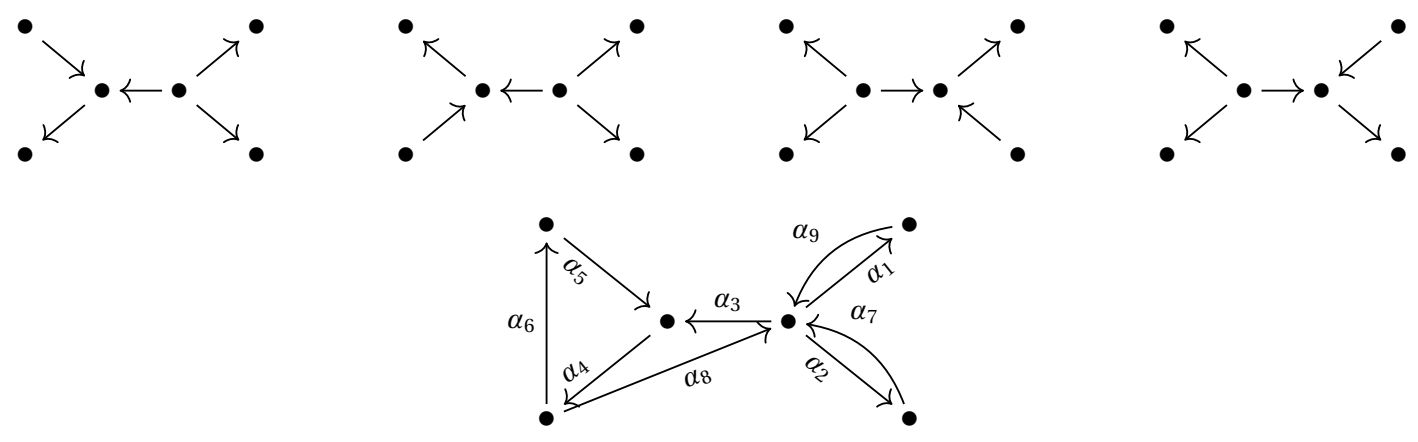

As relações do tipo 2 são: $r 1=\alpha_{3} \alpha_{4} \alpha_{6}, r 2=\alpha_{5} \alpha_{4} \alpha_{8}, r 3=\alpha_{8} \alpha_{1}, r 4=\alpha_{8} \alpha_{2}, r 5=$ $\alpha_{9} \alpha_{3}, r 6=\alpha_{7} \alpha_{3}, r 7=\alpha_{9} \alpha_{2}$ e $r 8=\alpha_{7} \alpha_{1}$. Os ciclos elementares são: $C_{1}=\alpha_{5} \alpha_{4} \alpha_{6}$, $C_{2}=\alpha_{3} \alpha_{4} \alpha_{8}, C_{3}=\alpha_{2} \alpha_{7}$ e $C_{4}=\alpha_{1} \alpha_{9}$. E por último, não temos solução também. 

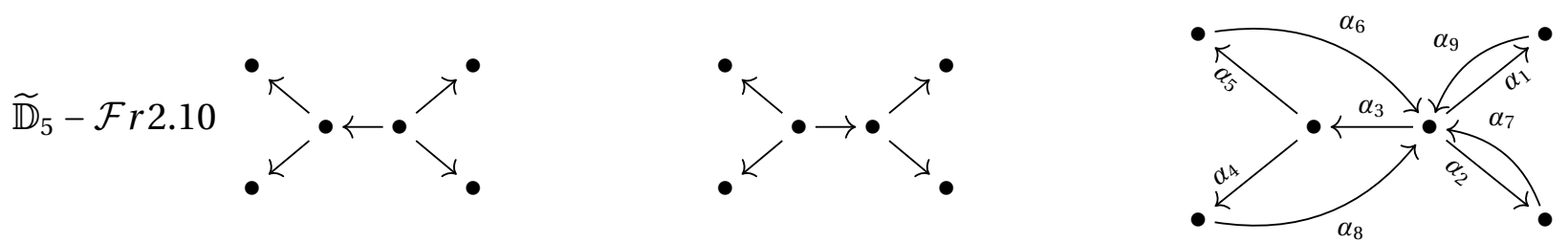

As relações do tipo 2 são: $r 1=\alpha_{8} \alpha_{3} \alpha_{5}, r 2=\alpha_{6} \alpha_{3} \alpha_{4}, r 3=\alpha_{8} \alpha_{1}, r 4=\alpha_{8} \alpha_{2}$, $r 5=\alpha_{9} \alpha_{3}, r 6=\alpha_{7} \alpha_{3}, r 7=\alpha_{6} \alpha_{1}, r 8=\alpha_{6} \alpha_{2}, r 9=\alpha_{9} \alpha_{2}$ e $r 10=\alpha_{7} \alpha_{1}$. Os ciclos elementares são: $C_{1}=\alpha_{3} \alpha_{5} \alpha_{6}, C_{2}=\alpha_{3} \alpha_{4} \alpha_{8}, C_{3}=\alpha_{2} \alpha_{7}$ e $C_{4}=\alpha_{1} \alpha_{9}$. Essa extensão trivial tem um único corte que origina a Phia dual da álgebra 8.

Depois de descrever as famílias de Phias ANS de tipo $\widetilde{\mathbb{D}}_{4}$ e $\widetilde{\mathbb{D}}_{5}$, conseguimos generalizar a demonstração e descrever a família de Phias ANS de tipo $\widetilde{\mathbb{D}}_{n}$ para $n \geq 6$.

O seguinte lema é usado várias vezes no teorema que descreve a família de Phias ANS de tipo $\widetilde{\mathbb{D}}_{n}$.

Lema 5.39. Dada a extensão trivial $\Gamma$ da álgebra hereditária de tipo $A_{n}$, com no mínimo dois ciclos elementares, consideramos o seu diagrama abaixo, a menos de ordem dos ciclos:

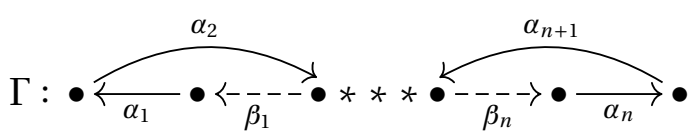

Usaremos a flecha tracejada representando um caminho em que a presença na aljava é opcional. Mais ainda, o símbolo ** * na aljava representa a possibilidade de colocar subaljavas da forma $\bullet \bar{K}^{--\rightarrow \bullet} \longrightarrow$ ou na forma dual.

i) Seja $\Sigma$ um corte sobre $\Gamma$. Portanto, existem cortes $\Sigma$ de $\Gamma$ tais que $K Q_{\Gamma} /\left\langle I_{\Gamma} \cup \Sigma\right\rangle$ são as seguintes álgebras de incidência:

ii) Supomos ainda que existe um ciclo elementar com três flechas ou mais em $\Gamma$.

Se $\alpha_{1} \in \Sigma$ ou $\alpha_{n} \in \Sigma$ então $K Q_{\Gamma} /<I_{\Gamma} \cup \Sigma>$ não é uma álgebra de incidência. Se $\alpha_{2} \in \Sigma$ ou $\alpha_{n+1} \in \Sigma$ então temos que $K Q_{\Gamma} /<I_{\Gamma} \cup \Sigma>$ é uma álgebra de incidência, com a aljava acima.

Demonstração $O$ objetivo é buscar um corte $\Sigma$ de $\Gamma$ em que $K Q_{\Gamma} /\left\langle I_{\Gamma} \cup \Sigma>\right.$ é uma álgebra de incidência. Primeiro vamos supor que existe um ciclo elementar com três flechas ou mais.

Mostraremos a existência de $\Sigma$ por indução no número de ciclos elementares. Para ter mais clareza, começaremos o primeiro passo do processo de indução com dois ciclos elementares a seguir.

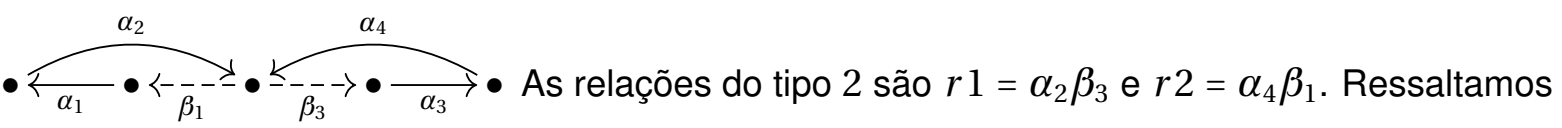


que a relação $r 2$ é definida com a flecha do caminho $\beta_{1}$ que faça sentido ter $\alpha_{4} \beta_{1}$. Caso não exista o caminho $\beta_{1}$, a relação vai ser $r 2=\alpha_{4} \alpha_{1}$. Os ciclos elementares são $C_{1}=\alpha_{2} \beta_{1} \alpha_{1}$ e $C_{2}=\alpha_{4} \beta_{3} \alpha_{3}$.

Sem perda de generalidade, supomos que existe o caminho $\beta_{1}$. E também, começamos a construção do conjunto $\Sigma$ escolhendo a flecha do ciclo elementar $C_{1}$.

Consideramos $\alpha_{2} \in \Sigma$. Então, para eliminar a relação $r 2=\alpha_{4} \beta_{1}$, obtemos que $\alpha_{4} \in \Sigma$. Portanto, usando $\Sigma=\left\{\alpha_{2}, \alpha_{4}\right\}$ temos a álgebra de incidência:

$$
K Q_{\Gamma} /<I_{\Gamma} \cup \Sigma>: \bullet \longleftarrow \bullet \bullet---\bullet--->\bullet \longrightarrow \bullet
$$

Seja $\Gamma$ a extensão trivial do lema com $m+1$ ciclos elementares. Denominamos o conjunto $\Sigma=\left\{\alpha_{2}, \alpha_{4}, \ldots, \alpha_{2 m}\right\}$ para $m$ ciclos elementares, em que satisfaz a hipótese de indução. Faltando definir qual a flecha do ciclo elementar $C_{m+1}$ completaria o corte $\Sigma$ em $\Gamma$ :

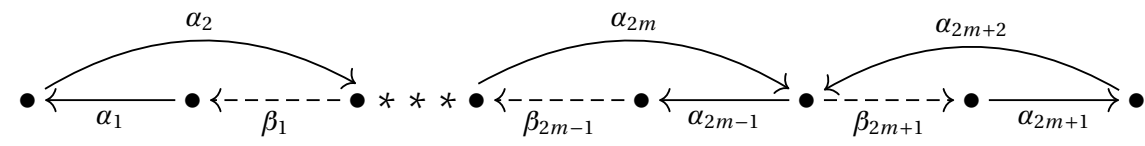

As relações do tipo 2 são: $r 1=\alpha_{2} \beta_{3}, r 2=\alpha_{4} \beta_{1}, \ldots, r k=\alpha_{2 m} \beta_{2 m+1}$ e $r(k+1)=\alpha_{2 m+2} \alpha_{2 m-1}$, para algum número natural $k$. E os ciclos elementares dessa extensão trivial são: $C_{1}=\beta_{1} \alpha_{1} \alpha_{2}, \ldots$, $C_{m+1}=\beta_{2 m+1} \alpha_{2 m+1} \alpha_{2 m+2}$.

Portando, pela relação $r(k+1)=\alpha_{2 m+2} \alpha_{2 m-1}$, determinamos a flecha $\alpha_{2 m+2} \in \Sigma$. Chegando na conclusão que queríamos, e a prova é análoga para $\alpha_{n+1} \in \Sigma$.

Agora, se a extensão trivial $\Gamma$ tem apenas ciclos elementares com duas flechas:

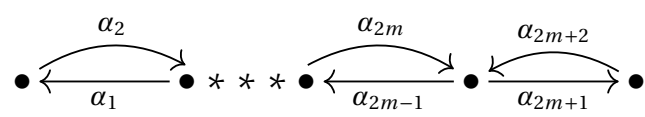

As relações do tipo 2 são: $r 1=\alpha_{2} \alpha_{3}, r 2=\alpha_{4} \alpha_{1}, \ldots, r k=\alpha_{2 m} \alpha_{2 m+1}$ e $r(k+1)=\alpha_{2 m+2} \alpha_{2 m-1}$, para algum número natural $k$. Os ciclos elementares são: $C_{1}=\alpha_{1} \alpha_{2}, \ldots, C_{m+1}=\alpha_{2 m+1} \alpha_{2 m+2}$.

O processo é análogo para conseguir um corte $\Sigma=\left\{\alpha_{2}, \alpha_{4}, \ldots, \alpha_{2 m+2}\right\}$ tal que resulte numa álgebra de incidência. E podemos verificar que o conjunto $\Sigma=\left\{\alpha_{1}, \alpha_{3}, \ldots, \alpha_{2 m+1}\right\}$ implica na álgebra de incidência dual.

Falta a última parte. Supomos que existe um ciclo elementar com três flechas ou mais em $\Gamma$, e $\alpha_{1} \in \Sigma$ ou $\alpha_{n} \in \Sigma$. Sem perda de generalidade, supomos que existe o caminho $\beta_{1}$. Vamos destacar a subaljava

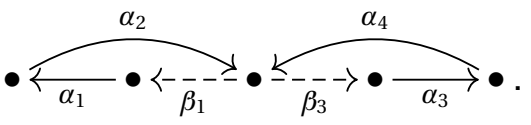

As relações do tipo 2 são $r 1=\alpha_{2} \beta_{3}$ e $r 2=\alpha_{4} \beta_{1}$. Os ciclos elementares são $C_{1}=\alpha_{2} \beta_{1} \alpha_{1}$ e $C_{2}=\beta_{3} \alpha_{3} \alpha_{4}$ 
Por hipótese, $\alpha_{1} \in \Sigma$. Então, falta escolher uma flecha do ciclo elementar $C_{2}=\beta_{3} \alpha_{3} \alpha_{4}$. Não importando a escolha, não conseguimos descartar a relação $r 1=\alpha_{2} \beta_{3}$ ou $r 2=\alpha_{4} \beta_{1}$. Portanto, $K Q_{\Gamma} /<I_{\Gamma} \cup \Sigma>$ não é uma álgebra de incidência. Analogamente, o resultado é o mesmo se partimos de $\alpha_{n} \in \Sigma$.

A seguir, exibiremos a família de Phias ANS de tipo $\widetilde{\mathbb{D}}_{n}$. A parte mais difícil da demonstração é no caso da álgebra $A$ hereditária de tipo $\widetilde{\mathbb{D}}_{n}$. As possibilidades referente a aljava de sua álgebra de caminhos é grande, resultando na enorme quantidade de extensões triviais de $A$.

Teorema 5.40. As álgebras associadas com as aljavas com relações abaixo:

1.

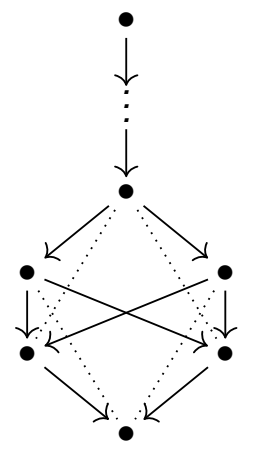

3.

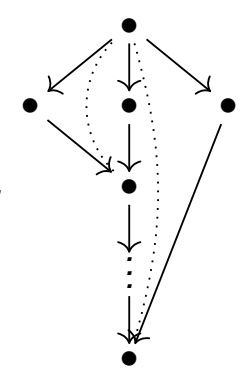

5.
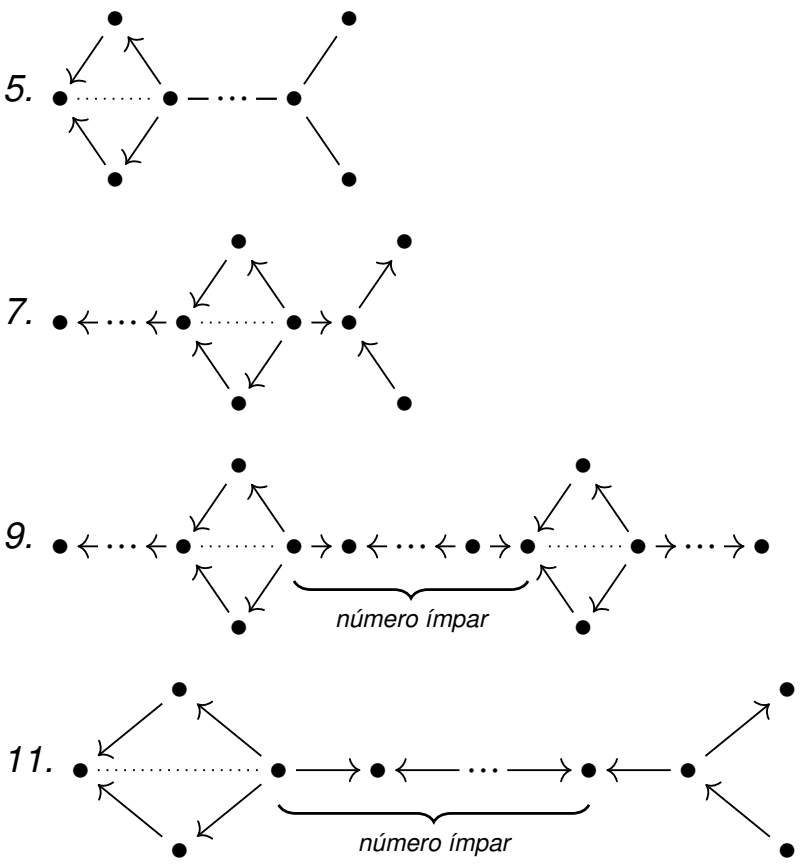

2
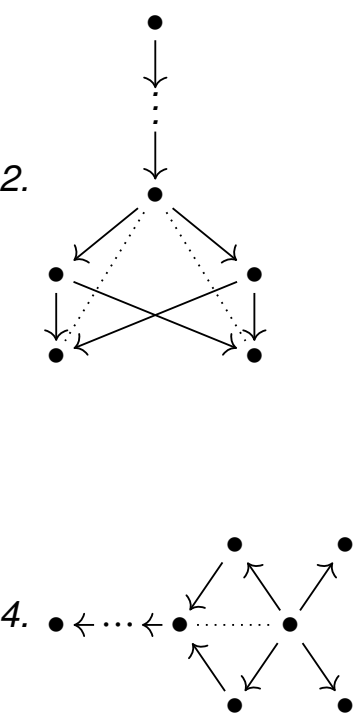

6.

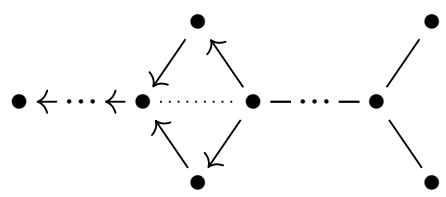

8.

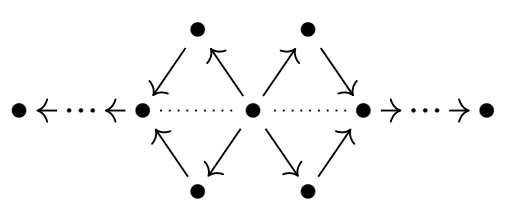

10.

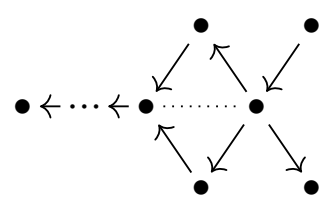


12.

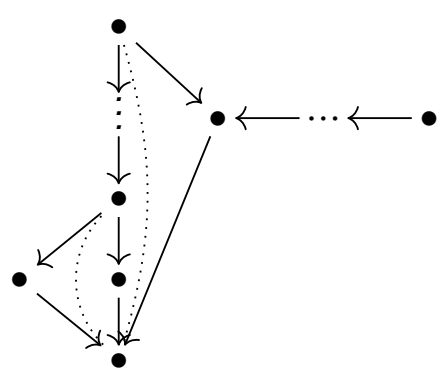

Tem dois casos na substituição da subaljava Se tivermos um número par de ares-

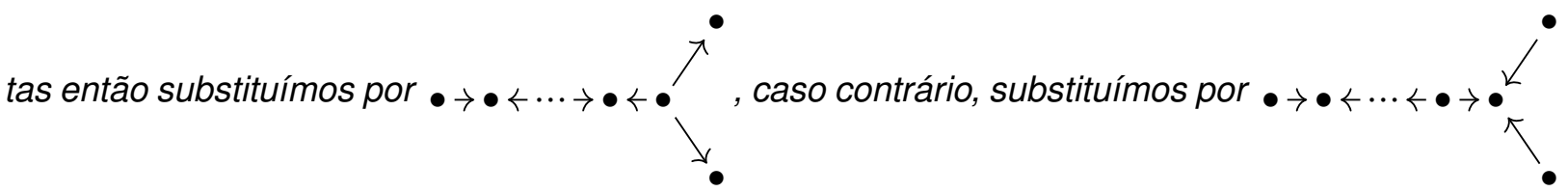

são Phias da família ANS de tipo $\widetilde{\mathbb{D}}_{n}$ para $n \geq 6$.

Demonstração Descrevemos o roteiro da prova de cada álgebra dessa família de Phias ANS:

- consideramos cada álgebra $A$ disfarçada schurian de tipo $\widetilde{\mathbb{D}}_{n}$;

- descrevemos as extensões triviais de $A$;

- analisamos os cortes que originam uma álgebra de incidência $K \Delta$;

- usamos o teorema 5.35, provando que $K \Delta$ é uma Phia de tipo $\widetilde{\mathbb{D}}_{n}$.

Começamos com a álgebra $A$ hereditária de tipo $\widetilde{\mathbb{D}}_{n}$, com $n \geq 6$. Primeiro, coletamos as configurações dos ciclos elementares das extensões triviais de $A$ de tipo $\widetilde{\mathbb{D}}_{4}$ e $\widetilde{\mathbb{D}}_{5}$. Em seguida, aplicamos nas extensões triviais da álgebra $A$ hereditária de tipo $\widetilde{\mathbb{D}}_{n}$. Assim, conseguimos listar todas essas extensões triviais, a menos de simetria ou álgebra oposta. Estamos enumerando com os caracteres $\mathcal{F} r 2$ referente ao frame da lista do capítulo XIV do livro "Elements of the representation theory of associative algebras" [SS07a].

A flecha tracejada simboliza um caminho de comprimento conforme o número $n$, podendo nem existir o caminho. Ressaltamos que nas relações das extensões triviais que possuem esses caminhos nas suas descrições, definimos essas relações na forma minimal. Caso não exista o caminho, a relação vai ser definida com a próxima flecha da aljava que tenha coerência na descrição.

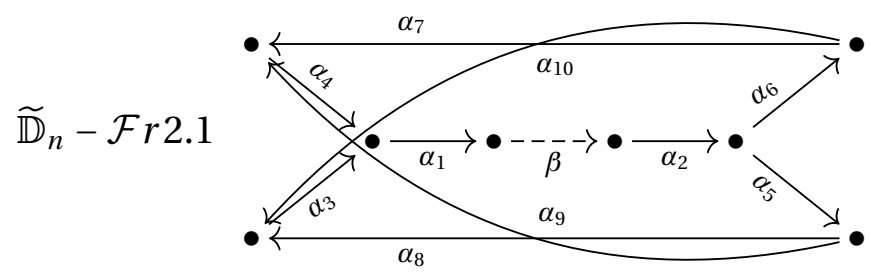


As relações do tipo 2 são:

$r 1=\alpha_{4} \alpha_{1} \beta \alpha_{2} \alpha_{6} \alpha_{10}, r 2=\alpha_{3} \alpha_{1} \beta \alpha_{2} \alpha_{6} \alpha_{7}, r 3=\alpha_{7} \alpha_{4} \alpha_{1} \beta \alpha_{2} \alpha_{5}, r 4=\alpha_{9} \alpha_{4} \alpha_{1} \beta \alpha_{2} \alpha_{6}$,

$r 5=\alpha_{10} \alpha_{3} \alpha_{1} \beta \alpha_{2} \alpha_{5}, r 6=\alpha_{8} \alpha_{3} \alpha_{1} \beta \alpha_{2} \alpha_{6}, r 7=\alpha_{3} \alpha_{1} \beta \alpha_{2} \alpha_{5} \alpha_{9}$ e $r 8=\alpha_{4} \alpha_{1} \beta \alpha_{2} \alpha_{5} \alpha_{8}$.

Os ciclos elementares são:

$C_{1}=\alpha_{6} \alpha_{10} \alpha_{3} \alpha_{1} \beta \alpha_{2}, C_{2}=\alpha_{5} \alpha_{8} \alpha_{3} \alpha_{1} \beta \alpha_{2}, C_{3}=\alpha_{5} \alpha_{9} \alpha_{4} \alpha_{1} \beta \alpha_{2}$ e $C_{4}=\alpha_{6} \alpha_{7} \alpha_{4} \alpha_{1} \beta \alpha_{2}$.

Como nos teoremas anteriores referentes ao tipo $\widetilde{\mathbb{D}}_{4}$ e $\widetilde{\mathbb{D}}_{5}$, existem quatro cortes gerando as Phias 1 e 2, e suas álgebras duais. A Phia 1 e 2 são originadas dos cortes $\left\{\alpha_{1}\right\}$ e $\left\{\alpha_{3}, \alpha_{4}\right\}$ respectivamente.

$\widetilde{\mathbb{D}}_{n}-\mathcal{F} r 2.2$ Na extensão trivial abaixo, consideramos $\beta$ o caminho de comprimento dependendo de $n$.

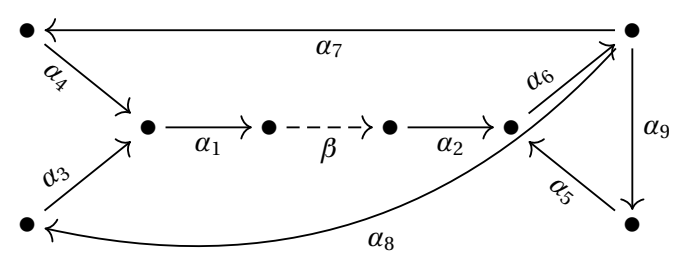

As relações do tipo 2 são: $r 1=\alpha_{4} \alpha_{1} \beta \alpha_{2} \alpha_{6} \alpha_{8}, r 2=\alpha_{3} \alpha_{1} \beta \alpha_{2} \alpha_{6} \alpha_{7}, r 3=\alpha_{2} \alpha_{6} \alpha_{9}$, $r 4=\alpha_{5} \alpha_{6} \alpha_{7}$ e $r 5=\alpha_{5} \alpha_{6} \alpha_{8}$. Os ciclos elementares são: $C_{1}=\alpha_{1} \beta \alpha_{2} \alpha_{6} \alpha_{7} \alpha_{4}, C_{2}=$ $\alpha_{1} \beta \alpha_{2} \alpha_{6} \alpha_{8} \alpha_{3}$ e $C_{3}=\alpha_{6} \alpha_{9} \alpha_{5}$.

Temos várias maneiras de mostrar que existem dois cortes que originam álgebras de incidência com relações de comutatividade. Uma maneira é ver a demonstração do teorema do caso tipo $\widetilde{\mathbb{D}}_{5}$, em que a aljava tem a mesma aparência.

Os cortes são $\left\{\alpha_{6}\right\}$ e $\left\{\alpha_{2}, \alpha_{5}\right\}$ em que são responsáveis pelas soluções 3 e 10 .

$\widetilde{\mathbb{D}}_{n}-\mathcal{F} r 2.3$ Seja $\beta$ o caminho de comprimento dependendo de $n$ do $\widetilde{\mathbb{D}}_{n}$.

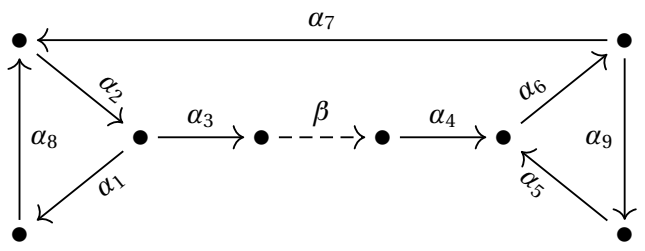

As relações do tipo 2 são: $r 1=\alpha_{4} \alpha_{6} \alpha_{9}, r 2=\alpha_{5} \alpha_{6} \alpha_{7}, r 3=\alpha_{7} \alpha_{2} \alpha_{1}$ e $r 4=\alpha_{8} \alpha_{2} \alpha_{3}$.

Os ciclos elementares são: $C_{1}=\alpha_{5} \alpha_{6} \alpha_{9}, C_{2}=\alpha_{2} \alpha_{1} \alpha_{8}$ e $C_{3}=\alpha_{2} \alpha_{3} \beta \alpha_{4} \alpha_{6} \alpha_{7}$.

Conforme os tipos $\widetilde{\mathbb{D}}_{4}$ e $\widetilde{\mathbb{D}}_{5}$, essa extensão trivial não tem corte que desejamos.

$\widetilde{\mathbb{D}}_{n}-\mathcal{F}$ r2.4 Na extensão trivial abaixo, consideramos $\beta$ o caminho de comprimento dependendo de $n$.

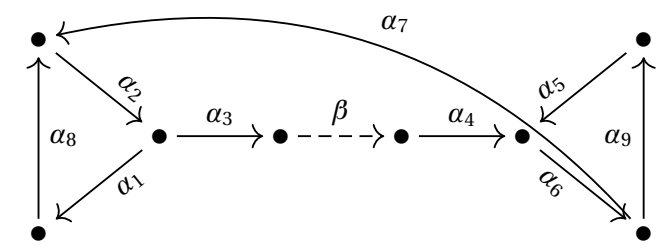


As relações do tipo 2 são: $r 1=\alpha_{4} \alpha_{6} \alpha_{9}, r 2=\alpha_{5} \alpha_{6} \alpha_{7}, r 3=\alpha_{7} \alpha_{2} \alpha_{1}$ e $r 4=\alpha_{8} \alpha_{2} \alpha_{3}$.

Os ciclos elementares são: $C_{1}=\alpha_{5} \alpha_{6} \alpha_{9}, C_{2}=\alpha_{2} \alpha_{1} \alpha_{8}$ e $C_{3}=\alpha_{2} \alpha_{3} \beta \alpha_{4} \alpha_{6} \alpha_{7}$.

Novamente, seguindo a orientação das demonstrações dos tipos $\widetilde{\mathbb{D}}_{4}$ e $\widetilde{\mathbb{D}}_{5}$, não temos solução.

$\widetilde{\mathbb{D}}_{n}-\mathcal{F}$ r2.5 Mostraremos uma extensão trivial da álgebra hereditária de tipo $\widetilde{\mathbb{D}}_{n}$ que não satisfaz o lema 5.22. Isto é, não existe corte que origina uma álgebra de incidência com relações de comutatividade.

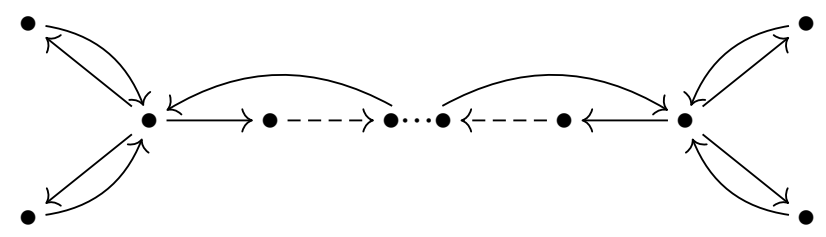

Durante a demonstração, usaremos a flecha tracejada representando um caminho em que é opcional na aljava. Caso não exista o caminho, trocaremos $\bullet---\rightarrow \bullet$ por $\bullet$. Mais ainda, o símbolo $* * *$ na aljava representa a possibilidade de colocar subaljavas da forma $\bullet \bar{K}^{--\rightarrow \bullet \longrightarrow} \longrightarrow$ ou na forma dual. Por exemplo, substituiríamos $* * *$ por

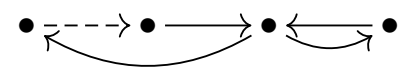

Na busca pelas Phias não hereditárias, nos cortes nas extensões triviais, estamos usando o lema 5.22. Esse resultado exige que uma flecha pertença a dois ciclos elementares, no mínimo. A etapa seguinte mostra as extensões triviais com duas características relacionadas com essa hipótese do lema 5.22:

- existe uma flecha pertencente a dois ciclos elementares;

- existe uma ou duas duplas de ciclos elementares com essa propriedade.

Mostraremos as configurações possíveis para essas duplas de ciclos elementares:
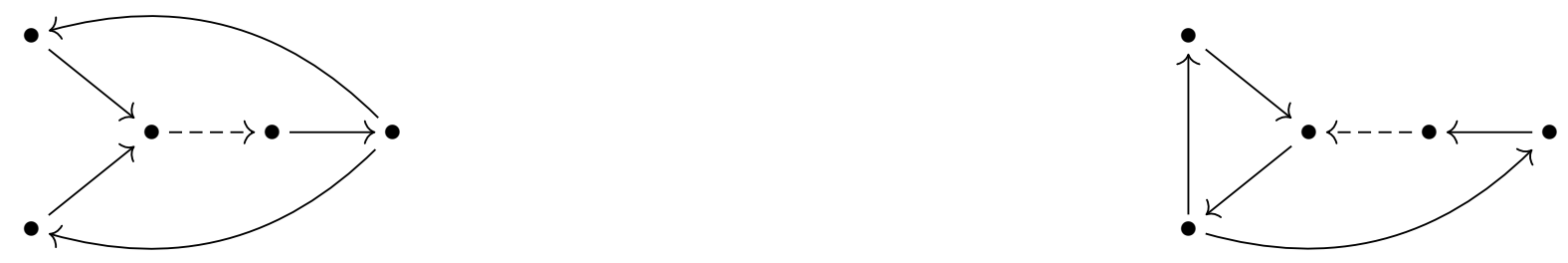

$\widetilde{\mathbb{D}}_{n}-\mathcal{F} r 2.6$ Seja $\Gamma$ a extensão trivial abaixo. Vamos procurar um corte $\Sigma$ de $\Gamma$ que resulte numa álgebra de incidência com relações de comutatividade.

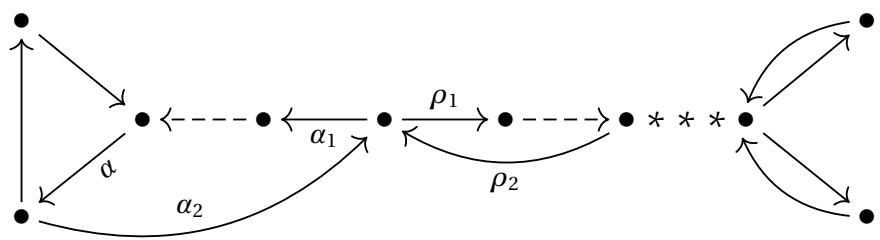


Seguindo o lema 5.22, $\alpha \in \Sigma$. Precisamos escolher uma flecha do ciclo elementar que contém as flechas $\rho_{1}$ e $\rho_{2}$. Caso escolhemos $\rho_{1} \in \Sigma$, a álgebra $K Q_{\Gamma} /\left\langle I_{\Gamma} \cup \Sigma>\right.$ tem a relação $\rho_{2} \alpha_{1}$ de tipo 2. Portanto, $K Q_{\Gamma} /<I_{\Gamma} \cup \Sigma>$ não é uma álgebra de incidência. O outro caso é análogo.

A conclusão é a mesma para o caso semelhante abaixo:

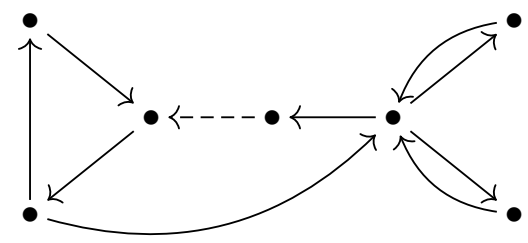

Logo a extensão trivial $\Gamma$ não tem corte $\Sigma$ tal que $K Q_{\Gamma} /\left\langle I_{\Gamma} \cup \Sigma\right\rangle$ seja uma álgebra de incidência com relações de comutatividade.

Observamos que o estudo dessa extensão trivial, se aplica similarmente a seguinte extensão trivial:

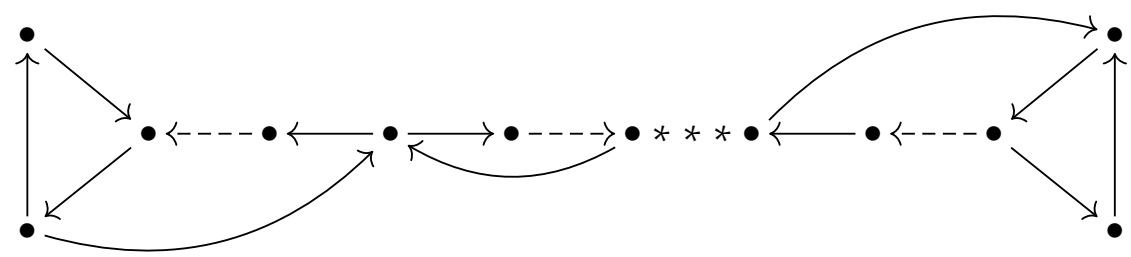

Como também sobre as extensões triviais que possuem as formas duais ou simétricas da subaljava $\Gamma^{\prime}: \mathfrak{\uparrow}^{\bullet} \bullet \bullet \bullet \bullet$. Por exemplo, observamos que na extensão trivial anterior tem no lado direito a forma dual e simétrica da subaljava $\Gamma^{\prime}$.

$\widetilde{\mathbb{D}}_{n}-\mathcal{F} r 2.7$ Essa extensão trivial tem um detalhe diferente da anterior:

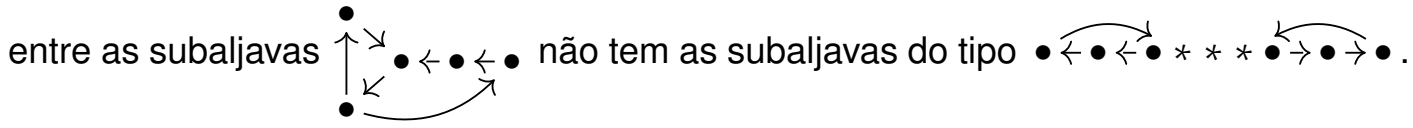

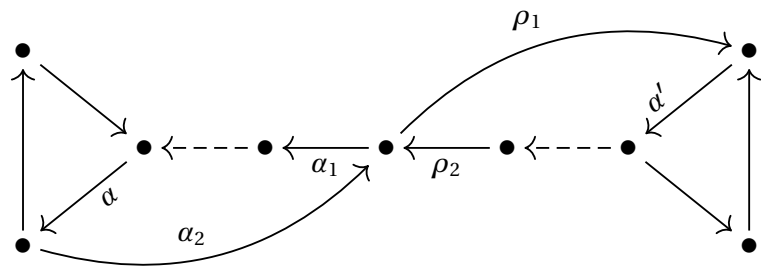

Consideramos um corte $\Sigma$. A partir do lema 5.22, $\alpha \in \Sigma$. Temos duas opções. A primeira é escolher uma flecha do ciclo elementar que contém as flechas $\rho_{1}, \rho_{2}$ e $\alpha^{\prime}$. Caso escolhemos $\rho_{2} \in \Sigma$, a álgebra $K Q_{\Gamma} /\left\langle I \cup \Sigma>\right.$ tem a relação $\alpha_{2} \rho_{1}$ de tipo 2 . Os outros casos desse ciclo elementar são análogos. Portanto, $K Q_{\Gamma} /<I_{\Gamma} \cup \Sigma>$ não é uma álgebra de incidência. A segunda opção é escolher a flecha $\alpha^{\prime}$ que pertence a dupla de ciclos elementares. Mas recaímos a conclusão da primeira opção. Observamos que a análise é semelhante quando começamos com $\alpha^{\prime} \in \Sigma$. 
Logo não temos solução para essa extensão trivial e os seus variantes em relação a

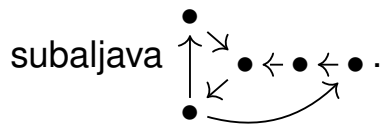

$\widetilde{\mathbb{D}}_{n}-\mathcal{F}$ r2.8 Nesse caso, começaremos a estudar as extensões triviais com a subaljava:

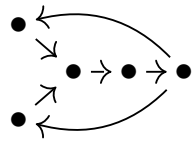

Começaremos com uma extensão trivial com apenas quatro ciclos elementares.

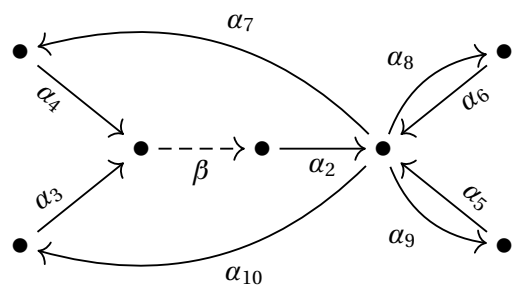

As relações do tipo 2 são: $r 1=\alpha_{4} \beta \alpha_{2} \alpha_{10}, r 2=\alpha_{3} \beta \alpha_{2} \alpha_{7}, r 3=\alpha_{2} \alpha_{8}, r 4=\alpha_{2} \alpha_{9}$, $r 5=\alpha_{6} \alpha_{7}, r 6=\alpha_{6} \alpha_{10}, r 7=\alpha_{5} \alpha_{7}, r 8=\alpha_{5} \alpha_{10}, r 9=\alpha_{6} \alpha_{9}$ e $r 10=\alpha_{5} \alpha_{8}$. Os ciclos elementares são: $C_{1}=\beta \alpha_{2} \alpha_{7} \alpha_{4}, C_{2}=\beta \alpha_{2} \alpha_{10} \alpha_{3}, C_{3}=\alpha_{6} \alpha_{8}$ e $C_{4}=\alpha_{5} \alpha_{9}$.

Consideramos o estudo das extensões triviais dos casos $\widetilde{\mathbb{D}}_{4}$ e $\widetilde{\mathbb{D}}_{5}$ com a mesma configuração, temos único corte $\left\{\alpha_{2}, \alpha_{5}, \alpha_{6}\right\}$. Provando a solução 4 .

$\widetilde{\mathbb{D}}_{n}-\mathcal{F} r 2.9$ Agora, a extensão trivial tem no mínimo cinco ciclos elementares. Nessa parte, começaremos a usar fortemente o lema 5.39.

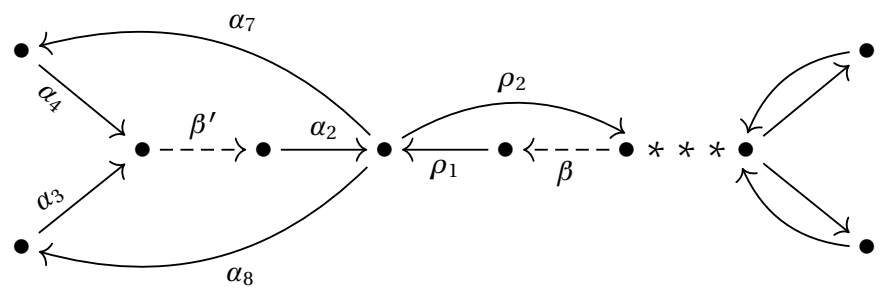

As relações do tipo 2 são: $r 1=\alpha_{4} \beta^{\prime} \alpha_{2} \alpha_{8}, r 2=\alpha_{3} \beta^{\prime} \alpha_{2} \alpha_{7}, r 3=\alpha_{2} \rho_{2}, r 4=\rho_{1} \alpha_{7}$, $r 5=\rho_{1} \alpha_{8}$ e os demais faz parte da subaljava abaixo:

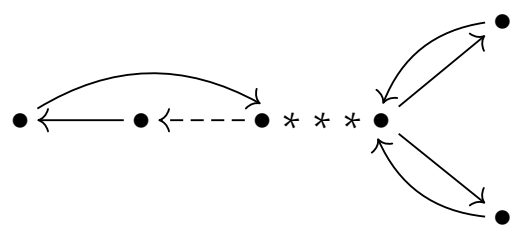

Os ciclos elementares são: $C_{1}=\beta^{\prime} \alpha_{2} \alpha_{7} \alpha_{4}, C_{2}=\beta^{\prime} \alpha_{2} \alpha_{8} \alpha_{3}$ e os demais faz parte da subaljava anterior.

Agora, vamos estudar as opções de configurações pertencentes a essa extensão trivial $\Gamma$. 
Primeiro supomos que o caminho $\beta$ tem um comprimento maior ou igual a 1 ou no lugar do símbolo $* * *$ for colocado algum ciclo elementar com três flechas ou mais. Buscaremos um corte $\Sigma$ tal que $K Q_{\Gamma} /\left\langle I_{\Gamma} \cup \Sigma>\right.$ é uma álgebra de incidência não hereditária. Para isso, precisamos ter $\alpha_{2} \in \Sigma$. Caso contrário, se o caminho $\beta^{\prime}$ tiver comprimento diferente de zero, então qualquer flecha do caminho $\beta^{\prime}$ em $\Sigma$ teríamos a relação $\alpha_{2} \rho_{2}$ ou $\rho_{1} \alpha_{7}$ em $K Q_{\Gamma} /<I_{\Gamma} \cup \Sigma>$. Como $\alpha_{2} \in \Sigma$, implica que $\rho_{1} \in \Sigma$. Portanto, pelo lema 5.39, obtemos que $K Q_{\Gamma} /\left\langle I_{\Gamma} \cup \Sigma>\right.$ não é uma álgebra de incidência.

Falta ver as configurações em que não existe o caminho $\beta$, e no lugar do símbolo *** for colocado apenas ciclo(s) elementar(es) com duas flechas. Como visto no parágrafo anterior, precisamos ter $\alpha_{2} \in \Sigma$. Implicando que $\rho_{1} \in \Sigma$. Novamente usamos o lema 5.39, implicando num único corte. Consequentemente obtemos uma solução.

A solução tem a aljava conforme o comprimento do caminho $\beta^{\prime}$ e a quantidade de ciclos elementares no meio da extensão trivial $\Gamma$. Por exemplo, se $\Gamma$ tem o caminho $\beta^{\prime}$ com comprimento um e apenas um ciclo elementar no meio de $\Gamma$, então obtemos a seguinte Phia:

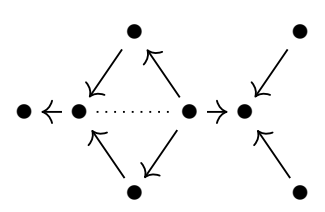

Separamos em duas soluções. Caso exista o caminho $\beta^{\prime}$ então temos a solução 6 . Caso contrário, obtemos a Phia 5.

$\widetilde{\mathbb{D}}_{n}-\mathcal{F} r 2.10$ As extensões triviais da álgebra hereditária de tipo $\widetilde{\mathbb{D}}_{n}$ têm outras configurações com a subaljava

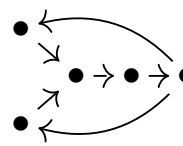
Vejamos uma delas abaixo:

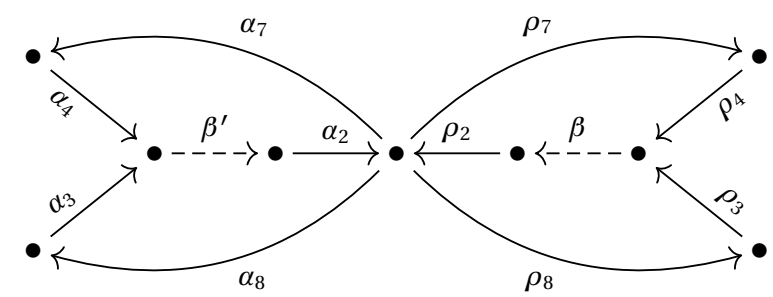

As relações do tipo 2 são: $r 1=\alpha_{3} \beta^{\prime} \alpha_{2} \alpha_{7}, r 2=\alpha_{4} \beta^{\prime} \alpha_{2} \alpha_{8}, r 3=\alpha_{2} \rho_{7}, r 4=\alpha_{2} \rho_{8}$, $r 5=\rho_{2} \alpha_{7}, r 6=\rho_{3} \beta \rho_{2} \rho_{7}, r 7=\rho_{2} \alpha_{8}$ e $r 8=\rho_{4} \beta \rho_{2} \rho_{8}$. Os ciclos elementares são: $C_{1}=\beta^{\prime} \alpha_{2} \alpha_{7} \alpha_{4}, C_{2}=\beta^{\prime} \alpha_{2} \alpha_{8} \alpha_{3}, C_{3}=\rho_{2} \rho_{7} \rho_{4} \beta$ e $C_{4}=\rho_{2} \rho_{8} \rho_{3} \beta$.

Utilizando o estudo das extensões triviais dos casos $\widetilde{\mathbb{D}}_{4}$ e $\widetilde{\mathbb{D}}_{5}$ com a mesma configuração, temos o único corte $\left\{\alpha_{2}, \rho_{2}\right\}$. Provando a solução 8.

$\widetilde{\mathbb{D}}_{n}-\mathcal{F}$ r2.11 Agora, veremos uma extensão trivial muito parecida com a anterior. A diferença está no meio da aljava em que tem as subaljavas do tipo $\bullet \overleftarrow{\leftarrow \bullet \leftarrow \bullet} * * * \bullet \rightarrow \bullet \bullet \bullet$. 
Vamos analisar dois casos. O primeiro é quando tem apenas ciclo(s) elementar(es) com duas flechas no meio da aljava. Mais ainda, possui um número ímpar de ciclo(s) elementar(es) desse tipo.

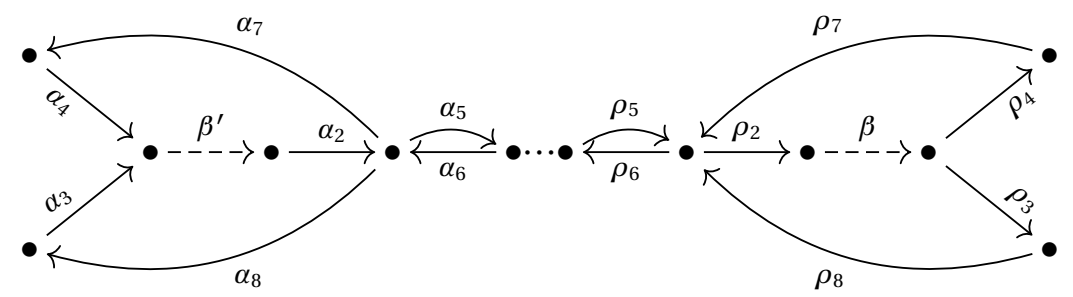

As relações do tipo 2 são: $r 1=\alpha_{3} \beta^{\prime} \alpha_{2} \alpha_{7}, r 2=\alpha_{4} \beta^{\prime} \alpha_{2} \alpha_{8}, r 3=\alpha_{2} \alpha_{5}, r 4=\alpha_{6} \alpha_{7}$, $r 5=\alpha_{6} \alpha_{8}, r 6=\rho_{5} \rho_{2}, r 7=\rho_{7} \rho_{6}, r 8=\rho_{8} \rho_{6}, r 9=\rho_{8} \rho_{2} \beta \rho_{4}, r 10=\rho_{7} \rho_{2} \beta \rho_{3} \mathrm{e}$ mais as relações do tipo 2 da subaljava abaixo:

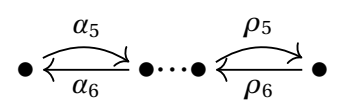

Os ciclos elementares são: $C_{1}=\beta^{\prime} \alpha_{2} \alpha_{7} \alpha_{4}, C_{2}=\beta^{\prime} \alpha_{2} \alpha_{8} \alpha_{3}, C_{3}=\rho_{2} \beta \rho_{4} \rho_{7}, C_{4}=$ $\rho_{2} \beta \rho_{3} \rho_{8}$ e mais os ciclos elementares da subaljava anterior.

Seja $\Sigma$ o corte que buscamos para as soluções. A análise das extensões triviais do item $\widetilde{\mathbb{D}}_{n}-\mathcal{F}$ r 2.9 é uma referência para a explicação dos dois casos. Vimos que não importando a existência ou não do caminho $\beta^{\prime}$, a flecha $\alpha_{2}$ precisa pertencer a $\Sigma$. Consequentemente, obtemos que $\alpha_{6} \in \Sigma$.

Sabendo que $\alpha_{6} \in \Sigma$, pelo lema 5.39, encadeia um dos dois cortes para o caso que tem apenas ciclos elementares com duas flechas. Pelo número ímpar de ciclos elementares com duas flechas, temos $\rho_{6} \in \Sigma$. Pela presença da relação $r 6=\rho_{5} \rho_{2}$, finalizamos com a flecha $\rho_{2} \in \Sigma$ e chegamos na conclusão que $K Q_{\Gamma} /<I_{\Gamma} \cup \Sigma>$ é a Phia 9.

O segundo caso é na presença de um ciclo elementar com três flechas ou mais no meio da aljava.

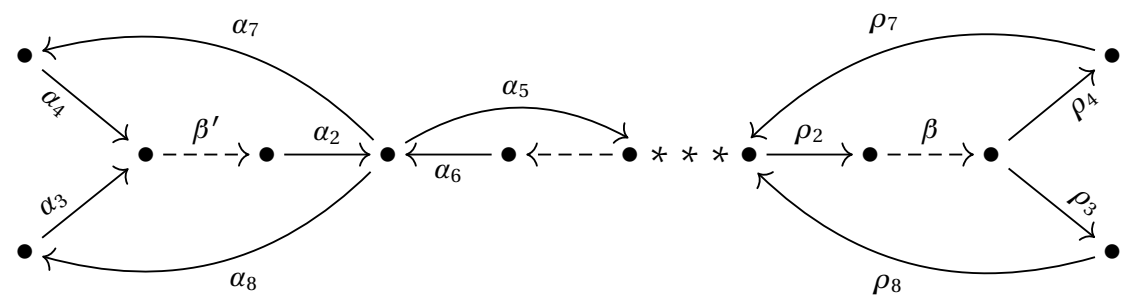

Pelos argumentos anteriores, temos que ter $\alpha_{6} \in \Sigma$. Portanto, pelo lema 5.39, concluimos que não temos uma Phia da família ANS.

$\widetilde{\mathbb{D}}_{n}-\mathcal{F} r 2.12$ Começamos a estudar as extensões triviais com a combinação das duas subaljavas:

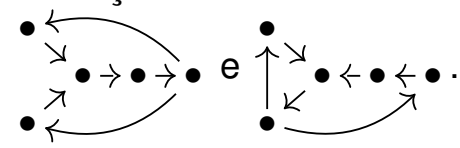




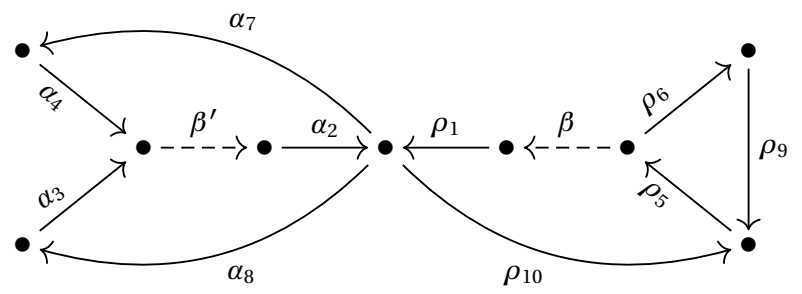

As relações do tipo 2 são: $r 1=\alpha_{3} \beta^{\prime} \alpha_{2} \alpha_{7}, r 2=\alpha_{4} \beta^{\prime} \alpha_{2} \alpha_{8}, r 3=\rho_{10} \rho_{5} \rho_{6}, r 4=$ $\rho_{9} \rho_{5} \beta, r 5=\alpha_{2} \rho_{10}, r 6=\rho_{1} \alpha_{7}$ e $r 7=\rho_{1} \alpha_{8}$. Os ciclos elementares são: $C_{1}=$ $\beta^{\prime} \alpha_{2} \alpha_{7} \alpha_{4}, C_{2}=\beta^{\prime} \alpha_{2} \alpha_{8} \alpha_{3}, C_{3}=\rho_{1} \rho_{10} \rho_{5} \beta$ e $C_{4}=\rho_{5} \rho_{6} \rho_{9}$.

A presença de uma solução, depende da existência do caminho $\beta$.

Caso não exista o caminho $\beta$, a relação $r 4=\rho_{9} \rho_{5} \beta$ se tornaria $r 4=\rho_{9} \rho_{5} \rho_{1}$. E o mais importante, conforme o estudo das extensões triviais dos casos $\widetilde{\mathbb{D}}_{4}$ e $\widetilde{\mathbb{D}}_{5}$ com a mesma configuração, existe apenas um corte $\left\{\alpha_{2}, \rho_{1}, \rho_{6}\right\}$. Provando a solução 7 .

Caso exista o caminho $\beta$, a relação $r 4=\rho_{9} \rho_{5} \beta$ impede ter a solução usando o corte $\left\{\alpha_{2}, \rho_{1}, \rho_{6}\right\}$. Nessa situação, tentaremos achar outro corte $\Sigma$ tal que $K Q_{\Gamma} /\left\langle I_{\Gamma} \cup \Sigma\right\rangle$ é uma álgebra de incidência não hereditária. Como o corte $\left\{\alpha_{2}, \rho_{1}, \rho_{6}\right\}$ não deu certo, outra possibilidade seria $\left\{\alpha_{2}, \rho_{1}, \rho_{9}\right\}$. Nesse corte, não eliminaria a relação $r 3=\rho_{10} \rho_{5} \rho_{6}$. Caso escolhemos o corte $\left\{\alpha_{2}, \rho_{5}\right\}$, teríamos a relação $r 6=\rho_{1} \alpha_{7}$ em $K Q_{\Gamma} /<I_{\Gamma} \cup \Sigma>$.

Se não colocasse $\alpha_{2}$ em $\Sigma$, conforme o lema 5.22, teríamos $\rho_{5} \in \Sigma$. De forma semeIhante as outras tentativas, não chegaríamos a uma solução.

$\widetilde{\mathbb{D}}_{n}-\mathcal{F} r 2.13$ Esse é o último conjunto de extensões triviais da álgebra hereditária de tipo $\widetilde{\mathbb{D}}_{n}$. No caso anterior, não temos nenhum ciclo entre as duplas de subaljavas. Aqui, teremos ciclos elementares no meio da aljava da extensão trivial.

Iniciamos na situação com apenas ciclo(s) elementar(es) com duas flechas no meio da aljava. Mais ainda, é um número ímpar de ciclos elementares.

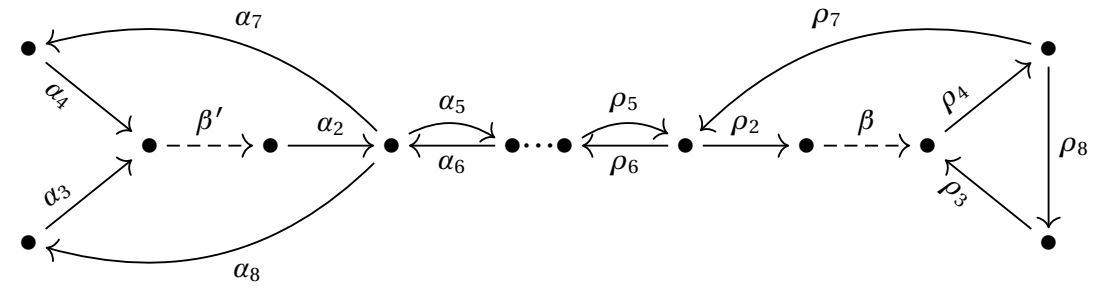

As relações do tipo 2 são: $r 1=\alpha_{3} \beta^{\prime} \alpha_{2} \alpha_{7}, r 2=\alpha_{4} \beta^{\prime} \alpha_{2} \alpha_{8}, r 3=\alpha_{2} \alpha_{5}, r 4=\alpha_{6} \alpha_{7}$, $r 5=\alpha_{6} \alpha_{8}, r 6=\rho_{5} \rho_{2}, r 7=\rho_{7} \rho_{6}, r 8=\rho_{3} \rho_{4} \rho_{7}, r 9=\beta \rho_{4} \rho_{8}$ e as relações do tipo 2 da subaljava:

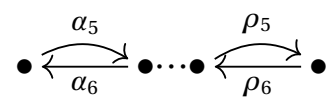

Os ciclos elementares são: $C_{1}=\beta^{\prime} \alpha_{2} \alpha_{7} \alpha_{4}, C_{2}=\beta^{\prime} \alpha_{2} \alpha_{8} \alpha_{3}, C_{3}=\rho_{2} \beta \rho_{4} \rho_{7}, C_{4}=$ $\rho_{8} \rho_{3} \rho_{4}$ e os ciclos elementares da subaljava anterior. 
Seja $\Sigma$ o corte em que resulta numa solução.

Como foi visto na parte $\widetilde{\mathbb{D}}_{n}-\mathcal{F} r 2.9$, a flecha $\alpha_{2}$ precisa pertencer a $\Sigma$. Implicando

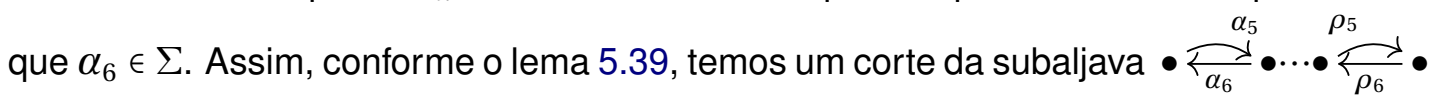
resultando numa subaljava sem relações.

Consequentemente, escolhemos $\rho_{2} \in \Sigma$ para eliminar a relação $r 6=\rho_{5} \rho_{2}$. Falta determinar uma flecha do ciclo elementar $C_{4}=\rho_{8} \rho_{3} \rho_{4}$. Temos apenas duas escolhas: $\rho_{3}$ e $\rho_{8}$. Se escolher $\rho_{8}$, então não eliminaríamos a relação $r 8=\rho_{3} \rho_{4} \rho_{7}$. Portanto, com $\rho_{3} \in \Sigma$ temos o corte completo com duas conclusões possíveis dependendo da presença do caminho $\beta$. Caso exista o caminho $\beta$, implica que $r 9=\beta \rho_{4} \rho_{8} \epsilon<$ $I_{\Gamma} \cup \Sigma>$. Caso contrário, a relação $r 9=\beta \rho_{4} \rho_{8}$ é escrita da forma $r 9=\rho_{2} \rho_{4} \rho_{8}$ e conseguimos ter a solução 11 . Podemos reparar nos argumentos que é a única solução para esse caso.

O segundo caso é na presença de um ciclo elementar com três flechas ou mais no meio da aljava.

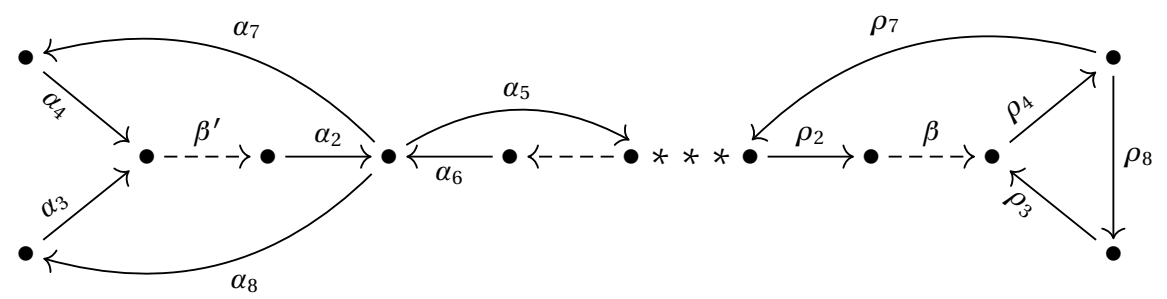

Pelos argumento anteriores, $\alpha_{6} \in \sum$. Sendo assim, pelo lema 5.39, não temos uma solução para esse caso.

$\widetilde{\mathbb{D}}_{n}-\mathcal{F} r 4$ Começamos a nossa análise sobre o penúltimo frame schurian das álgebras disfarçadas de tipo $\widetilde{\mathbb{D}}_{n}$. Mostraremos várias extensões triviais das álgebras resultantes das operações admissíveis sobre o frame $\mathcal{F} r 4$. Buscaremos através dos cortes, as Phias que são diferentes das álgebras disfarçadas.

Observamos que os conjuntos de extensões triviais são a menos de aljavas simétricas ou aljavas duais.

$\widetilde{\mathbb{D}}_{n}-\mathcal{F} r 4.1$ Colocamos no lado esquerdo a álgebra disfarçada originada do frame $\mathcal{F} r 4$. No lado direito, a extensão trivial da álgebra em que tem quatro ciclos elementares:
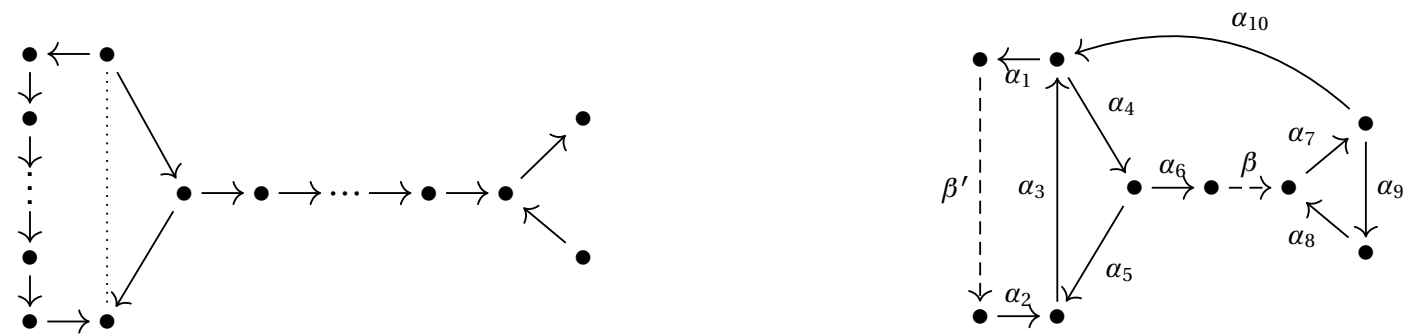

A seguir, deixamos registrado a outra álgebra disfarçada com uma pequena diferença na subaljava $\bullet_{\searrow}^{\bullet}$, em que teremos o mesmo estudo na busca pelas Phias da família 
ANS.
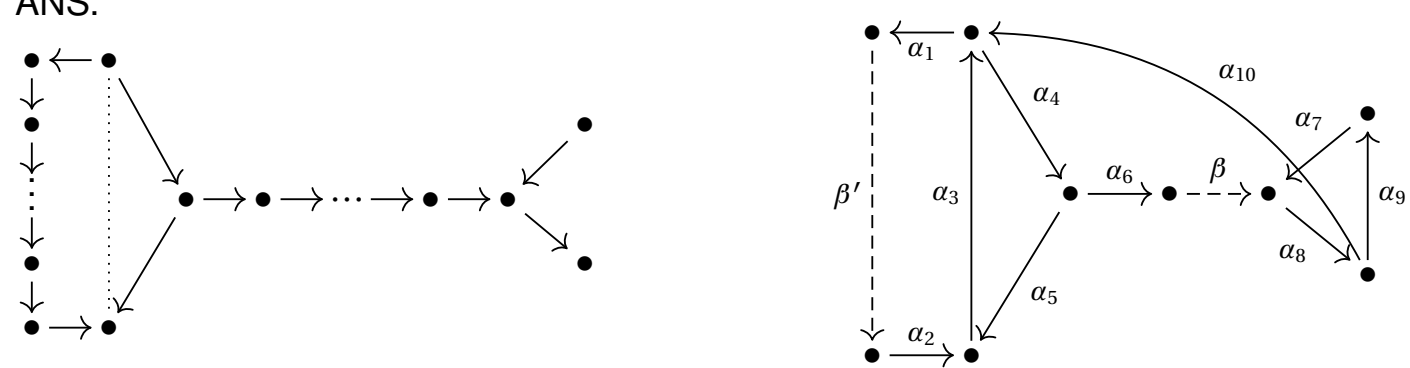

Como a configuração da extensão trivial é a mesma não importando o tipo $\widetilde{\mathbb{D}}_{n}$, faremos as contas para uma extensão trivial de uma álgebra disfarçada de tipo $\widetilde{\mathbb{D}}_{7}$ :

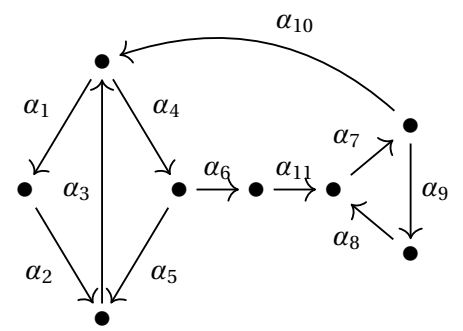

As relações do tipo 2 são: $r 1=\alpha_{2} \alpha_{3} \alpha_{4}, r 2=\alpha_{5} \alpha_{3} \alpha_{1}, r 3=\alpha_{3} \alpha_{4} \alpha_{6}, r 4=\alpha_{10} \alpha_{4} \alpha_{5}$, $r 5=\alpha_{10} \alpha_{1}, r 6=\alpha_{11} \alpha_{7} \alpha_{9}$ e $r 7=\alpha_{8} \alpha_{7} \alpha_{10}$. Os ciclos elementares são: $C_{1}=\alpha_{3} \alpha_{1} \alpha_{2}$, $C_{2}=\alpha_{3} \alpha_{4} \alpha_{5}, C_{3}=\alpha_{4} \alpha_{6} \alpha_{11} \alpha_{7} \alpha_{10}$ e $C_{4}=\alpha_{8} \alpha_{7} \alpha_{9}$.

Portanto, usando o programa, temos o corte $\left\{\alpha_{3}, \alpha_{9}, \alpha_{10}\right\}$. A partir desse corte, resulta na própria álgebra disfarçada de tipo $\widetilde{\mathbb{D}}_{7}$. Assim, concluímos que não temos nenhuma Phia diferente da álgebra disfarçada de tipo $\widetilde{\mathbb{D}}_{n}$.

$\widetilde{\mathbb{D}}_{n}-\mathcal{F}$ r4.2 Seja outra álgebra disfarçada originada do frame $\mathcal{F} r 4$. Veremos se existe uma Phia diferente da álgebra disfarçada.
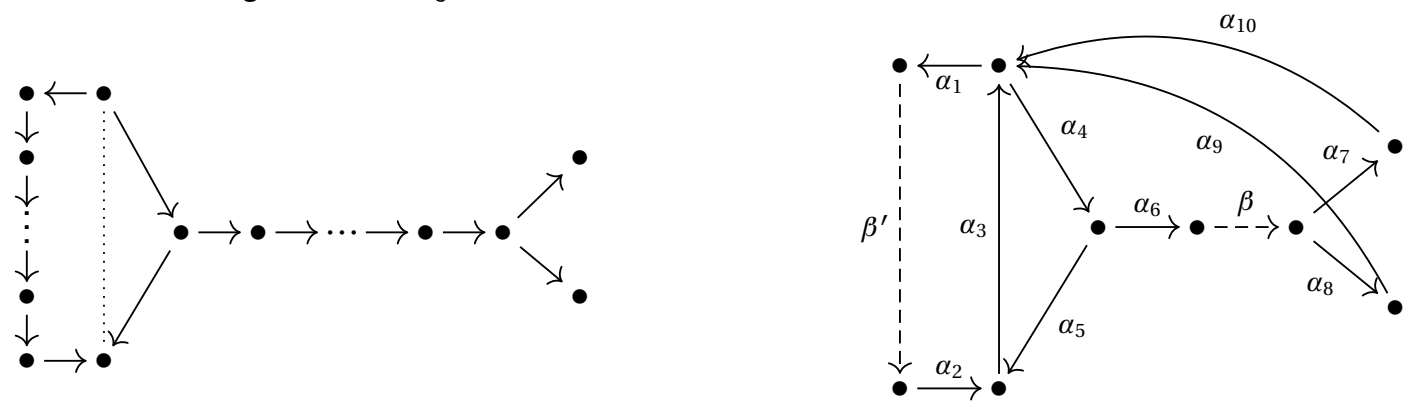

Com a mesma razão do caso anterior, calcularemos a possibilidade da existência de cortes que queremos para uma extensão trivial de uma álgebra disfarçada de tipo $\widetilde{\mathbb{D}}_{7}$ :

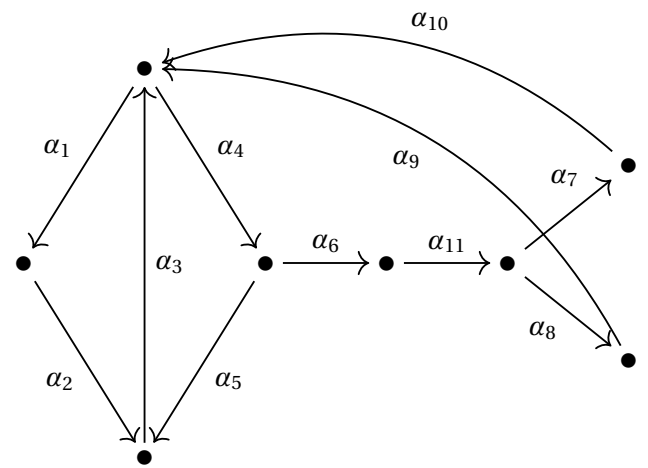


As relações do tipo 2 são: $r 1=\alpha_{2} \alpha_{3} \alpha_{4}, r 2=\alpha_{5} \alpha_{3} \alpha_{1}, r 3=\alpha_{3} \alpha_{4} \alpha_{6}, r 4=\alpha_{10} \alpha_{4} \alpha_{5}$, $r 5=\alpha_{9} \alpha_{4} \alpha_{5}, r 6=\alpha_{10} \alpha_{1}, r 7=\alpha_{9} \alpha_{1}, r 8=\alpha_{9} \alpha_{4} \alpha_{6} \alpha_{11} \alpha_{7}$ e $r 9=\alpha_{10} \alpha_{4} \alpha_{6} \alpha_{11} \alpha_{8}$. Os ciclos elementares são: $C_{1}=\alpha_{3} \alpha_{1} \alpha_{2}, C_{2}=\alpha_{3} \alpha_{4} \alpha_{5}, C_{3}=\alpha_{4} \alpha_{6} \alpha_{11} \alpha_{7} \alpha_{10}$ e $C_{4}=$ $\alpha_{4} \alpha_{6} \alpha_{11} \alpha_{8} \alpha_{9}$

O programa nos retornou os cortes $\left\{\alpha_{3}, \alpha_{9}, \alpha_{10}\right\}$ e $\left\{\alpha_{4}, \alpha_{1}\right\}$. Logo, obtemos apenas a solução 12 a partir do corte $\left\{\alpha_{4}, \alpha_{1}\right\}$.

$\widetilde{\mathbb{D}}_{n}-\mathcal{F}$ r4.3 Inspirado nas extensões triviais da álgebra hereditária de tipo $\widetilde{\mathbb{D}}_{n}$, mostraremos o restante das variações das extensões triviais da álgebra disfarçada originada do frame $\mathcal{F}$ r4. Ressaltamos que estamos supondo apenas aplicações das operações admissíveis 1 no frame $\mathcal{F} r 4$.

Antes de descrever as aljavas das extensões triviais, vamos mostrar os cortes $\Sigma$ que procuramos sobre a subaljava abaixo:

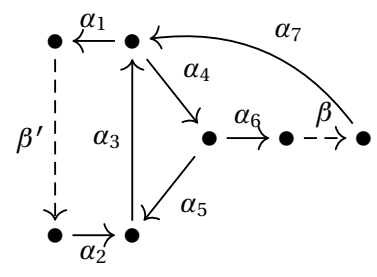

As relações do tipo 2 são: $r 1=\alpha_{2} \alpha_{3} \alpha_{4}, r 2=\alpha_{5} \alpha_{3} \alpha_{1}, r 3=\alpha_{7} \alpha_{4} \alpha_{5}, r 4=\alpha_{7} \alpha_{1}$ e $r 5=$ $\alpha_{3} \alpha_{4} \alpha_{6}$. Os ciclos elementares são: $C_{1}=\alpha_{3} \beta^{\prime} \alpha_{1} \alpha_{2}, C_{2}=\alpha_{3} \alpha_{4} \alpha_{5}$ e $C_{3}=\alpha_{4} \alpha_{6} \beta \alpha_{7}$. Reparamos que os caminhos $\beta$ e $\beta^{\prime}$ não participam das relações do tipo 2 . Sendo assim, a existência desses caminhos não é importante para a busca dos cortes $\Sigma$.

Conforme o lema 5.22, supomos $\alpha_{3} \in \Sigma$. Isso elimina as relações $r 1, r 2$ e $r 5$. Faltando escolher uma flecha do ciclo $C_{3}$ que não seja $\alpha_{4}$. Planejando em eliminar as relações $r 3$ e $r 4$, temos que ter $\alpha_{7} \in \Sigma$. Logo $\Sigma=\left\{\alpha_{3}, \alpha_{7}\right\}$ é um corte que buscamos.

Agora, se $\alpha_{4} \in \Sigma$ então precisaríamos escolher uma flecha do ciclo $C_{1}$, menos $\alpha_{3}$. Qualquer escolha que façamos, não conseguiríamos eliminar a relação $r 1$ ou $r 2$. Portanto, esse corte está descartado.

Isso vai ser importante na análise da aljava abaixo:

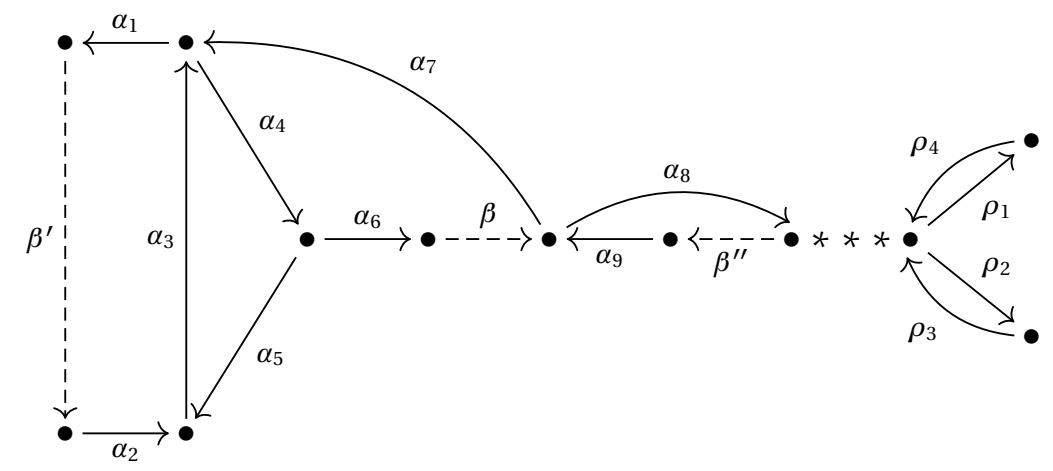

As relações do tipo 2 são: $r 1=\alpha_{2} \alpha_{3} \alpha_{4}, r 2=\alpha_{5} \alpha_{3} \alpha_{1}, r 3=\alpha_{7} \alpha_{4} \alpha_{5}, r 4=\alpha_{7} \alpha_{1}$, $r 5=\beta \alpha_{8}, r 6=\alpha_{9} \alpha_{7}, r 7=\alpha_{3} \alpha_{4} \alpha_{6}$ e da subaljava: 


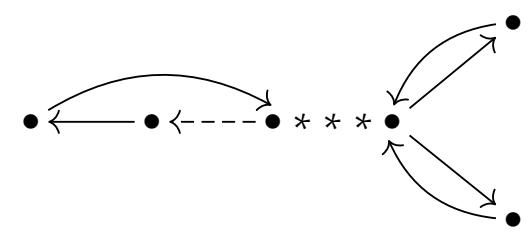

Os ciclos elementares são: $C_{1}=\alpha_{3} \alpha_{1} \beta^{\prime} \alpha_{2}, C_{2}=\alpha_{3} \alpha_{4} \alpha_{5}, C_{3}=\alpha_{4} \alpha_{6} \beta \alpha_{7}$ e os ciclos elementares da subaljava anterior.

Mostraremos que existe apenas o corte $\Sigma$ que resulta numa álgebra de incidência não hereditária. Primeiro, usaremos o estudo da subaljava da esquerda em que concluímos que $\alpha_{3}, \alpha_{7} \in \Sigma$. Isso implica que $\alpha_{8} \in \Sigma$ pois precisamos eliminar a relação $r 5=\beta \alpha_{8}$. Sabendo que $\alpha_{8} \in \Sigma$, implica que não importa a quantidade de flechas nos ciclos elementares, o lema 5.39 completa o corte $\Sigma$. Entretanto, a álgebra $K Q_{\Gamma} /<I_{\Gamma} \cup \Sigma>$ é uma álgebra disfarçada.

$\widetilde{\mathbb{D}}_{n}-\mathcal{F}$ r4.4 Essa extensão trivial tem uma diferença do caso anterior, a presença da subaljava

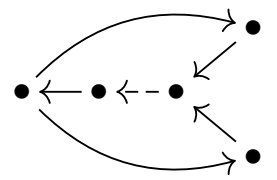
na parte direita da aljava:

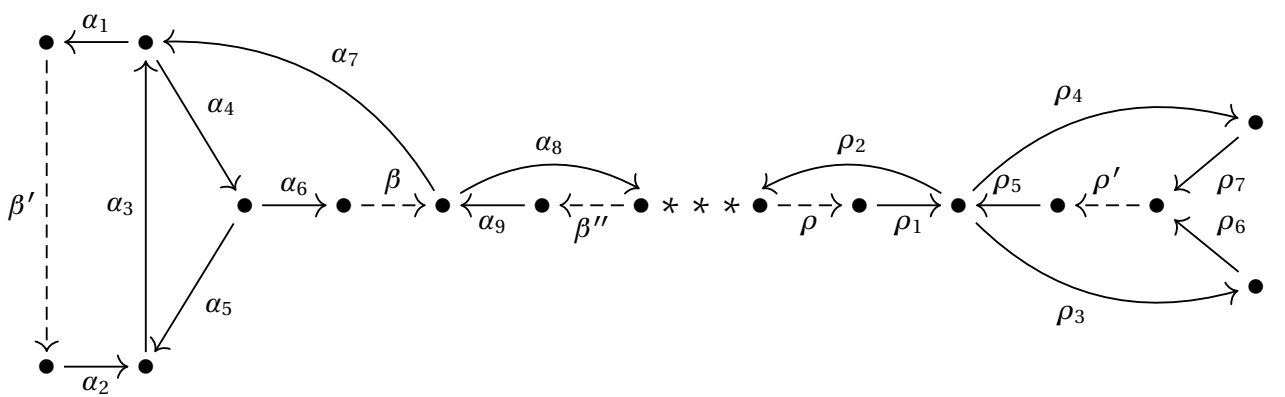

As relações do tipo 2 são: $r 1=\alpha_{2} \alpha_{3} \alpha_{4}, r 2=\alpha_{5} \alpha_{3} \alpha_{1}, r 3=\alpha_{7} \alpha_{4} \alpha_{5}, r 4=\alpha_{7} \alpha_{1}$, $r 5=\beta \alpha_{8}, r 6=\alpha_{9} \alpha_{7}, r 7=\rho_{1} \rho_{4}, r 8=\rho_{1} \rho_{3}, r 9=\rho_{5} \rho_{2}, r 10=\rho_{6} \rho^{\prime} \rho_{5} \rho_{4}, r 11=$ $\rho_{7} \rho^{\prime} \rho_{5} \rho_{3}, r 12=\alpha_{3} \alpha_{4} \alpha_{6}$ e da subaljava:

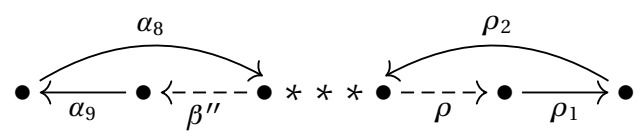

Os ciclos elementares são: $C_{1}=\alpha_{3} \alpha_{1} \beta^{\prime} \alpha_{2}, C_{2}=\alpha_{3} \alpha_{4} \alpha_{5}, C_{3}=\alpha_{4} \alpha_{6} \beta \alpha_{7}, C_{4}=$ $\rho_{6} \rho^{\prime} \rho_{5} \rho_{3}, C_{5}=\rho_{4} \rho_{7} \rho^{\prime} \rho_{5}$ e os ciclos elementares da subaljava anterior.

Reparamos pelas direções das flechas $\alpha_{9}$ e $\rho_{1}$ que o símbolo *** pode ser substituído por pares de ciclos elementares do tipo $\bullet \leftarrow \bullet \leftarrow \bullet-\longrightarrow \bullet \rightarrow \bullet$.

Dividiremos em duas etapas. A primeira etapa é começar as escolhas das flechas para compor o corte pelo lado esquerdo da aljava. Isto é, aproveitaremos os argumentos da parte anterior, tentando buscar o corte $\Sigma$ desejado. Portanto, começamos com $\alpha_{3}, \alpha_{7} \epsilon$ $\Sigma$. Pela relação $r 5=\beta \alpha_{8}$, implica que $\alpha_{8} \in \Sigma$. Pelo lema 5.39, a presença da flecha $\alpha_{8}$

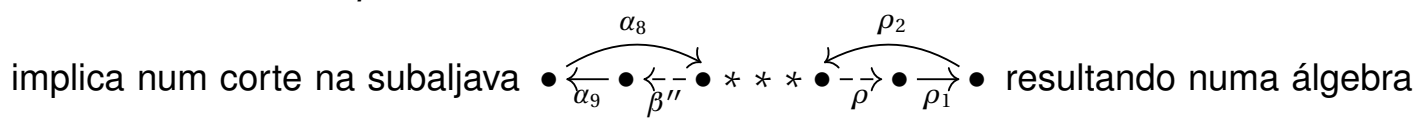


de incidência. Sabendo que $\rho_{2} \in \Sigma$, implica que $\rho_{4}, \rho_{3} \in \Sigma$. Consequentemente, a álgebra $K Q_{\Gamma} /<I_{\Gamma} \cup \Sigma>$ é uma álgebra disfarçada.

A segunda etapa é começar as escolhas dos elementos do corte pelo lado direito da aljava. Para satisfazer o lema 5.22, precisamos escolher uma flecha do caminho $\rho^{\prime}$ ou a flecha $\rho_{5}$ para pertencer ao $\Sigma$. Observamos que qualquer escolha da flecha do caminho $\rho^{\prime}$ para o conjunto $\Sigma$, implicaria na manutenção da relação $r 8=\rho_{1} \rho_{3}$ ou $r 9=\rho_{5} \rho_{2}$. Portanto, $\rho_{5} \in \Sigma$.

Supomos que existe algum ciclo elementar com três flechas ou mais. Sem perda de generalidade, supomos que existe o caminho $\rho$. Continuando as escolhas para o conjunto $\Sigma$, concluímos que $\rho_{1} \in \Sigma$ pois temos as relações $r 7=\rho_{1} \rho_{4}$ e $r 8=$ $\rho_{1} \rho_{3}$. A partir disso, pelo lema 5.39, não temos um corte desejado na subaljava

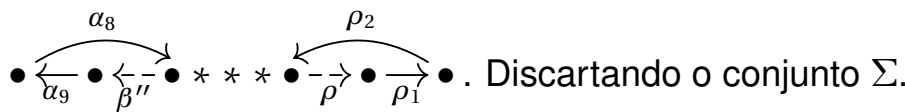

O próximo passo é não supor a presença de um ciclo elementar de três flechas ou mais. Ou seja, estamos supondo que existe apenas ciclos elementares de duas flechas no meio da aljava da extensão trivial. Novamente, iniciamos com $\rho_{5} \in \Sigma$. Implicando em

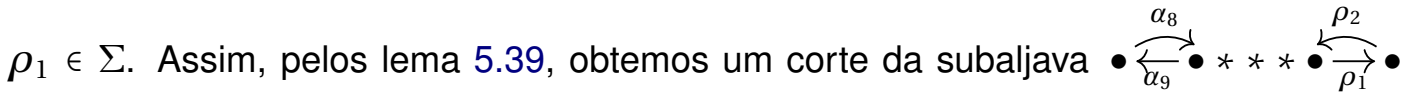
que resulta numa álgebra de incidência.

Em seguida, para completar o corte, escolheremos as flechas dos ciclos elementares $C_{1}, C_{2}$ e $C_{3}$. O intuito é fazer as escolhas conforme a presença das relações de tipo 2: $r 1=\alpha_{2} \alpha_{3} \alpha_{4}, r 2=\alpha_{5} \alpha_{3} \alpha_{1}, r 3=\alpha_{7} \alpha_{4} \alpha_{5}, r 4=\alpha_{7} \alpha_{1}, r 5=\beta \alpha_{8}, r 6=\alpha_{9} \alpha_{7} \mathrm{e}$ $r 12=\alpha_{3} \alpha_{4} \alpha_{6}$.

Sabendo que $\alpha_{9} \in \Sigma$, escolheremos uma flecha $\beta_{1} \in \Sigma$ do caminho $\beta$ tal que a concatenação com $\alpha_{8}$ seja diferente de zero. Isso implica que a relação $r 5$ foi eliminada em $K Q_{\Gamma} /<I_{\Gamma} \cup \Sigma>$ mas continuamos com a relação $r 12$. Caso não exista o caminho $\beta$, colocaremos $\alpha_{6}$ em $\sum$ implicando no corte da relação $r 12=\alpha_{3} \alpha_{4} \alpha_{6}$.

Ainda temos no mínimo as relações $r 1=\alpha_{2} \alpha_{3} \alpha_{4}, r 2=\alpha_{5} \alpha_{3} \alpha_{1}, r 3=\alpha_{7} \alpha_{4} \alpha_{5}$ e $r 4=\alpha_{7} \alpha_{1}$, e as escolhas dos ciclos elementares $C_{1}$ e $C_{2}$. A relação $r 4=\alpha_{7} \alpha_{1}$ determina a opção do ciclo elementar $C_{1}$, isto é, $\alpha_{1} \in \Sigma$. Por último, a única flecha do ciclo elementar $C_{2}$ que não está em $C_{1}$ e $C_{3}$ é $\alpha_{5} \in \Sigma$.

Com o conjunto $\Sigma$ completo, temos $K Q_{\Gamma} /<I_{\Gamma} \cup \Sigma>$ com a relação $r 1=\alpha_{2} \alpha_{3} \alpha_{4}$, isto é, não é uma álgebra de incidência. Portanto, não temos solução nesse caso.

$\widetilde{\mathbb{D}}_{n}-\mathcal{F} r 4.5$ A última extensão trivial da parte $\widetilde{\mathbb{D}}_{n}-\mathcal{F} r 4$. 


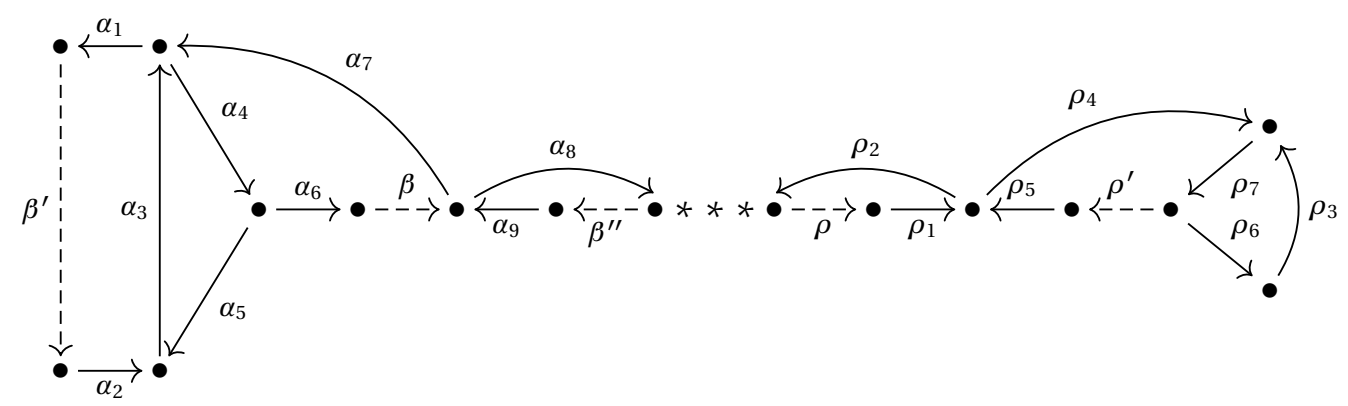

As relações do tipo 2 são: $r 1=\alpha_{2} \alpha_{3} \alpha_{4}, r 2=\alpha_{5} \alpha_{3} \alpha_{1}, r 3=\alpha_{7} \alpha_{4} \alpha_{5}, r 4=\alpha_{7} \alpha_{1}, r 5=$ $\beta \alpha_{8}, r 6=\alpha_{9} \alpha_{7}, r 7=\rho_{1} \rho_{4}, r 8=\rho_{5} \rho_{2}, r 9=\rho_{3} \rho_{7} \rho^{\prime}, r 10=\rho_{4} \rho_{7} \rho_{6}, r 11=\alpha_{3} \alpha_{4} \alpha_{6}$ e as relações do tipo 2 da subaljava:

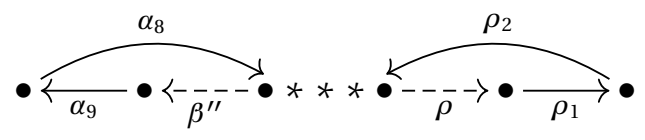

Os ciclos elementares são: $C_{1}=\alpha_{3} \alpha_{1} \beta^{\prime} \alpha_{2}, C_{2}=\alpha_{3} \alpha_{4} \alpha_{5}, C_{3}=\alpha_{4} \alpha_{6} \beta \alpha_{7}, C_{4}=$ $\rho_{6} \rho_{3} \rho_{7}, C_{5}=\rho_{4} \rho_{7} \rho^{\prime} \rho_{5}$ e os ciclos elementares da subaljava anterior.

Faremos o mesmo roteiro da parte anterior. Como já visto, o símbolo $* * *$ pode ser substituído por pares de ciclos elementares do tipo $\bullet \leftarrow \bullet<\bullet-\neg \bullet \rightarrow \bullet$.

Dividiremos em duas etapas na busca pelo corte $\Sigma$ desejado. A primeira etapa é começar as escolhas das flechas para compor o conjunto $\Sigma$ pelo lado esquerdo da aljava. Sendo assim, temos que $\alpha_{3}, \alpha_{7} \in \Sigma$. Consequentemente, pela relação $r 5=$ $\beta \alpha_{8}$, implica que $\alpha_{8} \in \Sigma$. Pelo lema 5.39, temos um corte aceitável na subaljava

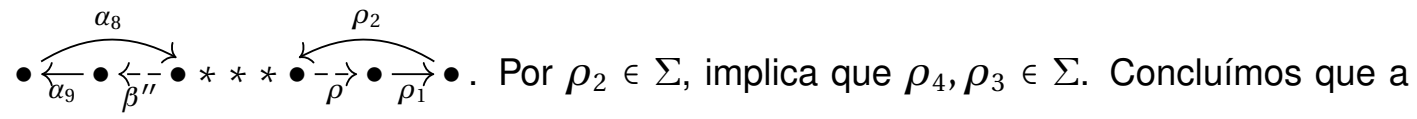
álgebra $K Q_{\Gamma} /<I_{\Gamma} \cup \Sigma>$ é uma álgebra disfarçada.

A segunda etapa é começar as escolhas dos elementos de $\Sigma$ pelo lado direito da aljava. Para satisfazer o lema 5.22, temos que ter a flecha $\rho_{7}$ no conjunto $\Sigma$.

Em seguida, escolheremos uma flecha do ciclo elementar $\rho_{2} \rho \rho_{1}$. Pelas relações $r 7=\rho_{1} \rho_{4}$ e $r 8=\rho_{5} \rho_{2}$, não importa a escolha que façamos nesse ciclo elementar, implicaria na manutenção de uma dessas relações de tipo 2. Portanto, podemos parar nessa etapa pois não conseguiremos um corte $\Sigma$ tal que $K Q_{\Gamma} /<I_{\Gamma} \cup \Sigma>$ seja uma álgebra de incidência.

Logo, terminamos essa parte sem nenhuma solução.

$\widetilde{\mathbb{D}}_{n}-\mathcal{F} r 5$ Esse é o último frame schurian das álgebras disfarçadas de tipo $\widetilde{\mathbb{D}}_{n}$ a ser estudado. Buscaremos através dos cortes, as Phias que são diferentes das álgebras disfarçadas. Observamos que os conjuntos de extensões triviais são a menos de aljavas simétricas ou aljavas duais.

$\widetilde{\mathbb{D}}_{n}-\mathcal{F} r 5.1$ Colocamos no lado esquerdo a álgebra disfarçada originada do frame $\mathcal{F} r 5$. No lado direito, a extensão trivial $\Gamma$ da álgebra em que tem cinco ciclos elementares: 


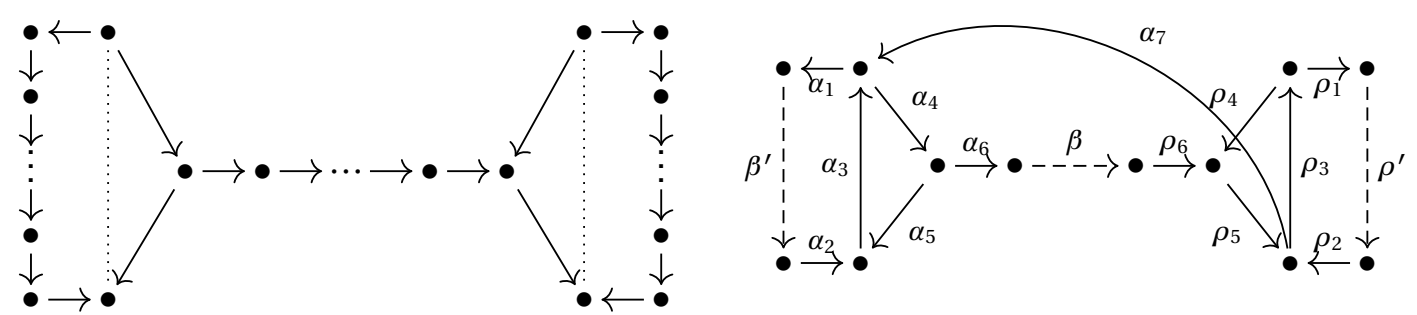

Não importando os comprimentos dos caminhos, a configuração da extensão trivial é a mesma. Portanto, faremos as contas para uma extensão trivial de uma álgebra disfarçada de tipo $\widetilde{\mathbb{D}}_{7}$.

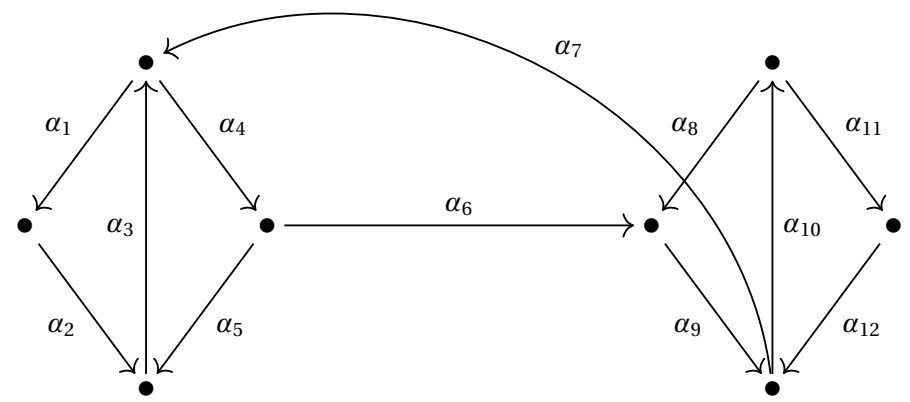

As relações do tipo 2 são: $r 1=\alpha_{2} \alpha_{3} \alpha_{4}, r 2=\alpha_{5} \alpha_{3} \alpha_{1}, r 3=\alpha_{3} \alpha_{4} \alpha_{6}, r 4=\alpha_{9} \alpha_{10} \alpha_{11}$, $r 5=\alpha_{12} \alpha_{10} \alpha_{8}, r 6=\alpha_{7} \alpha_{1}, r 7=\alpha_{7} \alpha_{4} \alpha_{5}, r 8=\alpha_{6} \alpha_{9} \alpha_{10}, r 9=\alpha_{12} \alpha_{7}$ e $r 10=\alpha_{8} \alpha_{9} \alpha_{7}$. Os ciclos elementares são: $C_{1}=\alpha_{3} \alpha_{1} \alpha_{2}, C_{2}=\alpha_{3} \alpha_{4} \alpha_{5}, C_{3}=\alpha_{4} \alpha_{6} \alpha_{9} \alpha_{7}, C_{4}=\alpha_{8} \alpha_{9} \alpha_{10}$ e $C_{5}=\alpha_{10} \alpha_{11} \alpha_{12}$.

Portanto, o programa nos mostra apenas o corte $\Sigma=\left\{\alpha_{3}, \alpha_{7}, \alpha_{10}\right\}$ tal que $K Q_{\Gamma} /<$ $I_{\Gamma} \cup \Sigma>$ é uma álgebra disfarçada.

$\widetilde{\mathbb{D}}_{n}-\mathcal{F} r 5.2$ Abaixo, no lado esquerdo tem álgebra disfarçada originada do frame $\mathcal{F} r 5$. No lado direito, a extensão trivial $\Gamma$ da álgebra em que tem seis ciclos elementares:

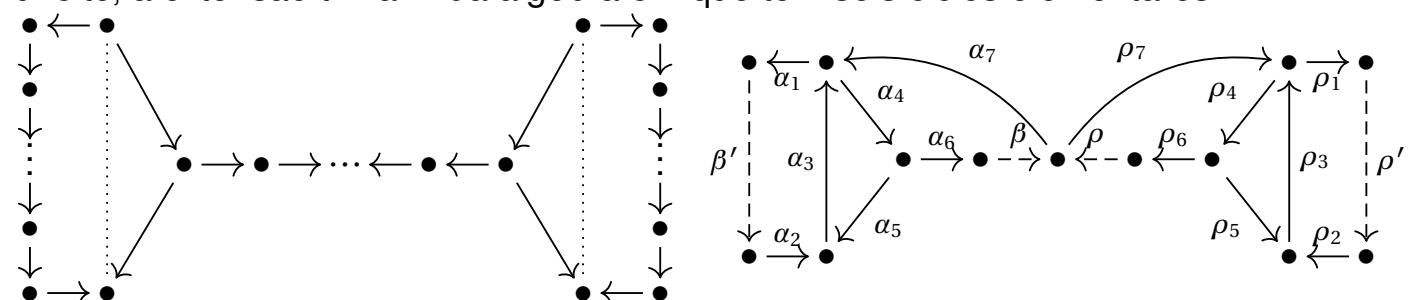

Pela mesma razão do caso anterior, faremos as contas para uma extensão trivial de uma álgebra disfarçada de tipo $\widetilde{\mathbb{D}}_{8}$.

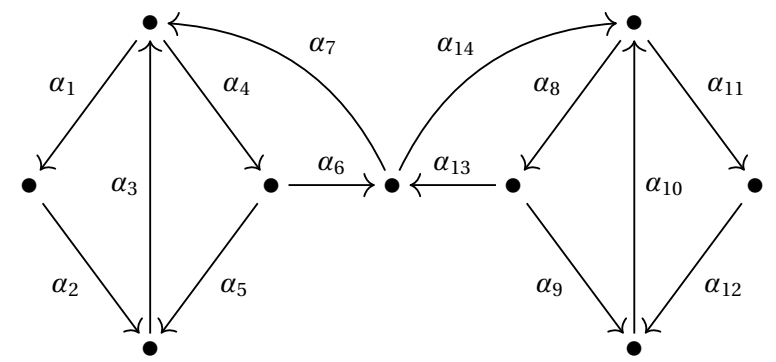

As relações do tipo 2 são: $r 1=\alpha_{2} \alpha_{3} \alpha_{4}, r 2=\alpha_{5} \alpha_{3} \alpha_{1}, r 3=\alpha_{3} \alpha_{4} \alpha_{6}, r 4=\alpha_{9} \alpha_{10} \alpha_{11}$, 
$r 5=\alpha_{12} \alpha_{10} \alpha_{8}, r 6=\alpha_{7} \alpha_{1}, r 7=\alpha_{7} \alpha_{4} \alpha_{5}, r 8=\alpha_{6} \alpha_{14}, r 9=\alpha_{13} \alpha_{7}, r 10=\alpha_{14} \alpha_{11}$, $r 11=\alpha_{14} \alpha_{8} \alpha_{9}$ e $r 12=\alpha_{10} \alpha_{8} \alpha_{13}$. Os ciclos elementares são: $C_{1}=\alpha_{3} \alpha_{1} \alpha_{2}, C_{2}=$ $\alpha_{3} \alpha_{4} \alpha_{5}, C_{3}=\alpha_{4} \alpha_{6} \alpha_{7}, C_{4}=\alpha_{8} \alpha_{9} \alpha_{10}, C_{5}=\alpha_{10} \alpha_{11} \alpha_{12}$ e $C_{6}=\alpha_{13} \alpha_{14} \alpha_{8}$.

Portanto, o mesmo resultado do caso anterior, o programa exibe apenas o corte $\Sigma=$ $\left\{\alpha_{3}, \alpha_{7}, \alpha_{10}, \alpha_{14}\right\}$ tal que $K Q_{\Gamma} /<I_{\Gamma} \cup \Sigma>$ é uma álgebra disfarçada.

$\widetilde{\mathbb{D}}_{n}-\mathcal{F} r 5.3$ Seja $\Gamma$ a última extensão trivial da parte $\widetilde{\mathbb{D}}_{n}-\mathcal{F} r 5$.

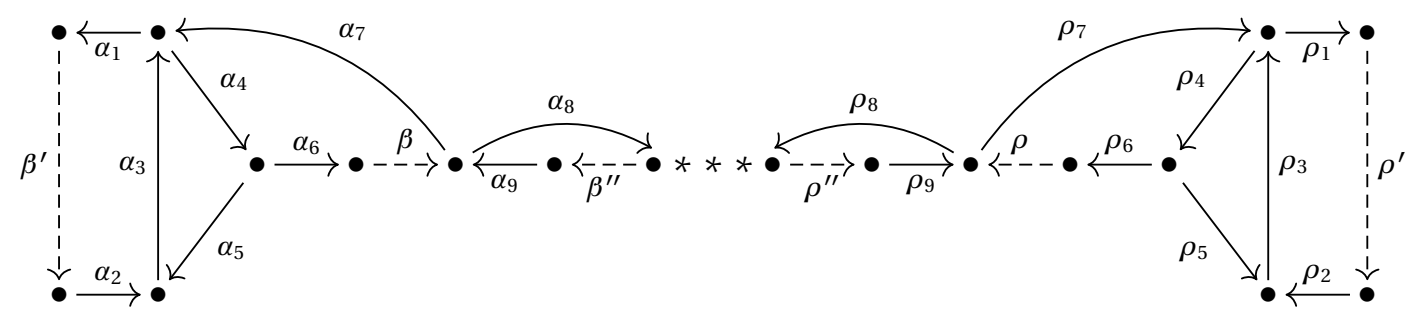

As relações do tipo 2 são: $r 1=\alpha_{2} \alpha_{3} \alpha_{4}, r 2=\alpha_{5} \alpha_{3} \alpha_{1}, r 3=\alpha_{3} \alpha_{4} \alpha_{6}, r 4=\alpha_{7} \alpha_{4} \alpha_{5}$, $r 5=\alpha_{7} \alpha_{1}, r 6=\beta \alpha_{8}, r 7=\alpha_{9} \alpha_{7}, r 8=\rho_{9} \rho_{7}, r 9=\rho \rho_{8}, r 10=\rho_{2} \rho_{3} \rho_{4}, r 11=$ $\rho_{5} \rho_{3} \rho_{1}, r 12=\rho_{7} \rho_{4} \rho_{5}, r 13=\rho_{7} \rho_{1}, r 14=\rho_{3} \rho_{4} \rho_{6}$ e as relações do tipo 2 da subaljava:

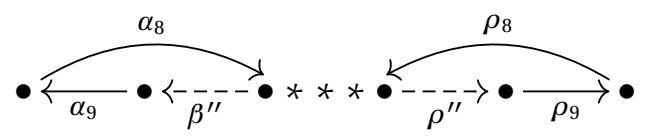

Os ciclos elementares são: $C_{1}=\alpha_{3} \alpha_{1} \beta^{\prime} \alpha_{2}, C_{2}=\alpha_{3} \alpha_{4} \alpha_{5}, C_{3}=\alpha_{4} \alpha_{6} \beta \alpha_{7}, C_{4}=$ $\rho_{1} \rho^{\prime} \rho_{2} \rho_{3}, C_{5}=\rho_{3} \rho_{4} \rho_{5}, C_{6}=\rho_{4} \rho_{6} \rho \rho_{7}$ e os ciclos elementares da subaljava anterior. Como foi visto na parte $\widetilde{\mathbb{D}}_{n}-\mathcal{F} r 4.4$, o símbolo $* * *$ pode ser substituído por pares de ciclos elementares do tipo $\bullet \leftarrow \bullet \leftarrow \bullet \bullet-\leftarrow \bullet \rightarrow \bullet$.

Ressaltamos que a demonstração de $\widetilde{\mathbb{D}}_{n}-\mathcal{F} r 5$ é válida para as variações seguintes de $\Gamma$ :

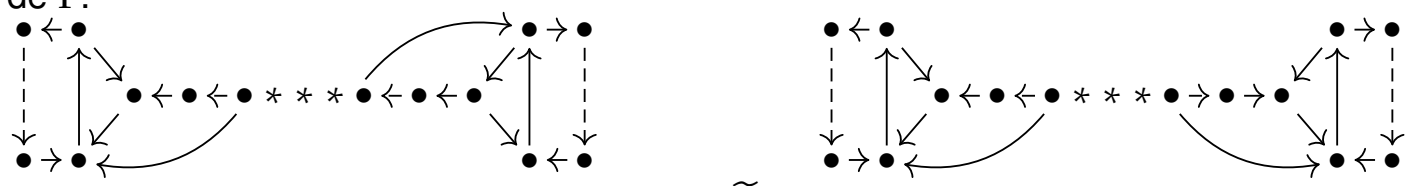

Seja $\Sigma$ o corte desejado. Conforme a parte $\widetilde{\mathbb{D}}_{n}-\mathcal{F} r 4.3$, temos que $\alpha_{3}, \alpha_{7} \in \Sigma$. Consequentemente, pela relação $r 5=\beta \alpha_{8}$, implica que $\alpha_{8} \in \Sigma$. Pelo lema 5.39, temos

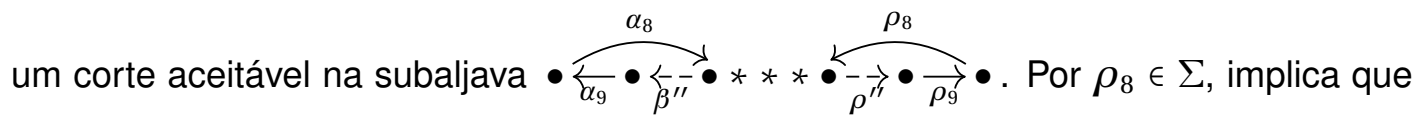
$\rho_{7} \in \Sigma$.

Para completar o conjunto $\Sigma$, precisamos escolher a(s) flecha(s) dos ciclos elementares $C_{4}=\rho_{1} \rho^{\prime} \rho_{2} \rho_{3}$ e $C_{5}=\rho_{3} \rho_{4} \rho_{5}$. A flecha $\rho_{4}$ não pode ser selecionada, pois pertence ao ciclo elementar $C_{6}$. Com objetivo de eliminar as relações $r 9=\rho_{2} \rho_{3} \rho_{4}, r 10=$ $\rho_{5} \rho_{3} \rho_{1}$ e $r 14=\rho_{3} \rho_{4} \rho_{6}$, a única escolha é $\rho_{3}$. Então o corte $\Sigma$ resultará numa álgebra disfarçada. 
Nessa parte, iniciaremos a sequência de dois teoremas referentes a descrição da família de Phias ANS de tipo $\widetilde{\mathbb{E}}_{6}$ e $\widetilde{\mathbb{E}}_{7}$.

Teorema 5.41. As álgebras associadas com as aljavas com relações abaixo:

1.<smiles>CC1(C)CCCCC1</smiles>

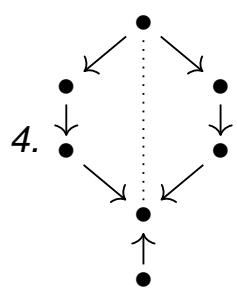

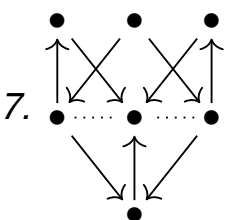

10.

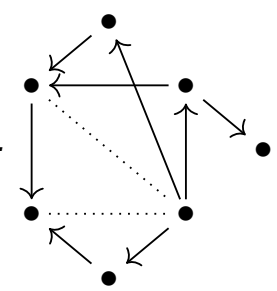

2.

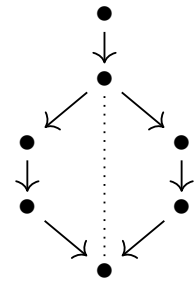

5.

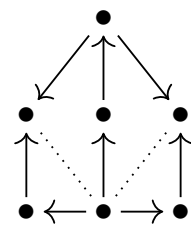

8.

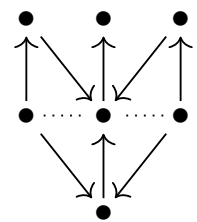

11.

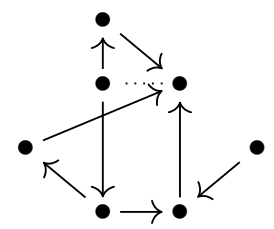

3.

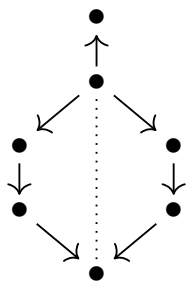

6.

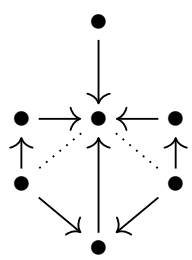

9.

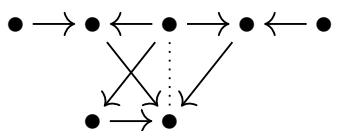

são Phias da família ANS de tipo $\widetilde{\mathbb{E}}_{6}$.

Demonstração A lista tem cinco frames: $\mathcal{F} r 6, \mathcal{F} r 7, \mathcal{F} r 8, \mathcal{F} r 9$ e $\mathcal{F} r 10$. Analisaremos apenas os frames schurian, satisfazendo a hipótese principal da teoria da seção $\mathbb{E}_{7}$ das Phias Dynkin. Faremos a extensão trivial da álgebra originada de cada frame. Isto é, dado um frame $\mathcal{F} r$, aplicaremos a operação admissível e depois mostraremos a extensão trivial dessa álgebra. Assim, colocaremos as informações necessárias de cada extensão trivial no programa e exibiremos as soluções não hereditárias.

Observamos que sempre a álgebra disfarçada do tipo $\widetilde{\mathbb{E}}_{6}$ é uma das soluções e iremos omitir ela. 


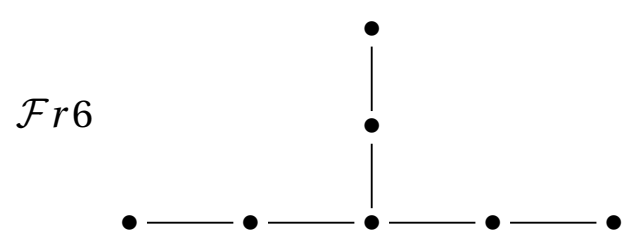

A aljava adjacente euclidiana $\widetilde{\mathbb{E}}_{6}$ tem $2^{6}$ de possibilidades de operações admissíveis um, ou seja, podemos ter $2^{6}$ grafos euclidianos $\widetilde{\mathbb{E}}_{6}$. Agora, o nosso objetivo é analisar a extensão trivial de cada grafo e ver se existe algum corte gerando uma Phia não hereditária. Para isso, na extensão trivial, precisamos ter no mínimo dois ciclos elementares que tenha pelo menos uma flecha em comum conforme 0 lema 5.22.

Assim, temos que ter dois caminhos maximais que tenham comprimento dois, no mínimo, e uma flecha em comum. A menos de dualidade, existem três casos:

1.

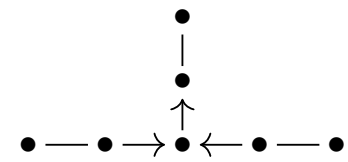

2.

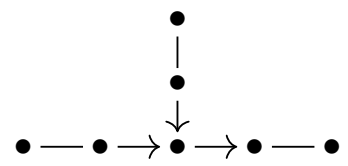

3.

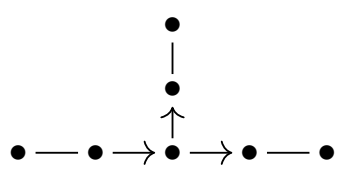

A menos de simetria, faremos todas as combinações de operações admissíveis 1 dos três casos anteriores.

$\mathcal{F} r 6.1$
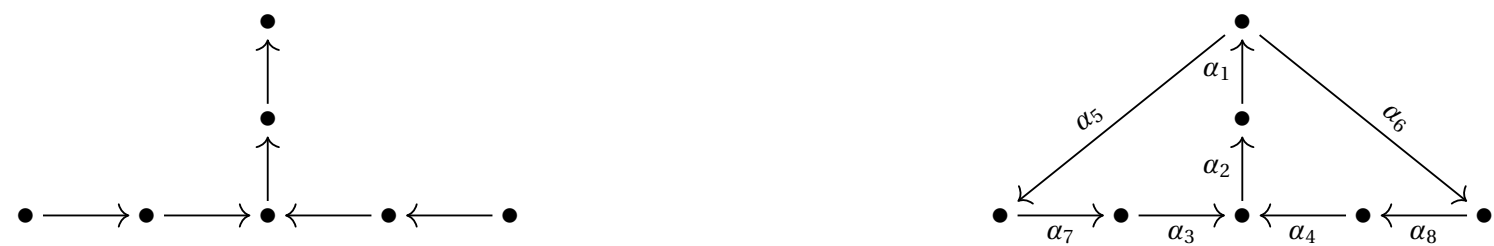

As relações do tipo 2 são: $r 1=\alpha_{3} \alpha_{2} \alpha_{1} \alpha_{6}$ e $r 2=\alpha_{4} \alpha_{2} \alpha_{1} \alpha_{5}$. Os ciclos elementares são: $C_{1}=\alpha_{7} \alpha_{3} \alpha_{2} \alpha_{1} \alpha_{5}$ e $C_{2}=\alpha_{8} \alpha_{4} \alpha_{2} \alpha_{1} \alpha_{6}$. Logo, o programa nos mostra os cortes que geram as álgebras 1 e 2 do teorema.

$\mathcal{F} r 6.2$
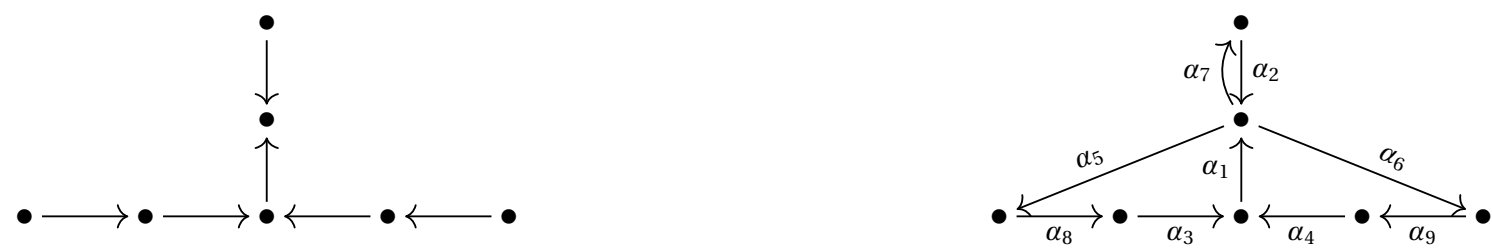

As relações do tipo 2 são: $r 1=\alpha_{3} \alpha_{1} \alpha_{6}, r 2=\alpha_{4} \alpha_{1} \alpha_{5}, r 3=\alpha_{2} \alpha_{5}, r 4=\alpha_{2} \alpha_{6}$ e $r 5=\alpha_{1} \alpha_{7}$. Os ciclos elementares são: $C_{1}=\alpha_{1} \alpha_{5} \alpha_{8} \alpha_{3}, C_{2}=\alpha_{1} \alpha_{6} \alpha_{9} \alpha_{4}$ e $C_{3}=\alpha_{2} \alpha_{7}$. Assim, temos a álgebra 3.

$\mathcal{F} r 6.3$
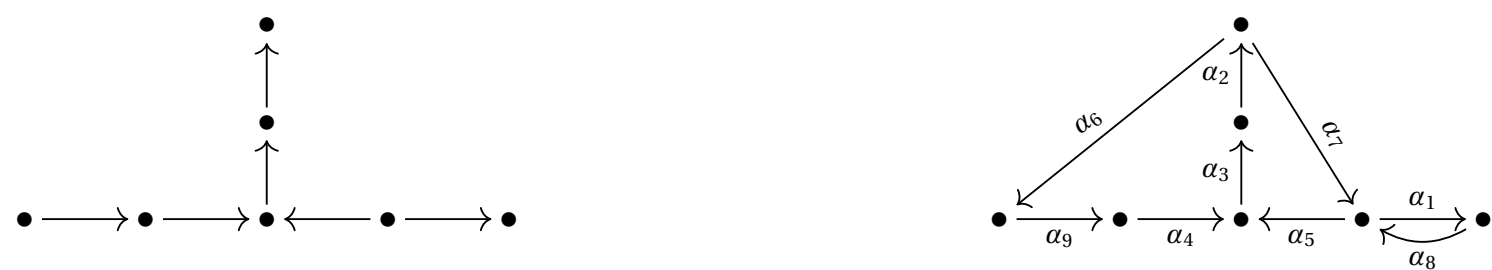

As relações do tipo 2 são: $r 1=\alpha_{4} \alpha_{3} \alpha_{2} \alpha_{7}, r 2=\alpha_{5} \alpha_{3} \alpha_{2} \alpha_{6}, r 3=\alpha_{8} \alpha_{5}$ e $r 4=\alpha_{7} \alpha_{1}$. Os ciclos elementares são: $C_{1}=\alpha_{2} \alpha_{6} \alpha_{9} \alpha_{4} \alpha_{3}, C_{2}=\alpha_{2} \alpha_{7} \alpha_{5} \alpha_{3}$ e $C_{3}=\alpha_{1} \alpha_{8}$. Com essa extensão trivial, não obtemos nenhum corte que gere uma Phia não hereditária. 
$\mathcal{F} r 6.4$
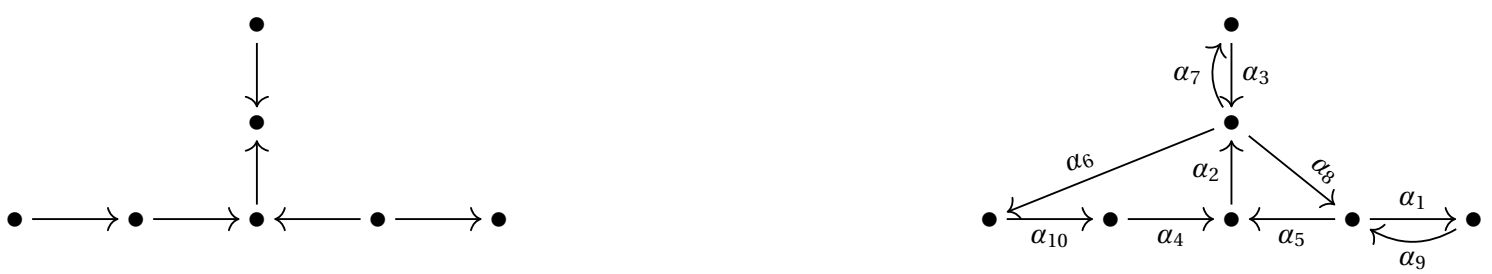

As relações do tipo 2 são: $r 1=\alpha_{4} \alpha_{2} \alpha_{8}, r 2=\alpha_{5} \alpha_{2} \alpha_{6}, r 3=\alpha_{3} \alpha_{6}, r 4=\alpha_{3} \alpha_{8}, r 5=\alpha_{2} \alpha_{7}$, $r 6=\alpha_{8} \alpha_{1}$ e $r 7=\alpha_{9} \alpha_{5}$. Os ciclos elementares são: $C_{1}=\alpha_{2} \alpha_{6} \alpha_{10} \alpha_{4}, C_{2}=\alpha_{2} \alpha_{8} \alpha_{5}$, $C_{3}=\alpha_{1} \alpha_{9}$ e $C_{4}=\alpha_{3} \alpha_{7}$. Também não temos nenhuma solução que gere uma Phia não hereditária.

$\mathcal{F}$ r6.5
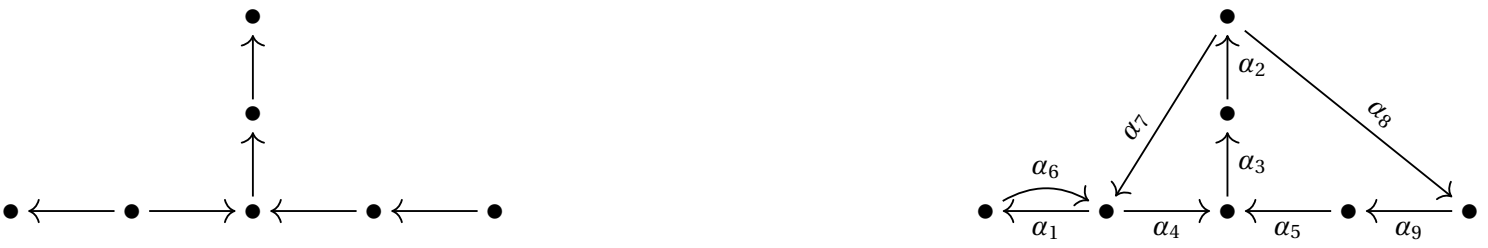

As relações do tipo 2 são: $r 1=\alpha_{4} \alpha_{3} \alpha_{2} \alpha_{8}, r 2=\alpha_{5} \alpha_{3} \alpha_{2} \alpha_{7}, r 3=\alpha_{6} \alpha_{4}$ e $r 4=\alpha_{7} \alpha_{1}$. Os ciclos elementares são: $C_{1}=\alpha_{2} \alpha_{7} \alpha_{4} \alpha_{3}, C_{2}=\alpha_{2} \alpha_{8} \alpha_{9} \alpha_{5} \alpha_{3}$ e $C_{3}=\alpha_{6} \alpha_{7}$. O programa exibe duas soluções hereditárias. Observamos que essa extensão trivial é a mesma extensão trivial da $\mathcal{F}$ r6.3.

$\mathcal{F} r 6.6$
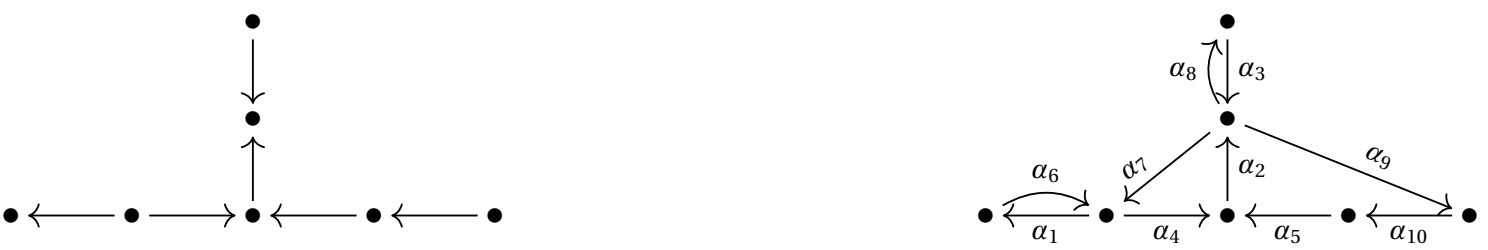

O $\mathcal{F}$ r6.4 é igual a esse $\mathcal{F}$ r6.6, então teremos a mesma extensão trivial e os mesmos resultados.

$\mathcal{F} r 6.7$
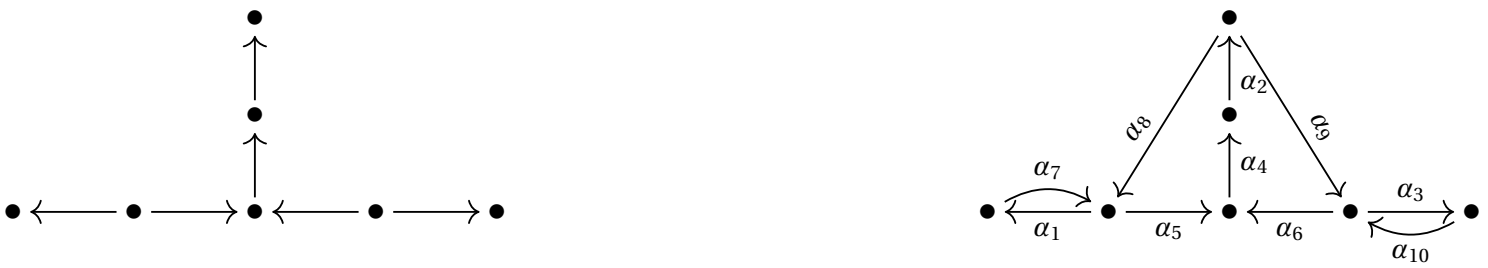

As relações do tipo 2 são: $r 1=\alpha_{5} \alpha_{4} \alpha_{2} \alpha_{9}, r 2=\alpha_{6} \alpha_{4} \alpha_{2} \alpha_{8}, r 3=\alpha_{7} \alpha_{5}, r 4=\alpha_{8} \alpha_{1}, r 5=$ $\alpha_{10} \alpha_{6}$ e $r 6=\alpha_{9} \alpha_{3}$. Os ciclos elementares são: $C_{1}=\alpha_{2} \alpha_{8} \alpha_{5} \alpha_{4}, C_{2}=\alpha_{2} \alpha_{9} \alpha_{6} \alpha_{4}, C_{3}=\alpha_{7} \alpha_{1}$ e $C_{4}=\alpha_{10} \alpha_{3}$. O programa exibe duas soluções hereditárias.

$\mathcal{F} r 6.8$
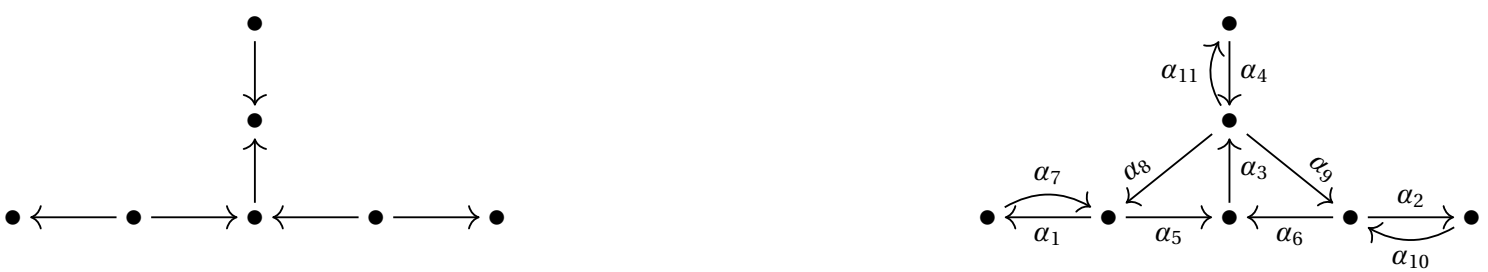

As relações do tipo 2 são: $r 1=\alpha_{5} \alpha_{3} \alpha_{9}, r 2=\alpha_{6} \alpha_{3} \alpha_{8}, r 3=\alpha_{7} \alpha_{5}, r 4=\alpha_{8} \alpha_{1}, r 5=\alpha_{9} \alpha_{2}$, $r 6=\alpha_{10} \alpha_{6}, r 7=\alpha_{4} \alpha_{8}, r 8=\alpha_{4} \alpha_{9}$ e $r 9=\alpha_{3} \alpha_{11}$. Os ciclos elementares são: $C_{1}=$ $\alpha_{2} \alpha_{8} \alpha_{5} \alpha_{4}, C_{2}=\alpha_{2} \alpha_{9} \alpha_{6} \alpha_{4}, C_{3}=\alpha_{7} \alpha_{1}, C_{4}=\alpha_{10} \alpha_{3}$ e $C_{5}=\alpha_{10} \alpha_{3}$. Portanto, obtemos apenas 
a solução hereditária.

Começamos o estudo de todas as extensões triviais do segundo caso.

$\mathcal{F} r 6.9$
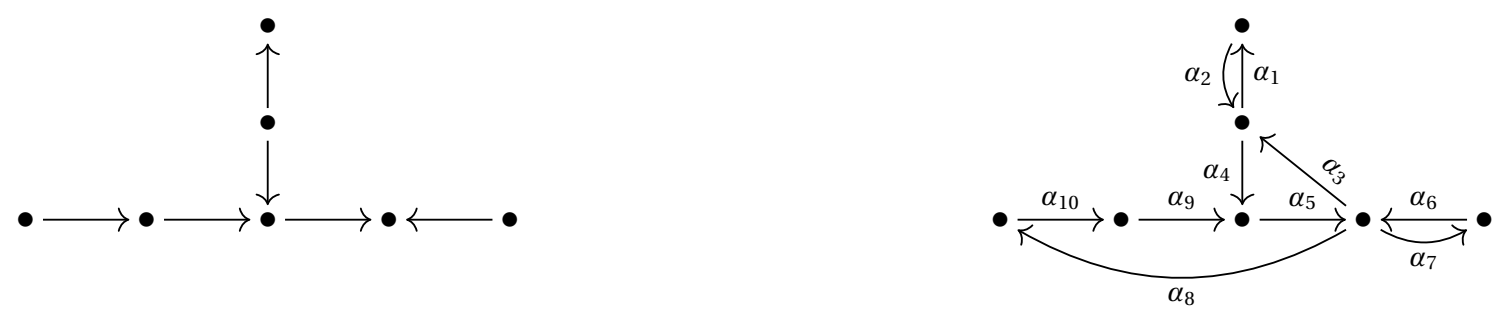

As relações do tipo 2 são: $r 1=\alpha_{2} \alpha_{4}, r 2=\alpha_{3} \alpha_{1}, r 3=\alpha_{6} \alpha_{3}, r 4=\alpha_{6} \alpha_{8}, r 5=\alpha_{5} \alpha_{7}$, $r 6=\alpha_{4} \alpha_{5} \alpha_{8}$ e $r 7=\alpha_{9} \alpha_{5} \alpha_{3}$. Os ciclos elementares são: $C_{1}=\alpha_{2} \alpha_{1}, C_{2}=\alpha_{6} \alpha_{7}, C_{3}=$ $\alpha_{4} \alpha_{5} \alpha_{3}$ e $C_{4}=\alpha_{5} \alpha_{8} \alpha_{10} \alpha_{9}$. Portanto, o programa nos mostra apenas um corte $\Sigma$ tal que $K Q_{\Gamma} /<I_{\Gamma} \cup \Sigma>$ é uma álgebra hereditária.

$\mathcal{F}$ r6.10
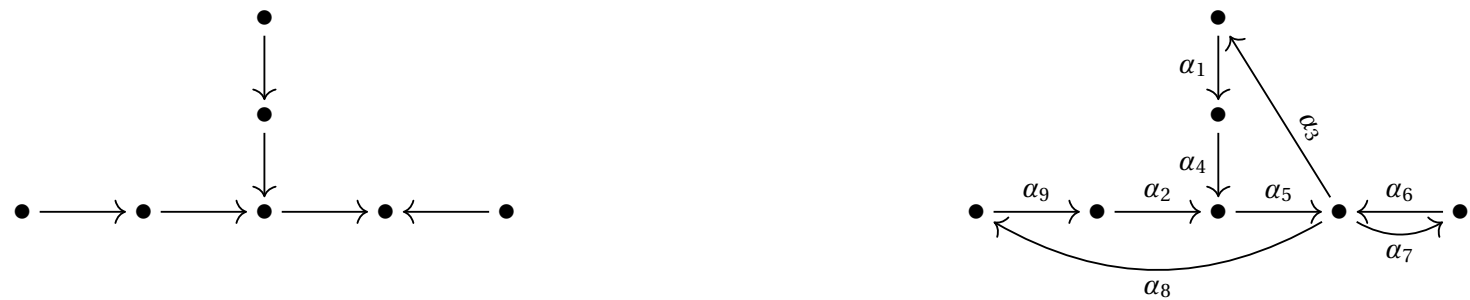

As relações do tipo 2 dessa extensão trivial $\Gamma$ são: $r 1=\alpha_{6} \alpha_{3}, r 2=\alpha_{6} \alpha_{8}, r 3=\alpha_{5} \alpha_{7}$, $r 4=\alpha_{2} \alpha_{5} \alpha_{3}$ e $r 5=\alpha_{4} \alpha_{5} \alpha_{8}$. Os ciclos elementares são: $C_{1}=\alpha_{6} \alpha_{7}, C_{2}=\alpha_{1} \alpha_{4} \alpha_{5} \alpha_{3}$ e $C_{3}=\alpha_{5} \alpha_{8} \alpha_{9} \alpha_{2}$. Obtemos um corte $\Sigma$ tal que $K Q_{\Gamma} /<I_{\Gamma} \cup \Sigma>$ é a solução 3 .

$\mathcal{F}$ r6.11
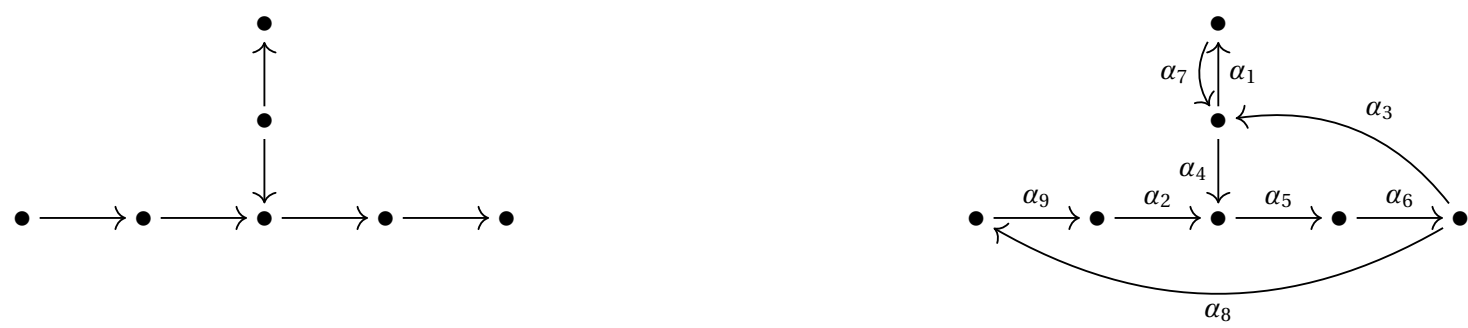

As relações do tipo 2 dessa extensão trivial são: $r 1=\alpha_{7} \alpha_{4}, r 2=\alpha_{3} \alpha_{1}, r 3=\alpha_{4} \alpha_{5} \alpha_{6} \alpha_{8}$ e $r 4=\alpha_{2} \alpha_{5} \alpha_{6} \alpha_{3}$. Os ciclos elementares são: $C_{1}=\alpha_{7} \alpha_{1}, C_{2}=\alpha_{4} \alpha_{5} \alpha_{6} \alpha_{3}$ e $C_{3}=\alpha_{5} \alpha_{6} \alpha_{8} \alpha_{9} \alpha_{2}$. Com essa extensão trivial, não obtemos nenhum corte que gere uma Phia não hereditária.

$\mathcal{F}$ r6.12
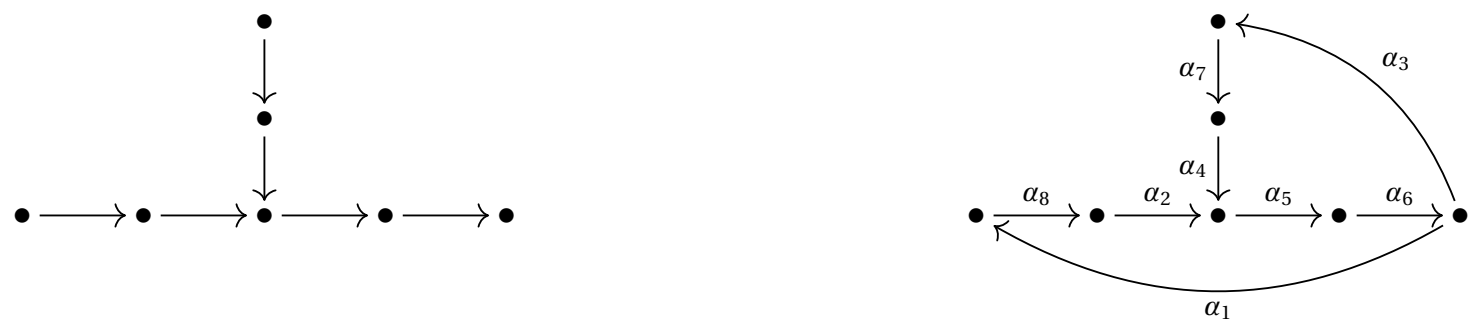

As relações do tipo 2 dessa extensão trivial $\Gamma$ são: $r 1=\alpha_{4} \alpha_{5} \alpha_{6} \alpha_{1}$ e $r 2=\alpha_{2} \alpha_{5} \alpha_{6} \alpha_{3}$. Os ciclos elementares são: $C_{1}=\alpha_{7} \alpha_{4} \alpha_{5} \alpha_{6} \alpha_{3}$ e $C_{2}=\alpha_{5} \alpha_{6} \alpha_{1} \alpha_{8} \alpha_{2}$. O programa exibe os cortes $\Sigma_{1}=\left\{\alpha_{6}\right\}$ e $\Sigma_{2}=\left\{\alpha_{5}\right\}$. Portanto, $K Q_{\Gamma} /<I_{\Gamma} \cup \Sigma_{i}>$ são as soluções $i$, para $i=1,2$. 
$\mathcal{F} r 6.13$
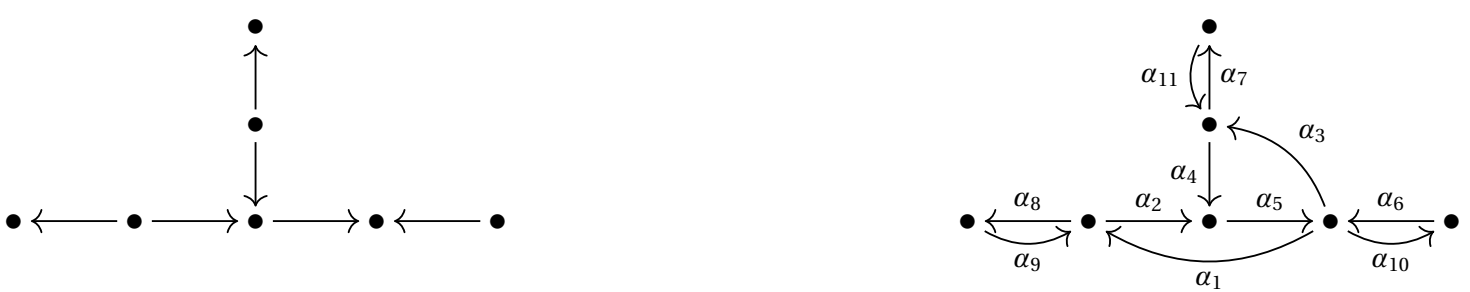

As relações do tipo 2 são: $r 1=\alpha_{6} \alpha_{3}, r 2=\alpha_{6} \alpha_{1}, r 3=\alpha_{5} \alpha_{10}, r 4=\alpha_{11} \alpha_{4}, r 5=\alpha_{3} \alpha_{7}$, $r 6=\alpha_{4} \alpha_{5} \alpha_{1}, r 7=\alpha_{2} \alpha_{5} \alpha_{3}, r 8=\alpha_{9} \alpha_{2}$ e $r 9=\alpha_{1} \alpha_{8}$. Os ciclos elementares são: $C_{1}=\alpha_{7} \alpha_{11}$, $C_{2}=\alpha_{6} \alpha_{10}, C_{3}=\alpha_{4} \alpha_{5} \alpha_{3}, C_{4}=\alpha_{5} \alpha_{1} \alpha_{2}$ e $C 5=\alpha_{8} \alpha_{9}$. O programa exibe uma solução hereditária.

$\mathcal{F} r 6.14$
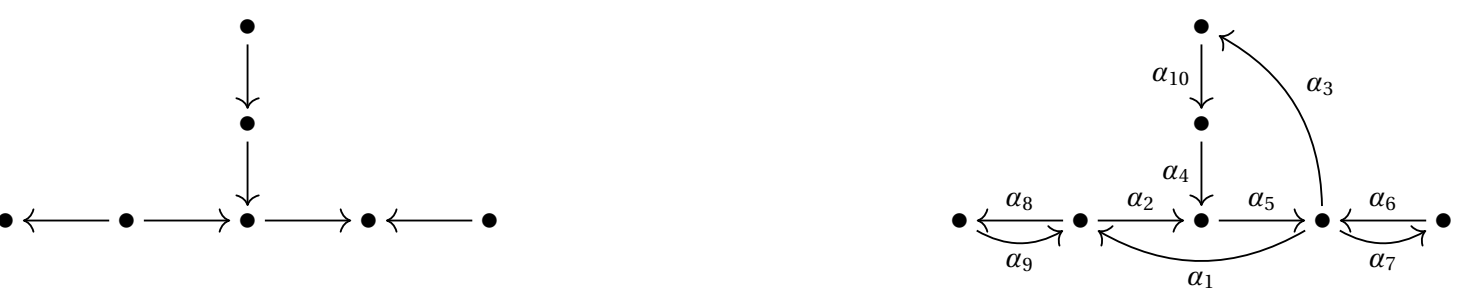

As relações do tipo 2 são: $r 1=\alpha_{6} \alpha_{3}, r 2=\alpha_{6} \alpha_{1}, r 3=\alpha_{5} \alpha_{7}, r 4=\alpha_{9} \alpha_{2}, r 5=\alpha_{1} \alpha_{8}, r 6=$ $\alpha_{4} \alpha_{5} \alpha_{1}$ e $r 7=\alpha_{2} \alpha_{5} \alpha_{3}$. Os ciclos elementares são: $C_{1}=\alpha_{6} \alpha_{7}, C_{2}=\alpha_{8} \alpha_{9}, C_{3}=\alpha_{10} \alpha_{4} \alpha_{5} \alpha_{3}$ e $C_{4}=\alpha_{5} \alpha_{1} \alpha_{2}$. Portanto, obtemos apenas a solução hereditária.

$\mathcal{F} r 6.15$
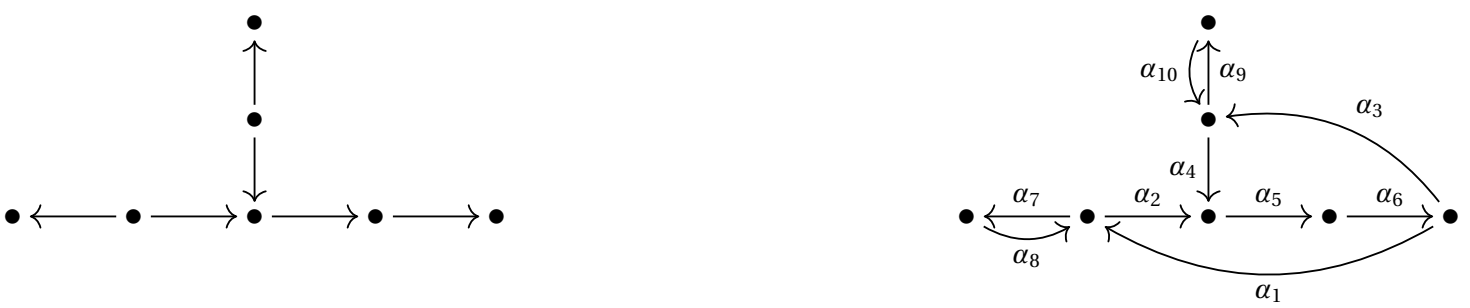

As relações do tipo 2 são: $r 1=\alpha_{10} \alpha_{4}, r 2=\alpha_{3} \alpha_{9}, r 3=\alpha_{8} \alpha_{2}, r 4=\alpha_{1} \alpha_{7}, r 5=\alpha_{4} \alpha_{5} \alpha_{6} \alpha_{1}$ e $r 6=\alpha_{2} \alpha_{5} \alpha_{6} \alpha_{3}$. Os ciclos elementares são: $C_{1}=\alpha_{7} \alpha_{8}, C_{2}=\alpha_{10} \alpha_{9}, C_{3}=\alpha_{4} \alpha_{5} \alpha_{6} \alpha_{3}$ e $C_{4}=\alpha_{2} \alpha_{5} \alpha_{6} \alpha_{1}$. O programa exibe duas soluções hereditárias.

$\mathcal{F} r 6.16$
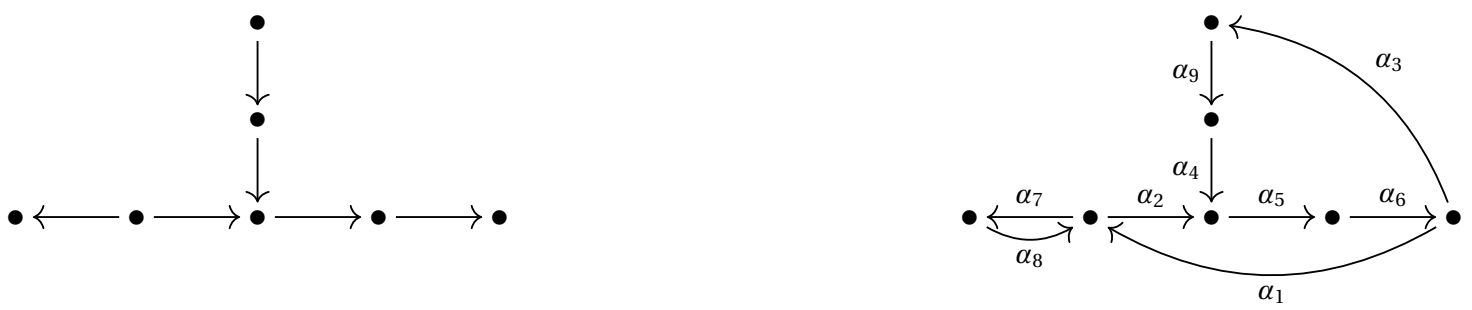

As relações do tipo 2 são: $r 1=\alpha_{8} \alpha_{2}, r 2=\alpha_{1} \alpha_{7}, r 3=\alpha_{4} \alpha_{5} \alpha_{6} \alpha_{1}$ e $r 4=\alpha_{2} \alpha_{5} \alpha_{6} \alpha_{3}$. Os ciclos elementares são: $C_{1}=\alpha_{7} \alpha_{8}, C_{2}=\alpha_{4} \alpha_{5} \alpha_{6} \alpha_{3} \alpha_{9}$ e $C_{3}=\alpha_{2} \alpha_{5} \alpha_{6} \alpha_{1}$. Novamente, obtemos dois cortes que originam duas álgebras hereditárias.

Essa parte é o estudo de todas as extensões triviais do terceiro caso. 
$\mathcal{F} r 6.17$
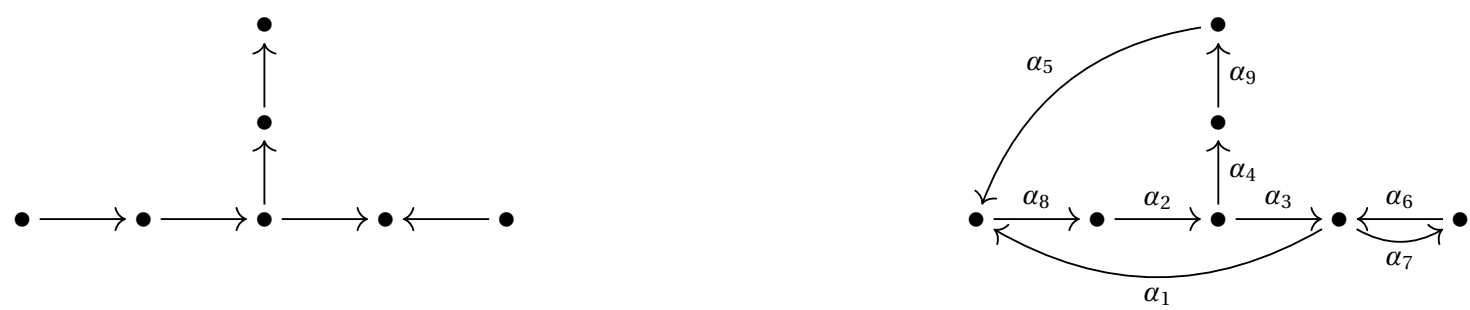

As relações do tipo 2 são: $r 1=\alpha_{6} \alpha_{1}, r 2=\alpha_{3} \alpha_{7}, r 3=\alpha_{1} \alpha_{8} \alpha_{2} \alpha_{4}$ e $r 4=\alpha_{5} \alpha_{8} \alpha_{2} \alpha_{3}$. Os ciclos elementares são: $C_{1}=\alpha_{6} \alpha_{7}, C_{2}=\alpha_{4} \alpha_{9} \alpha_{5} \alpha_{8} \alpha_{2}$ e $C_{3}=\alpha_{8} \alpha_{2} \alpha_{3} \alpha_{1}$. O programa exibe duas soluções hereditárias.

$\mathcal{F}$ r6.18
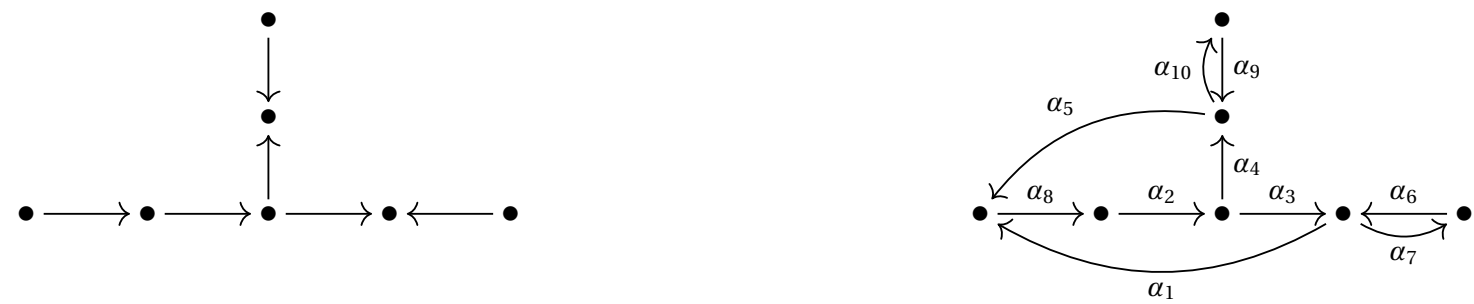

As relações do tipo 2 são: $r 1=\alpha_{6} \alpha_{1}, r 2=\alpha_{3} \alpha_{7}, r 3=\alpha_{1} \alpha_{8} \alpha_{2} \alpha_{4}, r 4=\alpha_{5} \alpha_{8} \alpha_{2} \alpha_{3}, r 5=$ $\alpha_{4} \alpha_{10}$ e $r 6=\alpha_{9} \alpha_{5}$. Os ciclos elementares são: $C_{1}=\alpha_{6} \alpha_{7}, C_{2}=\alpha_{4} \alpha_{5} \alpha_{8} \alpha_{2}, C_{3}=\alpha_{8} \alpha_{2} \alpha_{3} \alpha_{1}$ e $C_{4}=\alpha_{9} \alpha_{10}$. Temos a mesma conclusão do caso anterior.

$\mathcal{F}$ r6.19
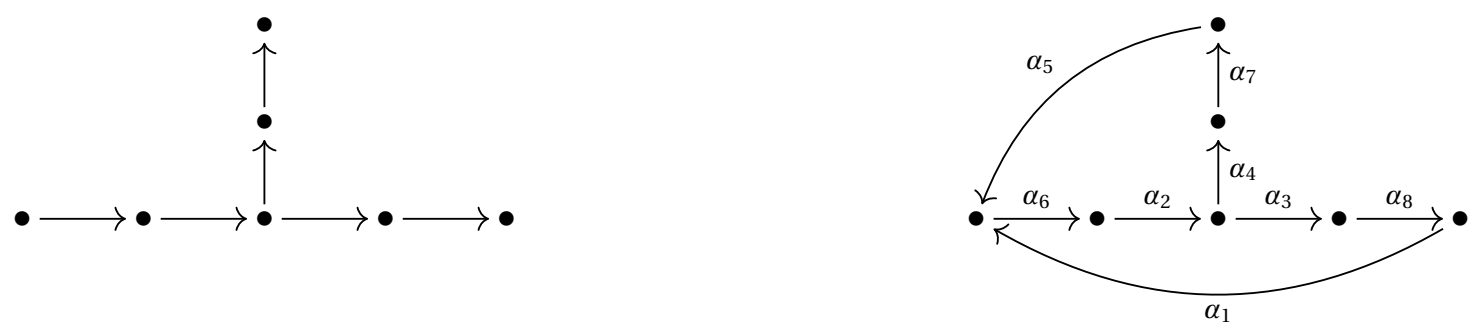

As relações do tipo 2 são: $r 1=\alpha_{1} \alpha_{6} \alpha_{2} \alpha_{4}$ e $r 2=\alpha_{5} \alpha_{6} \alpha_{2} \alpha_{3}$. Os ciclos elementares são: $C_{1}=\alpha_{4} \alpha_{7} \alpha_{5} \alpha_{6} \alpha_{2}$ e $C_{2}=\alpha_{6} \alpha_{2} \alpha_{3} \alpha_{8} \alpha_{1}$. O programa exibe quatro cortes. Dois deles originam álgebras hereditárias. Os outros dois cortes originam as soluções 1 e 2 .

$\mathcal{F} r 6.20$
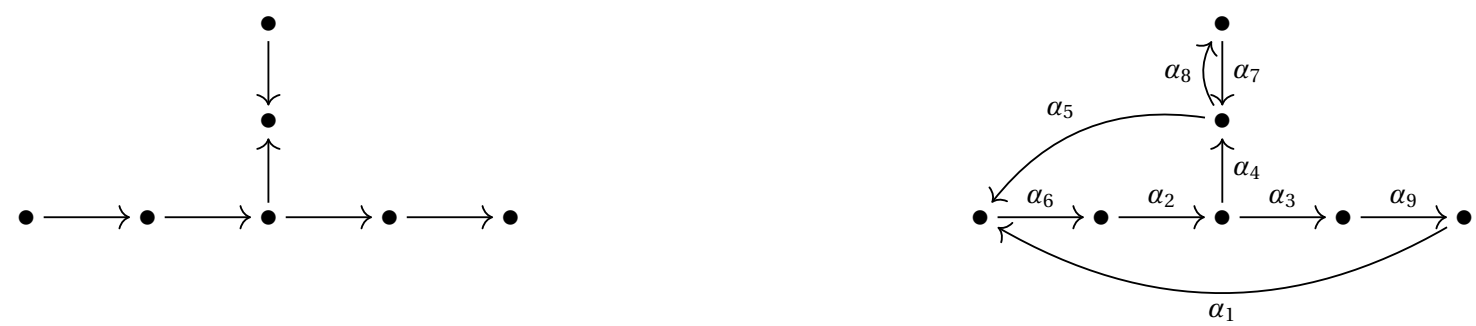

As relações do tipo 2 são: $r 1=\alpha_{7} \alpha_{5}, r 2=\alpha_{4} \alpha_{8}, r 3=\alpha_{1} \alpha_{6} \alpha_{2} \alpha_{4}$ e $r 4=\alpha_{5} \alpha_{6} \alpha_{2} \alpha_{3}$. Os ciclos elementares são: $C_{1}=\alpha_{8} \alpha_{7}, C_{2}=\alpha_{4} \alpha_{5} \alpha_{6} \alpha_{2}$ e $C_{3}=\alpha_{6} \alpha_{2} \alpha_{3} \alpha_{9} \alpha_{1}$. Dessa extensão trivial, o programa exibe apenas cortes que originam álgebras hereditárias.

$\mathcal{F}$ r6.21
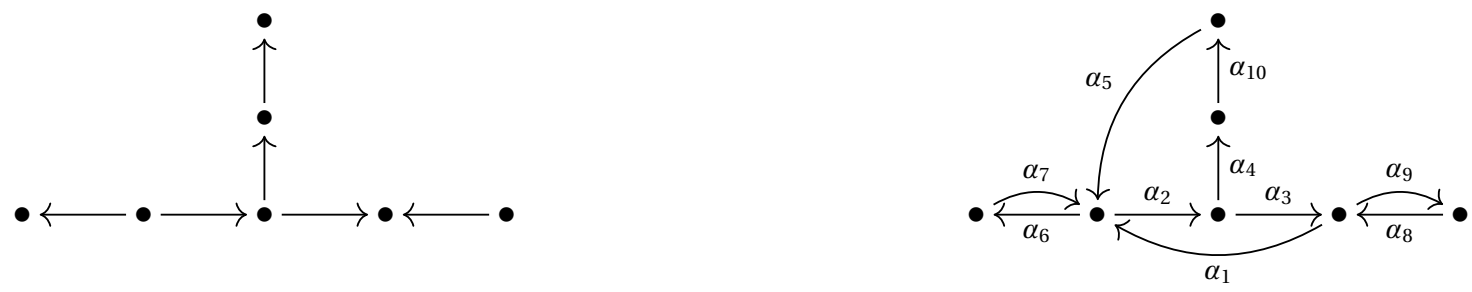
As relações do tipo 2 são: $r 1=\alpha_{8} \alpha_{1}, r 2=\alpha_{3} \alpha_{9}, r 3=\alpha_{1} \alpha_{2} \alpha_{4}, r 4=\alpha_{5} \alpha_{2} \alpha_{3}, r 5=\alpha_{7} \alpha_{2}$, $r 6=\alpha_{5} \alpha_{6}$ e $r 7=\alpha_{1} \alpha_{6}$. Os ciclos elementares são: $C_{1}=\alpha_{8} \alpha_{9}, C_{2}=\alpha_{4} \alpha_{10} \alpha_{5} \alpha_{2}, C_{3}=$ $\alpha_{2} \alpha_{3} \alpha_{1}$ e $C 4=\alpha_{7} \alpha_{6}$. Novamente, obtemos apenas álgebras hereditárias.

$\mathcal{F} r 6.22$
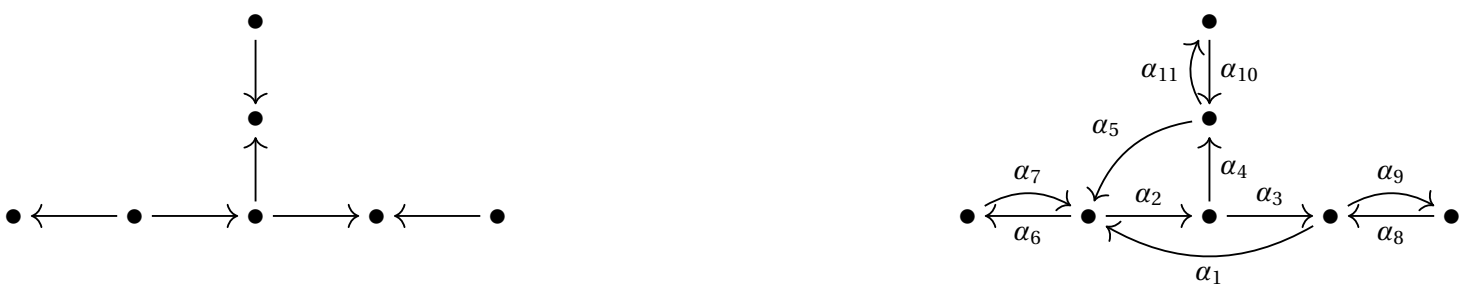

As relações do tipo 2 são: $r 1=\alpha_{8} \alpha_{1}, r 2=\alpha_{3} \alpha_{9}, r 3=\alpha_{1} \alpha_{2} \alpha_{4}, r 4=\alpha_{5} \alpha_{2} \alpha_{3}, r 5=\alpha_{7} \alpha_{2}$, $r 6=\alpha_{5} \alpha_{6}, r 7=\alpha_{1} \alpha_{6}, r 8=\alpha_{10} \alpha_{5}$ e $r 9=\alpha_{5} \alpha_{6}$. Os ciclos elementares são: $C_{1}=\alpha_{8} \alpha_{9}$, $C_{2}=\alpha_{4} \alpha_{5} \alpha_{2}, C_{3}=\alpha_{2} \alpha_{3} \alpha_{1}, C_{4}=\alpha_{7} \alpha_{6}$ e $C_{5}=\alpha_{10} \alpha_{11}$. O programa mostra apenas um corte em que origina uma álgebra hereditária.

$\mathcal{F} r 6.23$
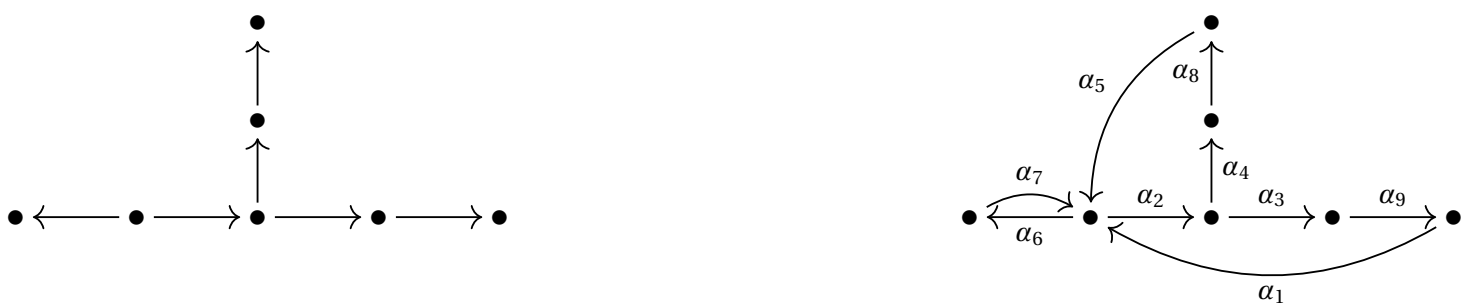

As relações do tipo 2 são: $r 1=\alpha_{1} \alpha_{2} \alpha_{4}, r 2=\alpha_{5} \alpha_{2} \alpha_{3}, r 3=\alpha_{7} \alpha_{2}, r 4=\alpha_{5} \alpha_{6}$ e $r 5=\alpha_{1} \alpha_{6}$. Os ciclos elementares são: $C_{1}=\alpha_{7} \alpha_{6}, C_{2}=\alpha_{4} \alpha_{8} \alpha_{5} \alpha_{2}$ e $C_{3}=\alpha_{2} \alpha_{3} \alpha_{9} \alpha_{1}$. Dessa extensão trivial, o programa nos mostra dois cortes. Um deles implica numa álgebra hereditária. $\mathrm{O}$ outro corte $\Sigma=\left\{\alpha_{2} \alpha_{6}\right\}$ implica que $K Q_{\Gamma} /<I_{\Gamma} \cup \Sigma>$ é a solução 4 .

$\mathcal{F} r 6.24$
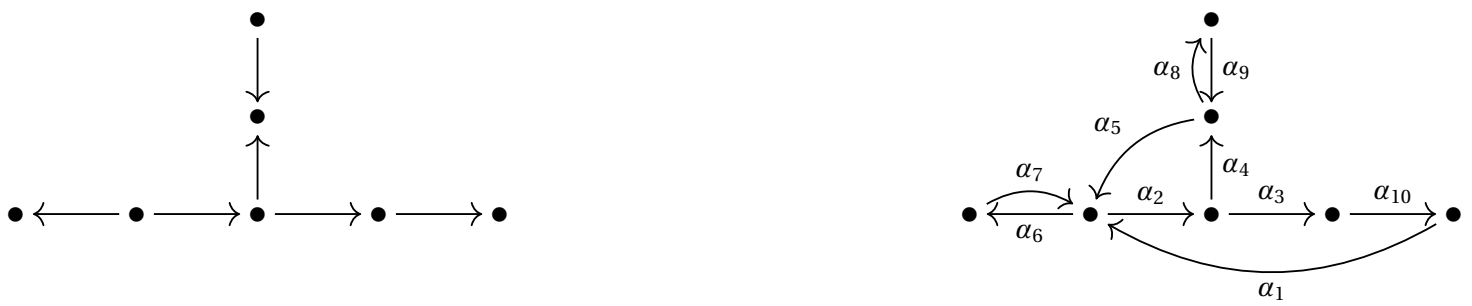

As relações do tipo 2 são: $r 1=\alpha_{1} \alpha_{2} \alpha_{4}, r 2=\alpha_{5} \alpha_{2} \alpha_{3}, r 3=\alpha_{7} \alpha_{2}, r 4=\alpha_{5} \alpha_{6}, r 5=\alpha_{1} \alpha_{6}$, $r 6=\alpha_{9} \alpha_{5}$ e $r 7=\alpha_{4} \alpha_{8}$. Os ciclos elementares são: $C_{1}=\alpha_{7} \alpha_{6}, C_{2}=\alpha_{4} \alpha_{5} \alpha_{2}, C_{3}=$ $\alpha_{2} \alpha_{3} \alpha_{10} \alpha_{1}$ e $C_{4}=\alpha_{9} \alpha_{8}$. O programa exibe apenas um corte em que implica numa álgebra hereditária.

$\mathcal{F} r 7.1$
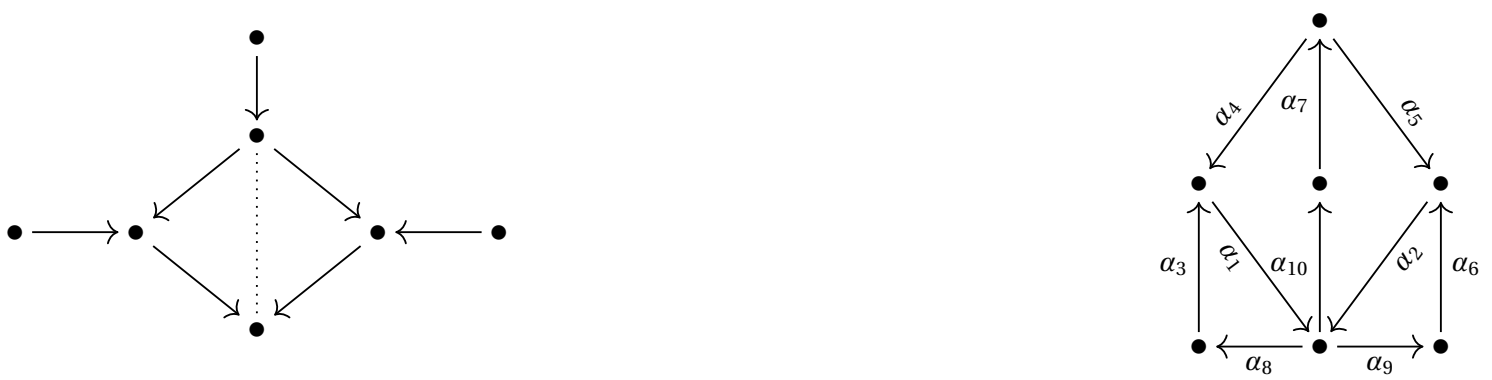

As relações do tipo 2 são: $r 1=\alpha_{3} \alpha_{1} \alpha_{10}, r 2=\alpha_{4} \alpha_{1} \alpha_{8}, r 3=\alpha_{1} \alpha_{10} \alpha_{7} \alpha_{5}, r 4=\alpha_{2} \alpha_{10} \alpha_{7} \alpha_{4}$, 
$r 5=\alpha_{5} \alpha_{2} \alpha_{9}, r 6=\alpha_{6} \alpha_{2} \alpha_{10}, r 7=\alpha_{1} \alpha_{9}$ e $r 8=\alpha_{2} \alpha_{8} \alpha_{7}$. Os ciclos elementares são: $C_{1}=\alpha_{3} \alpha_{1} \alpha_{8}, C_{2}=\alpha_{4} \alpha_{1} \alpha_{10} \alpha_{7}, C_{3}=\alpha_{5} \alpha_{2} \alpha_{10} \alpha_{7}$ e $C_{4}=\alpha_{6} \alpha_{2} \alpha_{9}$. Portanto temos a solução 5 do teorema.
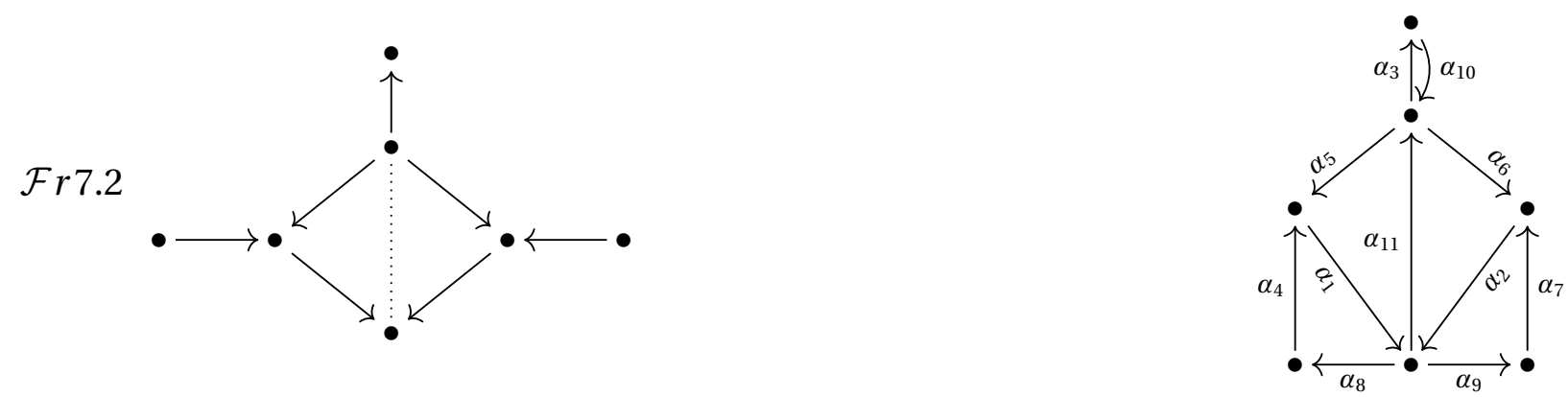

As relações do tipo 2 são: $r 1=\alpha_{4} \alpha_{1} \alpha_{11}, r 2=\alpha_{5} \alpha_{1} \alpha_{8}, r 3=\alpha_{1} \alpha_{11} \alpha_{6}, r 4=\alpha_{2} \alpha_{11} \alpha_{5}, r 5=$ $\alpha_{6} \alpha_{2} \alpha_{9}, r 6=\alpha_{7} \alpha_{2} \alpha_{11}, r 7=\alpha_{1} \alpha_{9}, r 8=\alpha_{2} \alpha_{8}, r 9=\alpha_{10} \alpha_{5}, r 10=\alpha_{10} \alpha_{6}$ e $r 11=\alpha_{11} \alpha_{3}$. Os ciclos elementares são: $C_{1}=\alpha_{1} \alpha_{8} \alpha_{4}, C_{2}=\alpha_{2} \alpha_{9} \alpha_{7}, C_{3}=\alpha_{5} \alpha_{1} \alpha_{11}, C_{4}=\alpha_{6} \alpha_{2} \alpha_{11} \mathrm{e}$ $C_{4}=\alpha_{3} \alpha_{10}$. A única solução é a álgebra disfarçada.

$\mathcal{F} r 7.3$
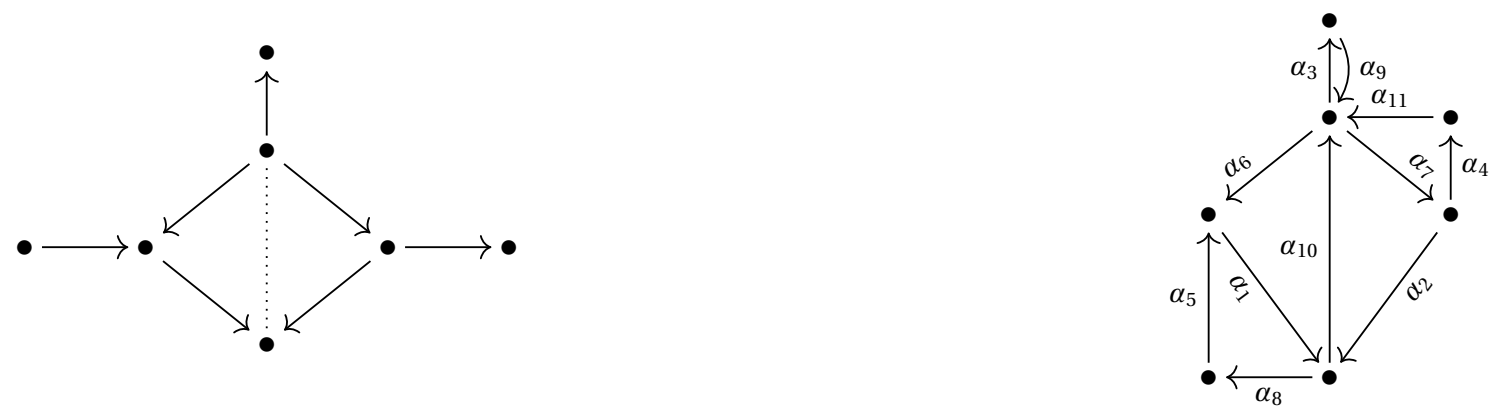

As relações do tipo 2 são: $r 1=\alpha_{5} \alpha_{1} \alpha_{10}, r 2=\alpha_{6} \alpha_{1} \alpha_{8}, r 3=\alpha_{1} \alpha_{10} \alpha_{7}, r 4=\alpha_{2} \alpha_{10} \alpha_{6}$, $r 5=\alpha_{10} \alpha_{7} \alpha_{4}, r 6=\alpha_{11} \alpha_{7} \alpha_{2}, r 7=\alpha_{2} \alpha_{8}, r 8=\alpha_{9} \alpha_{6}, r 9=\alpha_{9} \alpha_{7}, r 10=\alpha_{10} \alpha_{3}, r 11=\alpha_{11} \alpha_{3}$ e $r 12=\alpha_{11} \alpha_{6}$. Os ciclos elementares são: $C_{1}=\alpha_{1} \alpha_{8} \alpha_{5}, C_{2}=\alpha_{1} \alpha_{10} \alpha_{6}, C_{3}=\alpha_{10} \alpha_{7} \alpha_{2}$, $C_{4}=\alpha_{7} \alpha_{4} \alpha_{11}$ e $C_{5}=\alpha_{9} \alpha_{3}$. A única solução é a álgebra disfarçada.

$\mathcal{F} r 7.4$
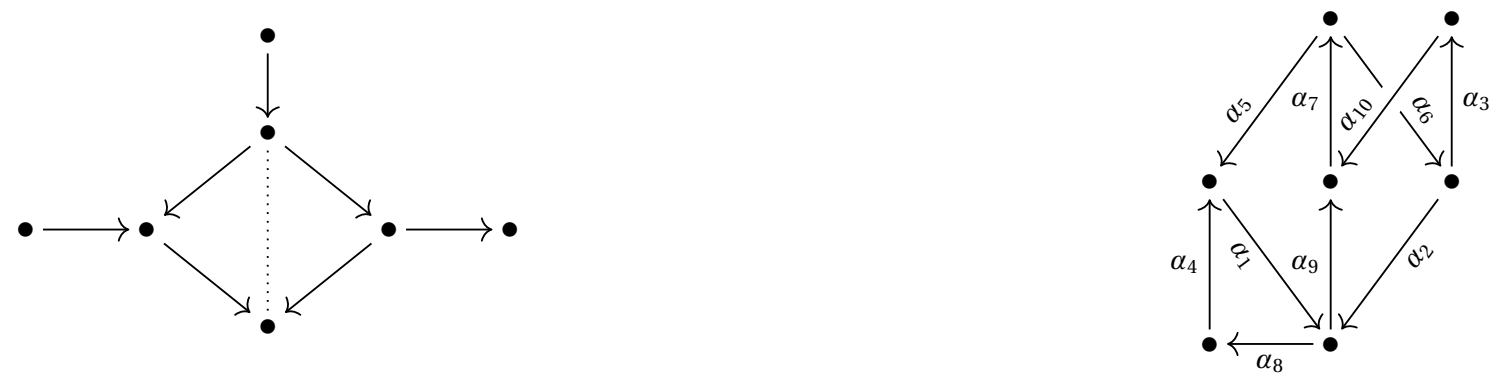

As relações do tipo 2 são: $r 1=\alpha_{4} \alpha_{1} \alpha_{9}, r 2=\alpha_{5} \alpha_{1} \alpha_{8}, r 3=\alpha_{1} \alpha_{9} \alpha_{7} \alpha_{6}, r 4=\alpha_{2} \alpha_{9} \alpha_{7} \alpha_{5}$, $r 5=\alpha_{10} \alpha_{7} \alpha_{6} \alpha_{2}, r 6=\alpha_{9} \alpha_{7} \alpha_{6} \alpha_{3}, r 7=\alpha_{10} \alpha_{7} \alpha_{5}$ e $r 8=\alpha_{2} \alpha_{8}$. Os ciclos elementares são: $C_{1}=\alpha_{1} \alpha_{8} \alpha_{4}, C_{2}=\alpha_{1} \alpha_{9} \alpha_{7} \alpha_{5}, C_{3}=\alpha_{9} \alpha_{7} \alpha_{6} \alpha_{2}$ e $C_{4}=\alpha_{3} \alpha_{10} \alpha_{7} \alpha_{6}$. Novamente, a única solução é a álgebra disfarçada. 

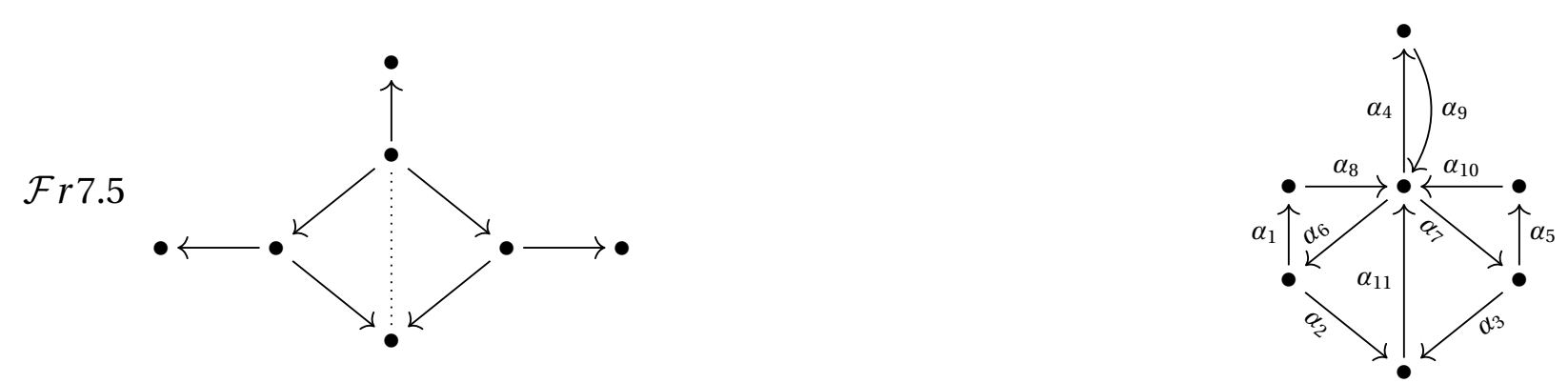

As relações do tipo 2 são: $r 1=\alpha_{8} \alpha_{6} \alpha_{2}, r 2=\alpha_{11} \alpha_{6} \alpha_{1}, r 3=\alpha_{3} \alpha_{11} \alpha_{6}, r 4=\alpha_{2} \alpha_{11} \alpha_{7}$, $r 5=\alpha_{11} \alpha_{7} \alpha_{5}, r 6=\alpha_{10} \alpha_{7} \alpha_{3}, r 7=\alpha_{9} \alpha_{6}, r 8=\alpha_{9} \alpha_{7}, r 9=\alpha_{8} \alpha_{4}, r 10=\alpha_{10} \alpha_{4}, r 11=\alpha_{11} \alpha_{4}$, $r 12=\alpha_{10} \alpha_{6}$ e $r 13=\alpha_{8} \alpha_{7}$. Os ciclos elementares são: $C_{1}=\alpha_{4} \alpha_{9}, C_{2}=\alpha_{1} \alpha_{8} \alpha_{6}, C_{3}=$ $\alpha_{10} \alpha_{7} \alpha_{5}, C_{4}=\alpha_{6} \alpha_{2} \alpha_{11}$ e $C_{5}=\alpha_{11} \alpha_{7} \alpha_{3}$. Portanto, temos a solução 6 .

$\mathcal{F} r 7.6$
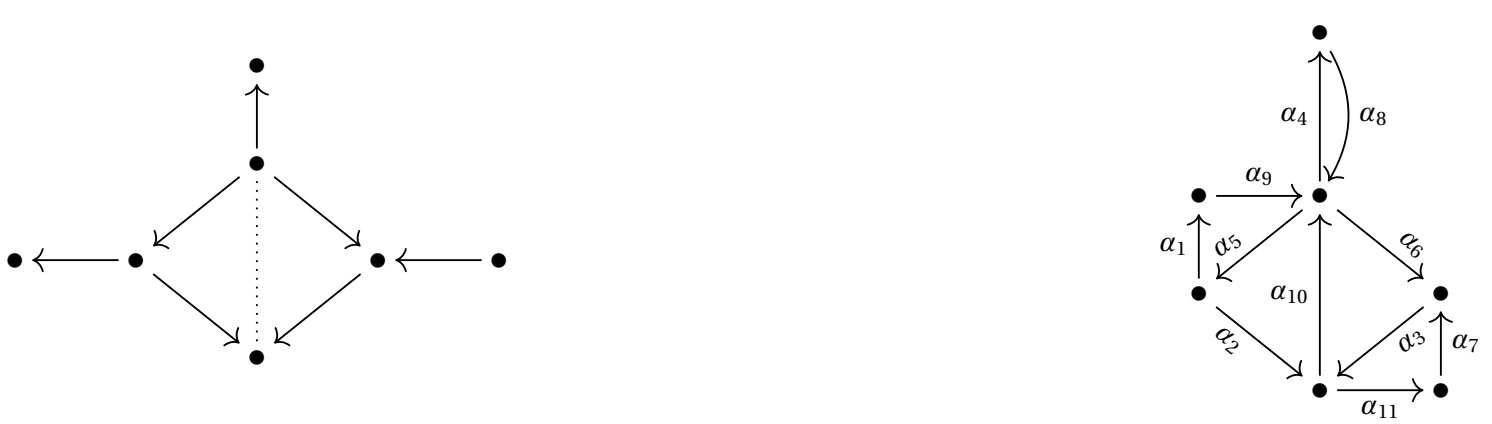

As relações do tipo 2 são: $r 1=\alpha_{9} \alpha_{5} \alpha_{2}, r 2=\alpha_{10} \alpha_{5} \alpha_{1}, r 3=\alpha_{2} \alpha_{10} \alpha_{6}, r 4=\alpha_{3} \alpha_{10} \alpha_{5}$, $r 5=\alpha_{6} \alpha_{3} \alpha_{11}, r 6=\alpha_{7} \alpha_{3} \alpha_{10}, r 7=\alpha_{9} \alpha_{4}, r 8=\alpha_{10} \alpha_{4}, r 9=\alpha_{8} \alpha_{5}, r 10=\alpha_{8} \alpha_{6}, r 11=\alpha_{2} \alpha_{11}$ e $r 12=\alpha_{9} \alpha_{6}$. Os ciclos elementares são: $C_{1}=\alpha_{5} \alpha_{1} \alpha_{9}, C_{2}=\alpha_{4} \alpha_{8}, C_{3}=\alpha_{5} \alpha_{2} \alpha_{10}$, $C_{4}=\alpha_{10} \alpha_{6} \alpha_{3}$ e $C_{5}=\alpha_{3} \alpha_{11} \alpha_{7}$. Somente temos a solução que origina a álgebra disfarçada.

$\mathcal{F} r 7.7$
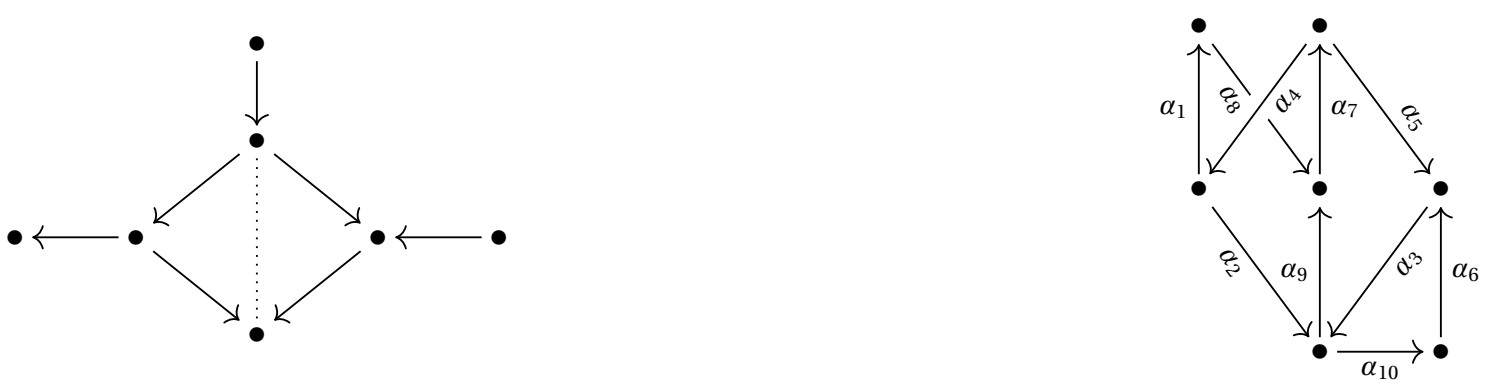

As relações do tipo 2 são: $r 1=\alpha_{6} \alpha_{3} \alpha_{9}, r 2=\alpha_{5} \alpha_{3} \alpha_{10}, r 3=\alpha_{3} \alpha_{9} \alpha_{7} \alpha_{4}, r 4=\alpha_{2} \alpha_{9} \alpha_{7} \alpha_{5}$, $r 5=\alpha_{8} \alpha_{7} \alpha_{4} \alpha_{2}, r 6=\alpha_{9} \alpha_{7} \alpha_{4} \alpha_{1}, r 7=\alpha_{8} \alpha_{7} \alpha_{5}$ e $r 8=\alpha_{2} \alpha_{10}$. Os ciclos elementares são: $C_{1}=\alpha_{5} \alpha_{3} \alpha_{9} \alpha_{7}, C_{2}=\alpha_{3} \alpha_{10} \alpha_{6}, C_{3}=\alpha_{7} \alpha_{4} \alpha_{1} \alpha_{8}$ e $C_{4}=\alpha_{7} \alpha_{4} \alpha_{2} \alpha_{9}$. Apenas exibe a solução da álgebra disfarçada.
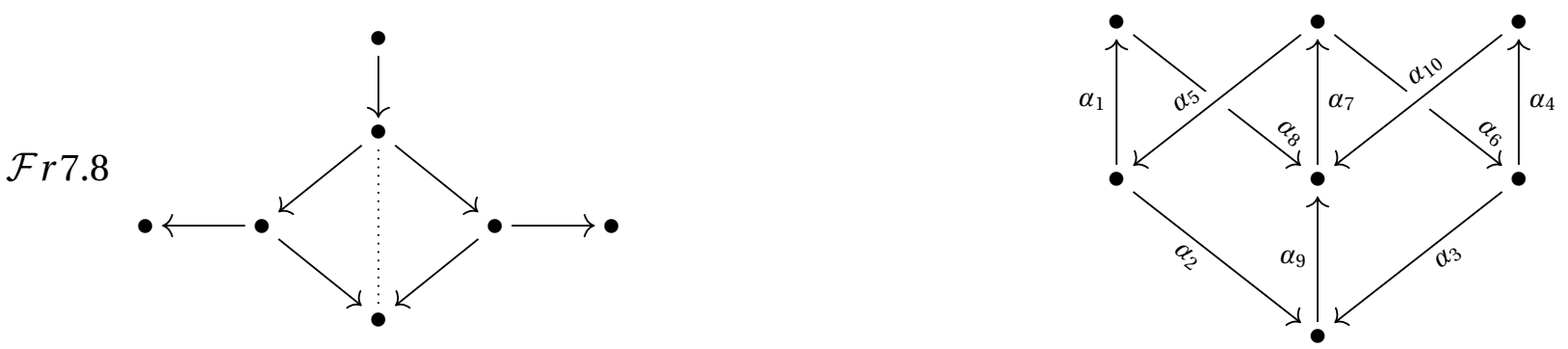

As relações do tipo 2 são: $r 1=\alpha_{3} \alpha_{9} \alpha_{7} \alpha_{5}, r 2=\alpha_{2} \alpha_{9} \alpha_{7} \alpha_{6}, r 3=\alpha_{8} \alpha_{7} \alpha_{5} \alpha_{2}, r 4=\alpha_{9} \alpha_{7} \alpha_{5} \alpha_{1}$, 
$r 5=\alpha_{8} \alpha_{7} \alpha_{6}, r 6=\alpha_{9} \alpha_{7} \alpha_{6} \alpha_{4}, r 7=\alpha_{10} \alpha_{7} \alpha_{6} \alpha_{3}$ e $r 8=\alpha_{10} \alpha_{7} \alpha_{5}$. Os ciclos elementares são: $C_{1}=\alpha_{7} \alpha_{5} \alpha_{2} \alpha_{9}, C_{2}=\alpha_{7} \alpha_{6} \alpha_{3} \alpha_{9}, C_{3}=\alpha_{7} \alpha_{6} \alpha_{4} \alpha_{10}$ e $C_{4}=\alpha_{7} \alpha_{5} \alpha_{1} \alpha_{8}$. O programa exibe três soluções: as álgebras 7,8 e a álgebra disfarçada.

$\mathcal{F} r 8.1$
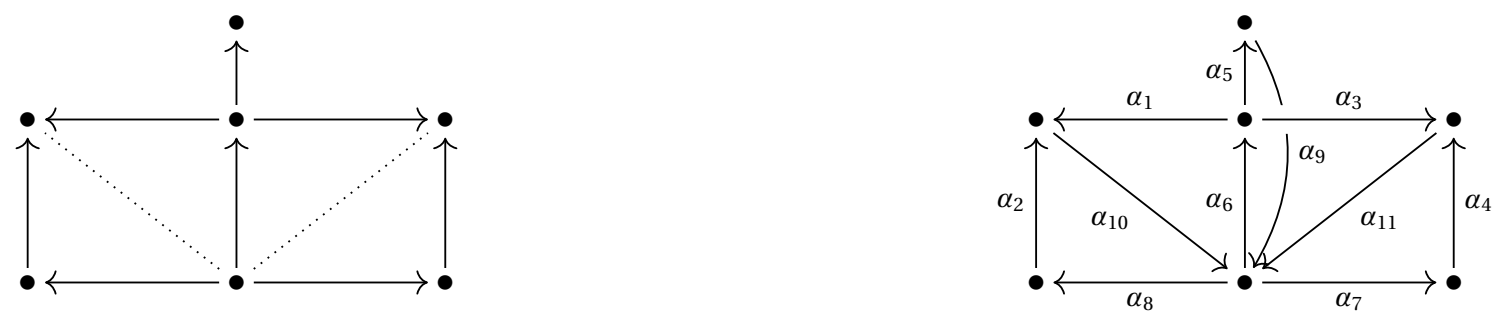

As relações do tipo 2 são: $r 1=\alpha_{9} \alpha_{7}, r 2=\alpha_{9} \alpha_{8}, r 3=\alpha_{11} \alpha_{8}, r 4=\alpha_{10} \alpha_{7}, r 5=\alpha_{2} \alpha_{10} \alpha_{6}$, $r 6=\alpha_{1} \alpha_{10} \alpha_{8}, r 7=\alpha_{4} \alpha_{11} \alpha_{6}, r 8=\alpha_{3} \alpha_{11} \alpha_{7}, r 9=\alpha_{11} \alpha_{6} \alpha_{5}, r 10=\alpha_{9} \alpha_{6} \alpha_{3}, r 11=\alpha_{10} \alpha_{6} \alpha_{5}$, $r 12=\alpha_{9} \alpha_{6} \alpha_{1}, r 13=\alpha_{11} \alpha_{6} \alpha_{1}$ e $r 14=\alpha_{10} \alpha_{6} \alpha_{3}$. Os ciclos elementares são: $C_{1}=\alpha_{6} \alpha_{5} \alpha_{9}$, $C_{2}=\alpha_{6} \alpha_{1} \alpha_{10}, C_{3}=\alpha_{8} \alpha_{2} \alpha_{10}, C_{4}=\alpha_{6} \alpha_{3} \alpha_{11}$ e $C_{5}=\alpha_{7} \alpha_{4} \alpha_{11}$. Assim, obtemos a solução 9 do teorema.

$\mathcal{F} r 8.2$
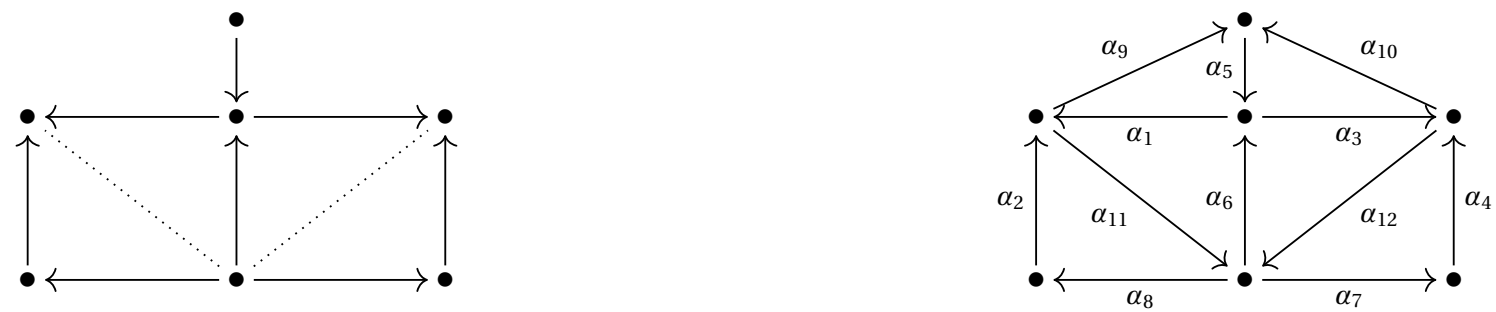

As relações do tipo 2 são: $r 1=\alpha_{2} \alpha_{9}, r 2=\alpha_{4} \alpha_{10}, r 3=\alpha_{12} \alpha_{8}, r 4=\alpha_{11} \alpha_{7}, r 5=\alpha_{2} \alpha_{11} \alpha_{6}$, $r 6=\alpha_{1} \alpha_{11} \alpha_{8}, r 7=\alpha_{4} \alpha_{12} \alpha_{6}, r 8=\alpha_{3} \alpha_{12} \alpha_{7}, r 9=\alpha_{5} \alpha_{1} \alpha_{11}, r 10=\alpha_{6} \alpha_{1} \alpha_{9}, r 11=\alpha_{5} \alpha_{3} \alpha_{12}$, $r 12=\alpha_{6} \alpha_{3} \alpha_{10}, r 13=\alpha_{10} \alpha_{5} \alpha_{1}, r 14=\alpha_{9} \alpha_{5} \alpha_{3}, r 15=\alpha_{12} \alpha_{6} \alpha_{1}$ e $r 16=\alpha_{11} \alpha_{6} \alpha_{3}$. Os ciclos elementares são: $C_{1}=\alpha_{5} \alpha_{1} \alpha_{9}, C_{2}=\alpha_{5} \alpha_{3} \alpha_{10}, C_{3}=\alpha_{6} \alpha_{1} \alpha_{11}, C_{4}=\alpha_{8} \alpha_{2} \alpha_{11}, C_{5}=\alpha_{7} \alpha_{4} \alpha_{12}$ e $C_{6}=\alpha_{6} \alpha_{3} \alpha_{12}$. Não temos solução diferente da álgebra disfarçada.

$\mathcal{F} r 9.1$
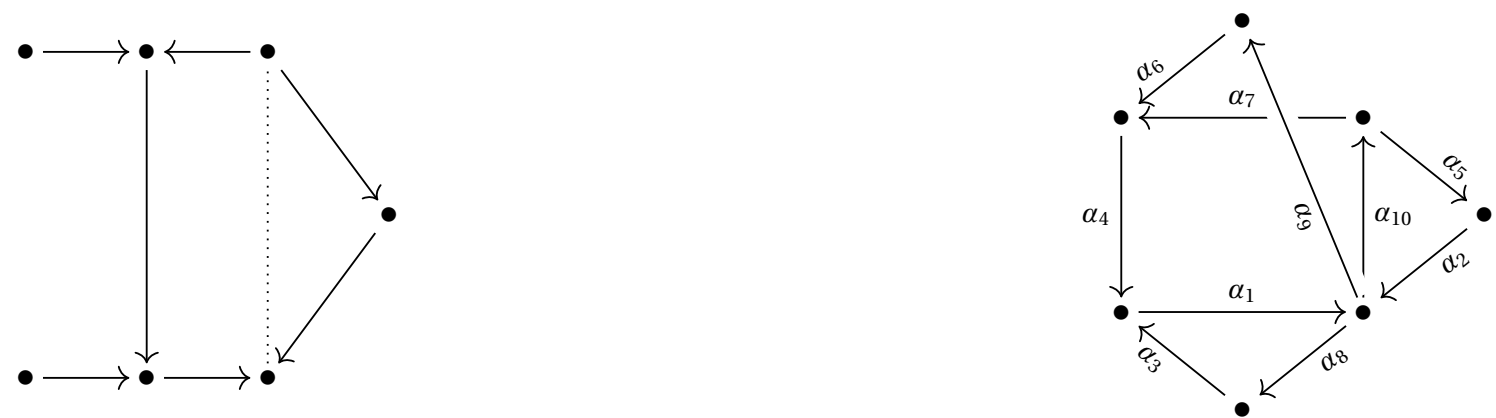

As relações do tipo 2 são: $r 1=\alpha_{3} \alpha_{1} \alpha_{10}, r 2=\alpha_{4} \alpha_{1} \alpha_{8}, r 3=\alpha_{1} \alpha_{10} \alpha_{5}, r 4=\alpha_{2} \alpha_{10} \alpha_{7}$, $r 5=\alpha_{6} \alpha_{4} \alpha_{1} \alpha_{10}, r 6=\alpha_{7} \alpha_{4} \alpha_{1} \alpha_{9}, r 7=\alpha_{3} \alpha_{1} \alpha_{9}, r 8=\alpha_{2} \alpha_{9}$ e $r 9=\alpha_{2} \alpha_{8}$. Os ciclos elementares são: $C_{1}=\alpha_{1} \alpha_{8} \alpha_{3}, C_{2}=\alpha_{1} \alpha_{9} \alpha_{6} \alpha_{4}, C_{3}=\alpha_{1} \alpha_{10} \alpha_{7} \alpha_{4}$ e $C_{4}=\alpha_{10} \alpha_{5} \alpha_{2}$. Logo, o programa mostra a solução 10 . 
$\mathcal{F} r 9.2$
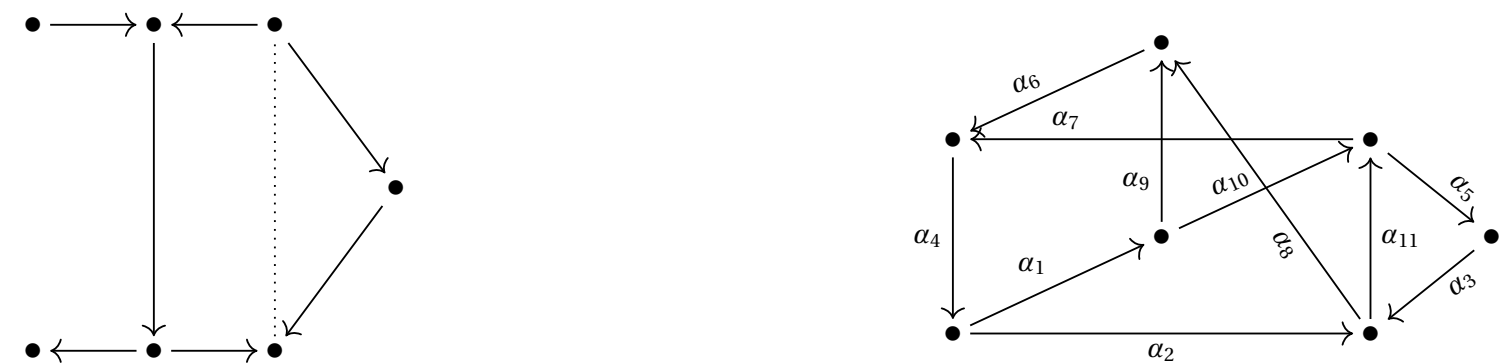

As relações do tipo 2 são: $r 1=\alpha_{3} \alpha_{11} \alpha_{7}, r 2=\alpha_{2} \alpha_{11} \alpha_{5}, r 3=\alpha_{11} \alpha_{7} \alpha_{4} \alpha_{1}, r 4=\alpha_{10} \alpha_{7} \alpha_{4} \alpha_{2}$, $r 5=\alpha_{7} \alpha_{4} \alpha_{2} \alpha_{8}, r 6=\alpha_{6} \alpha_{4} \alpha_{2} \alpha_{11}, r 7=\alpha_{7} \alpha_{4} \alpha_{1} \alpha_{9}, r 8=\alpha_{6} \alpha_{4} \alpha_{1} \alpha_{10}, r 9=\alpha_{9} \alpha_{6} \alpha_{4} \alpha_{2}$, $r 10=\alpha_{8} \alpha_{6} \alpha_{4} \alpha_{1}, r 11=\alpha_{3} \alpha_{8}$ e $r 12=\alpha_{10} \alpha_{5}$. Os ciclos elementares são: $C_{1}=\alpha_{3} \alpha_{11} \alpha_{5}$, $C_{2}=\alpha_{11} \alpha_{7} \alpha_{4} \alpha_{2}, C_{3}=\alpha_{7} \alpha_{4} \alpha_{1} \alpha_{10}, C_{4}=\alpha_{6} \alpha_{4} \alpha_{1} \alpha_{9}$ e $C_{5}=\alpha_{6} \alpha_{4} \alpha_{2} \alpha_{8}$. Não temos solução diferente da álgebra disfarçada.

$\mathcal{F} r 9.3$
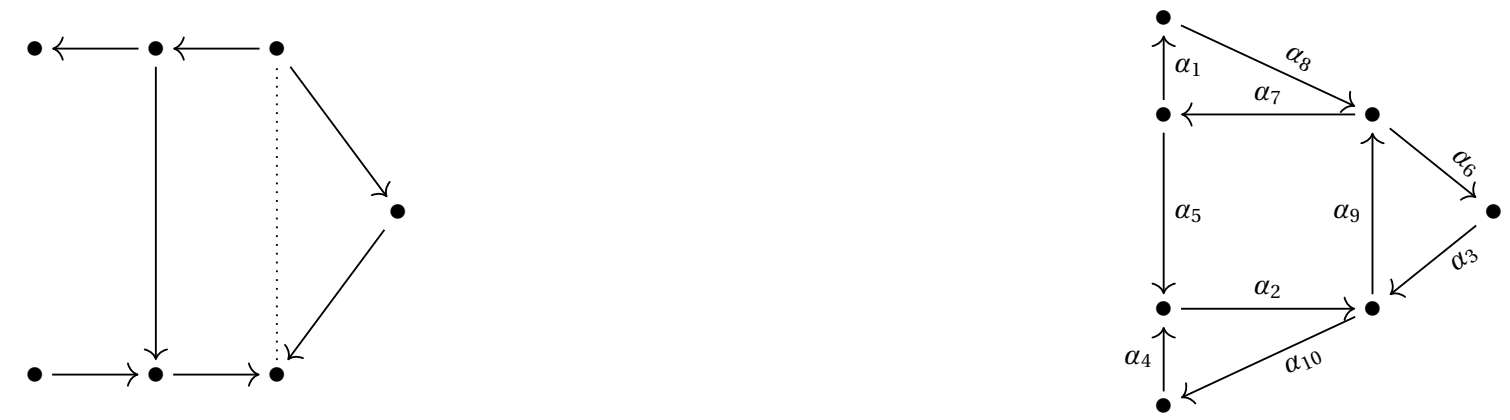

As relações do tipo 2 são: $r 1=\alpha_{8} \alpha_{7} \alpha_{5}, r 2=\alpha_{9} \alpha_{7} \alpha_{1}, r 3=\alpha_{2} \alpha_{9} \alpha_{6}, r 4=\alpha_{3} \alpha_{9} \alpha_{7}, r 5=$ $\alpha_{4} \alpha_{2} \alpha_{9}, r 6=\alpha_{5} \alpha_{2} \alpha_{10}, r 7=\alpha_{3} \alpha_{10}$ e $r 8=\alpha_{8} \alpha_{6}$. Os ciclos elementares são: $C_{1}=\alpha_{7} \alpha_{1} \alpha_{8}$, $C_{2}=\alpha_{7} \alpha_{5} \alpha_{2} \alpha_{9}, C_{3}=\alpha_{9} \alpha_{6} \alpha_{3}$ e $C_{4}=\alpha_{2} \alpha_{10} \alpha_{4}$. O programa apenas mostra o corte que origina a álgebra disfarçada.

$\mathcal{F} r 9.4$
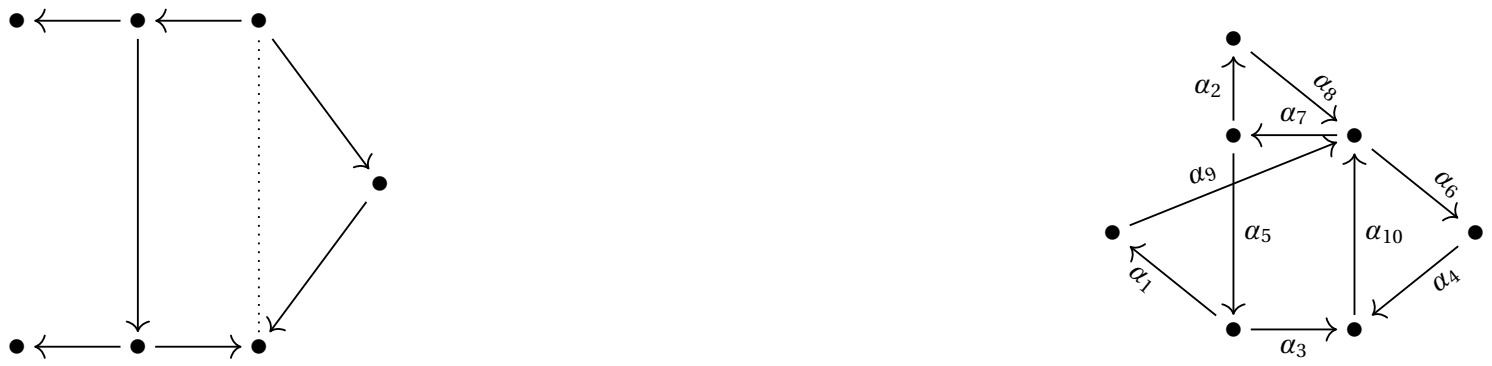

As relações do tipo 2 são: $r 1=\alpha_{4} \alpha_{10} \alpha_{7}, r 2=\alpha_{3} \alpha_{10} \alpha_{6}, r 3=\alpha_{10} \alpha_{7} \alpha_{2}, r 4=\alpha_{8} \alpha_{7} \alpha_{5}$, $r 5=\alpha_{10} \alpha_{7} \alpha_{5} \alpha_{1}, r 6=\alpha_{9} \alpha_{7} \alpha_{5} \alpha_{3}, r 7=\alpha_{9} \alpha_{7} \alpha_{2}, r 8=\alpha_{8} \alpha_{6}$ e $r 9=\alpha_{9} \alpha_{6}$. Os ciclos elementares são: $C_{1}=\alpha_{7} \alpha_{2} \alpha_{8}, C_{2}=\alpha_{7} \alpha_{5} \alpha_{3} \alpha_{10}, C_{3}=\alpha_{10} \alpha_{6} \alpha_{4}$ e $C_{4}=\alpha_{7} \alpha_{5} \alpha_{1} \alpha_{9}$. Portanto, temos a álgebra 11. 


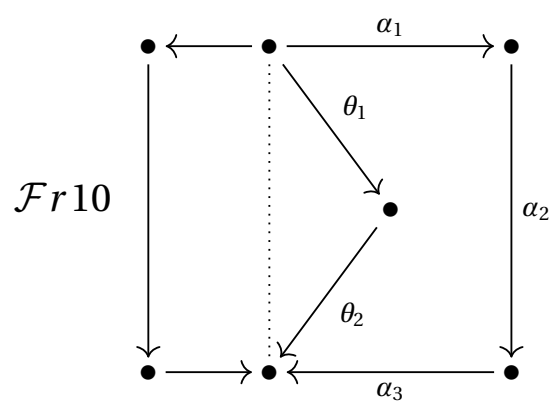

Como $\theta_{1} \theta_{2}$ é diferente do caminho $\alpha_{1} \alpha_{2} \alpha_{3}$, esse frame não é uma álgebra schurian. Então não podemos usar a teoria da extensão trivial da seção $\mathbb{E}_{7}$.

Antes de enunciar o teorema da família de Phias ANS de tipo $\widetilde{\mathbb{E}}_{7}$, vamos mostrar um lema que usaremos nas demonstrações dos próximos dois teoremas.

Lema 5.42. Seja $\Gamma$ uma extensão trivial de uma álgebra schurian com no mínimo três ciclos elementares com as seguintes condições:

a) dois ciclos elementares têm no mínimo uma flecha em comum, denominaremos essa intersecção por um caminho $\lambda$;

b) o terceiro ciclo elementar tem um vértice em comum com um dos dois ciclos elementares anteriores e não tem intersecção com o outro;

c) o vértice em comum da condição anterior, não pertence ao caminho $\lambda$;

Para melhor entendimento dessas condições, exibimos o seguinte exemplo:

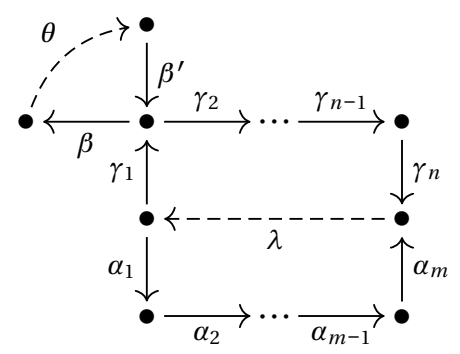

Seja $\Sigma$ um corte de $\Gamma$. Se alguma flecha de $\lambda$ pertencer a $\Sigma$ então $K Q_{\Gamma} /<I_{\Gamma} \cup \Sigma>$ não é uma álgebra de incidência.

Demonstração Sem perda de generalidade, consideraremos $\Gamma$ como uma extensão trivial com três ciclos elementares satisfazendo as condições do enunciado do lema:

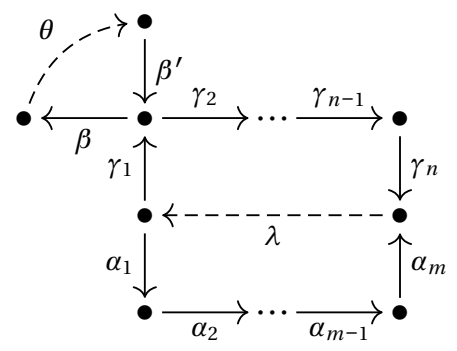

Denominaremos $\theta$ como um caminho opcional. 
Assim, as relações do tipo 2 são: $r 1=\gamma_{1} \beta, r 2=\beta^{\prime} \gamma_{2}, r 3=\alpha_{m} \lambda \gamma_{1}$ e $r 4=\gamma_{n} \lambda \alpha_{1}$. Os ciclos elementares são: $C_{1}=\alpha_{1} \alpha_{2} \ldots \alpha_{m-1} \alpha_{m} \lambda, C_{2}=\lambda \gamma_{1} \gamma_{2} \ldots \gamma_{n-1} \gamma_{n}$ e $C_{3}=\theta \beta^{\prime} \beta$.

Supondo que alguma flecha do caminho $\lambda$ pertence ao corte $\Sigma$. Essa hipótese implica que já tem um representante dos ciclos $C_{1}$ e $C_{2}$ no conjunto $\Sigma$. Veremos que não importando a escolha da flecha do ciclo elementar $C_{3}$, a álgebra resultante não é de incidência.

Supomos que $\beta^{\prime} \in \Sigma$, então a relação $r 1$ pertence a $<I_{\Gamma} \cup \Sigma>$. Consequentemente, $K Q_{\Gamma} /<$ $I_{\Gamma} \cup \Sigma>$ não é uma álgebra de incidência.

De forma análoga, chegamos ao mesmo resultado se $\beta \in \Sigma$ ou qualquer flecha de $\theta$ pertença ao corte.

Esse lema vai ser importante no estudo das extensões triviais das álgebras hereditárias de tipo $\widetilde{\mathbb{E}}_{p}$, como veremos a seguir.

Teorema 5.43. As álgebras associadas com as aljavas com relações abairo:
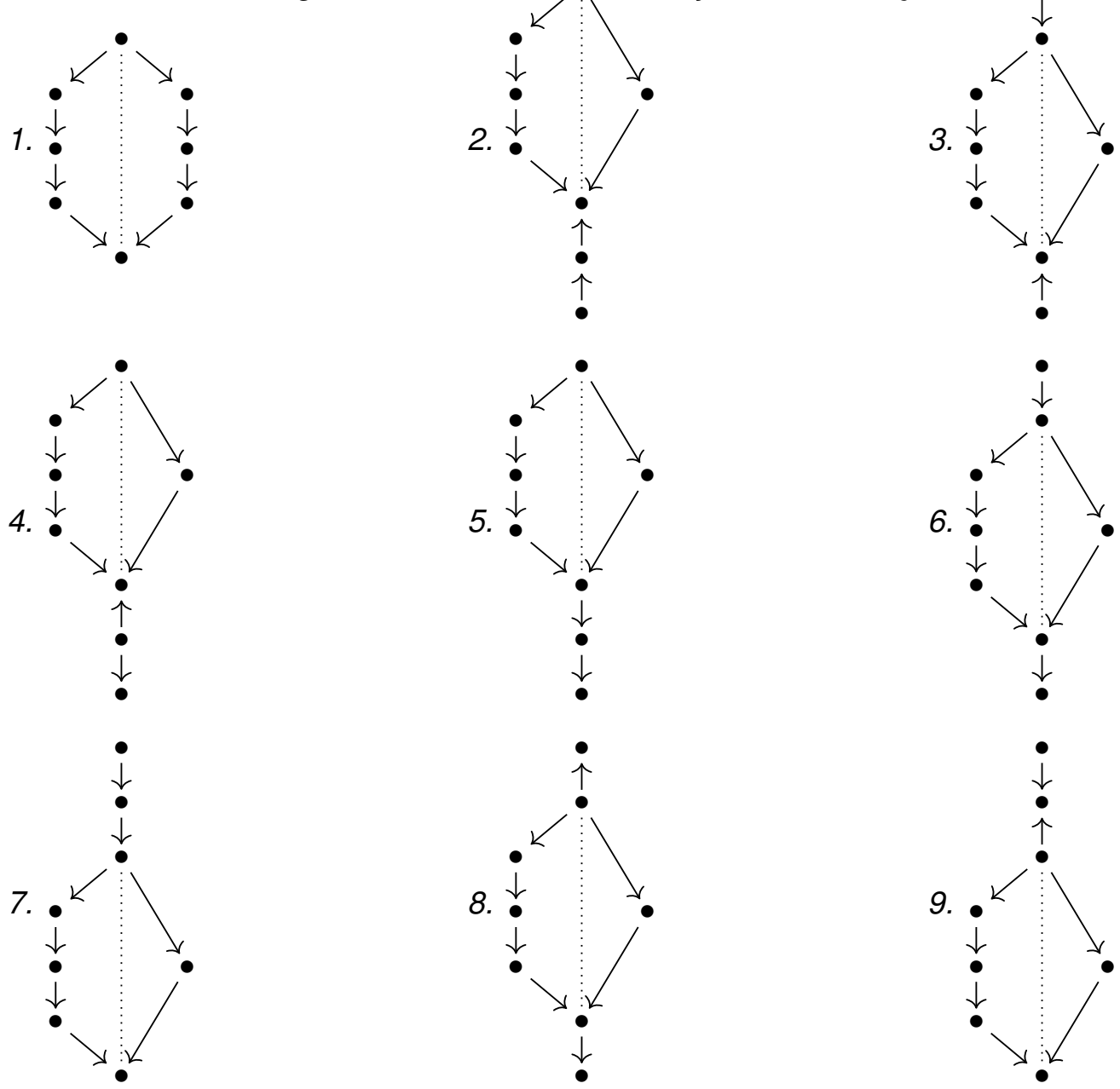

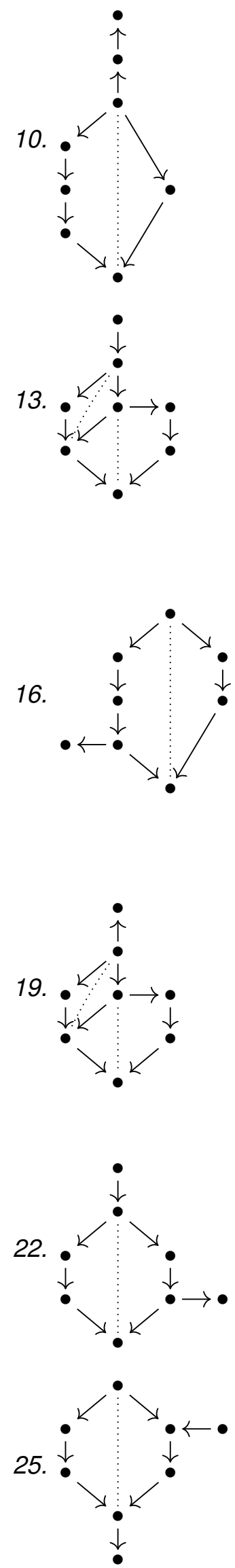
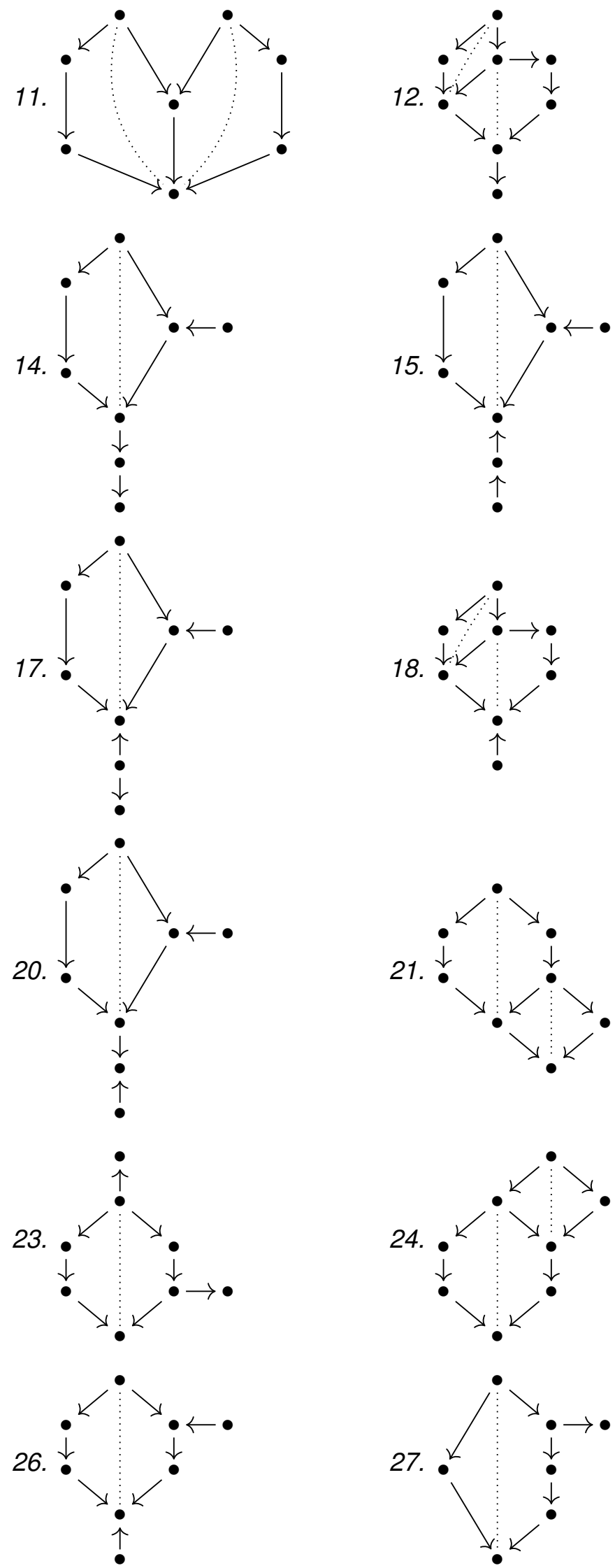
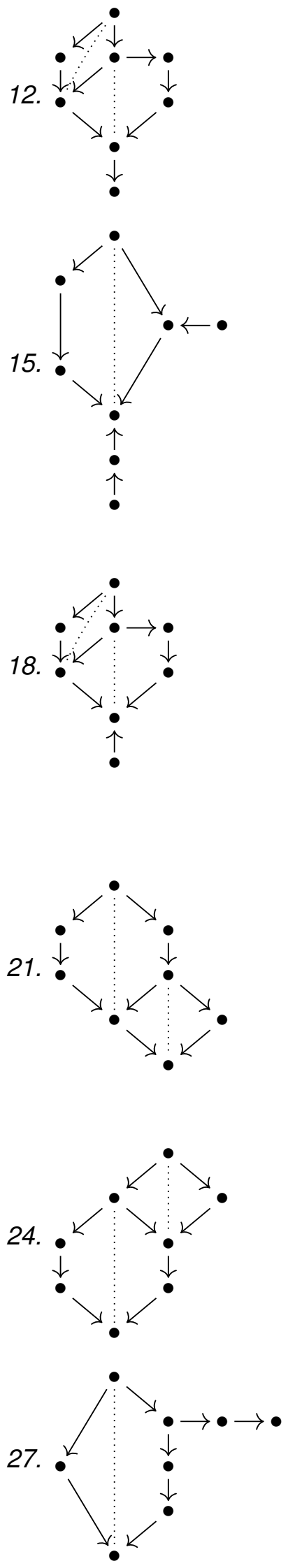
28.

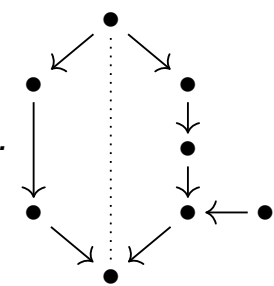

31.

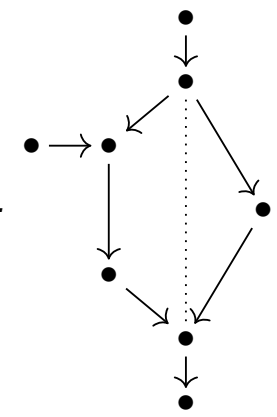

34.

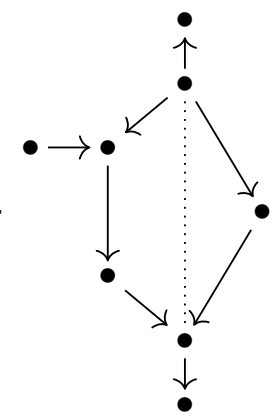

37.

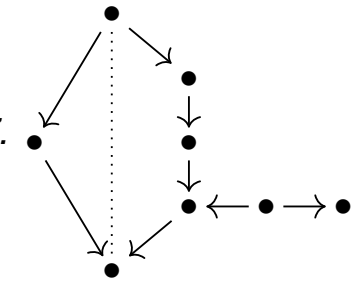

40.

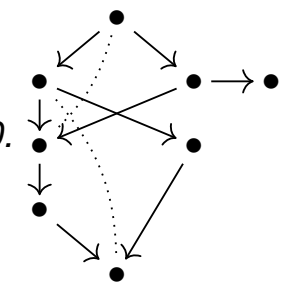

43.

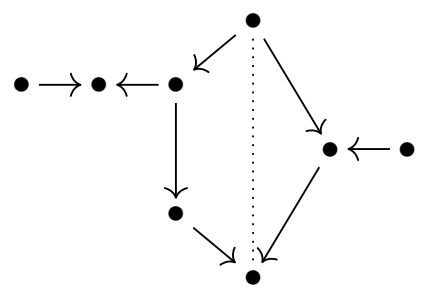

29.

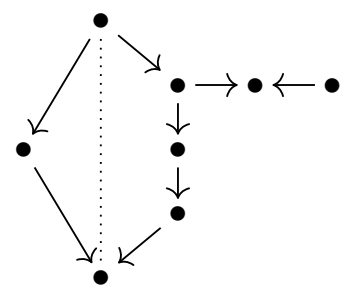

32.

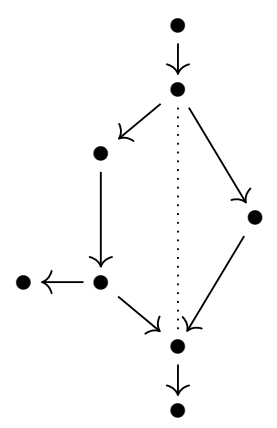

38.

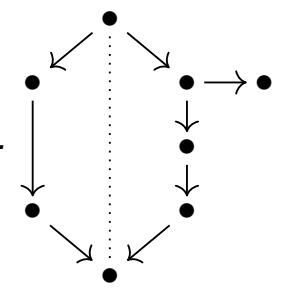

41.

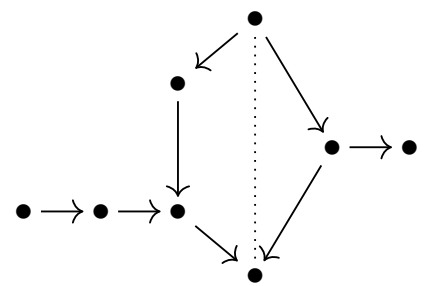

44.
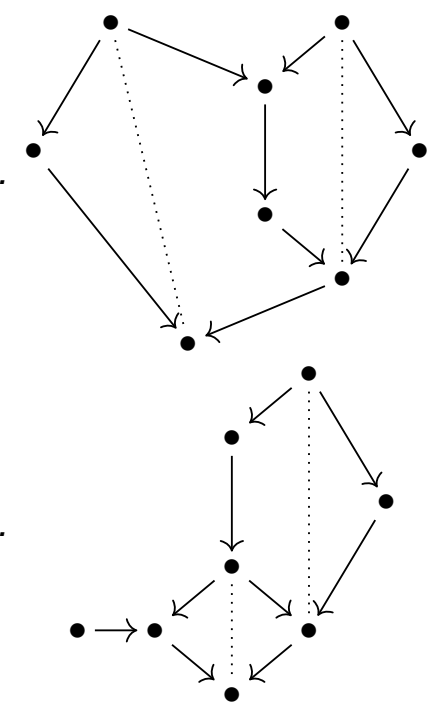

30.

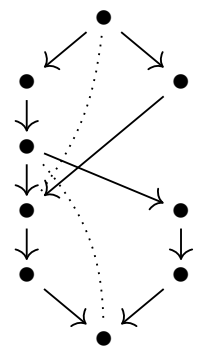

33.

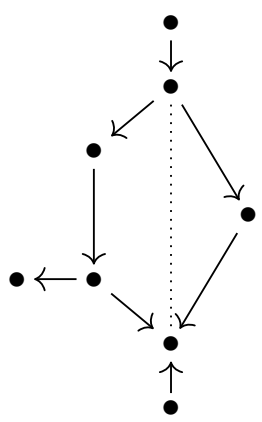

39.

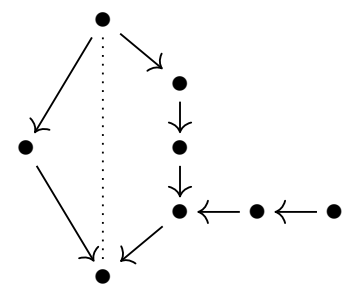

42.

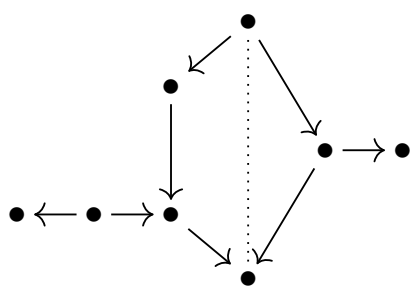

45.

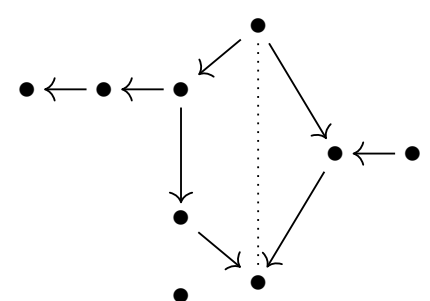

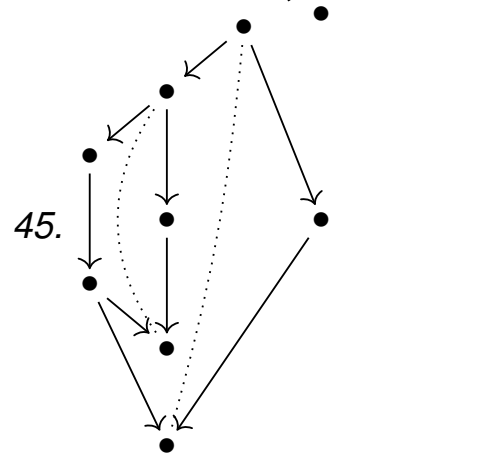


46.

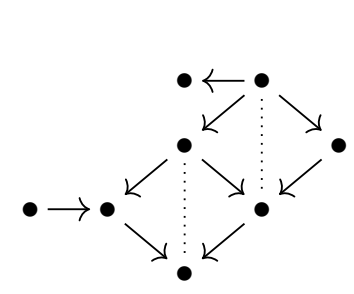

49.

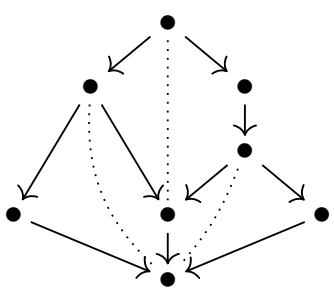

52.

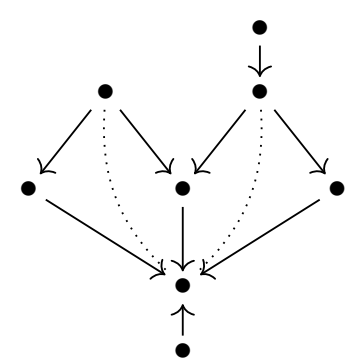

55.

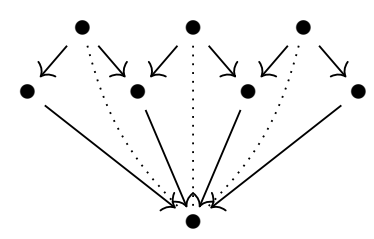

61.
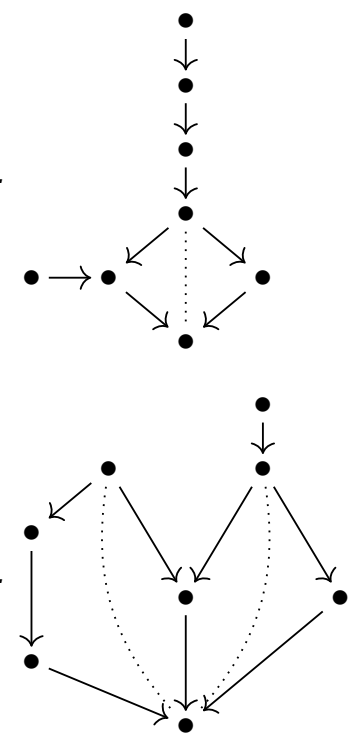

47.

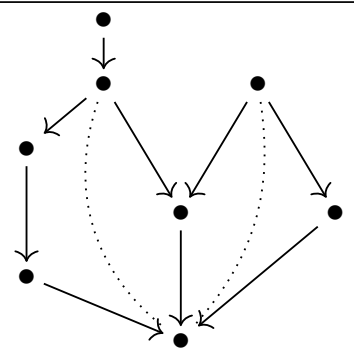

50.

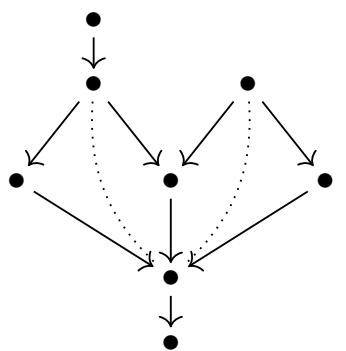

53.

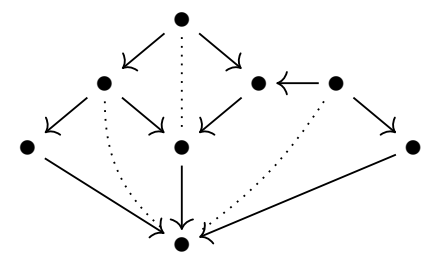

56.

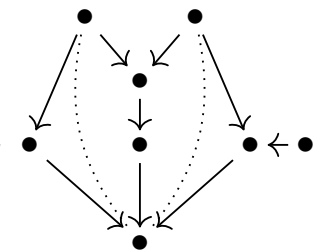

59.

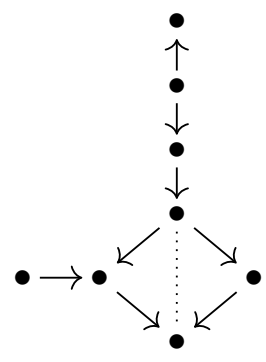

62.

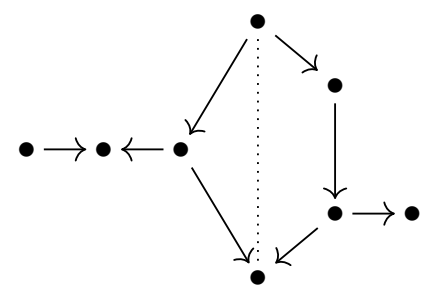

48.

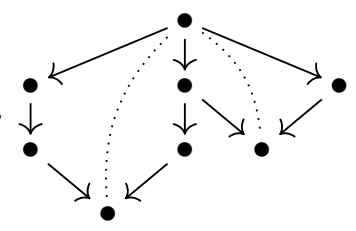

51.

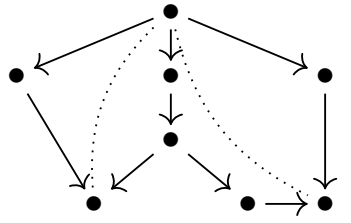

54.

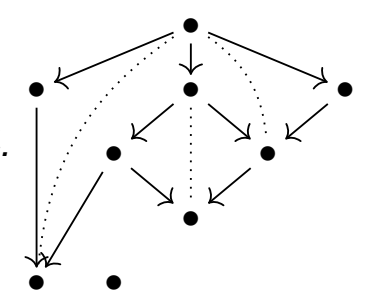

57.

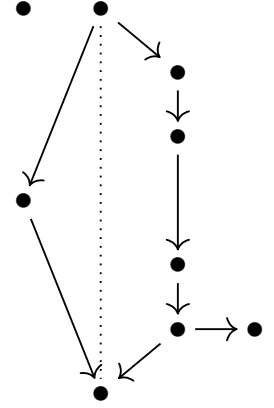

60.

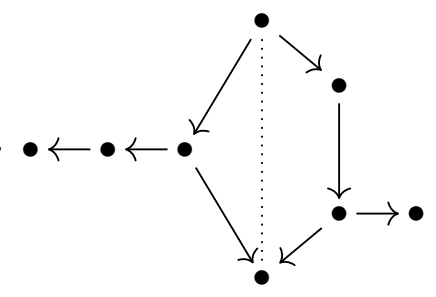

63.

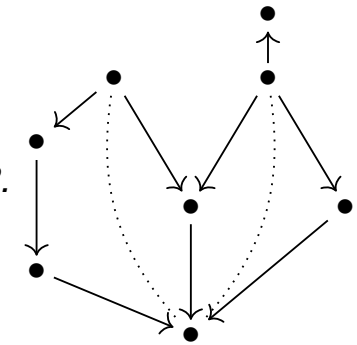


64.

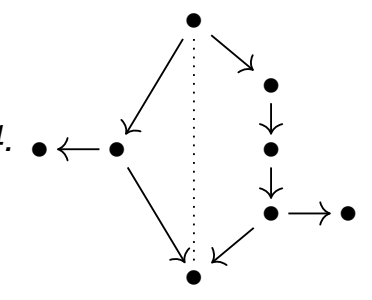

67.

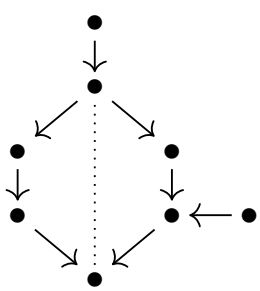

70.

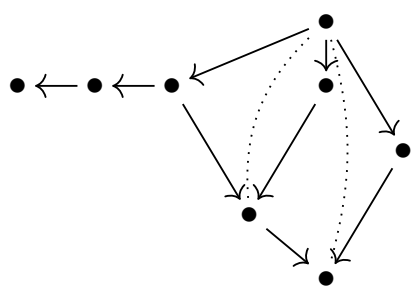

73.

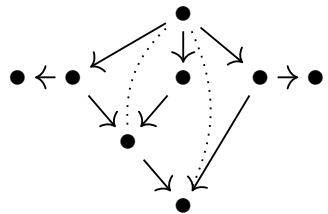

76.

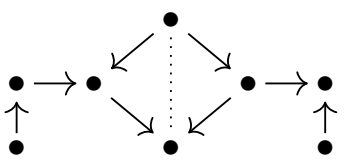

65.

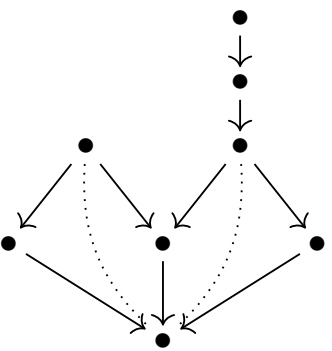

68.

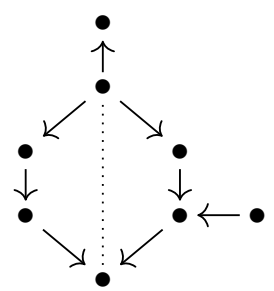

71.

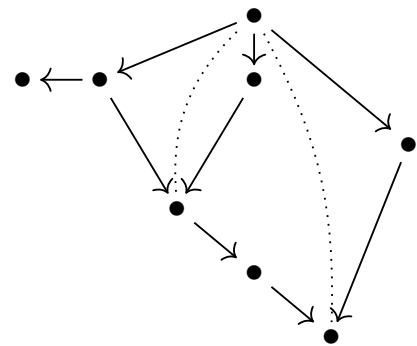

74.
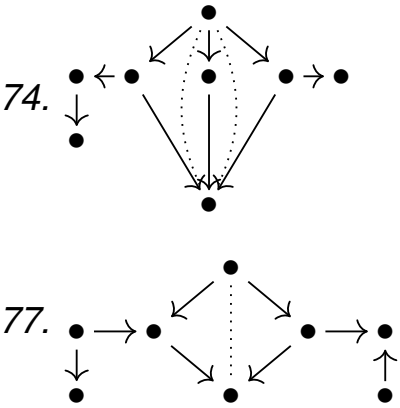

66.

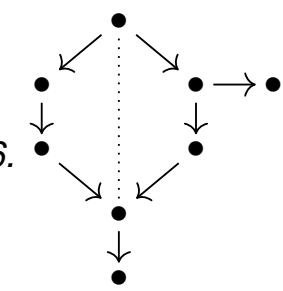

69.

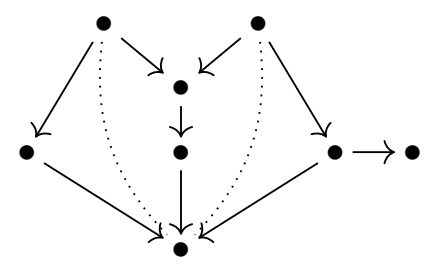

72.

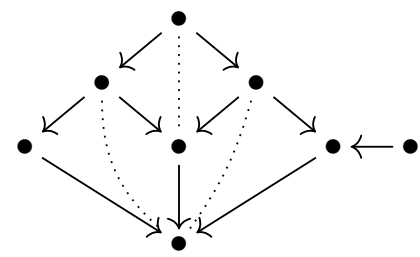

75.

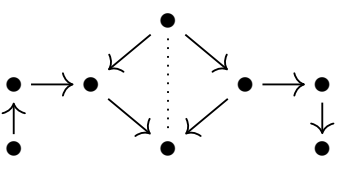

78.

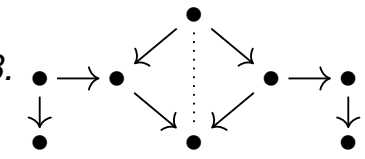

são Phias da família ANS de tipo $\widetilde{\mathbb{E}}_{7}$.

Demonstração Consideramos a lista dos frames das álgebras disfarçadas mansas. Na parte dos frames de tipo $\widetilde{\mathbb{E}}_{7}$ tem 23 frames: $\mathcal{F} r 11, \ldots, \mathcal{F} r 32$. Como explicamos no início dessa seção, utilizaremos apenas os frames schurian. Para cada frame, faremos a operação admissível resultando numa álgebra disfarçada schurian $A$. Em seguida, colocaremos as informações necessárias da extensão trivial $T(A)$ no programa. Finalmente, os integrantes dessa família ANS serão as álgebras $B$ originadas dos cortes de $T(A)$ satisfazendo duas características: uma álgebra de incidência, e não é disfarçada. 
$\mathcal{F} r 11$

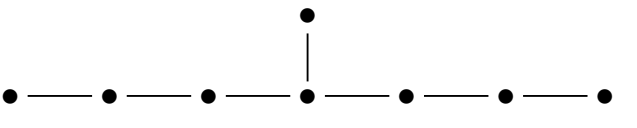

Aplicamos a operação admissível 1 no frame euclidiano $\widetilde{\mathbb{E}}_{7}$, resultando na álgebra hereditária de tipo $\widetilde{\mathbb{E}}_{7}$, trivialmente é uma álgebra disfarçada. Graças ao teorema 5.35, analisaremos a extensão trivial da álgebra disfarçada reparando na existência de algum corte gerando uma Phia não hereditária. Para isso, na extensão trivial, precisamos ter no mínimo dois ciclos elementares que tenha pelo menos uma flecha em comum conforme o lema 5.22.

Portanto, precisamos ter dois caminhos maximais nas álgebras hereditárias que tenham no mínimo comprimento dois e uma flecha em comum. A menos de grafos duais, as possibilidades são:

1.

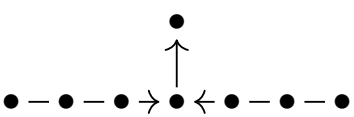

2.

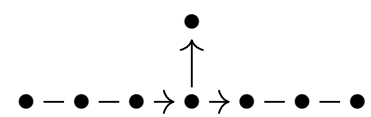

3.

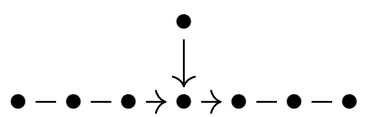

$\mathcal{F} r 11.1$ Investigaremos todas as extensões triviais do caso 1 . Vamos deixar de lado as extensões triviais que satisfazem as hipóteses do lema 5.42:
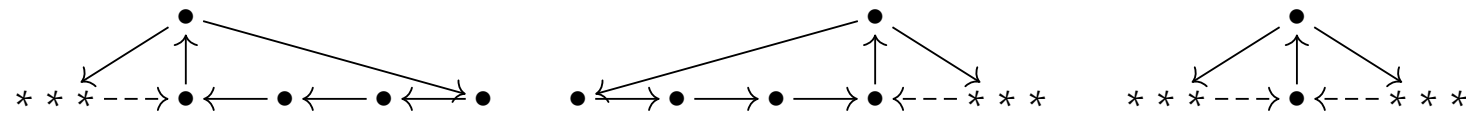

O símbolo $* * *$ pode ser substituído por $\bullet \longleftarrow \bullet$, ou $\bullet \bigsqcup^{\natural} \longleftarrow \bullet$, ou $\bullet \longleftarrow \bullet \longleftarrow \bullet$, ou $\bullet \longrightarrow \bullet$ de tal forma faça sentido na álgebra.

Mais uma observação, o caminho •-.-.-• tem comprimento um ou dois.

Portanto, resultado desse filtro, obtemos apenas a extensão trivial:
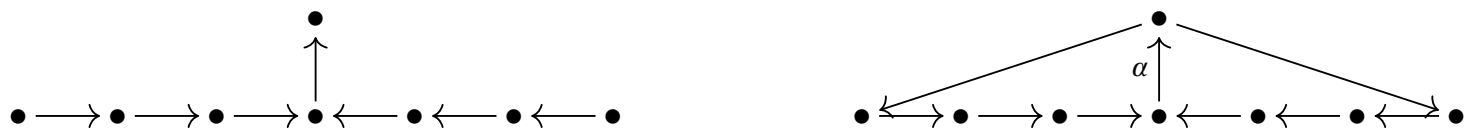

Uma aplicação direta do lema 5.22 , temos o corte $\{\alpha\}$ resultando na solução 1 .

$\mathcal{F} r 11.2$ Essa parte mostra a análise das extensões triviais do caso 2. Excluiremos as extensões triviais que satisfazem as hipóteses do lema 5.42:

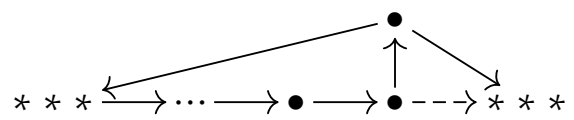

O símbolo $* * *$ pode ser substituído por $\bullet \longleftarrow \bullet$, ou $\bullet \longleftarrow \bullet \longleftarrow \bullet$, ou $\bullet \longleftarrow \bullet \longleftarrow \bullet$, ou $\bullet \longleftrightarrow \bullet$ de tal forma faça sentido na álgebra.

Outro detalhe é que o caminho $\bullet--\rightarrow \bullet$ tem comprimento um ou dois.

Consequentemente, sobraram quatro extensões triviais:
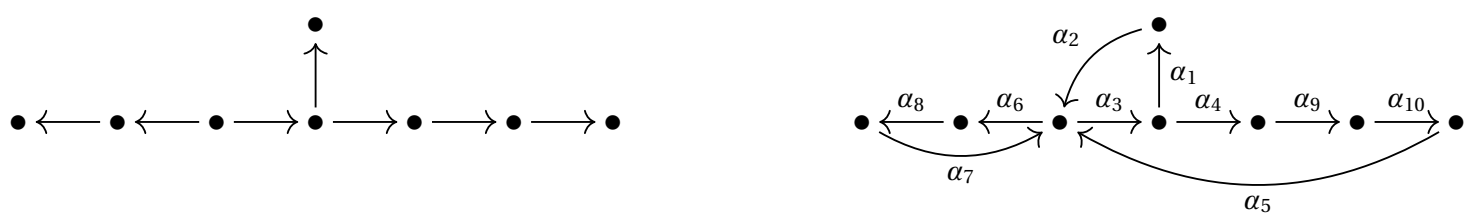
As relações do tipo 2 são: $r 1=\alpha_{2} \alpha_{3} \alpha_{4}, r 2=\alpha_{5} \alpha_{3} \alpha_{1}, r 3=\alpha_{2} \alpha_{6}, r 4=\alpha_{5} \alpha_{6}$ e $r 5=\alpha_{7} \alpha_{3}$. Os ciclos elementares são: $C_{1}=\alpha_{1} \alpha_{2} \alpha_{3}, C_{2}=\alpha_{3} \alpha_{4} \alpha_{9} \alpha_{10} \alpha_{5}$ e $C_{3}=\alpha_{6} \alpha_{8} \alpha_{7}$. O programa exibe o corte $\left\{\alpha_{3}, \alpha_{6}\right\}$ tal que origina a álgebra 2 .
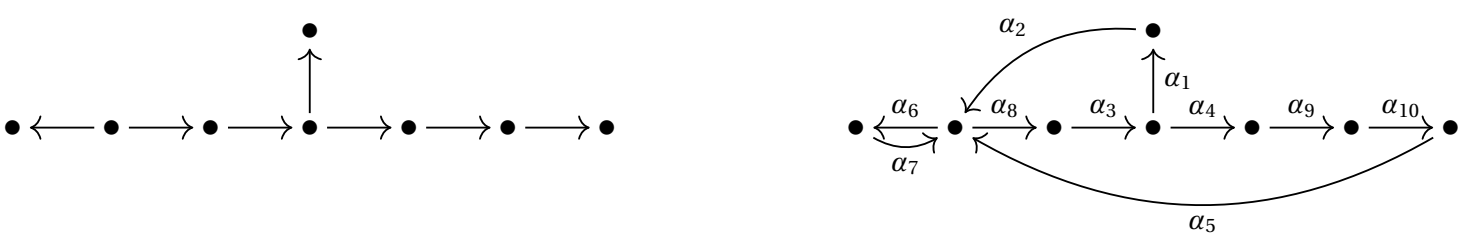

As relações do tipo 2 são: $r 1=\alpha_{2} \alpha_{8} \alpha_{3} \alpha_{4}, r 2=\alpha_{5} \alpha_{8} \alpha_{3} \alpha_{1}, r 3=\alpha_{2} \alpha_{6}, r 4=\alpha_{5} \alpha_{6}$ e $r 5=\alpha_{7} \alpha_{8}$. Os ciclos elementares são: $C_{1}=\alpha_{1} \alpha_{2} \alpha_{8} \alpha_{3}, C_{2}=\alpha_{3} \alpha_{4} \alpha_{9} \alpha_{10} \alpha_{5} \alpha_{8}$ e $C_{3}=\alpha_{6} \alpha_{7}$. Logo, a partir do programa, obtemos o corte $\left\{\alpha_{8}, \alpha_{6}\right\}$ em que resulta na álgebra 3 .
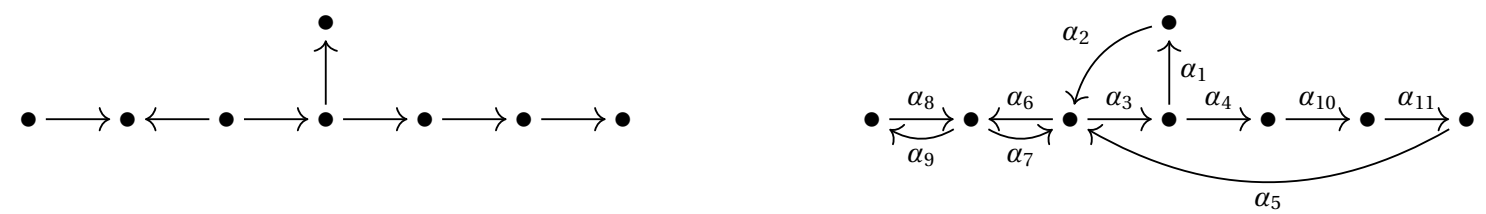

As relações do tipo 2 são: $r 1=\alpha_{2} \alpha_{3} \alpha_{4}, r 2=\alpha_{5} \alpha_{3} \alpha_{1}, r 3=\alpha_{2} \alpha_{6}, r 4=\alpha_{5} \alpha_{6}, r 5=\alpha_{7} \alpha_{3}$, $r 6=\alpha_{6} \alpha_{9}$ e $r 7=\alpha_{8} \alpha_{7}$. Os ciclos elementares são: $C_{1}=\alpha_{1} \alpha_{2} \alpha_{3}, C_{2}=\alpha_{3} \alpha_{4} \alpha_{10} \alpha_{11} \alpha_{5}$, $C_{3}=\alpha_{6} \alpha_{7}$ e $C_{4}=\alpha_{8} \alpha_{9}$. Portanto temos o corte $\left\{\alpha_{8}, \alpha_{6}, \alpha_{3}\right\}$ implicando na álgebra 4 .
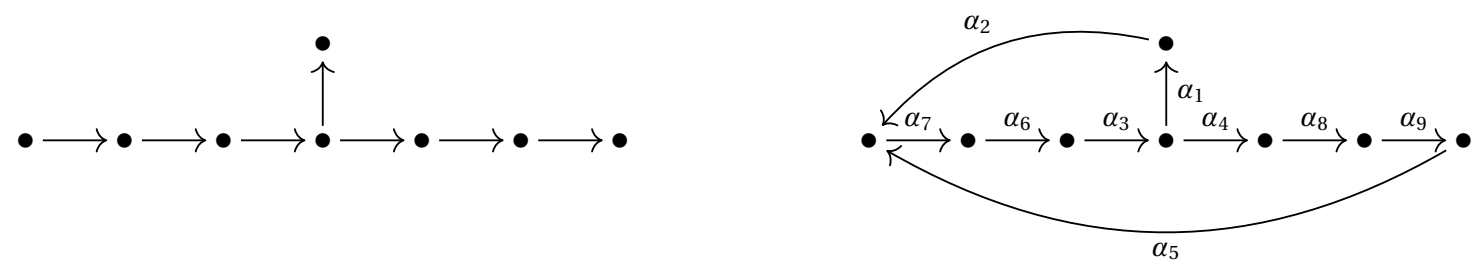

As relações do tipo 2 são: $r 1=\alpha_{2} \alpha_{7} \alpha_{6} \alpha_{3} \alpha_{4}$ e $r 2=\alpha_{5} \alpha_{7} \alpha_{6} \alpha_{3} \alpha_{1}$. Os ciclos elementares são: $C_{1}=\alpha_{1} \alpha_{2} \alpha_{7} \alpha_{6} \alpha_{3}$ e $C_{2}=\alpha_{7} \alpha_{6} \alpha_{3} \alpha_{4} \alpha_{8} \alpha_{9} \alpha_{5}$. Aplicação direta do lema 5.22, temos os cortes $\left\{\alpha_{7}\right\},\left\{\alpha_{6}\right\}$ e $\left\{\alpha_{3}\right\}$ implicando nas álgebras 5,6 e 7 , respectivamente.

$\mathcal{F} r 11.3$ Seguindo a mesma linha de raciocínio dos casos anteriores, graças ao lema 5.42, temos a forma geral da extensão trivial que não iremos estudar:

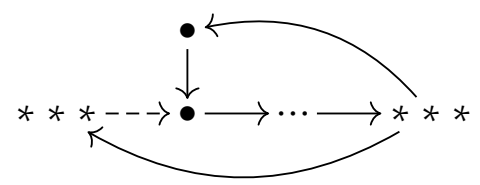

O símbolo $* * *$ pode ser substituído por $\bullet \longleftarrow \bullet$, ou $\bullet \longleftarrow \bullet \longleftarrow \bullet$, ou $\bullet \longleftarrow \bullet \longleftarrow \bullet$, ou $\bullet \longmapsto \bullet$ de tal forma faça sentido na álgebra.

Outro detalhe é que o caminho •-.- • tem comprimento um ou dois.

Portanto, restam quatro extensões triviais para analisar: 

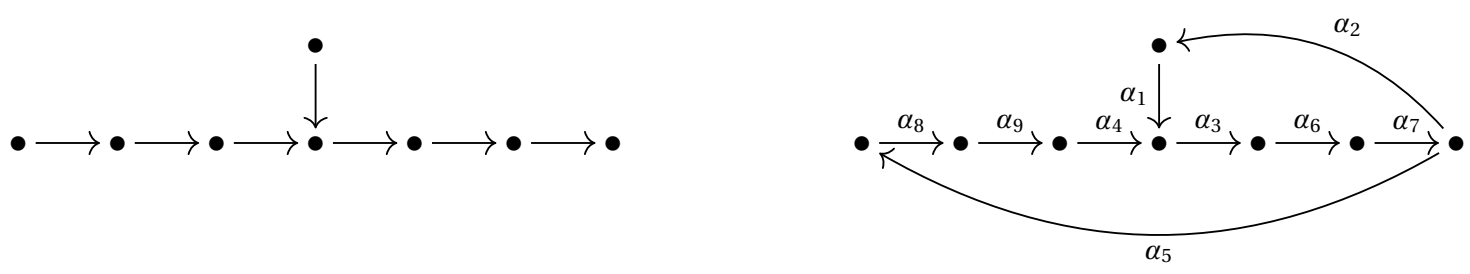

As relações do tipo 2 são: $r 1=\alpha_{1} \alpha_{3} \alpha_{6} \alpha_{7} \alpha_{5}$ e $r 2=\alpha_{4} \alpha_{3} \alpha_{6} \alpha_{7} \alpha_{2}$. Os ciclos elementares são: $C_{1}=\alpha_{2} \alpha_{1} \alpha_{3} \alpha_{6} \alpha_{7}$ e $C_{2}=\alpha_{5} \alpha_{8} \alpha_{9} \alpha_{4} \alpha_{3} \alpha_{6} \alpha_{7}$. Através do lema 5.22, obtemos três cortes $\left\{\alpha_{3}\right\},\left\{\alpha_{6}\right\}$ e $\left\{\alpha_{7}\right\}$ originando as álgebra 5,6 e 7 .
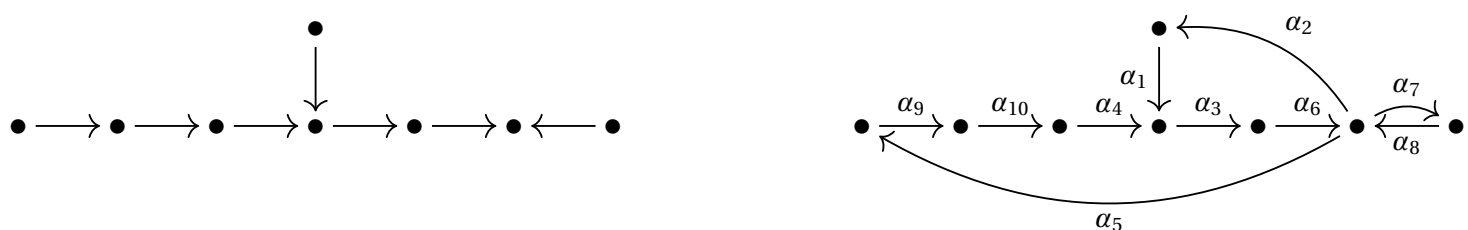

As relações do tipo 2 são: $r 1=\alpha_{1} \alpha_{3} \alpha_{6} \alpha_{5}, r 2=\alpha_{4} \alpha_{3} \alpha_{6} \alpha_{2}, r 3=\alpha_{6} \alpha_{7}, r 4=\alpha_{8} \alpha_{2}$ e $r 5=\alpha_{8} \alpha_{5}$. Os ciclos elementares são: $C_{1}=\alpha_{2} \alpha_{1} \alpha_{3} \alpha_{6}, C_{2}=\alpha_{5} \alpha_{9} \alpha_{10} \alpha_{4} \alpha_{3} \alpha_{6}$ e $C_{3}=\alpha_{7} \alpha_{8}$. Usamos o programa, obtendo o corte $\left\{\alpha_{8}, \alpha_{6}\right\}$ em que resulta na álgebra 8.
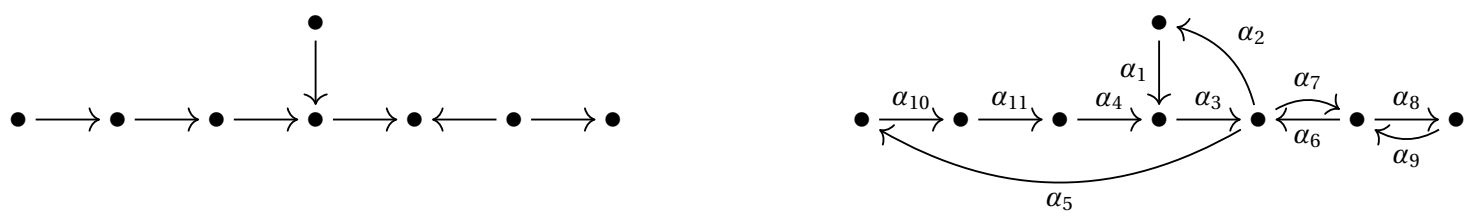

As relações do tipo 2 são: $r 1=\alpha_{1} \alpha_{3} \alpha_{5}, r 2=\alpha_{4} \alpha_{3} \alpha_{2}, r 3=\alpha_{3} \alpha_{7}, r 4=\alpha_{6} \alpha_{2}, r 5=\alpha_{6} \alpha_{5}$, $r 6=\alpha_{9} \alpha_{6}$ e $r 7=\alpha_{7} \alpha_{8}$. Os ciclos elementares são: $C_{1}=\alpha_{2} \alpha_{1} \alpha_{3}, C_{2}=\alpha_{5} \alpha_{10} \alpha_{11} \alpha_{4} \alpha_{3}$, $C_{3}=\alpha_{6} \alpha_{7}$ e $C_{4}=\alpha_{8} \alpha_{9}$. Portanto temos o corte $\left\{\alpha_{8}, \alpha_{6}, \alpha_{3}\right\}$ implicando na solução 9 .
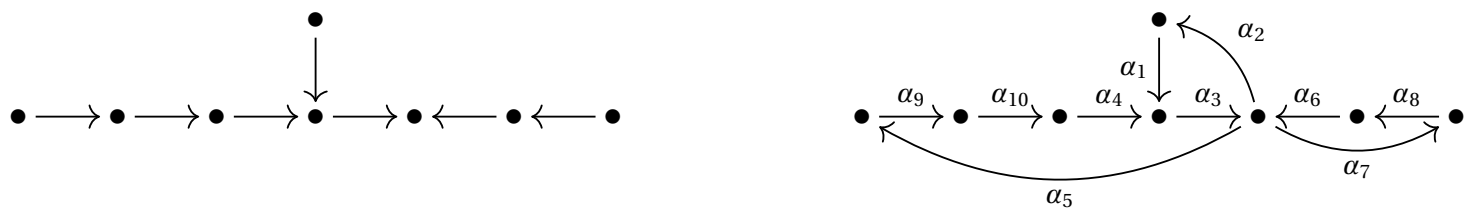

As relações do tipo 2 são: $r 1=\alpha_{1} \alpha_{3} \alpha_{5}, r 2=\alpha_{4} \alpha_{3} \alpha_{2}, r 3=\alpha_{3} \alpha_{7}, r 4=\alpha_{6} \alpha_{2}$ e $r 5=\alpha_{6} \alpha_{5}$. Os ciclos elementares são: $C_{1}=\alpha_{2} \alpha_{1} \alpha_{3}, C_{2}=\alpha_{5} \alpha_{9} \alpha_{10} \alpha_{4} \alpha_{3}$ e $C_{3}=\alpha_{6} \alpha_{7} \alpha_{8}$. O programa mostra o corte $\left\{\alpha_{3}, \alpha_{6}\right\}$ tal que origina a álgebra 10.

O frame $\mathcal{F} r 12$ tem no total 16 possibilidades de operações admissíveis 1 , mais 8 possibilidades com operações admissíveis 1 e 2, e a álgebra resultante da operação admissível 2 apenas. Não importando a operação admissível escolhida, a álgebra resultante é uma álgebra schurian.

$\mathcal{F} r 12.1$
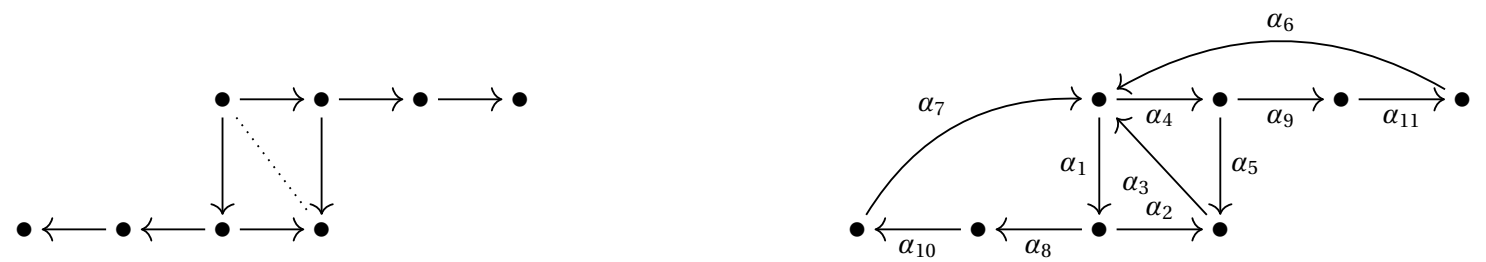

As relações do tipo 2 são: $r 1=\alpha_{2} \alpha_{3} \alpha_{4}, r 2=\alpha_{5} \alpha_{3} \alpha_{1}, r 3=\alpha_{7} \alpha_{1} \alpha_{2}, r 4=\alpha_{3} \alpha_{1} \alpha_{8}, r 5=$ 
$\alpha_{7} \alpha_{4}, r 6=\alpha_{3} \alpha_{4} \alpha_{9}, r 7=\alpha_{6} \alpha_{4} \alpha_{5}$ e $r 8=\alpha_{6} \alpha_{1}$. Os ciclos elementares são: $C_{1}=\alpha_{1} \alpha_{2} \alpha_{3}$, $C_{2}=\alpha_{3} \alpha_{4} \alpha_{5}, C_{3}=\alpha_{4} \alpha_{9} \alpha_{11} \alpha_{6}$ e $C_{4}=\alpha_{7} \alpha_{1} \alpha_{8} \alpha_{10}$. O programa mostra dois cortes: um origina a álgebra disfarçada e $\left\{\alpha_{1}, \alpha_{4}\right\}$ que resulta na solução 11 .

$\mathcal{F} r 12.2$
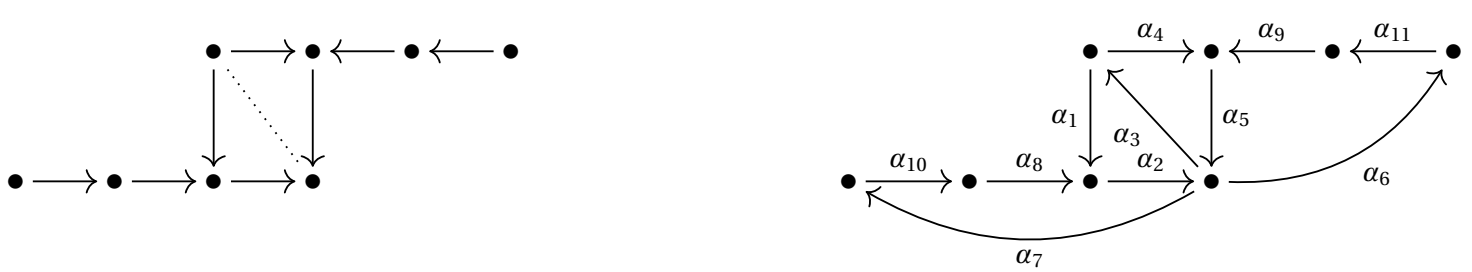

As relações do tipo 2 são: $r 1=\alpha_{2} \alpha_{3} \alpha_{4}, r 2=\alpha_{5} \alpha_{3} \alpha_{1}, r 3=\alpha_{1} \alpha_{2} \alpha_{7}, r 4=\alpha_{8} \alpha_{2} \alpha_{3}, r 5=$ $\alpha_{5} \alpha_{7}, r 6=\alpha_{4} \alpha_{5} \alpha_{6}, r 7=\alpha_{9} \alpha_{5} \alpha_{3}$ e $r 8=\alpha_{2} \alpha_{6}$. Os ciclos elementares são: $C_{1}=\alpha_{1} \alpha_{2} \alpha_{3}$, $C_{2}=\alpha_{3} \alpha_{4} \alpha_{5}, C_{3}=\alpha_{5} \alpha_{6} \alpha_{11} \alpha_{9}$ e $C_{4}=\alpha_{8} \alpha_{2} \alpha_{7} \alpha_{10}$. O programa mostra o corte $\left\{\alpha_{2}, \alpha_{5}\right\}$ que resulta na álgebra dual da álgebra 11 .

$\mathcal{F} r 12.3$

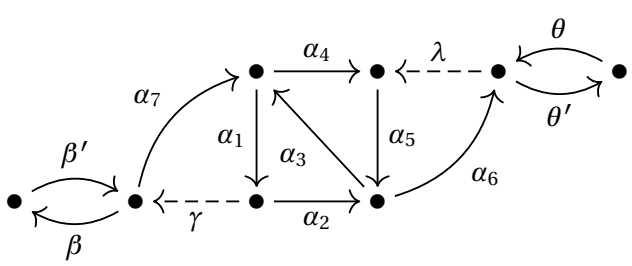

As relações do tipo 2 são: $r 1=\alpha_{2} \alpha_{3} \alpha_{4}, r 2=$ $\alpha_{5} \alpha_{3} \alpha_{1}, r 3=\alpha_{7} \alpha_{1} \alpha_{2}, r 4=\alpha_{3} \alpha_{1} \gamma, r 5=\alpha_{7} \alpha_{4}$, $r 6=\alpha_{4} \alpha_{5} \alpha_{6}, r 7=\lambda \alpha_{5} \alpha_{3}, r 8=\alpha_{2} \alpha_{6}, r 9=\theta \lambda$, $r 10=\alpha_{6} \theta^{\prime}, r 11=\beta^{\prime} \alpha_{7}$ e $r 12=\gamma \beta$. Os ciclos elementares são: $C_{1}=\alpha_{1} \alpha_{2} \alpha_{3}, C_{2}=\alpha_{3} \alpha_{4} \alpha_{5}$, $C_{3}=\lambda \alpha_{5} \alpha_{6}, C_{4}=\alpha_{7} \alpha_{1} \gamma, C_{5}=\theta^{\prime} \theta$ e $C_{6}=\beta^{\prime} \beta$.

A extensão trivial anterior é um esquema representando 4 possibilidades de operações admissíveis 1 sobre o frame $\mathcal{F} r$ 12. A flecha tracejada representa um caminho de comprimento um ou dois. Se o caminho $\gamma$ tiver comprimento dois, então não existe o ciclo elementar $C_{6}=\beta^{\prime} \beta$. Caso contrário, a presença do ciclo elementar $C_{6}$ é assegurada. Condição análoga para o caminho $\lambda$.

Mostraremos que não iremos obter um corte originando uma Phia não disfarçada. Seja $\Sigma$ um corte da extensão trivial anterior. Vamos dividir em três casos.

O primeiro caso começa com $\alpha_{1} \in \Sigma$. Se existir o ciclo elementar $C_{6}$, então não teremos uma Phia não disfarçada, pelo lema 5.42. Supomos que não existe o ciclo elementar $C_{6}$. Pelas relações $r 5=\alpha_{7} \alpha_{4}$ e $r 8=\alpha_{2} \alpha_{6}$, precisamos escolher $\alpha_{4}, \alpha_{6} \in \Sigma$. Consequentemente, não tem como eliminar a relação $r 1=\alpha_{2} \alpha_{3} \alpha_{4}$ pois já escolhemos as flechas dos ciclos elementares $C_{1}, C_{2}$. Portanto, concluímos o desejado.

O segundo caso começa com $\alpha_{5} \in \Sigma$. O raciocínio é análogo ao caso anterior.

O terceiro caso consideramos com $\alpha_{3} \in \Sigma$. Pelas relações $r 5=\alpha_{7} \alpha_{4}$ e $r 8=\alpha_{2} \alpha_{6}$, precisamos escolher $\alpha_{7}, \alpha_{6} \in \Sigma$. Não importando a presença dos ciclos elementares $C_{5}, C_{6}$, completamos um corte $\Sigma$ que resulta na álgebra disfarçada.

Essa mesma análise serve para o restante das possibilidades de operações admissíveis 1 no frame $\mathcal{F} r 12$ representado abaixo:
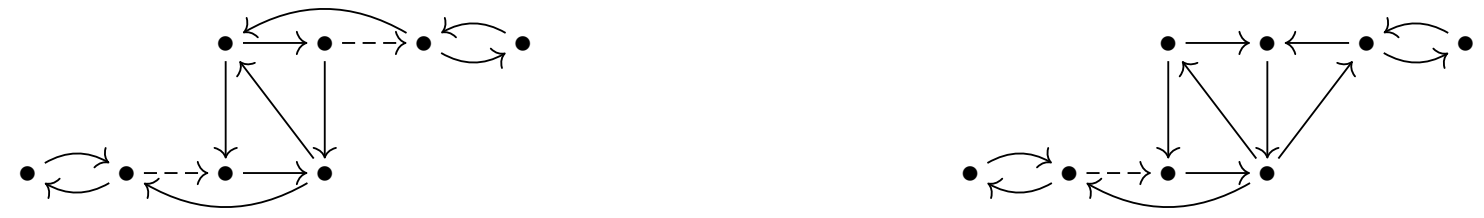

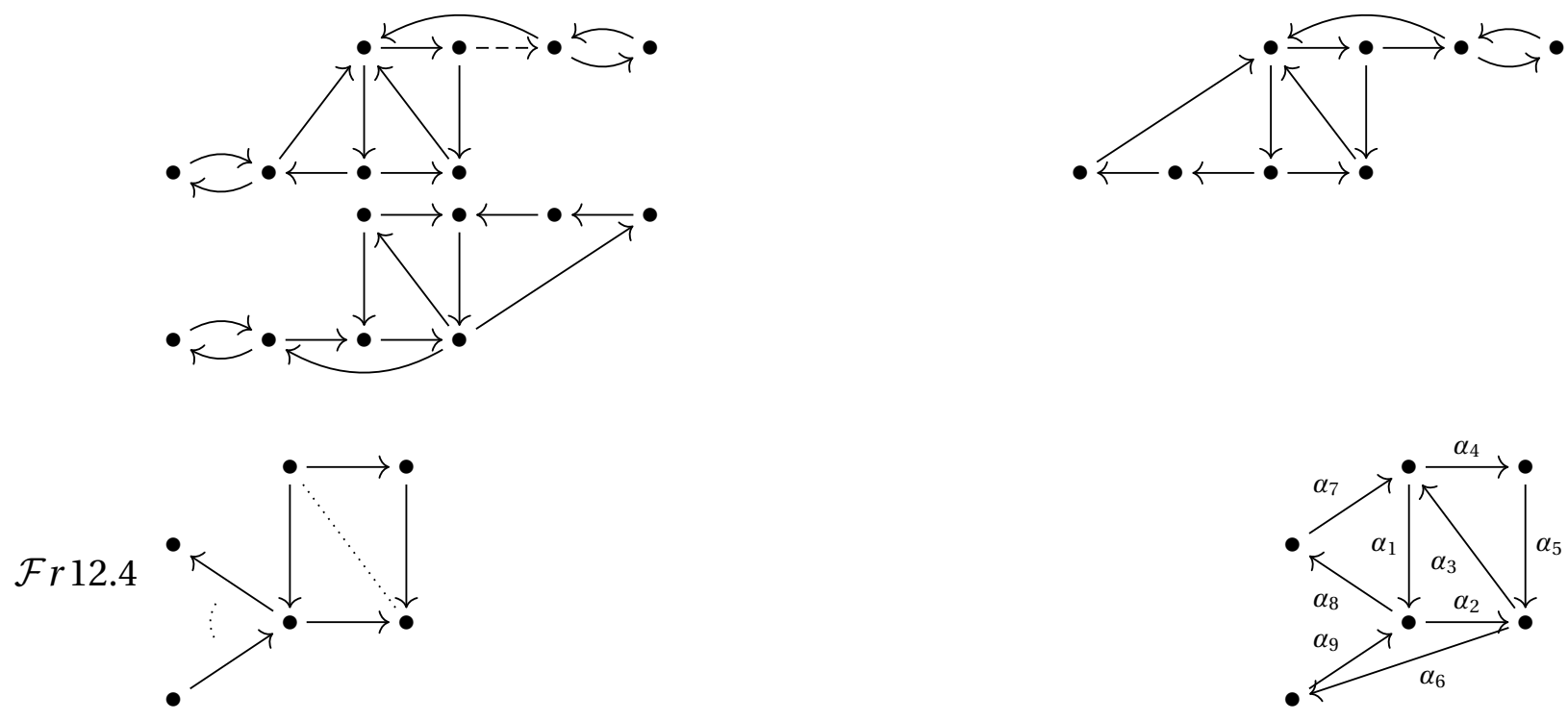

As relações do tipo 2 são: $r 1=\alpha_{2} \alpha_{3} \alpha_{4}, r 2=\alpha_{5} \alpha_{3} \alpha_{1}, r 3=\alpha_{7} \alpha_{4}, r 4=\alpha_{3} \alpha_{1} \alpha_{8}, r 5=$ $\alpha_{5} \alpha_{6}, r 6=\alpha_{9} \alpha_{2} \alpha_{3}, r 7=\alpha_{9} \alpha_{8}, r 8=\alpha_{7} \alpha_{1} \alpha_{2}$ e $r 9=\alpha_{1} \alpha_{2} \alpha_{6}$. Os ciclos elementares são: $C_{1}=\alpha_{1} \alpha_{2} \alpha_{3}, C_{2}=\alpha_{3} \alpha_{4} \alpha_{5}, C_{3}=\alpha_{1} \alpha_{8} \alpha_{7}$ e $C_{4}=\alpha_{2} \alpha_{6} \alpha_{9}$.

O estudo dessa subaljava se aplica na extensões triviais das álgebras oriundas das cinco possibilidades de operações admissíveis 2 e 1 no frame $\mathcal{F} r 12$. Vamos dividir em dois casos tendo como referência a eliminação da relação $r 7=\alpha_{9} \alpha_{8}$. Seja $\Sigma$ um corte dessa subaljava.

O primeiro caso é $\alpha_{9} \in \Sigma$. Pela relação $r 5=\alpha_{5} \alpha_{6}$, implica que $\alpha_{5} \in \Sigma$. Então não podemos escolher as flechas dos ciclos elementares $C_{4}$ e $C_{2}$. Agora, pela relação $r 3=\alpha_{7} \alpha_{4}$, temos que $\alpha_{7} \in \Sigma$. Completando o corte e sobrando a relação $r 1=\alpha_{2} \alpha_{3} \alpha_{4}$. Concluindo que não tem corte que resulte numa Phia não hereditária.

Já no segundo caso tem $\alpha_{8} \in \Sigma$. Pela relação $r 3=\alpha_{7} \alpha_{4}$, precisamos ter $\alpha_{4} \in \Sigma$. Sendo assim, não podemos escolher as flechas dos ciclos elementares $C_{3}$ e $C_{2}$. Agora, pela relação $r 5=\alpha_{5} \alpha_{6}$, implica que $\alpha_{6} \in \Sigma$. Completando o corte e ainda tendo a relação $r 2=\alpha_{5} \alpha_{3} \alpha_{1}$. Portando não tem corte que origina numa Phia não hereditária.

Essa mesma análise é válida para as outras extensões triviais originadas das quatro possibilidades de operações admissíveis 2 e 1 no frame $\mathcal{F} r 12$ em que tem a subaljava abaixo:
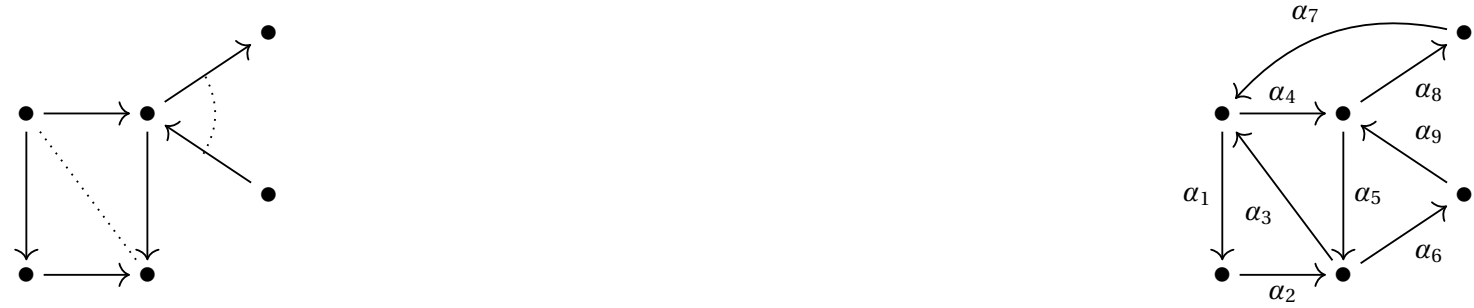

O frame $\mathcal{F} r 13$ tem a mesma quantidade de álgebras oriundas das operações admissíveis 1 ou 2 que o frame anterior. Entretanto a estratégia vai ser diferente pois temos mais soluções que o anterior. 
$\mathcal{F} r 13.1$
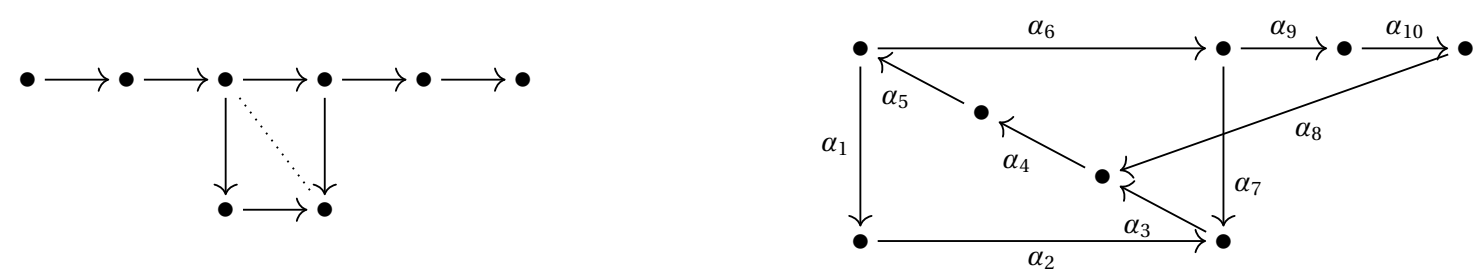

As relações do tipo 2 são: $r 1=\alpha_{2} \alpha_{3} \alpha_{4} \alpha_{5} \alpha_{6}, r 2=\alpha_{7} \alpha_{3} \alpha_{4} \alpha_{5} \alpha_{1}, r 3=\alpha_{3} \alpha_{4} \alpha_{5} \alpha_{6} \alpha_{9}$, $r 4=\alpha_{8} \alpha_{4} \alpha_{5} \alpha_{1}$ e $r 5=\alpha_{8} \alpha_{4} \alpha_{5} \alpha_{6} \alpha_{7}$. Os ciclos elementares são: $C_{1}=\alpha_{1} \alpha_{2} \alpha_{3} \alpha_{4} \alpha_{5}$, $C_{2}=\alpha_{3} \alpha_{4} \alpha_{5} \alpha_{6} \alpha_{7}$ e $C_{3}=\alpha_{4} \alpha_{5} \alpha_{6} \alpha_{9} \alpha_{10} \alpha_{8}$. O programa nos mostra quatro cortes $\Sigma_{i}$ com $i=1,2,3,4$. O $\Sigma_{1}=\left\{\alpha_{4}\right\}$ resulta na solução $13, \circ \Sigma_{2}=\left\{\alpha_{5}\right\}$ origina a solução 12 , $\Sigma_{3}=\left\{\alpha_{6}, \alpha_{1}\right\}$ faz parte da álgebra 14 e o último origina a álgebra disfarçada acima.

$\mathcal{F} r 13.2$
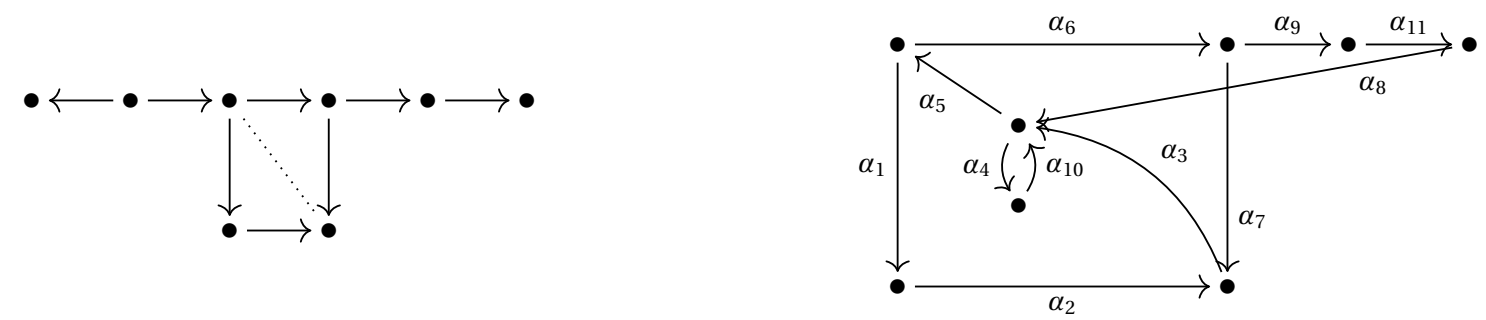

As relações do tipo 2 são: $r 1=\alpha_{2} \alpha_{3} \alpha_{5} \alpha_{6}, r 2=\alpha_{7} \alpha_{3} \alpha_{5} \alpha_{1}, r 3=\alpha_{3} \alpha_{5} \alpha_{6} \alpha_{9}, r 4=\alpha_{8} \alpha_{5} \alpha_{1}$, $r 5=\alpha_{8} \alpha_{5} \alpha_{6} \alpha_{7}, r 6=\alpha_{10} \alpha_{5}, r 7=\alpha_{8} \alpha_{4}$ e $r 8=\alpha_{3} \alpha_{4}$. Os ciclos elementares são: $C_{1}=$ $\alpha_{1} \alpha_{2} \alpha_{3} \alpha_{5}, C_{2}=\alpha_{3} \alpha_{5} \alpha_{6} \alpha_{7}, C_{3}=\alpha_{5} \alpha_{6} \alpha_{9} \alpha_{11} \alpha_{8}$ e $C_{4}=\alpha_{4} \alpha_{10}$. Portanto, temos a Phia 18 através do corte $\left\{\alpha_{5}, \alpha_{4}\right\}$.

$\mathcal{F} r 13.3$
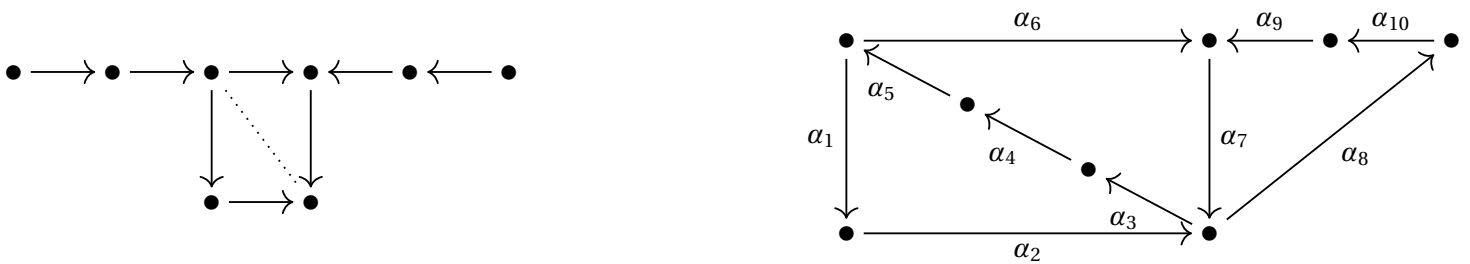

As relações do tipo 2 são: $r 1=\alpha_{2} \alpha_{3} \alpha_{4} \alpha_{5} \alpha_{6}, r 2=\alpha_{7} \alpha_{3} \alpha_{4} \alpha_{5} \alpha_{1}, r 3=\alpha_{9} \alpha_{7} \alpha_{3}, r 4=$ $\alpha_{6} \alpha_{7} \alpha_{8}$ e $r 5=\alpha_{2} \alpha_{8}$. Os ciclos elementares são: $C_{1}=\alpha_{1} \alpha_{2} \alpha_{3} \alpha_{4} \alpha_{5}, C_{2}=\alpha_{3} \alpha_{4} \alpha_{5} \alpha_{6} \alpha_{7}$ e $C_{3}=\alpha_{7} \alpha_{8} \alpha_{10} \alpha_{9}$. Obtemos apenas a solução 16 através do corte $\left\{\alpha_{7}, \alpha_{2}\right\}$.

$\mathcal{F} r 13.4$
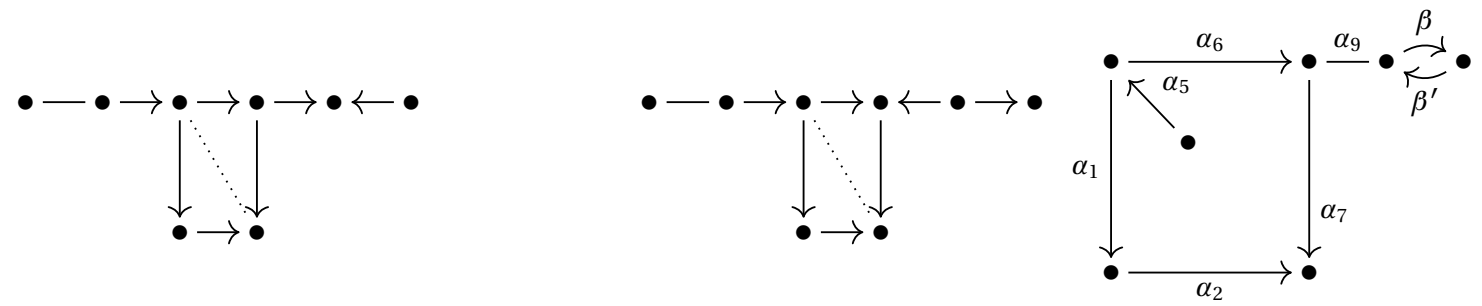

Essa parte resume quatro possibilidades de operações admissíveis 1 representado pelas arestas das frames. Mostraremos que não tem solução. A aljava da direita é uma subaljava da extensão trivial de qualquer possibilidade que for aplicada. Observamos que o ciclo elementar $\beta \beta^{\prime}$ e os dois ciclos elementares tais que um deles contém o caminho $\alpha_{5} \alpha_{6} \alpha_{7}$ e o outro a flecha $\alpha_{9}$ satisfazem a maioria das hipóteses do lema 5.42. Faltando verificar apenas a hipótese sobre o corte, isso é garantido pelas respostas dos itens $\mathcal{F} r 13.1, \mathcal{F} r 13.2$ 
e $\mathcal{F} r$ 13.3. Portanto, concluímos que não tem corte desejado.

$\mathcal{F} r 13.5$
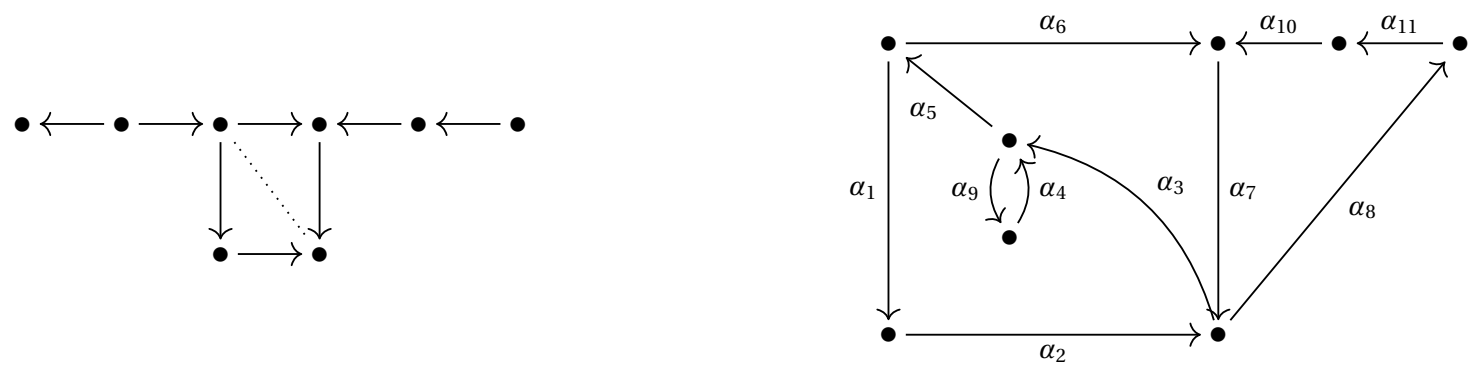

As relações do tipo 2 são: $r 1=\alpha_{2} \alpha_{3} \alpha_{5} \alpha_{6}, r 2=\alpha_{7} \alpha_{3} \alpha_{5} \alpha_{1}, r 3=\alpha_{10} \alpha_{7} \alpha_{3}, r 4=\alpha_{6} \alpha_{7} \alpha_{8}$, $r 5=\alpha_{2} \alpha_{8}, r 5=\alpha_{3} \alpha_{9}$ e $r 5=\alpha_{4} \alpha_{5}$. Os ciclos elementares são: $C_{1}=\alpha_{1} \alpha_{2} \alpha_{3} \alpha_{5}, C_{2}=$ $\alpha_{3} \alpha_{5} \alpha_{6} \alpha_{7}, C_{3}=\alpha_{7} \alpha_{8} \alpha_{11} \alpha_{10}$ e $C_{4}=\alpha_{4} \alpha_{9}$. O programa nos mostra apenas a solução disfarçada.

$\mathcal{F} r 13.6$
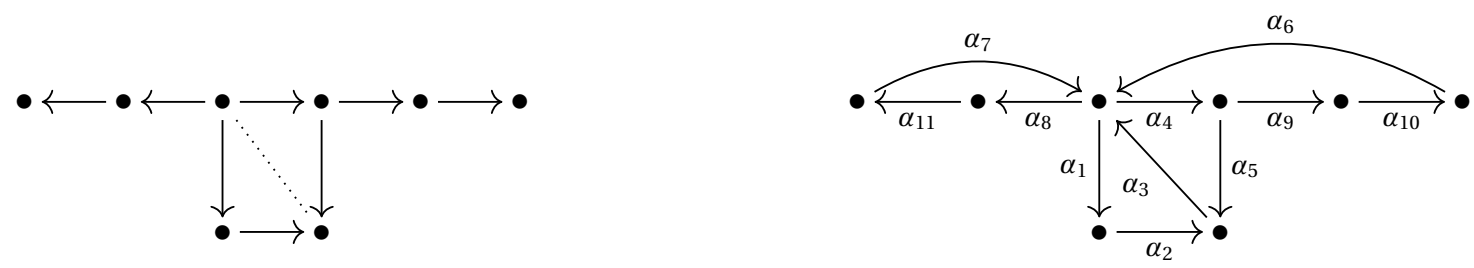

As relações do tipo 2 são: $r 1=\alpha_{2} \alpha_{3} \alpha_{4}, r 2=\alpha_{5} \alpha_{3} \alpha_{1}, r 3=\alpha_{7} \alpha_{1}, r 4=\alpha_{3} \alpha_{8}, r 5=$ $\alpha_{6} \alpha_{1}, r 6=\alpha_{6} \alpha_{4} \alpha_{5}, r 7=\alpha_{3} \alpha_{4} \alpha_{9}, r 8=\alpha_{6} \alpha_{8}$ e $r 9=\alpha_{7} \alpha_{4}$. Os ciclos elementares são: $C_{1}=\alpha_{1} \alpha_{2} \alpha_{3}, C_{2}=\alpha_{3} \alpha_{4} \alpha_{5}, C_{3}=\alpha_{4} \alpha_{9} \alpha_{10} \alpha_{6}$ e $C_{4}=\alpha_{7} \alpha_{8} \alpha_{11}$. Informando esses dados ao programa, temos o corte $\left\{\alpha_{4}, \alpha_{1}, \alpha_{8}\right\}$ resultando na Phia 15 e a álgebra disfarçada acima.

$\mathcal{F} r 13.7$
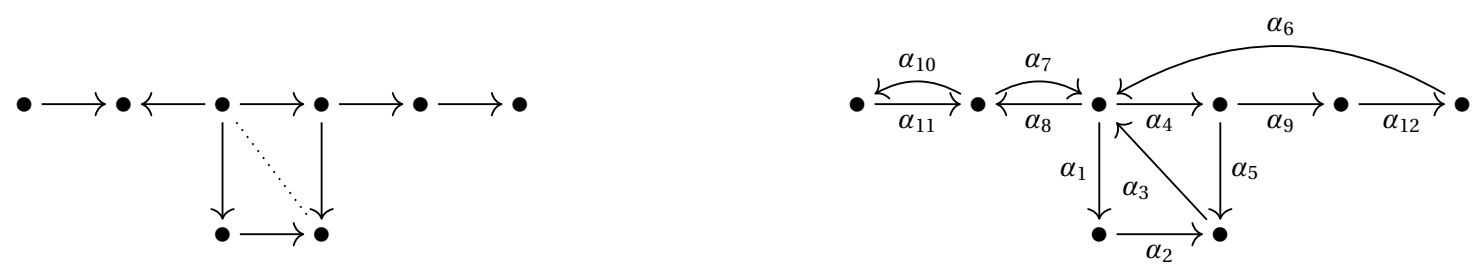

As relações do tipo 2 são: $r 1=\alpha_{2} \alpha_{3} \alpha_{4}, r 2=\alpha_{5} \alpha_{3} \alpha_{1}, r 3=\alpha_{7} \alpha_{1}, r 4=\alpha_{3} \alpha_{8}, r 5=\alpha_{6} \alpha_{1}$, $r 6=\alpha_{6} \alpha_{4} \alpha_{5}, r 7=\alpha_{3} \alpha_{4} \alpha_{9}, r 8=\alpha_{6} \alpha_{8}, r 9=\alpha_{7} \alpha_{4}, r 10=\alpha_{8} \alpha_{10}$ e $r 11=\alpha_{11} \alpha_{7}$. Os ciclos elementares são: $C_{1}=\alpha_{1} \alpha_{2} \alpha_{3}, C_{2}=\alpha_{3} \alpha_{4} \alpha_{5}, C_{3}=\alpha_{4} \alpha_{9} \alpha_{12} \alpha_{6}, C_{4}=\alpha_{7} \alpha_{8}$ e $C_{5}=\alpha_{11} \alpha_{10}$. O programa nos mostra o corte $\left\{\alpha_{4}, \alpha_{1}, \alpha_{8}, \alpha_{11}\right\}$ que origina a Phia 17.

$\mathcal{F} r 13.8$
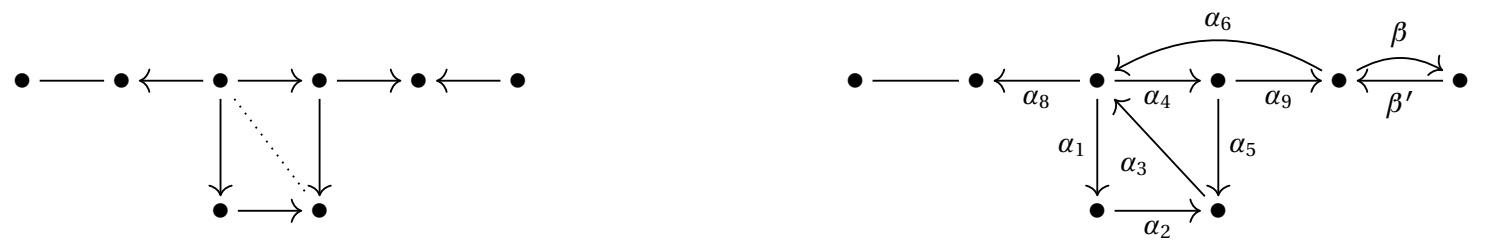

Como fizemos no item $\mathcal{F} r$ 13.4, essa análise é sobre duas possibilidades de operações admissíveis 1 representado pela aresta do frame. Provaremos que não tem corte que origina uma álgebra da família ANS. A aljava da direita é uma subaljava da extensão trivial de qualquer possibilidade que for aplicada no frame da esquerda. Vejamos que temos as hipóteses do lema 5.42 satisfeitas com os ciclos elementares $\beta \beta^{\prime}, \alpha_{4} \alpha_{9} \alpha_{6}$ e $\alpha_{3} \alpha_{4} \alpha_{5}$. 
Faltando verificar apenas a hipótese sobre o corte, isso é garantido pelas respostas dos itens $\mathcal{F} r 13.6$ e $\mathcal{F} r$ 13.7. Portanto, concluímos que não tem corte desejado.

$\mathcal{F} r 13.9$
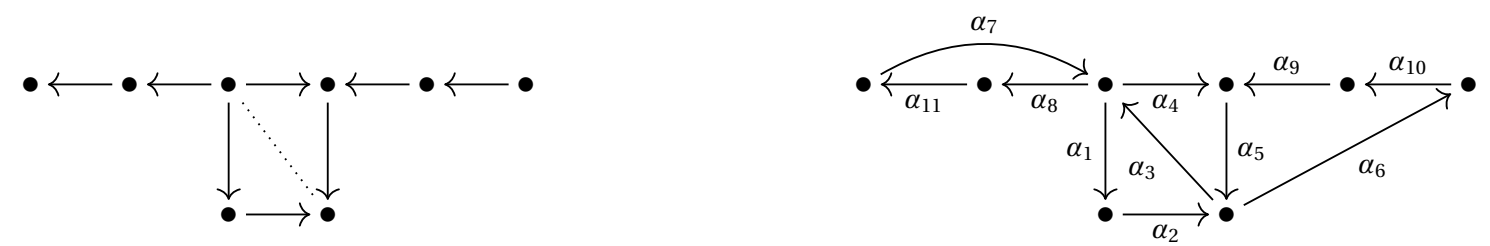

As relações do tipo 2 são: $r 1=\alpha_{2} \alpha_{3} \alpha_{4}, r 2=\alpha_{5} \alpha_{3} \alpha_{1}, r 3=\alpha_{7} \alpha_{1}, r 4=\alpha_{3} \alpha_{8}, r 5=\alpha_{2} \alpha_{6}$, $r 6=\alpha_{9} \alpha_{5} \alpha_{2}, r 7=\alpha_{4} \alpha_{5} \alpha_{6}$ e $r 8=\alpha_{7} \alpha_{4}$. Os ciclos elementares são: $C_{1}=\alpha_{1} \alpha_{2} \alpha_{3}$, $C_{2}=\alpha_{3} \alpha_{4} \alpha_{5}, C_{3}=\alpha_{5} \alpha_{6} \alpha_{10} \alpha_{9}$ e $C_{4}=\alpha_{7} \alpha_{8} \alpha_{11}$.

Mostraremos que não temos solução. Para isso, dividiremos em dois casos. Seja $\Sigma$ um corte da extensão trivial anterior.

O primeiro caso é quando $\alpha_{3} \in \Sigma$. Pelas relações $r 3=\alpha_{7} \alpha_{1}$ e $r 5=\alpha_{2} \alpha_{6}$, precisamos escolher $\alpha_{7}, \alpha_{6} \in \Sigma$. Completando o corte e originando a álgebra disfarçada.

$\mathrm{O}$ segundo caso é quando $\alpha_{5} \in \Sigma$. Pelas relações $r 8=\alpha_{7} \alpha_{4}$ e $r 5=\alpha_{2} \alpha_{6}$, obtemos que $\alpha_{7}, \alpha_{2} \in \Sigma$. Já escolhido o representante de cada ciclo elementar, não conseguimos eliminar a relação $r 4=\alpha_{3} \alpha_{8}$.

Portando, não conseguimos um corte que resulte numa Phia integrante da família ANS.

Essa análise serve para essas três possibilidades:

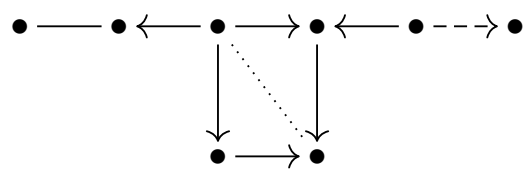

$\mathcal{F} r 13.10$
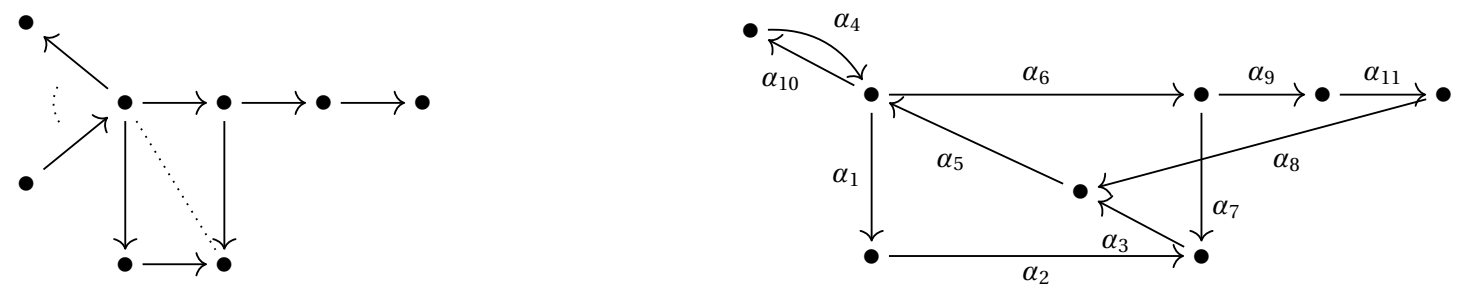

As relações do tipo 2 são: $r 1=\alpha_{2} \alpha_{3} \alpha_{5} \alpha_{6}, r 2=\alpha_{7} \alpha_{3} \alpha_{5} \alpha_{1}, r 3=\alpha_{3} \alpha_{5} \alpha_{6} \alpha_{9}, r 4=\alpha_{8} \alpha_{5} \alpha_{1}$, $r 5=\alpha_{8} \alpha_{5} \alpha_{6} \alpha_{7}, r 6=\alpha_{5} \alpha_{10}, r 7=\alpha_{4} \alpha_{1}$ e $r 8=\alpha_{4} \alpha_{6}$. Os ciclos elementares são: $C_{1}=$ $\alpha_{1} \alpha_{2} \alpha_{3} \alpha_{5}, C_{2}=\alpha_{3} \alpha_{5} \alpha_{6} \alpha_{7}, C_{3}=\alpha_{5} \alpha_{6} \alpha_{9} \alpha_{11} \alpha_{8}$ e $C_{4}=\alpha_{4} \alpha_{10}$. Portanto, o programa nos mostra dois cortes $\left\{\alpha_{5}, \alpha_{4}\right\}$ e $\left\{\alpha_{6}, \alpha_{1}, \alpha_{10}\right\}$ resultando nas Phias 19 e 20 respectivamente.
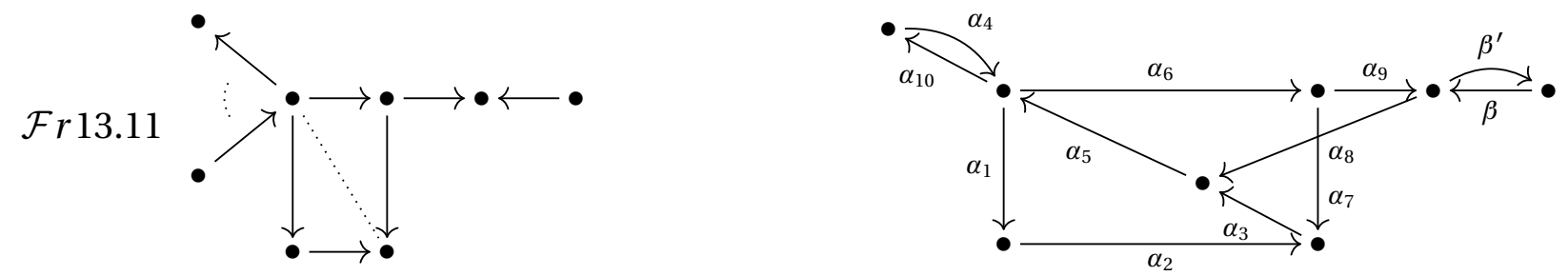

Esse caso não tem solução. A justificativa é análoga aos itens $\mathcal{F} r 13.4$ e $\mathcal{F} r 13.8$ usando o item anterior. 
$\mathcal{F} r 13.12$
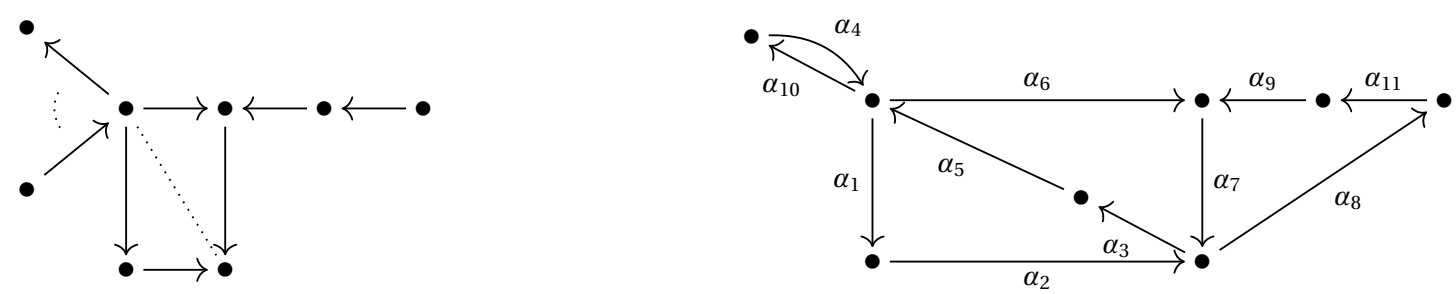

As relações do tipo 2 são: $r 1=\alpha_{2} \alpha_{3} \alpha_{5} \alpha_{6}, r 2=\alpha_{7} \alpha_{3} \alpha_{5} \alpha_{1}, r 3=\alpha_{6} \alpha_{7} \alpha_{8}, r 4=\alpha_{9} \alpha_{7} \alpha_{3}$, $r 5=\alpha_{2} \alpha_{8}, r 6=\alpha_{5} \alpha_{10}, r 7=\alpha_{4} \alpha_{1}$ e $r 8=\alpha_{4} \alpha_{6}$. Os ciclos elementares são: $C_{1}=\alpha_{1} \alpha_{2} \alpha_{3} \alpha_{5}, C_{2}=\alpha_{3} \alpha_{5} \alpha_{6} \alpha_{7}, C_{3}=\alpha_{7} \alpha_{8} \alpha_{11} \alpha_{9}$ e $C_{4}=\alpha_{4} \alpha_{10}$. O programa nos mostra nenhuma solução. Lembrando que o programa exibe apenas os cortes que originam álgebras de incidência.
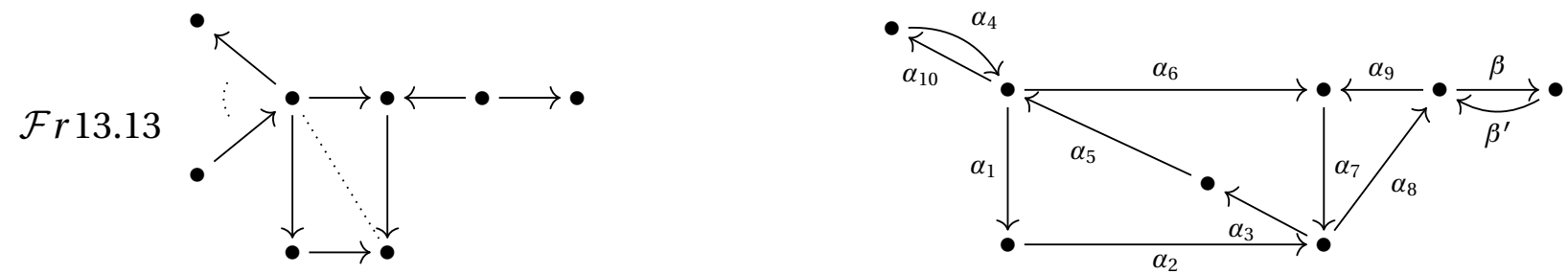

Esse caso também não tem integrante da família ANS. A justificativa é semelhante aos itens $\mathcal{F} r 13.4$ e $\mathcal{F} r 13.8$ usando o item anterior.

$\mathcal{F} r 13.14$ As extensões triviais restantes não tem solução. Para isso, usamos a mesma demonstração que o caso $\mathcal{F}$ r 12.4 para analisar as cinco possibilidades restantes de operações admissíveis 2 e 1 no frame $\mathcal{F} r 13$ em que tem a subaljava abaixo:
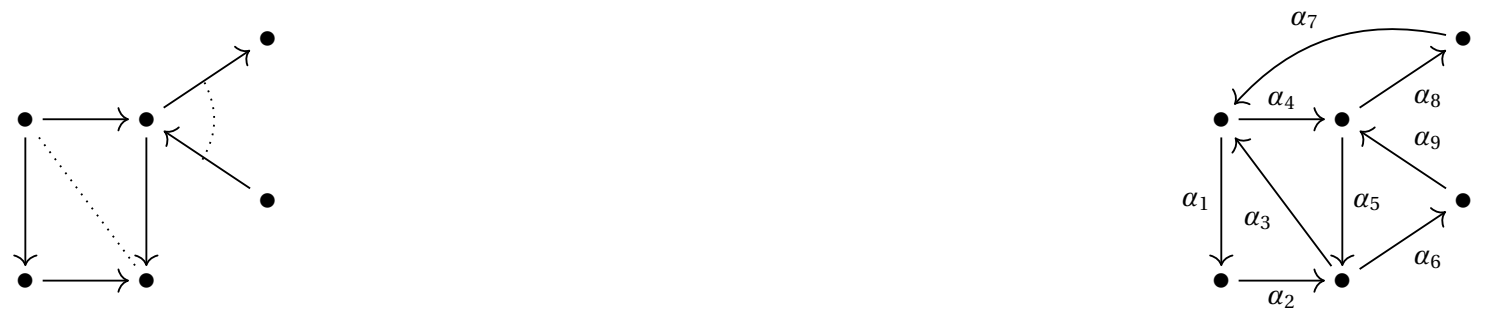

O frame $\mathcal{F} r 14$ tem uma possibilidade de usar a operação admissível 2 diferente dos dois frames anteriores mesmo tendo a mesma quantidade de arestas. Isso garante 20 casos para analisar.

$\mathcal{F} r 14.1$
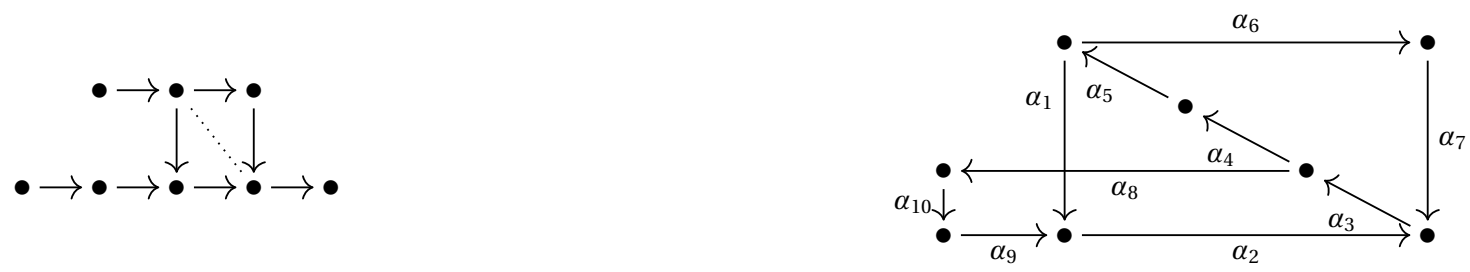

As relações do tipo 2 são: $r 1=\alpha_{2} \alpha_{3} \alpha_{4} \alpha_{5} \alpha_{6}, r 2=\alpha_{7} \alpha_{3} \alpha_{4} \alpha_{5} \alpha_{1}, r 3=\alpha_{9} \alpha_{2} \alpha_{3} \alpha_{4}$, $r 4=\alpha_{1} \alpha_{2} \alpha_{3} \alpha_{8}$ e $r 5=\alpha_{7} \alpha_{3} \alpha_{8}$. Os ciclos elementares são: $C_{1}=\alpha_{1} \alpha_{2} \alpha_{3} \alpha_{4} \alpha_{5}$, $C_{2}=\alpha_{3} \alpha_{4} \alpha_{5} \alpha_{6} \alpha_{7}$ e $C_{3}=\alpha_{2} \alpha_{3} \alpha_{8} \alpha_{10} \alpha_{9}$. Logo, através do programa, obtemos os cortes $\left\{\alpha_{3}\right\}$ e $\left\{\alpha_{2}, \alpha_{7}\right\}$ que originam as soluções 21 e 22 respectivamente.

Usando essas respostas, conforme fizemos em $\mathcal{F} r 13.4$ e $\mathcal{F} r 13.8$, podemos aplicar o lema 5.42 para descartar esses casos: 

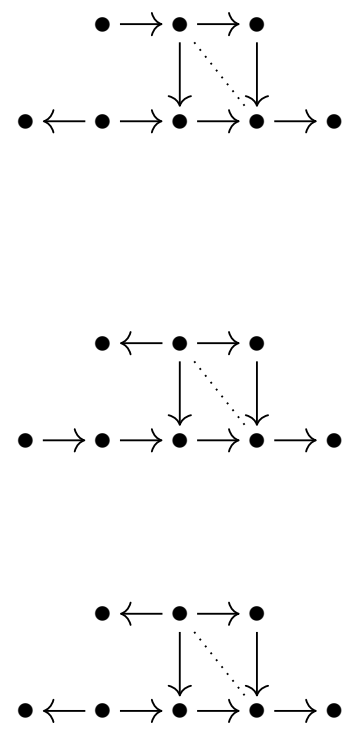

$\mathcal{F} r 14.2$

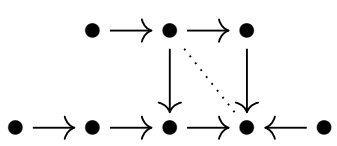

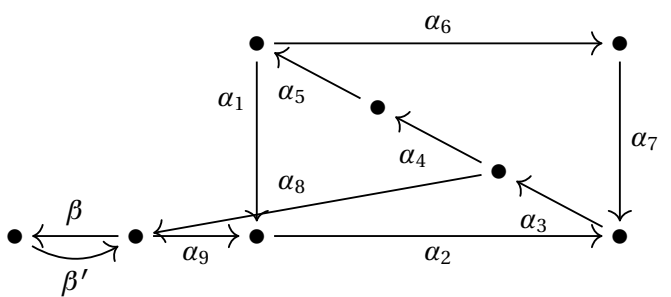
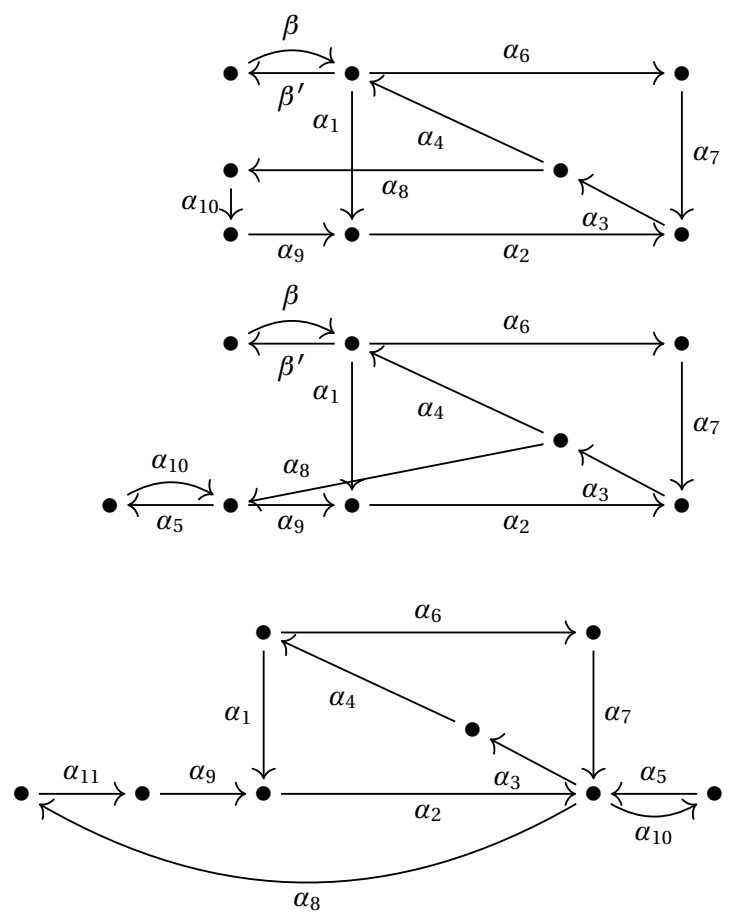

As relações do tipo 2 são: $r 1=\alpha_{2} \alpha_{3} \alpha_{4} \alpha_{6}, r 2=\alpha_{7} \alpha_{3} \alpha_{4} \alpha_{1}, r 3=\alpha_{9} \alpha_{2} \alpha_{3}, r 4=\alpha_{1} \alpha_{2} \alpha_{8}$, $r 5=\alpha_{7} \alpha_{8}, r 6=\alpha_{2} \alpha_{10}, r 7=\alpha_{7} \alpha_{10}, r 8=\alpha_{5} \alpha_{3}$ e $r 9=\alpha_{5} \alpha_{8}$. Os ciclos elementares são: $C_{1}=\alpha_{1} \alpha_{2} \alpha_{3} \alpha_{4}, C_{2}=\alpha_{7} \alpha_{3} \alpha_{4} \alpha_{6}, C_{3}=\alpha_{2} \alpha_{8} \alpha_{11} \alpha_{9}$ e $C_{4}=\alpha_{5} \alpha_{10}$. Portanto, conseguimos o corte $\left\{\alpha_{2}, \alpha_{5}, \alpha_{7}\right\}$ responsável pela solução 23 .

Sabendo dessas respostas, podemos usar uma justificativa análoga de $\mathcal{F} r 13.4$ e $\mathcal{F} r 13.8$ para aplicar o lema 5.42 e eliminar essas possibilidades:
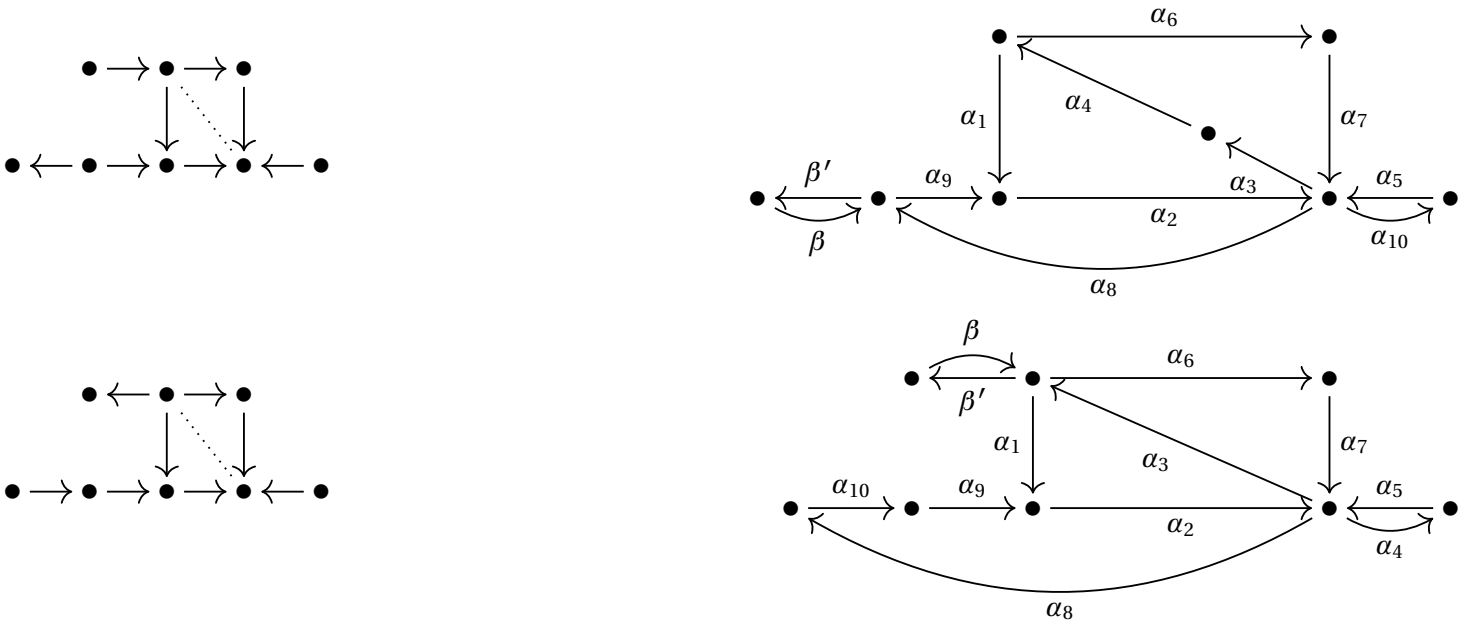

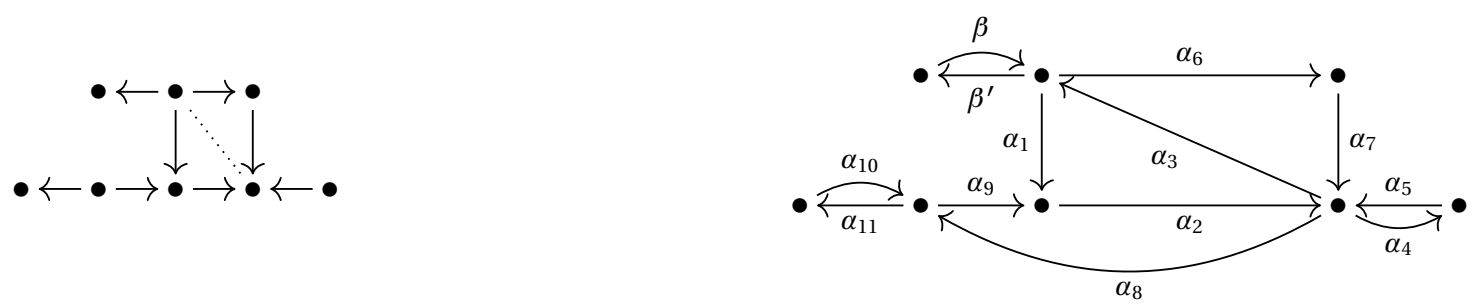

$\mathcal{F} r 14.3$
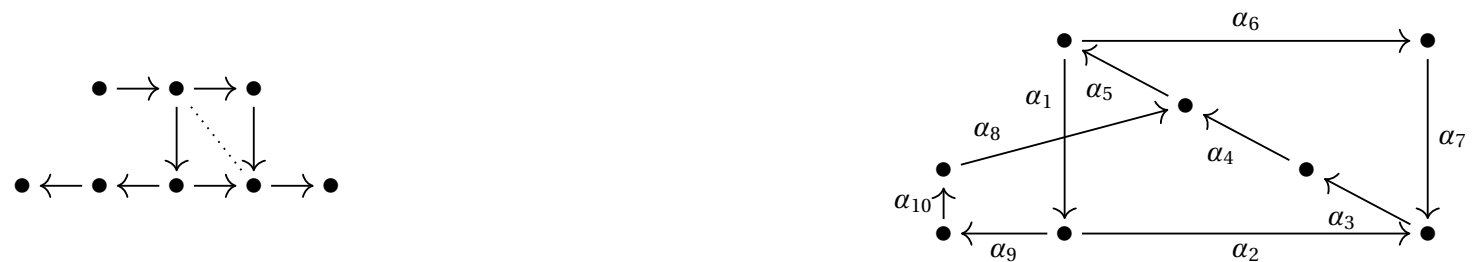

As relações do tipo 2 são: $r 1=\alpha_{2} \alpha_{3} \alpha_{4} \alpha_{5} \alpha_{6}, r 2=\alpha_{7} \alpha_{3} \alpha_{4} \alpha_{5} \alpha_{1}, r 3=\alpha_{8} \alpha_{5} \alpha_{6}$, $r 4=\alpha_{4} \alpha_{5} \alpha_{1} \alpha_{9}$ e $r 5=\alpha_{8} \alpha_{5} \alpha_{1} \alpha_{2}$. Os ciclos elementares são: $C_{1}=\alpha_{1} \alpha_{2} \alpha_{3} \alpha_{4} \alpha_{5}$, $C_{2}=\alpha_{3} \alpha_{4} \alpha_{5} \alpha_{6} \alpha_{7}$ e $C_{3}=\alpha_{5} \alpha_{1} \alpha_{9} \alpha_{10} \alpha_{8}$. O programa nos mostra os cortes $\left\{\alpha_{5}\right\}$ e $\left\{\alpha_{1}, \alpha_{6}\right\}$ que resultam nas Phias 24 e 25 respectivamente.

Utilizando essas respostas e a justificativa análoga aos itens $\mathcal{F} r 13.4$ e $\mathcal{F} r$ 13.8, descartamos esses casos:
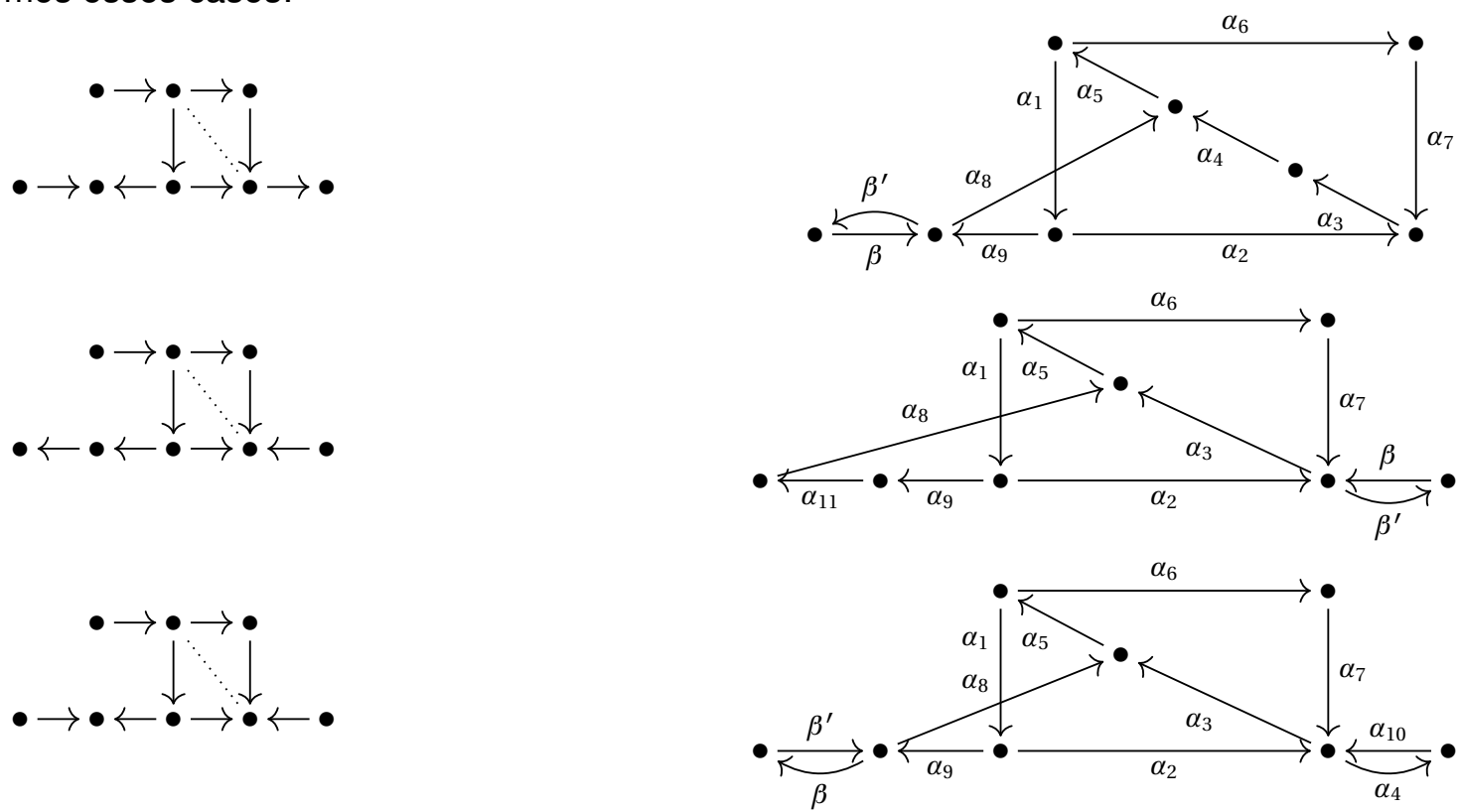

$\mathcal{F} r 14.4$
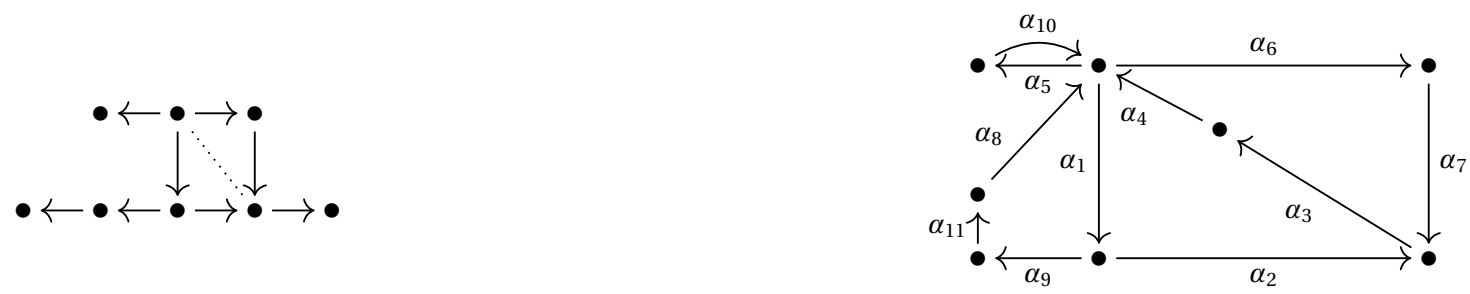

As relações do tipo 2 são: $r 1=\alpha_{2} \alpha_{3} \alpha_{4} \alpha_{6}, r 2=\alpha_{7} \alpha_{3} \alpha_{4} \alpha_{1}, r 3=\alpha_{8} \alpha_{6}, r 4=\alpha_{4} \alpha_{1} \alpha_{9}$, $r 5=\alpha_{8} \alpha_{1} \alpha_{2}, r 6=\alpha_{10} \alpha_{1}, r 7=\alpha_{10} \alpha_{6}, r 8=\alpha_{4} \alpha_{5}$ e $r 9=\alpha_{8} \alpha_{5}$. Os ciclos elementares são: $C_{1}=\alpha_{1} \alpha_{2} \alpha_{3} \alpha_{4}, C_{2}=\alpha_{3} \alpha_{4} \alpha_{6} \alpha_{7}, C_{3}=\alpha_{1} \alpha_{9} \alpha_{11} \alpha_{8}$ e $C_{4}=\alpha_{5} \alpha_{10}$. Portanto, obtemos o corte $\left\{\alpha_{1}, \alpha_{5}, \alpha_{6}\right\}$ que resulta na álgebra 26 .

Sabendo dessa resposta, vamos aplicar o lema 5.42 igual fizemos em $\mathcal{F} r 13.4$ e $\mathcal{F} r 13.8$, 
para eliminar os seguintes casos:
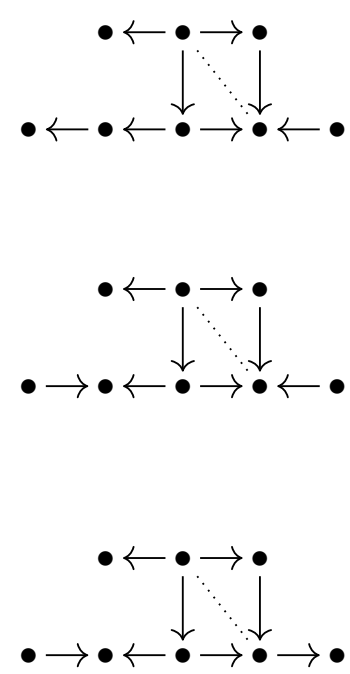

$\mathcal{F} r 14.5$

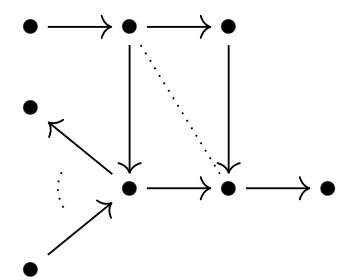

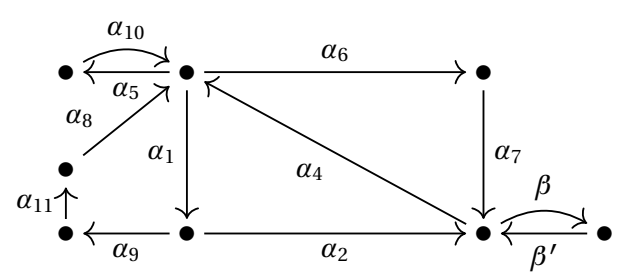
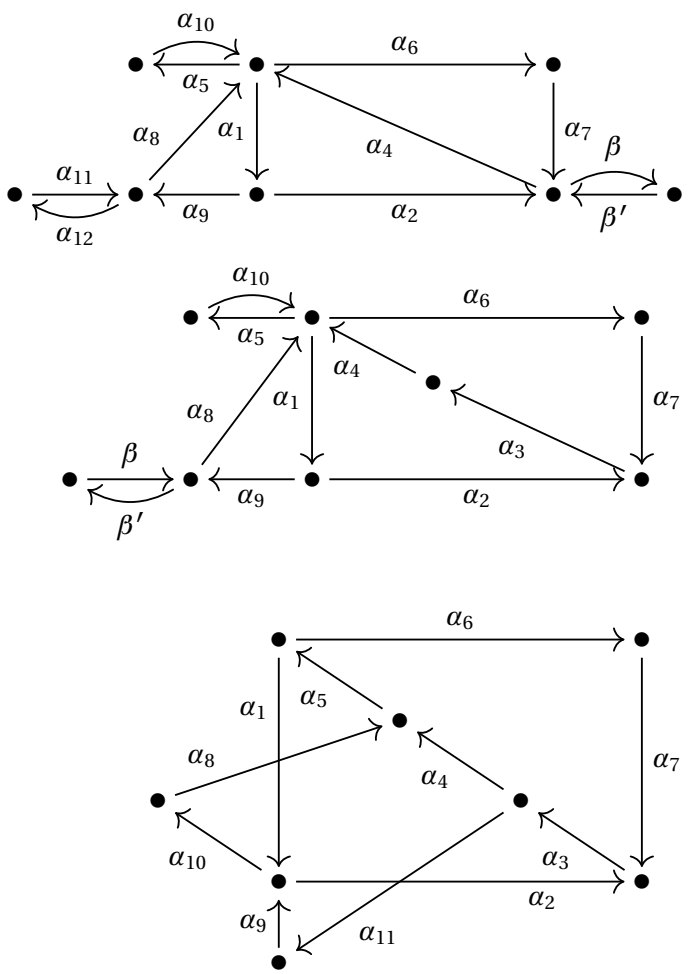

As relações do tipo 2 são: $r 1=\alpha_{2} \alpha_{3} \alpha_{4} \alpha_{5} \alpha_{6}, r 2=\alpha_{7} \alpha_{3} \alpha_{4} \alpha_{5} \alpha_{1}, r 3=\alpha_{8} \alpha_{5} \alpha_{6}, r 4=$ $\alpha_{8} \alpha_{5} \alpha_{1} \alpha_{2}, r 5=\alpha_{4} \alpha_{5} \alpha_{1} \alpha_{10}, r 6=\alpha_{9} \alpha_{2} \alpha_{3} \alpha_{4}, r 7=\alpha_{7} \alpha_{3} \alpha_{11}, r 8=\alpha_{1} \alpha_{2} \alpha_{3} \alpha_{11}$ e $r 9=$ $\alpha_{9} \alpha_{10}$. Os ciclos elementares são: $C_{1}=\alpha_{1} \alpha_{2} \alpha_{3} \alpha_{4} \alpha_{5}, C_{2}=\alpha_{3} \alpha_{4} \alpha_{5} \alpha_{6} \alpha_{7}, C_{3}=\alpha_{5} \alpha_{1} \alpha_{10} \alpha_{8}$ e $C_{4}=\alpha_{9} \alpha_{2} \alpha_{3} \alpha_{11}$. O programa mostra que não tem solução.

$\mathcal{F} r 14.6$
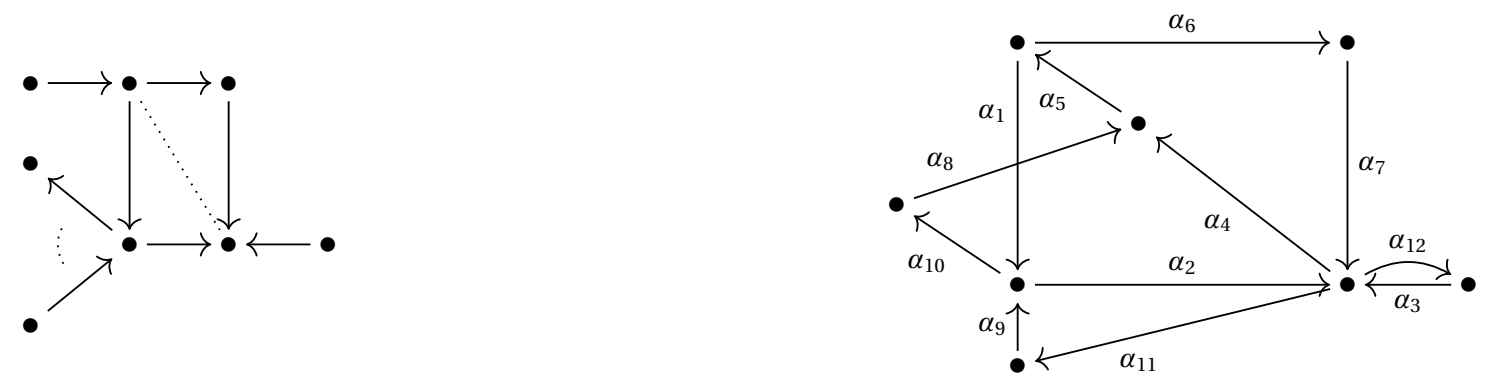

As relações do tipo 2 são: $r 1=\alpha_{2} \alpha_{4} \alpha_{5} \alpha_{6}, r 2=\alpha_{7} \alpha_{4} \alpha_{5} \alpha_{1}, r 3=\alpha_{8} \alpha_{5} \alpha_{6}, r 4=\alpha_{8} \alpha_{5} \alpha_{1} \alpha_{2}$, $r 5=\alpha_{4} \alpha_{5} \alpha_{1} \alpha_{10}, r 6=\alpha_{9} \alpha_{2} \alpha_{4}, r 7=\alpha_{7} \alpha_{11}, r 8=\alpha_{1} \alpha_{2} \alpha_{11}, r 9=\alpha_{9} \alpha_{10}, r 10=\alpha_{2} \alpha_{12}$, $r 11=\alpha_{7} \alpha_{12}, r 12=\alpha_{3} \alpha_{11}$ e $r 13=\alpha_{3} \alpha_{4}$. Os ciclos elementares são: $C_{1}=\alpha_{1} \alpha_{2} \alpha_{4} \alpha_{5}$, $C_{2}=\alpha_{4} \alpha_{5} \alpha_{6} \alpha_{7}, C_{3}=\alpha_{5} \alpha_{1} \alpha_{10} \alpha_{8}, C_{4}=\alpha_{9} \alpha_{2} \alpha_{11}$ e $C_{5}=\alpha_{3} \alpha_{12}$.

Mostraremos que também não tem solução. Seja $\Sigma$ o corte da extensão trivial acima. Vamos dividir em dois casos tendo como referência a eliminação da relação $r 9=\alpha_{9} \alpha_{10}$. $O$ primeiro caso é quando $\alpha_{9} \in \Sigma$. Pela relação $r 12=\alpha_{3} \alpha_{11}$, precisamos ter $\alpha_{3} \in \Sigma$. Então não podemos escolher flecha dos ciclos $C_{4}$ e $C_{5}$. Isso implica que não tem como eliminar a 
relação $r 10=\alpha_{2} \alpha_{12}$, portanto não tem solução nesse caso.

O último caso é se $\alpha_{10} \in \Sigma$. Pela relação $r 10=\alpha_{2} \alpha_{12}$, obtemos que $\alpha_{12} \in \Sigma$. Isso implica que não podemos escolher flecha dos ciclos $C_{3}$ e $C_{5}$. Entretanto, a existência da relação $r 13=\alpha_{3} \alpha_{4}$ é garantida. Portanto, esse corte não resulta em um integrante da família ANS. Logo, provamos que esse caso não tem corte que procuramos. Uma análise análoga é aplicada aos outros dois casos restantes desse frame.
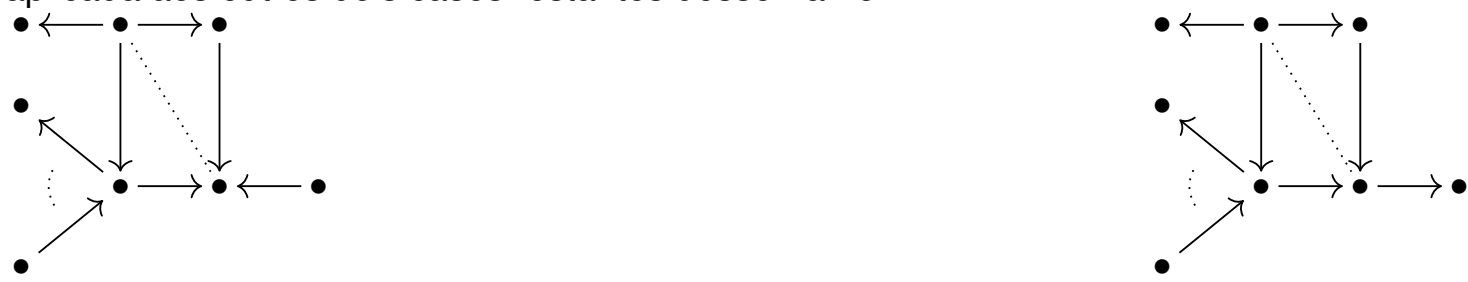

Qualquer operação admissível no frame $\mathcal{F} r 15$ não vai resultar em uma álgebra de incidência. Entretanto é uma álgebra schurian. A seguir, vamos analisar cada caso dos 25 possíveis.

$\mathcal{F} r 15.1$
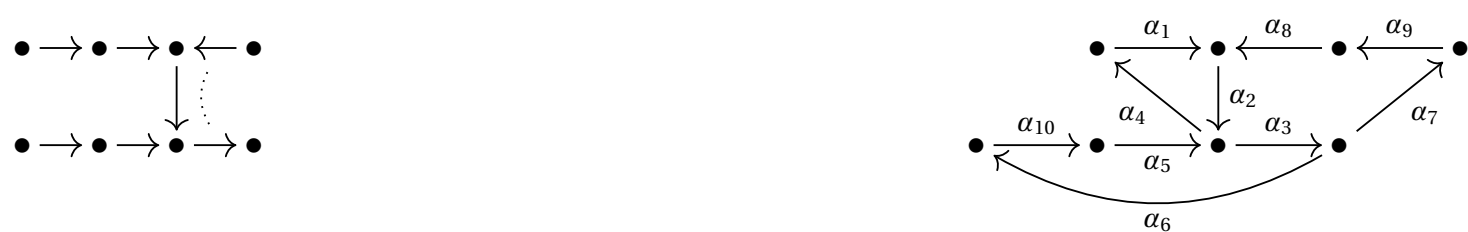

As relações do tipo 2 são: $r 1=\alpha_{1} \alpha_{2} \alpha_{3}, r 2=\alpha_{8} \alpha_{2} \alpha_{4}, r 3=\alpha_{5} \alpha_{4}, r 4=\alpha_{5} \alpha_{3} \alpha_{7}$ e $r 5=$ $\alpha_{2} \alpha_{3} \alpha_{6}$. Os ciclos elementares são: $C_{1}=\alpha_{1} \alpha_{2} \alpha_{4}, C_{2}=\alpha_{2} \alpha_{3} \alpha_{7} \alpha_{9} \alpha_{8}$ e $C_{3}=\alpha_{3} \alpha_{6} \alpha_{10} \alpha_{5}$. Entrando com esses dados no programa, temos os cortes $\left\{\alpha_{2}, \alpha_{5}\right\}$ e $\left\{\alpha_{3}, \alpha_{4}\right\}$ obtendo as soluções 27 e 28 , respectivamente.

Utilizando essa resposta, aplicamos o lema 5.42 como fizemos em $\mathcal{F} r 13.4$ e $\mathcal{F} r 13.8$, e concluímos que os seguintes casos não têm solução:
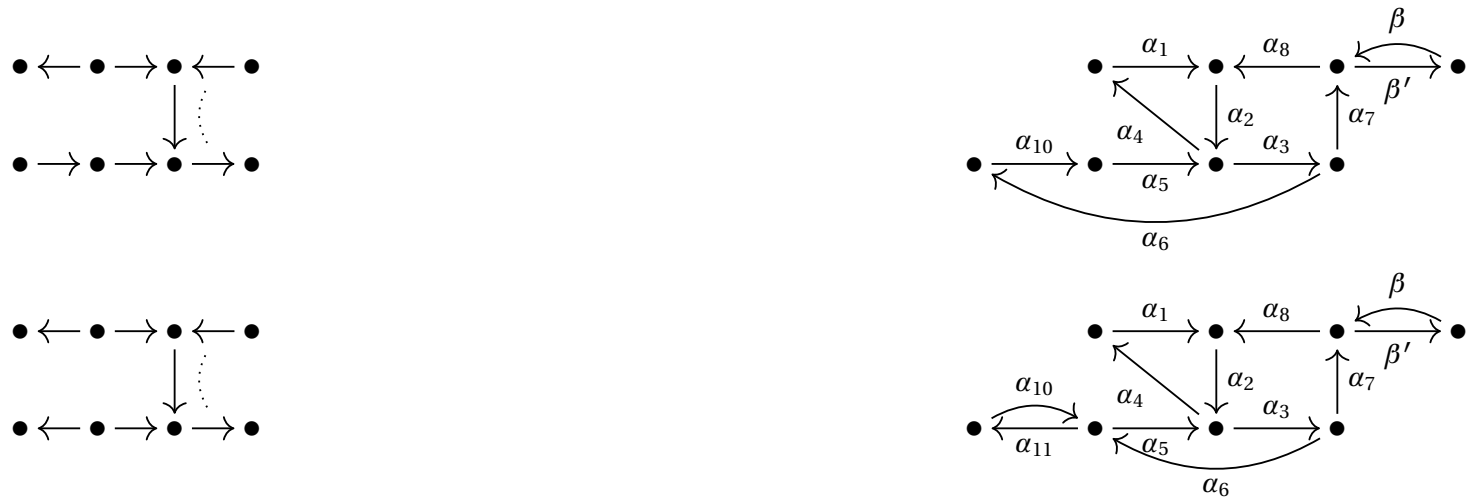

$\mathcal{F} r 15.2$
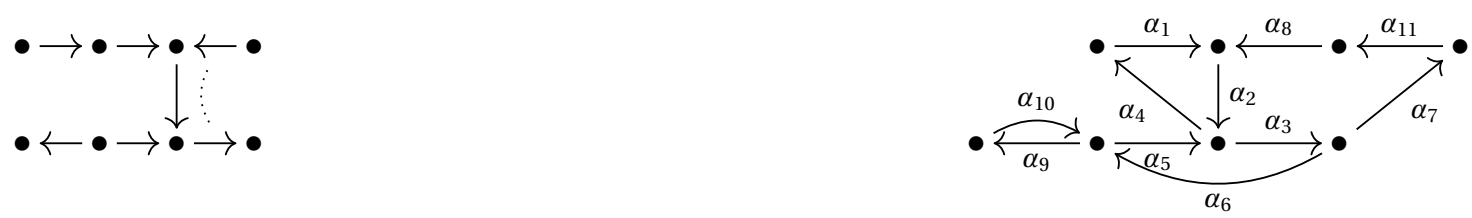

As relações do tipo 2 são: $r 1=\alpha_{1} \alpha_{2} \alpha_{3}, r 2=\alpha_{8} \alpha_{2} \alpha_{4}, r 3=\alpha_{5} \alpha_{4}, r 4=\alpha_{5} \alpha_{3} \alpha_{7}$, $r 5=\alpha_{2} \alpha_{3} \alpha_{6}, r 6=\alpha_{10} \alpha_{5}$ e $r 7=\alpha_{6} \alpha_{9}$. Os ciclos elementares são: $C_{1}=\alpha_{1} \alpha_{2} \alpha_{4}$, $C_{2}=\alpha_{2} \alpha_{3} \alpha_{7} \alpha_{11} \alpha_{8}, C_{3}=\alpha_{3} \alpha_{6} \alpha_{5}$ e $C_{4}=\alpha_{9} \alpha_{10}$. Sendo assim, obtemos o corte $\left\{\alpha_{2}, \alpha_{5}, \alpha_{9}\right\}$ tendo a Phia 29. 
$\mathcal{F} r 15.3$
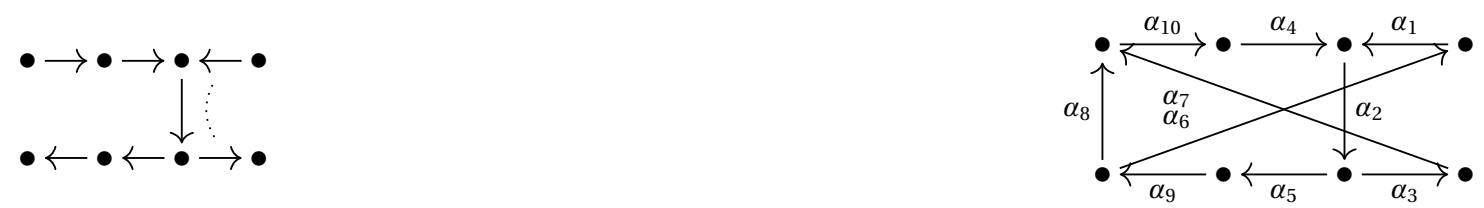

As relações do tipo 2 são: $r 1=\alpha_{1} \alpha_{2} \alpha_{3}, r 2=\alpha_{4} \alpha_{2} \alpha_{5} \alpha_{9} \alpha_{6}, r 3=\alpha_{1} \alpha_{2} \alpha_{5} \alpha_{9} \alpha_{8}$, $r 4=\alpha_{7} \alpha_{10} \alpha_{4} \alpha_{2} \alpha_{5}$ e $r 5=\alpha_{8} \alpha_{10} \alpha_{4} \alpha_{2} \alpha_{3}$. Os ciclos elementares são: $C_{1}=\alpha_{1} \alpha_{2} \alpha_{5} \alpha_{9} \alpha_{6}$, $C_{2}=\alpha_{10} \alpha_{4} \alpha_{2} \alpha_{3} \alpha_{7}$ e $C_{3}=\alpha_{10} \alpha_{4} \alpha_{2} \alpha_{5} \alpha_{9} \alpha_{8}$. Logo, o programa nos mostra os cortes $\left\{\alpha_{2}\right\}$, $\left\{\alpha_{5}, \alpha_{3}\right\}$ e $\left\{\alpha_{1}, \alpha_{4}\right\}$ resultando nas Phias 30,31 e 32 , respectivamente.

$\mathcal{F} r 15.4$
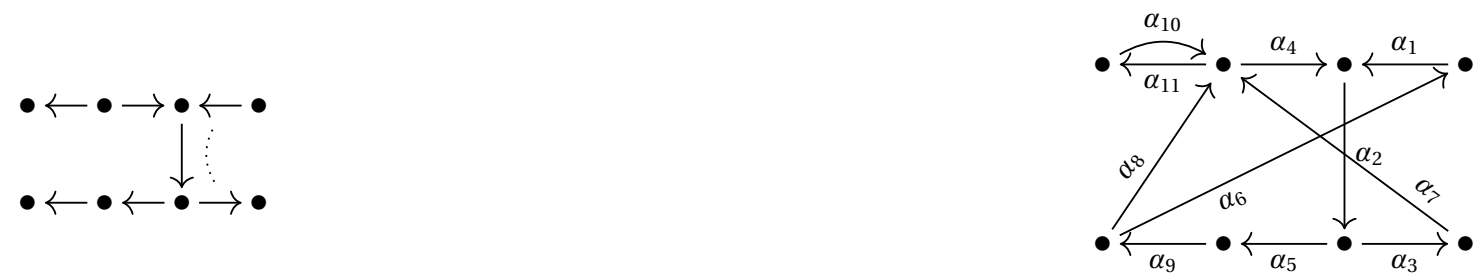

As relações do tipo 2 são: $r 1=\alpha_{1} \alpha_{2} \alpha_{3}, r 2=\alpha_{4} \alpha_{2} \alpha_{5} \alpha_{9} \alpha_{6}, r 3=\alpha_{1} \alpha_{2} \alpha_{5} \alpha_{9} \alpha_{8}, r 4=$ $\alpha_{7} \alpha_{4} \alpha_{2} \alpha_{5}, r 5=\alpha_{8} \alpha_{4} \alpha_{2} \alpha_{3}, r 6=\alpha_{10} \alpha_{4}, r 7=\alpha_{2} \alpha_{11}$ e $r 8=\alpha_{8} \alpha_{11}$. Os ciclos elementares são: $C_{1}=\alpha_{1} \alpha_{2} \alpha_{5} \alpha_{9} \alpha_{6}, C_{2}=\alpha_{4} \alpha_{2} \alpha_{3} \alpha_{7}, C_{3}=\alpha_{4} \alpha_{2} \alpha_{5} \alpha_{9} \alpha_{8}$ e $C_{4}=\alpha_{10} \alpha_{11}$. O programa exibe o corte $\left\{\alpha_{1}, \alpha_{4}, \alpha_{11}\right\}$ resultando na solução 33 .

$\mathcal{F} r 15.5$
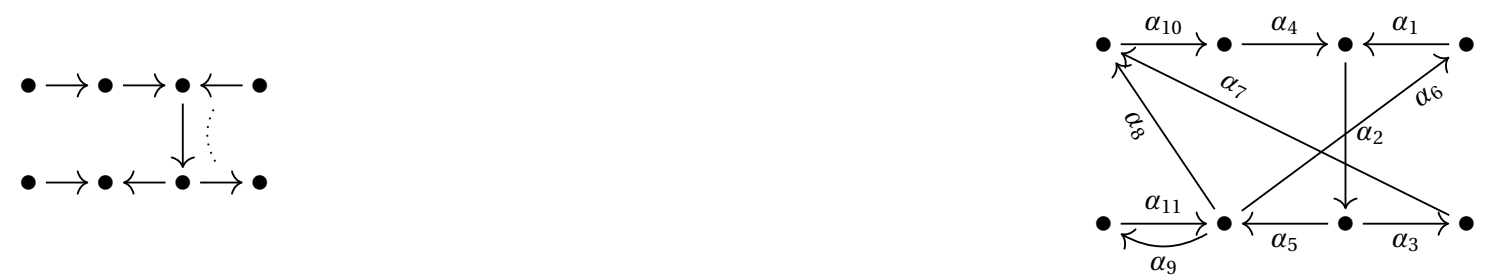

As relações do tipo 2 são: $r 1=\alpha_{1} \alpha_{2} \alpha_{3}, r 2=\alpha_{4} \alpha_{2} \alpha_{5} \alpha_{6}, r 3=\alpha_{1} \alpha_{2} \alpha_{5} \alpha_{8}, r 4=$ $\alpha_{7} \alpha_{10} \alpha_{4} \alpha_{2} \alpha_{5}, r 5=\alpha_{8} \alpha_{10} \alpha_{4} \alpha_{2} \alpha_{3}, r 6=\alpha_{11} \alpha_{8}, r 7=\alpha_{11} \alpha_{6}$ e $r 8=\alpha_{5} \alpha_{9}$. Os ciclos elementares são: $C_{1}=\alpha_{1} \alpha_{2} \alpha_{5} \alpha_{6}, C_{2}=\alpha_{4} \alpha_{2} \alpha_{3} \alpha_{7} \alpha_{10}, C_{3}=\alpha_{4} \alpha_{2} \alpha_{5} \alpha_{8} \alpha_{10}$ e $C_{4}=\alpha_{9} \alpha_{11}$. Consequentemente, obtemos o corte $\left\{\alpha_{5}, \alpha_{3}, \alpha_{11}\right\}$ originando a Phia 34 .

$\mathcal{F} r 15.6$
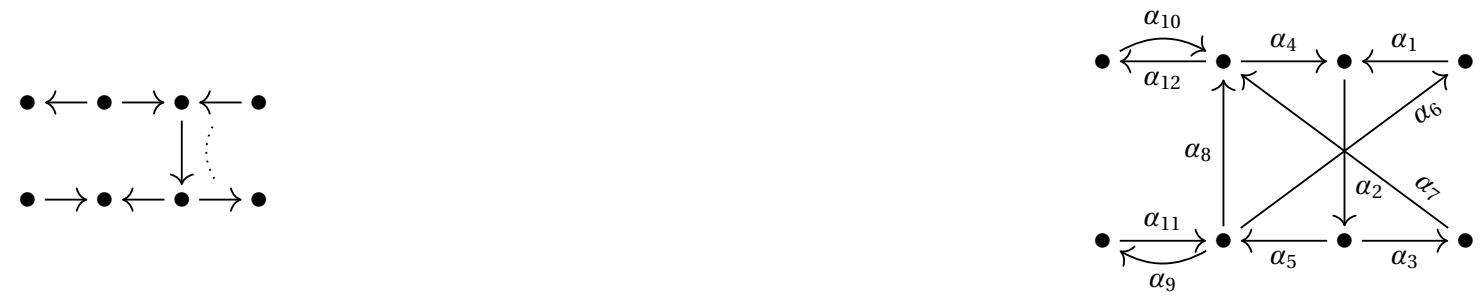

As relações do tipo 2 são: $r 1=\alpha_{1} \alpha_{2} \alpha_{3}, r 2=\alpha_{4} \alpha_{2} \alpha_{5} \alpha_{6}, r 3=\alpha_{1} \alpha_{2} \alpha_{5} \alpha_{8}, r 4=\alpha_{7} \alpha_{4} \alpha_{2} \alpha_{5}$, $r 5=\alpha_{8} \alpha_{4} \alpha_{2} \alpha_{3}, r 6=\alpha_{11} \alpha_{8}, r 7=\alpha_{11} \alpha_{6}, r 8=\alpha_{5} \alpha_{9}, r 9=\alpha_{10} \alpha_{4}, r 10=\alpha_{7} \alpha_{12}$ e $r 11=$ $\alpha_{8} \alpha_{12}$. Os ciclos elementares são: $C_{1}=\alpha_{1} \alpha_{2} \alpha_{5} \alpha_{6}, C_{2}=\alpha_{4} \alpha_{2} \alpha_{3} \alpha_{7}, C_{3}=\alpha_{4} \alpha_{2} \alpha_{5} \alpha_{8}$, $C_{4}=\alpha_{9} \alpha_{11}$ e $C_{5}=\alpha_{10} \alpha_{12}$. Logo, não tem corte que queremos. 
$\mathcal{F} r 15.7$
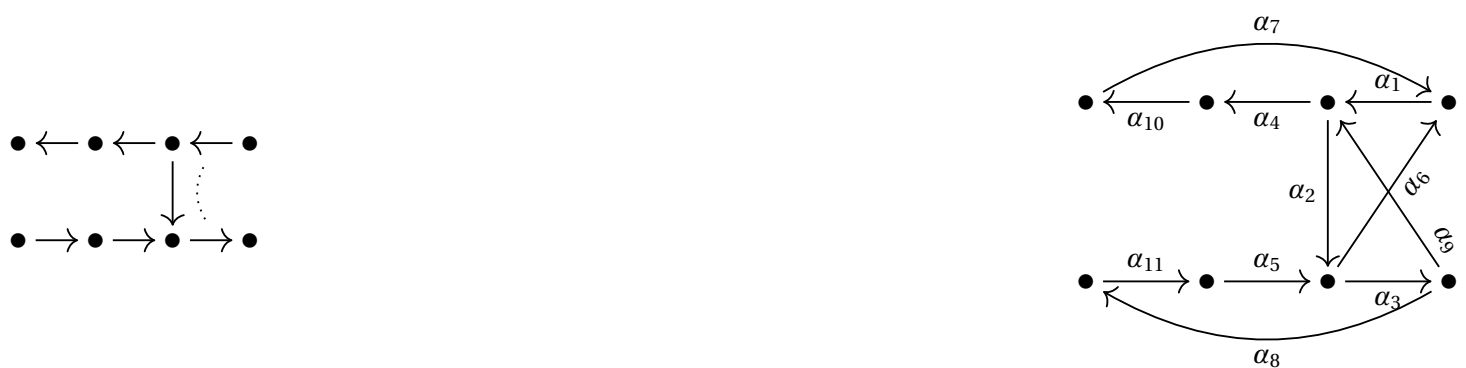

As relações do tipo 2 são: $r 1=\alpha_{1} \alpha_{2} \alpha_{3}, r 2=\alpha_{5} \alpha_{6}, r 3=\alpha_{6} \alpha_{1} \alpha_{4}, r 4=\alpha_{7} \alpha_{1} \alpha_{2}, r 5=$ $\alpha_{9} \alpha_{2} \alpha_{6}, r 6=\alpha_{2} \alpha_{3} \alpha_{8}, r 7=\alpha_{5} \alpha_{3} \alpha_{9}$ e $r 8=\alpha_{9} \alpha_{4}$. Os ciclos elementares são: $C_{1}=\alpha_{1} \alpha_{2} \alpha_{6}$, $C_{2}=\alpha_{1} \alpha_{4} \alpha_{10} \alpha_{7}, C_{3}=\alpha_{11} \alpha_{5} \alpha_{3} \alpha_{8}$ e $C_{4}=\alpha_{2} \alpha_{3} \alpha_{9}$.

Entrando com esses dados no programa, temos a solução 75 através do corte $\left\{\alpha_{2}, \alpha_{4}, \alpha_{5}\right\}$.

$\mathcal{F} r 15.8$
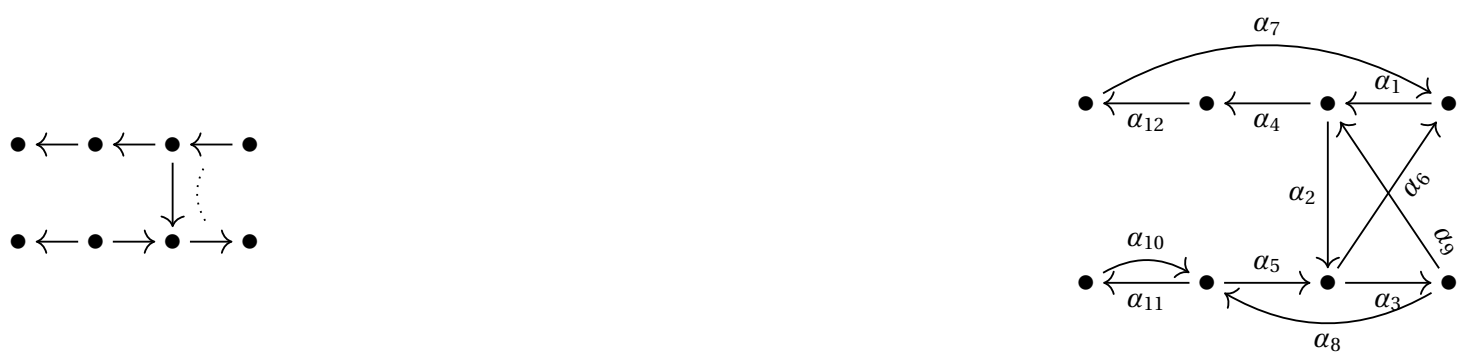

As relações do tipo 2 são: $r 1=\alpha_{1} \alpha_{2} \alpha_{3}, r 2=\alpha_{5} \alpha_{6}, r 3=\alpha_{6} \alpha_{1} \alpha_{4}, r 4=\alpha_{7} \alpha_{1} \alpha_{2}, r 5=$ $\alpha_{9} \alpha_{2} \alpha_{6}, r 6=\alpha_{2} \alpha_{3} \alpha_{8}, r 7=\alpha_{5} \alpha_{3} \alpha_{9}, r 8=\alpha_{9} \alpha_{4}, r 9=\alpha_{8} \alpha_{11}$ e $r 10=\alpha_{10} \alpha_{5}$. Os ciclos elementares são: $C_{1}=\alpha_{1} \alpha_{2} \alpha_{6}, C_{2}=\alpha_{1} \alpha_{4} \alpha_{12} \alpha_{7}, C_{3}=\alpha_{5} \alpha_{3} \alpha_{8}, C_{4}=\alpha_{2} \alpha_{3} \alpha_{9}$ e $C_{5}=$ $\alpha_{11} \alpha_{10}$.

Tem a solução 76 através do corte $\left\{\alpha_{2}, \alpha_{4}, \alpha_{5}, \alpha_{11}\right\}$.

$\mathcal{F} r 15.9$
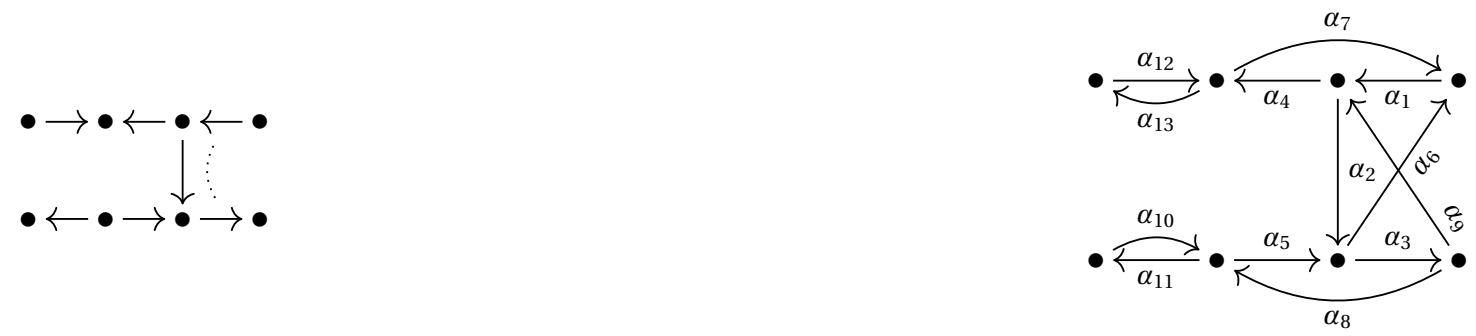

As relações do tipo 2 são: $r 1=\alpha_{1} \alpha_{2} \alpha_{3}, r 2=\alpha_{5} \alpha_{6}, r 3=\alpha_{6} \alpha_{1} \alpha_{4}, r 4=\alpha_{7} \alpha_{1} \alpha_{2}, r 5=$ $\alpha_{9} \alpha_{2} \alpha_{6}, r 6=\alpha_{2} \alpha_{3} \alpha_{8}, r 7=\alpha_{5} \alpha_{3} \alpha_{9}, r 8=\alpha_{9} \alpha_{4}, r 9=\alpha_{8} \alpha_{11}, r 10=\alpha_{10} \alpha_{5}, r 11=\alpha_{4} \alpha_{13}$ e $r 12=\alpha_{12} \alpha_{7}$. Os ciclos elementares são: $C_{1}=\alpha_{1} \alpha_{2} \alpha_{6}, C_{2}=\alpha_{1} \alpha_{4} \alpha_{7}, C_{3}=\alpha_{5} \alpha_{3} \alpha_{8}$, $C_{4}=\alpha_{2} \alpha_{3} \alpha_{9}, C_{5}=\alpha_{11} \alpha_{10}$ e $C_{6}=\alpha_{12} \alpha_{13}$.

Obtemos a Phia 77 através do corte $\left\{\alpha_{2}, \alpha_{4}, \alpha_{5}, \alpha_{11}, \alpha_{12}\right\}$.

$\mathcal{F} r 15.10$
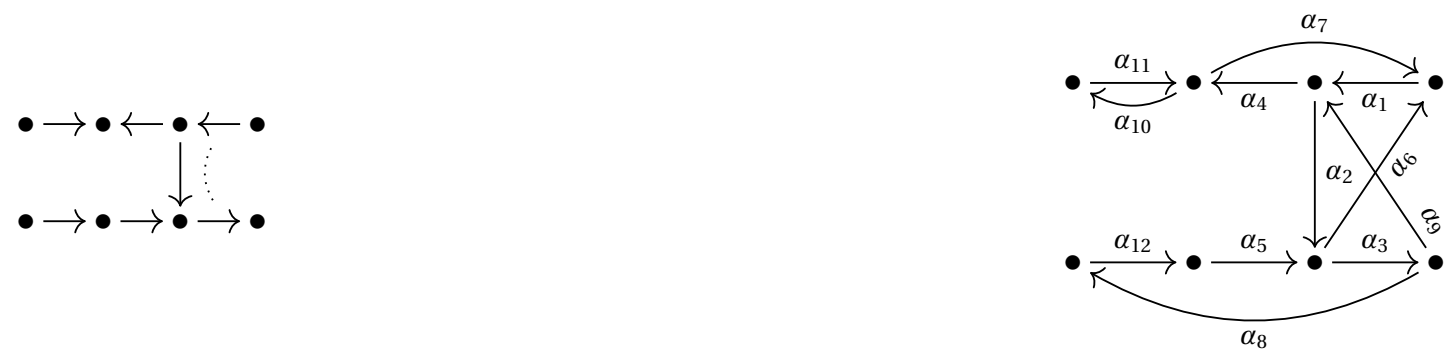
As relações do tipo 2 são: $r 1=\alpha_{1} \alpha_{2} \alpha_{3}, r 2=\alpha_{5} \alpha_{6}, r 3=\alpha_{6} \alpha_{1} \alpha_{4}, r 4=\alpha_{7} \alpha_{1} \alpha_{2}, r 5=$ $\alpha_{9} \alpha_{2} \alpha_{6}, r 6=\alpha_{2} \alpha_{3} \alpha_{8}, r 7=\alpha_{5} \alpha_{3} \alpha_{9}, r 8=\alpha_{9} \alpha_{4}, r 9=\alpha_{4} \alpha_{10}$ e $r 10=\alpha_{11} \alpha_{7}$. Os ciclos elementares são: $C_{1}=\alpha_{1} \alpha_{2} \alpha_{6}, C_{2}=\alpha_{1} \alpha_{4} \alpha_{7}, C_{3}=\alpha_{12} \alpha_{5} \alpha_{3} \alpha_{8}, C_{4}=\alpha_{2} \alpha_{3} \alpha_{9}$ e $C_{5}=$ $\alpha_{11} \alpha_{10}$.

Tem o corte $\left\{\alpha_{2}, \alpha_{4}, \alpha_{5}, \alpha_{11}\right\}$ responsável pela Phia 78 .

$\mathcal{F} r 15.11$
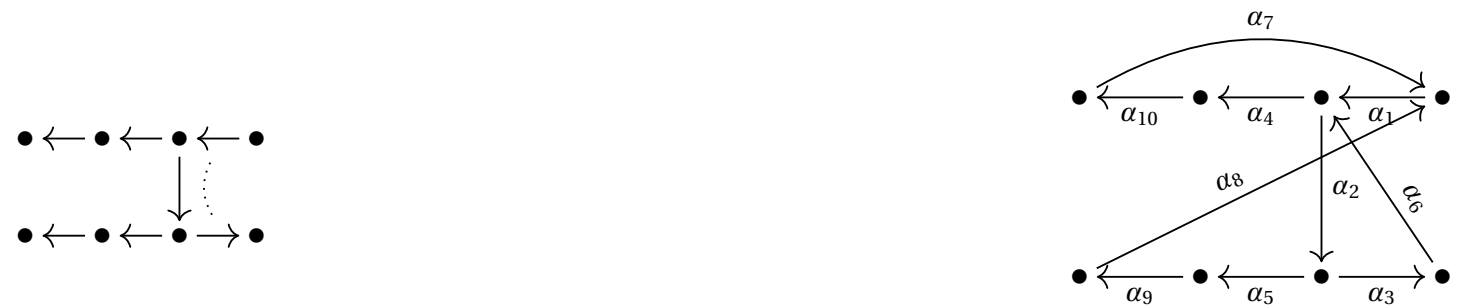

As relações do tipo 2 são: $r 1=\alpha_{8} \alpha_{1} \alpha_{4}, r 2=\alpha_{7} \alpha_{1} \alpha_{2}, r 3=\alpha_{1} \alpha_{2} \alpha_{3}, r 4=\alpha_{6} \alpha_{2} \alpha_{5}$ e $r 5=\alpha_{6} \alpha_{4}$. Os ciclos elementares são: $C_{1}=\alpha_{1} \alpha_{2} \alpha_{5} \alpha_{9} \alpha_{8}, C_{2}=\alpha_{1} \alpha_{4} \alpha_{10} \alpha_{7}$ e $C_{3}=\alpha_{2} \alpha_{3} \alpha_{6}$. Portanto, entrando com esses dados no programa, obtemos dois cortes $\left\{\alpha_{1}, \alpha_{6}\right\}$ e $\left\{\alpha_{4}, \alpha_{2}\right\}$ resultando nas soluções 35 e 36 , respectivamente.

$\mathcal{F} r 15.12$
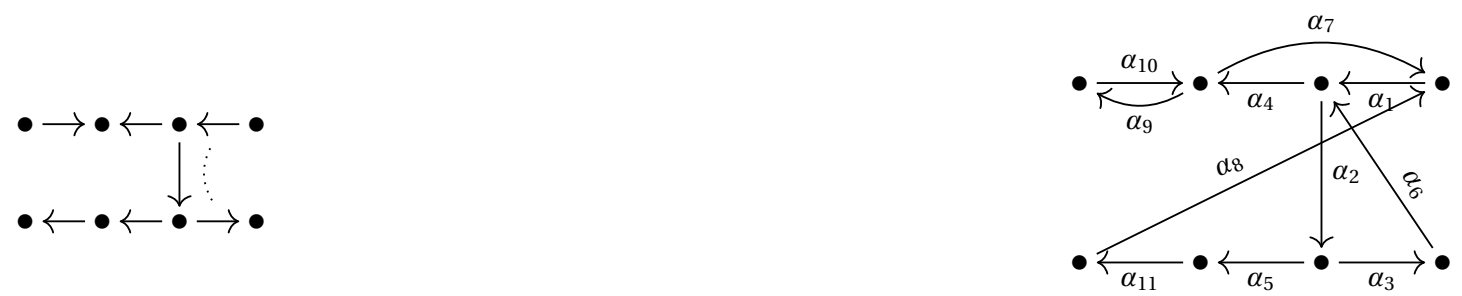

As relações do tipo 2 são: $r 1=\alpha_{8} \alpha_{1} \alpha_{4}, r 2=\alpha_{7} \alpha_{1} \alpha_{2}, r 3=\alpha_{1} \alpha_{2} \alpha_{3}, r 4=\alpha_{6} \alpha_{2} \alpha_{5}$, $r 5=\alpha_{6} \alpha_{4}, r 6=\alpha_{4} \alpha_{9}$ e $r 7=\alpha_{10} \alpha_{7}$. Os ciclos elementares são: $C_{1}=\alpha_{1} \alpha_{2} \alpha_{5} \alpha_{11} \alpha_{8}$, $C_{2}=\alpha_{1} \alpha_{4} \alpha_{7}, C_{3}=\alpha_{2} \alpha_{3} \alpha_{6}$ e $C_{4}=\alpha_{10} \alpha_{9}$. Logo, obtemos o corte $\left\{\alpha_{4}, \alpha_{2}, \alpha_{10}\right\}$ resultando na Phia 37.

$\mathcal{F} r 15.13$
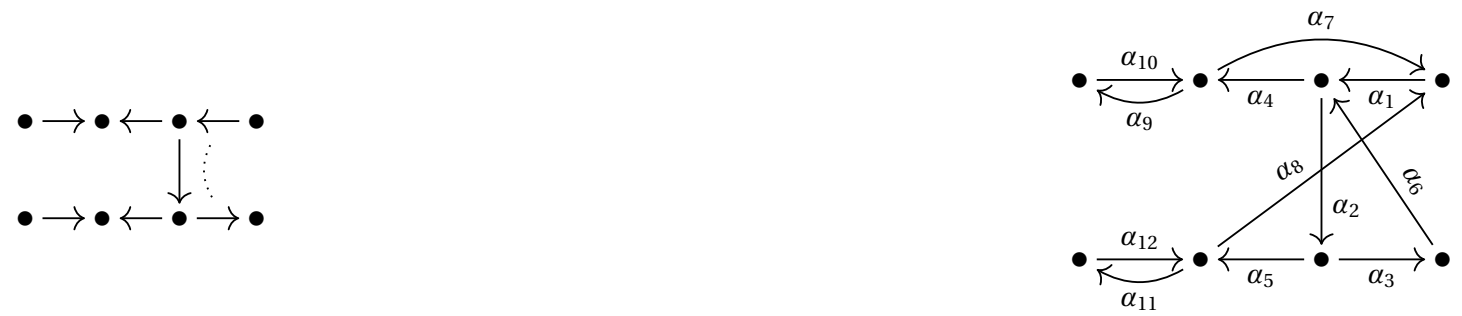

As relações do tipo 2 são: $r 1=\alpha_{8} \alpha_{1} \alpha_{4}, r 2=\alpha_{7} \alpha_{1} \alpha_{2}, r 3=\alpha_{1} \alpha_{2} \alpha_{3}, r 4=\alpha_{6} \alpha_{2} \alpha_{5}$, $r 5=\alpha_{6} \alpha_{4}, r 6=\alpha_{4} \alpha_{9}, r 7=\alpha_{10} \alpha_{7}, r 8=\alpha_{12} \alpha_{8}$ e $r 9=\alpha_{5} \alpha_{11}$. Os ciclos elementares são: $C_{1}=\alpha_{1} \alpha_{2} \alpha_{5} \alpha_{8}, C_{2}=\alpha_{1} \alpha_{4} \alpha_{7}, C_{3}=\alpha_{2} \alpha_{3} \alpha_{6}, C_{4}=\alpha_{10} \alpha_{9}$ e $C_{5}=\alpha_{11} \alpha_{12}$. O programa nos mostra que não tem corte resultando num integrante da família ANS.

$\mathcal{F} r 15.14$
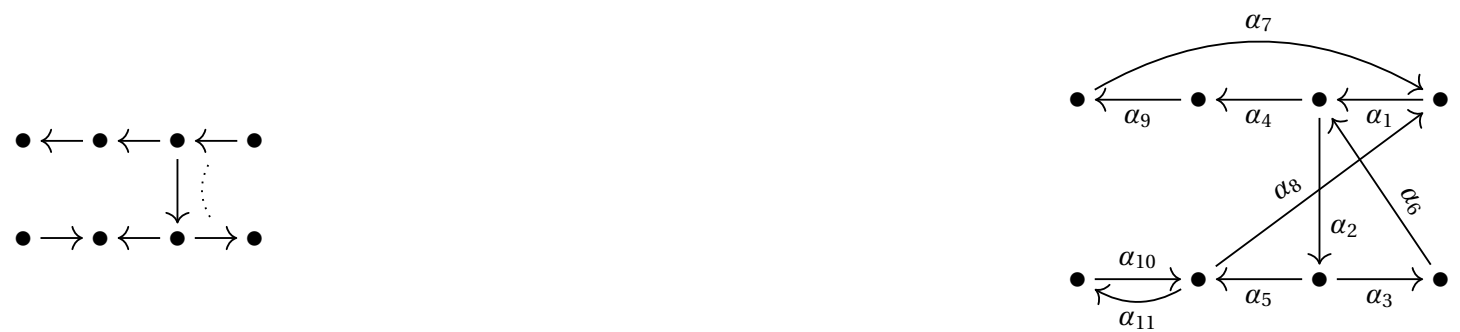
As relações do tipo 2 são: $r 1=\alpha_{8} \alpha_{1} \alpha_{4}, r 2=\alpha_{7} \alpha_{1} \alpha_{2}, r 3=\alpha_{1} \alpha_{2} \alpha_{3}, r 4=\alpha_{6} \alpha_{2} \alpha_{5}$, $r 5=\alpha_{6} \alpha_{4}, r 6=\alpha_{10} \alpha_{8}$ e $r 7=\alpha_{5} \alpha_{9}$. Os ciclos elementares são: $C_{1}=\alpha_{1} \alpha_{2} \alpha_{5} \alpha_{8}, C_{2}=$ $\alpha_{1} \alpha_{4} \alpha_{11} \alpha_{7}, C_{3}=\alpha_{2} \alpha_{3} \alpha_{6}$ e $C_{4}=\alpha_{10} \alpha_{9}$. Portanto, o programa mostra que não tem solução.

$\mathcal{F} r 15.15$
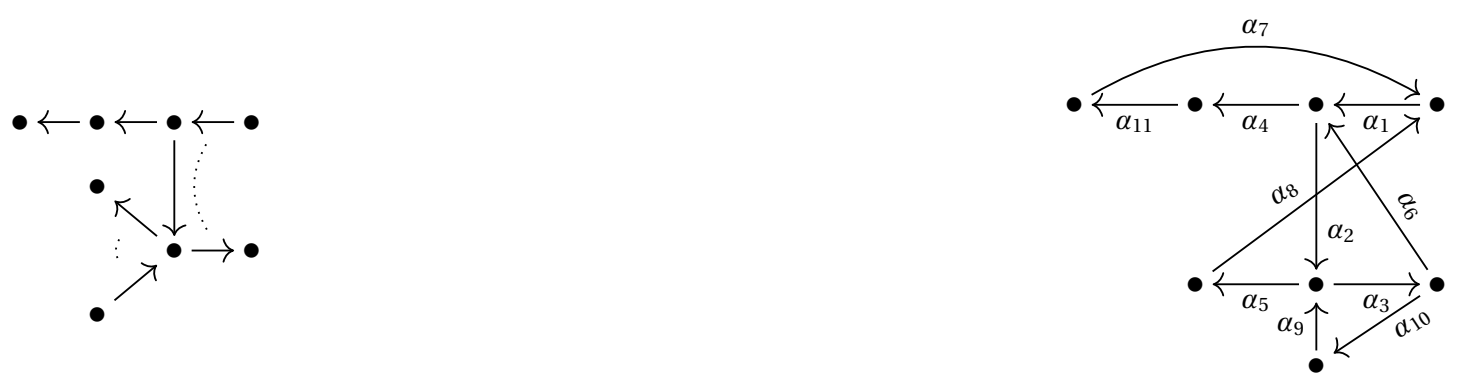

As relações do tipo 2 são: $r 1=\alpha_{8} \alpha_{1} \alpha_{4}, r 2=\alpha_{7} \alpha_{1} \alpha_{2}, r 3=\alpha_{1} \alpha_{2} \alpha_{3}, r 4=\alpha_{6} \alpha_{2} \alpha_{5}$, $r 5=\alpha_{6} \alpha_{4}, r 6=\alpha_{9} \alpha_{5}, r 7=\alpha_{9} \alpha_{3} \alpha_{6}$ e $r 8=\alpha_{2} \alpha_{3} \alpha_{10}$. Os ciclos elementares são: $C_{1}=$ $\alpha_{1} \alpha_{2} \alpha_{5} \alpha_{8}, C_{2}=\alpha_{1} \alpha_{4} \alpha_{11} \alpha_{7}, C_{3}=\alpha_{2} \alpha_{3} \alpha_{6}$ e $C_{4}=\alpha_{10} \alpha_{9} \alpha_{3}$. Consequentemente, obtemos o corte $\left\{\alpha_{2}, \alpha_{4}, \alpha_{9}\right\}$ originando a Phia 38 .

$\mathcal{F} r 15.16$
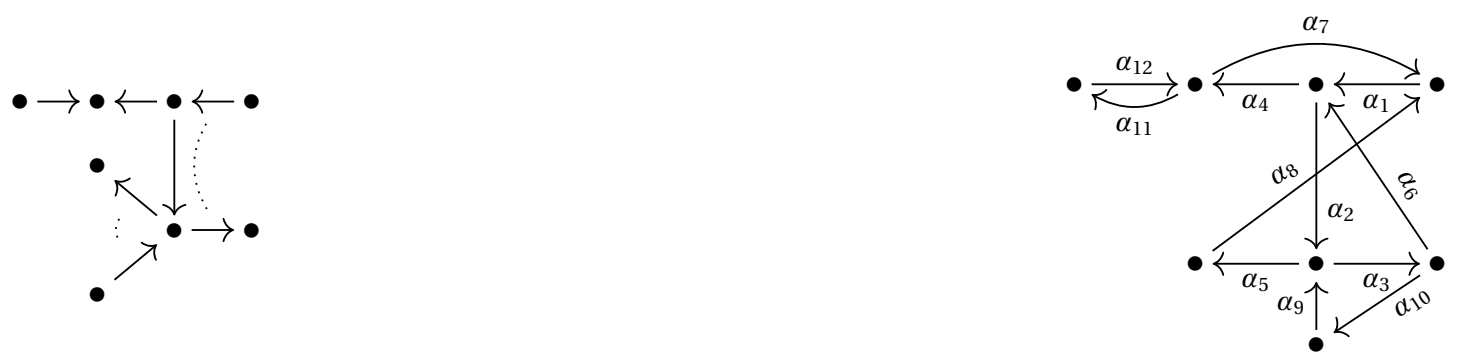

As relações do tipo 2 são: $r 1=\alpha_{8} \alpha_{1} \alpha_{4}, r 2=\alpha_{7} \alpha_{1} \alpha_{2}, r 3=\alpha_{1} \alpha_{2} \alpha_{3}, r 4=\alpha_{6} \alpha_{2} \alpha_{5}$, $r 5=\alpha_{6} \alpha_{4}, r 6=\alpha_{9} \alpha_{5}, r 7=\alpha_{9} \alpha_{3} \alpha_{6}, r 8=\alpha_{2} \alpha_{3} \alpha_{10}, r 9=\alpha_{4} \alpha_{11}$ e $r 10=\alpha_{12} \alpha_{7}$. Os ciclos elementares são: $C_{1}=\alpha_{1} \alpha_{2} \alpha_{5} \alpha_{8}, C_{2}=\alpha_{1} \alpha_{4} \alpha_{7}, C_{3}=\alpha_{2} \alpha_{3} \alpha_{6}, C_{4}=\alpha_{10} \alpha_{9} \alpha_{3}$ e $C_{5}=\alpha_{11} \alpha_{12}$. Sendo assim, temos o corte $\left\{\alpha_{2}, \alpha_{4}, \alpha_{9}\right\}$ resultando na solução 39 .

$\mathcal{F} r 15.17$
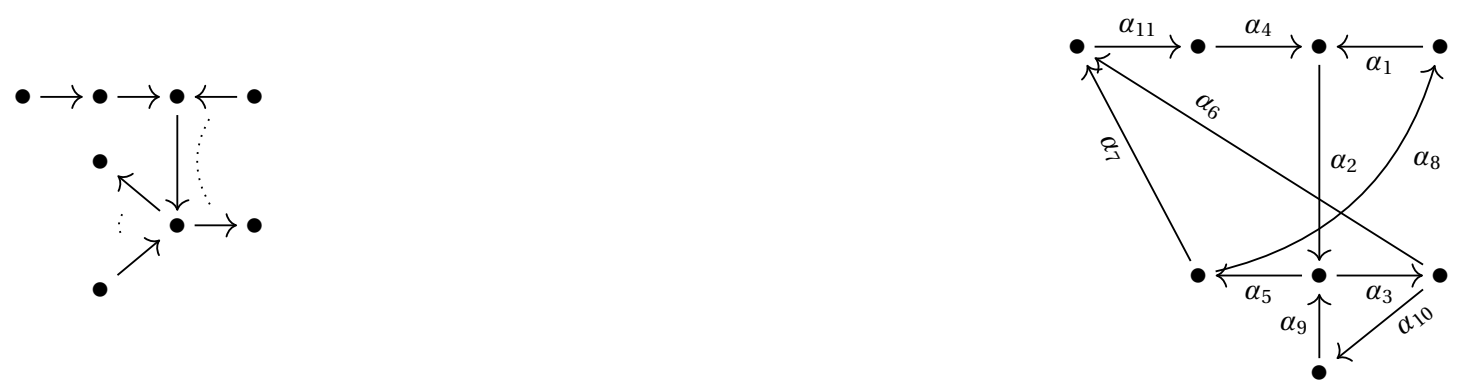

As relações do tipo 2 são: $r 1=\alpha_{1} \alpha_{2} \alpha_{3}, r 2=\alpha_{1} \alpha_{2} \alpha_{5} \alpha_{7}, r 3=\alpha_{4} \alpha_{2} \alpha_{5} \alpha_{8}, r 4=$ $\alpha_{7} \alpha_{11} \alpha_{4} \alpha_{2} \alpha_{3}, r 5=\alpha_{2} \alpha_{3} \alpha_{10}, r 6=\alpha_{6} \alpha_{11} \alpha_{4} \alpha_{2} \alpha_{5}, r 7=\alpha_{9} \alpha_{5}$ e $r 8=\alpha_{9} \alpha_{3} \alpha_{6}$. Os ciclos elementares são: $C_{1}=\alpha_{1} \alpha_{2} \alpha_{5} \alpha_{8}, C_{2}=\alpha_{11} \alpha_{4} \alpha_{2} \alpha_{5} \alpha_{7}, C_{3}=\alpha_{11} \alpha_{4} \alpha_{2} \alpha_{3} \alpha_{6}$ e $C_{4}=\alpha_{9} \alpha_{3} \alpha_{10}$. Logo, temos os cortes $\left\{\alpha_{2} \alpha_{9}\right\}$ e $\left\{\alpha_{3} \alpha_{5}\right\}$ obtendo as soluções 40 e 41 . 
$\mathcal{F} r 15.18$
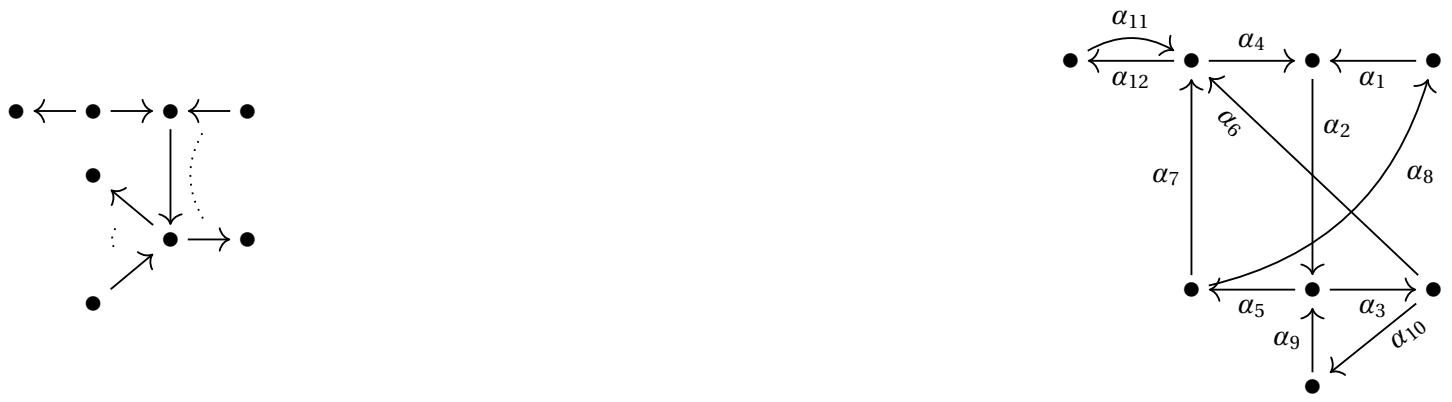

As relações do tipo 2 são: $r 1=\alpha_{1} \alpha_{2} \alpha_{3}, r 2=\alpha_{1} \alpha_{2} \alpha_{5} \alpha_{7}, r 3=\alpha_{4} \alpha_{2} \alpha_{5} \alpha_{8}, r 4=\alpha_{7} \alpha_{4} \alpha_{2} \alpha_{3}$, $r 5=\alpha_{2} \alpha_{3} \alpha_{10}, r 6=\alpha_{6} \alpha_{4} \alpha_{2} \alpha_{5}, r 7=\alpha_{9} \alpha_{5}, r 8=\alpha_{9} \alpha_{3} \alpha_{6}, r 9=\alpha_{11} \alpha_{4}, r 10=\alpha_{7} \alpha_{12} \mathrm{e}$ $r 11=\alpha_{6} \alpha_{12}$. Os ciclos elementares são: $C_{1}=\alpha_{1} \alpha_{2} \alpha_{5} \alpha_{8}, C_{2}=\alpha_{4} \alpha_{2} \alpha_{5} \alpha_{7}, C_{3}=\alpha_{4} \alpha_{2} \alpha_{3} \alpha_{6}$, $C_{4}=\alpha_{9} \alpha_{3} \alpha_{10}$ e $C_{5}=\alpha_{11} \alpha_{12}$. Entrando com essas informações no programa, não temos solução.

$\mathcal{F} r 15.19$
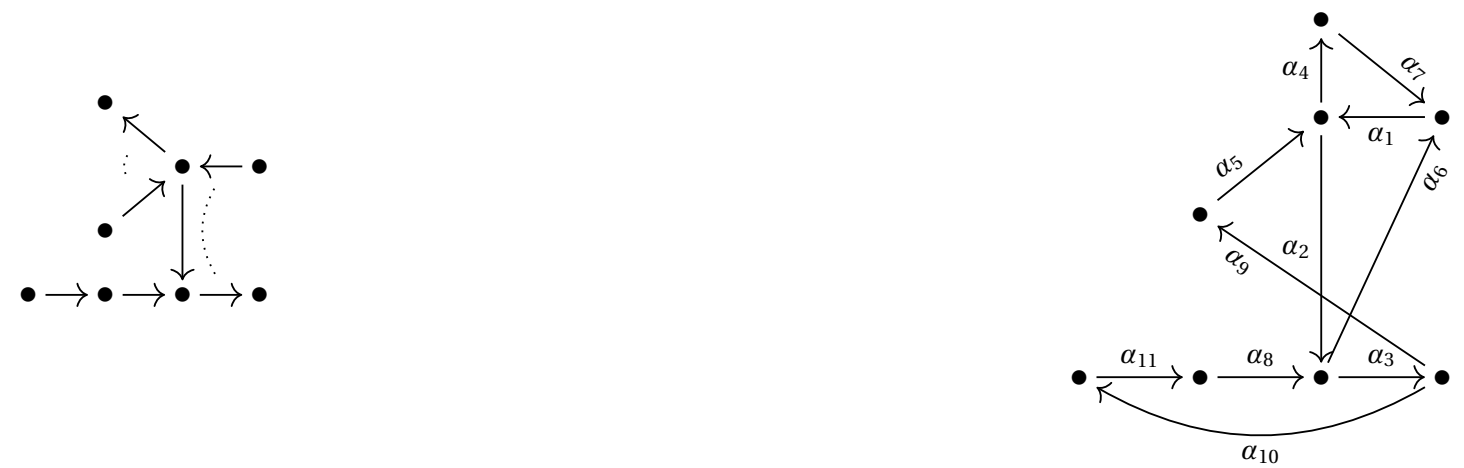

As relações do tipo 2 são: $r 1=\alpha_{7} \alpha_{1} \alpha_{2}, r 2=\alpha_{6} \alpha_{1} \alpha_{4}, r 3=\alpha_{5} \alpha_{4}, r 4=\alpha_{5} \alpha_{2} \alpha_{6}, r 5=$ $\alpha_{2} \alpha_{3} \alpha_{10}, r 6=\alpha_{1} \alpha_{2} \alpha_{3}, r 7=\alpha_{8} \alpha_{6}$ e $r 8=\alpha_{8} \alpha_{3} \alpha_{9}$. Os ciclos elementares são: $C_{1}=$ $\alpha_{1} \alpha_{4} \alpha_{7}, C_{2}=\alpha_{5} \alpha_{2} \alpha_{3} \alpha_{9}, C_{3}=\alpha_{1} \alpha_{2} \alpha_{6}$ e $C_{4}=\alpha_{11} \alpha_{8} \alpha_{3} \alpha_{10}$. Consequentemente, essa extensão trivial nos trouxe o corte $\left\{\alpha_{2}, \alpha_{4}, \alpha_{8}\right\}$ que resulta na Phia 42.

$\mathcal{F} r 15.20$
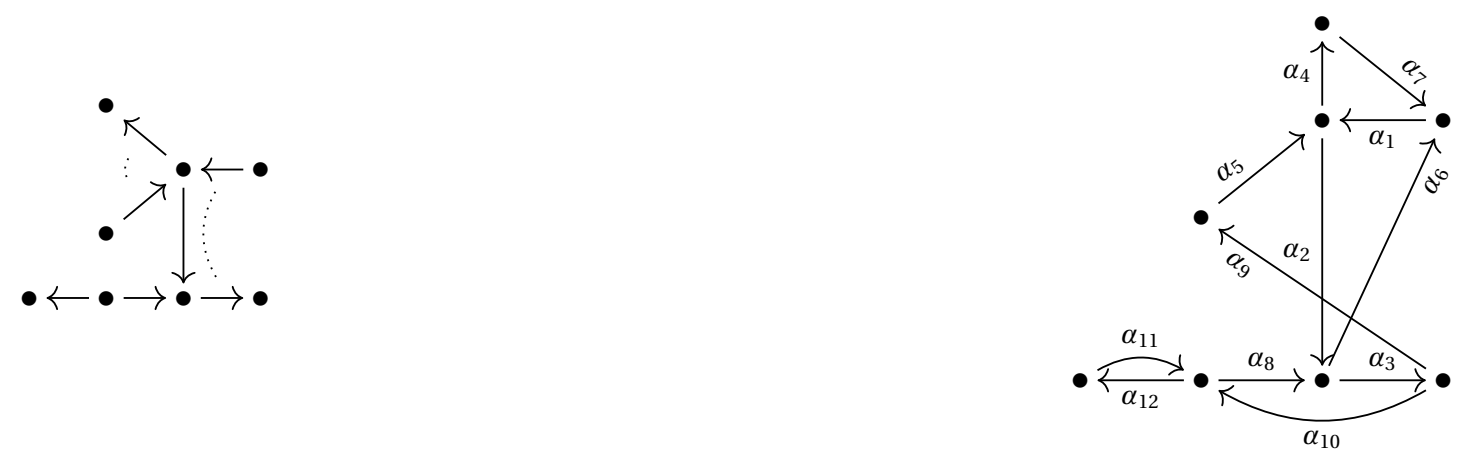

As relações do tipo 2 são: $r 1=\alpha_{7} \alpha_{1} \alpha_{2}, r 2=\alpha_{6} \alpha_{1} \alpha_{4}, r 3=\alpha_{5} \alpha_{4}, r 4=\alpha_{5} \alpha_{2} \alpha_{6}, r 5=$ $\alpha_{2} \alpha_{3} \alpha_{10}, r 6=\alpha_{1} \alpha_{2} \alpha_{3}, r 7=\alpha_{8} \alpha_{6}, r 8=\alpha_{8} \alpha_{3} \alpha_{9}, r 9=\alpha_{11} \alpha_{8}$ e $r 10=\alpha_{10} \alpha_{12}$. Os ciclos elementares são: $C_{1}=\alpha_{1} \alpha_{4} \alpha_{7}, C_{2}=\alpha_{5} \alpha_{2} \alpha_{3} \alpha_{9}, C_{3}=\alpha_{1} \alpha_{2} \alpha_{6}, C_{4}=\alpha_{8} \alpha_{3} \alpha_{10}$ e $C_{5}=\alpha_{11} \alpha_{12}$. Portanto, o programa exibe o corte $\left\{\alpha_{2}, \alpha_{4}, \alpha_{8} \alpha_{12}\right\}$ que resulta na Phia 43. 
$\mathcal{F} r 15.21$
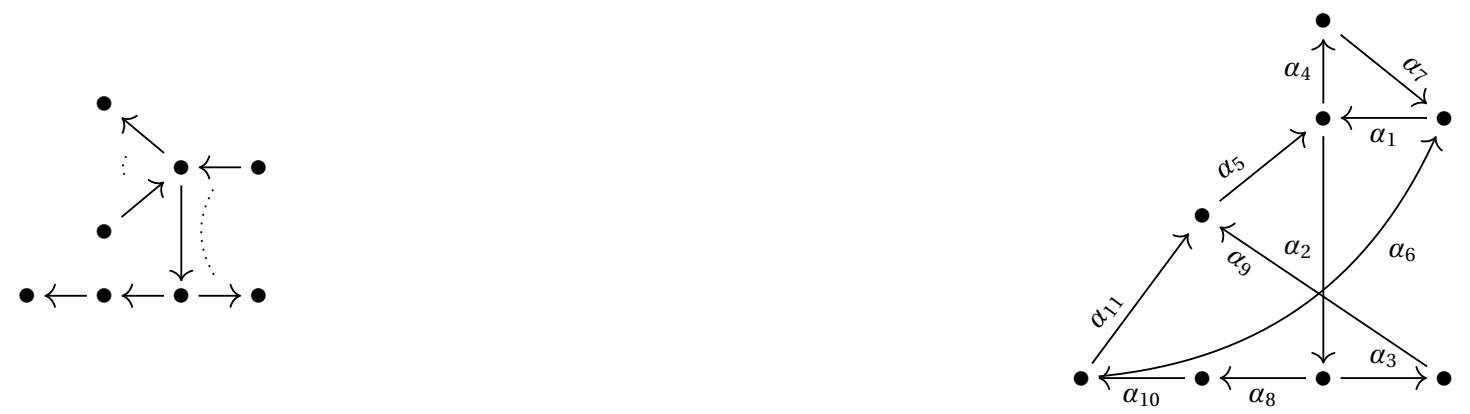

As relações do tipo 2 são: $r 1=\alpha_{7} \alpha_{1} \alpha_{2}, r 2=\alpha_{6} \alpha_{1} \alpha_{4}, r 3=\alpha_{5} \alpha_{4}, r 4=\alpha_{9} \alpha_{5} \alpha_{2} \alpha_{8}, r 5=$ $\alpha_{1} \alpha_{2} \alpha_{8} \alpha_{10} \alpha_{11}, r 6=\alpha_{1} \alpha_{2} \alpha_{3}, r 7=\alpha_{11} \alpha_{5} \alpha_{2} \alpha_{3}$ e $r 8=\alpha_{5} \alpha_{2} \alpha_{8} \alpha_{10} \alpha_{6}$.Os ciclos elementares são: $C_{1}=\alpha_{1} \alpha_{4} \alpha_{7}, C_{2}=\alpha_{5} \alpha_{2} \alpha_{3} \alpha_{9}, C_{3}=\alpha_{1} \alpha_{2} \alpha_{8} \alpha_{10} \alpha_{6}$ e $C_{4}=\alpha_{5} \alpha_{2} \alpha_{8} \alpha_{10} \alpha_{11}$.

Sendo assim, temos os cortes $\left\{\alpha_{2}, \alpha_{4}\right\}$ e $\left\{\alpha_{1}, \alpha_{5}\right\}$ que originam as soluções 44 e 45 , respectivamente.

$\mathcal{F} r 15.22$
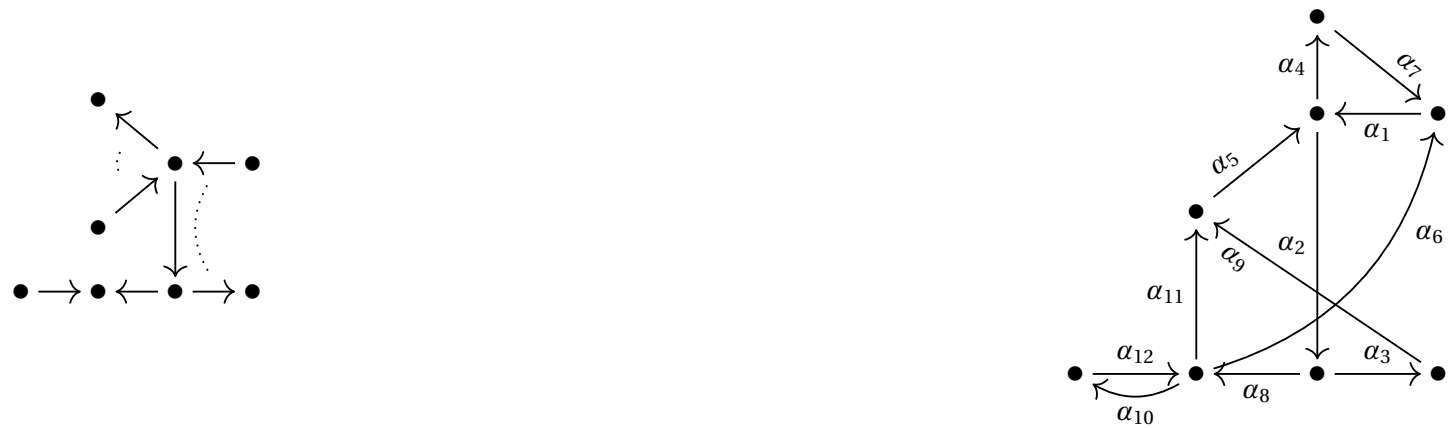

As relações do tipo 2 são: $r 1=\alpha_{7} \alpha_{1} \alpha_{2}, r 2=\alpha_{6} \alpha_{1} \alpha_{4}, r 3=\alpha_{5} \alpha_{4}, r 4=\alpha_{9} \alpha_{5} \alpha_{2} \alpha_{8}, r 5=$ $\alpha_{1} \alpha_{2} \alpha_{8} \alpha_{11}, r 6=\alpha_{1} \alpha_{2} \alpha_{3}, r 7=\alpha_{11} \alpha_{5} \alpha_{2} \alpha_{3}, r 8=\alpha_{5} \alpha_{2} \alpha_{8} \alpha_{6}, r 9=\alpha_{12} \alpha_{11}, r 10=\alpha_{12} \alpha_{6} \mathrm{e}$ $r 11=\alpha_{8} \alpha_{10}$. Os ciclos elementares são: $C_{1}=\alpha_{1} \alpha_{4} \alpha_{7}, C_{2}=\alpha_{5} \alpha_{2} \alpha_{3} \alpha_{9}, C_{3}=\alpha_{1} \alpha_{2} \alpha_{8} \alpha_{6}$, $C_{4}=\alpha_{5} \alpha_{2} \alpha_{8} \alpha_{11}$ e $C_{5}=\alpha_{12} \alpha_{10}$. Essa extensão trivial não tem corte que procuramos.

$\mathcal{F} r 15.23$
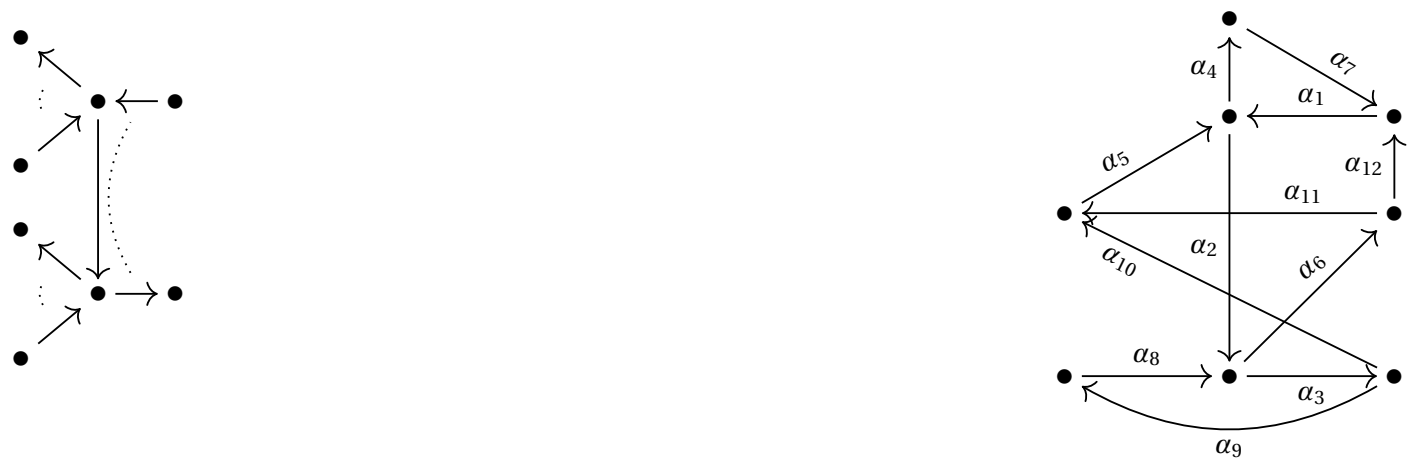

As relações do tipo 2 são: $r 1=\alpha_{5} \alpha_{4}, r 2=\alpha_{8} \alpha_{6}, r 3=\alpha_{7} \alpha_{1} \alpha_{2}, r 4=\alpha_{2} \alpha_{3} \alpha_{9}, r 5=$ $\alpha_{12} \alpha_{1} \alpha_{4}, r 6=\alpha_{1} \alpha_{2} \alpha_{3}, r 7=\alpha_{1} \alpha_{2} \alpha_{6} \alpha_{11}, r 8=\alpha_{11} \alpha_{5} \alpha_{2} \alpha_{3}, r 9=\alpha_{5} \alpha_{2} \alpha_{6} \alpha_{12}$ e $r 10=$ $\alpha_{8} \alpha_{3} \alpha_{10}$. Os ciclos elementares são: $C_{1}=\alpha_{1} \alpha_{4} \alpha_{7}, C_{2}=\alpha_{5} \alpha_{2} \alpha_{3} \alpha_{10}, C_{3}=\alpha_{1} \alpha_{2} \alpha_{6} \alpha_{12}, C_{4}=$ $\alpha_{5} \alpha_{2} \alpha_{6} \alpha_{11}$ e $C_{5}=\alpha_{8} \alpha_{3} \alpha_{9}$. Consequentemente, obtemos o corte $\left\{\alpha_{2}, \alpha_{4}, \alpha_{8}\right\}$ originando a Phia 46.

O frame $\mathcal{F} r 16$ tem uma aresta a menos que os três anteriores, com apenas uma possibilidade de operação admissível 2. Calculando todas as combinações, temos 10 casos para estudar. 
$\mathcal{F} r 16.1$
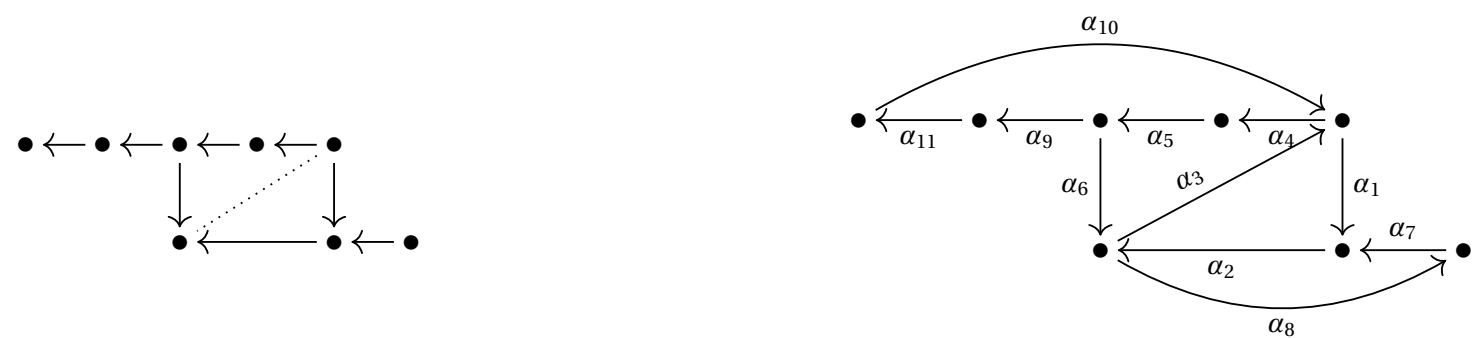

As relações do tipo 2 são: $r 1=\alpha_{2} \alpha_{3} \alpha_{4}, r 2=\alpha_{1} \alpha_{2} \alpha_{8}, r 3=\alpha_{6} \alpha_{3} \alpha_{1}, r 4=\alpha_{6} \alpha_{8}, r 5=$ $\alpha_{3} \alpha_{4} \alpha_{5} \alpha_{9}, r 6=\alpha_{7} \alpha_{2} \alpha_{3}, r 7=\alpha_{10} \alpha_{4} \alpha_{5} \alpha_{6}$ e $r 8=\alpha_{10} \alpha_{1}$. Os ciclos elementares são: $C_{1}=\alpha_{1} \alpha_{2} \alpha_{3}, C_{2}=\alpha_{3} \alpha_{4} \alpha_{5} \alpha_{6}, C_{3}=\alpha_{7} \alpha_{2} \alpha_{8}$ e $C_{4}=\alpha_{4} \alpha_{5} \alpha_{9} \alpha_{11} \alpha_{10}$. Sendo assim, o programa exibe apenas o corte $\left\{\alpha_{3}, \alpha_{8}, \alpha_{10}\right\}$ que origina a álgebra disfarçada.

Utilizando essa resposta, podemos usar analogamente no caso seguinte e também descartá-lo:
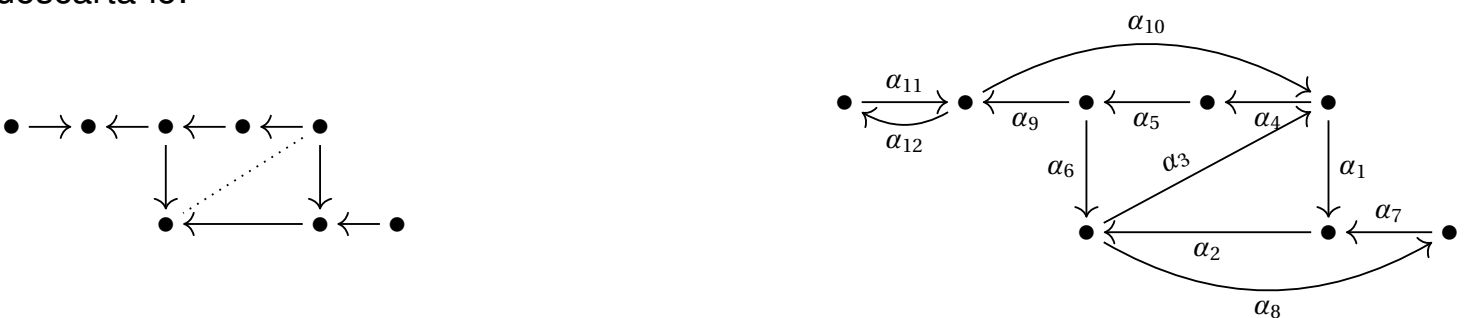

$\mathcal{F} r 16.2$
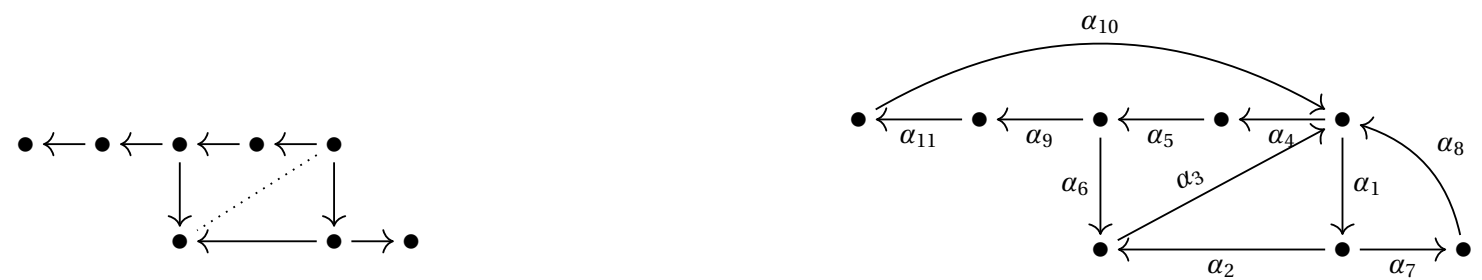

As relações do tipo 2 são: $r 1=\alpha_{2} \alpha_{3} \alpha_{4}, r 2=\alpha_{3} \alpha_{1} \alpha_{7}, r 3=\alpha_{6} \alpha_{3} \alpha_{1}, r 4=\alpha_{8} \alpha_{4}, r 5=$ $\alpha_{3} \alpha_{4} \alpha_{5} \alpha_{9}, r 6=\alpha_{8} \alpha_{1} \alpha_{2}, r 7=\alpha_{10} \alpha_{4} \alpha_{5} \alpha_{6}$ e $r 8=\alpha_{10} \alpha_{1}$. Os ciclos elementares são: $C_{1}=\alpha_{1} \alpha_{2} \alpha_{3}, C_{2}=\alpha_{3} \alpha_{4} \alpha_{5} \alpha_{6}, C_{3}=\alpha_{7} \alpha_{8} \alpha_{1}$ e $C_{4}=\alpha_{4} \alpha_{5} \alpha_{9} \alpha_{11} \alpha_{10}$. Informando esses dados ao programa, obtemos que existe o corte $\left\{\alpha_{1}, \alpha_{4}\right\}$ resultando na solução 47 .

Essa resposta e o lema 5.42 implicam que podemos eliminar o caso seguinte:

$\mathcal{F} r 16.3$
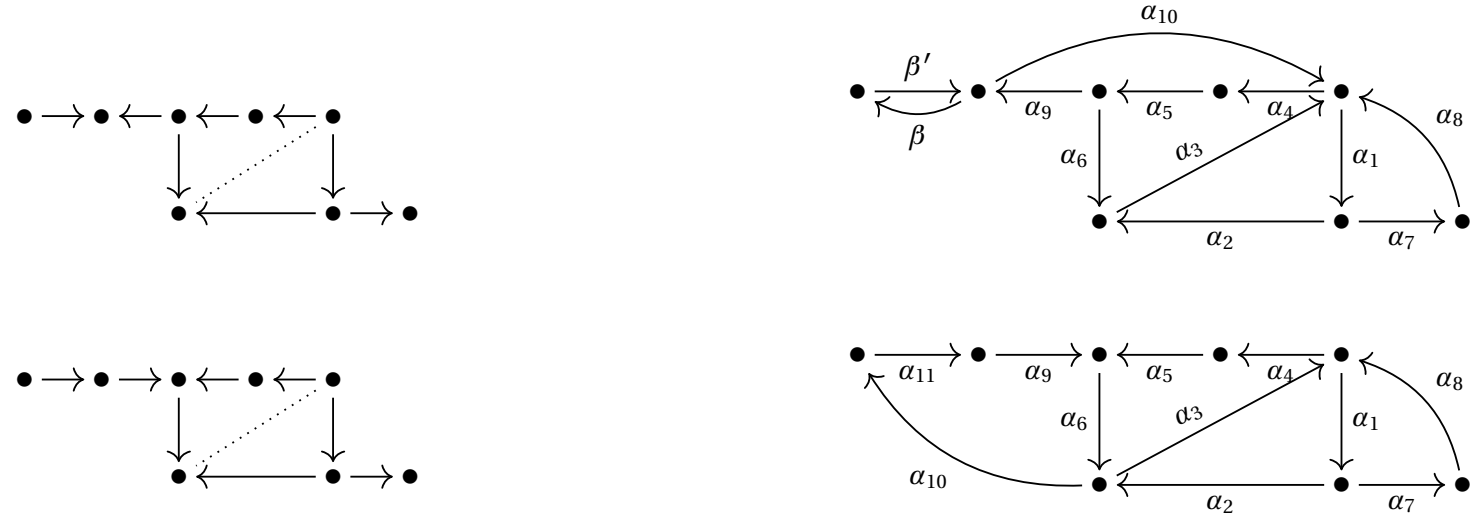

As relações do tipo 2 são: $r 1=\alpha_{2} \alpha_{3} \alpha_{4}, r 2=\alpha_{3} \alpha_{1} \alpha_{7}, r 3=\alpha_{6} \alpha_{3} \alpha_{1}, r 4=\alpha_{8} \alpha_{4}, r 5=$ $\alpha_{2} \alpha_{10}, r 6=\alpha_{8} \alpha_{1} \alpha_{2}, r 7=\alpha_{5} \alpha_{6} \alpha_{10}$ e $r 8=\alpha_{9} \alpha_{6} \alpha_{3}$. Os ciclos elementares são: $C_{1}=$ $\alpha_{1} \alpha_{2} \alpha_{3}, C_{2}=\alpha_{3} \alpha_{4} \alpha_{5} \alpha_{6}, C_{3}=\alpha_{7} \alpha_{8} \alpha_{1}$ e $C_{4}=\alpha_{6} \alpha_{10} \alpha_{11} \alpha_{9}$. Implicando que tem apenas o 
corte $\left\{\alpha_{3}, \alpha_{8}, \alpha_{10}\right\}$ que origina a álgebra disfarçada.

Utilizando essa resposta, veremos que o seguinte caso também tem a mesma solução:
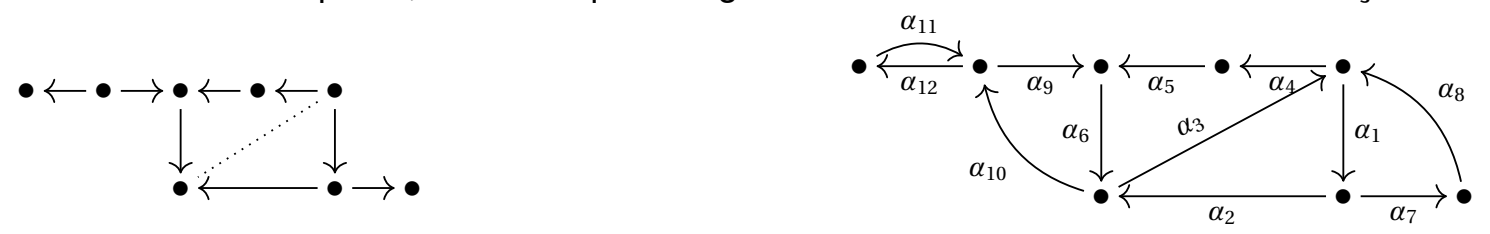

$\mathcal{F} r 16.4$
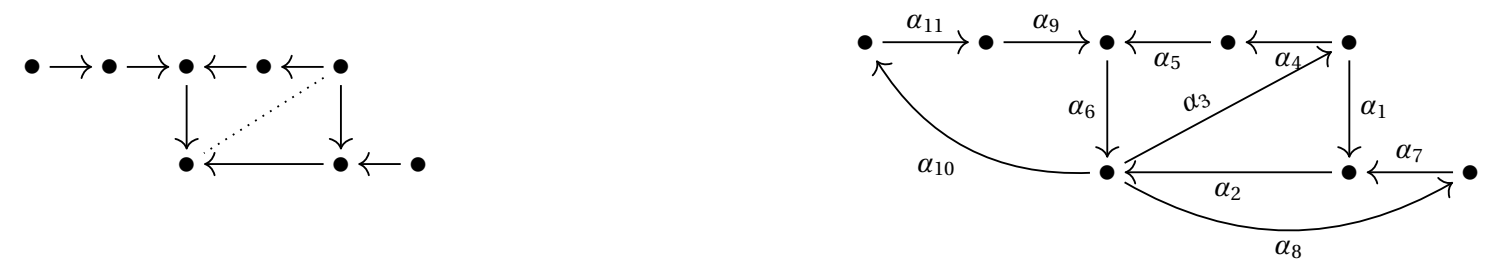

As relações do tipo 2 são: $r 1=\alpha_{2} \alpha_{3} \alpha_{4}, r 2=\alpha_{1} \alpha_{2} \alpha_{8}, r 3=\alpha_{6} \alpha_{3} \alpha_{1}, r 4=\alpha_{6} \alpha_{8}, r 5=$ $\alpha_{2} \alpha_{10}, r 6=\alpha_{7} \alpha_{2} \alpha_{3}, r 7=\alpha_{5} \alpha_{6} \alpha_{10}$ e $r 8=\alpha_{9} \alpha_{6} \alpha_{3}$. Os ciclos elementares são: $C_{1}=$ $\alpha_{1} \alpha_{2} \alpha_{3}, C_{2}=\alpha_{3} \alpha_{4} \alpha_{5} \alpha_{6}, C_{3}=\alpha_{7} \alpha_{2} \alpha_{8}$ e $C_{4}=\alpha_{6} \alpha_{10} \alpha_{11} \alpha_{9}$. O programa exibe o corte $\left\{\alpha_{2}, \alpha_{6}\right\}$ em que resulta na Phia 48.

O seguinte caso pode ser eliminado usando essa resposta juntamente com o lema 5.42.
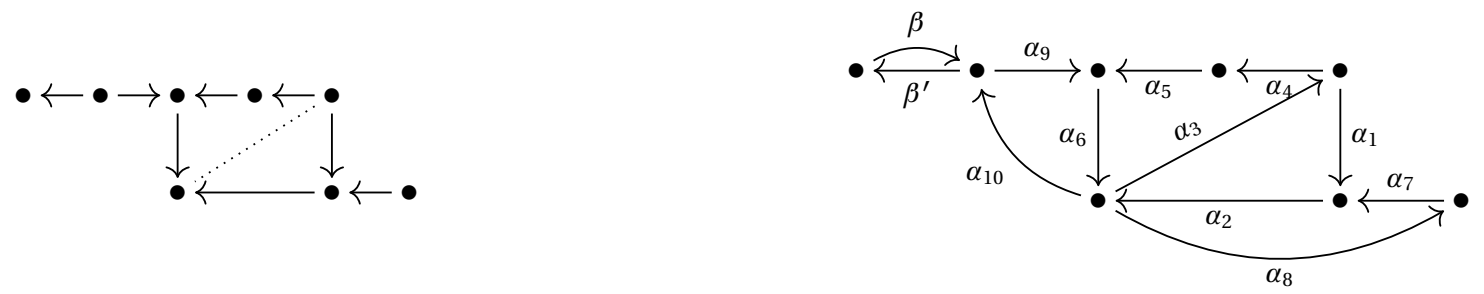

$\mathcal{F} r 16.5$
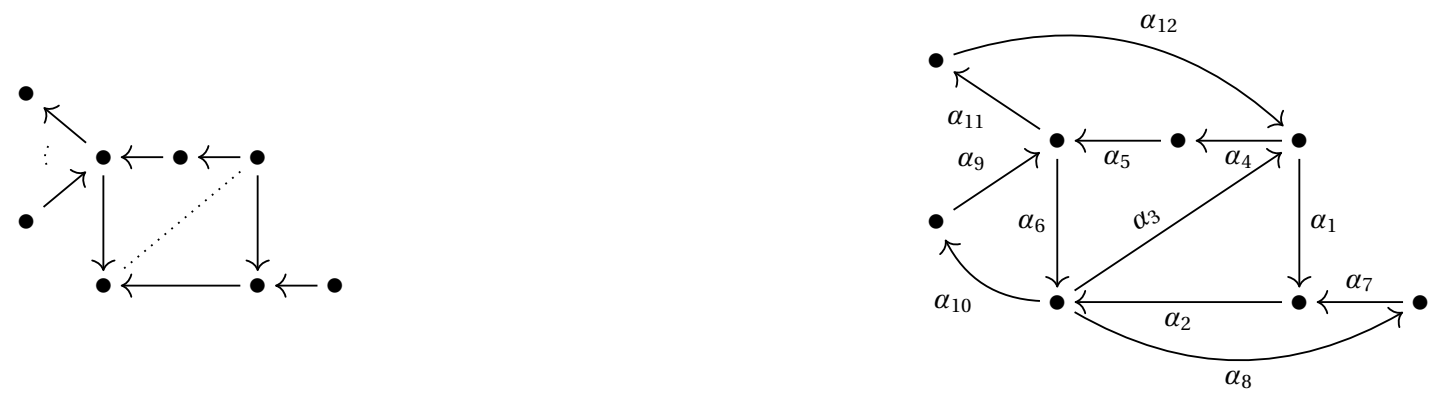

As relações do tipo 2 são: $r 1=\alpha_{2} \alpha_{3} \alpha_{4}, r 2=\alpha_{1} \alpha_{2} \alpha_{8}, r 3=\alpha_{6} \alpha_{3} \alpha_{1}, r 4=\alpha_{6} \alpha_{8}, r 5=$ $\alpha_{2} \alpha_{10}, r 6=\alpha_{7} \alpha_{2} \alpha_{3}, r 7=\alpha_{5} \alpha_{6} \alpha_{10}, r 8=\alpha_{9} \alpha_{6} \alpha_{3}, r 9=\alpha_{9} \alpha_{11}, r 10=\alpha_{12} \alpha_{4} \alpha_{5} \alpha_{6}, r 11=$ $\alpha_{3} \alpha_{4} \alpha_{5} \alpha_{11}$ e $r 12=\alpha_{12} \alpha_{1}$. Os ciclos elementares são: $C_{1}=\alpha_{1} \alpha_{2} \alpha_{3}, C_{2}=\alpha_{3} \alpha_{4} \alpha_{5} \alpha_{6}$, $C_{3}=\alpha_{7} \alpha_{2} \alpha_{8}, C_{4}=\alpha_{6} \alpha_{10} \alpha_{9}$ e $C_{5}=\alpha_{4} \alpha_{5} \alpha_{11} \alpha_{12}$. Essa extensão trivial não tem corte em que origine uma álgebra de incidência.

$\mathcal{F} r 16.6$
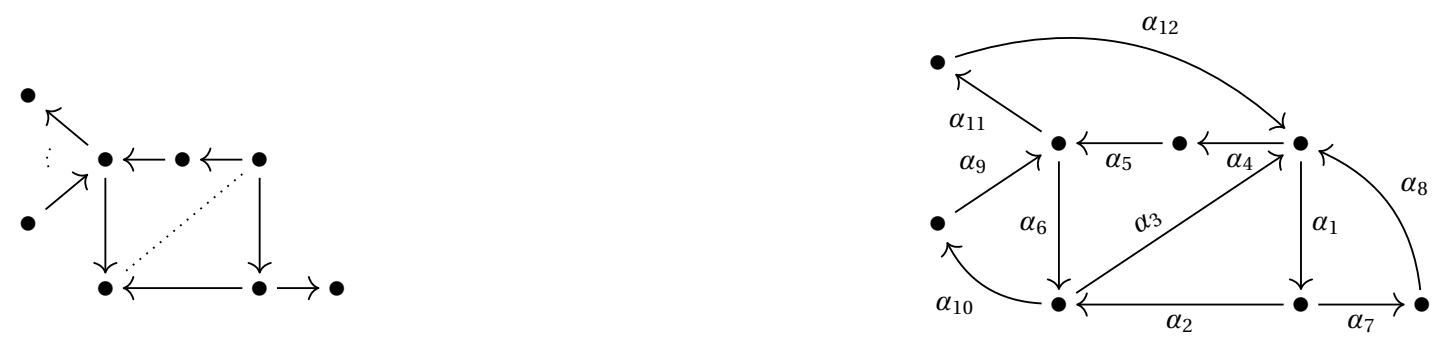
As relações do tipo 2 são: $r 1=\alpha_{2} \alpha_{3} \alpha_{4}, r 2=\alpha_{3} \alpha_{1} \alpha_{7}, r 3=\alpha_{6} \alpha_{3} \alpha_{1}, r 4=\alpha_{8} \alpha_{4}, r 5=$ $\alpha_{2} \alpha_{10}, r 6=\alpha_{8} \alpha_{1} \alpha_{2}, r 7=\alpha_{5} \alpha_{6} \alpha_{10}, r 8=\alpha_{9} \alpha_{6} \alpha_{3}, r 9=\alpha_{9} \alpha_{11}, r 10=\alpha_{12} \alpha_{4} \alpha_{5} \alpha_{6}, r 11=$ $\alpha_{3} \alpha_{4} \alpha_{5} \alpha_{11}$ e $r 12=\alpha_{12} \alpha_{1}$. Os ciclos elementares são: $C_{1}=\alpha_{1} \alpha_{2} \alpha_{3}, C_{2}=\alpha_{3} \alpha_{4} \alpha_{5} \alpha_{6}$, $C_{3}=\alpha_{7} \alpha_{8} \alpha_{1}, C_{4}=\alpha_{6} \alpha_{10} \alpha_{9}$ e $C_{5}=\alpha_{4} \alpha_{5} \alpha_{11} \alpha_{12}$. Também, não temos solução para esse caso.

$\mathcal{F} r 17.1$
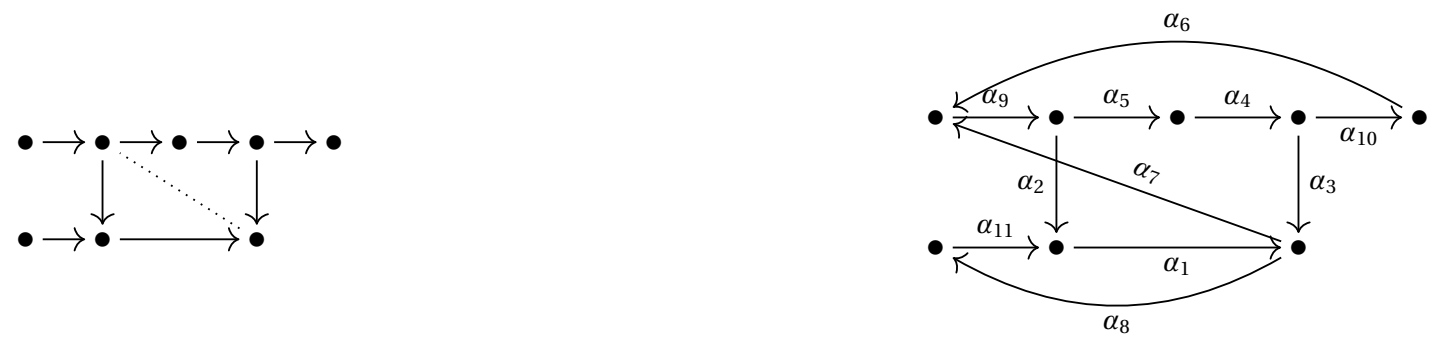

As relações do tipo 2 são: $r 1=\alpha_{7} \alpha_{9} \alpha_{5} \alpha_{4} \alpha_{10}, r 2=\alpha_{6} \alpha_{9} \alpha_{2}, r 3=\alpha_{6} \alpha_{9} \alpha_{5} \alpha_{4} \alpha_{3}$, $r 4=\alpha_{2} \alpha_{1} \alpha_{8}, r 5=\alpha_{3} \alpha_{8}, r 6=\alpha_{11} \alpha_{1} \alpha_{7}, r 7=\alpha_{3} \alpha_{7} \alpha_{9} \alpha_{2}$ e $r 8=\alpha_{1} \alpha_{7} \alpha_{9} \alpha_{5}$.Os ciclos elementares são: $C_{1}=\alpha_{9} \alpha_{2} \alpha_{1} \alpha_{7}, C_{2}=\alpha_{9} \alpha_{5} \alpha_{4} \alpha_{3} \alpha_{7}, C_{3}=\alpha_{9} \alpha_{5} \alpha_{4} \alpha_{10} \alpha_{6}$ e $C_{4}=\alpha_{11} \alpha_{1} \alpha_{8}$. Essa extensão trivial possui apenas o corte $\left\{\alpha_{6}, \alpha_{7}, \alpha_{8}\right\}$ que resulta na álgebra disfarçada.

$\mathcal{F} r 17.2$
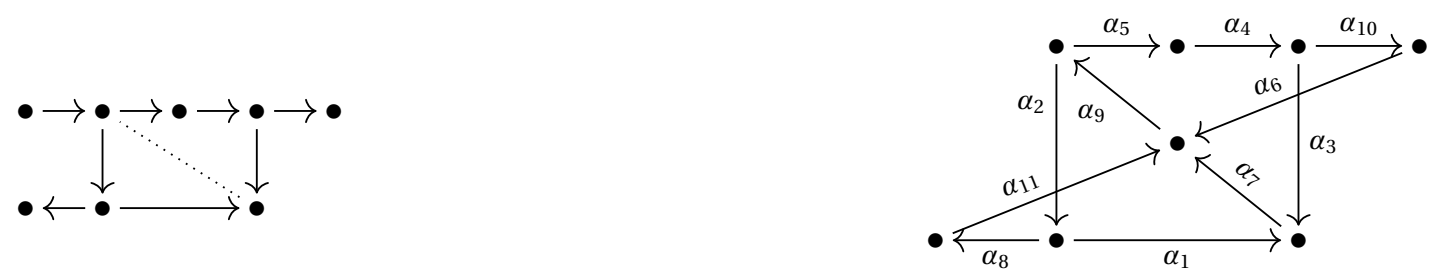

As relações do tipo 2 são: $r 1=\alpha_{7} \alpha_{9} \alpha_{5} \alpha_{4} \alpha_{10}, r 2=\alpha_{6} \alpha_{9} \alpha_{2}, r 3=\alpha_{6} \alpha_{9} \alpha_{5} \alpha_{4} \alpha_{3}$, $r 4=\alpha_{11} \alpha_{9} \alpha_{5}, r 5=\alpha_{7} \alpha_{9} \alpha_{2} \alpha_{8}, r 6=\alpha_{11} \alpha_{9} \alpha_{2} \alpha_{1}, r 7=\alpha_{3} \alpha_{7} \alpha_{9} \alpha_{2}$ e $r 8=\alpha_{1} \alpha_{7} \alpha_{9} \alpha_{5}$. Os ciclos elementares são: $C_{1}=\alpha_{9} \alpha_{2} \alpha_{1} \alpha_{7}, C_{2}=\alpha_{9} \alpha_{5} \alpha_{4} \alpha_{3} \alpha_{7}, C_{3}=\alpha_{9} \alpha_{5} \alpha_{4} \alpha_{10} \alpha_{6}$ e $C_{4}=\alpha_{11} \alpha_{9} \alpha_{2} \alpha_{8}$

Entramos com essas informações no programa e obtivemos três cortes $\left\{\alpha_{9}\right\},\left\{\alpha_{2}, \alpha_{5}\right\}$ e $\left\{\alpha_{6}, \alpha_{7}, \alpha_{11}\right\}$ resultando nas soluções 49,50 e a álgebra disfarçada, respectivamente.

$\mathcal{F} r 17.3$
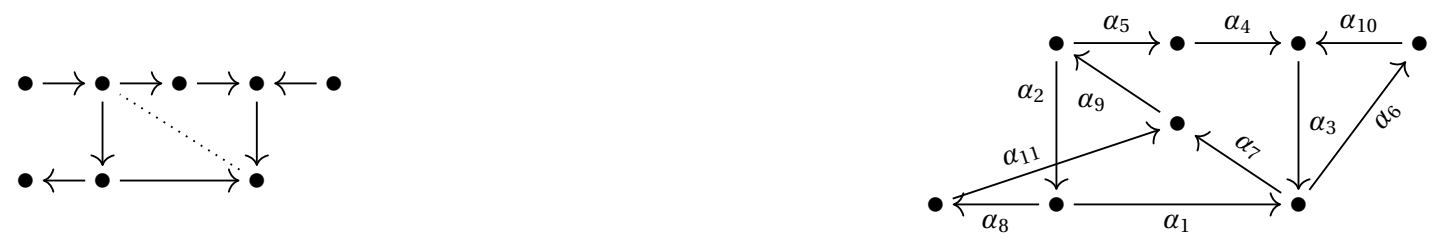

As relações do tipo 2 são: $r 1=\alpha_{1} \alpha_{7} \alpha_{9} \alpha_{5}, r 2=\alpha_{7} \alpha_{9} \alpha_{2} \alpha_{8}, r 3=\alpha_{1} \alpha_{6}, r 4=\alpha_{3} \alpha_{7} \alpha_{9} \alpha_{2}$, $r 5=\alpha_{4} \alpha_{3} \alpha_{6}, r 6=\alpha_{10} \alpha_{3} \alpha_{7}, r 7=\alpha_{11} \alpha_{9} \alpha_{2} \alpha_{1}$ e $r 8=\alpha_{11} \alpha_{9} \alpha_{5}$.Os ciclos elementares são: $C_{1}=\alpha_{9} \alpha_{2} \alpha_{1} \alpha_{7}, C_{2}=\alpha_{9} \alpha_{5} \alpha_{4} \alpha_{3} \alpha_{7}, C_{3}=\alpha_{10} \alpha_{3} \alpha_{6}$ e $C_{4}=\alpha_{11} \alpha_{9} \alpha_{2} \alpha_{8}$

Novamente, temos uma extensão trivial que não tem corte que origina um integrante da família ANS. 
$\mathcal{F} r 17.4$
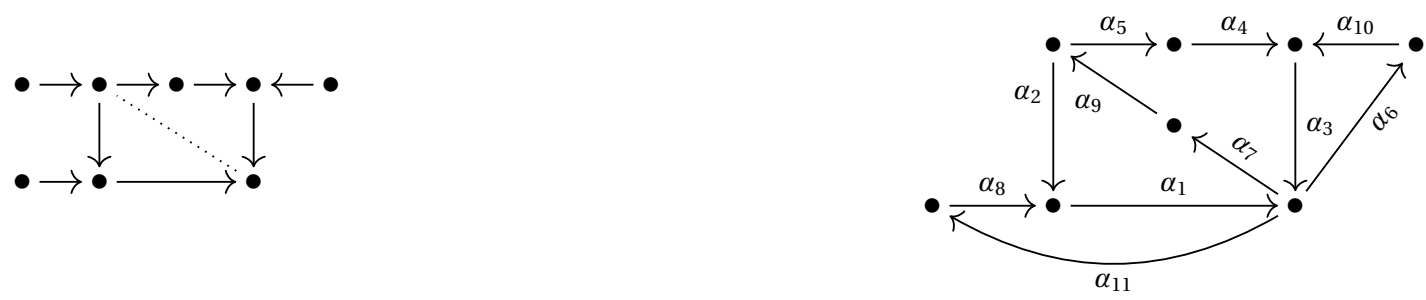

As relações do tipo 2 são: $r 1=\alpha_{1} \alpha_{7} \alpha_{9} \alpha_{5}, r 2=\alpha_{2} \alpha_{1} \alpha_{11}, r 3=\alpha_{1} \alpha_{6}, r 4=\alpha_{3} \alpha_{7} \alpha_{9} \alpha_{2}$, $r 5=\alpha_{4} \alpha_{3} \alpha_{6}, r 6=\alpha_{10} \alpha_{3} \alpha_{7}, r 7=\alpha_{8} \alpha_{1} \alpha_{7}$ e $r 8=\alpha_{3} \alpha_{11}$.Os ciclos elementares são: $C_{1}=\alpha_{9} \alpha_{2} \alpha_{1} \alpha_{7}, C_{2}=\alpha_{9} \alpha_{5} \alpha_{4} \alpha_{3} \alpha_{7}, C_{3}=\alpha_{10} \alpha_{3} \alpha_{6}$ e $C_{4}=\alpha_{11} \alpha_{8} \alpha_{1}$.

Entrando com esses dados no programa, obtemos o corte $\left\{\alpha_{1}, \alpha_{3}\right\}$ resultando na solução 51.

$\mathcal{F} r 17.5$
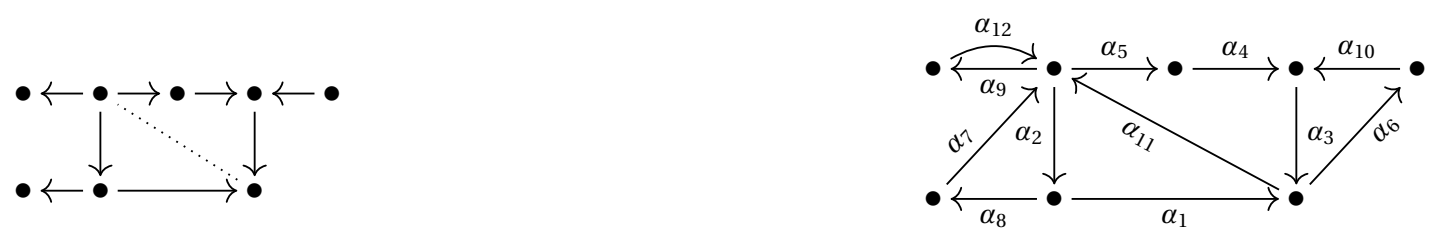

As relações do tipo 2 são: $r 1=\alpha_{4} \alpha_{3} \alpha_{6}, r 2=\alpha_{10} \alpha_{3} \alpha_{11}, r 3=\alpha_{3} \alpha_{11} \alpha_{2}, r 4=\alpha_{1} \alpha_{11} \alpha_{5}$, $r 5=\alpha_{1} \alpha_{6}, r 6=\alpha_{11} \alpha_{2} \alpha_{8}, r 7=\alpha_{7} \alpha_{2} \alpha_{1}, r 8=\alpha_{7} \alpha_{5}, r 9=\alpha_{7} \alpha_{9}, r 10=\alpha_{11} \alpha_{9}, r 11=\alpha_{12} \alpha_{2}$ e $r 12=\alpha_{12} \alpha_{5}$. Os ciclos elementares são: $C_{1}=\alpha_{9} \alpha_{12}, C_{2}=\alpha_{2} \alpha_{1} \alpha_{11}, C_{3}=\alpha_{5} \alpha_{4} \alpha_{3} \alpha_{11}$, $C_{4}=\alpha_{10} \alpha_{3} \alpha_{6}$ e $C_{5}=\alpha_{2} \alpha_{8} \alpha_{7}$.

Portanto, o programa mostra apenas o corte que resulta na álgebra disfarçada.

$\mathcal{F} r 17.6$
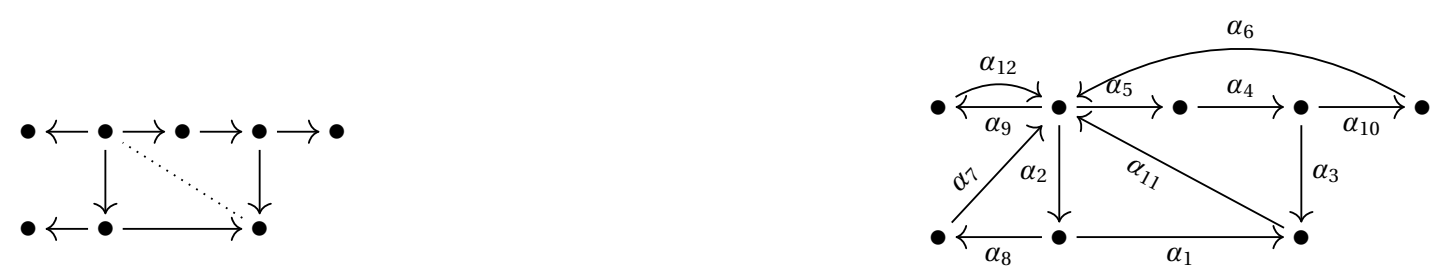

As relações do tipo 2 são: $r 1=\alpha_{11} \alpha_{5} \alpha_{4} \alpha_{10}, r 2=\alpha_{6} \alpha_{5} \alpha_{4} \alpha_{3}, r 3=\alpha_{3} \alpha_{11} \alpha_{2}, r 4=\alpha_{1} \alpha_{11} \alpha_{5}$, $r 5=\alpha_{6} \alpha_{2}, r 6=\alpha_{11} \alpha_{2} \alpha_{8}, r 7=\alpha_{7} \alpha_{2} \alpha_{1}, r 8=\alpha_{7} \alpha_{5}, r 9=\alpha_{7} \alpha_{9}, r 10=\alpha_{11} \alpha_{9}, r 11=\alpha_{12} \alpha_{2}$, $r 12=\alpha_{12} \alpha_{5}$ e $r 13=\alpha_{6} \alpha_{9}$. Os ciclos elementares são: $C_{1}=\alpha_{9} \alpha_{12}, C_{2}=\alpha_{2} \alpha_{1} \alpha_{11}$, $C_{3}=\alpha_{5} \alpha_{4} \alpha_{3} \alpha_{11}, C_{4}=\alpha_{10} \alpha_{6} \alpha_{5} \alpha_{4}$ e $C_{5}=\alpha_{2} \alpha_{8} \alpha_{7}$

Logo, temos o corte $\left\{\alpha_{5}, \alpha_{2}, \alpha_{9}\right\}$ resultando na solução 52 .

$\mathcal{F} r 17.7$
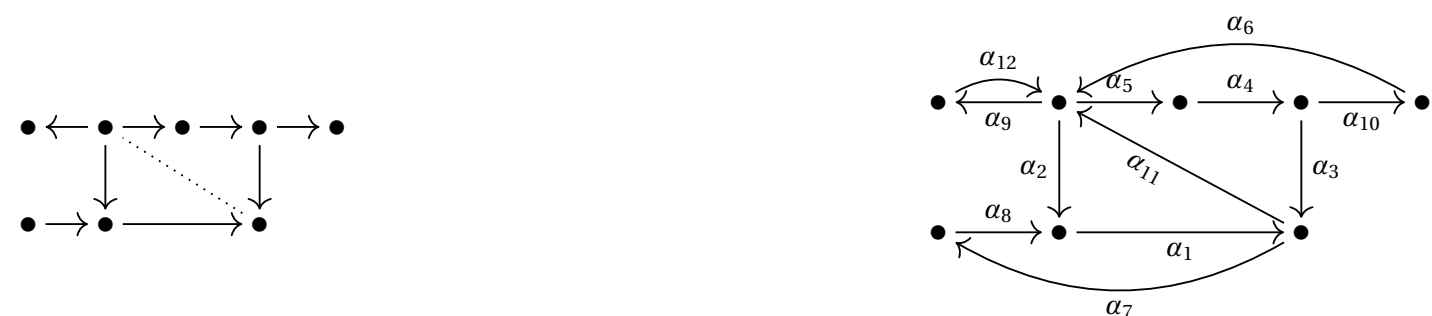

As relações do tipo 2 são: $r 1=\alpha_{11} \alpha_{5} \alpha_{4} \alpha_{10}, r 2=\alpha_{6} \alpha_{5} \alpha_{4} \alpha_{3}, r 3=\alpha_{3} \alpha_{11} \alpha_{2}, r 4=\alpha_{1} \alpha_{11} \alpha_{5}$, $r 5=\alpha_{6} \alpha_{2}, r 6=\alpha_{2} \alpha_{1} \alpha_{7}, r 7=\alpha_{8} \alpha_{1} \alpha_{11}, r 8=\alpha_{3} \alpha_{7}, r 9=\alpha_{6} \alpha_{9}, r 10=\alpha_{11} \alpha_{9}, r 11=\alpha_{12} \alpha_{2}$ e $r 12=\alpha_{12} \alpha_{5}$.Os ciclos elementares são: $C_{1}=\alpha_{9} \alpha_{12}, C_{2}=\alpha_{2} \alpha_{1} \alpha_{11}, C_{3}=\alpha_{5} \alpha_{4} \alpha_{3} \alpha_{11}$, $C_{4}=\alpha_{10} \alpha_{6} \alpha_{5} \alpha_{4}$ e $C_{5}=\alpha_{7} \alpha_{8} \alpha_{1}$. 
Logo, não temos solução para essa extensão trivial.

$\mathcal{F} r 17.8$
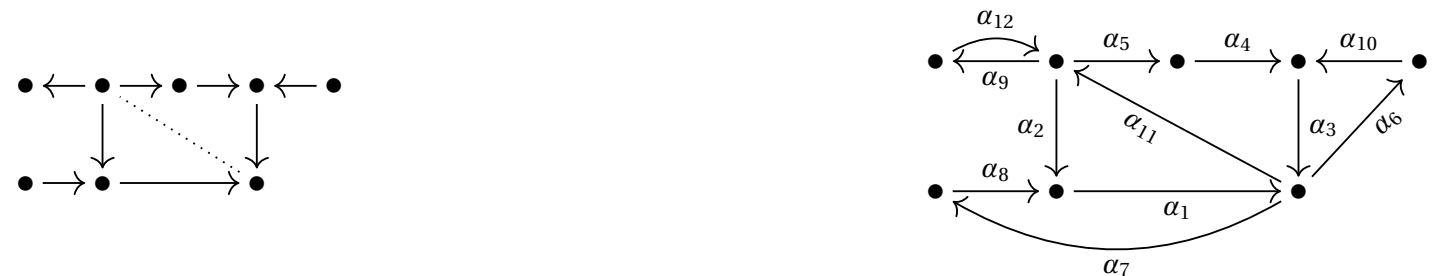

As relações do tipo 2 são: $r 1=\alpha_{4} \alpha_{3} \alpha_{6}, r 2=\alpha_{10} \alpha_{3} \alpha_{11}, r 3=\alpha_{3} \alpha_{11} \alpha_{2}, r 4=\alpha_{1} \alpha_{11} \alpha_{5}, r 5=$ $\alpha_{1} \alpha_{6}, r 6=\alpha_{2} \alpha_{1} \alpha_{7}, r 7=\alpha_{8} \alpha_{1} \alpha_{11}, r 8=\alpha_{3} \alpha_{7}, r 9=\alpha_{11} \alpha_{9}, r 10=\alpha_{12} \alpha_{2}$ e $r 11=\alpha_{12} \alpha_{5}$.Os ciclos elementares são: $C_{1}=\alpha_{9} \alpha_{12}, C_{2}=\alpha_{2} \alpha_{1} \alpha_{11}, C_{3}=\alpha_{5} \alpha_{4} \alpha_{3} \alpha_{11}, C_{4}=\alpha_{10} \alpha_{3} \alpha_{6}$ e $C_{5}=\alpha_{7} \alpha_{8} \alpha_{1}$.

O último caso desse frame, não temos um integrante da família ANS.

$\mathcal{F} r 18.1$
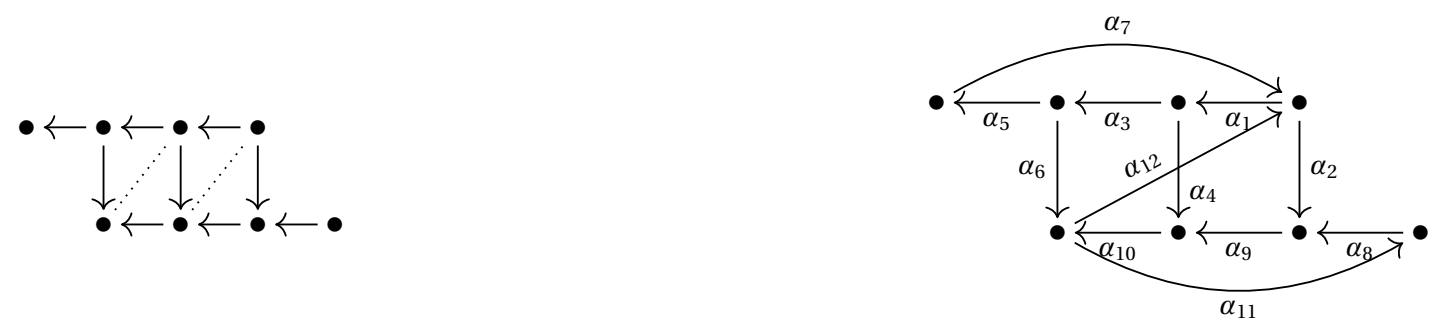

As relações do tipo 2 são: $r 1=\alpha_{7} \alpha_{2}, r 2=\alpha_{7} \alpha_{1} \alpha_{3} \alpha_{6}, r 3=\alpha_{7} \alpha_{1} \alpha_{4}, r 4=\alpha_{8} \alpha_{9} \alpha_{10} \alpha_{12}$, $r 5=\alpha_{6} \alpha_{11}, r 6=\alpha_{4} \alpha_{10} \alpha_{11}, r 7=\alpha_{2} \alpha_{9} \alpha_{10} \alpha_{11}, r 8=\alpha_{6} \alpha_{12} \alpha_{1} \alpha_{4}, r 9=\alpha_{6} \alpha_{12} \alpha_{2}$, $r 10=\alpha_{10} \alpha_{12} \alpha_{1} \alpha_{3}, r 11=\alpha_{4} \alpha_{10} \alpha_{12} \alpha_{2}, r 12=\alpha_{9} \alpha_{10} \alpha_{12} \alpha_{1}$ e $r 13=\alpha_{12} \alpha_{1} \alpha_{3} \alpha_{5}$. Os ciclos elementares são: $C_{1}=\alpha_{1} \alpha_{3} \alpha_{5} \alpha_{7}, C_{2}=\alpha_{8} \alpha_{9} \alpha_{10} \alpha_{11}, C_{3}=\alpha_{1} \alpha_{3} \alpha_{6} \alpha_{12}, C_{4}=\alpha_{1} \alpha_{4} \alpha_{10} \alpha_{12} \mathrm{e}$ $C_{5}=\alpha_{2} \alpha_{9} \alpha_{10} \alpha_{12}$.

Logo, obtemos apenas o corte que origina a álgebra disfarçada acima.

$\mathcal{F} r 18.2$
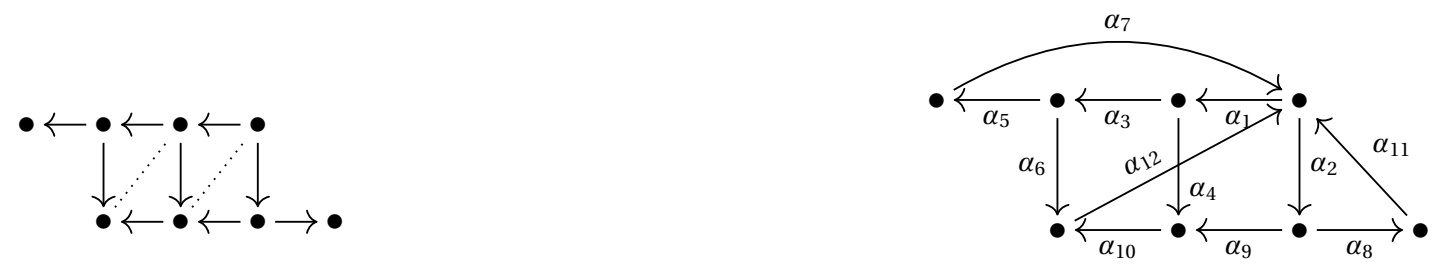

As relações do tipo 2 são: $r 1=\alpha_{7} \alpha_{2}, r 2=\alpha_{7} \alpha_{1} \alpha_{3} \alpha_{6}, r 3=\alpha_{7} \alpha_{1} \alpha_{4}, r 4=\alpha_{11} \alpha_{2} \alpha_{9}$, $r 5=\alpha_{11} \alpha_{1}, r 6=\alpha_{12} \alpha_{2} \alpha_{8}, r 7=\alpha_{6} \alpha_{12} \alpha_{1} \alpha_{4}, r 8=\alpha_{6} \alpha_{12} \alpha_{2}, r 9=\alpha_{10} \alpha_{12} \alpha_{1} \alpha_{3}, r 10=$ $\alpha_{4} \alpha_{10} \alpha_{12} \alpha_{2}, r 11=\alpha_{9} \alpha_{10} \alpha_{12} \alpha_{1}, r 12=\alpha_{12} \alpha_{1} \alpha_{3} \alpha_{5}$ e $r 13=\alpha_{7} \alpha_{2} \alpha_{8}$. Os ciclos elementares são: $C_{1}=\alpha_{1} \alpha_{3} \alpha_{5} \alpha_{7}, C_{2}=\alpha_{2} \alpha_{8} \alpha_{11}, C_{3}=\alpha_{1} \alpha_{3} \alpha_{6} \alpha_{12}, C_{4}=\alpha_{1} \alpha_{4} \alpha_{10} \alpha_{12}$ e $C_{5}=\alpha_{2} \alpha_{9} \alpha_{10} \alpha_{12}$. Sendo assim, temos o corte $\left\{\alpha_{2}, \alpha_{1}\right\}$ que origina a solução 53 .

$\mathcal{F} r 18.3$
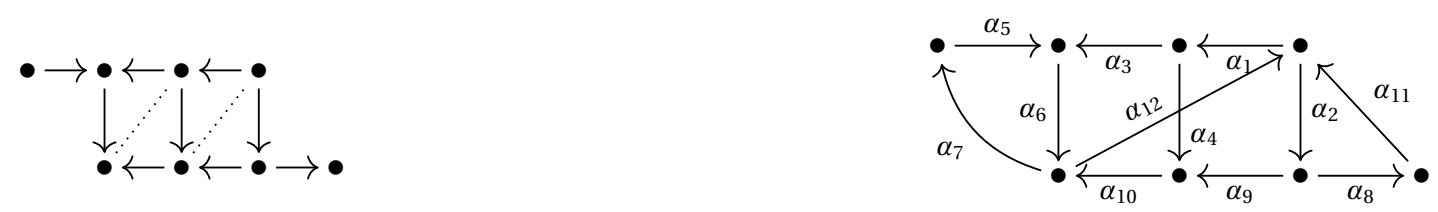

As relações do tipo 2 são: $r 1=\alpha_{5} \alpha_{6} \alpha_{12}, r 2=\alpha_{10} \alpha_{7}, r 3=\alpha_{3} \alpha_{6} \alpha_{7}, r 4=\alpha_{11} \alpha_{2} \alpha_{9}$, 
$r 5=\alpha_{11} \alpha_{1}, r 6=\alpha_{12} \alpha_{2} \alpha_{8}, r 7=\alpha_{6} \alpha_{12} \alpha_{1} \alpha_{4}, r 8=\alpha_{6} \alpha_{12} \alpha_{2}, r 9=\alpha_{10} \alpha_{12} \alpha_{1} \alpha_{3}, r 10=$ $\alpha_{4} \alpha_{10} \alpha_{12} \alpha_{2}$ e $r 11=\alpha_{9} \alpha_{10} \alpha_{12} \alpha_{1}$.Os ciclos elementares são: $C_{1}=\alpha_{5} \alpha_{6} \alpha_{7}, C_{2}=\alpha_{2} \alpha_{8} \alpha_{11}$, $C_{3}=\alpha_{1} \alpha_{3} \alpha_{6} \alpha_{12}, C_{4}=\alpha_{1} \alpha_{4} \alpha_{10} \alpha_{12}$ e $C_{5}=\alpha_{2} \alpha_{9} \alpha_{10} \alpha_{12}$.

Logo, temos apenas a álgebra disfarçada acima.

$\mathcal{F} r 18.4$
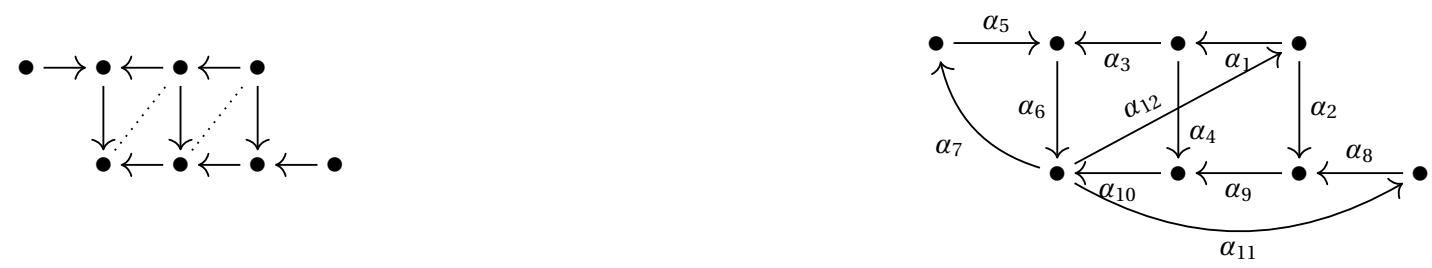

As relações do tipo 2 são: $r 1=\alpha_{5} \alpha_{6} \alpha_{12}, r 2=\alpha_{10} \alpha_{7}, r 3=\alpha_{3} \alpha_{6} \alpha_{7}, r 4=\alpha_{8} \alpha_{9} \alpha_{10} \alpha_{12}$, $r 5=\alpha_{6} \alpha_{11}, r 6=\alpha_{4} \alpha_{10} \alpha_{11}, r 7=\alpha_{6} \alpha_{12} \alpha_{1} \alpha_{4}, r 8=\alpha_{6} \alpha_{12} \alpha_{2}, r 9=\alpha_{10} \alpha_{12} \alpha_{1} \alpha_{3}, r 10=$ $\alpha_{4} \alpha_{10} \alpha_{12} \alpha_{2}, r 11=\alpha_{9} \alpha_{10} \alpha_{12} \alpha_{1}$ e $r 12=\alpha_{2} \alpha_{9} \alpha_{10} \alpha_{11}$. Os ciclos elementares são: $C_{1}=$ $\alpha_{5} \alpha_{6} \alpha_{7}, C_{2}=\alpha_{8} \alpha_{9} \alpha_{10} \alpha_{11}, C_{3}=\alpha_{1} \alpha_{3} \alpha_{6} \alpha_{12}, C_{4}=\alpha_{1} \alpha_{4} \alpha_{10} \alpha_{12}$ e $C_{5}=\alpha_{2} \alpha_{9} \alpha_{10} \alpha_{12}$.

O programa exibe o corte $\left\{\alpha_{10}, \alpha_{6}\right\}$ que origina a Phia 54 .

$\mathcal{F} r 19.1$
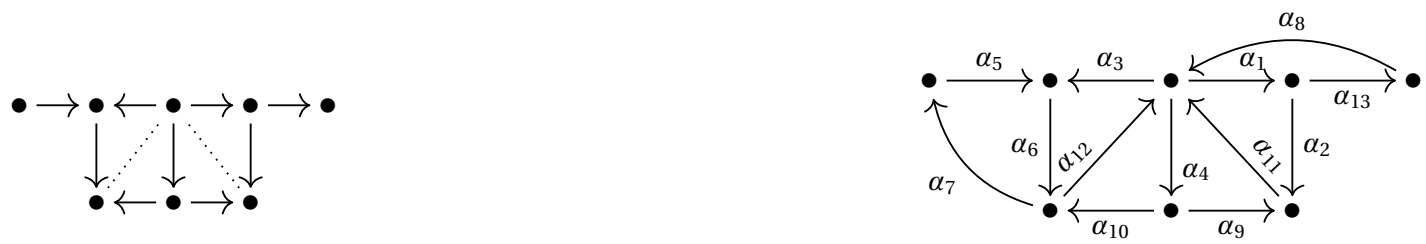

As relações do tipo 2 são: $r 1=\alpha_{6} \alpha_{12} \alpha_{4}, r 2=\alpha_{10} \alpha_{12} \alpha_{3}, r 3=\alpha_{12} \alpha_{4} \alpha_{9}, r 4=\alpha_{11} \alpha_{4} \alpha_{10}$, $r 5=\alpha_{9} \alpha_{11} \alpha_{1}, r 6=\alpha_{2} \alpha_{11} \alpha_{4}, r 7=\alpha_{5} \alpha_{6} \alpha_{12}, r 8=\alpha_{3} \alpha_{6} \alpha_{7}, r 9=\alpha_{8} \alpha_{1} \alpha_{2}, r 10=\alpha_{11} \alpha_{1} \alpha_{13}$, $r 11=\alpha_{10} \alpha_{7}, r 12=\alpha_{12} \alpha_{1}, r 13=\alpha_{8} \alpha_{4}, r 14=\alpha_{8} \alpha_{3}$ e $r 15=\alpha_{11} \alpha_{3}$. Os ciclos elementares são: $C_{1}=\alpha_{3} \alpha_{6} \alpha_{12}, C_{2}=\alpha_{4} \alpha_{10} \alpha_{12}, C_{3}=\alpha_{1} \alpha_{2} \alpha_{11}, C_{4}=\alpha_{4} \alpha_{9} \alpha_{11}, C_{5}=\alpha_{1} \alpha_{13} \alpha_{8}$ e $C_{6}=$ $\alpha_{5} \alpha_{6} \alpha_{7}$.

O programa exibe apenas o corte responsável pela álgebra disfarçada acima.

$\mathcal{F} r 19.2$
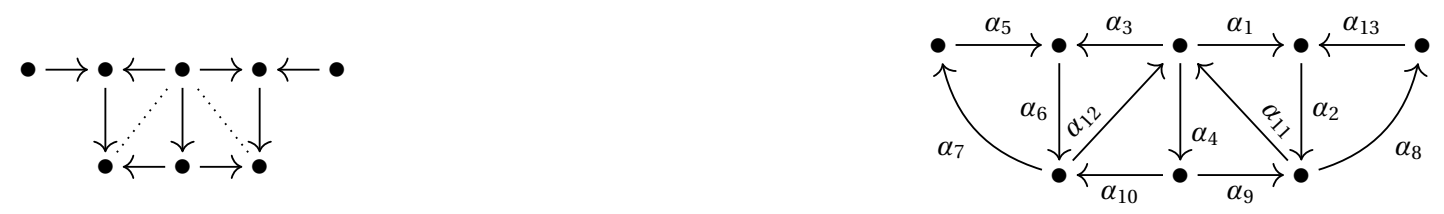

As relações do tipo 2 são: $r 1=\alpha_{6} \alpha_{12} \alpha_{4}, r 2=\alpha_{10} \alpha_{12} \alpha_{3}, r 3=\alpha_{12} \alpha_{4} \alpha_{9}, r 4=\alpha_{11} \alpha_{4} \alpha_{10}$, $r 5=\alpha_{9} \alpha_{11} \alpha_{1}, r 6=\alpha_{2} \alpha_{11} \alpha_{4}, r 7=\alpha_{5} \alpha_{6} \alpha_{12}, r 8=\alpha_{3} \alpha_{6} \alpha_{7}, r 9=\alpha_{1} \alpha_{2} \alpha_{8}, r 10=\alpha_{13} \alpha_{1} \alpha_{11}$, $r 11=\alpha_{10} \alpha_{7}, r 12=\alpha_{12} \alpha_{1}, r 13=\alpha_{9} \alpha_{8}$ e $r 14=\alpha_{11} \alpha_{3}$. Os ciclos elementares são: $C_{1}=\alpha_{3} \alpha_{6} \alpha_{12}, C_{2}=\alpha_{4} \alpha_{10} \alpha_{12}, C_{3}=\alpha_{1} \alpha_{2} \alpha_{11}, C_{4}=\alpha_{4} \alpha_{9} \alpha_{11}, C_{5}=\alpha_{13} \alpha_{2} \alpha_{8}$ e $C_{6}=\alpha_{5} \alpha_{6} \alpha_{7}$.

Novamente, temos a mesma resposta do caso anterior. 
$\mathcal{F} r 19.3$
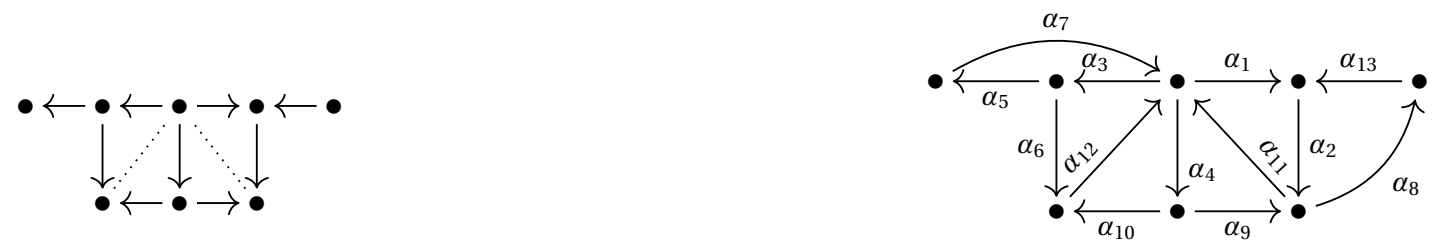

As relações do tipo 2 são: $r 1=\alpha_{6} \alpha_{12} \alpha_{4}, r 2=\alpha_{10} \alpha_{12} \alpha_{3}, r 3=\alpha_{12} \alpha_{4} \alpha_{9}, r 4=\alpha_{11} \alpha_{4} \alpha_{10}$, $r 5=\alpha_{9} \alpha_{11} \alpha_{1}, r 6=\alpha_{2} \alpha_{11} \alpha_{4}, r 7=\alpha_{7} \alpha_{3} \alpha_{6}, r 8=\alpha_{12} \alpha_{3} \alpha_{5}, r 9=\alpha_{1} \alpha_{2} \alpha_{8}, r 10=\alpha_{13} \alpha_{2} \alpha_{11}$, $r 11=\alpha_{7} \alpha_{4}, r 12=\alpha_{12} \alpha_{1}, r 13=\alpha_{9} \alpha_{8}, r 14=\alpha_{11} \alpha_{3}$ e $r 15=\alpha_{7} \alpha_{1}$. Os ciclos elementares são: $C_{1}=\alpha_{3} \alpha_{6} \alpha_{12}, C_{2}=\alpha_{4} \alpha_{10} \alpha_{12}, C_{3}=\alpha_{1} \alpha_{2} \alpha_{11}, C_{4}=\alpha_{4} \alpha_{9} \alpha_{11}, C_{5}=\alpha_{13} \alpha_{2} \alpha_{8}$ e $C_{6}=$ $\alpha_{5} \alpha_{7} \alpha_{3}$.

Também não tem integrante da família ANS.

$\mathcal{F} r 19.4$
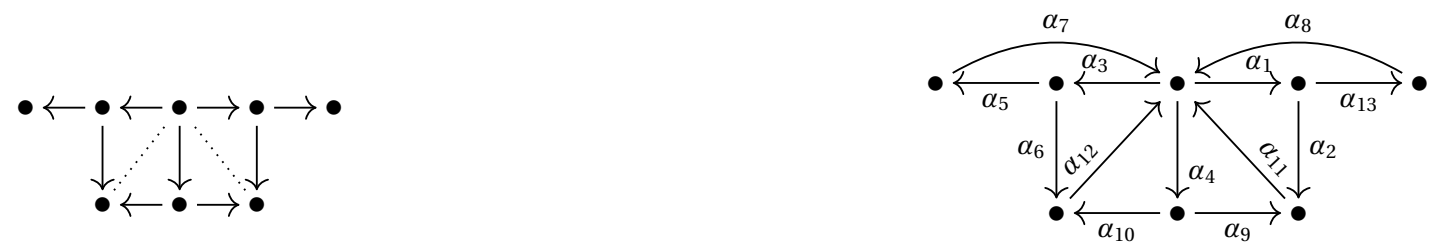

As relações do tipo 2 são: $r 1=\alpha_{6} \alpha_{12} \alpha_{4}, r 2=\alpha_{10} \alpha_{12} \alpha_{3}, r 3=\alpha_{12} \alpha_{4} \alpha_{9}, r 4=\alpha_{11} \alpha_{4} \alpha_{10}$, $r 5=\alpha_{9} \alpha_{11} \alpha_{1}, r 6=\alpha_{2} \alpha_{11} \alpha_{4}, r 7=\alpha_{7} \alpha_{3} \alpha_{6}, r 8=\alpha_{12} \alpha_{3} \alpha_{5}, r 9=\alpha_{11} \alpha_{1} \alpha_{13}, r 10=\alpha_{8} \alpha_{1} \alpha_{2}$, $r 11=\alpha_{7} \alpha_{4}, r 12=\alpha_{12} \alpha_{1}, r 13=\alpha_{8} \alpha_{4}, r 14=\alpha_{11} \alpha_{3}, r 15=\alpha_{7} \alpha_{1}$ e $r 15=\alpha_{8} \alpha_{3}$. Os ciclos elementares são: $C_{1}=\alpha_{3} \alpha_{6} \alpha_{12}, C_{2}=\alpha_{4} \alpha_{10} \alpha_{12}, C_{3}=\alpha_{1} \alpha_{2} \alpha_{11}, C_{4}=\alpha_{4} \alpha_{9} \alpha_{11}$, $C_{5}=\alpha_{13} \alpha_{8} \alpha_{1}$ e $C_{6}=\alpha_{5} \alpha_{7} \alpha_{3}$.

Informamos esses dados ao programa e obtemos o corte $\left\{\alpha_{1}, \alpha_{3}, \alpha_{4}\right\}$ responsável pela Phia 55.

$\mathcal{F} r 20.1$
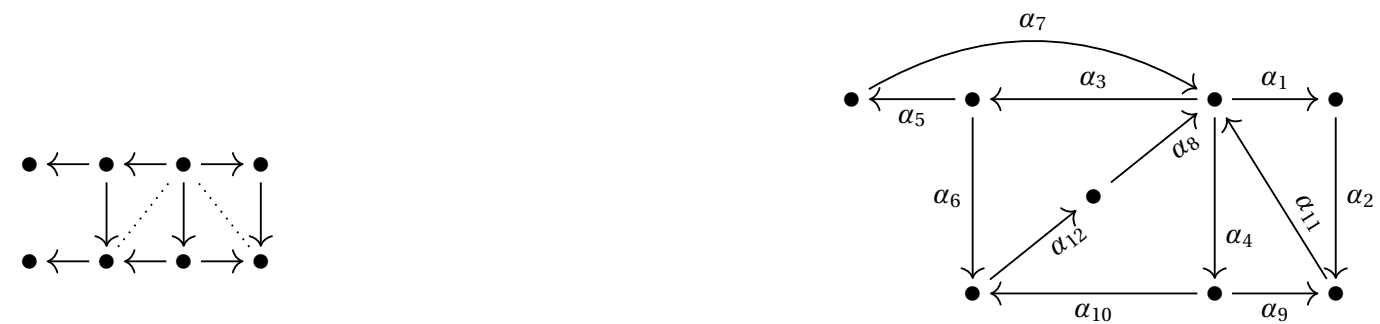

As relações do tipo 2 são: $r 1=\alpha_{2} \alpha_{11} \alpha_{4}, r 2=\alpha_{9} \alpha_{11} \alpha_{1}, r 3=\alpha_{11} \alpha_{4} \alpha_{10}, r 4=\alpha_{8} \alpha_{4} \alpha_{9}$, $r 5=\alpha_{10} \alpha_{12} \alpha_{8} \alpha_{3}, r 6=\alpha_{6} \alpha_{12} \alpha_{8} \alpha_{4}, r 7=\alpha_{8} \alpha_{3} \alpha_{5}, r 8=\alpha_{7} \alpha_{3} \alpha_{6}, r 9=\alpha_{8} \alpha_{1}, r 10=\alpha_{11} \alpha_{3}$, $r 11=\alpha_{7} \alpha_{4}$ e $r 12=\alpha_{7} \alpha_{1}$. Os ciclos elementares são: $C_{1}=\alpha_{1} \alpha_{2} \alpha_{11}, C_{2}=\alpha_{4} \alpha_{9} \alpha_{11}$, $C_{3}=\alpha_{4} \alpha_{10} \alpha_{12} \alpha_{8}, C_{4}=\alpha_{12} \alpha_{8} \alpha_{3} \alpha_{6}$ e $C_{5}=\alpha_{3} \alpha_{5} \alpha_{7}$

Portanto, temos o corte $\left\{\alpha_{1}, \alpha_{3}, \alpha_{4}\right\}$ que origina a Phia 56.

Eliminamos o caso seguinte, aproveitando essa resposta e utilizando o lema 5.42: 

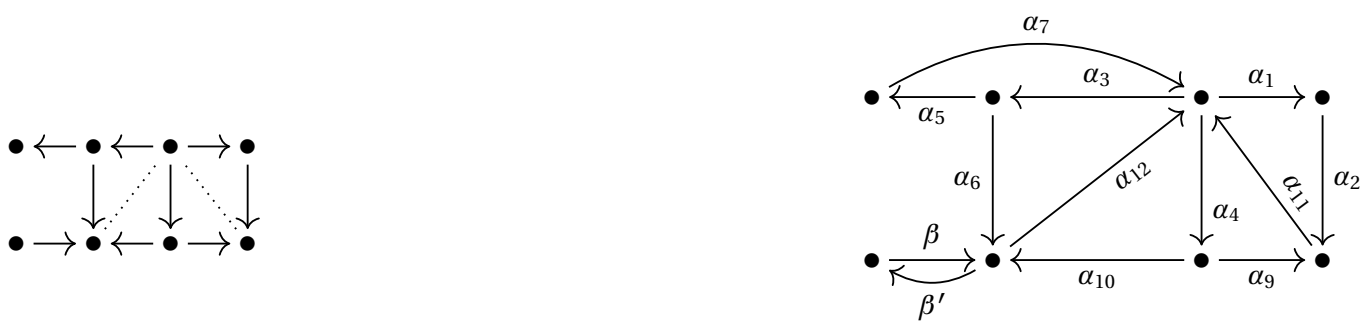

$\mathcal{F} r 20.2$
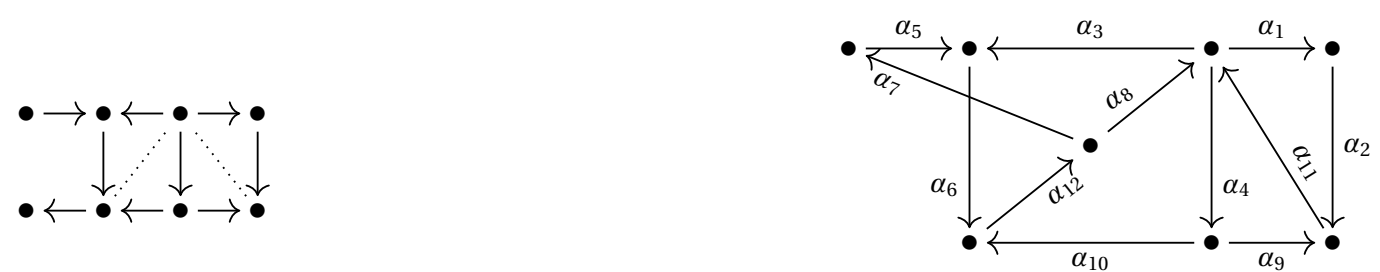

As relações do tipo 2 são: $r 1=\alpha_{2} \alpha_{11} \alpha_{4}, r 2=\alpha_{9} \alpha_{11} \alpha_{1}, r 3=\alpha_{11} \alpha_{4} \alpha_{10}, r 4=\alpha_{8} \alpha_{4} \alpha_{9}$, $r 5=\alpha_{10} \alpha_{12} \alpha_{8} \alpha_{3}, r 6=\alpha_{6} \alpha_{12} \alpha_{8} \alpha_{4}, r 7=\alpha_{10} \alpha_{12} \alpha_{7}, r 8=\alpha_{5} \alpha_{6} \alpha_{12} \alpha_{8}, r 9=\alpha_{6} \alpha_{12} \alpha_{7}$, $r 10=\alpha_{8} \alpha_{1}$ e $r 11=\alpha_{11} \alpha_{3}$. Os ciclos elementares são: $C_{1}=\alpha_{1} \alpha_{2} \alpha_{11}, C_{2}=\alpha_{4} \alpha_{9} \alpha_{11}$, $C_{3}=\alpha_{4} \alpha_{10} \alpha_{12} \alpha_{8}, C_{4}=\alpha_{12} \alpha_{8} \alpha_{3} \alpha_{6}$ e $C_{5}=\alpha_{7} \alpha_{5} \alpha_{6} \alpha_{12}$.

O programa mostra apenas o corte que resulta na álgebra disfarçada acima.

$\mathcal{F} r 20.3$
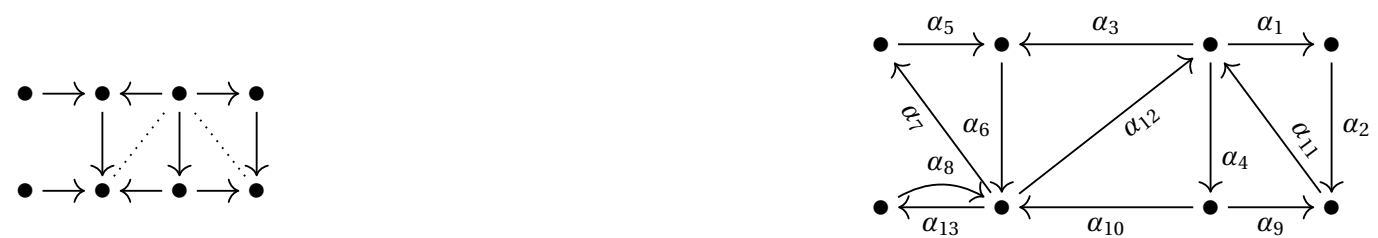

As relações do tipo 2 são: $r 1=\alpha_{2} \alpha_{11} \alpha_{4}, r 2=\alpha_{9} \alpha_{11} \alpha_{1}, r 3=\alpha_{11} \alpha_{4} \alpha_{10}, r 4=\alpha_{12} \alpha_{4} \alpha_{9}$, $r 5=\alpha_{10} \alpha_{12} \alpha_{3}, r 6=\alpha_{6} \alpha_{12} \alpha_{4}, r 7=\alpha_{3} \alpha_{6} \alpha_{7}, r 8=\alpha_{5} \alpha_{6} \alpha_{12}, r 9=\alpha_{10} \alpha_{7}, r 10=\alpha_{8} \alpha_{12}$, $r 11=\alpha_{11} \alpha_{3}, r 12=\alpha_{12} \alpha_{1}, r 13=\alpha_{8} \alpha_{7}, r 14=\alpha_{10} \alpha_{13}$ e $r 15=\alpha_{6} \alpha_{13}$. Os ciclos elementares são: $C_{1}=\alpha_{1} \alpha_{2} \alpha_{11}, C_{2}=\alpha_{4} \alpha_{9} \alpha_{11}, C_{3}=\alpha_{4} \alpha_{10} \alpha_{12}, C_{4}=\alpha_{12} \alpha_{3} \alpha_{6}, C_{5}=\alpha_{7} \alpha_{5} \alpha_{6}$ e $C_{6}=\alpha_{8} \alpha_{13}$.

Também não temos integrante da família ANS.

$\mathcal{F} r 21.1$
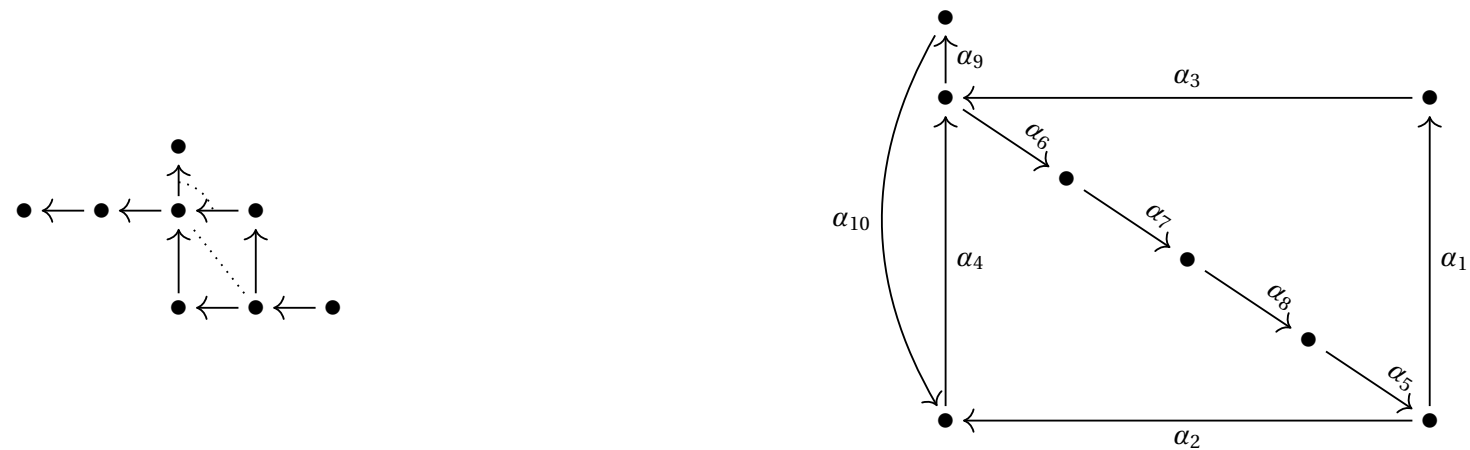

As relações do tipo 2 são: $r 1=\alpha_{3} \alpha_{6} \alpha_{7} \alpha_{8} \alpha_{5} \alpha_{2}, r 2=\alpha_{4} \alpha_{6} \alpha_{7} \alpha_{8} \alpha_{5} \alpha_{1}, r 3=\alpha_{3} \alpha_{9}$, $r 4=\alpha_{2} \alpha_{4} \alpha_{9}$ e $r 5=\alpha_{10} \alpha_{4} \alpha_{6}$. Os ciclos elementares são: $C_{1}=\alpha_{1} \alpha_{3} \alpha_{6} \alpha_{7} \alpha_{8} \alpha_{5}, C_{2}=$ $\alpha_{2} \alpha_{4} \alpha_{6} \alpha_{7} \alpha_{8} \alpha_{5}$ e $C_{3}=\alpha_{4} \alpha_{9} \alpha_{10}$. 
O programa nos mostra os cortes $\left\{\alpha_{4}, \alpha_{3}\right\}$ e $\left\{\alpha_{6}, \alpha_{9}\right\}$ que originam as Phias 57 e 58, respectivamente.

Fazendo uso dessas respostas juntamente com o lema 5.42, descartamos os seguintes casos:
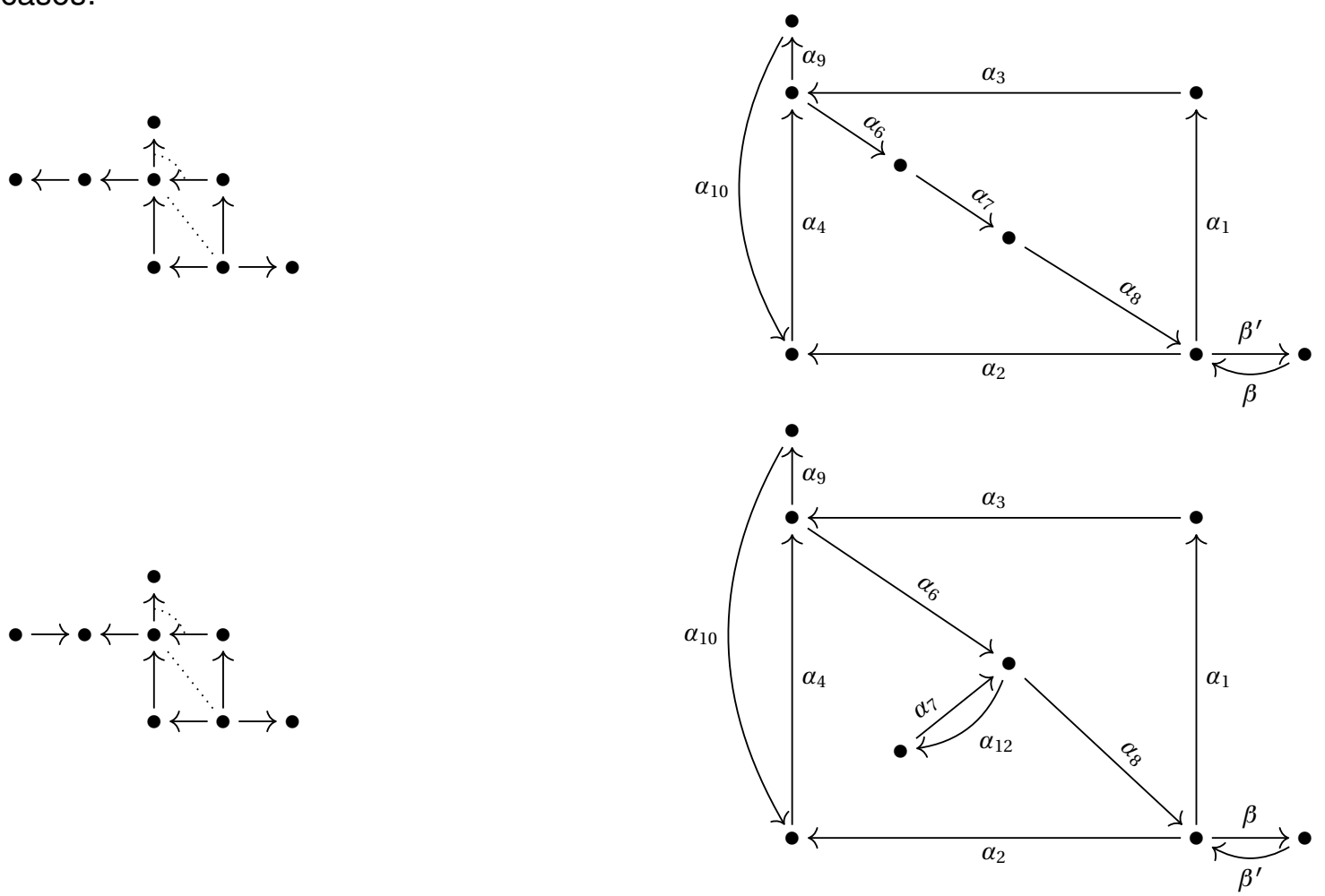

$\mathcal{F} r 21.2$
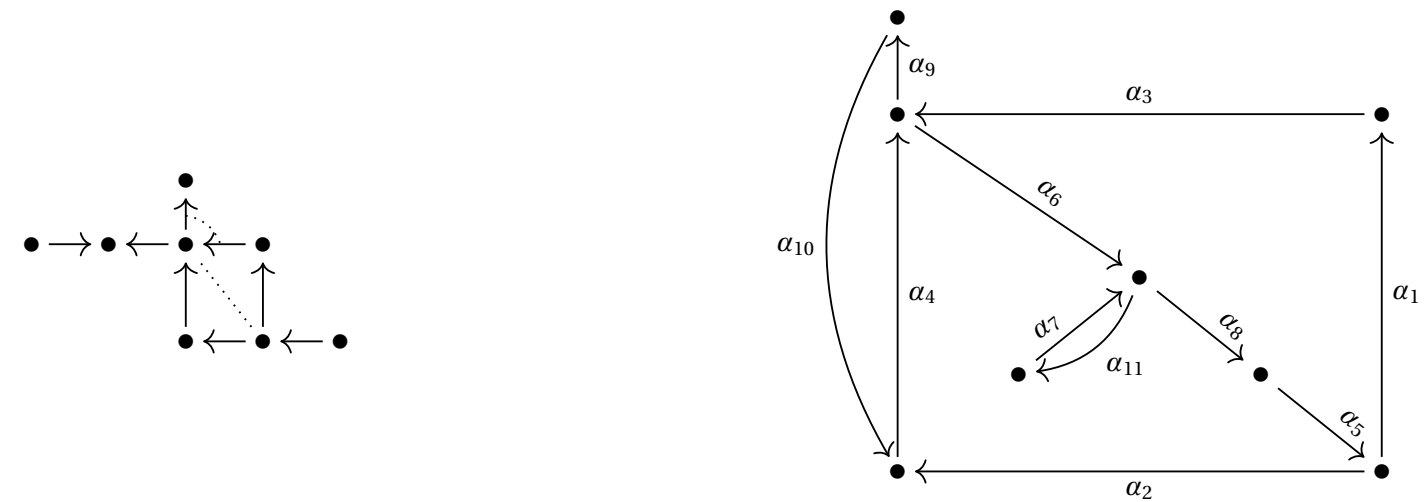

As relações do tipo 2 são: $r 1=\alpha_{3} \alpha_{6} \alpha_{8} \alpha_{5} \alpha_{2}, r 2=\alpha_{4} \alpha_{6} \alpha_{8} \alpha_{5} \alpha_{1}, r 3=\alpha_{3} \alpha_{9}, r 4=\alpha_{2} \alpha_{4} \alpha_{9}$, $r 5=\alpha_{10} \alpha_{4} \alpha_{6}, r 6=\alpha_{7} \alpha_{8}$ e $r 7=\alpha_{6} \alpha_{11}$.Os ciclos elementares são: $C_{1}=\alpha_{1} \alpha_{3} \alpha_{6} \alpha_{8} \alpha_{5}$, $C_{2}=\alpha_{2} \alpha_{4} \alpha_{6} \alpha_{8} \alpha_{5}, C_{3}=\alpha_{4} \alpha_{9} \alpha_{10}$ e $C_{4}=\alpha_{7} \alpha_{11}$.

Portanto, obtemos o integrante 59 da família ANS através do corte $\left\{\alpha_{6}, \alpha_{7}, \alpha_{9}\right\}$.

$\mathcal{F} r 21.3$
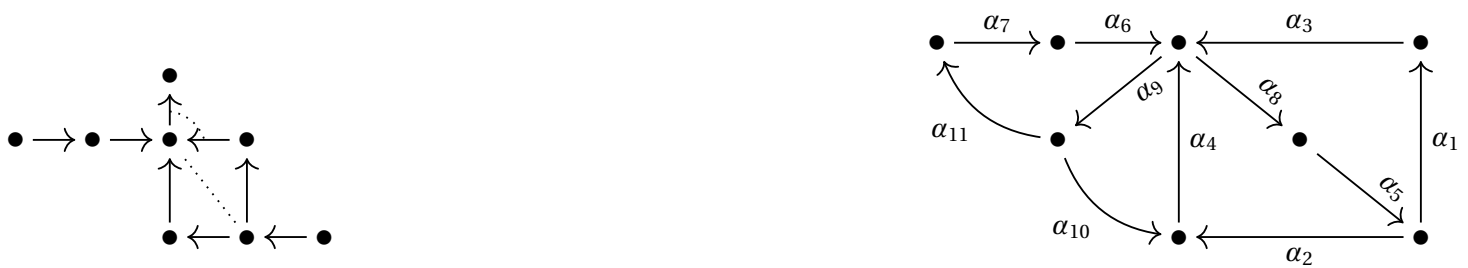
As relações do tipo 2 são: $r 1=\alpha_{3} \alpha_{8} \alpha_{5} \alpha_{2}, r 2=\alpha_{4} \alpha_{8} \alpha_{5} \alpha_{1}, r 3=\alpha_{3} \alpha_{9}, r 4=\alpha_{2} \alpha_{4} \alpha_{9}$, $r 5=\alpha_{10} \alpha_{4} \alpha_{8}, r 6=\alpha_{6} \alpha_{8}, r 7=\alpha_{6} \alpha_{9} \alpha_{10}$ e $r 8=\alpha_{4} \alpha_{9} \alpha_{11}$. Os ciclos elementares são: $C_{1}=\alpha_{1} \alpha_{3} \alpha_{8} \alpha_{5}, C_{2}=\alpha_{2} \alpha_{4} \alpha_{8} \alpha_{5}, C_{3}=\alpha_{4} \alpha_{9} \alpha_{10}$ e $C_{4}=\alpha_{7} \alpha_{6} \alpha_{9} \alpha_{11}$.

Sendo assim, temos as soluções 60 e 61 através dos cortes $\left\{\alpha_{3}, \alpha_{4}, \alpha_{6}\right\}$ e $\left\{\alpha_{8}, \alpha_{9}\right\}$, respectivamente.

$\mathcal{F} r 21.4$
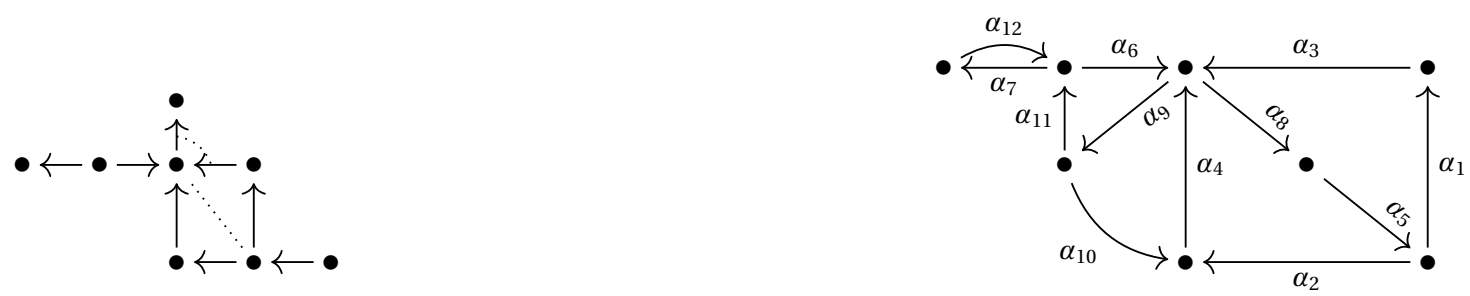

As relações do tipo 2 são: $r 1=\alpha_{3} \alpha_{8} \alpha_{5} \alpha_{2}, r 2=\alpha_{4} \alpha_{8} \alpha_{5} \alpha_{1}, r 3=\alpha_{3} \alpha_{9}, r 4=\alpha_{2} \alpha_{4} \alpha_{9}$, $r 5=\alpha_{10} \alpha_{4} \alpha_{8}, r 6=\alpha_{6} \alpha_{8}, r 7=\alpha_{6} \alpha_{9} \alpha_{10}, r 8=\alpha_{4} \alpha_{9} \alpha_{11}, r 9=\alpha_{12} \alpha_{6}$ e $r 10=\alpha_{11} \alpha_{7}$.Os ciclos elementares são: $C_{1}=\alpha_{1} \alpha_{3} \alpha_{8} \alpha_{5}, C_{2}=\alpha_{2} \alpha_{4} \alpha_{8} \alpha_{5}, C_{3}=\alpha_{4} \alpha_{9} \alpha_{10}, C_{4}=\alpha_{6} \alpha_{9} \alpha_{11}$ e $C_{5}=\alpha_{7} \alpha_{12}$.

Portanto, obtemos a Phia 62 através do corte $\left\{\alpha_{3}, \alpha_{4}, \alpha_{6}, \alpha_{7}\right\}$.

O caso seguinte pode ser descartado aplicando o lema 5.42 e a resposta é o corte $\left\{\alpha_{3}, \alpha_{4}, \alpha_{6}, \alpha_{7}\right\}$.
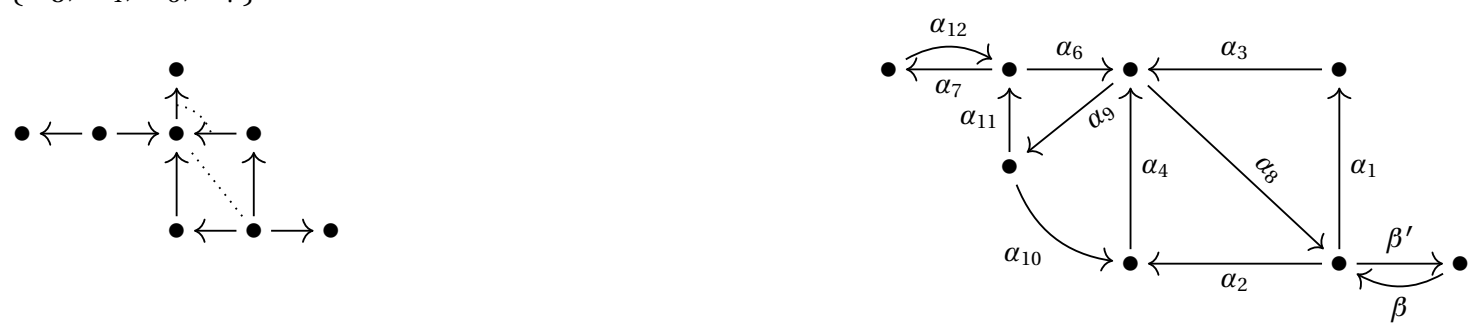

$\mathcal{F} r 21.5$
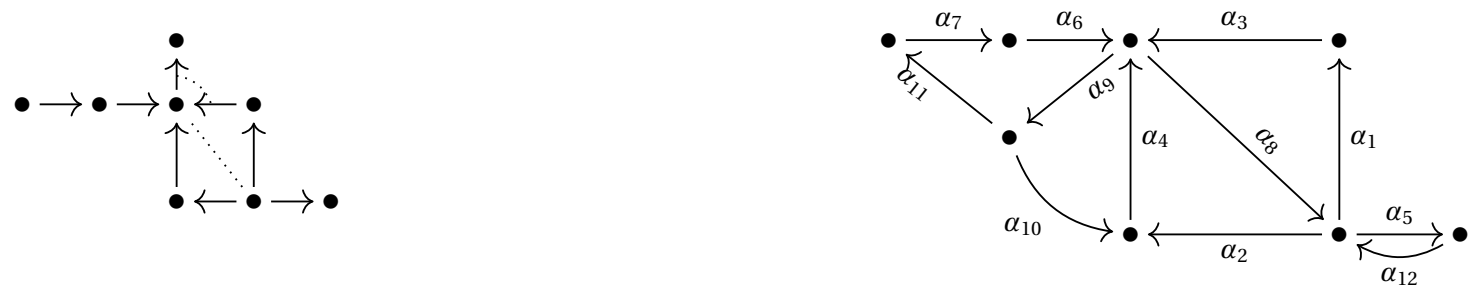

As relações do tipo 2 são: $r 1=\alpha_{3} \alpha_{8} \alpha_{2}, r 2=\alpha_{4} \alpha_{8} \alpha_{1}, r 3=\alpha_{3} \alpha_{9}, r 4=\alpha_{2} \alpha_{4} \alpha_{9}$, $r 5=\alpha_{10} \alpha_{4} \alpha_{8}, r 6=\alpha_{6} \alpha_{8}, r 7=\alpha_{6} \alpha_{9} \alpha_{10}, r 8=\alpha_{4} \alpha_{9} \alpha_{11}, r 9=\alpha_{12} \alpha_{2}, r 10=\alpha_{12} \alpha_{1}$ e $r 11=\alpha_{8} \alpha_{5}$.Os ciclos elementares são: $C_{1}=\alpha_{1} \alpha_{3} \alpha_{8}, C_{2}=\alpha_{2} \alpha_{4} \alpha_{8}, C_{3}=\alpha_{4} \alpha_{9} \alpha_{10}$, $C_{4}=\alpha_{7} \alpha_{6} \alpha_{9} \alpha_{11}$ e $C_{5}=\alpha_{5} \alpha_{12}$.

Logo, o programa mostra a solução 63 através do corte $\left\{\alpha_{8}, \alpha_{9}, \alpha_{12}\right\}$.

$\mathcal{F} r 21.6$
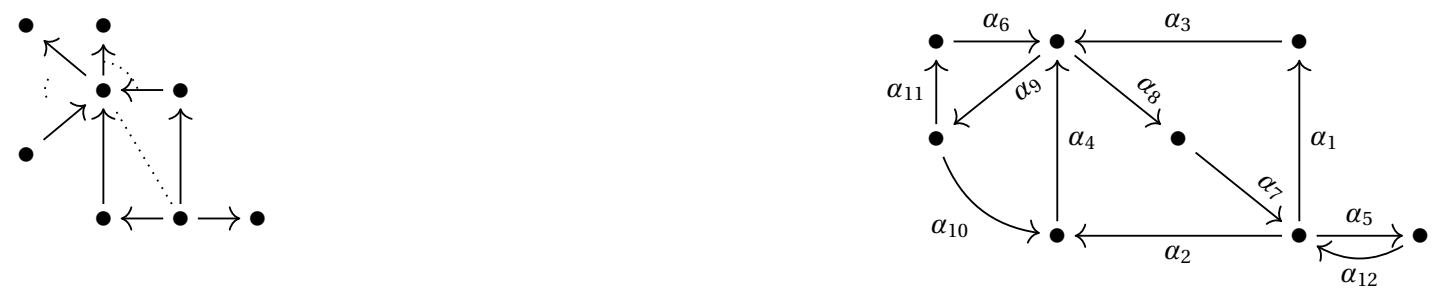
As relações do tipo 2 são: $r 1=\alpha_{3} \alpha_{8} \alpha_{7} \alpha_{2}, r 2=\alpha_{4} \alpha_{8} \alpha_{7} \alpha_{1}, r 3=\alpha_{3} \alpha_{9}, r 4=\alpha_{2} \alpha_{4} \alpha_{9}$, $r 5=\alpha_{10} \alpha_{4} \alpha_{8}, r 6=\alpha_{6} \alpha_{8}, r 7=\alpha_{6} \alpha_{9} \alpha_{10}, r 8=\alpha_{4} \alpha_{9} \alpha_{11}, r 9=\alpha_{12} \alpha_{2}, r 10=\alpha_{12} \alpha_{1} \mathrm{e}$ $r 11=\alpha_{7} \alpha_{5}$.Os ciclos elementares são: $C_{1}=\alpha_{1} \alpha_{3} \alpha_{8} \alpha_{7}, C_{2}=\alpha_{2} \alpha_{4} \alpha_{8} \alpha_{7}, C_{3}=\alpha_{4} \alpha_{9} \alpha_{10}$, $C_{4}=\alpha_{6} \alpha_{9} \alpha_{11}$ e $C_{5}=\alpha_{5} \alpha_{12}$.

Entramos com essas informações e não temos solução.

$\mathcal{F} r 21.7$
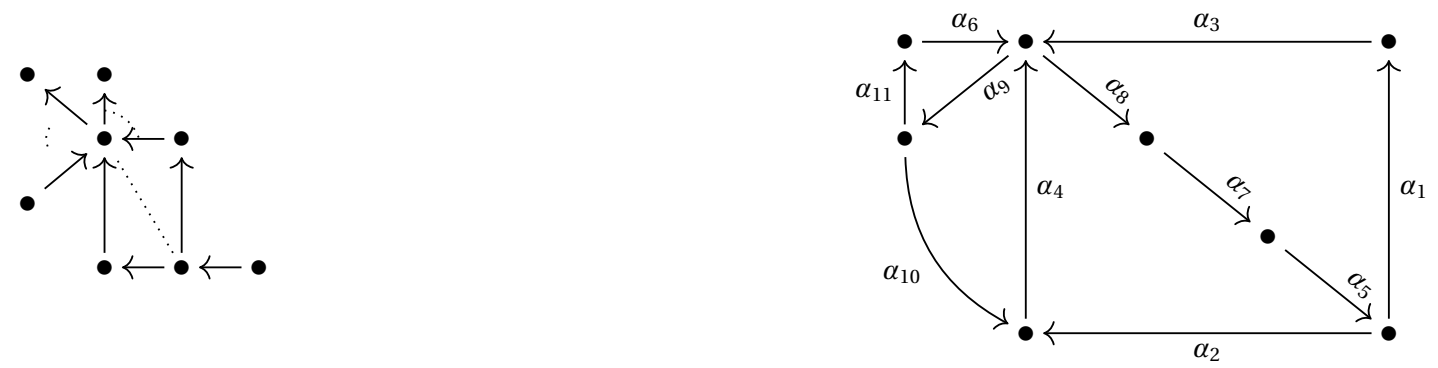

As relações do tipo 2 são: $r 1=\alpha_{3} \alpha_{8} \alpha_{7} \alpha_{5} \alpha_{2}, r 2=\alpha_{4} \alpha_{8} \alpha_{7} \alpha_{5} \alpha_{1}, r 3=\alpha_{3} \alpha_{9}, r 4=\alpha_{2} \alpha_{4} \alpha_{9}$, $r 5=\alpha_{10} \alpha_{4} \alpha_{8}, r 6=\alpha_{6} \alpha_{8}, r 7=\alpha_{6} \alpha_{9} \alpha_{10}$ e $r 8=\alpha_{4} \alpha_{9} \alpha_{11}$.Os ciclos elementares são: $C_{1}=\alpha_{1} \alpha_{3} \alpha_{8} \alpha_{7} \alpha_{5}, C_{2}=\alpha_{2} \alpha_{4} \alpha_{8} \alpha_{7} \alpha_{5}, C_{3}=\alpha_{4} \alpha_{9} \alpha_{10}$ e $C_{4}=\alpha_{6} \alpha_{9} \alpha_{11}$.

Entramos com essas informações no programa, obtemos as Phias 64 e 65 através dos cortes $\left\{\alpha_{3}, \alpha_{4}, \alpha_{6}\right\}$ e $\left\{\alpha_{8}, \alpha_{9}\right\}$, respectivamente.

$\mathcal{F} r 22.1$
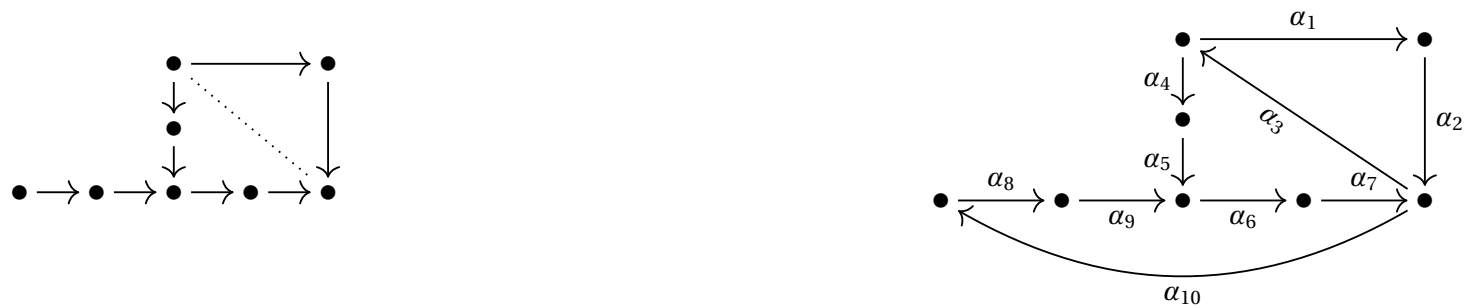

As relações do tipo 2 são: $r 1=\alpha_{2} \alpha_{3} \alpha_{4}, r 2=\alpha_{7} \alpha_{3} \alpha_{1}, r 3=\alpha_{5} \alpha_{6} \alpha_{7} \alpha_{8}, r 4=\alpha_{9} \alpha_{6} \alpha_{7} \alpha_{3}$ e $r 5=\alpha_{2} \alpha_{8}$.Os ciclos elementares são: $C_{1}=\alpha_{1} \alpha_{2} \alpha_{3}, C_{2}=\alpha_{3} \alpha_{4} \alpha_{5} \alpha_{6} \alpha_{7}$ e $C_{3}=$ $\alpha_{10} \alpha_{9} \alpha_{6} \alpha_{7} \alpha_{8}$.

Logo, obtemos a solução 66 pelo corte $\left\{\alpha_{7}, \alpha_{2}\right\}$. Utilizamos essa resposta em conjunto com o lema 5.42 e descartamos o seguinte caso:
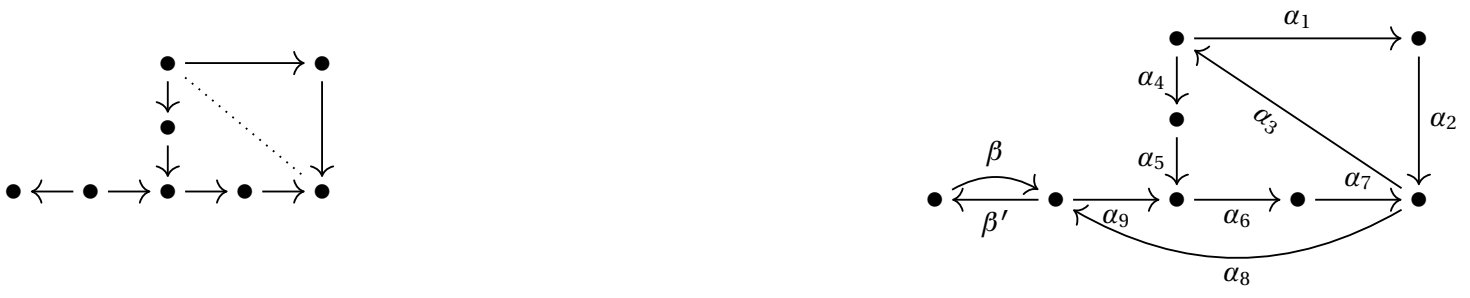

$\mathcal{F} r 22.2$
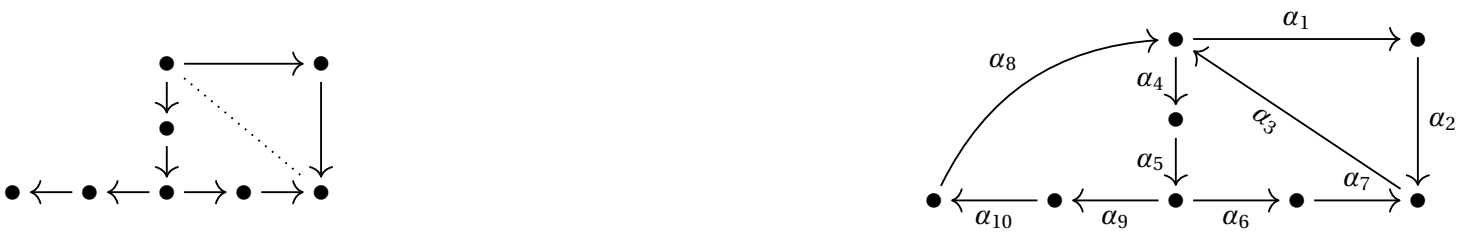

As relações do tipo 2 são: $r 1=\alpha_{2} \alpha_{3} \alpha_{4}, r 2=\alpha_{7} \alpha_{3} \alpha_{1}, r 3=\alpha_{3} \alpha_{4} \alpha_{5} \alpha_{9}, r 4=\alpha_{8} \alpha_{4} \alpha_{5} \alpha_{6}$ e $r 5=\alpha_{8} \alpha_{1}$. Os ciclos elementares são: $C_{1}=\alpha_{1} \alpha_{2} \alpha_{3}, C_{2}=\alpha_{3} \alpha_{4} \alpha_{5} \alpha_{6} \alpha_{7}$ e $C_{3}=$ 
$\alpha_{10} \alpha_{8} \alpha_{4} \alpha_{5} \alpha_{9}$

Portanto mostramos a solução 67 pelo corte $\left\{\alpha_{4}, \alpha_{1}\right\}$. Utilizamos essa resposta em conjunto com o lema 5.42 e desconsideramos o último caso:
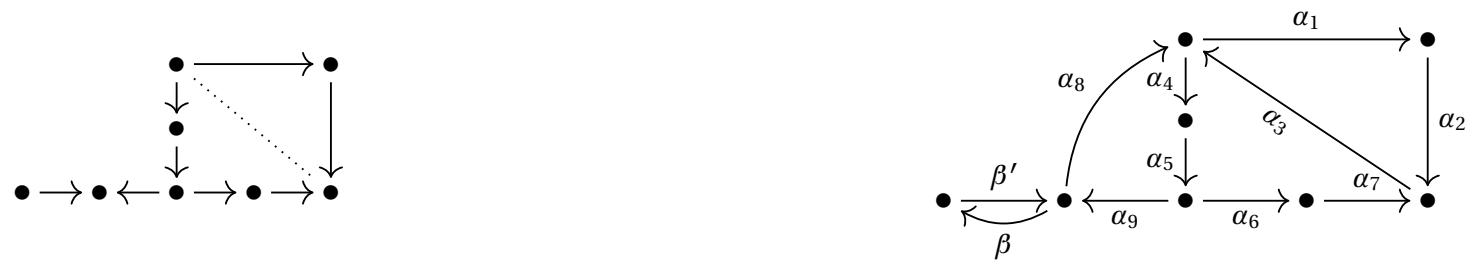

$\mathcal{F} r 23.1$
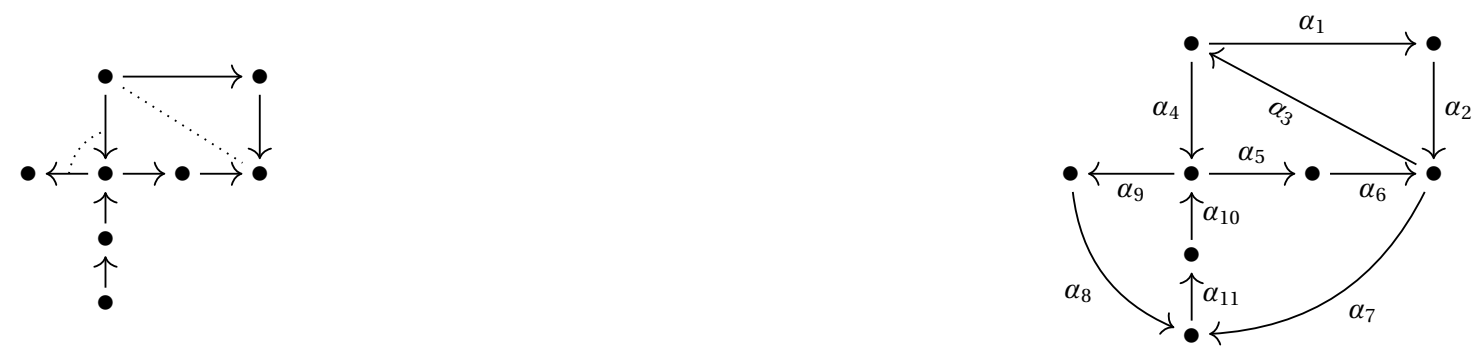

As relações do tipo 2 são: $r 1=\alpha_{2} \alpha_{3} \alpha_{4}, r 2=\alpha_{6} \alpha_{3} \alpha_{1}, r 3=\alpha_{4} \alpha_{5} \alpha_{6} \alpha_{7}, r 4=\alpha_{10} \alpha_{5} \alpha_{6} \alpha_{3}$, $r 5=\alpha_{2} \alpha_{7}, r 6=\alpha_{7} \alpha_{11} \alpha_{10} \alpha_{9}, r 7=\alpha_{8} \alpha_{11} \alpha_{10} \alpha_{5}$ e $r 8=\alpha_{4} \alpha_{9}$.Os ciclos elementares são: $C_{1}=\alpha_{1} \alpha_{2} \alpha_{3}, C_{2}=\alpha_{3} \alpha_{4} \alpha_{5} \alpha_{6}, C_{3}=\alpha_{11} \alpha_{10} \alpha_{5} \alpha_{6} \alpha_{7}$ e $C_{4}=\alpha_{11} \alpha_{10} \alpha_{9} \alpha_{8}$.

Entrando com essas informações no programa, mostramos que não tem solução. Aproveitamos essa resposta e de forma análoga, podemos eliminar esse caso:
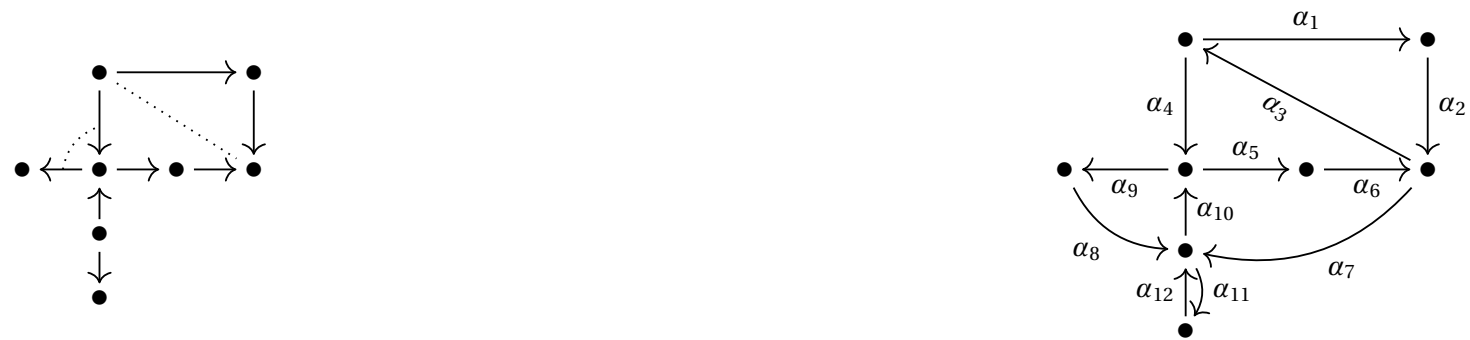

$\mathcal{F} r 23.2$
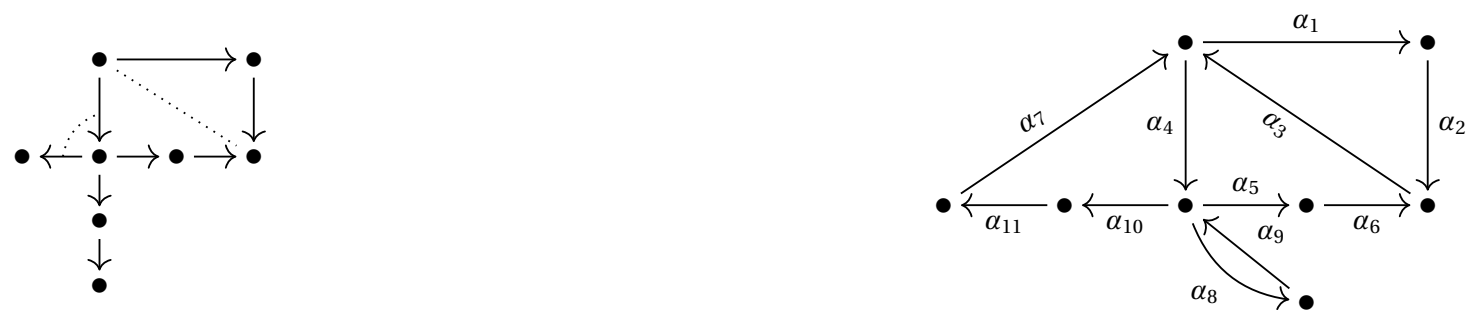

As relações do tipo 2 são: $r 1=\alpha_{2} \alpha_{3} \alpha_{4}, r 2=\alpha_{6} \alpha_{3} \alpha_{1}, r 3=\alpha_{3} \alpha_{4} \alpha_{10}, r 4=\alpha_{7} \alpha_{4} \alpha_{5}$, $r 5=\alpha_{7} \alpha_{1}, r 6=\alpha_{4} \alpha_{8}, r 7=\alpha_{9} \alpha_{5}$ e $r 8=\alpha_{9} \alpha_{10}$.Os ciclos elementares são: $C_{1}=\alpha_{1} \alpha_{2} \alpha_{3}$, $C_{2}=\alpha_{3} \alpha_{4} \alpha_{5} \alpha_{6}, C_{3}=\alpha_{10} \alpha_{11} \alpha_{7} \alpha_{4}$ e $C_{4}=\alpha_{9} \alpha_{8}$.

Logo, mostramos a existência da Phia 68 através do corte $\left\{\alpha_{1}, \alpha_{4}, \alpha_{9}\right\}$. Mais ainda, pelo lema 5.42 e com esse corte, podemos descartar o seguinte caso: 

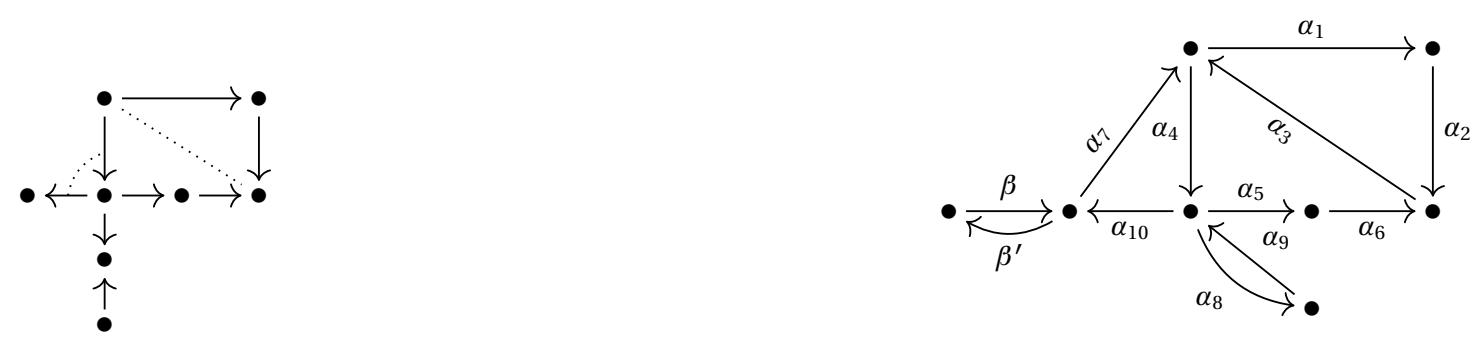

$\mathcal{F} r 23.3$
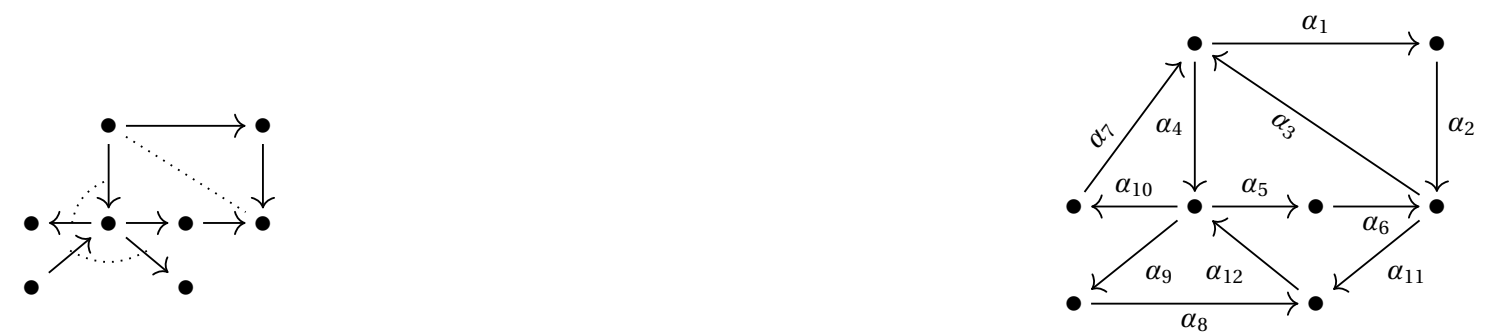

As relações do tipo 2 são: $r 1=\alpha_{2} \alpha_{3} \alpha_{4}, r 2=\alpha_{6} \alpha_{3} \alpha_{1}, r 3=\alpha_{3} \alpha_{4} \alpha_{10}, r 4=\alpha_{7} \alpha_{4} \alpha_{5}$, $r 5=\alpha_{7} \alpha_{1}, r 6=\alpha_{4} \alpha_{9}, r 7=\alpha_{8} \alpha_{12} \alpha_{5}, r 8=\alpha_{11} \alpha_{12} \alpha_{9}, r 9=\alpha_{2} \alpha_{11}, r 10=\alpha_{12} \alpha_{10}, r 11=$ $\alpha_{4} \alpha_{5} \alpha_{6} \alpha_{11}$ e $r 12=\alpha_{12} \alpha_{5} \alpha_{6} \alpha_{3}$. Os ciclos elementares são: $C_{1}=\alpha_{1} \alpha_{2} \alpha_{3}, C_{2}=\alpha_{3} \alpha_{4} \alpha_{5} \alpha_{6}$, $C_{3}=\alpha_{10} \alpha_{7} \alpha_{4}, C_{4}=\alpha_{9} \alpha_{8} \alpha_{12}$ e $C_{5}=\alpha_{12} \alpha_{5} \alpha_{6} \alpha_{11}$.

Entretanto, essa extensão trivial não tem corte que desejamos.

$\mathcal{F} r 24.1$
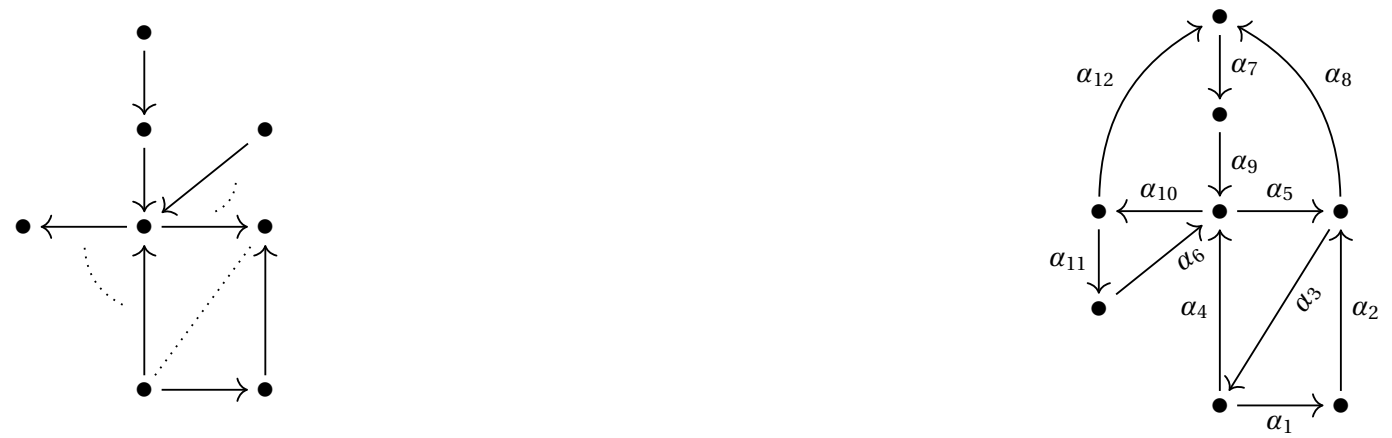

As relações do tipo 2 são: $r 1=\alpha_{2} \alpha_{3} \alpha_{4}, r 2=\alpha_{5} \alpha_{3} \alpha_{1}, r 3=\alpha_{6} \alpha_{5}, r 4=\alpha_{4} \alpha_{10}, r 5=$ $\alpha_{9} \alpha_{5} \alpha_{3}, r 6=\alpha_{2} \alpha_{8}, r 7=\alpha_{8} \alpha_{7} \alpha_{9} \alpha_{10}, r 8=\alpha_{12} \alpha_{7} \alpha_{9} \alpha_{5}, r 9=\alpha_{6} \alpha_{10} \alpha_{12}, r 10=\alpha_{4} \alpha_{5} \alpha_{8} \mathrm{e}$ $r 11=\alpha_{9} \alpha_{10} \alpha_{11}$.Os ciclos elementares são: $C_{1}=\alpha_{1} \alpha_{2} \alpha_{3}, C_{2}=\alpha_{3} \alpha_{4} \alpha_{5}, C_{3}=\alpha_{8} \alpha_{7} \alpha_{9} \alpha_{5}$, $C_{4}=\alpha_{6} \alpha_{10} \alpha_{11}$ e $C_{5}=\alpha_{10} \alpha_{12} \alpha_{7} \alpha_{9}$.

Portanto, essa extensão trivial tem o corte $\left\{\alpha_{2}, \alpha_{5}, \alpha_{10}\right\}$ originando a Phia 69. Usando essa resposta em conjunto com o lema 5.42, podemos eliminar o seguinte caso:
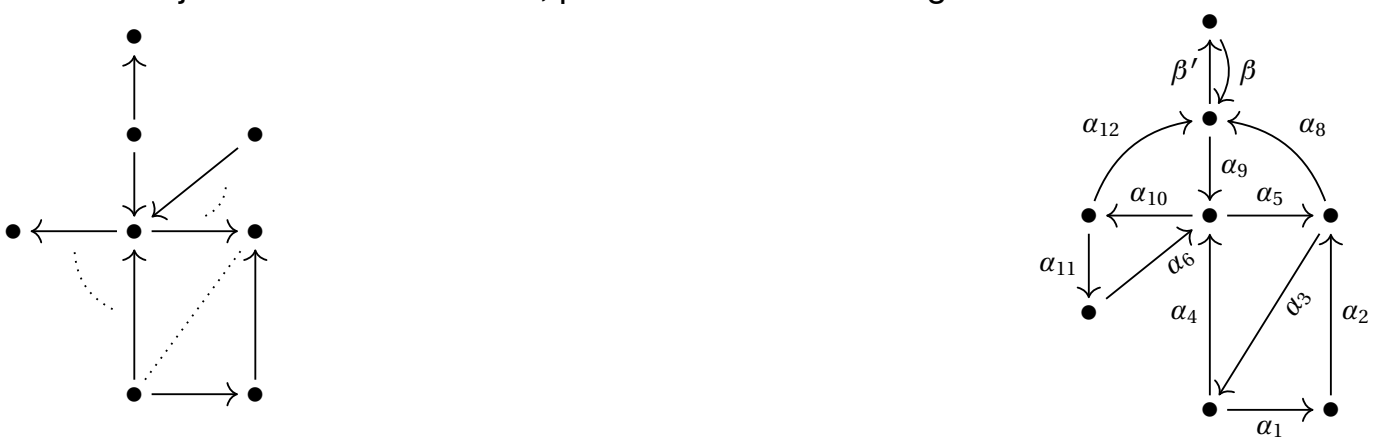
$\mathcal{F} r 24.2$
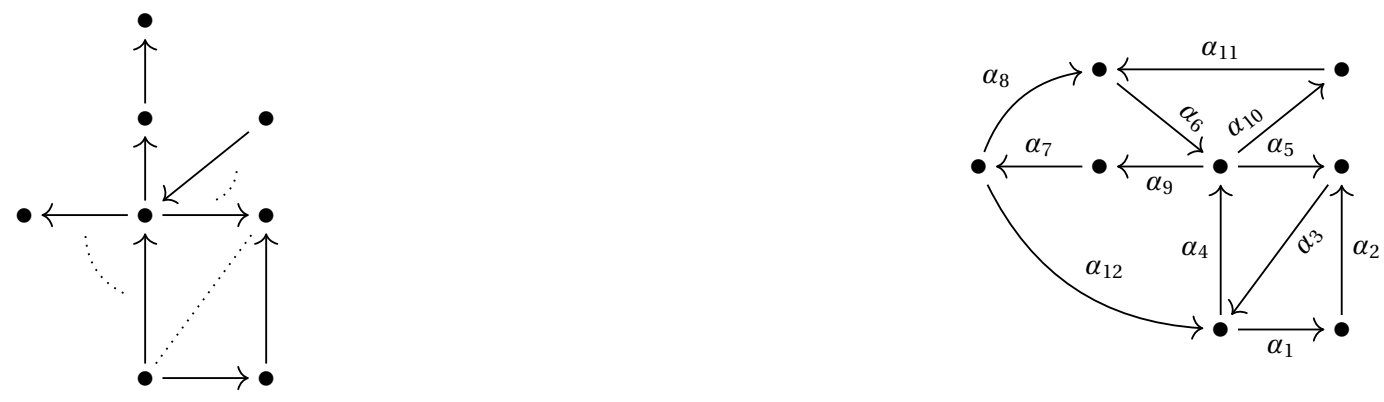

As relações do tipo 2 são: $r 1=\alpha_{2} \alpha_{3} \alpha_{4}, r 2=\alpha_{5} \alpha_{3} \alpha_{1}, r 3=\alpha_{6} \alpha_{5}, r 4=\alpha_{4} \alpha_{10}, r 5=$ $\alpha_{3} \alpha_{4} \alpha_{9}, r 6=\alpha_{12} \alpha_{4} \alpha_{5}, r 7=\alpha_{12} \alpha_{1}, r 8=\alpha_{4} \alpha_{9} \alpha_{7} \alpha_{8}, r 9=\alpha_{6} \alpha_{9} \alpha_{7} \alpha_{12}, r 10=\alpha_{8} \alpha_{6} \alpha_{10} \mathrm{e}$ $r 11=\alpha_{11} \alpha_{6} \alpha_{9}$.Os ciclos elementares são: $C_{1}=\alpha_{1} \alpha_{2} \alpha_{3}, C_{2}=\alpha_{3} \alpha_{4} \alpha_{5}, C_{3}=\alpha_{8} \alpha_{6} \alpha_{9} \alpha_{7}$, $C_{4}=\alpha_{6} \alpha_{10} \alpha_{11}$ e $C_{5}=\alpha_{12} \alpha_{4} \alpha_{9} \alpha_{7}$.

Sendo assim, temos a solução dual da Phia 69 através do corte $\left\{\alpha_{1}, \alpha_{4}, \alpha_{6}\right\}$. O lema 5.42 e juntamente com essa resposta, podemos desconsiderar o caso abaixo:
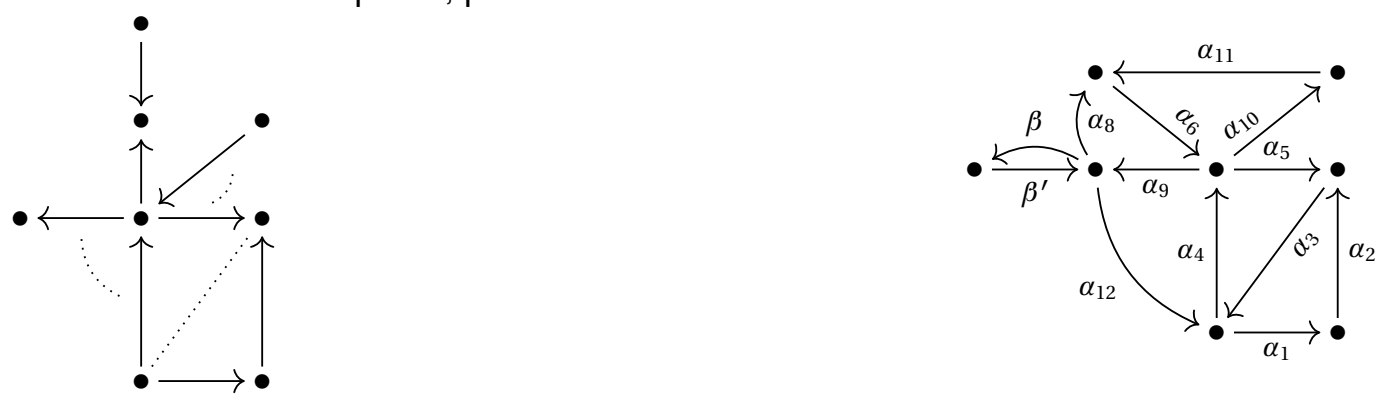

$\mathcal{F} r 24.3$
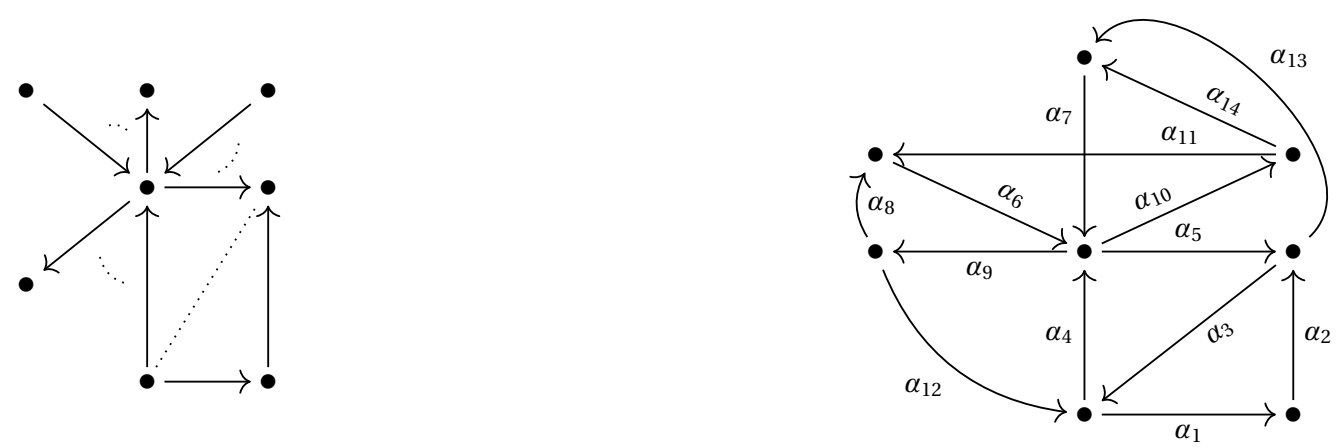

As relações do tipo 2 são: $r 1=\alpha_{2} \alpha_{3} \alpha_{4}, r 2=\alpha_{5} \alpha_{3} \alpha_{1}, r 3=\alpha_{6} \alpha_{5}, r 4=\alpha_{4} \alpha_{10}, r 5=$ $\alpha_{3} \alpha_{4} \alpha_{9}, r 6=\alpha_{12} \alpha_{4} \alpha_{5}, r 7=\alpha_{12} \alpha_{1}, r 8=\alpha_{4} \alpha_{9} \alpha_{8}, r 9=\alpha_{6} \alpha_{9} \alpha_{12}, r 10=\alpha_{8} \alpha_{6} \alpha_{10}, r 11=$ $\alpha_{11} \alpha_{6} \alpha_{9}, r 12=\alpha_{7} \alpha_{9}, r 13=\alpha_{7} \alpha_{10} \alpha_{11}, r 14=\alpha_{6} \alpha_{10} \alpha_{14}, r 15=\alpha_{4} \alpha_{5} \alpha_{13}, r 16=\alpha_{7} \alpha_{5} \alpha_{3}$ e $r 17=\alpha_{2} \alpha_{13}$. Os ciclos elementares são: $C_{1}=\alpha_{1} \alpha_{2} \alpha_{3}, C_{2}=\alpha_{3} \alpha_{4} \alpha_{5}, C_{3}=\alpha_{8} \alpha_{6} \alpha_{9}$, $C_{4}=\alpha_{6} \alpha_{10} \alpha_{11}, C_{5}=\alpha_{12} \alpha_{4} \alpha_{9}, C_{6}=\alpha_{7} \alpha_{10} \alpha_{14}$ e $C_{7}=\alpha_{7} \alpha_{5} \alpha_{13}$.

Logo, o programa não mostra solução.

$\mathcal{F} r 25.1$
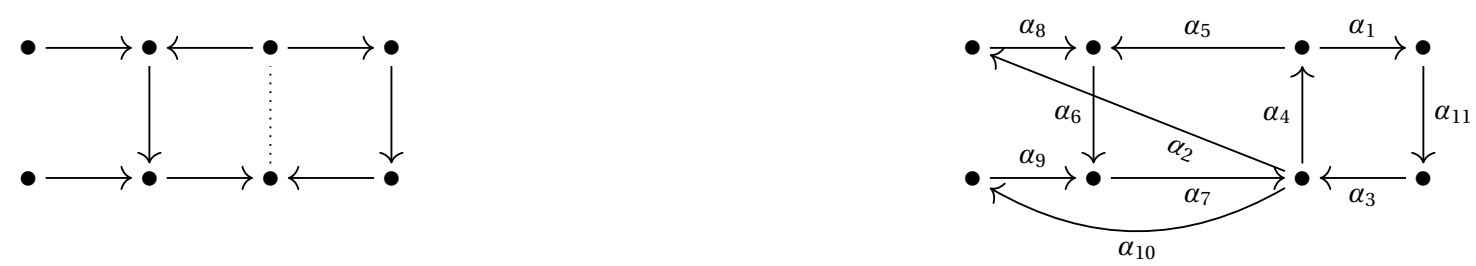

As relações do tipo 2 são: $r 1=\alpha_{3} \alpha_{4} \alpha_{5}, r 2=\alpha_{7} \alpha_{4} \alpha_{1}, r 3=\alpha_{8} \alpha_{6} \alpha_{7} \alpha_{4}, r 4=\alpha_{5} \alpha_{6} \alpha_{7} \alpha_{2}$, 
$r 5=\alpha_{3} \alpha_{2}, r 6=\alpha_{3} \alpha_{10}, r 7=\alpha_{9} \alpha_{7} \alpha_{4}, r 8=\alpha_{6} \alpha_{7} \alpha_{10}$ e $r 9=\alpha_{9} \alpha_{7} \alpha_{2}$.Os ciclos elementares são: $C_{1}=\alpha_{1} \alpha_{11} \alpha_{3} \alpha_{4}, C_{2}=\alpha_{5} \alpha_{6} \alpha_{7} \alpha_{4}, C_{3}=\alpha_{9} \alpha_{7} \alpha_{10}$ e $C_{4}=\alpha_{8} \alpha_{6} \alpha_{7} \alpha_{2}$.

Portanto, o programa exibe o corte $\left\{\alpha_{3}, \alpha_{7}\right\}$ que origina a Phia 70.

$\mathcal{F} r 25.2$
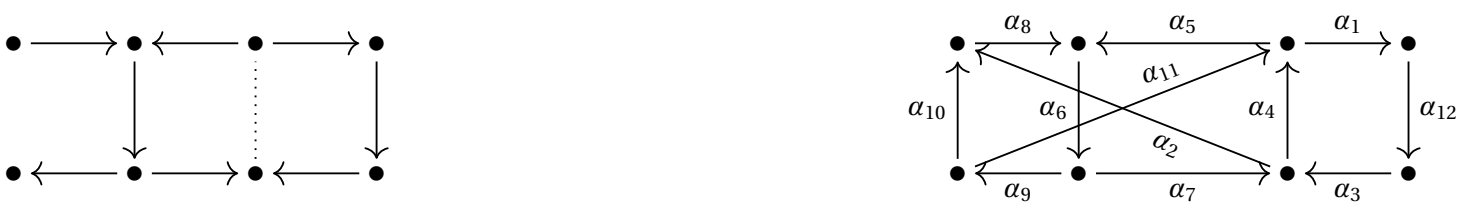

As relações do tipo 2 são: $r 1=\alpha_{3} \alpha_{4} \alpha_{5}, r 2=\alpha_{7} \alpha_{4} \alpha_{1}, r 3=\alpha_{8} \alpha_{6} \alpha_{7} \alpha_{4}, r 4=\alpha_{5} \alpha_{6} \alpha_{7} \alpha_{2}$, $r 5=\alpha_{3} \alpha_{2}, r 6=\alpha_{5} \alpha_{6} \alpha_{9} \alpha_{10}, r 7=\alpha_{10} \alpha_{8} \alpha_{6} \alpha_{7}, r 8=\alpha_{2} \alpha_{8} \alpha_{6} \alpha_{9}, r 9=\alpha_{11} \alpha_{1}$, $r 10=\alpha_{11} \alpha_{5} \alpha_{6} \alpha_{7}$ e $r 11=\alpha_{4} \alpha_{5} \alpha_{6} \alpha_{9}$. Os ciclos elementares são: $C_{1}=\alpha_{1} \alpha_{12} \alpha_{3} \alpha_{4}$, $C_{2}=\alpha_{5} \alpha_{6} \alpha_{7} \alpha_{4}, C_{3}=\alpha_{9} \alpha_{10} \alpha_{8} \alpha_{6}, C_{4}=\alpha_{8} \alpha_{6} \alpha_{7} \alpha_{2}$ e $C_{5}=\alpha_{5} \alpha_{6} \alpha_{9} \alpha_{11}$.

Portanto, o programa não exibe solução diferente da álgebra disfarçada.

$\mathcal{F} r 25.3$
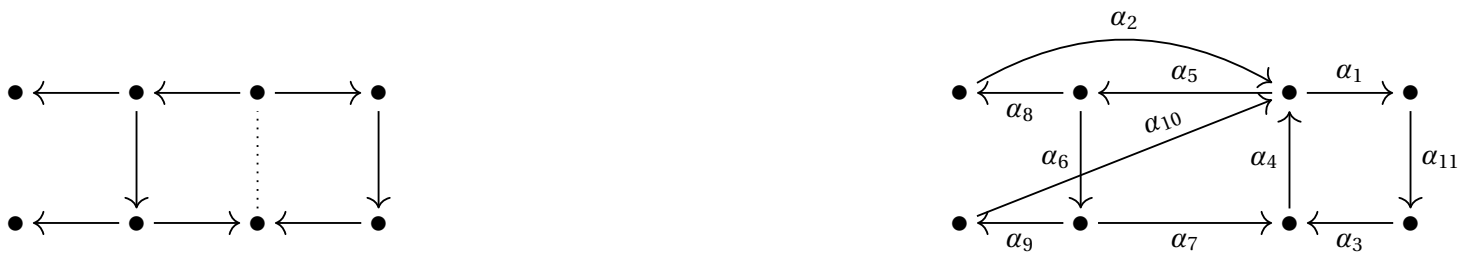

As relações do tipo 2 são: $r 1=\alpha_{3} \alpha_{4} \alpha_{5}, r 2=\alpha_{7} \alpha_{4} \alpha_{1}, r 3=\alpha_{10} \alpha_{5} \alpha_{6} \alpha_{7}, r 4=\alpha_{4} \alpha_{5} \alpha_{6} \alpha_{9}$, $r 5=\alpha_{10} \alpha_{1}, r 6=\alpha_{2} \alpha_{5} \alpha_{6}, r 7=\alpha_{4} \alpha_{5} \alpha_{8}, r 8=\alpha_{2} \alpha_{1}$ e $r 9=\alpha_{10} \alpha_{5} \alpha_{8}$.Os ciclos elementares são: $C_{1}=\alpha_{1} \alpha_{11} \alpha_{3} \alpha_{4}, C_{2}=\alpha_{5} \alpha_{6} \alpha_{7} \alpha_{4}, C_{3}=\alpha_{9} \alpha_{10} \alpha_{5} \alpha_{6}$ e $C_{4}=\alpha_{5} \alpha_{8} \alpha_{2}$.

Entrando com essas informações, obtemos uma solução dual da 70 através do corte $\left\{\alpha_{1}, \alpha_{5}\right\}$.

$\mathcal{F} r 25.4$
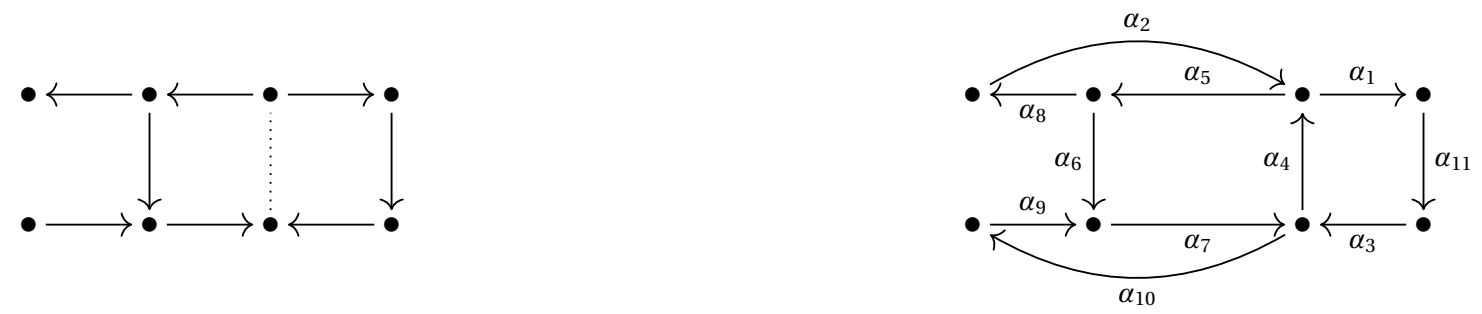

As relações do tipo 2 são: $r 1=\alpha_{3} \alpha_{4} \alpha_{5}, r 2=\alpha_{7} \alpha_{4} \alpha_{1}, r 3=\alpha_{9} \alpha_{7} \alpha_{4}, r 4=\alpha_{6} \alpha_{7} \alpha_{10}$, $r 5=\alpha_{3} \alpha_{10}, r 6=\alpha_{2} \alpha_{5} \alpha_{6}, r 7=\alpha_{4} \alpha_{5} \alpha_{8}$ e $r 8=\alpha_{2} \alpha_{1}$.Os ciclos elementares são: $C_{1}=$ $\alpha_{1} \alpha_{11} \alpha_{3} \alpha_{4}, C_{2}=\alpha_{5} \alpha_{6} \alpha_{7} \alpha_{4}, C_{3}=\alpha_{9} \alpha_{7} \alpha_{10}$ e $C_{4}=\alpha_{5} \alpha_{8} \alpha_{2}$.

Último caso desse frame, não temos um integrante da família ANS.

Observamos que o frame $\mathcal{F} r 26$ é bem semelhante ao anterior. Essa semelhança implica na extensão trivial. As respostas do frame $\mathcal{F} r 25$ se aplicam diretamente nesse frame, juntamente com as extensões triviais, seus ciclos elementares e relações do tipo 2 . Portanto, obtemos apenas a Phia 71. 
$\mathcal{F} r 26.1$
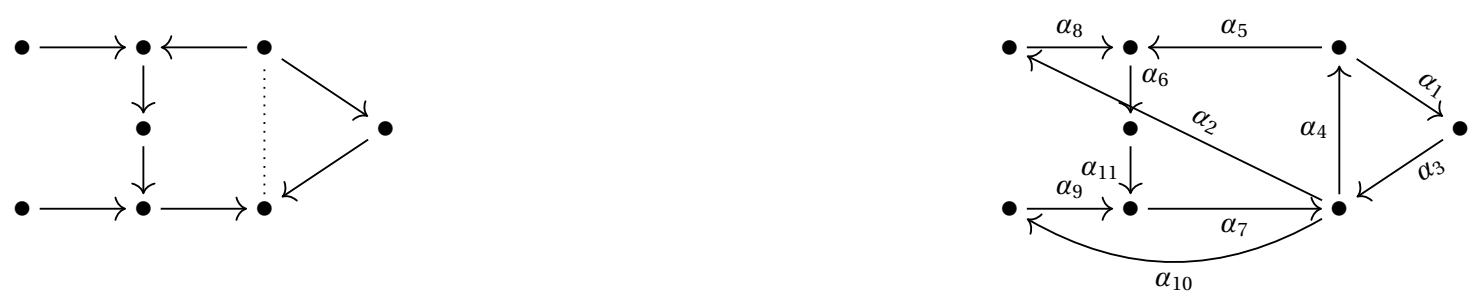

As relações do tipo 2 são: $r 1=\alpha_{3} \alpha_{4} \alpha_{5}, r 2=\alpha_{7} \alpha_{4} \alpha_{1}, r 3=\alpha_{8} \alpha_{6} \alpha_{11} \alpha_{7} \alpha_{4}, r 4=$ $\alpha_{5} \alpha_{6} \alpha_{11} \alpha_{7} \alpha_{2}, r 5=\alpha_{3} \alpha_{2}, r 6=\alpha_{3} \alpha_{10}, r 7=\alpha_{9} \alpha_{7} \alpha_{4}, r 8=\alpha_{11} \alpha_{7} \alpha_{10}$ e $r 9=\alpha_{9} \alpha_{7} \alpha_{2}$.Os ciclos elementares são: $C_{1}=\alpha_{1} \alpha_{3} \alpha_{4}, C_{2}=\alpha_{5} \alpha_{6} \alpha_{11} \alpha_{7} \alpha_{4}, C_{3}=\alpha_{9} \alpha_{7} \alpha_{10}$ e $C_{4}=$ $\alpha_{8} \alpha_{6} \alpha_{11} \alpha_{7} \alpha_{2}$.

Igual ao caso $\mathcal{F} r 25.1$, obtemos o corte $\left\{\alpha_{3}, \alpha_{7}\right\}$ que origina a Phia 71.
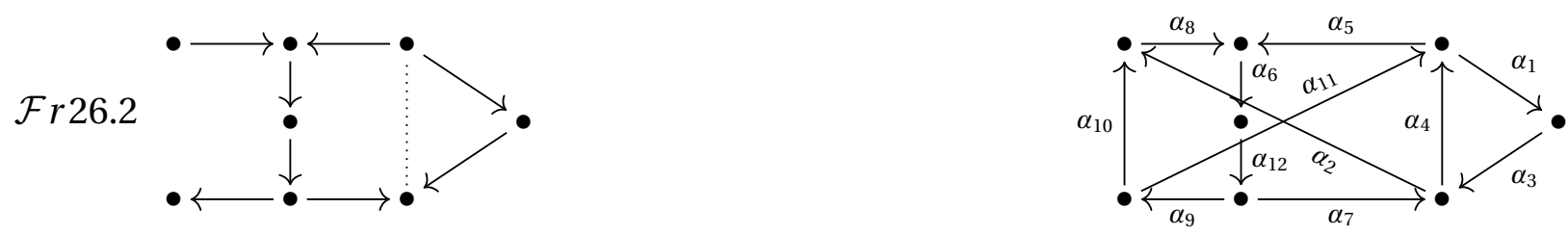

As relações do tipo 2 são: $r 1=\alpha_{3} \alpha_{4} \alpha_{5}, r 2=\alpha_{7} \alpha_{4} \alpha_{1}, r 3=\alpha_{8} \alpha_{6} \alpha_{12} \alpha_{7} \alpha_{4}, r 4=$ $\alpha_{5} \alpha_{6} \alpha_{12} \alpha_{7} \alpha_{2}, r 5=\alpha_{3} \alpha_{2}, r 6=\alpha_{5} \alpha_{6} \alpha_{12} \alpha_{9} \alpha_{10}, r 7=\alpha_{10} \alpha_{8} \alpha_{6} \alpha_{12} \alpha_{7}, r 8=\alpha_{2} \alpha_{8} \alpha_{6} \alpha_{12} \alpha_{9}$, $r 9=\alpha_{11} \alpha_{1}, r 10=\alpha_{11} \alpha_{5} \alpha_{6} \alpha_{12} \alpha_{7}$ e $r 11=\alpha_{4} \alpha_{5} \alpha_{6} \alpha_{12} \alpha_{9}$.Os ciclos elementares são: $C_{1}=$ $\alpha_{1} \alpha_{3} \alpha_{4}, C_{2}=\alpha_{5} \alpha_{6} \alpha_{12} \alpha_{7} \alpha_{4}, C_{3}=\alpha_{9} \alpha_{10} \alpha_{8} \alpha_{6} \alpha_{12}, C_{4}=\alpha_{8} \alpha_{6} \alpha_{12} \alpha_{7} \alpha_{2}$ e $C_{5}=\alpha_{5} \alpha_{6} \alpha_{12} \alpha_{9} \alpha_{11}$.

$\mathcal{F} r 26.3$
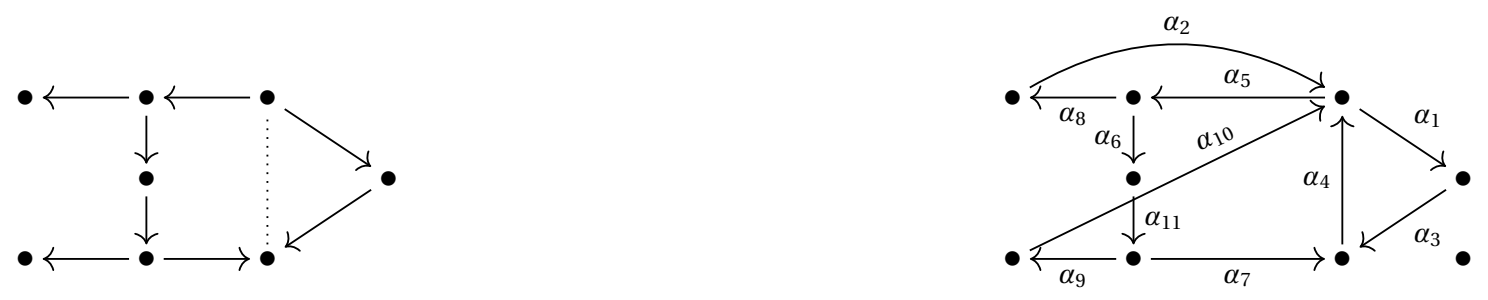

As relações do tipo 2 são: $r 1=\alpha_{3} \alpha_{4} \alpha_{5}, r 2=\alpha_{7} \alpha_{4} \alpha_{1}, r 3=\alpha_{10} \alpha_{5} \alpha_{6} \alpha_{11} \alpha_{7}, r 4=$ $\alpha_{4} \alpha_{5} \alpha_{6} \alpha_{11} \alpha_{9}, r 5=\alpha_{10} \alpha_{1}, r 6=\alpha_{2} \alpha_{5} \alpha_{6}, r 7=\alpha_{4} \alpha_{5} \alpha_{8}, r 8=\alpha_{2} \alpha_{1}$ e $r 9=\alpha_{10} \alpha_{5} \alpha_{8}$.Os ciclos elementares são: $C_{1}=\alpha_{1} \alpha_{3} \alpha_{4}, C_{2}=\alpha_{5} \alpha_{6} \alpha_{11} \alpha_{7} \alpha_{4}, C_{3}=\alpha_{9} \alpha_{10} \alpha_{5} \alpha_{6} \alpha_{11} \mathrm{e}$ $C_{4}=\alpha_{5} \alpha_{8} \alpha_{2}$.

$\mathcal{F} r 26.4$
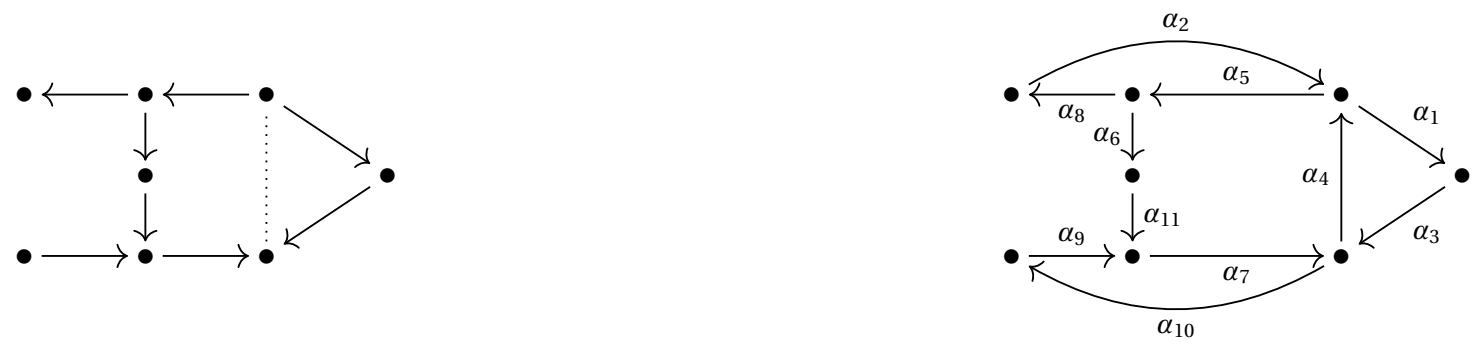

As relações do tipo 2 são: $r 1=\alpha_{3} \alpha_{4} \alpha_{5}, r 2=\alpha_{7} \alpha_{4} \alpha_{1}, r 3=\alpha_{9} \alpha_{7} \alpha_{4}, r 4=\alpha_{11} \alpha_{7} \alpha_{10}, r 5=$ $\alpha_{3} \alpha_{10}, r 6=\alpha_{2} \alpha_{5} \alpha_{6}, r 7=\alpha_{4} \alpha_{5} \alpha_{8}$ e $r 8=\alpha_{2} \alpha_{1}$. Os ciclos elementares são: $C_{1}=\alpha_{1} \alpha_{3} \alpha_{4}$, $C_{2}=\alpha_{5} \alpha_{6} \alpha_{11} \alpha_{7} \alpha_{4}, C_{3}=\alpha_{9} \alpha_{7} \alpha_{10}$ e $C_{4}=\alpha_{5} \alpha_{8} \alpha_{2}$. 
$\mathcal{F} r 27$
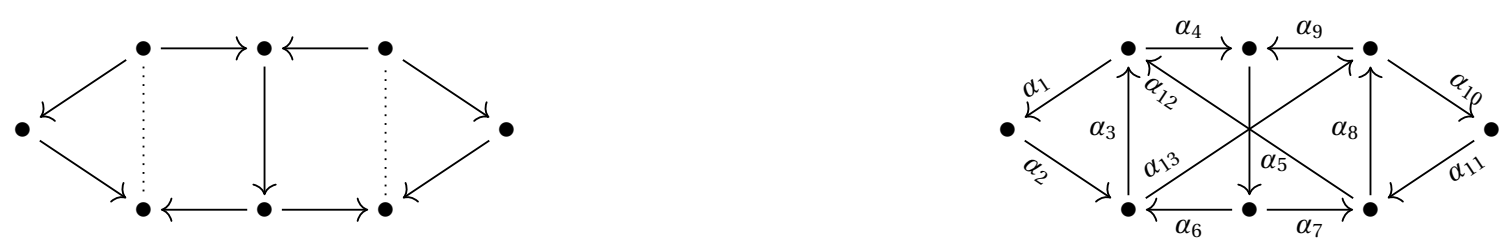

As relações do tipo 2 são: $r 1=\alpha_{2} \alpha_{3} \alpha_{4}, r 2=\alpha_{6} \alpha_{3} \alpha_{1}, r 3=\alpha_{3} \alpha_{4} \alpha_{5} \alpha_{7}, r 4=\alpha_{9} \alpha_{5} \alpha_{6} \alpha_{3}$, $r 5=\alpha_{7} \alpha_{8} \alpha_{10}, r 6=\alpha_{11} \alpha_{8} \alpha_{9}, r 7=\alpha_{12} \alpha_{4} \alpha_{5} \alpha_{6}, r 8=\alpha_{13} \alpha_{9} \alpha_{5} \alpha_{7}, r 9=\alpha_{4} \alpha_{5} \alpha_{6} \alpha_{13}, r 10=$ $\alpha_{9} \alpha_{5} \alpha_{7} \alpha_{12}, r 11=\alpha_{13} \alpha_{10}, r 12=\alpha_{11} \alpha_{12}, r 13=\alpha_{12} \alpha_{1}, r 14=\alpha_{2} \alpha_{13}, r 15=\alpha_{4} \alpha_{5} \alpha_{7} \alpha_{8} \mathrm{e}$ $r 16=\alpha_{8} \alpha_{9} \alpha_{5} \alpha_{6}$.Os ciclos elementares são: $C_{1}=\alpha_{1} \alpha_{2} \alpha_{3}, C_{2}=\alpha_{3} \alpha_{4} \alpha_{5} \alpha_{6}, C_{3}=\alpha_{5} \alpha_{7} \alpha_{8} \alpha_{9}$, $C_{4}=\alpha_{8} \alpha_{10} \alpha_{11}, C_{5}=\alpha_{4} \alpha_{5} \alpha_{7} \alpha_{12}$ e $C_{6}=\alpha_{5} \alpha_{6} \alpha_{13} \alpha_{9}$.

Entrando com esses dados no programa, não temos solução.

$\mathcal{F} r 28$

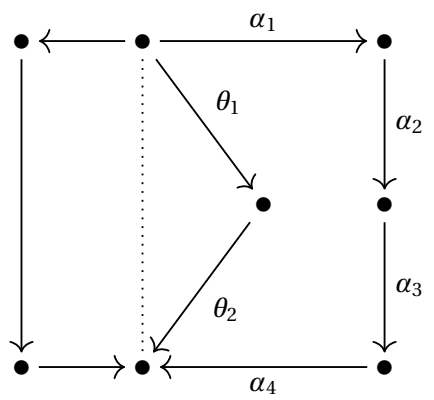

Como $\theta_{1} \theta_{2}$ é diferente do caminho $\alpha_{1} \alpha_{2} \alpha_{3} \alpha_{4}$, esse frame não é uma álgebra schurian. Então não podemos analisar.
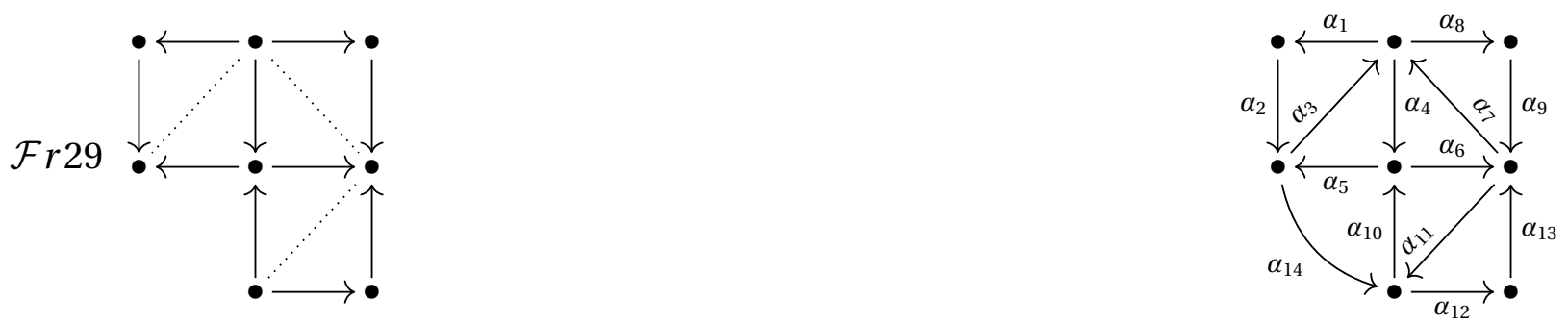

As relações do tipo 2 são: $r 1=\alpha_{2} \alpha_{3} \alpha_{4}, r 2=\alpha_{5} \alpha_{3} \alpha_{1}, r 3=\alpha_{3} \alpha_{4} \alpha_{6}, r 4=\alpha_{7} \alpha_{4} \alpha_{5}, r 5=$ $\alpha_{6} \alpha_{7} \alpha_{8}, r 6=\alpha_{9} \alpha_{7} \alpha_{4}, r 7=\alpha_{4} \alpha_{6} \alpha_{11}, r 8=\alpha_{10} \alpha_{6} \alpha_{7}, r 9=\alpha_{6} \alpha_{11} \alpha_{12}, r 10=\alpha_{13} \alpha_{11} \alpha_{10}$, $r 11=\alpha_{3} \alpha_{8}, r 12=\alpha_{7} \alpha_{1}, r 13=\alpha_{13} \alpha_{7}, r 14=\alpha_{9} \alpha_{11}, r 15=\alpha_{10} \alpha_{5} \alpha_{3}, r 16=\alpha_{4} \alpha_{5} \alpha_{14}$, $r 17=\alpha_{14} \alpha_{10} \alpha_{6}, r 18=\alpha_{11} \alpha_{10} \alpha_{5}, r 19=\alpha_{14} \alpha_{12}$ e $r 20=\alpha_{2} \alpha_{14}$. Os ciclos elementares são: $C_{1}=\alpha_{1} \alpha_{2} \alpha_{3}, C_{2}=\alpha_{3} \alpha_{4} \alpha_{5}, C_{3}=\alpha_{4} \alpha_{6} \alpha_{7}, C_{4}=\alpha_{7} \alpha_{8} \alpha_{9}, C_{5}=\alpha_{6} \alpha_{11} \alpha_{10}, C_{6}=\alpha_{11} \alpha_{12} \alpha_{13} \mathrm{e}$ $C_{7}=\alpha_{10} \alpha_{5} \alpha_{14}$.

Portanto, não temos solução para essa extensão trivial.

$\mathcal{F} r 30.1$
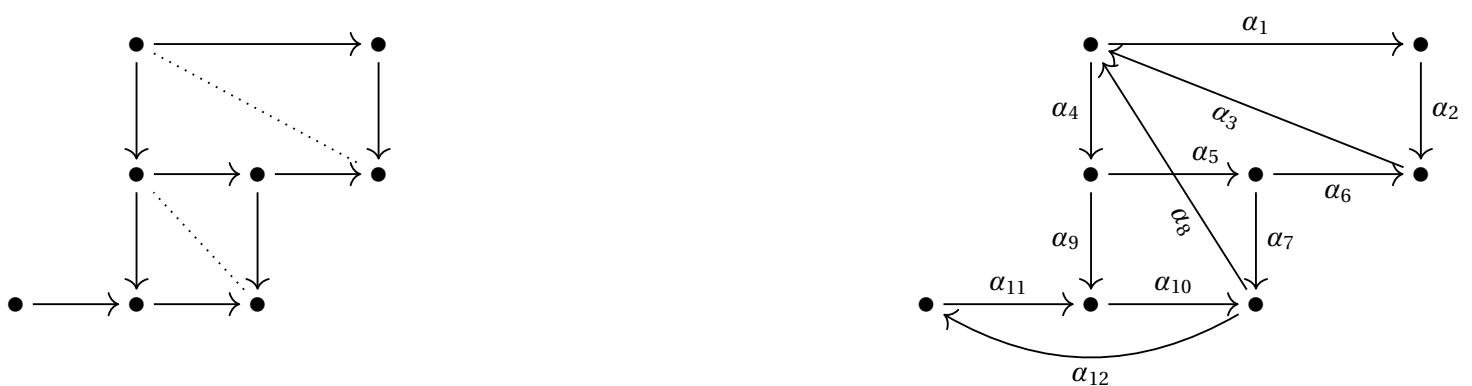

As relações do tipo 2 são: $r 1=\alpha_{2} \alpha_{3} \alpha_{4}, r 2=\alpha_{6} \alpha_{3} \alpha_{1}, r 3=\alpha_{3} \alpha_{4} \alpha_{5} \alpha_{7}, r 4=\alpha_{8} \alpha_{4} \alpha_{5} \alpha_{6}$, 
$r 5=\alpha_{3} \alpha_{4} \alpha_{9}, r 6=\alpha_{7} \alpha_{8} \alpha_{4} \alpha_{9}, r 7=\alpha_{10} \alpha_{8} \alpha_{4} \alpha_{5}, r 8=\alpha_{11} \alpha_{10} \alpha_{8}, r 9=\alpha_{9} \alpha_{10} \alpha_{12}, r 10=$ $\alpha_{8} \alpha_{1}$ e $r 11=\alpha_{7} \alpha_{12}$.Os ciclos elementares são: $C_{1}=\alpha_{1} \alpha_{2} \alpha_{3}, C_{2}=\alpha_{3} \alpha_{4} \alpha_{5} \alpha_{6}, C_{3}=$ $\alpha_{4} \alpha_{5} \alpha_{7} \alpha_{8}, C_{4}=\alpha_{4} \alpha_{9} \alpha_{10} \alpha_{8}$ e $C_{5}=\alpha_{11} \alpha_{10} \alpha_{12}$.

Logo, essa extensão trivial não tem corte que desejamos.

$\mathcal{F} r 30.2$
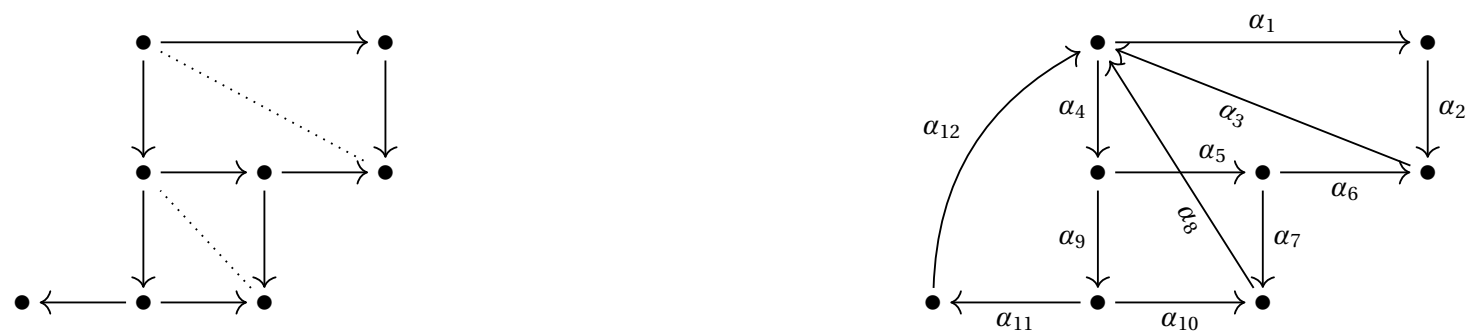

As relações do tipo 2 são: $r 1=\alpha_{2} \alpha_{3} \alpha_{4}, r 2=\alpha_{6} \alpha_{3} \alpha_{1}, r 3=\alpha_{3} \alpha_{4} \alpha_{5} \alpha_{7}, r 4=\alpha_{8} \alpha_{4} \alpha_{5} \alpha_{6}$, $r 5=\alpha_{3} \alpha_{4} \alpha_{9}, r 6=\alpha_{7} \alpha_{8} \alpha_{4} \alpha_{9}, r 7=\alpha_{10} \alpha_{8} \alpha_{4} \alpha_{5}, r 8=\alpha_{12} \alpha_{4} \alpha_{5}, r 9=\alpha_{3} \alpha_{4} \alpha_{9} \alpha_{11}, r 10=$ $\alpha_{8} \alpha_{1}, r 11=\alpha_{12} \alpha_{1}, r 12=\alpha_{12} \alpha_{4} \alpha_{9} \alpha_{10}$ e $r 13=\alpha_{8} \alpha_{4} \alpha_{9} \alpha_{11}$.Os ciclos elementares são: $C_{1}=\alpha_{1} \alpha_{2} \alpha_{3}, C_{2}=\alpha_{3} \alpha_{4} \alpha_{5} \alpha_{6}, C_{3}=\alpha_{4} \alpha_{5} \alpha_{7} \alpha_{8}, C_{4}=\alpha_{4} \alpha_{9} \alpha_{10} \alpha_{8}$ e $C_{5}=\alpha_{4} \alpha_{9} \alpha_{11} \alpha_{12}$.

Obtemos a Phia 72 através do corte $\left\{\alpha_{1}, \alpha_{4}\right\}$.

$\mathcal{F} r 31.1$
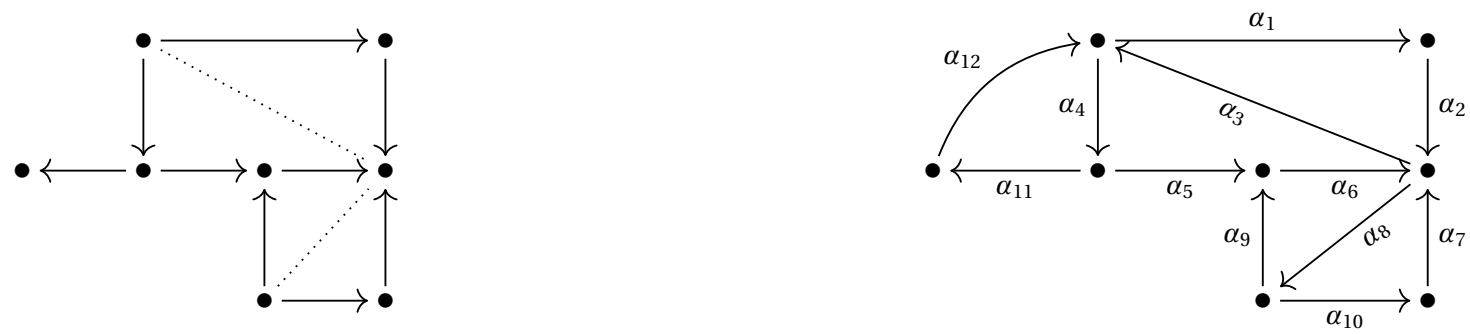

As relações do tipo 2 são: $r 1=\alpha_{2} \alpha_{3} \alpha_{4}, r 2=\alpha_{6} \alpha_{3} \alpha_{1}, r 3=\alpha_{5} \alpha_{6} \alpha_{8}, r 4=\alpha_{9} \alpha_{6} \alpha_{3}$, $r 5=\alpha_{6} \alpha_{8} \alpha_{10}, r 6=\alpha_{7} \alpha_{8} \alpha_{9}, r 7=\alpha_{3} \alpha_{4} \alpha_{11}, r 8=\alpha_{12} \alpha_{4} \alpha_{5}, r 9=\alpha_{2} \alpha_{8}, r 10=\alpha_{7} \alpha_{3} \mathrm{e}$ $r 11=\alpha_{12} \alpha_{1}$.Os ciclos elementares são: $C_{1}=\alpha_{1} \alpha_{2} \alpha_{3}, C_{2}=\alpha_{3} \alpha_{4} \alpha_{5} \alpha_{6}, C_{3}=\alpha_{9} \alpha_{6} \alpha_{8}$, $C_{4}=\alpha_{7} \alpha_{8} \alpha_{10}$ e $C_{5}=\alpha_{4} \alpha_{11} \alpha_{12}$.

Logo, temos apenas o corte que origina a álgebra disfarçada acima.

$\mathcal{F} r 31.2$
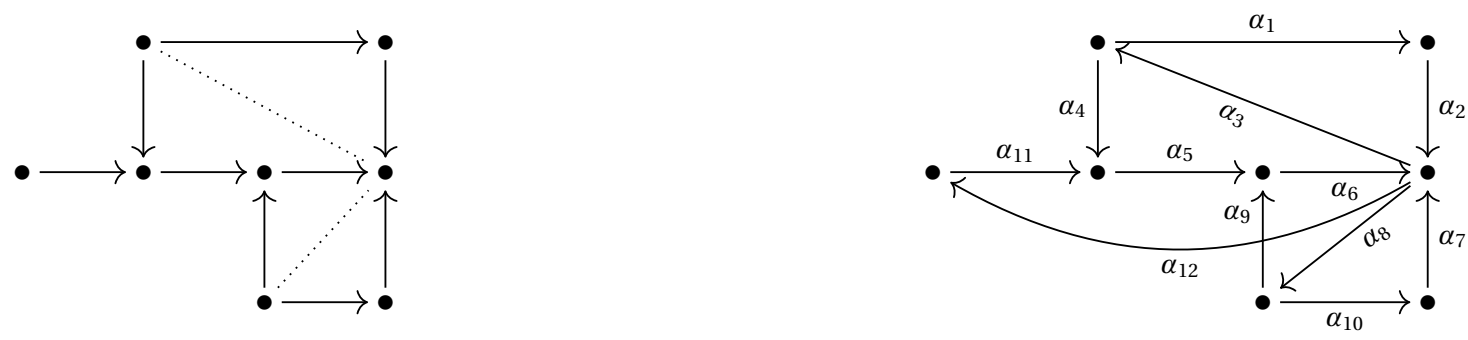

As relações do tipo 2 são: $r 1=\alpha_{2} \alpha_{3} \alpha_{4}, r 2=\alpha_{6} \alpha_{3} \alpha_{1}, r 3=\alpha_{5} \alpha_{6} \alpha_{8}, r 4=\alpha_{9} \alpha_{6} \alpha_{3}$, $r 5=\alpha_{6} \alpha_{8} \alpha_{10}, r 6=\alpha_{7} \alpha_{8} \alpha_{9}, r 7=\alpha_{4} \alpha_{5} \alpha_{6} \alpha_{12}, r 8=\alpha_{11} \alpha_{5} \alpha_{6} \alpha_{3}, r 9=\alpha_{9} \alpha_{6} \alpha_{12}, r 10=$ $\alpha_{7} \alpha_{3}, r 11=\alpha_{2} \alpha_{8}, r 12=\alpha_{7} \alpha_{12}$ e $r 13=\alpha_{2} \alpha_{12}$.Os ciclos elementares são: $C_{1}=\alpha_{1} \alpha_{2} \alpha_{3}$, $C_{2}=\alpha_{3} \alpha_{4} \alpha_{5} \alpha_{6}, C_{3}=\alpha_{9} \alpha_{6} \alpha_{8}, C_{4}=\alpha_{7} \alpha_{8} \alpha_{10}$ e $C_{5}=\alpha_{6} \alpha_{12} \alpha_{11} \alpha_{5}$.

Sendo assim, o programa exibe a Phia 73 através do corte $\left\{\alpha_{6}, \alpha_{7}, \alpha_{2}\right\}$. 

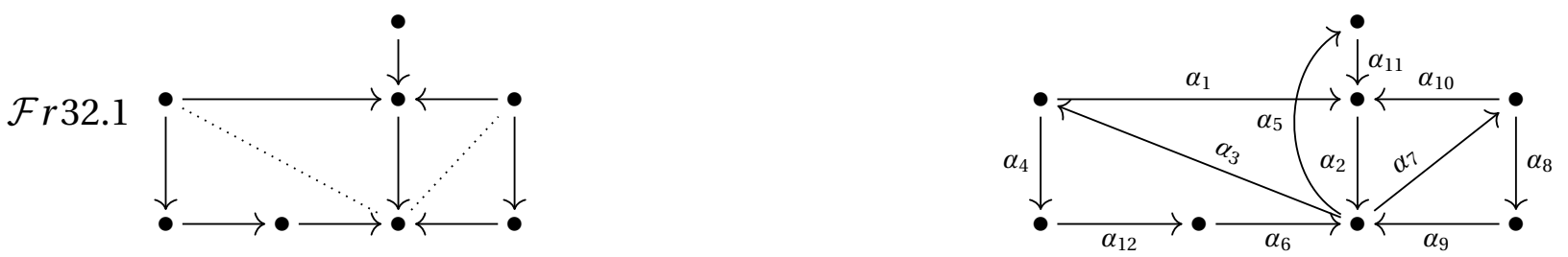

As relações do tipo 2 são: $r 1=\alpha_{2} \alpha_{3} \alpha_{4}, r 2=\alpha_{6} \alpha_{3} \alpha_{1}, r 3=\alpha_{1} \alpha_{2} \alpha_{7}, r 4=\alpha_{10} \alpha_{2} \alpha_{3}$, $r 5=\alpha_{2} \alpha_{7} \alpha_{8}, r 6=\alpha_{9} \alpha_{7} \alpha_{10}, r 7=\alpha_{10} \alpha_{2} \alpha_{5}, r 8=\alpha_{11} \alpha_{2} \alpha_{7}, r 9=\alpha_{11} \alpha_{2} \alpha_{3}, r 10=\alpha_{1} \alpha_{2} \alpha_{5}$, $r 11=\alpha_{6} \alpha_{7}, r 12=\alpha_{9} \alpha_{3}, r 13=\alpha_{6} \alpha_{5}$ e $r 14=\alpha_{9} \alpha_{5}$.Os ciclos elementares são: $C_{1}=$ $\alpha_{1} \alpha_{2} \alpha_{3}, C_{2}=\alpha_{3} \alpha_{4} \alpha_{12} \alpha_{6}, C_{3}=\alpha_{7} \alpha_{8} \alpha_{9}, C_{4}=\alpha_{7} \alpha_{10} \alpha_{2}$ e $C_{5}=\alpha_{11} \alpha_{2} \alpha_{5}$.

Entrando com essas informações no programa, temos a solução 74 através do corte $\left\{\alpha_{2}, \alpha_{6}, \alpha_{9}\right\}$.

$\mathcal{F} r 32.2$
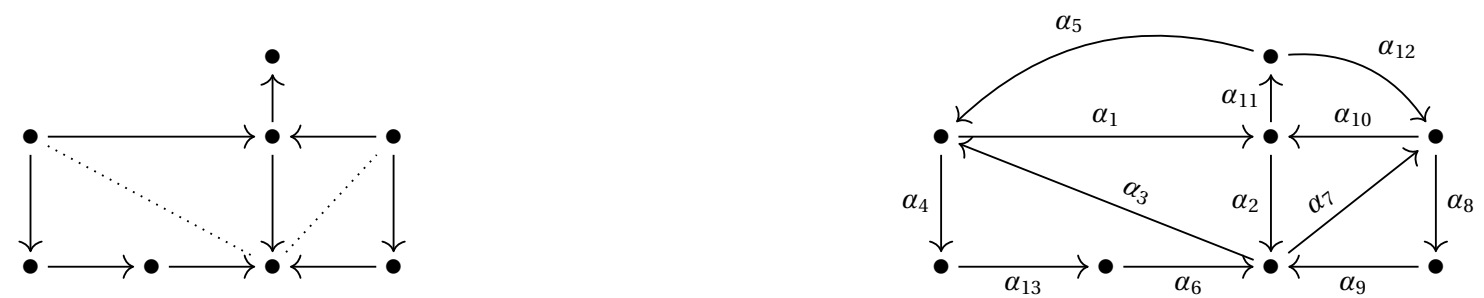

As relações do tipo 2 são: $r 1=\alpha_{2} \alpha_{3} \alpha_{4}, r 2=\alpha_{6} \alpha_{3} \alpha_{1}, r 3=\alpha_{1} \alpha_{2} \alpha_{7}, r 4=\alpha_{10} \alpha_{2} \alpha_{3}, r 5=$ $\alpha_{2} \alpha_{7} \alpha_{8}, r 6=\alpha_{9} \alpha_{7} \alpha_{10}, r 7=\alpha_{7} \alpha_{10} \alpha_{11}, r 8=\alpha_{12} \alpha_{10} \alpha_{2}, r 9=\alpha_{10} \alpha_{11} \alpha_{5}, r 10=\alpha_{1} \alpha_{11} \alpha_{12}$, $r 11=\alpha_{6} \alpha_{7}, r 12=\alpha_{9} \alpha_{3}, r 13=\alpha_{12} \alpha_{8}, r 14=\alpha_{5} \alpha_{4}, r 15=\alpha_{5} \alpha_{1} \alpha_{2}$ e $r 16=\alpha_{3} \alpha_{1} \alpha_{11}$.Os ciclos elementares são: $C_{1}=\alpha_{1} \alpha_{2} \alpha_{3}, C_{2}=\alpha_{3} \alpha_{4} \alpha_{13} \alpha_{6}, C_{3}=\alpha_{7} \alpha_{8} \alpha_{9}, C_{4}=\alpha_{7} \alpha_{10} \alpha_{2}$, $C_{5}=\alpha_{11} \alpha_{12} \alpha_{10}$ e $C_{6}=\alpha_{11} \alpha_{5} \alpha_{1}$.

O último caso dessa demonstração, não temos integrante Phia da família ANS.

Um trabalho a fazer depois da tese é aplicar o teorema 5.35 nos frames $\mathcal{F} r 33, \ldots, \mathcal{F} r 149$ da lista de Happel e Vossieck [HV83] com uma demonstração análoga do teorema anterior. Isso resolveria a descrição das Phias da família ANS de tipo $\widetilde{\mathbb{E}}_{8}$.

\subsection{O tipo selvagem}

São poucos trabalhos explorando as álgebras derivadamente equivalentes à álgebras hereditárias selvagens. Podemos citar os artigos "Tilting wild algebras" e "Wild tilted algebras" ambos do Kerner.

Nessa seção, exibiremos alguns exemplos de Phias de tipo selvagem. Mostraremos que a extensão por um ponto é um recurso útil para determinar Phias desse tipo.

Exemplo 5.44. Mostraremos alguns exemplos de Phias de tipo selvagem. Consideramos $A=K Q$ uma álgebra de caminhos de tipo selvagem abaixo: 


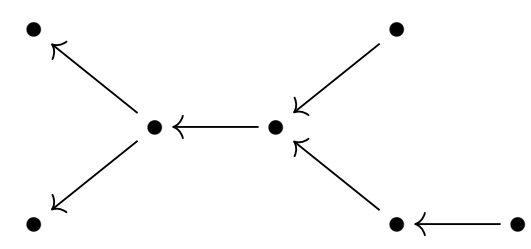

Em seguida, as aljavas de Phias de tipo selvagem derivadamente equivalente a A:
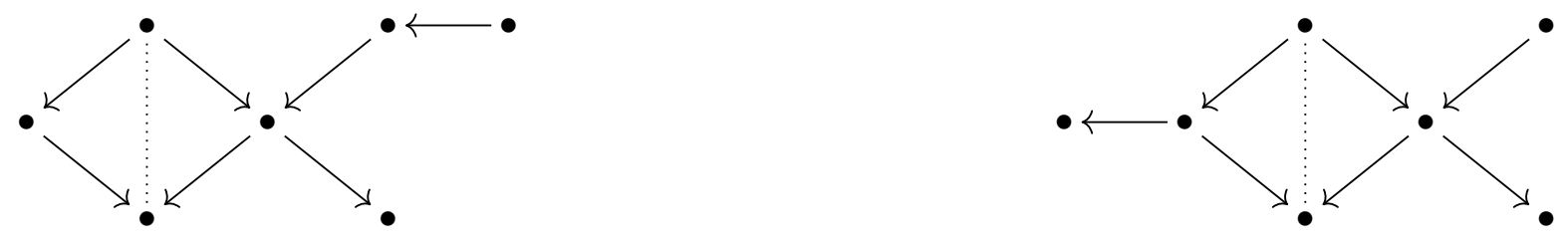

fonte [SSOTb].

Vamos lembrar a definição da extensão por um ponto.

\section{Definição 5.45}

Sejam A uma $K$-álgebra, e $M$ um módulo sobre $A$. A extensão por um ponto de $A$ por $M$ é a álgebra

$$
\left[\begin{array}{cc}
A & 0 \\
{ }_{K} M_{A} & K
\end{array}\right],
$$

o qual denotamos por $A[M]$. As operações de adição e a de multiplicação são as usuais das matrizes.

A extensão por um ponto é um caminho para determinar Phias de tipo selvagem. Uma maneira de aplicação vai ser visto no próximo capítulo, mais exatamente no teorema 6.14 , o qual poderemos ter uma gama de Phias de tipo selvagem.

A outra maneira é utilizando a próxima proposição.

Proposição 5.46 (Lenzing-la Peña [LD08]). Seja A uma K -álgebra que é derivadamente equivalente a uma álgebra de caminhos de uma aljava Dynkin Q. Seja B uma extensão ou coextensão por um ponto de um A-módulo indecomponível $M$. Então $B$ é derivadamente equivalente a uma álgebra de caminhos de uma aljava $Q^{\prime}$ obtida de $Q$ adicionando um novo vértice e uma nova flecha.

Como determina a nova flecha e o novo vértice em $Q$ ? A resposta dessa pergunta está na demonstração do resultado [LD08]. Colocaremos a ideia do método. Seja o funtor que faz a equivalência triangulada:

$$
F: D^{b}(A) \rightarrow D^{b}(K Q) .
$$

Seja $M$ o $A$-módulo indecomponível tal que $B=A[M]$. Como $M$ é indecomponível e $K Q$ é hereditária, temos que $F(M)=N$ é um $K Q$-módulo indecomponível, a menos de translação. Sendo assim, $N=\lambda^{-t} P(a)$ para algum $t \in \mathbb{N}$, em que $\lambda$ é a translação de Auslander-Reiten de $D^{b}(K Q)$ e $a \in Q_{0}$. 
A partir de $N=\lambda^{-t} P(a)$, descobrimos que a nova flecha vai ser da forma $* \longrightarrow a$ em que * é o novo vértice. A pergunta que podemos fazer é quando podemos determinar o $A$-módulo indecomponível $M$ tal que $A[M]$ é uma Phia de tipo selvagem? 


\section{Capítulo 6}

\section{Álgebra de incidência hereditária por partes de tipo feixes}

Em 1987, Geigle e Lenzing introduziram a categoria Coh $\mathbb{X}$ chamada de categoria dos feixes coerentes sobre uma reta projetiva com pesos [GL87]. Essa categoria tem uma relação importante com a categoria de módulos sobre uma álgebra canônica, Coh $\mathbb{X}$ é derivadamente equivalente a categoria de módulos sobre uma álgebra canônica $C(p, \lambda)$ [GL87]. Algumas referências para esse tipo de álgebra são: "Tame algebras and integral quadratic forms" [Rin84] e "Elements of the representation theory of associative algebras, volume três" [SS07b].

\section{Definição 6.1 (álgebra canônica)}

Seja $m \geq 2$ um número inteiro. Tome $p=\left(p_{1}, \cdots, p_{m}\right)$ uma $m$-úpla de números inteiros positivos em que é chamado de sequência de pesos, e $\lambda=\left(\lambda_{3}, \cdots, \lambda_{m}\right)$ uma $m$-2-úpla de coordenadas distintas da reta projetiva $\mathbb{P}_{1}(K)$ em que é chamado de sequência de parâmetros. Depois, considere a aljava $Q(p)$ :

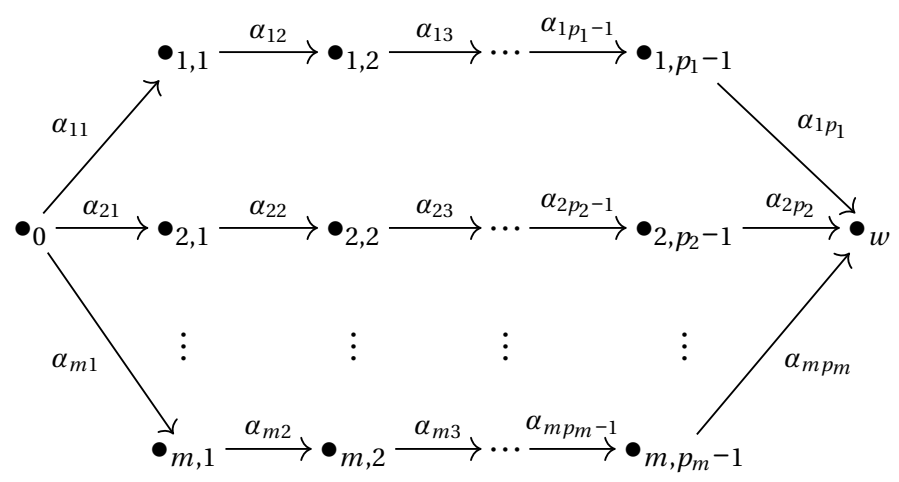

A álgebra $C(p, \lambda)$ é definida da seguinte forma:

a) para $m=2$, define-se $C(p, \lambda)=K Q(p)$.

b) para $m \geq 3$, assume-se que $p_{1} \geq 2, \cdots, p_{m} \geq 2$, define-se

$$
C(p, \lambda)=K Q(p) / I(p, \lambda)
$$


em que $I(p, \lambda)$ é o ideal da álgebra de caminhos $K Q(p)$ gerado pelos elementos

$$
\alpha_{j p_{j}} \cdots \alpha_{j 1}+\alpha_{1 p_{1}} \cdots \alpha_{11}+\lambda_{j} \alpha_{2 p_{2}} \cdots \alpha_{21} \operatorname{com} j \in\{3, \cdots, m\}
$$

Ao iniciarmos nossos estudos sobre as Phias $K \Delta \cong^{\prime}$ Coh $\mathbb{X}$, nos deparamos primeiramente com o artigo "Which canonical algebras are derived equivalent to incidence algebras of posets?" [Lad08b] de Ladkani, que em parte, se relaciona com a nossa tese.

Esse artigo foi publicado em 2008, e apresenta um estudo direto das Phias de tipo feixes ou Phias de tipo canônico, nomenclatura influenciada pela equivalência derivada entre as categorias $\operatorname{Coh}(\mathbb{X})$ e a categoria de módulos sobre a álgebra canônica $C(p, \lambda)$. A seguir veremos o principal teorema desse artigo:

Teorema 6.2 (Ladkani [Lad08b]). Seja $K \Delta$ uma álgebra de incidência. Se $C(p, \lambda) \cong \cong^{\prime} K \Delta$ então temos $p=\left(p_{1}, p_{2}, p_{3}\right)$ ou $p=\left(p_{1}, p_{2}\right)$, com $p_{i} \geq 2$. Para cada reta projetiva com pesos com $p=\left(p_{1}, p_{2}, p_{3}\right)$ ou $p=\left(p_{1}, p_{2}\right)$, em que $p_{i} \geq 2$, existe uma álgebra de incidência $K \Delta$ tal que $C(p, \lambda) \cong \cong^{\prime} K \Delta$.

Não temos uma descrição completa das aljavas com relações das Phias de tipo feixes, mas, por esse resultado, sabemos que essas Phias são de tipos $\operatorname{Coh}(\mathbb{X})$ com no máximo três pesos.

Denominaremos $\operatorname{Coh}(\mathbb{X})=\mathcal{C}$ a título de simplificar a notação. Revemos agora alguns fatos da teoria de feixes coerentes de retas projetivas com peso.

As categorias do tipo $\mathcal{C}$ acima se dividem em 3 classes disjuntas que são: aljava, tubular e selvagem. Essas classes são caracterizadas conforme o número de Euler $\mathcal{X}(\mathbb{X})$ da reta projetiva com pesos, seja $\mathbb{X}=\left(p_{1}, \ldots, p_{n} ; \lambda_{3}, \ldots, \lambda_{n}\right)$ definimos o número de Euler como:

$$
\mathcal{X}(\mathbb{X})=2-\sum_{i=1}^{n}\left(1-\frac{1}{p_{i}}\right)
$$

Se $\mathcal{C}$ tem o número de Euler maior que zero então $\mathcal{C}$ é do tipo aljava, ou seja, é derivadamente equivalente a categoria dos módulos sobre a álgebra hereditária do tipo Dynkin estendido. Se a categoria dos feixes tem o número de Euler igual a zero então é da família tubular. Por último, a família selvagem tem o número de Euler negativo.

Exemplo 6.3. Alguns exemplos de Phias de tipo feixes aljava, tubular e selvagem respectivamente. $A$ Phia de tipo $\widetilde{\mathbb{E}}_{6}$ é uma álgebra disfarçada do capítulo anterior. A Phia de tipo feixe tubular é associada ao peso $(3,3,3)$ e a Phia de tipo feixe selvagem é correspondente ao peso $(3,3,4)$, ambas retiradas do artigo do Ladkani [Lad08b]. 

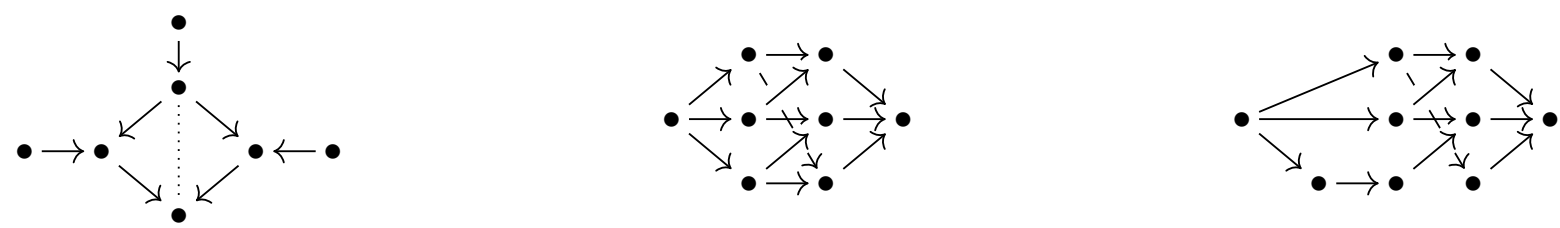

Lembrando que as álgebras acima têm as relações definidas de tal forma que dois caminhos paralelos são iguais

$\mathrm{O}$ artigo "A class of weighted projective curves arising in representation theory of finite dimensional algebras" [GL87] de Geigle e Lenzing, e o trabalho "Introduction to coherent sheaves on weighted projective line" [CK10] de Chen e Krause são importantes textos para uma introdução a teoria da categoria dos feixes coerentes sobre uma reta projetiva com pesos. Entretanto, utilizaremos a caracterização da categoria $\mathcal{C}$ exposto no "Hereditary noetherian categories with a tilting complex" [Len97] de Lenzing.

Teorema 6.4 (Lenzing [Len97]). Seja $K$ um corpo algebricamente fechado. Seja $\mathcal{H}$ uma $K$-categoria esqueletamente pequena, conexa, abeliana e com espaços de extensões e morfismos de dimensão finita. As seguintes afirmações são equivalentes:

(i) $\mathcal{H}$ é equivalente a $\operatorname{Coh}(\mathbb{X})$;

(ii) cada objeto de $\mathcal{H}$ é noetheriano. $\mathcal{H}$ é hereditário, não tem projetivos não nulos, e admite um objeto inclinante;

(iii) cada objeto de $\mathcal{H}$ é noetheriano, mais ainda

(a) existe uma equivalência $\tau: \mathcal{H} \rightarrow \mathcal{H}$ (translação de Auslanter-Reiten) tal que $\operatorname{Ext}^{1}(X, Y) \cong$ $D \operatorname{Hom}(Y, \tau X)$ assegurada funtorialmente para $X, Y$ de $\mathcal{H}$;

(b) o grupo de Grothedieck $K_{0}(\mathcal{H})$ é livre, finitamente gerado, e tem a forma de Euler abaixo:

$$
\begin{aligned}
\langle-,-\rangle: K_{0}(\mathcal{H}) \times K_{0}(\mathcal{H}) & \longrightarrow \mathbb{Z} \\
([X],[Y]) & \longrightarrow\langle[X],[Y]\rangle=\operatorname{dim}_{K} \operatorname{Hom}(X, Y)-\operatorname{dim}_{K} \operatorname{Ext}^{1}(X, Y) .
\end{aligned}
$$

(c) $\mathcal{H}$ tem um objeto sem autoextensões e que não tem comprimento finito.

Com intuito de adicionar mais informações sobre a categoria $\mathcal{C}$, incluiremos mais três lemas de Lenzing. Seja $\mathcal{C}_{0}$ a subcategoria plena consistindo dos objetos de $\mathcal{C}$ com comprimento finito.

Lema 6.5 (Lenzing [Len97]). Assumimos que $\mathcal{C}$ satisfaz a condição (iii)(a). Então $\mathcal{C}_{0}$ é uniserial, isto é, cada objeto de $X \in \mathcal{C}_{0}$ tem uma única série de composição finita. De acordo, para algum conjunto $\mathbb{X}$ de índices, a categoria $\mathcal{C}_{0}$ decompõe em um coproduto $\mathcal{C}_{0}=\prod_{x \in \mathbb{X}} \mathcal{U}_{x}$ de categorias conexas uniseriais $\mathcal{U}_{x}$, cujos indecomponíveis formam os tubos estáveis.

Cada objeto $X$ de $\mathcal{C}$ tem um subobjeto maximal $t X$ de comprimento finito (que chamaremos parte de torção), e $X / t X$ não possui submódulo simples, isto é, $X / t X$ é livre de torção. A subcategoria plena de objetos livres de torção denominaremos de $\mathcal{C}_{+}$, os quais são chamados também de fibrados. 
Lema 6.6 (Lenzing [Len97]). Cada objeto indecomponível $X$ de $\mathcal{C}$ é um fibrado ou tem comprimento finito. Mais ainda, o único morfismo de um objeto com comprimento finito a um fibrado é o nulo.

Assim, podemos dizer que a categoria $\mathcal{C}$ tem uma bisecção $\mathcal{C}_{+} \vee \mathcal{C}_{0}$

Lema 6.7 (Lenzing [Len97]). Existe uma aplicação linear sobrejetora rk: $K_{0}(\mathcal{C}) \rightarrow \mathbb{Z}$, chamada função rank, que tem as características:

(i) $\tau$ estável, isto é, $\operatorname{rk}(\tau X)=\operatorname{rk}(X)$;

(ii) a função é nula nos objetos com comprimento finito;

(iii) os fibrados têm rank estritamente positivo, exceto o objeto zero;

(iv) existe um fibrado indecomponível com rank um.

Agora, podemos entender o número zero da notação $\mathcal{C}_{0}$ e também o símbolo + da notação $\mathcal{C}_{+}$.

\subsection{Extensão por um ponto}

Com a finalidade de mostrar algumas famílias de Phias de tipo feixes, estudaremos extensão por um ponto. Os trabalhos de Barot, De la Peña e Lenzing ([BL98], [BL03] e [LD08]) fornecem condições para as álgebras $A$ hereditárias por partes e para os seus módulos $M$ que resultam na hereditariedade por partes das álgebras $A[M]$.

$\mathrm{Na}$ definição da extensão por um ponto, mais detalhes na seção 5.7, reparamos na liberdade da escolha do módulo $M$. Em breve, nas hipóteses dos teoremas, veremos as condições sobre o módulo $M$. Entretanto, focaremos em um módulo especial sobre a álgebra de incidência $K \Delta=$ $K Q / I$, o módulo sincero canônico $M 3.3$ que é o módulo associado à seguinte representação:

a) para cada vértice $a$ de $Q$ associamos $K$;

b) para cada flecha $\alpha: a \rightarrow b$ de $Q$ associamos a identidade $1: K \rightarrow K$.

Exemplo 6.8. Vejamos um exemplo de extensão por um ponto $K \Delta[M]$, em que $M$ é o módulo sincero canônico:
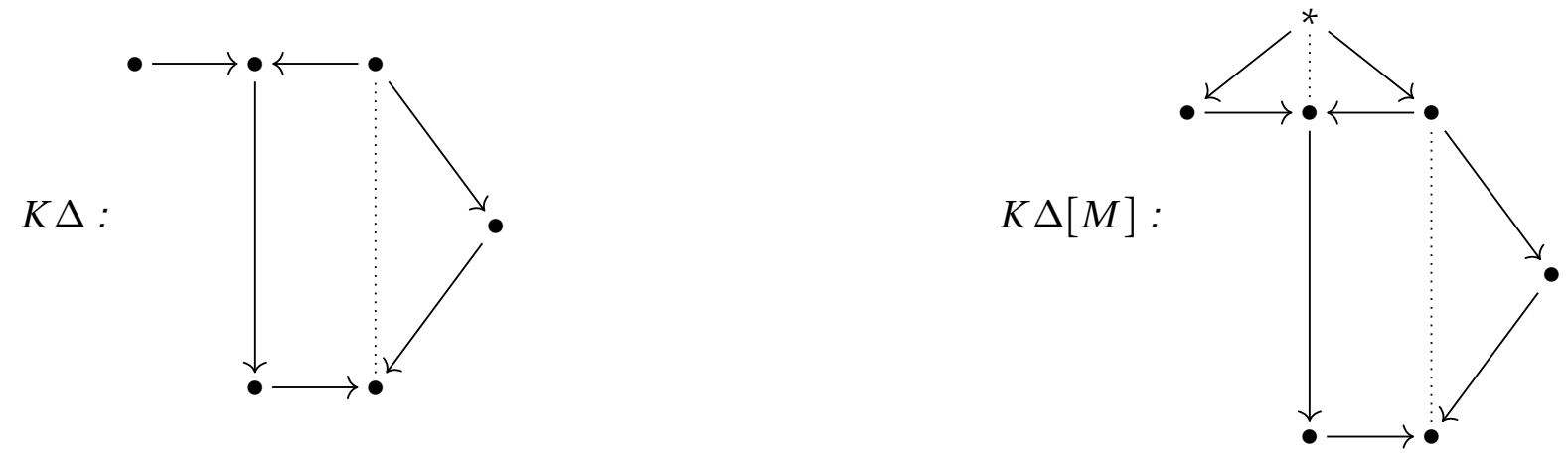
Observamos que $K \Delta[M]$ é uma álgebra de incidência que tem um poset com um único elemento maximal representado pelo vertice *. A extensão por um ponto pelo módulo sincero canônico $M$ nos remete a uma técnica muito usada no estudo de posets, adicionar um elemento maximal único.

Seja o funtor que faz a equivalência triangulada:

$$
F: D^{b}(K \Delta) \rightarrow D^{b}(\mathcal{C})
$$

Uma condição a ser verificada no módulo sincero canônico $M$ é se o objeto $F M$ é um fibrado.

O trabalho "Quasi-tilted algebras of canonical type" [LS96] é um caminho a ser seguido para mostrar que o módulo $M$ é associado a um fibrado. Lenzing e Skowroński conseguem caracterizar cada módulo indecomponível sobre uma álgebra $A$ quase-inclinada de tipo feixe e de tipo de representação infinito.

Sabemos que $D^{b}(A)$ é equivalente como categoria triangulada a $D^{b}(\mathcal{C})$, então existe um objeto inclinante $T$ em $D^{b}(\mathcal{C})$ tal que $\left(\operatorname{End}_{D^{b}(\mathcal{C})}(T)\right)^{o p}=A$. Lenzing e Skowroński decompõem $T$ em uma soma direta $T_{0}^{\prime}[-1] \oplus T_{+} \oplus T_{0}^{\prime \prime}$. Através disso, identificam $\bmod (A)$ com uma subcategoria plena de $D^{b}(\mathcal{C})$ cujos os objetos $X$ satisfazem $\operatorname{Hom}_{D^{b}(\mathcal{C})}(T, X[n])=0$ para todo inteiro $n \neq 0$.

Pela hereditariedade de $\mathcal{C}, \bmod (A)$ está contido em $\operatorname{add}\left(\mathcal{C}^{\prime}[-1] \vee \mathcal{C}_{+} \vee \mathcal{C}_{0} \vee \mathcal{C}_{+}[1] \vee \mathcal{C}_{0}^{\prime \prime}[1]\right)$ Agora, podemos enunciar o resultado abaixo.

Proposição 6.9. Seja $A=K Q / I$ uma álgebra sincera, quase-inclinada de tipo feixe e de tipo de representação infinito. Considere $M$ o módulo sincero indecomponível. Então $M$ está em $\bmod _{+}(A)$.

Demonstração Começaremos a analisar o módulo $M$ através da equivalência triangulada das categorias derivadas. Como já sabemos, existe um funtor que faz a equivalência triangulada:

$$
F: D^{b}(A) \rightarrow D^{b}(\mathcal{C})
$$

Por $M$ ser sincero, temos que $\operatorname{Hom}_{A}\left(P_{x}, M\right) \neq 0$ para $P_{x}$ projetivo indecomponível e para todo vértice $x$ de $Q$. Assim, dado um vértice $x$ de $Q$, usando o funtor $F$ obtemos

$$
\operatorname{Hom}_{A}\left(P_{x}, M\right) \cong \operatorname{Hom}_{D^{b}(A)}\left(P_{x}, M\right) \cong \operatorname{Hom}_{D^{b}(\mathcal{C})}\left(F P_{x}, F M\right)
$$

Sejam $F P_{x}=P_{x}^{\prime}[p]$ e $F M=Y[m]$, os quais $P_{x}^{\prime}, Y$ pertencem a $\mathcal{C}[0]$. Portanto,

$$
\operatorname{Hom}_{D^{b}(\mathcal{C})}\left(F P_{x}, F M\right) \cong \operatorname{Hom}_{D^{b}(\mathcal{C})}\left(P_{x}^{\prime}[p], Y[m]\right) .
$$

Dessa forma, $\operatorname{Hom}_{D^{b}(\mathcal{C})}\left(P_{x}^{\prime}[p], Y[m]\right)$ é não nulo.

Precisamos relacionar $P_{x}^{\prime}[p]$ com os indecomponíveis de $T$, em que $\left(\operatorname{End}_{D^{b}(\mathcal{C})}(T)\right)^{o p}=A$. 
Graças ao funtor $G$ quasi-inverso de $F$

$$
G: D^{b}(\mathcal{C}) \rightarrow D^{b}(A) \text { tal que } G T=A,
$$

conseguimos que $F P_{x_{1}} \oplus \ldots \oplus F P_{x_{n}}$ é isomorfo a $T=T_{0}^{\prime}[-1] \oplus T_{+} \oplus T_{0}^{\prime \prime}$, para cada $x_{i} \in Q$ com $i=1, \ldots n$. Ou seja, $P_{x_{i}}^{\prime}[p]$ é isomorfo a um objeto indecomponível de $T$ consequentemente $p=0$ ou $p=-1$. Assim estamos preparados para utilizar a seguinte proposição:

Proposição 6.10 (Lenzing-Skowroński [LS96]). Seja A uma álgebra quase inclinada de tipo feixe e com tipo de representação infinito. Então podemos dividir a subcategoria $\bmod A$ nas seguintes subcategorias plenas.

a) $\bmod _{0}^{l}(A)$ que tem como objetos $Y[-1]$ em que $Y$ está em $\mathcal{C}_{0}^{\prime}$ satisfazendo $\operatorname{Hom}_{\mathcal{C}}\left(T_{+}, Y\right)=0$ $e \operatorname{Ext}_{\mathcal{C}}^{1}\left(T_{0}^{\prime}, Y\right)=0$;

b) $\bmod _{+}(A)$ que tem como objetos $Y$ que está em $T_{0}^{\prime \prime \perp} \cap \mathcal{C}_{+}$satisfazendo $\operatorname{Ext}_{\mathcal{C}}^{1}\left(T_{+}, Y\right)=0$;

c) $\bmod _{0}^{c}(A)$ que tem como objetos $Y$ pertencendo $a \mathcal{C}_{0} \operatorname{com}_{\mathcal{C}}\left(T_{0}^{\prime}, Y\right)=0$ e $\operatorname{Ext}_{\mathcal{C}}^{1}\left(T_{0}^{\prime \prime}, Y\right)=$ 0 ;

d) $\bmod _{-}(A)$ que tem como objetos $Z[1]$ em que $Z$ está em $T_{0}^{\prime \perp} \cap \mathcal{C}_{+}$tendo $\operatorname{Hom}_{\mathcal{C}}\left(T_{+}, Z\right)=0$;

e) $\bmod _{0}^{r}(A)$ que tem como objetos $Z[1]$ em que $Z$ está em $\mathcal{C}_{0}$ satisfazendo $\operatorname{Hom}_{\mathcal{C}}\left(T_{+}, Y\right)=0$ $e \operatorname{Hom}_{\mathcal{C}}\left(T_{0}^{\prime \prime}, Y\right)=0$.

Por existir $x \in Q$ tal que $F P_{x}=P_{x}^{\prime}[0]$ e $\operatorname{Hom}_{D^{b}(\mathcal{C})}\left(P_{x}^{\prime}, F M\right) \neq 0$ então $F M$ não é isomorfo a $Y[-1]$ eliminando o caso a). De forma análoga, por $\operatorname{Hom}_{D^{b}(\mathcal{C})}\left(P_{x}^{\prime}[-1], F M\right) \neq 0$ então $F M$ não é isomorfo a $Y[1]$ eliminando os casos d) e e). Sobrando os casos b) e c) tal que $F M \cong$ $Y$. Supomos que é válido o caso c) para o módulo $M$. Então $\operatorname{Hom}_{\mathcal{C}}\left(T_{0}^{\prime}, F M\right)=0$, ou seja, $\operatorname{Hom}_{D^{b}(\mathcal{C})}\left(P_{x}^{\prime}, F M\right)=0$ para algum $x \in Q$ contradizendo o fato de que $M$ é sincero. Portanto o caso c) não é válido para $M$. Logo $M$ está em $\bmod _{+}(A)$.

Lembramos que as Phias são álgebras sinceras. O passo final é demonstrar que o módulo $M$ sincero canônico é excepcional. Relembramos a sua definição:

\section{Definição 6.11 (excepcional)}

Um objeto $E$ em uma $K$-categoria triangulada $\mathcal{T}$ é chamada de excepcional se $\operatorname{End}(E)=K$ e, mais ainda, $E$ não tem auto-extensões, ou seja, $\operatorname{Hom}_{\mathcal{T}}(E, E[n])=0$ para cada número inteiro não nulo $n$.

Correspondente, seja A uma $K$-álgebra de dimensão finita, o A-módulo E é chamado de excepcional se $\operatorname{End}(E)=K, \operatorname{ext}_{A}^{1}(E, E)=0$.

Novamente a proposição final para esse fim não é específica para as álgebras de incidência. $E$ apenas garantimos para as categorias $\mathcal{C}$ de tipo aljava. 
Proposição 6.12. Seja $A=K Q / I$ uma álgebra sincera, quase-inclinada canônica de tipo aljava e de representação infinito. Considere $M$ o módulo sincero indecomponível. Então $M$ é excepcional.

Demonstração Demonstrando que $F M \cong Y$ é um objeto excepcional, ou seja, $\operatorname{Ext}_{\mathcal{C}}^{1}(Y, Y)=0$, obtemos que $M$ é excepcional por meio das igualdades abaixo:

$$
\operatorname{Ext}_{\mathcal{C}}^{1}(Y, Y)=\operatorname{Hom}_{D^{b}(\mathcal{C})}(Y, Y[1]) \cong \operatorname{Hom}_{D^{b}(A)}(M, M[1])=\operatorname{Ext}_{A}^{1}(M, M)
$$

Pela proposição anterior, $Y$ está em $\mathcal{C}_{+}$. Portanto, $Y$ é um fibrado. A proposição que usaremos a seguir pode ter sido provada anteriormente ao trabalho de Lenzing e Reiten [LR06], a citação é útil pela sua demonstração cuidadosa.

Proposição 6.13 (Lenzing-Reiten [LR06]). SejaC de tipo aljava, então cada fibrado indecomponível é excepcional.

Logo, o módulo sincero indecomponível $M$ é excepcional.

Portanto, o módulo sincero canônico sobre as Phias quase-inclinada canônica de tipo aljava e de representação infinito são fibrados excepcionais, via equivalência derivada. Assim, enunciaremos o teorema de Lenzing e De la Peña para o nosso interesse.

Teorema 6.14 (Lenzing-De la Peña [LD08]). Seja A uma álgebra derivadamente equivalente a uma álgebra canônica $\Lambda$ com peso $\left(p_{1}, \ldots, p_{n}\right)$. Fixamos uma equivalência-triangulada $D^{b}(A) \cong D^{b}(\mathcal{C})$ para uma reta projetiva com pesos $\mathbb{X}$ corresponde a $\Lambda$. Sejam $M$ um A-módulo excepcional e $A[M]$ a extensão por um ponto de $A$ por $M$.

A menos de translação de $D^{b}(\mathcal{C})$, se o módulo $M$ corresponde a um fibrado excepcional então obtemos:

Se $\mathcal{X}(\mathbb{X})>0$, então implica que $A[M]$ é derivadamente equivalente a uma álgebra hereditária selvagem.

Sendo assim, aproveitando as descrições das aljavas com relações das Phias $K \Delta$ de tipo Dynkin estendido, podemos produzir vários exemplos de Phias $K \Delta[M]$ de tipo selvagem em que $M$ é o módulo sincero canônico.

Exemplo 6.15. Entre as Phias de tipo Dynkin estendido, escolhemos as álgebras disfarçadas. Como vimos no capítulo anterior, as Phias disfarçadas são de tipo de representação infinito satisfazendo todas as hipóteses dos resultados dessa seção. O exemplo é de uma Phia de tipo $\widetilde{\mathbb{E}}_{6}$.
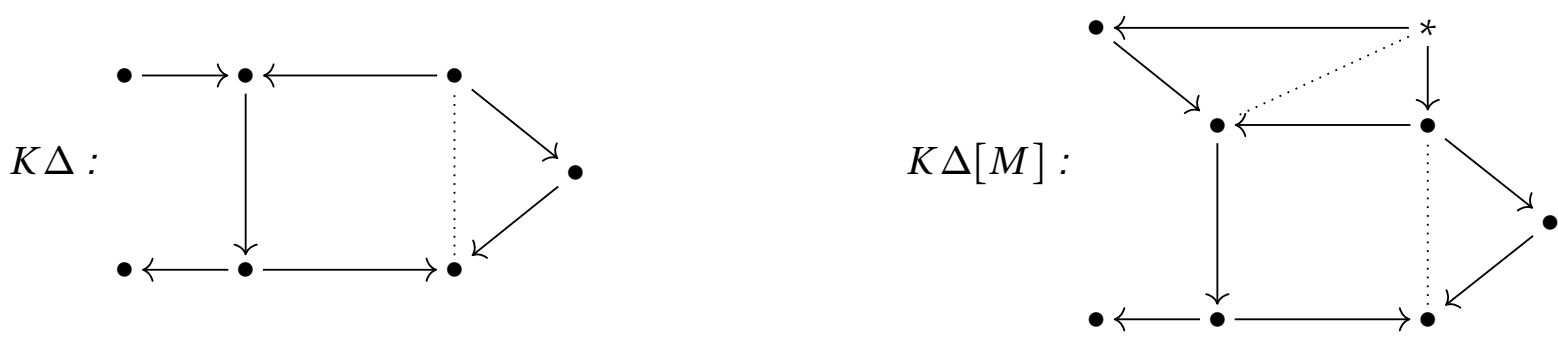
Quando buscamos o objetivo de saber a hereditariedade por partes de $K \Delta[M]$ em que $K \Delta$ é uma Phia de tipo feixes tubular ou selvagem, podemos nos deparar com mais exigências do módulo M. Os trabalhos ([BL98], [BL03] e [LD08]) tratam dessas situações. A hipótese mais presente sobre $M$ é que o módulo seja derivadamente simples.

\section{Definição 6.16 (derivadamente simples)}

Seja X um módulo indecomponível sobre uma álgebra A de dimensão global finita. Podemos chamar $X$ de derivadamente simples, caso satisfaça as condições:

1. o "termo do meio" E do triângulo de Auslander-Reiten

$$
\tau X \rightarrow E \rightarrow X \rightarrow \tau X[1]
$$

é indecomponível;

2. $X$ é $\tau$-periódico em $D^{b}(A)$.

No percurso da investigação sobre hereditariedade por partes de $K \Delta[M]$ surgiu uma questão, será o módulo sincero canônico $M$ excepcional? Seja $K \Delta$ uma Phia quase-inclinada canônica de tipo aljava. Trabalhamos com $K \Delta$ de tipo de representação infinito, mostrando que $M$ é excepcional. A próxima proposição responde positivamente para o caso de tipo de representação finito para as Phias de tipo feixes aljava e tubular.

Proposição 6.17. Seja $K \Delta$ uma Phia de tipo feixes aljava, ou tubular, e de tipo de representação finito. Considere $M$ o módulo sincero indecomponível. Então $M$ é excepcional.

Demonstração $O$ primeiro passo é mostrar que $K \Delta$ é simplesmente conexa. Usando o seguinte teorema:

Teorema 6.18 (Assem-Skowroński [AS88]). Se A é uma álgebra derivadamente equivalente a uma álgebra canônica tubular ou a uma álgebra hereditária euclidiana, então A é simplesmente conexa.

Obtemos que $K \Delta$ é simplesmente conexa.

O segundo passo é provar que $K \Delta$ é fortemente simplesmente conexa.

Teorema 6.19 (Bretscher-Gabriel [BG83]). Uma álgebra de tipo de representação finito é fortemente simplesmente conexa se, e somente se, a álgebra é simplesmente conexa.

O passo final é usar a proposição abaixo.

Proposição 6.20 (Gastaminza-De la Peña-Platzeck-Redondo-Trepode [GDP+99]). Seja $K \Delta=K Q / I$ uma álgebra de incidência fortemente simplesmente conexa. Considere $M$ único A-módulo indecomponível satisfazendo $\operatorname{dim}_{K} M(x)=1$ para cada $x \in Q$ e $M(x)$ da sua representação de módulo. Então 

1. $\operatorname{dp} M \leq 1$;
2. $\operatorname{Ext}_{K \Delta}^{1}(M, M)=0$.

Portanto o módulo $M$ sincero canônico é excepcional.

Lembramos que uma Phia fortemente simplesmente conexa é quase-inclinada, pela proposição 3.11 .

Para $K \Delta$ Phia quase-inclinada de tipo feixes aljava e de tipo de representação finito, provamos que o $K \Delta$-módulo sincero canônico $M$ é excepcional e $K \Delta[M]$ é uma Phia. Assim sendo, pelas duas proposições anteriores, asseguramos que o $M$ é excepcional somente para:

1. $K \Delta$ quase-inclinada de tipo canônico euclidiano;

2. $K \Delta$ quase-inclinada de tipo canônico tubular e de representação finito. 


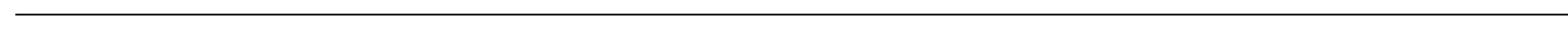




\section{Apêndice A}

\section{Programa}

\section{A.1 O código fonte da página principal do site}

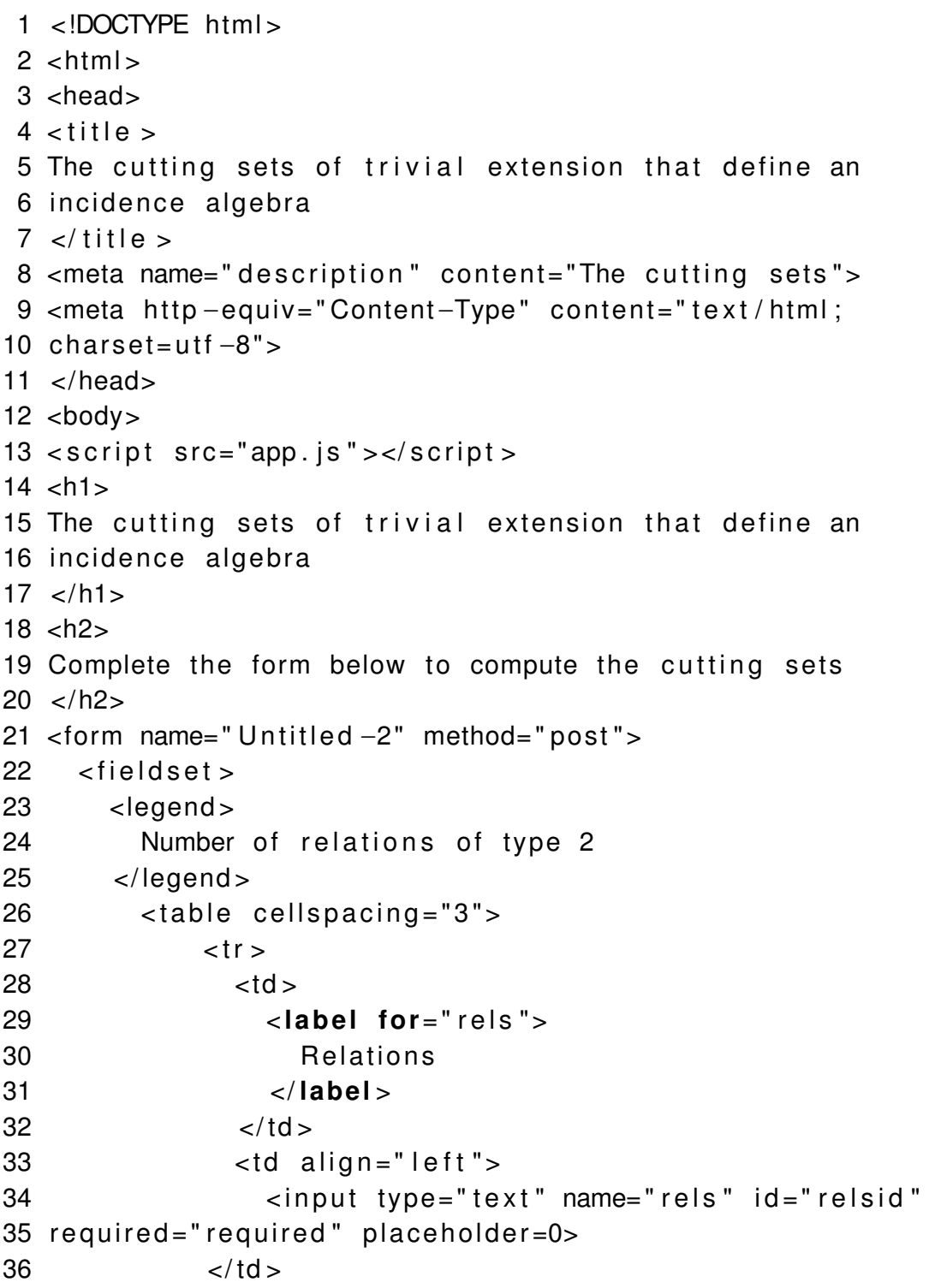




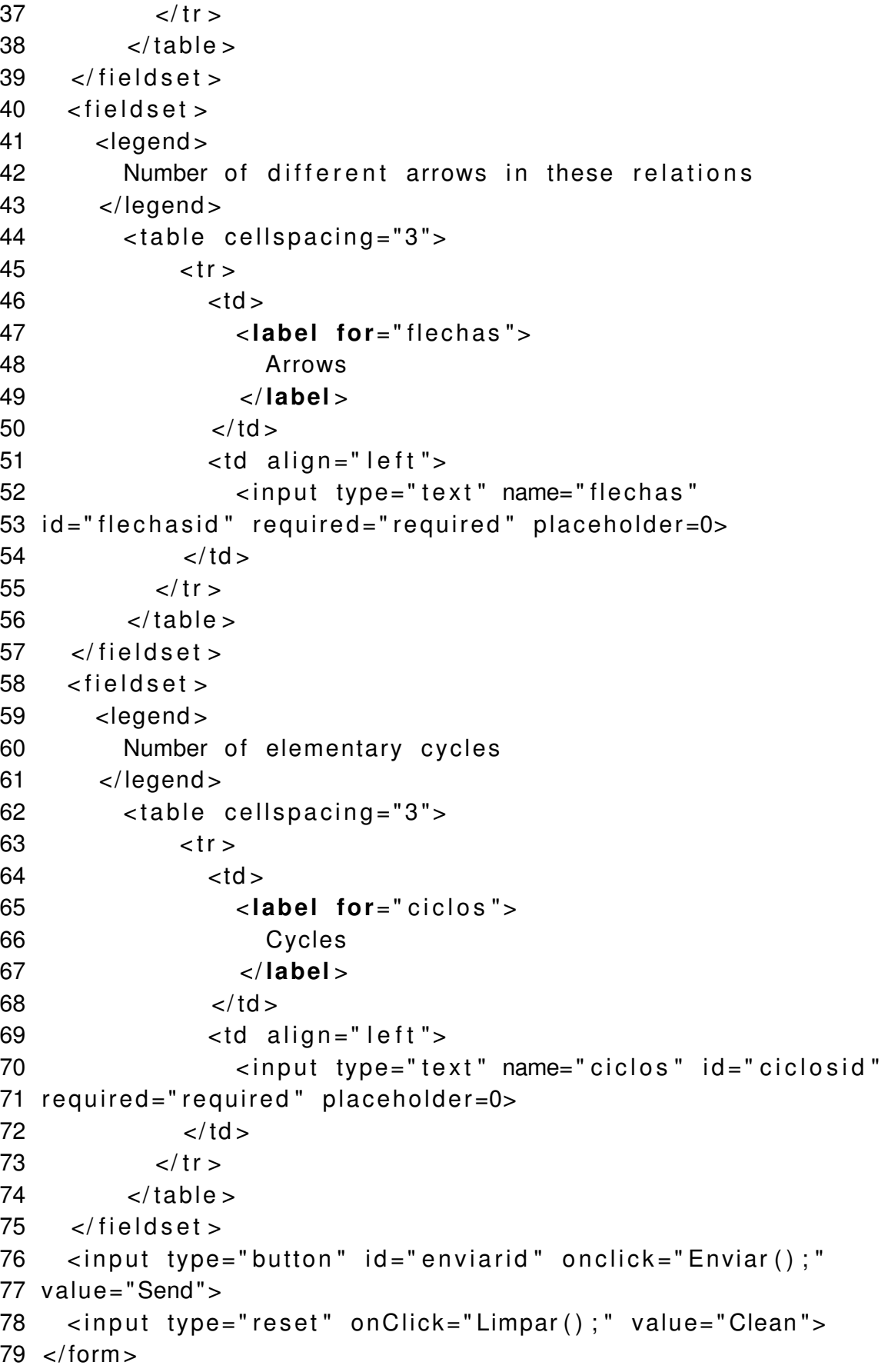

\section{A.2 O código fonte do app.js}

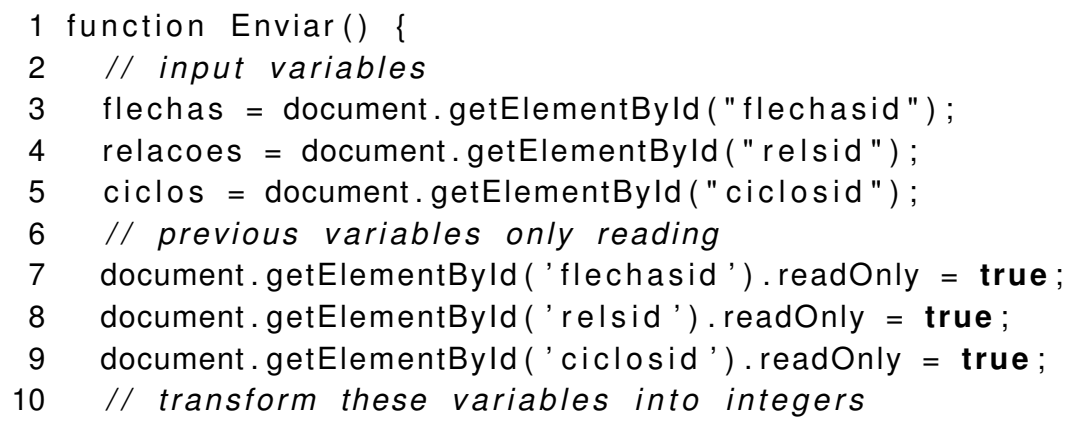




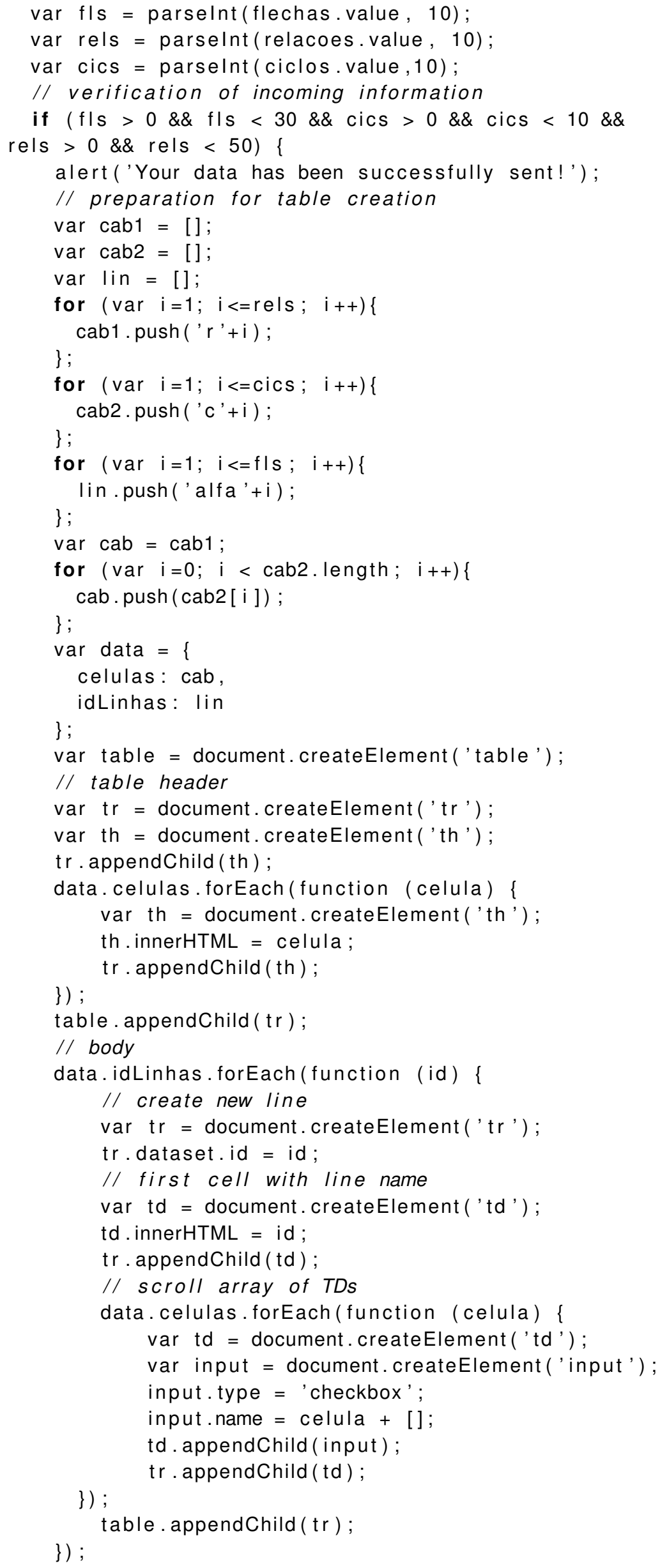




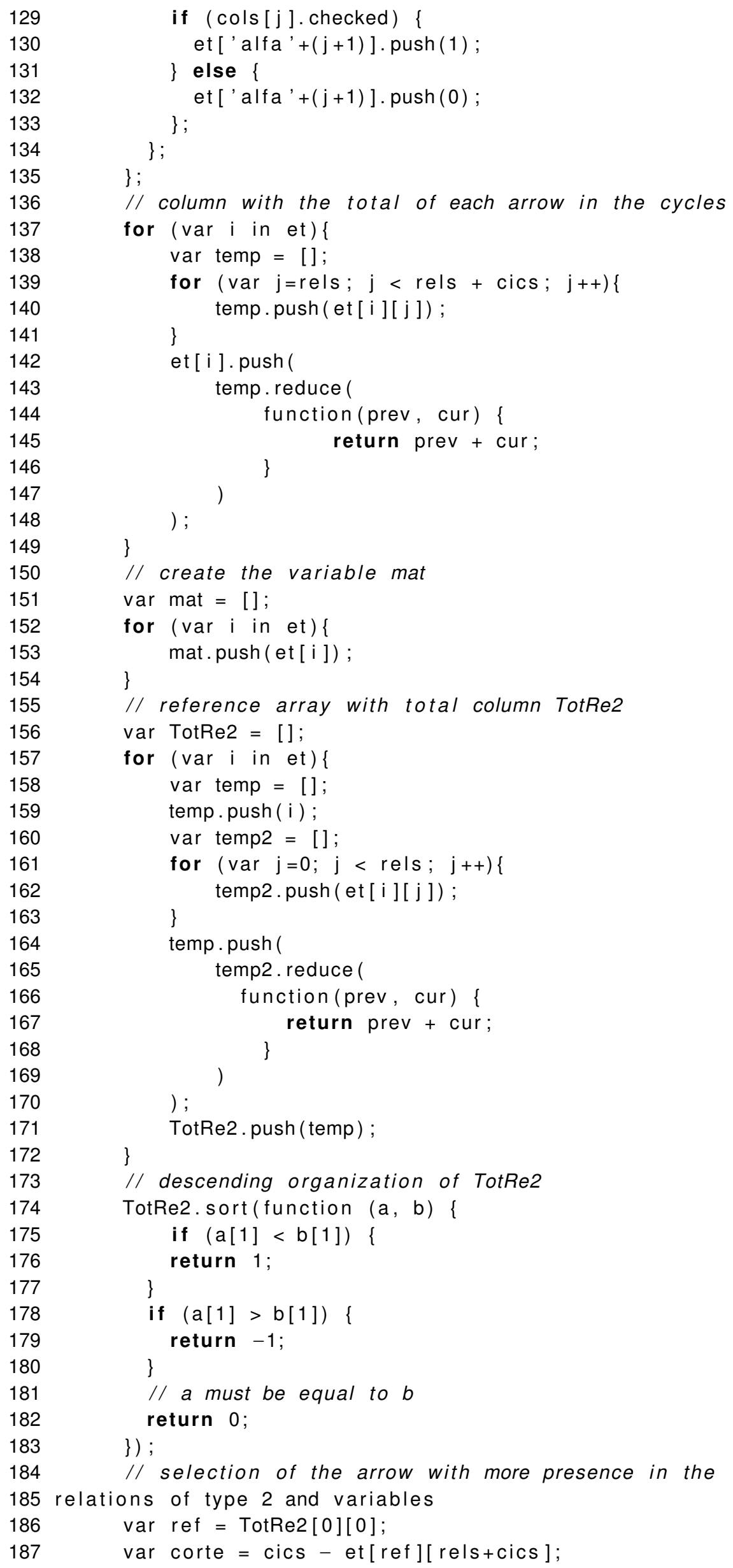




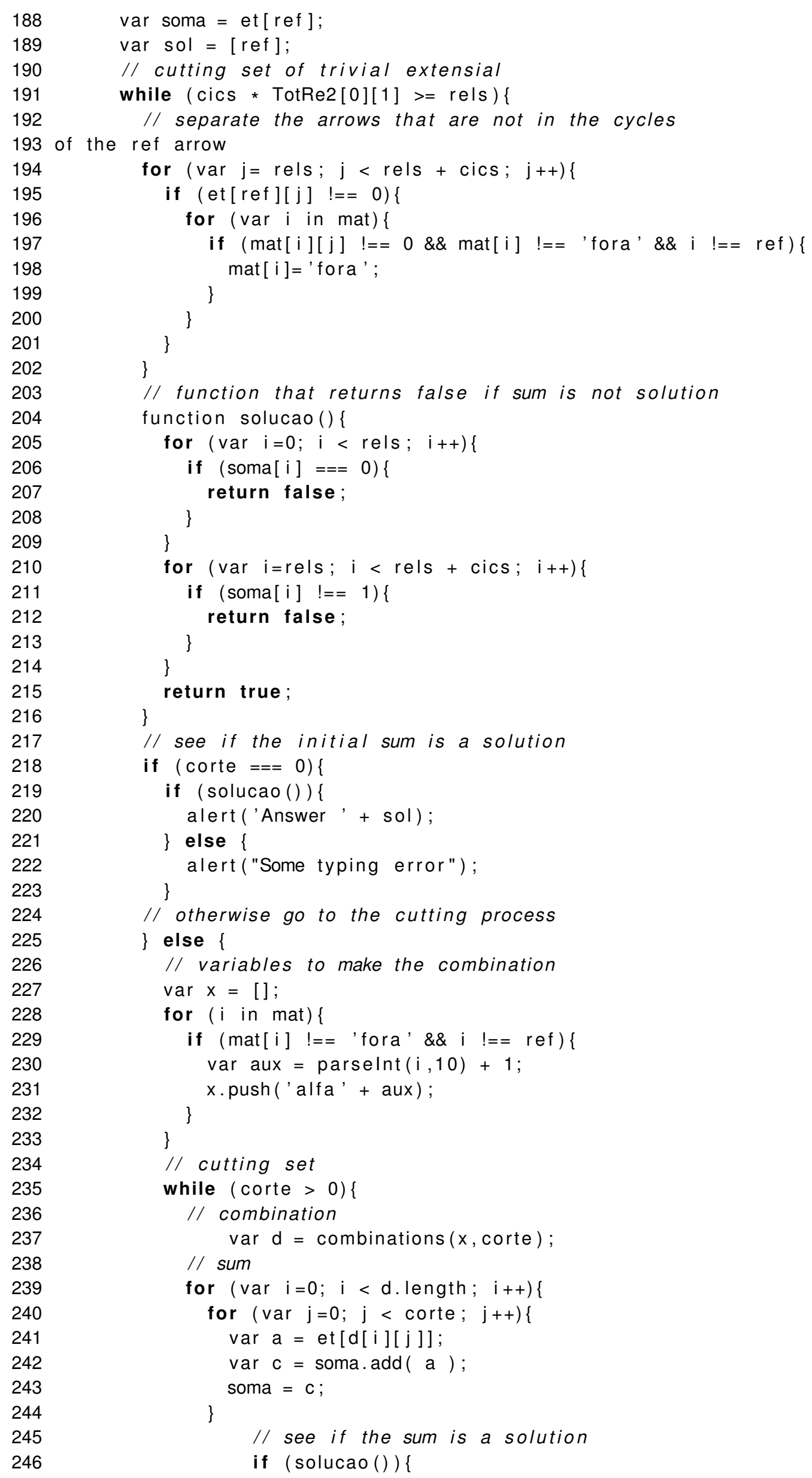




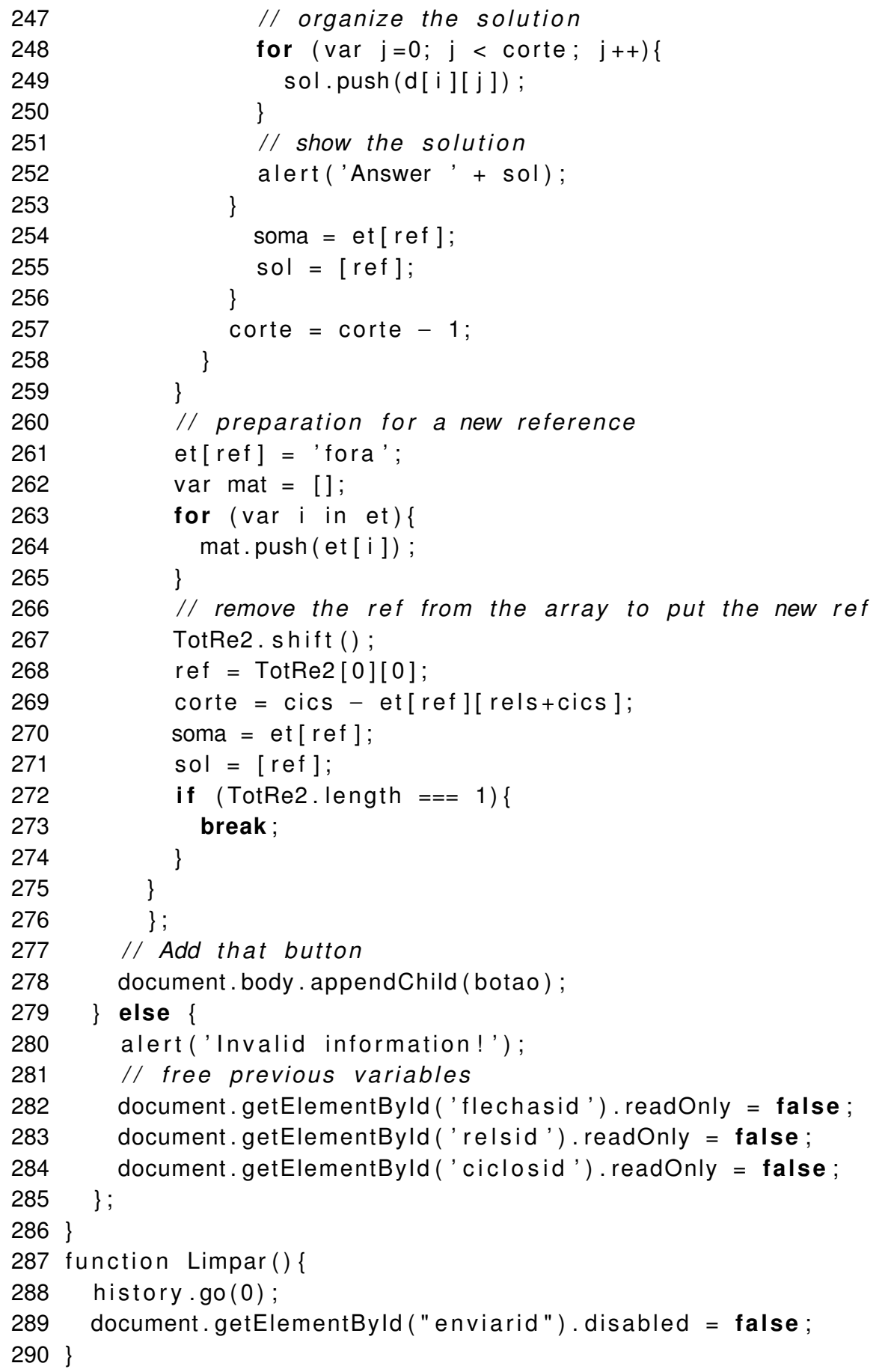




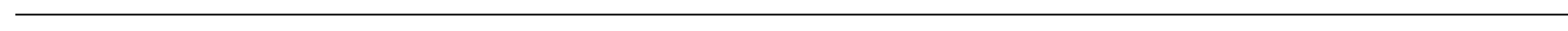




\section{Referências Bibliográficas}

[ACMT05] I. Assem, D. Castonguay, E.N. Marcos e S. Trepode. On strongly simply connected algebras and multiplicative bases. Journal of Algebra, 283(1):161-189, Janeiro 2005. 28

[AHR84] I. Assem, D. Happel e O. Roldán. Representation-finite trivial extension algebras. Journal of Pure and Applied Algebra, (33):235-242, 1984. 37

[AMD01] I. Assem, E.N. Marcos e J.A. De la Peña. The simple connectedness of a tame tilted algebra. Journal of Algebra, 237(2):647-656, 2001. 158

[ANS89] I. Assem, J. Nehring e A. Skowroński. Domestic trivial extensions of simply connected algebras. Tsukuba Journal of Mathematics, 13(1):31-72, 1989. 158

[AS88] I. Assem e A. Skowroński. On some classes of simply connected algebras. Proceedings of the London Mathematical Society, s3-56(3):417-450, 1988. 248

[ASS06] I. Assem, D. Simson e A. Skowroński. Elements of the representation theory of associative algebras, volume 65 of London Math. Soc. Student Text. Cambridge Univ. Press, 2006. 33

[BFT11] N. Bordino, E. Fernández e S. Trepode. A criterion for global dimension two for strongly simply connected schurian algebras. arXiv:1110.6160v1 [math.RA], Outubro 2011. 26, 27

[BG83] O. Bretscher e P. Gabriel. The standard form of a representation-finite algebra. Bulletin de la Société Mathématique de France, 111:21-40, 1983. 248

[BKL10] M. Barot, D. Kussin e H. Lenzing. The cluster category of a canonical algebra. Transactions of the American Mathematical Society, 362(8):4313-4330, Agosto 2010. 19

[BL98] M. Barot e H. Lenzing. Derived canonical algebras as one-point extensions. Contemporary Mathematics, 229:7-15, 1998. 244, 248

[BL03] M. Barot e H. Lenzing. One-point extensions and derived equivalence. Journal of Algebra, 264(1):1-5, Junho 2003. 244, 248

[BMR ${ }^{+}$06] A.B Buan, R. Marsh, M. Reineke, I. Reiten e G. Todorov. Tilting theory and cluster combinatorics. Advances in Mathematics, 204:572-618, 2006. 19

[Bon84] K. Bongartz. Critical simply connected algebras. Manuscripta Mathematica, (46):117136, 1984. 152

[Bus02] J.C. Bustamante. On the fundamental group of a schurian algebra. Communications in Algebra, 30(11):5307-5329, 2002. 13 
[CK10] X. Chen e H. Krause. Introduction to coherent sheaves on weighted projective line. techreport, arXiv:0911.4473v3 [math.RT], Setembro 2010. 243

[DS01] J.A. De la Peña e M. Saorín. On the first hochschild cohomology group of an algebra. Manuscripta Mathematica, (104):431-442, 2001. 15

[Fer99] E. Fernández. Extensiones triviales y Álgebras inclinadas iteradas. Tese de Doutorado, Universidad Nacional del Sur, 1999. 1, 35, 36, 38, 39, 41, 42, 43, 48, 50, 56, 68, 79, 95

[FP06] E. Fernández e M.I. Platzeck. Isomorphic trivial extensions of finite dimensional algebras. Journal of Pure and Applied Algebra, (204):9-20, 2006. 40

[Fre64] Peter Freyd. Abelian categories. Harper \& Row and John Weatherhill, 1964. 8

[GB82] P. Gabriel e K. Bongartz. Covering spaces in representation theory. Inventiones mathematicae, 65:331-378, 1982. 16

[GDP+99] S. Gastaminza, J.A. De la Peña, M.I. Platzeck, M.J. Redondo e S. Trepode. Finite dimensional algebras with vanishing hochschild cohomology. Journal of Algebra, 212(1):1-16, Fevereiro 1999. 248

[GL87] W. Geigle e H. Lenzing. A class of weighted projective curves arising in representation theory of finite dimensional algebras, volume 1273 of Lectures Notes in Mathematics. Springer, 1987. 241, 243

[H8̈9] T. Hübner. Classification of indecomposable vector bundles on weighted curves. Tese de Doutorado, Diplomarbeit, Paderborn, 1989. 151

[Hap85] D. Happel. Tilting sets on cylinders. Proceedings of the London Mathematical Society, 3(51):21-55, 1985. 35

[Hap87] D. Happel. On the derived category of a finite-dimensional algebra. Commentarii Mathematici Helvetici, (62):339-389, 1987. 25

[Hap01] D. Happel. A characterization of hereditary categories with tilting object. Inventiones mathematicae, 144(2):381-398, Maio 2001. 1, 9

[HKL12] L.A. Hügel, S. Koenig e Q. Liu. Jordan-hölder theorems for derived module categories of piecewise hereditary algebras. Journal of Algebra, 352(1):361-381, Fevereiro 2012. 30

[HR82] D. Happel e C.M. Ringel. Tilted algebras. Transactions of the American Mathematical Society, 274(2):399-443, Dezembro 1982. 1, 26

[HRS88] D. Happel, J. Rickard e A. Schofield. Piecewise hereditary algebras. Bulletin London Mathematical Society, 20(1):23-28, 1988. 10

[HRS96] D. Happel, I. Reiten e S. Smalø. Piecewise hereditary algebras. Archiv der Mathematik, 66(3):182-186, 1996. 23

[HU05] D. Happel e L. Unger. On the set of tilting objects in hereditary categories. Fields Institute Communications, 45:141-159, 2005. 19

[HV83] D. Happel e D. Vossieck. Minimal algebras of inifinite representation type with preprojective component. Manuscripta Mathematica, (42):221-243, 1983. 152, 154, 155, 158, 238 
[HZ08] D. Happel e D. Zacharia. A homological characterization of piecewise hereditary algebras. Mathematische Zeitschrift, 260(1):177-185, Setembro 2008. 1, 29, 30

[Kel91] B. Keller. Algébres héréditaires par morceaux de type $d_{n}$. Comptes Rendus de l'Académie des Sciences Paris, 312(Serie I):483-486, 1991. 1, 35

[Lad08a] S. Ladkani. Bounds on the global dimension of certain piecewise hereditary categories. Journal of Pure and Applied Algebra, 212(9):2140-2145, 2008. 23, 24, 25, 32

[Lad08b] S. Ladkani. Which canonical algebras are derived equivalent to incidence algebras of posets? Communications in Algebra, 36(12):4599-4606, 2008. 242

[LD08] H. Lenzing e J.A. De la Peña. Spectral analysis of finite dimensional algebras and singularities, páginas 541-588. Series of Congress Reports. 2008. 239, 244, 247, 248

[Le 11] P. Le Meur. Topological invariants of piecewise hereditary algebras. Transactions of the American Mathematical Society, 363(4):2143-2170, Abril 2011. 16, 19, 20, 21, 22

[Len97] $\mathrm{H}$. Lenzing. Hereditary noetherian categories with a tilting complex. Proceedings of the American Mathematical Society, 125(7):1893-1901, Julho 1997. 243, 244

[LR06] H. Lenzing e I. Reiten. Hereditary noetherian categories of positive euler characteristic. Mathematische Zeitschrift, (254):133-171, 2006. 247

[LS96] H. Lenzing e A. Skowroński. Quasi-tilted algebras of canonical type. Colloquium Mathematicum, 71(2):161-181, 1996. 245, 246

[MLA14] E.N. Marcos, P. Le Meur e E.R. Alvares. The strong global dimension of piecewise hereditary algebras. arXiv:1305.5213v2 [math.RT], Dezembro 2014. 30

[Rin84] C.M. Ringel. Tame algebras and integral quadratic forms, volume 1099 of Lectures Notes in Mathematics. Springer, 1984. 25, 241

[Rog95] B. Roggon. Selfinjective and iterated tilted algebras of type $E_{6}, E_{7}, E_{8}$. University of Bielefeld, 1995. 36

[SS07a] D. Simson e A. Skowroński. Elements of the representation theory of associative algebras, volume 71 of London Math. Soc. Student Text. Cambridge Univ. Press, 2007. 154, 168

[SS07b] D. Simson e A. Skowroński. Elements of the representation theory of associative algebras, volume 72 of London Math. Soc. Student Text. Cambridge Univ. Press, 2007. 239, 241

[SZ03] J. Schröer e A. Zimmermann. Stable endomorphism algebras of modules over special biserial algebras. Mathematische Zeitschrift, 244(3):515-530, 2003. 35

[ZH09] D. Zacharia e D. Happel. Homological properties of piecewise hereditary algebras. Journal of Algebra, 323:1139-1154, Novembro 2009. 31 


\section{Índice Remissivo}

$G(\mathcal{A}), 23$

$\operatorname{Coh}(\mathbb{X}), 151,242$

álgebra

simplesmente conexa, 13, 16

autoinjetiva, 37

canônica, 151, 242

crítica, 26

de caminhos, 6

de incidência, 7

dirigida, 25

disfarçada, 151

extensão por um ponto, 239, 244

extensão trivial, 158

fortemente simplesmente conexa, 26, 248

gentil, 33

hereditária $A_{n}, 165$

hereditária selvagem, 247

inclinada iterada, 10

Kronecker generalizada, 152

minimal de t.r. infinito, 152

quase inclinada, 245

resultante do corte, 40

schurian, 38, 158

simplesmente conexa, 158, 248

sincera, 24

triangular, 41

aljava, 5

aljava estável de Auslander-Reiten, 37

caminho

maximal, 38

orientado, 6

categoria

conexa, 18

localmente limitada, 16

abeliana, 8

de comprimento finito, 23

derivada, 8

hereditária, 9

hereditária por partes, 9 ciclo elementar, 38, 159

classe de Cartan, 37

cohomologia de Hochschild, 13

componente posprojetiva, 152

comprimento do complexo limitado, 29

corte, 40, 159

elemento minimal, 30

extensão trivial, 37

função rank, 244

funtor

push-down, 18

recobrimento, 16

grafo dos objetos inclinantes, 18

grupo fundamental, 12

módulo

derivadamente simple, 248

dirigido, 25

excepcional, 246

inclinante, 9

sincero, 24, 244

número de Euler, 242

objeto

excepcional, 246

inclinante, 9

passeio, 11

Phias

de tipo canônico, 151, 242

de tipo Dynkin estendido, 247

de tipo feixes, 242

de tipo selvagem, 247

poset, 1

elemento maximal, 245

propriedades galosianas, 20

recobrimento de Galois, 17

relação 
de homotopia, 11

minimal, 11

relações de tipo 1,2 e 3, 40

suplemento de um caminho, 38 